\title{
DUAL INTERFEROMETER SYSTEM FOR MEASURING INDEX OF REFRACTION
}

by

Eric Peter Goodwin

\author{
A Dissertation Submitted to the Faculty of the \\ COMMITTEE ON OPTICAL SCIENCES (GRADUATE) \\ In Partial Fulfillment of the Requirements \\ For the Degree of \\ DOCTOR OF PHILOSOPHY \\ In the Graduate College \\ THE UNIVERSITY OF ARIZONA
}




\section{THE UNIVERSITY OF ARIZONA GRADUATE COLLEGE}

As members of the Dissertation Committee, we certify that we have read the dissertation prepared by Eric Peter Goodwin entitled Dual Interferometer System for Measuring Index of Refraction and recommend that it be accepted as fulfilling the dissertation requirement for the Degree of Doctor of Philosophy

Date: March 23, 2007

John Greivenkamp

Date: March 23, 2007

Jose Sasian

Date: March 23, 2007

James Wyant

Final approval and acceptance of this dissertation is contingent upon the candidate's submission of the final copies of the dissertation to the Graduate College.

I hereby certify that I have read this dissertation prepared under my direction and recommend that it be accepted as fulfilling the dissertation requirement.

Date: March 23, 2007

Dissertation Director: John Greivenkamp 


\section{STATEMENT BY AUTHOR}

This dissertation has been submitted in partial fulfillment of requirements for an advanced degree at the University of Arizona and is deposited in the University Library to be made available to borrowers under rules of the Library.

Brief quotations from this dissertation are allowable without special permission, provided that accurate acknowledgment of source is made. Requests for permission for extended quotation from or reproduction of this manuscript in whole or in part may be granted by the head of the major department or the Dean of the Graduate College when in his or her judgment the proposed use of the material is in the interests of scholarship. In all other instances, however, permission must be obtained from the author.

SIGNED: $\quad$ Eric Peter Goodwin 


\section{ACKNOWLEDGMENTS}

The author would like to thank the corporate project sponsor for providing funding and equipment for the development of this instrumentation. 


\section{TABLE OF CONTENTS}

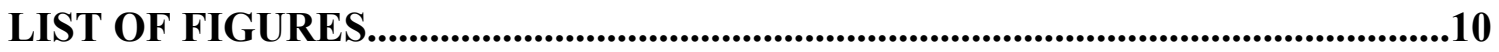

LIST OF TABLES..............................................................................................24

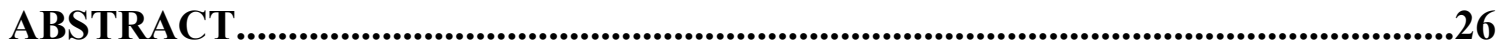

1 INTRODUCTION TO REFRACTIVE INDEX MEASUREMENTS..................28

1.1 Refractive Index Definitions.........................................................................30

1.2 Measurement Considerations......................................................................32

1.2.1 Accuracy Requirements................................................................................32

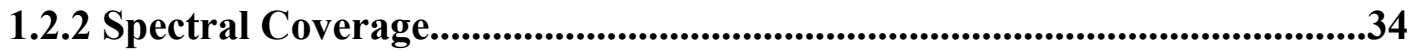

1.2.3 Material Properties..................................................................................35

1.3 The Glass Standard.......................................................................................40

1.4 Existing Refractive Index Measurement Techniques and Hydrogels..............43

1.4.1 Prism Spectrometer..................................................................................................43

1.4.2 Abbe Refractometer...................................................................................44

1.4.3 Index Matching Fluids.....................................................................46

1.4.4 Ellipsometry................................................................................................................47

1.4.5 Confocal Microscopy.............................................................................49

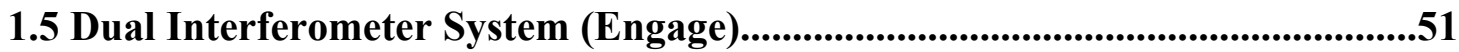

2 THICKNESS MEASUREMENT - THEORY ........................................................60

2.1 Basic Low Coherence Interferometry...............................................................60

2.2 The Low Coherence Interferometer for Engage..................................................66

2.3 Detailed Theory of LCI..............................................................................................71 


\section{TABLE OF CONTENTS - Continued}

2.3.1 Origins of the Low Coherence Signal...........................................................72

2.3.2 Sources of LCI Signal Degradation.............................................................79

2.3.3 Derivation of the Effects of Dispersion on the LCI Signal............................84

2.4 Dispersion Effects versus Index, Material and Source Characteristics............90

3 DETERMINING SURFACE LOCATION FROM THE LCI DATA CUBE.......101

3.1 The Fourier Transform Method.................................................................105

3.2 The Curve Fitting Method.........................................................................117

3.3 Least Squares Fit to Modulation.............................................................129

3.3.1 Incorporating Spatial Information................................................................132

3.3.2 Spatial Modulation Technique and the Saline/Contact Interface............146

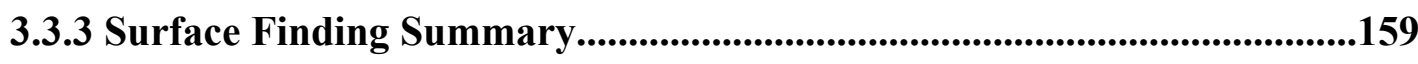

3.4 Determining the Sample Thickness..............................................................160

4 HARDWARE CONSIDERATIONS FOR LCI...................................................164

4.1 The Light Source...................................................................................164

4.2 Reference Arm Components of the LCI.................................................173

4.3 Moving the Reference Surface..........................................................................175

4.3.1 Post-correction of Stage Position Values..................................................186

4.3.2 Pre and Post-correction of Stage Position Values.........................................188

4.4 Test Arm Components of the LCI............................................................191

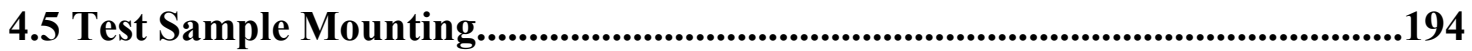

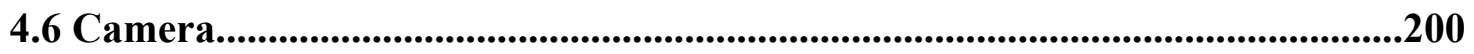




\section{TABLE OF CONTENTS - Continued}

4.7 Data Collection Procedures............................................................................................204

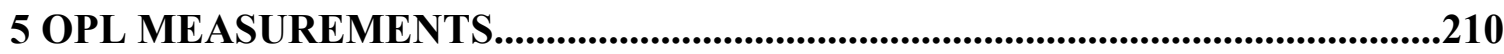

5.1 Measuring the Phase in Transmission.....................................................................213

5.1.1 Boundary Determination...................................................................................215

5.1.2 Unwrapping the Phase.........................................................................................216

5.1.3 Fitting a Plane to the Phase...................................................................................222

5.2 Interferometer Background Error................................................................223

5.3 Determining Correct Value for ' $m$ ' '............................................................................228

5.4 Hardware for the Mach-Zehnder Interferometer................................................228

5.5 Data Collection...................................................................................................................235

6 DETERMINING REFRACTIVE INDEX.................................................................238

6.1 Multiple Thickness Technique....................................................................................244

6.2 Practical Considerations for the Multiple Thickness Technique.......................249

6.2.1 Wedged Samples..........................................................................................................250

6.2.2 Slope Limitations....................................................................................................252

6.2.3 Contact Lenses as Test Samples.......................................................................254

6.3 Index Calculation - Saline........................................................................................256

6.4 Index Calculation - Contact Lens Materials.........................................................265

6.5 Removing Bad Regions from the Measurement...................................................276

6.6 Graphical User Interface (GUI)............................................................................280

6.6.1 GUI Menu Items..................................................................................................282 
TABLE OF CONTENTS - Continued

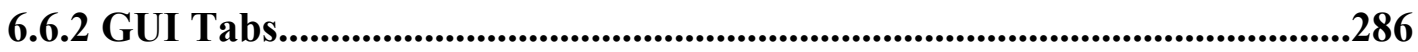

7 SYSTEM CALIBRATION PROCEDURES......................................................292

7.1 Glass Calibration Samples.....................................................................292

7.2 Calibration with the Hilger-Chance Refractometer......................................306

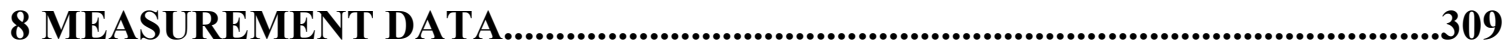

8.1 Thickness Measurements.......................................................................................310

8.2 Saline Measurements..............................................................................311

8.3 Contact Lens Material Measurements.............................................................313

9 ANALYSES OF SYSTEM ERRORS....................................................................316

9.1 Allowable Thickness Errors.........................................................................316

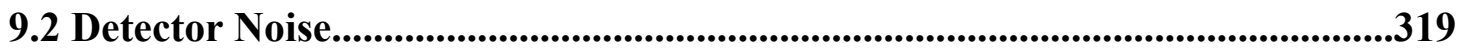

9.3 Group Index Errors...........................................................................322

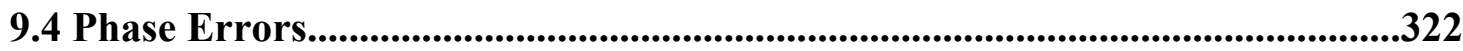

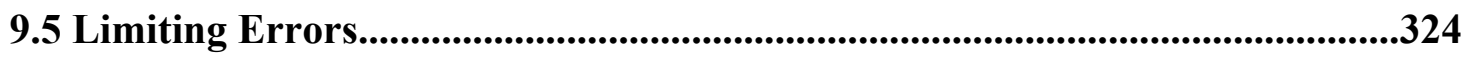

10 FUTURE WORK AND CONCLUSIONS................................................................325

10.1 System Capabilities and Limitations.......................................................325

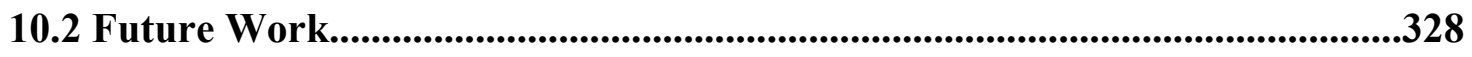

10.2.1 Accuracy Improvement.....................................................................................328

10.2.2 Decreasing Data Collection Time..............................................................329

10.2.3 Decreasing Data Processing Time........................................................330

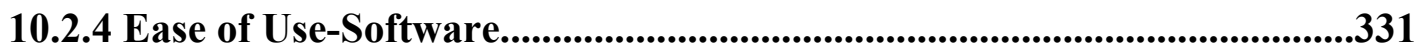


TABLE OF CONTENTS - Continued

10.2.5 Ease of Use-Sample Mounting.........................................................................332

10.2.6 Contact Lens Testing...........................................................................................332

10.3 Conclusions.................................................................................................................333

APPENDIX A: SUPERLUMINESCENT DIODE DATA SHEET..............................334

APPENDIX B: CUSTOM MOUNT FOR SLD FIBER OUTPUT...............................335

APPENDIX C: DETERMINING CORRECT TILT OF COMPENSATING

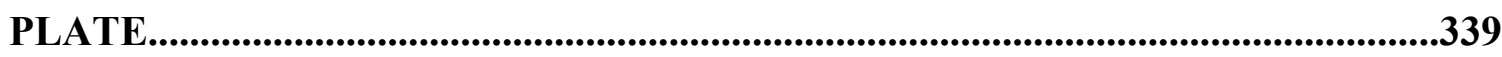

APPENDIX D: IDL FUNCTION POSITION_MAP_FILE.PRO..................................341

APPENDIX E: CAMERA SPECIFICATION SHEET...................................................343

APPENDIX F: REFERENCE DISPERSION CURVE FOR SALINE......................345

APPENDIX G: EXAMPLE SAMPLE MOUNTING IMAGES...................................346

APPENDIX H: REFRACTIVE INDEX OF GLASS CALIBRATION

SAMPLES........................................................................................................................352

REFERENCES......................................................................................................................353 


\section{LIST OF FIGURES}

FIGURE 1.1 - Contact lens material must be tested while hydrated. .36

FIGURE 1.2 - The meniscus shape of a contact lens is determined by the shape of the eye.

FIGURE 1.3 - Initial concept for contact lens material sample geometry. .38

FIGURE 1.4 - Contact angle of a water droplet on a solid material.

FIGURE 1.5 - Prism spectrometer for characterizing index of glass prisms (Sears, 1949).

FIGURE 1.6 - FIGURE 1.6 - Photograph of Mark II Abbe refractometer from Leica Microsystems (Leica website, 2007).

FIGURE 1.7 - FIGURE 1.7 - Refraction in an Abbe refractometer when measuring a) a liquid and b) a solid (Wyant, 2007).

FIGURE 1.8 - FIGURE 1.8 - A glass lens in a) a mismatched index liquid, and b), a matched index liquid (Seong, 2007).

FIGURE 1.9 - Schematic of $\mathrm{p}$ and s polarization incident on an interface.

FIGURE 1.10 - Confocal microscope focused a) on top of dispersive sample, and b) on bottom of dispersive sample.

FIGURE 1.11 - Origins of the instrument name, "Engage".

FIGURE 1.12 - Basic schematic of Engage, showing the light paths for the LCI thickness measurement.

FIGURE 1.13 - Basic schematic of Engage, showing the light paths for the MachZehnder interferometer measurement of the sample OPL. .55

FIGURE 1.14 - Picture of protective Engage housing. The door opens to allow access to the cuvette holder. .56

FIGURE 1.15 - Picture of optical layout of Engage with LCI beam paths. .57

FIGURE 1.16 - Mach-Zehnder beam paths superimposed on a picture of the optical layout of Engage. 


\section{LIST OF FIGURES - Continued}

FIGURE 2.1 - Basic Twyman-Green with scanning reference surface.

61

FIGURE 2.2 - Irradiance versus OPD for a modeled LCI signal.

FIGURE 2.3 - FIGURE 2.3 - Schematic of a SWLI system with a converging test beam

(Goodwin, 2006). .63

FIGURE 2.4 - Relationship between focus (ray paths) and fringe visibility for a wellconstructed interferometric objective with the test beam in air.

FIGURE 2.5 - As the SWLI scans through a dispersive media, the focus plane and the coherence plane move apart.

FIGURE 2.6 - Measuring the sample in a cell with a converging test beam. ..................66

FIGURE 2.7 - Layout of the low coherence interferometer used in Engage.

FIGURE 2.8 - Two views of the sample cuvette: a) Side view, and b) top view illustrating spatially multiplexing concept and camera footprint. .68

FIGURE 2.9 - Determining $\mathrm{t}_{1}(\mathrm{x}, \mathrm{y})$, the cuvette thickness, using LCI. .............................68

FIGURE 2.10 - Second LCI scan, to determine the group index of saline, $\mathrm{N}_{\mathrm{g}, \text { sal }}$...........70

FIGURE 2.11 - Third LCI scan, to determine the sample thickness, $\mathrm{t}_{\mathrm{sam}}(\mathrm{x}, \mathrm{y}) \ldots \ldots \ldots \ldots \ldots . . .70$

FIGURE 2.12 - The LCI used in Engage, indicating the test and reference arms of the interferometer. .73

FIGURE 2.13 - Reflection from the five interfaces of the measurement cuvette. …......74

FIGURE 2.14 - An example of a power spectrum of an LCI source. ..............................75

FIGURE 2.15 - Gaussian power spectrum and associated irradiance pattern, I(z). Superimposed dotted envelope indicates the coherence envelope. …………………........78

FIGURE 2.16 - Power spectrum and I(z) for a laser. ..................................................79

FIGURE 2.17 - Since $\mathrm{n}$ is larger at $\lambda=675 \mathrm{~nm}$, that wavelength has more OPL through the glass plate. 


\section{LIST OF FIGURES - Continued}

FIGURE 2.18 - The fringe pattern shifts according to the OPD while the coherence envelope shifts by the OGD. This is shown with a modeled LCI signal a) without dispersive material and $b$ ) with dispersive material.

FIGURE 2.19 - Second order dispersion leads to variations in the local slope of refractive index which leads to a distortion of the coherence envelope.

FIGURE 2.20 - Comparison of coherence envelope for linear and real refractive index versus wavelength: a) envelopes plotted together, and b) difference plot. Note the lack of symmetry for the real index.

FIGURE 2.21 - Plot of index vs. wavelength for BK7.

FIGURE 2.22 - Glass plate in test arm of a Twyman-Green Interferometer. The beamsplitter has zero thickness.

FIGURE 2.23 - Plots of a) n vs. $\lambda(400-430 \mathrm{~nm})$; b) Difference for linear and quadratic models of index (400-430 nm); c) n vs. $\lambda(665-695 \mathrm{~nm})$; d) Difference for linear and quadratic models of index $(665-695 \mathrm{~nm}$.

FIGURE $2.24-$ Plot of second derivative of index $\left(\Delta \mathrm{n} / \mu \mathrm{m}^{2}\right)$ versus $\lambda$ for BK7.

FIGURE 2.25 - By matching the dispersive material in the test and reference arms of the Twyman-Green interferometer, LCI signal due to dispersion can be compensated. ........94

FIGURE 2.26 - Plot of position error in recovering the surface location using the Fourier transform algorithm (Chapter 3 ) from the modeled LCI signals as a function of the FWHM of the Gaussian power spectrum of a modeled LCI source for BK7 (left) and water (right).

FIGURE 2.27 - Position bias vs. plate thickness of BK7 for a source centered at $680 \mathrm{~nm}$ with a FWHM of $19 \mathrm{~nm}$, using Fourier transform method.

FIGURE 2.28 - SLD output versus wavelength.

.98

FIGURE 2.29 - Position error in recovering the surface location using the Fourier transform algorithm versus source FWHM, showing increasing measurement bias for larger spectral bandwidth sources. Note the positive error for the SLD used in Engage. 


\section{LIST OF FIGURES - Continued}

FIGURE 2.30 - Surface biases vs. plate thickness for SLD source used in Engage. Dashed line is linear fit to data with a slope of a) $22.1 \mathrm{~nm}$ bias $/ \mathrm{mm}$ plate thickness for BK7 and b) $28.1 \mathrm{~nm} / \mathrm{mm}$ for water.

FIGURE 3.1 - Data cube for a single surface measured with LCI, I(x,y,z). .101

FIGURE 3.2 - Continuous representation of I(z) for Engage SLD.

FIGURE 3.3 - Comparison of a) $\lambda / 4$ sampling and b) sub-Nyquist $5 \lambda / 4$ sampling of the

LCI signal.

FIGURE 3.4 - Actual data of irradiance as a function of depth, I(z). The data has been shifted to be roughly centered at zero.

FIGURE 3.5 - Plot of $\Phi(\mathrm{k})$ as defined in equation 3.5, for the data in Figure 3.4 ........108

FIGURE 3.6 - Weighting function for valid points for calculating $\mathrm{z}_{0}$.

FIGURE 3.7 - Determination of $z_{0}$ from phase of the Fourier transform of I(z).

FIGURE 3.8 - Reconstructed surface, $\mathrm{z}_{0}(\mathrm{x}, \mathrm{y})$ for a glass/air interface (best fit plane removed).

FIGURE 3.9 - Single LCI image from data cube, I(x,y), illustrating relationship between fringe orientation and features in the recovered surface in Figure 3.8.

FIGURE 3.10 - Profile of surface shown in Figure 3.8. Note the scale of the error. ....112

FIGURE 3.11 - Data for I(z) for the saline2 interface. Note the low fringe visibility. .113

FIGURE 3.12 - Recovered $z_{0}$ value for LCI data at interface saline2, weighted appropriately.

FIGURE 3.13 - Recovered saline2 surface using Fourier transform technique. .115

FIGURE 3.14 - Surface height of sub-section on a finer axial scale. .116

FIGURE 3.15 - Profile of the recovered surface in Figure 3.14. 116

FIGURE 3.16 - Raw data I(z) (black) and estimated LCI curve, $\mathrm{I}_{\text {est }}$, (pink) used to estimate $\mathrm{z}_{0}$. 


\section{LIST OF FIGURES - Continued}

FIGURE 3.17 - Plot of error function (Equation 3.9) versus $z_{0}$ to determine best $z_{0}$ value.

FIGURE 3.18 - Reconstructed surface, $\mathrm{z}_{0}(\mathrm{x}, \mathrm{y})$ for a glass/air interface using curve fitting technique. 121

FIGURE 3.19 - Profile of reconstructed surface shown in Figure 3.18.

FIGURE 3.20 - Grayscale image of reconstructed surface (left) and single image (right), $\mathrm{I}(\mathrm{x}, \mathrm{y})$, from the scan to show the background features. Red box indicates the region that is magnified in Figure 3.21.

FIGURE 3.21 - Magnified regions from two images in Figure 3.20.

FIGURE 3.22 - Raw data I(z) (black) and estimated LCI curve, $\mathrm{I}_{\text {est }}$, (purple) for saline/contact interface (saline2).

FIGURE 3.23 - Same surface sub-section from Figure 3.14 processed using the curve fitting technique, for comparison with the Fourier technique.

FIGURE 3.24 - Profile of the recovered surface using the curve fitting technique. ......128

FIGURE 3.25 - Using spatial information from the data cube should improve surface finding accuracy. 130

FIGURE 3.26 - Image from data cube showing tilt fringes from a glass/air interface...133

FIGURE 3.27 - Schematic of the LCI in Engage.

FIGURE 3.28 - Wrapped and unwrapped phase from the 5-step phase-shifting algorithm applied to LCI data. The slope of the phase surface is different than the slope of the actual surface in space.

FIGURE 3.29 - I(x) for a single image after the baseline has been removed. This signal is used to calculate the modulation for the center pixel $\left(\mathrm{x}_{\mathrm{c}}\right)$ at this frame, $\mathrm{z}_{\mathrm{i}}$...............136

FIGURE 3.30 - Modulation versus depth $\gamma(\mathrm{z})$ for LCI signal in Figure 3.4.

FIGURE 3.31 - Plots of calculated modulation, $\gamma(\mathrm{z})$ (black) and LSF to the natural log of the modulation (purple). 


\section{LIST OF FIGURES - Continued}

FIGURE 3.32 - Recovered surfaces using LSF to modulation with spatial information technique.

FIGURE 3.33 - Recovered air1 surface using spatial modulation technique (plane removed), shown on same axial scale as 3.8 and 3.18 for comparison.

FIGURE 3.34 - Recovered air1 surface using spatial modulation technique (plane removed), at a finer scale $(\mathrm{P}-\mathrm{V}=225 \mathrm{~nm})$.

FIGURE 3.35 - Profile of reconstructed surface in Figure 3.34, same region and scale as Figures 3.10 and 3.19. Noise increases slightly at the edges since only the surface height is only calculated once at the edges (along y).

FIGURE 3.36 - Typical interferogram for saline2 interface. Dark lines indicate boundaries between regions with different thicknesses.

FIGURE 3.37 - Difference between neighboring frames in depth to show fringes.

FIGURE 3.38 - Image of wrapped phase, $\varphi(\mathrm{x}, \mathrm{y})$ for a single frame, $\mathrm{z}_{\mathrm{i}}(\mathrm{left})$, and an image of the phase difference along $\mathrm{x}, \delta_{\mathrm{i}, \mathrm{x}}\left(\mathrm{x}, \mathrm{y}, \mathrm{z}_{\mathrm{i}}\right)$ (right). For each data cube, many such images are computed and averaged to determine $\delta_{\mathrm{i}, \mathrm{x}}(\mathrm{x}, \mathrm{y})$ for the whole surface.

FIGURE 3.39 - Array of irradiance values I(x, z). Each row is used to determine $\gamma$ at that depth $\mathrm{z}_{\mathrm{i}}$, for that pixel $(\mathrm{x}, \mathrm{y})$. Black pixel indicates a location with invalid data (insufficient fringe modulation). The software must determine which other pixel(s) must not be used to find $\gamma(\mathrm{z})$ for this location.

FIGURE 3.40 - Two methods for dealing with the bad pixel in Figure 3.39: a) remove the offending row, or b) remove the offending column and the column symmetric about the center column.

FIGURE 3.41 - Example data used to determine $\gamma(\mathrm{z})$ for a single pixel $(\mathrm{x}, \mathrm{y})$.

FIGURE 3.42 - Plot of the natural $\log$ of the modulation data (black) and the best fit parabola (purple).

FIGURE 3.43 - Single region of saline2 interface using spatial modulation technique, shown on the same scale as Figures 3.14 and 3.23.

FIGURE 3.44 - Profile of reconstructed saline2 surface using the same region and scale as Figures 3.15 and 3.24. 


\section{LIST OF FIGURES - Continued}

FIGURE 3.45 - Saline2 surface profile comparison for 3 methods. ...............................158

FIGURE 3.46 - Cartoon illustrating 5 required surfaces for measuring $\mathrm{t}_{\mathrm{sam}}(\mathrm{x}, \mathrm{y})$..........160

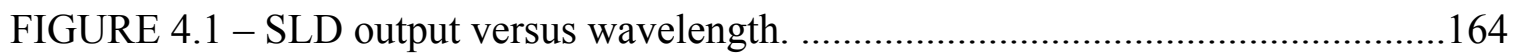

FIGURE 4.2 - Schematic of a SLD cavity (Park, 2006)...............................................166

FIGURE 4.3 - Coherence function (vendor supplied) for SLD, showing secondary modulation peaks due to reflection from the facets of the active region of the SLD. ....167

FIGURE 4.4 - Schematic of required components for SLD operation. ..........................168

FIGURE 4.5 - Since the fiber output is cleaved at $8^{\circ}$, a stage with large tip/tilt capabilities is required for mounting the fiber.

FIGURE 4.6 - Side view of Engage showing vertical orientation for SLD fiber. .........170

FIGURE 4.7 - Collimating lens for SLD source. .......................................................171

FIGURE 4.8 - Optical layout of the LCI portion of Engage. ........................................172

FIGURE 4.9 - The reference surface for LCI has a $3^{\circ}$ wedge. ......................................173

FIGURE 4.10 - Simulated fringe patters as the reference surface scans relative to the measurement cuvette. ............................................................................................... 175

FIGURE 4.11 - Schematic of required components for achieving motion of reference surface.

FIGURE 4.12 - Reported step distances for LCI stage, uncorrected. 178

FIGURE 4.13 - Measured (black) and modeled (pink) I(z) for stage positions shown in Figure 4.12 . 179

FIGURE 4.14 - Layout of the DMI for accurately monitoring stage position. 180

FIGURE 4.15 - Plot of $\varepsilon_{\mathrm{pos}}$ for the stage used in the Arizona Engage system. 182

FIGURE 4.16 - Magnified view of the curve in Figure 4.15 showing the high frequency optical encoder error. 


\section{LIST OF FIGURES - Continued}

FIGURE 4.17 - Four signals recovered from the two optical encoders used to determine

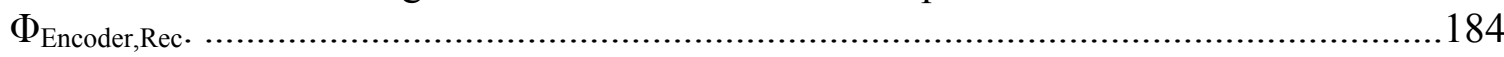

FIGURE 4.18 - Step distances for LCI stage, post-corrected using lookup table. ..........187

FIGURE 4.19 - Determining $\varepsilon_{\mathrm{OE}}$ for a $\mathrm{Z}_{\mathrm{OE}}$ that falls between points with known $\varepsilon_{\mathrm{OE}}$...187

FIGURE 4.20 - Measured (black) and modeled (pink) I(z) for stage positions shown in

Figure 4.18. 188

FIGURE 4.21 - Evenly spaced position goals (left) and pre-corrected position goals (right).

FIGURE 4.22 - Step distances for LCI stage, pre- and post-corrected using lookup table.

190

FIGURE 4.23 - Measured (black) and modeled (pink) I(z) for stage positions shown in

Figure 4.22.

FIGURE 4.24 - Minimum thickness for the cavity of the cuvette.

.192

FIGURE 4.25 - Drawing of the current measurement cuvette where the cavity is formed by $350 \mu \mathrm{m}$ diameter sapphire balls glued to the top plate. 193

FIGURE 4.26 - Four optical surfaces of the cavity for coating designations. .193

FIGURE 4.27 - Drawing of a) desired effects of the washer and b) actual results of the washer. 195

FIGURE 4.28 - Resulting a) raw image and b) image subtraction, showing LCI fringes, using a washer as a weight.

FIGURE 4.29 - Static fringe pattern from sample shown in Figure 4.30. Orange circle indicates the only region that is sticking to the glass plate.

FIGURE 4.30 - For a small enough saline bubble beneath the sample, the reflections from the two interfaces are coherent, causing a static fringe pattern (does not vary as the reference surface is moved).

FIGURE 4.31 - Fishing line as a support structure for suspending the sample in saline. 


\section{LIST OF FIGURES - Continued}

FIGURE 4.32 - Fishing line used to suspend the sample in the cuvette. In a), the dashed arrow indicates the fishing line on the bottom plate while b) shows the fringes obtained. ..

FIGURE 4.33 - Histogram of the difference in irradiance values between two frames during an LCI measurement ( $\sigma=30$ digital counts).

FIGURE 4.34 - Difference in recovered surface height between a) 8-bit and 12-bit, and

b) 10-bit and 12-bit. The profiles are for a saline/sample interface (saline2). .204

FIGURE 4.35 - Timing diagram for capturing a single image during an LCI scan. ......206

FIGURE 4.36 - The plane of OGD $=0$ should be here when the surface finding routine starts. .207

FIGURE 4.37 - Flowchart for finding the starting locations for the 5 LCI surfaces. ....208

FIGURE 5.1 - Optical layout of Engage, illustrating the beam paths for the MachZehnder interferometer.

FIGURE 5.2 - Measuring OPL $\mathrm{Cair}_{\text {and }}$ OPL $\mathrm{L}_{\text {saline }}$ for a cuvette containing air and saline..212

FIGURE 5.3 - The required mirror motion to achieve a $90^{\circ}$ phase shift is $\mathrm{d}_{\mathrm{i}}$..............214

FIGURE 5.4 - Fringe image $\mathrm{I}_{1}$ (left) and the wrapped phase (right). ...........................215

FIGURE 5.5 - User drawn polygon specifies the region of $\varphi(\mathrm{x}, \mathrm{y})$ containing air. ........216

FIGURE 5.6 - Profile of a) wrapped phase and b) unwrapped phase. ...........................217

FIGURE 5.7 - Typical user defined ROI's based on sample geometry. ........................218

FIGURE 5.8 - Gray region is the first subsection to be unwrapped. Black regions are invalid data. White regions are areas yet to be unwrapped. White ' $x$ ' is the overall starting point and each point on the red line is the starting point for that y-profile. .......219

FIGURE 5.9 - Three subsections were required to unwrap the region shown in Figure 5.8 .

FIGURE 5.10 - The image on the left shows discontinuities between unwrapped subsections. The image on the right shows result of correcting the regions. 


\section{LIST OF FIGURES - Continued}

FIGURE 5.11 - Image with a) raw unwrapped data and b) after most the errors have been removed automatically in software. The red box in b) indicates a suitable region for the user to remove for error-free unwrapping.

FIGURE 5.12 - Final unwrapped phase after the small region outlined by the red box in

Figure 5.11 has been removed by the user. .222

FIGURE 5.13 - A plane is fit to the phase data in the ROI containing air.

FIGURE 5.14 - Schematic of Mach-Zehnder interferometer without a cuvette. Ideally, the resulting interferogram should only contain tilt.

FIGURE $5.15-\mathrm{A} 5^{\text {th }}$ order polynomial fit to $\varphi_{\varepsilon}(\mathrm{x}, \mathrm{y})$, the Mach-Zehnder interferometer baseline error. .225

FIGURE 5.16 - Each fringe represents $1 / 50^{\text {th }}$ of a fringe in the collected images. ........226

FIGURE 5.17 - Picture of the tunable HeNe laser used in Engage (REO website). ......229

FIGURE 5.18 - Rotating the Littrow prism allows wavelength selection in the tunable

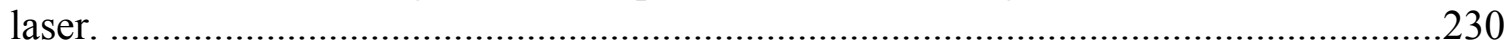

FIGURE 5.19 - Relevant pinout for GPIO2 connector of XPS controller. .....................233

FIGURE 5.20 - Schematic of the components used to control the PZT. ........................234

FIGURE 5.21 - Phase-stepping versus phase-ramping. ……………………………...235

FIGURE 5.22 - Timing diagram for data collection with the Mach-Zehnder interferometer. .236

FIGURE 6.1 - Wrapped phase from Mach-Zehnder interferometer showing the discontinuity between the air and saline regions. .240

FIGURE 6.2 - The interferogram from a discrete step measured in reflectance will have a discontinuity at the boundary. Which fringe on the right is fringe ' $a$ ' from the left? ....240

FIGURE 6.3 - Plots of a) refractive index vs. wavelength, b) first derivative of dispersion curve $(\Delta \mathrm{n} / \mathrm{nm})$ and c) second derivative of dispersion curve $\left(\Delta^{2} \mathrm{n} / \mathrm{nm}^{2}\right)$ for water. ........243

FIGURE 6.4 - A hypothetical sample with two different thicknesses. .245 


\section{LIST OF FIGURES - Continued}

FIGURE 6.5 - The calculated index value for two thickness regions of the same material will only agree at one index value, indicated here by the circle. .245

FIGURE 6.6 - Side and top views of an 'inverted wedding cake' sample.

FIGURE 6.7 - A wedged sample with a thickness gradient only along $\mathrm{x}$. .250

FIGURE 6.8 - Calculated $\mathrm{n}(\mathrm{x}, \mathrm{y})$ for $\Delta \mathrm{m}=\mathrm{a}) \Delta \mathrm{m}_{\text {correct, }}$ b) $\Delta \mathrm{m}_{\text {correct }}+1$ and c) $\Delta \mathrm{m}_{\text {correct }}+2$.

FIGURE 6.9 - A fringe pattern at the Nyquist limit has a phase change of $180^{\circ}$ or $\pi$ radians.

FIGURE 6.10 - The maximum wedge angle for a test sample is about $1^{\circ}$. .253

FIGURE 6.11 - Plots of a) NC vs. $t_{1}$, where $t_{2}=t_{1}+20$ (fixed thickness difference), and $b$ ) $\mathrm{NC}$ vs. $t_{1}$, where $t_{2}=1.2 * t_{1}$. The minimum thickness difference needed to satisfy the NC limits depends on $\mathrm{t}_{1}$. The dashed orange line is $\mathrm{NC}=0.0005$. .255

FIGURE 6.12 - A contact lens in its relaxed state has too large of a base curve. .255

FIGURE 6.13 - If an independent measurement is used to determine the correct value of $\Delta \mathrm{m}$ and find $\mathrm{n}_{\text {saline, }}$, it must be more accurate then $\Delta \mathrm{n}_{\Delta \mathrm{m}=1} / 2$. .258

FIGURE 6.14 - Measuring $\theta$ in a well-known V-block allows $\mathrm{n}$ to be calculated. .......259

FIGURE 6.15 - Christmas tree plot for selecting correct values for $\mathrm{n}_{\text {saline. }}$. .261

FIGURE 6.16 - A reference dispersion curve for saline can be used to determine $\Delta \mathrm{m}_{\text {correct. }}$ .264

FIGURE 6.17 - The product of the raw phase and the saline region mask gives the saline region that must be unwrapped to obtain $\varphi_{\text {saline. }}$ .268

FIGURE 6.18 - A plane is fit to $\varphi_{\text {saline }}$ after $\varphi_{\varepsilon}$ has been subtracted. .269

FIGURE 6.19 - The phase in each thickness region must be unwrapped individually...270 


\section{LIST OF FIGURES - Continued}

FIGURE 6.20 - Christmas tree plot for contact lens material. The orders outlined by the dashed box line up for all 4 thickness regions. Note how the index values for the multiple regions 'fan out' at $+/-1$ in $\Delta \mathrm{m}$.

FIGURE 6.21 - First plot shown after user clicks near a set of refractive index values in Figure 6.20, which shows the dispersion curves for all four regions.

FIGURE 6.22 - Second window, showing calculated index values for each wavelength and region.

FIGURE 6.23 - Clicking below x-axis recalculates possible index values at lower values for $\mathrm{n}$. The various $\mathrm{n}_{\text {periodic }}$ values for this data set prevent all of the orders from lining up at any value for $\mathrm{n}$ other than the correct one.

FIGURE 6.24 - The optic zone of a contact lens tested in Engage, along with the concentric ring structure for selecting multiple thickness regions.

FIGURE 6.25 - A saline bubble trapped beneath the sample will make $t_{\text {sample }}$ too large.

FIGURE 6.26 - Image from LCI data cube showing sample regions that are not sticking to the glass plate at the bottom of the cuvette.

FIGURE 6.27 - Masked standard deviation image. The circled regions show rapid variations in the standard deviations consistent with the varying phase relationship for the two reflected beams from a saline bubble. .280

FIGURE 6.28 - GUI used for both data collection and analysis for Engage. .281

FIGURE 6.29 - Screenshot of "Main" tab of Engage control GUI. .287

FIGURE 6.30 - Screenshot of "System Variable" GUI tab. ............................................28

FIGURE 6.31 - Screenshot of "Camera" tab of the Engage GUI. .................................289

FIGURE 6.32 - Screenshot of the "Pico/V output" tab of the GUI. ..............................290

FIGURE 6.33 - Screenshot of the "Analysis Options” tab of the Engage GUI. ............291

FIGURE 7.1 - Initial prism geometry of glass calibration samples. .293 


\section{LIST OF FIGURES - Continued}

FIGURE 7.2 - Two views of the final geometry of the glass calibration samples. ........295

FIGURE 7.3 - Testing geometry of the glass calibration samples.

FIGURE 7.4 - An a) LCI image and b) a frame-by-frame subtraction to show the fringes of data from the saline 2 interface of a calibration measurement.

296

FIGURE 7.5 - Wrapped phase in transmission for a glass calibration sample.

FIGURE 7.6 - Two thickness regions chosen from the area containing the glass calibration sample.

FIGURE 7.7 - The measured index of prism BK7-1 varies with measurement. The solid pink line is the value measured with the prism spectrometer and the dotted lines are $+/$ -

0.0001 from this accepted value.

.299

FIGURE 7.8 - Index difference versus measurement for all 5 laser wavelengths. Note each curve (wavelength) follows a similar trend.

FIGURE 7.9 - Mean measured thickness of glass sample (relative to measurement 0) versus measurement number.

FIGURE 7.10 - Plot of $\varepsilon_{\text {pos }}$ for the stage used in the Arizona Engage system. The width of the red box corresponds to the typical travel range used for a single LCI measurement. .303

FIGURE 7.11 - Spatial plot of refractive index of calibration sample. .305

FIGURE 9.1 - Standard deviation of irradiance noise versus number of averaged images, theoretical and measured. .320

FIGURE 9.2 - Standard deviation of the surface error as a function of the number of images averaged during the LCI scan.

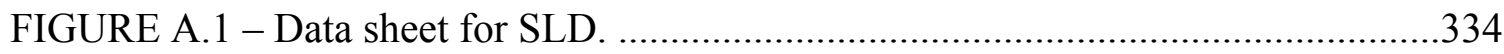

FIGURE B.1 - Photograph of the 561-tilt from Newport. ..........................................335

FIGURE B.2 - Drawings of the 561-FCH for holding the fiber. .................................336

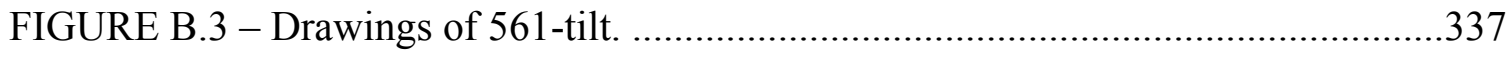




\section{LIST OF FIGURES - Continued}

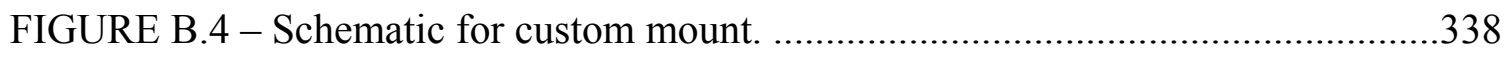

FIGURE C.1 - Tilt the compensating plate out of the beam to increase OGL. .............339

FIGURE E.1 - Page 1 of camera data sheet (Photometrics, 2006). ..............................343

FIGURE E.2 - Page 2 of camera data sheet (Photometrics, 2006). .............................344

FIGURE G.1 - Four example images of suitable spatial multiplexing. ........................347

FIGURE G.2 - Air bubbles appear as bright regions often surrounded by a circular

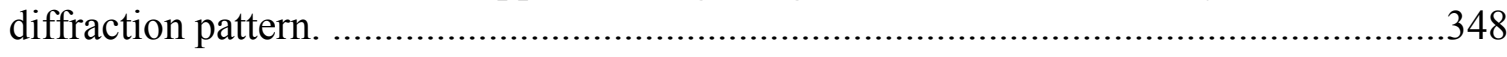

FIGURE G.3 - Blue polygons outline the static fringe patterns indicative of saline bubbles. The orange oval indicates debris.

FIGURE G.4 - At first glance, the region outlined in blue is sticking. The loss of static fringe visibility and the brightness of the region indicate that this area is not sticking...350

FIGURE G.5 - Another example of brightness variations. .350

FIGURE G.6 - This sample is mounted correctly, even though there are regions with defects. 


\section{LIST OF TABLES}

TABLE 1.1 - List of basic index measurement requirements. .52

TABLE 2.1 - Example reflectance coefficients for various interfaces in the sample cell.

TABLE 3.1 - Outline of steps for finding surface location $\mathrm{z}_{0}(\mathrm{x}, \mathrm{y})$ using the spatial modulation algorithm.

TABLE 3.2 - Processing time for Spatial Modulation surface finding technique.

TABLE 4.1 - Summary of surface statistics for the same LCI data for a glass/saline interface processed at 8,10 and 12 bits. The differences in the surface coefficients $(a, b, c)$ are negligible.

TABLE 4.2 - Table of surface name and number of required images to capture the full

LCI signal. .205

TABLE 5.1 - Laser wavelengths and their optical powers for the tunable laser. .229

TABLE 5.2 - Output voltages per $90^{\circ}$ for the five laser wavelengths. .233

TABLE 5.3 - Exposure times for the five laser wavelengths. .236

TABLE 6.1 - For a sample with two thicknesses, $\mathrm{n}_{\text {periodic }}$ and the noise ceiling cannot be simultaneously large. 248

TABLE 6.2 - Comparison of $n$ measured by HCR and Engage $\left(\mathrm{T}=24^{\circ} \mathrm{C}\right)$. .262

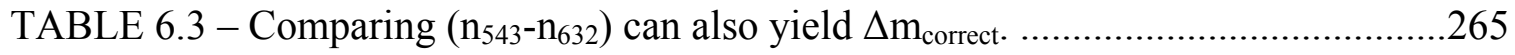

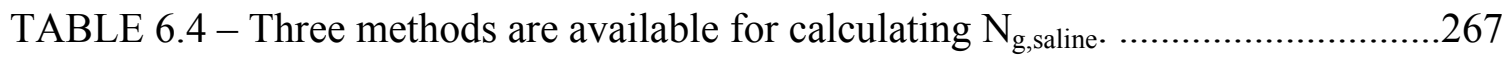

TABLE 6.5 - Average thicknesses of the 4 regions and $\Delta \mathrm{n}_{\Delta \mathrm{m}=1}$ for each. .....................270

TABLE 6.6 - Measured index of soft contact lens material as a function of wavelength.

TABLE 6.7 - Guide to the values in Engage configuration file, "config2.txt". .282

TABLE 7.1 - Refractive index of calibration sample BK7-1 as measured with Engage and with the prism spectrometer. 


\section{LIST OF TABLES - Continued}

TABLE 7.2 - Comparison of the index of a saline sample measured by the HCR and

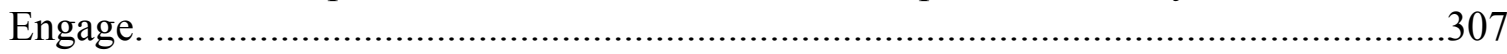

TABLE 8.1 - The coefficients of the best fit plane for 3 measurements using the axial positions from the DMI and the optical encoder. .311

TABLE $8.2-$ Index of saline comparison for $\lambda=593.93 \mathrm{~nm}$.

TABLE 8.3 - Index of refraction of soft contact lens material for 5 data sets using DMI positions.

TABLE 8.4 - Index of refraction of soft contact lens material for 5 data sets using OE positions.

TABLE 9.1 - Thickness errors that cause a $\Delta \mathrm{n}=0.0001$ for various materials. 318

TABLE 10.1 - Original refractive index measurement goals. 325

TABLE H.1 - Prism spectrometer data for the 7 glass calibration samples. .352 


\begin{abstract}
The optical power of a lens is determined by the surface curvature and the refractive index, n. Knowledge of the index is required for accurate lens design models and for examining material variations from sample to sample. The refractive index of glass can be accurately measured using a prism spectrometer, but measuring the index of soft contact lens materials presents many challenges. These materials are non-rigid, thin, and must remain hydrated in a saline solution during testing. Clearly an alternative to a prism spectrometer must be used to accurately measure index.
\end{abstract}

A Dual Interferometer System has been designed, built and characterized as a novel method for measuring the refractive index of transparent optical materials, including soft contact lens materials. The first interferometer is a Low Coherence Interferometer in a Twyman-Green configuration with a scanning reference mirror. The contact lens material sample is placed in a measurement cuvette, where it remains hydrated. By measuring the locations of the multiple optical interfaces, the physical thickness $t$ of the material is measured. A new algorithm has been developed for processing the low coherence signals obtained from the reflection at each optical interface.

The second interferometer is a Mach-Zehnder interferometer with a tunable HeNe laser light source. This interferometer measures the optical path length (OPL) of the test sample in the cuvette in transmission as a function of five wavelengths in the visible 
spectrum. This is done using phase-shifting interferometry. Multiple thickness regions are used to solve $2 \pi$ phase ambiguities in the OPL.

The outputs of the two interferometers are combined to determine the refractive index as a function of wavelength: $n(\lambda)=\operatorname{OPL}(\lambda) /$ t. Since both $t$ and OPL are measured using a detector array, $\mathrm{n}$ is measured at hundreds of thousands of data points. A measurement accuracy of 0.0001 in refractive index is achieved with this new instrument, which is verified using custom glass calibration samples. 


\section{INTRODUCTION TO REFRACTIVE INDEX MEASUREMENTS}

One of the goals of engineering is to solve the technical problems that arise from pushing the boundaries of science. The direction from which this push can come varies from the drive to investigate entire new fields of research to improving existing technologies. In optical engineering, as in any other engineering field, the push to improve covers many sub-disciplines. Of particular interest for the purposes of this dissertation and the group in which the research took place is the drive for improved metrology capabilities. The development of any optical instrumentation is always faced with three major hurdles in order to become a working tool or product. The system must be designed correctly; it must be possible to fabricate that design; and finally, it must be possible to verify that the fabrication is correct.

The specifics of these three requirements will vary with the application, but none of the three can be ignored. A logical chain of dependence is as follows; a system has been designed which, according to models, outperforms what currently exists. However, it requires improvements in existing fabrication techniques, so the fabrication techniques evolve. The new fabrication technique is now capable of creating parts that cannot be tested with existing metrology techniques, so the metrology is forced to improve. At first glance, this seems to complete the chain. In fact, the chain should be formed into a loop, where the available testing methods dictate the allowable design space. Otherwise, 
whatever optical system that looks great in the design phase may never be achievable because it cannot be tested.

If an engineer follows the chain without considering design, fabrication and testing simultaneously, the project may never be successful. At the same time, new tools and products should not be limited in design to what is currently possible, as this would halt progress in all three areas. Advances in any one area often act as a catalyst for improvement in the other areas.

The original push for this research work was to be able to measure the refractive index of contact lens materials. The Dual Interferometer system created for testing these contact lens materials turned into an independent project all of its own requiring a novel design, accurate fabrication, and a means of verification. Only after these three tasks had been adequately accomplished could the Dual Interferometer be used as a testing method for contact lenses. The universal applicability of design, fabrication and testing in regards to optical engineering becomes obvious.

The Dual Interferometer system is one of several testing instruments studied on behalf of the project sponsor, all of which relate to the testing of contact lenses or some step in the fabrication of contact lenses. References to the other instrumentation will be made when required, but the main goal of this dissertation is to convey the novel technology that 
went into the design, fabrication and testing of the Dual Interferometer system for measuring the refractive index of contact lens materials.

This chapter will begin with the origins of the specific requirements for this instrument. This will include some background on contact lenses, but only enough to understand the requirements imposed on the testing method. Several excellent sources are available for further explanation of the design and fabrication of contact lenses for the interested reader.

Armed with the testing requirements, existing methods for measuring refractive index will be discussed. There are commercially available methods along with techniques recently developed and published in the literature, and a thorough examination of them all would require volumes. Many of the more applicable techniques were considered as solutions to this particular problem in a master's report by a previous student (Brumfield, 2003). The methods chosen to be covered here are those that offer insight into what led to the final system design.

\subsection{Refractive Index Definitions}

The absolute refractive index of a material is a unit less ratio of the speed of light (c) in a vacuum to the speed of light in some material at wavelength $\lambda$.

$$
n_{\text {Absolute }}(\lambda)=\frac{c}{v_{\text {Material }}(\lambda)}
$$

Equation 1.1 
Therefore, the refractive index of a vacuum is defined to be 1.00 , exactly. In practice, refractive index $n$ refers to the index relative to $n_{\text {air }}=1.00$ (Born, 1999). The absolute refractive index of air is 1.00029 , so the difference is small but not negligible. Unless stated otherwise, refractive index values in this dissertation are relative to $\mathrm{n}_{\text {air }}=1.00$. The functional dependence of the index $n$ on wavelength $\lambda$ is referred to as dispersion. All transparent optical materials exhibit dispersion. Refractive index dictates the amount light bends at an interface between two materials of different indices, according to Snell's Law.

$$
n_{1} \sin (\theta)=n_{2} \sin \left(\theta^{\prime}\right)
$$

In the case of a lens, the refractive index is one of the two important parameters defining the optical power of the lens, the other being surface curvature (C). This is a manifestation of Snell's law on a curved surface. Here, $\varphi$ is the power of the lens in the thin lens equation:

$$
\phi_{\text {Lens, }, A i r}=(n-1)\left(C_{1}-C_{2}\right)=\Delta n \Delta C
$$

Optical path length (OPL) is the product of the physical thickness of a material $t$, and its index, $n$. This is analogous to the time delay for light traveling through a material with index greater than 1 .

$$
O P L=n t
$$

The quantitative importance of the index of refraction will be established shortly. 


\subsection{Measurement Considerations}

Knowing the refractive index of an optical element is important for any refractive system. A contact lens is no different in this regard. The major fabrication goal of a contact lens is to achieve a specified optical power, expressed in diopters $(1 / \mathrm{m})$. As is clear from Equation 1.3, the refractive index of the contact lens material comprises a major part of the power of the final product.

One previously completed project was to build an instrument capable of measuring the power of contact lenses, $\varphi_{\text {lens, }}$ in a Mach-Zehnder interferometer via transmitted wavefront testing, which will be referred to as CLOVER (Williby, 2003). As discussed by Williby, the accuracy of the measured power is only as good as the accuracy of the refractive index of the material and the saline solution in which it is immersed.

\subsubsection{Accuracy Requirements}

An important design goal for the measurement system is the required measurement accuracy. This number is not arbitrarily chosen but is the result of requirements on the ability to measure the power to some certain accuracy. There are two approaches to finding the required index accuracy.

The first approach is to consider the relation of refractive index to the recovered power from CLOVER. In order to measure the power of the contact lens, the lens is placed in a cuvette filled with saline solution such that the outer edge of the lens is in contact with 
the bottom plate of the cuvette. Light travels vertically through the lens in the interferometer. The power of the contact lens is reduced by a factor of $\sim 6$ because it is immersed in saline. For this analysis, the index of saline is assumed to be 1.336 and the index of the contact lens material is assumed to be 1.405 .

$$
\begin{array}{cc}
\phi_{\text {Lens in Air }} \approx\left(n_{\text {Lens }}-1\right) \Delta C & \text { Equation } 1.5 \\
\phi_{\text {Lens in Saline }} \approx\left(n_{\text {Lens }}-n_{\text {Saline }}\right) \Delta C & \text { Equation 1.6 } \\
\frac{\phi_{\text {Lens in Air }}}{\phi_{\text {Lens in Saline }}}=\frac{\left(n_{\text {Lens }}-1\right) \Delta C}{\left(n_{\text {Lens }}-n_{\text {Saline }}\right) \Delta C}=\frac{0.405}{0.069} \approx 5.9 & \text { Equation } 1.7
\end{array}
$$

This makes it possible to use the CLOVER interferometer to measure a large range of contact lens powers without adding part-dependent corrector optics (i.e. partial nulls). The contact lens prescription power, which is specified in air, is calculated using the above ratio and the measured power from the interferometer. Any errors in the values used for the index of saline or the index of the contact lens translate into errors in the measured power. Differentiating Equation 1.3 leads to:

$$
\frac{\delta \phi}{\phi}=\frac{\delta \Delta n}{\Delta n}+\frac{\delta \Delta C}{\Delta C}
$$

For a fixed curvature difference and nominal index values of $n_{\text {Lens }}=1.405$ and $n_{\text {Saline }}=$ $1.336, \Delta n$ is 0.069 . A $1 \%$ index error of 0.00069 gives a $1 \%$ error in the measured power. Therefore the index difference must be known to better than 0.00069 in order to measure a lens to less then $1 \%$ error.

A second approach to determining the required accuracy in determining the refractive index of the contact lens materials is driven by the ANSI specification on the tolerance 
for power of contact lenses of 0.25 diopters. Using this along with the sponsor specified desired precision to tolerance $(\mathrm{P} / \mathrm{T})$ ratio of $30 \%$ gives the allowed change in power of 0.025 diopters.

$$
\begin{array}{ll}
\frac{P}{T}=30 \%=\frac{3 \sigma}{0.25 D} & \text { Equation } 1.9 \\
\sigma=d \phi=0.025 D & \text { Equation } 1.10
\end{array}
$$

Here $\sigma$ is, of course, the standard deviation of the measurement. On a +10 diopter lens, this change in power corresponds to a $\delta \Delta$ n of 0.00017 .

$$
\begin{array}{cc}
\frac{\delta \phi}{\phi}=\frac{0.025 D}{10 D}=\frac{\delta \Delta n}{0.069} & \text { Equation } 1.11 \\
\delta \Delta n=0.00017 &
\end{array}
$$

This is a tougher requirement on the required measurement accuracy then obtained from an analysis of CLOVER data. Based on this analysis, the initial measurement goal was an accuracy of $+/-0.0001$ in refractive index, $n$.

\subsubsection{Spectral Coverage}

Any reported index of refraction value is not complete without including the corresponding wavelength for the index. The appropriate wavelength values vary depending on the application for the optical system. For an ophthalmic optical system such as a contact lens, the index of refraction in the visible $(\lambda=400$ to $700 \mathrm{~nm})$ seems best. In addition, the desired measurement output should complement other contact lens metrology instrumentation, such as CLOVER or the Hilger-Chance refractometer (Pixton, 2006). The Hilger-Chance refractometer measures the index of refraction of 
liquids, such as saline. The CLOVER interferometer used to measure the transmitted wavefront tests at the green line of a HeNe laser $(\lambda=543.4 \mathrm{~nm})$. This is also near the peak spectral response of the human eye of $555 \mathrm{~nm}$ (Greivenkamp, 2004).

Determining the refractive index at a single wavelength is useful, but testing at multiple visible wavelengths provides additional insight into the contact lens materials. The dispersion of the material can be found to varying accuracy, depending on the number of wavelengths used and their spectral separation. Therefore, the goal for this project is to measure the index of refraction of the contact lens materials to $+/-0.0001$ at multiple wavelengths in the visible portion of the spectrum.

\subsubsection{Material Properties}

During the process of finding the best measurements method for a given task, it is important to understand how the material properties of the measurement sample can affect the measurement technique. The contact lens materials considered to be tested fall into a class of materials called hydrogels, which are hydrophilic polymers plasticized by the water they absorb. They can be thought of as soft, elastic water containing gels (Tighe, 1997). In this case, water containing means that the water content is typically between $40-60 \%$, by weight.

$$
\text { Water Content }=100 \% \frac{\text { Weight of water }}{\text { Weight of hydrated gel }}=\mathrm{WC} \quad \text { Equation } 1.12
$$

Another way to think of these materials is as a thin sponge. When they dry, they become stiff and brittle. Also, in order to get an accurate gauge on the refractive index of the 
material, it must remain hydrated so that the sample maintains the desired physical state. One way to think about this is that the refractive index of the material is a weighted average of the two primary components; the polymer and the water or saline in which it is submerged.

$$
n_{\text {Hydrogel }}=\mathrm{WC} \cdot n_{\text {Water } / \text { Saline }}+(1-\mathrm{WC}) n_{\text {Polymer }}
$$

Testing the material in a dehydrated state would result in index values indicative of the polymer and not the lens in the state it will be used in the final optical system, i.e. the eye, where it is hydrated with tears. A dry hydrogel material would measure a higher index as the index of the polymer is higher then that of the saline solution.

The hydration's influence on index of refraction also requires that the sample not be tested under any physical deformation. Thinking again of the sample as a sponge, squishing the test sample would decrease the water content and change the refractive index. A logical requirement of the testing method is that it not squish or physically stress the sample, as this can change very thing that is being measured. In addition to changing the water content, making mechanical contact with the sample will change the physical dimensions. There are multiple reasons that physical contact with the hydrogel must be avoided as it will inevitably alter the material itself.

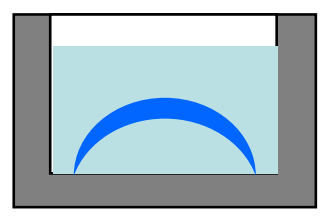

FIGURE 1.1 - Contact lens material must be tested while hydrated. 
The easiest method for keeping the contact lens material hydrated is to test it while completely submerged in a saline solution. This is how lens testing is done in CLOVER (Figure 1.1). Although this sample configuration is required for measuring refractive index, it makes other aspects of the test more difficult, as will be seen in the following chapters. One consequence is that whatever is used to hold the sample during testing must be able to contain a liquid as well.

Another important part of any optical test is the material geometry, both overall shape and surface quality. The shape of a contact lens is obviously dictated by the shape of the cornea, as they are designed to fit on the eye and are therefore in the shape of a meniscus lens (Figure 1.2). Their effectiveness in correcting the focal length of the eye is primarily achieved by changing the curvature of the outer corneal surface, which accounts for the majority of the optical power of the eye.

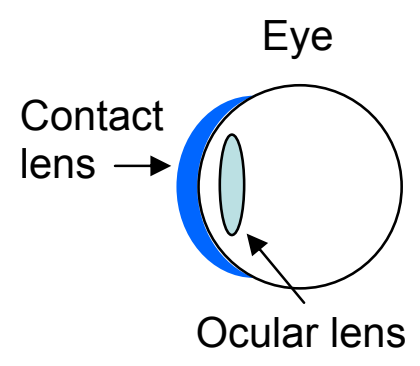

FIGURE 1.2 - The meniscus shape of a contact lens is determined by the shape of the eye. 
From a manufacturing and process monitoring standpoint, it would be ideal to measure the index of refraction of the contact lens in its final geometrical form. Unfortunately, a contact lens has steep curvatures, is thin, and will not maintain this form to a high accuracy during the course of a measurement. The steep curvatures make a reflective test extremely difficult. Because of the added difficulties that would be encountered in testing a thin meniscus lens, the initial goal of the measurement was to measure contact lens materials in some form, not necessarily in the final form of a lens. This simplifies the measurement approach for any test utilizing reflections off the material surfaces. A round, flat disk of contact lens material was considered the test sample geometry during the system design phase. Here, $t$ is considered to be on the order of a few hundred microns while D is approximately 10 millimeters (Figure 1.3).

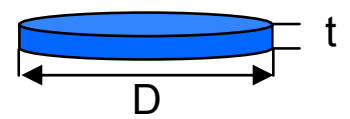

FIGURE 1.3 - Initial concept for contact lens material sample geometry.

\begin{abstract}
Although the steep curvatures are no longer present in this testing geometry, the sample must remain as thin as actual contact lenses. The main reason for this comes from the way in which the lenses are manufactured. The liquid chemicals are injected into a plastic mold. Once the mold is filled, the liquid is cured into the final sponge state using ultraviolet (UV)-curing (Tighe, 1997). Extending the thickness of the material beyond a few hundred microns might alter the curing properties and in turn influence the refractive index.
\end{abstract}


As anyone who has ever worn soft contact lens would know, these materials are very non-rigid when hydrated. Gravity is enough to deform the shape of a lens. Any testing methodology must either provide a means for keeping the lens stable during testing or take the necessary data quickly, so as to not be influenced the material moving during testing.

Another factor is the wetting of the contact lens materials. When fully hydrated, the contact lens materials become slippery, which can be important in relation to mounting and mechanical stability. The terms 'hydrophilic' and 'hydrophobic' are often used to refer to their relative water content. In terms of bulk properties, they mean 'water-loving' and 'water-hating', respectively (Tighe, 1997). As the surface of the lens dries, it becomes tacky and sticky, which can be useful in mounting of the samples.

A water droplet placed on a hydrophilic surface will spread out into a thin layer. Placed on a hydrophobic surface, that same drop would form a small droplet, the tangents of the sides of which are nearly normal to the hydrophobic surface.

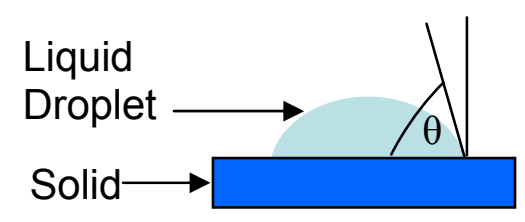

FIGURE 1.4 - Contact angle of a water droplet on a solid material. 
A convenient way of classifying a surface is by measuring the contact angle $\theta$; the larger

the angle, the more hydrophobic the surface (Figure 1.4). This will become important in a later chapter.

Summarizing the material properties relevant to measuring refractive index, the sample must be submersed in a saline solution during testing since the material has about $50 \%$ saline content; any measurement technique cannot put pressure on the sample that would eject saline or stress the sample; the sample will be thin (a few hundred microns), but is not required to have highly curved surfaces as in the lens geometry; and the sample must remain stationary during testing.

\subsection{The Glass Standard}

At first glance, it may seem strange that the specified sample geometry of the contact lens materials is a round, flat disk and not an actual contact lens, since this is the geometry in which the material is used. When it comes to index of refraction measurements, the most commonly measured materials are glasses used as optical elements. However, glass lenses are not measured directly to determine their index of refraction. For stock commercial lenses, the index of refraction is simply quoted from a lookup table for the appropriate material, as the index of glass is more stable then the index of hydrogel. For higher precision glass applications, a prism is poured from the same glass melt to be used as a reference. This is analogous to measuring index of contact lens materials on a disk instead of a lens. 
The index of a glass prism is measured using a prism spectrometer, shown in Figure 1.5. A finite spectral source, often a laser, is used to illuminate the entrance slit of the prism spectrometer. This light is collimated with the collimating lens in the figure. The long direction of the slit is orthogonal to the plane of rotation of the test prism. The angular location of the collimated light is located by lining up a reticule inside the eyepiece of the telescope and recording the angle on the scale. High end spectrometers report angles to the nearest arc-second or $1 / 10^{\text {th }}$ of an arc-second. The prism is then placed at the center of the rotation table. The telescope can be rotated around the prism, and its center of rotation is at the center of the rotation table as well. The tip and tilt of the prism spectrometer's rotation table is adjusted such that the prism faces are normal to the optical axis of the collimating system and the telescope.

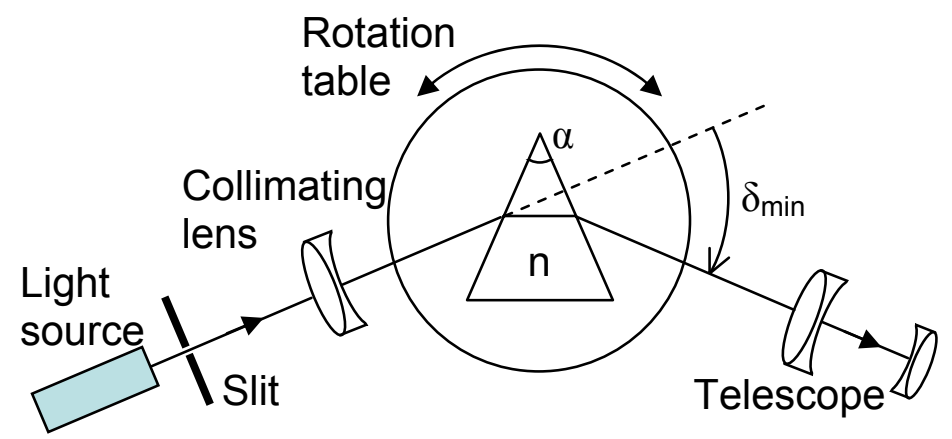

FIGURE 1.5 - Prism spectrometer for characterizing index of glass prisms (Sears, 1949).

The prism is rotated to the minimum deviation angle, where the light is least deviated through the prism (Figure 1.5). The reticule is aligned with the refracted slit image and the angle is recorded. The difference between these two angles is the minimum deviation angle, $\delta_{\min }$. 


$$
n(\lambda)=\frac{\sin \left(\frac{\alpha+\delta_{\min }(\lambda)}{2}\right)}{\sin \left(\frac{\alpha}{2}\right)}
$$

Here, $\alpha$ is the apex angle of the prism, as shown in Figure 1.5. The value of $\alpha$ is also determined using the prism spectrometer. A crosshair pattern is projected by the telescope onto the test prism. The telescope is rotated until the specular reflection from the front surface of the prism is coincident with the reticule in the telescope and the angle is recorded. Without disturbing the test prism, the telescope arm is rotated until the crosshair pattern reflects off the other prism face that makes up the angle $\alpha$. The difference between these angles is $\alpha$.

Consider measuring the refractive index of a BK $745^{\circ}-45^{\circ}-90^{\circ}$ prism, using one of the $45^{\circ}$ corners for $\alpha$. The test wavelength is $632.8 \mathrm{~nm}$, at which the catalog refractive index of BK7 is 1.51509 . For a perfect $45^{\circ}$ apex angle, $\delta_{\min }$ is $25^{\circ} 52^{\prime} 22.5^{\prime}$. To obtain an accuracy of 1 part in the $5^{\text {th }}$ decimal place $(0.00001)$, the minimum deviation angle $\delta_{\text {min }}$ must be measured to within about 2 arc-seconds. The measurement is more sensitive to errors in measuring $\alpha$, requiring 0.75 arc-seconds accuracy to obtain 1 part in the $5^{\text {th }}$ decimal place. These numbers can be achieved with good instrumentation and a lot of time, but this illustrates how difficult it can be to determine refractive index accurately.

An additional consideration for any refractive index measurement is the temperature of the prism. The change in index per degree Celsius of BK7 is $3 \times 10^{-6}$ (Yoder, 1993). 
Therefore, to obtain 0.00001 in index for glass, the temperature must be known to within $3^{\circ}$ Celsius. Glass is relatively stable with respect to temperature when compared to the $\mathrm{dn} / \mathrm{dT}$ of distilled water, which is about $-1 \times 10^{-4}$ (Tilton, 1938). It is important to accurately monitor the temperature when measuring the index of hydrogel materials because of their high water content and the large change in index with temperature of water.

\subsection{Existing Refractive Index Measurement Techniques and Hydrogels}

The prism spectrometer is not the only common method for measuring refractive index. Ideally, achieving a measurement accuracy of 0.0001 in index for hydrogel contact lens materials could be done using an existing, mature technology such as the prism spectrometer. One might expect existing approaches to work since the required accuracy is an order of magnitude less than what some methods are capable of. The following discussion is an overview some available techniques, explaining why they will not work for this particular measurement. This list is not meant to include all available measurement techniques, but will illustrate why a new method was developed to solve this problem. Applicable material parameters will be discussed for each method.

\subsubsection{Prism Spectrometer}

Measuring a hydrogel with a prism spectrometer would require making a prism out of the hydrogel. There are many problems with this; creating plane prism surfaces; maintaining 
a stable prism angle to the nearest few arc-seconds in the presence of gravity; and maintaining the proper hydration of the test sample, to name just a few. Additionally, the prism would likely be at least an order of magnitude thicker then a typical contact lens. The chemistry of the UV-curing process could be affected as well as the material properties in the center of the hydrogel prism. For these reasons, this method was never seriously considered.

\subsubsection{Abbe Refractometer}

A standard method for determining the index of refraction of liquids is the Abbe refractometer (Figure 1.6). This instrument is simple to use and certain models claim accuracies of 0.0001 at the D (Sodium) wavelength of $589 \mathrm{~nm}$. These models provide no dispersion information. Other models sacrifice accuracy at the D wavelength (typically $0.0005)$ to additionally provide the principal dispersion, $\mathrm{n}_{\mathrm{f}}-\mathrm{n}_{\mathrm{c}}$.

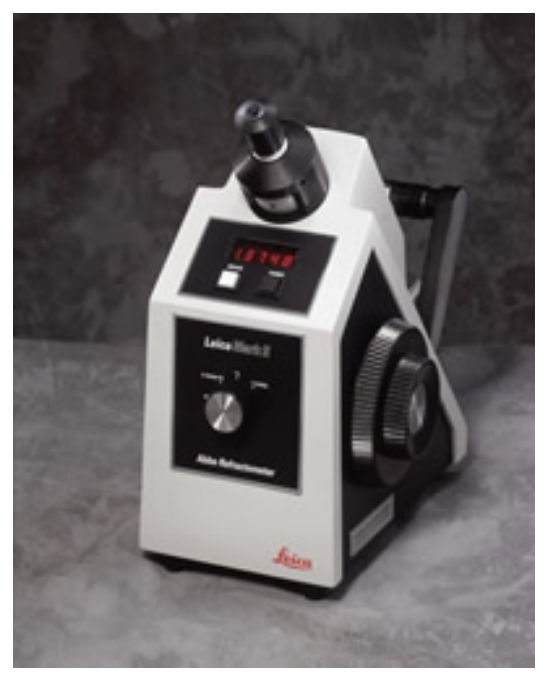

FIGURE 1.6 - Photograph of Mark II Abbe refractometer from Leica Microsystems (Leica website, 2007). 
Figure 1.7a illustrates the refractions that take place when measuring a liquid sample with an Abbe refractometer. A liquid is measured by placing it between two high-index prisms of known refractive index $\left(\mathrm{n}_{1}, \mathrm{n}_{3}\right)$. The index of refraction of these two prisms must be larger than the index of the fluid to be measured. Measuring the maximum angle $\theta_{3}$ corresponds to a $\theta_{2}$ of $90^{\circ}$, in which case Equation 1.15 can be solved for $\mathrm{n}_{2}$, the liquid index.

a)

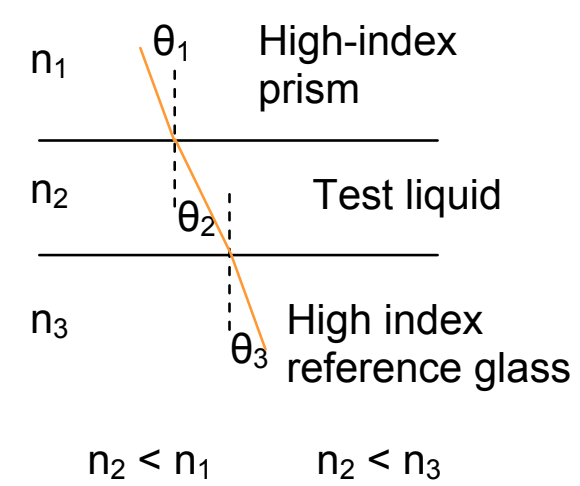

b)

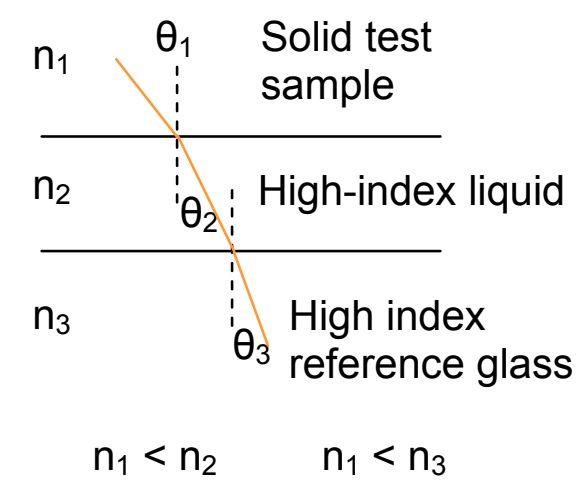

FIGURE 1.7 - Refraction in an Abbe refractometer when measuring a) a liquid and b) a solid (Wyant, 2007).

$$
n_{1} \sin \theta_{1}=n_{2} \sin \theta_{2}=n_{3} \sin \theta_{3}
$$

Equation 1.15

A solid can be measured if the solid test sample is placed on top of a fluid with a refractive index higher than the solid test sample (Figure 1.7b). In this case, the maximum angle $\theta_{3}$ corresponds to $\theta_{1}$ of $90^{\circ}$. This allows $n_{1}$ to be calculated.

There are two problems with this technique when testing contact lens materials. First, this technique measures the surface index since it is based off the refraction at the material boundary. The goal of this project is to measure the bulk refractive index. 
Second, the sample must be hydrated in saline during testing. The index of saline is lower than the index of the test sample which violates the measurement principle. Placing the sample in a high index fluid would change the index of the sample due to the high sample liquid content. A possible alternative would be squishing the sample with the top prism. This would eject saline, changing the sample liquid content and the refractive index.

Finally, the spectral coverage of the Abbe is poor. It either reports at the sodium D line to the required accuracy (neglecting the squishing problem and the skin index problem) or provides some dispersion information at lower accuracy levels. This method is inappropriate because of the squishing required and the insufficient accuracy and/or spectral coverage.

\subsubsection{Index Matching Fluids}

Measuring the refractive index of fluids to 0.0001 can be done with an Abbe or with other methods. One method that is sometimes used to determine the index of a glass lens is to submerge the lens in a liquid solution, and place the cell in the test beam of an interferometer. Initially, the refractive index of the glass lens and the liquid are different, and so the fringes are discontinuous at the edge of the lens (Figure 1.8a). The index of the liquid can be increased by adding a higher index liquid until the fringes line up at the edge of the lens (Figure 1.8b). In this case, the refractive indices of the lens and the 
liquid are equal. The index of the liquid can then be measured to determine the index of the glass lens.

a)

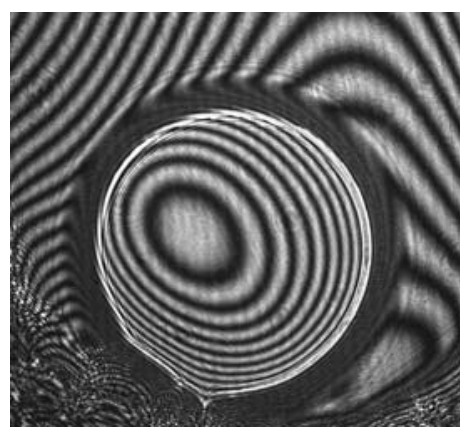

b)

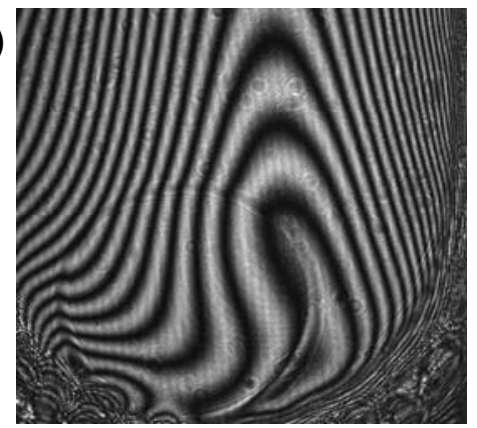

FIGURE 1.8 - A glass lens in a) a mismatched index liquid, and b), a matched index liquid (Seong, 2007).

The obvious problem with this method for testing contact lenses is that the contact lens will absorb whatever liquid in which it is submerged, which changes the very thing that being measured.

\subsubsection{Ellipsometry}

The theory of operation for ellipsometric measurements is based on the Fresnel reflection or transmission equations for polarized light encountering boundaries in planar multilayered materials (Figure 1.9, Hecht, 1998).

$$
\begin{aligned}
& r_{p}=\frac{n_{2} \cos \left[\theta_{1}\right]-n_{1} \cos \left[\theta_{2}\right]}{n_{1} \cos \left[\theta_{2}\right]+n_{2} \cos \left[\theta_{1}\right]} \\
& r_{s}=\frac{n_{1} \cos \left[\theta_{1}\right]-n_{2} \cos \left[\theta_{2}\right]}{n_{1} \cos \left[\theta_{1}\right]+n_{2} \cos \left[\theta_{2}\right]}
\end{aligned}
$$




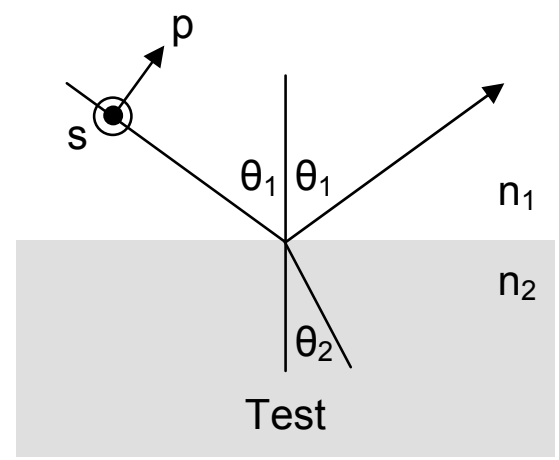

FIGURE 1.9 - Schematic of $\mathrm{p}$ and s polarization incident on an interface.

The ellipsometer measures $\tan (\Psi)$, the ratio of reflected amplitudes between $\mathrm{p}$ and $\mathrm{s}$ polarization, and $\Delta$, the phase difference between $\mathrm{p}$ and $\mathrm{s}$ polarization, in the following equation (Synowicki, 1998; Woollam, 1999). Here $r_{p}$ and $r_{s}$ are the complex Fresnel reflection coefficients of the sample for $p$ and s polarized light, and $\rho$ is the complex reflectance ratio.

$$
\rho=\frac{r_{p}}{r_{s}}=\tan (\Psi) e^{i \Delta}
$$

Ellipsometry measures intensity-independent complex quantities and is analogous to an impedance measurement. One of the two polarization states acts as a phase and amplitude reference for the other polarization state, removing a lot of error sources due to source instabilities (Azzam, 1988). Each ellipsometric measurement of $\rho$ is performed at a specific wavelength $\lambda$ and angle of incidence $\theta_{1}$. In the case of measuring a thin film (Guo, 1996) where only the film thickness ( $\mathrm{t})$ and index $\left(\mathrm{n}_{2}\right)$ are unknown, it would be ideal to have large, independent changes in $\rho$ for both $t$ and $\mathrm{n}_{2}$. Unfortunately, the two are usually correlated and single wavelength, single-angle measurement is not always possible. There are cases in the literature of measuring refractive index to close to the 
required accuracy $(0.0005)$, but only in special sample geometries or in cases where the thickness of the test sample is known from another technique. In the case of Guo, a 10 $\mu \mathrm{m}$ film was measured with a profilometer. The force of a mechanical contact probe on a hydrated hydrogel sample would change the thickness significantly during the course of a measurement.

Assuming the required accuracy could be reached, there are additional problems. The simplest testing geometry for an ellipsometric measurement would be to have light incident directly on the top surface of the test sample. Due to the hydration constraints, this would require the full system to be submerged in saline. Alternatively, a measurement could probe through a series of interfaces making up some sort of measurement cell. The reflection coefficient of the desired interface would be orders of magnitude lower then the other interfaces, causing the desired signal to be overwhelmed by other signals, degrading the accuracy. Finally, this technique measures the skin refractive index, not the desired bulk index. This technique was eliminated as a possible method for measuring contact lens materials.

\subsubsection{Confocal Microscopy}

Confocal microscopy utilizes a scanning pinhole to optically section a sample in depth. Spatial coverage can also be achieved by scanning. Consider a layered sample, such as a flat disk on contact lens material in a glass cell, surrounded by saline solution. The light 
is focused on one interface in the cell, reflected from that interface back through the microscope, and passes through the pinhole without being attenuated (Figure 1.10a).

a)

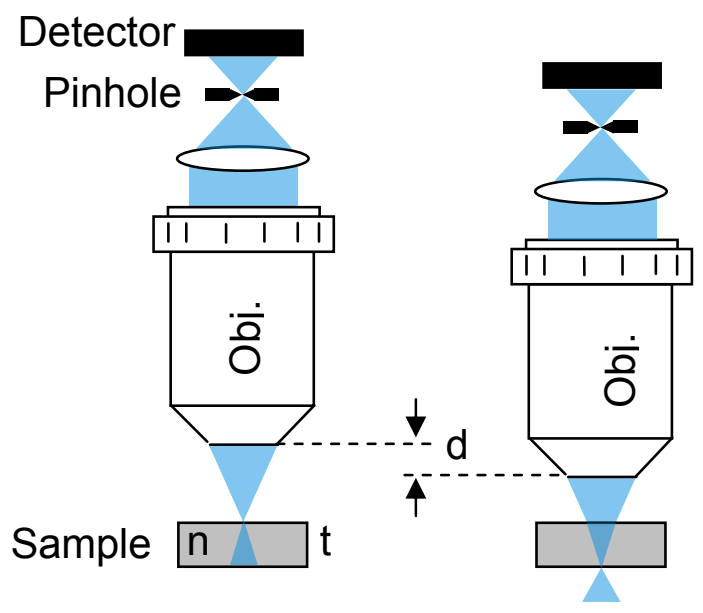

FIGURE 1.10 - Confocal microscope focused a) on top of dispersive sample, and b) on bottom of dispersive sample.

Light reflected from the neighboring interfaces is mostly blocked by the pinhole, so that only the in focus surface is inspected. Translating the sample a known distance $d$ will bring a new interface into focus, which will be transmitted by the pinhole. This distance $\mathrm{d}$ between the two interfaces is related to the refractive index and plate thickness.

$$
d \approx t\left(\frac{n-1}{n}\right)
$$

The location of each interface is determined by measuring the irradiance as a function of depth and finding the peak value. The higher the numerical aperture (NA) of the microscope objective, the narrower the full-width-half maximum (FWHM) of the irradiance versus depth and the more accurately the OPL is determined. 
High end confocal microscopes use objectives with an NA of 1.45, which can obtain a $330 \mathrm{~nm}$ FWHM (Blanca, 2002). As will be shown later, this is not sufficient to meet the index of refraction goal of 0.0001 . Translating through a dispersive material such as a piece of hydrogel will introduce spherical aberration (Sheppard, 2000). The higher the NA, the worse this problem becomes. So even though increasing the NA improves the axial resolution of a single surface, it decreases the allowable thickness that can be measured (Sheppard, 1991). In addition, the index cannot be found from Equation 1.19 without prior knowledge of the physical thickness of the sample, t. For contact lens material sample, $\mathrm{t}$ is not known without a separate measurement.

\subsection{Dual Interferometer System (Engage)}

The various techniques discussed above are all incapable of measuring the refractive index of these materials to the desired accuracy. Interferometry was not covered as one of these possible options, even though there are many different systems using interferometry to measure thickness, group refractive index and even phase index. Individually, each of these techniques falls short of the list of requirements shown in Table 1.1; they require prior knowledge of thickness, they measure the group index instead of the phase index (Hirai, 2003; Hirai, 2006; Murphy, 2000), they have insufficient accuracy (Fukano, 1999), they assume a dispersion curve (Hopler, 1991), the sample must be at least $1 \mathrm{~mm}$ thick (Haruna, 1998), or they are incapable of measuring a 
sample that must be in solution (Maruyama, 2002). Rather then discuss all of the various techniques and pointing out where they fall short of our measurement parameters, the novel technique used will be presented. Aspects of the other measurements that are used are noted where appropriate.

The system designed to accomplish this refractive index measurement is a Dual Interferometer system. It incorporates elements of previously published techniques that, on their own, were insufficient. Before the details of this system are discussed, the measurement requirements are shown in Table 1.1 for reference.

\begin{tabular}{|l|l|}
\hline Refractive index accuracy & 0.0001 \\
\hline Wavelengths & Visible $(\sim 400-700 \mathrm{~nm})$ \\
\hline Sample geometry & $\begin{array}{l}\text { Round disk }(\mathrm{t} \sim 200 \mu \mathrm{m}, \mathrm{D} \sim 10 \mathrm{~mm}), \\
\text { hydrated during testing }\end{array}$ \\
\hline Other considerations & Non-contact method \\
\hline
\end{tabular}

TABLE 1.1 - List of basic index measurement requirements.

The most basic description of this measurement technique starts from the definition of optical path length (OPL). The measurement will be broken into two steps; first, the physical thickness $t$ of the test sample will be measured using a Low Coherence Interferometer (LCI) in a Twyman-Green configuration. Second, the OPL in transmission is measured using a Mach-Zehnder interferometer with a tunable HeNe laser as a light source. Dividing OPL by thickness gives the desired index, $\mathrm{n}$. Measuring OPL 
through the sample guarantees that the bulk refractive index, not the skin index, is measured.

$$
O P L=n t \quad n(\lambda)=\frac{O P L(\lambda)}{t}
$$

The physical thickness is independent of wavelength and only needs to be measured once. To determine the index as a function of wavelength, the OPL must be measured at multiple wavelengths in the spectral region of interest, in this case the visible.

The two interferometers explain the origin of the name Dual Interferometer system. A less cumbersome name used to refer to the instrument is Engage, which comes from the goal of the project: to provide a "gauge" for measuring refractive index, or "n" (Figure $1.11)$.

\section{"n" + "gauge" $\Rightarrow$ "Engage"}

FIGURE 1.11 - Origins of the instrument name, "Engage".

Figure 1.12 and 1.13 are schematics of Engage with the beam paths for the LCI measurement and for the Mach-Zehnder interferometer, respectively. 


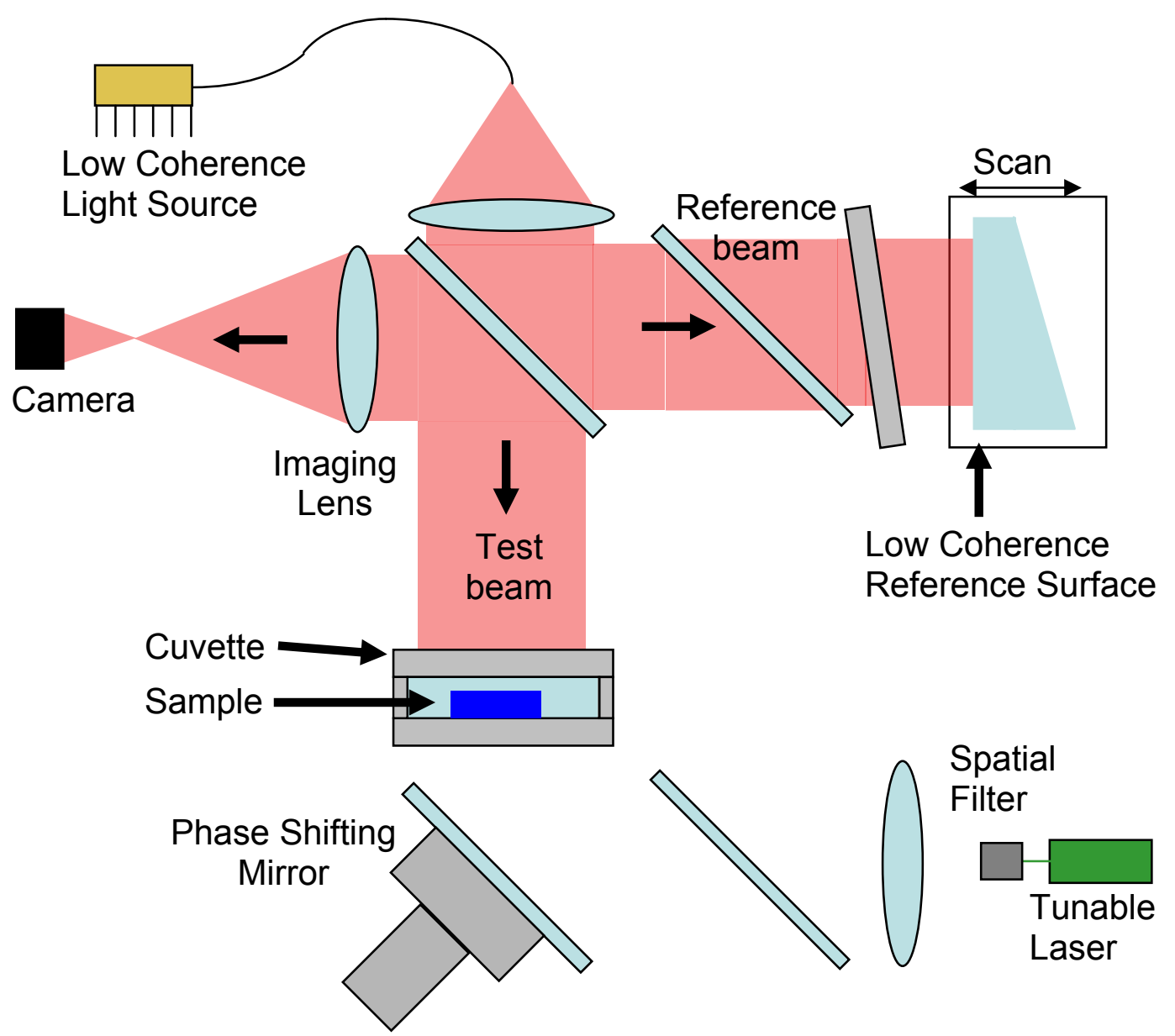

FIGURE 1.12 - Basic schematic of Engage, showing the light paths for the LCI thickness measurement. 


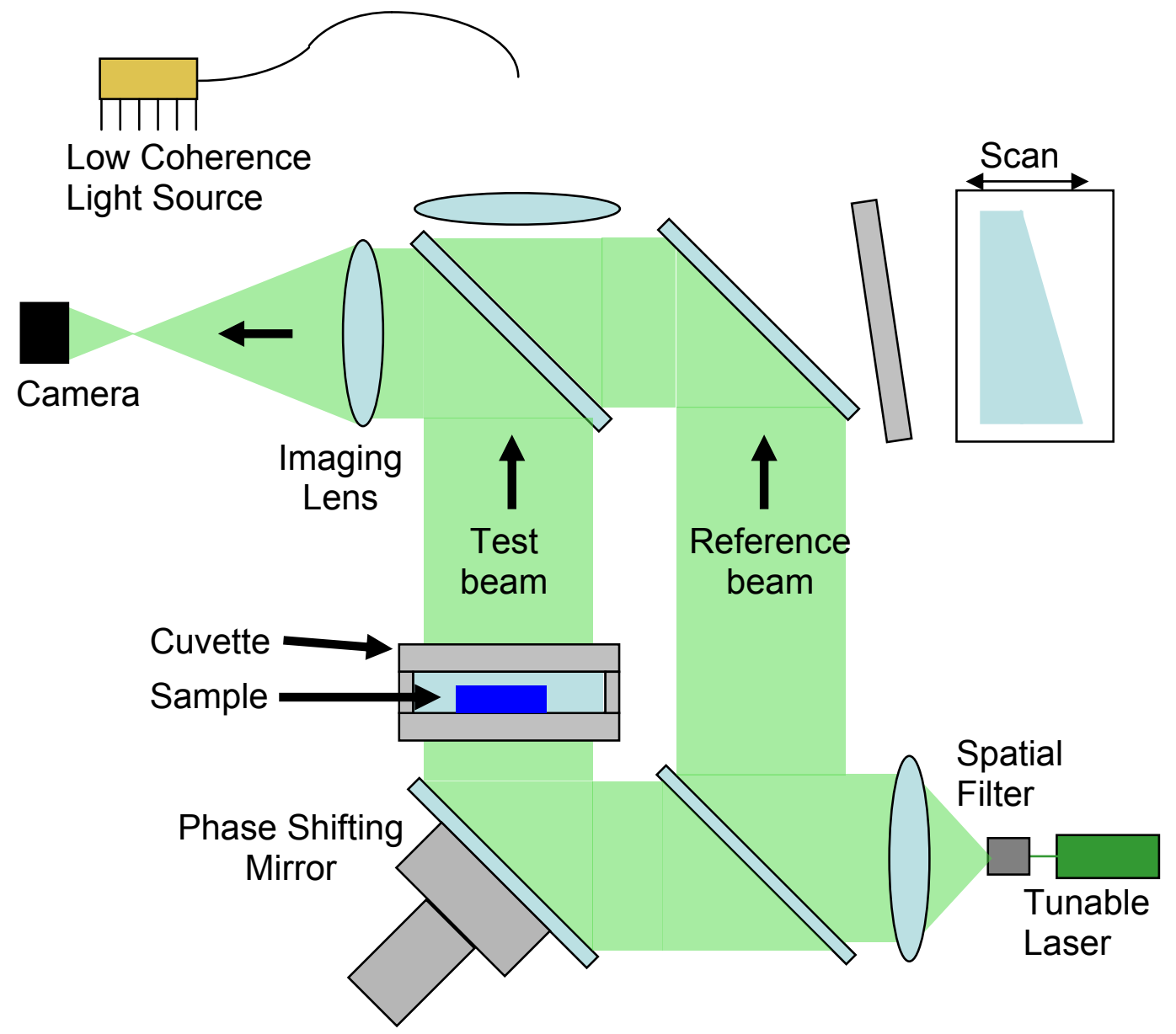

FIGURE 1.13 - Basic schematic of Engage, showing the light paths for the MachZehnder interferometer measurement of the sample OPL.

Note that several of the optical components are common to both interferometers. Figure 1.14 is a photograph of Engage enclosed by its protective housing. The access door swings open as indicated by the arrow to allow access to the cuvette holder. 


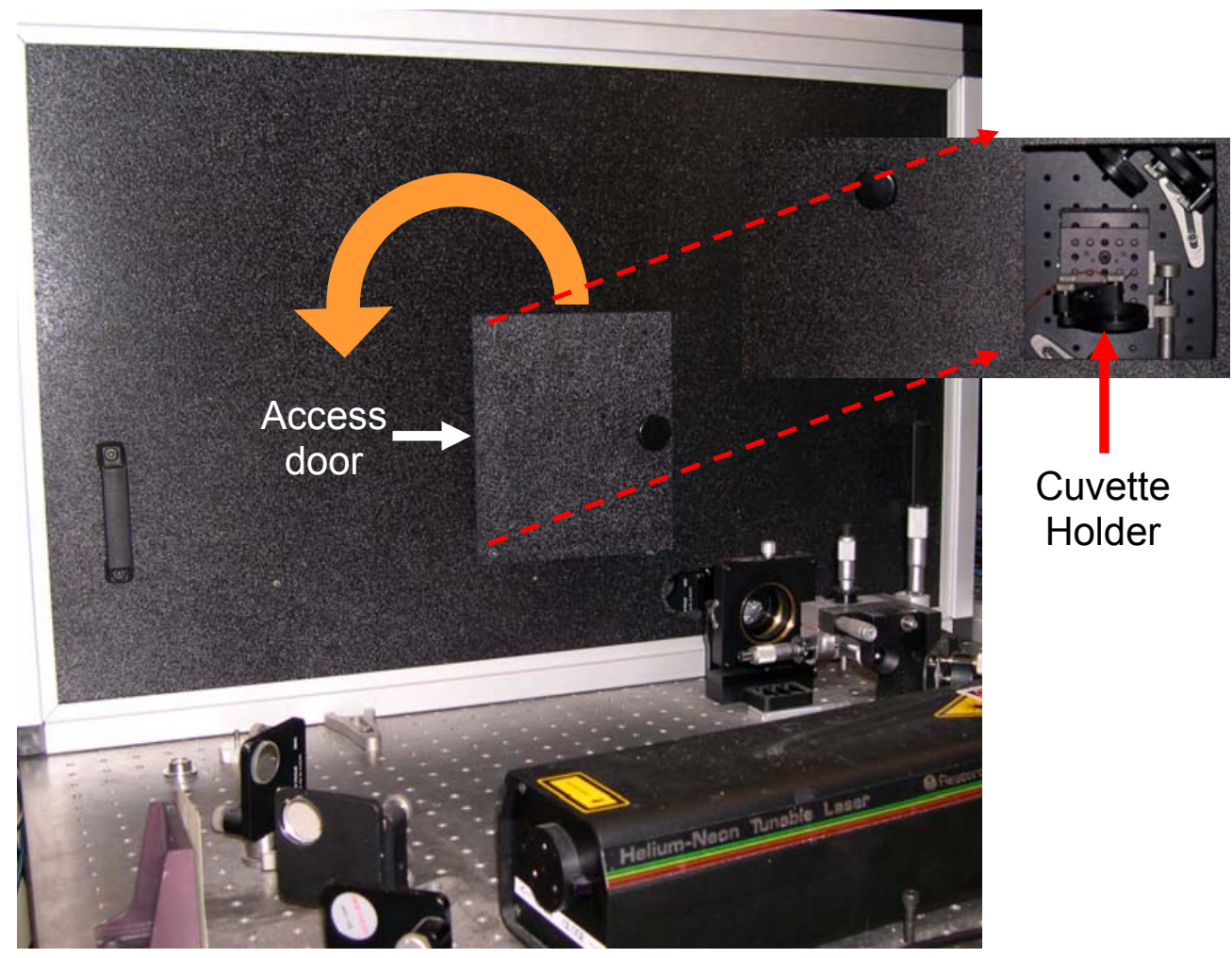

FIGURE 1.14 - Picture of protective Engage housing. The door opens to allow access to the cuvette holder.

Figure 1.15 is a photograph of the optical layout with the LCI beams artificially superimposed. 


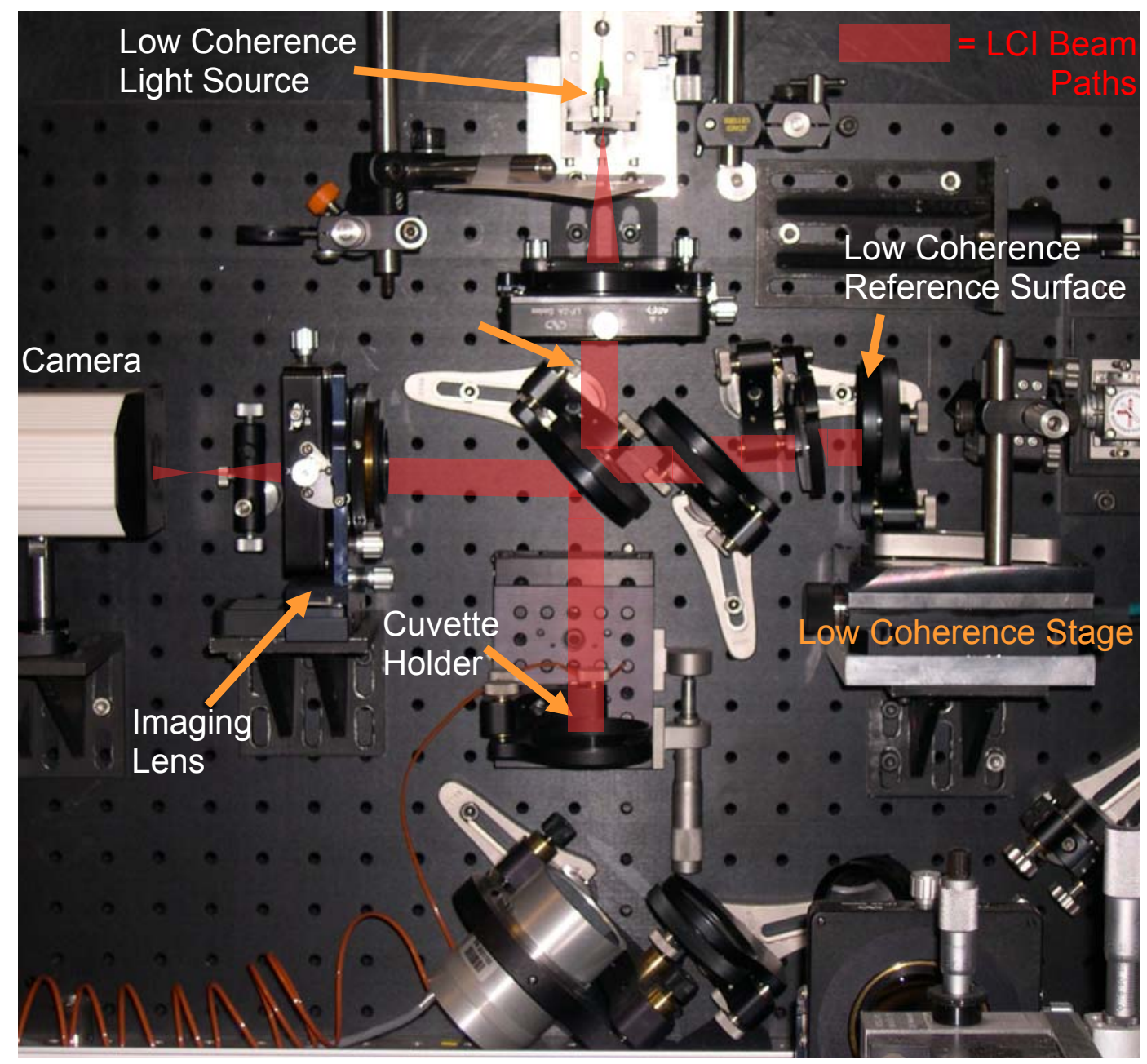

FIGURE 1.15 - Picture of optical layout of Engage with LCI beam paths.

Figure 1.16 does not include the beam steering optics or the spatial filter used with the laser, but it does show the beam paths for the test and reference beams of the MachZehnder interferometer. 


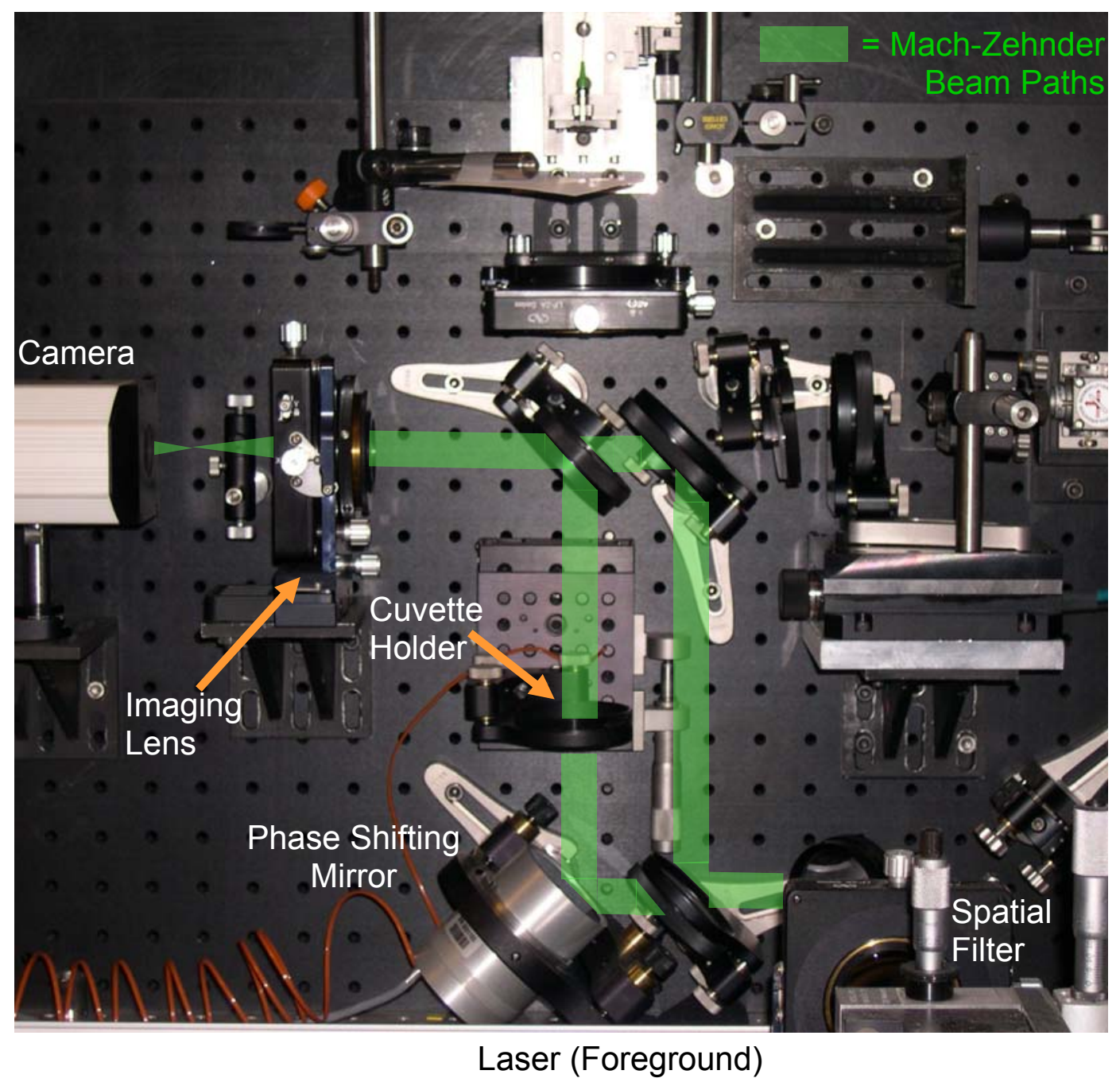

FIGURE 1.16 - Mach-Zehnder beam paths superimposed on a picture of the optical layout of Engage.

The theory, data collection, and data analysis are all broken down as two separate measurements (thickness and OPL), so the system description will follow along the same lines. Keep in mind that each half of the measurement is part of a larger picture. Chapter 2 covers the detailed theory behind the effects of the interferometer configuration on the LCI signal used for thickness measurement. Chapter 3 covers the task of converting the LCI signals into surface locations. Chapter 4 discusses the hardware considerations for 
the thickness measurement of the system. Chapter 5 explains the theory and hardware behind the OPL measurement. Chapter 6 combines the two measurement results to determine refractive index. Chapter 7 explains various means of calibrating the Engage system. Chapter 8 presents measurement results of contact lens material samples. Chapter 9 compares the influence of various system errors on the final refractive index output, and Chapter 10 concludes the dissertation and suggests possible future work for improving the system. 


\section{THICKNESS MEASUREMENT - THEORY}

The underlying technology used to measure the thickness of the hydrogel test material (sample) in Engage is low coherence interferometry, or LCI. The thickness is probed with light, so the requirement of a non-contact measurement is satisfied. LCI, which is sometimes referred to as white light interferometry, is a well established technique for precise, unambiguous surface profiling. Commercial instruments are available using this technique, such as the Wyko NT1100 (Wyko NT1100 website, 2007). Depending on the instrumental arrangement, height measurement accuracies are in the range of a few nanometers to sub-nanometer (Deck, 1994). The details for obtaining such a high accuracy are discussed at length in later sections.

\subsection{Basic Low Coherence Interferometry}

The basic idea employed by scanning white light interferometers (SWLI) is to find the relative height of a surface by finding the maximum fringe contrast versus depth (Deck, 1994). Consider a Twyman-Green interferometer configuration where the reference surface can be scanned along the axial direction, $\mathrm{z}$ (Figure 2.1). 


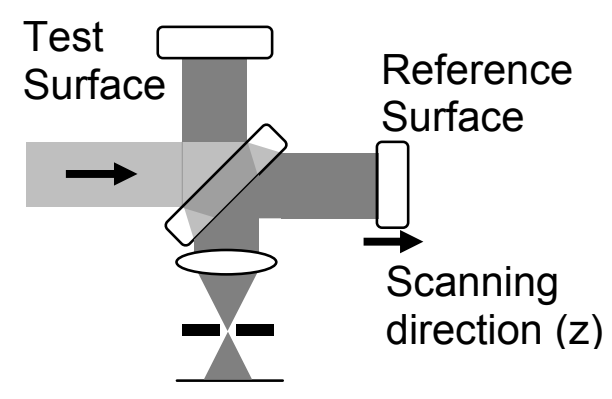

FIGURE 2.1 - Basic Twyman-Green with scanning reference surface.

In LCI, the source has limited temporal coherence. This means the source has a spectral bandwidth associated with it, and the coherence length, $1_{\mathrm{c}}$ is inversely proportional to the spectral bandwidth. If the coherence length of the source is $10 \mu \mathrm{m}$, then interference fringes will only be observed when the optical path difference (OPD) between the test and reference arms of the interferometer is less then the coherence length, or less then 10 $\mu \mathrm{m}$ (Equation 2.1). In practice, the transition between the two regimes is gradual.

$$
\begin{aligned}
& |O P D| \leq l_{c} ; \text { Fringes } \\
& |O P D|>l_{c} ; \text { No Fringes }
\end{aligned}
$$

The visibility, or contrast, of the fringes will be maximized when OPD equals 0 for a surface in air. Scanning the reference surface and recording the irradiance as a function of reference surface position might yield a pattern like that shown in Figure 2.2. 


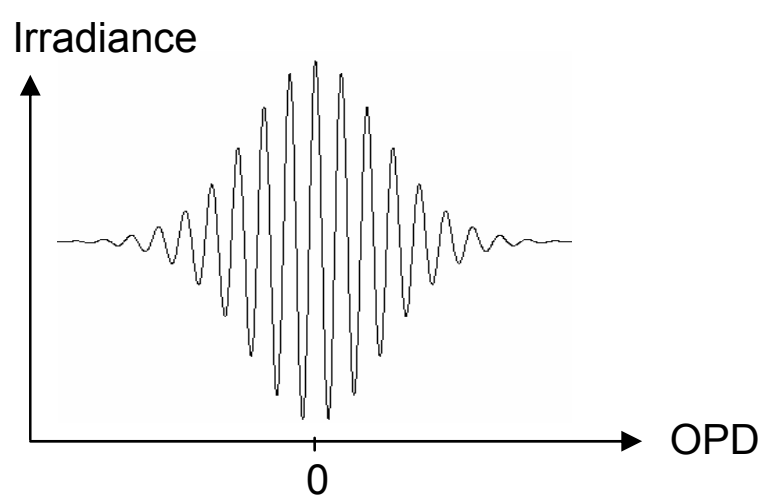

FIGURE 2.2 - Irradiance versus OPD for a modeled LCI signal.

Once such a signal is recorded, finding the center of the pattern gives the height of that point on the test surface relative to the neighboring points, which produce a similar signal, shifted along $\mathrm{z}$ according the local surface height. By using a detector array, such as a CCD camera, a 3D surface height map is produced from the three dimensional data set that was gathered.

Since the goal is to apply LCI to a thickness measurement, a second surface must also be measured. This is where commercial SWLI systems fail. Commercial SWLI systems are designed to examine a single surface, exposed to air. They are not usually capable of measuring the thickness of a material or profiling a 'buried' surface due to signal degradation from dispersion, the working distance of the interferometric objective, or other problems. Scanning through a small, thin sample to profile the back surface and find the thickness of the sample presents several complications. Perhaps the most obvious is that the scan distance will not simply be the physical thickness $t$, but a product of the thickness and group index of refraction, $\mathrm{N}_{\mathrm{g}}$ (Hopler, 1991). 


$$
N_{g}=n_{\lambda}-\left.\lambda \frac{d n}{d \lambda}\right|_{\lambda}
$$

In this context, the distinction between group index and phase index, $\mathrm{n}$, is secondary. The distinction is made for correctness. The primary problem is that to determine the thickness, the group index must be known so that the scan distance could be divided by $\mathrm{N}_{\mathrm{g}}$ to determine t. $\mathrm{N}_{\mathrm{g}}$ is certainly not known for the hydrogel samples. Somehow this information must be obtained for LCI to be a viable method for finding t. Other problems with measuring buried surfaces are due to higher order dispersion effects (Hirai, 2006), but they will be discussed later.

Most LCI systems use a converging wavefront incident on the test surface, as shown in Figure 2.3. Usually the scanning is accomplished by translating the entire interferometer relative to the sample, or vice versa.

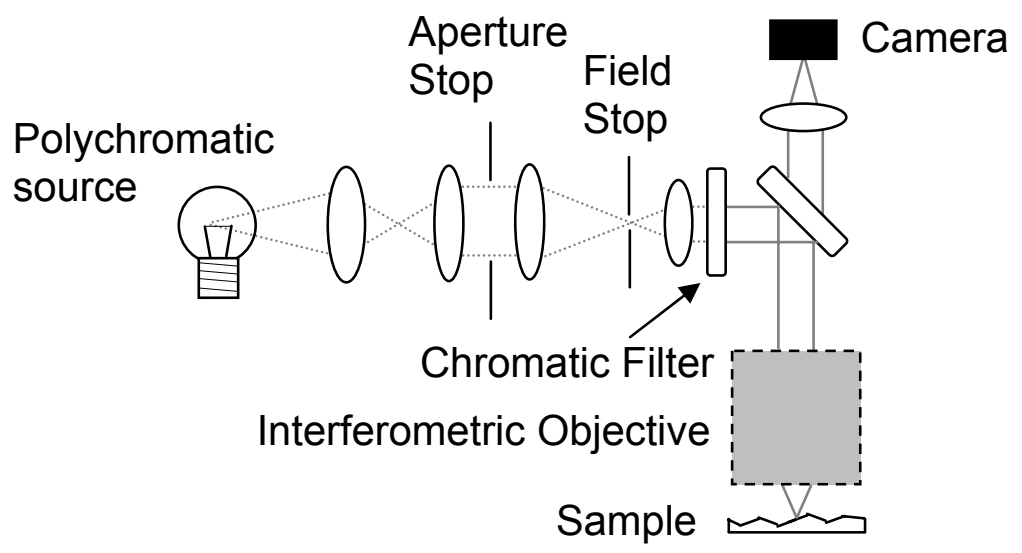

FIGURE 2.3 - Schematic of a SWLI system with a converging test beam (Goodwin, 2006). 
For a properly constructed interferometric objective, the coherence plane $(\mathrm{OPD}=0)$, which has the best visibility fringes, is axially coincident with the plane of best focus (Figure 2.4).

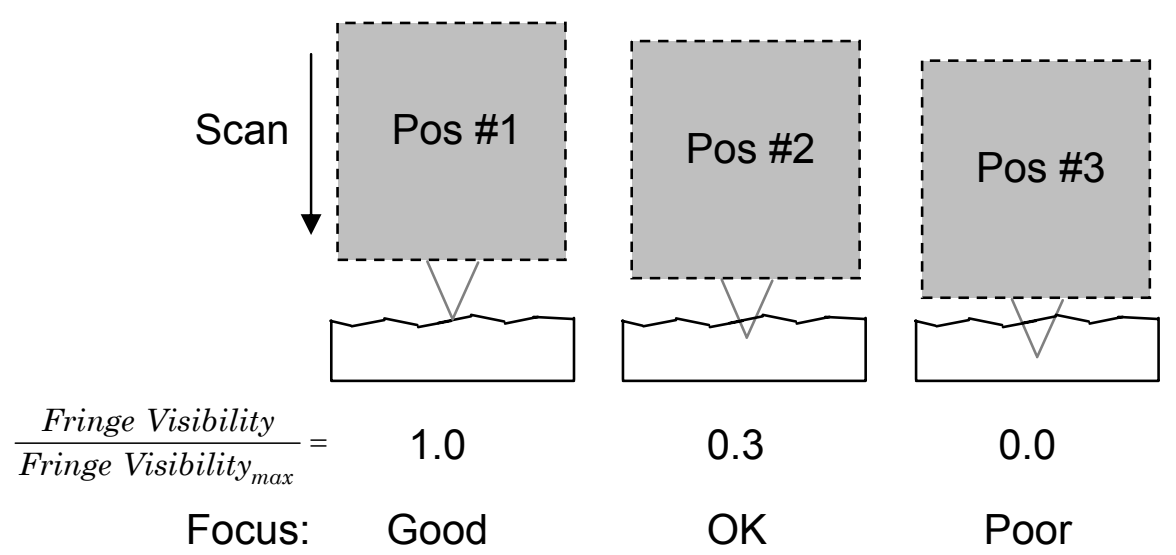

FIGURE 2.4 - Relationship between focus (ray paths) and fringe visibility for a well-constructed interferometric objective with the test beam in air.

In the case of examining a surface on the far side of a plane parallel plate of dispersive media, this relationship deteriorates. The problem is that, even while neglecting aberrations, the ideal focus position moves down according the OPL, which is further than the scan amount, as illustrated in Figure 2.5.

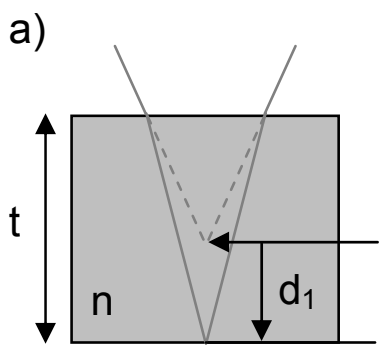

Focus position, no sample Focus position, with sample b)

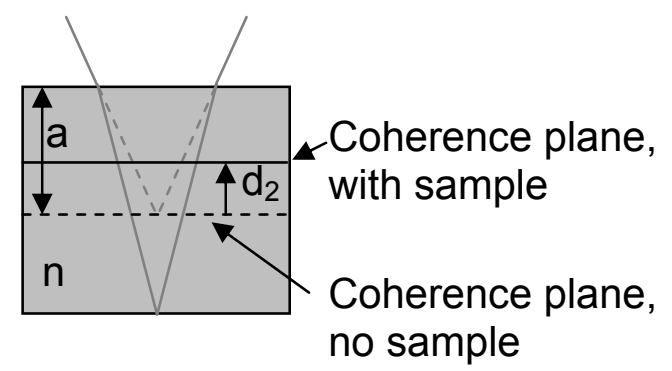

FIGURE 2.5 - As the SWLI scans through a dispersive media, the focus plane and the coherence plane move apart. 
Since the light travels the path shown above (solid line), the OPL of the light in the test arm of the interferometer (the light probing the test part) increases by a distance $d_{1}$. The equation for $d_{1}$ is using the approximation that $\tan (\theta)=\sin (\theta)$, where $\theta$ is the angle made by the converging ray incident on the plane parallel plate.

$$
d_{1}=t\left(\frac{n-1}{n}\right)
$$

In Figure 2.5b, the dashed lines indicate the focus location and the coherence plane with no dispersive sample. The horizontal solid lines represent the focus position (Figure 2.5a) and coherence plane (Figure 2.5b) after inserting the dispersive plane parallel plate. The location of the coherence plane $(\mathrm{OPD}=0)$, however, moves upward in the presence of dispersion a distance $\mathrm{d}_{2}$. This distance is a bit harder to define as it depends on the distance a, the distance from the top of the plane parallel plate to the original coherence plane (Figure 2.5b).

$$
d_{2}=a(n-1)
$$

Therefore, the more dispersive material there is in the test arm, the further the coherence plane and best focus will separate. For the case where the back surface of the plane parallel plate is the coherence plane with the sample in place, the ideal focus has moved a distance d:

$$
d=t\left(\frac{n-1}{n}\right)+t(n-1)
$$

For thin enough samples, this problem can be overcome somewhat. In the case of a hydrogel material, which must remain hydrated in some sort of cell, it becomes more difficult. In this case, the dispersive material includes the glass that makes up the top 
plate of the sample cell, the saline liquid that hydrates the material, and in the case of measuring the back surface, the sample itself (Figure 2.6).

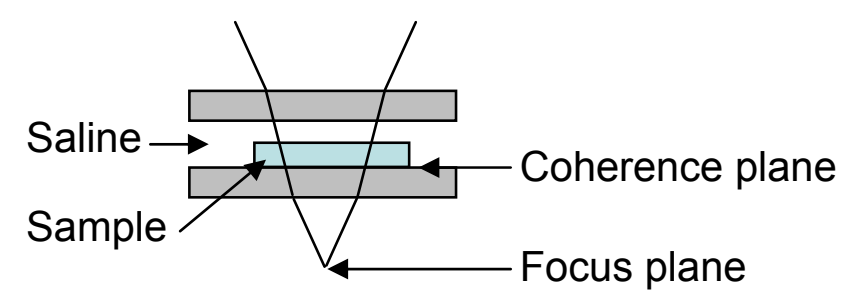

FIGURE 2.6 - Measuring the sample in a cell with a converging test beam.

The variation in blur with depth will degrade the spatial resolution on the sample. Microscopic spatial resolution of the test sample was not one of the requirements for this measurement, as the sample's spatial extent is relatively large $(\sim 10 \mathrm{~mm})$. By building a low coherence interferometer with a collimated test beam incident on the sample, the separation of the focus and coherence planes becomes less of an issue since the depth of focus (DOF) is directly dependent on the f/number of the imaging system.

\subsection{The Low Coherence Interferometer for Engage}

In order to adapt LCI to measuring the thickness of a hydrogel sample, the above issues must all be addressed. Engage uses a collimated low coherence beam in a TwymanGreen configuration, as shown in Figure 2.7. The entire interferometer is constructed vertically so that the liquid in the sample cell does not drain out. Instead of using an interferometric objective, the interferometer uses 2 " diameter optics, allowing for flexibility in sample size. 


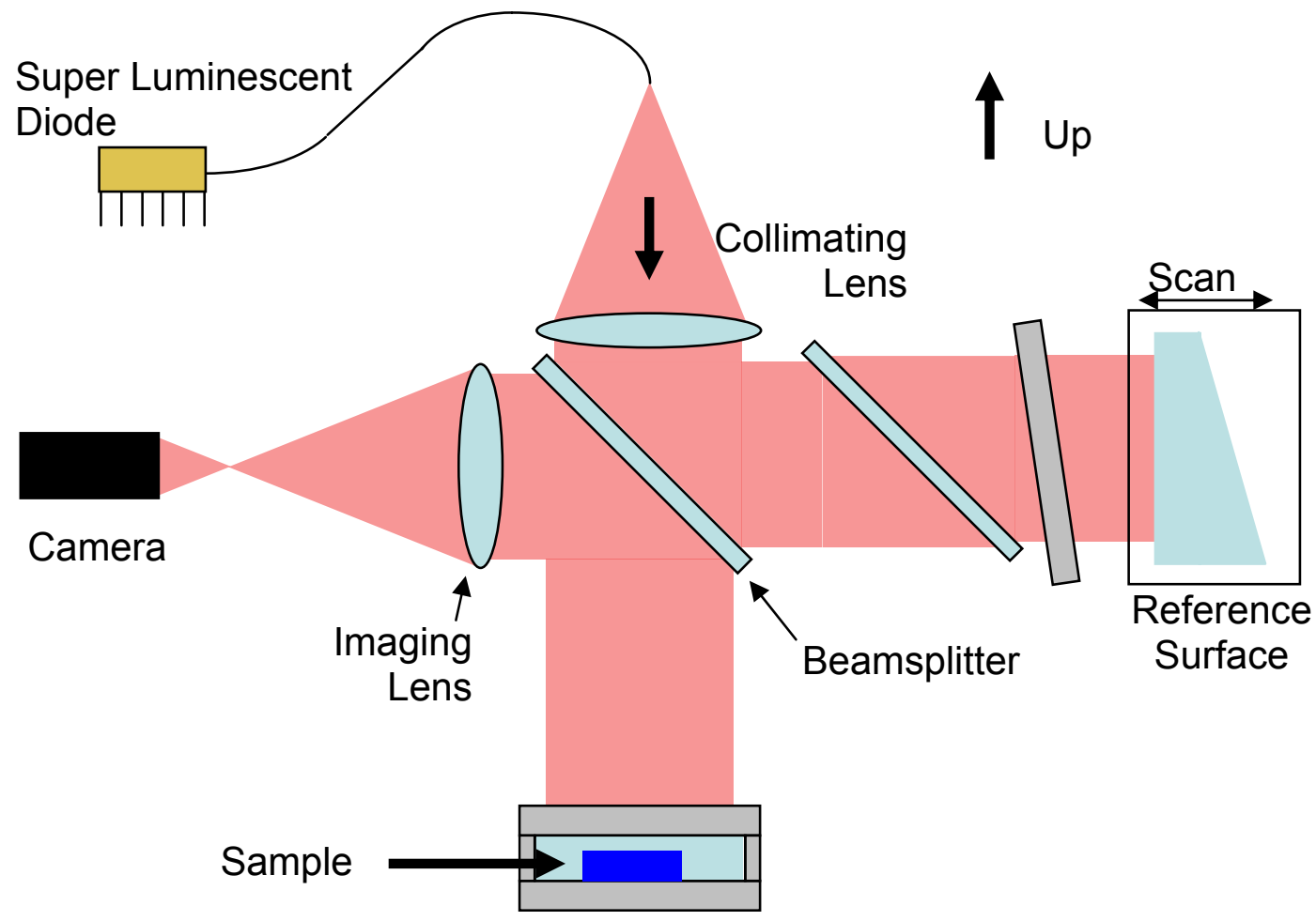

FIGURE 2.7 - Layout of the low coherence interferometer used in Engage.

The details of the interferometer hardware are discussed later; for now, the basic measurement principles and procedures are important. As mentioned in the previous section, simply locating the top and bottom surfaces of the test sample gives the product of the thickness $\mathrm{t}$ and group index $\mathrm{N}_{\mathrm{g}}$ : one equation and two unknowns. Therefore, the thickness measurement becomes a multi-step process.

Consider the measurement cell, or cuvette, to simply be a thin cavity between two glass plates. The bottom surface of the top plate (A) and the top surface of the bottom plate (B) are both well polished, flat $(\lambda / 20)$ optical surfaces (Figure 2.8a). Since the detector is 
a CCD camera, in this case with $1392 \times 1040$ pixels, an area on the cuvette is viewed. The dotted line on Figure $2.8 \mathrm{~b}$ shows the 'footprint' of the camera on the cuvette.

a)

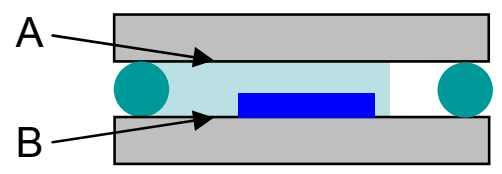

b)

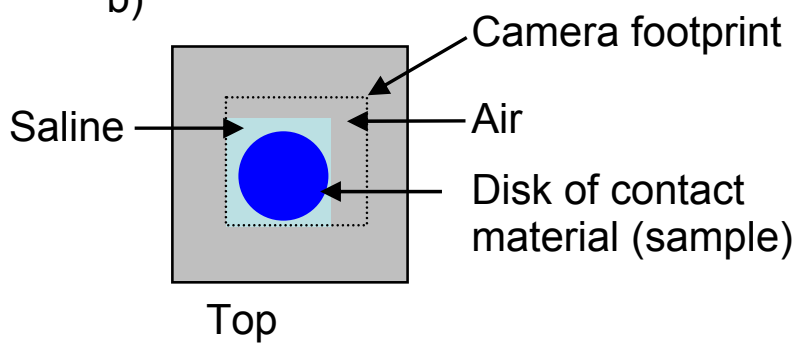

FIGURE 2.8 - Two views of the sample cuvette: a) Side view, and b) top view illustrating spatially multiplexing concept and camera footprint.

The area in the footprint is spatially multiplexed with three different materials to allow the physical thickness of the material to be determined. The area containing air is scanned first to determine the physical thickness of the cuvette, $\mathrm{t}_{1}(\mathrm{x}, \mathrm{y})$ (Figure 2.9).

Since the cuvette is made up of flats, the thickness in the air region will be a plane defined by a base thickness and slopes in the $\mathrm{x}$ and $\mathrm{y}$ directions (Equation 2.6), which can be extrapolated across the rest of the camera footprint.

$$
t_{1, f i t}(x, y)=a x+b y+c
$$

The extrapolation gives the physical thickness everywhere in the camera field of view.
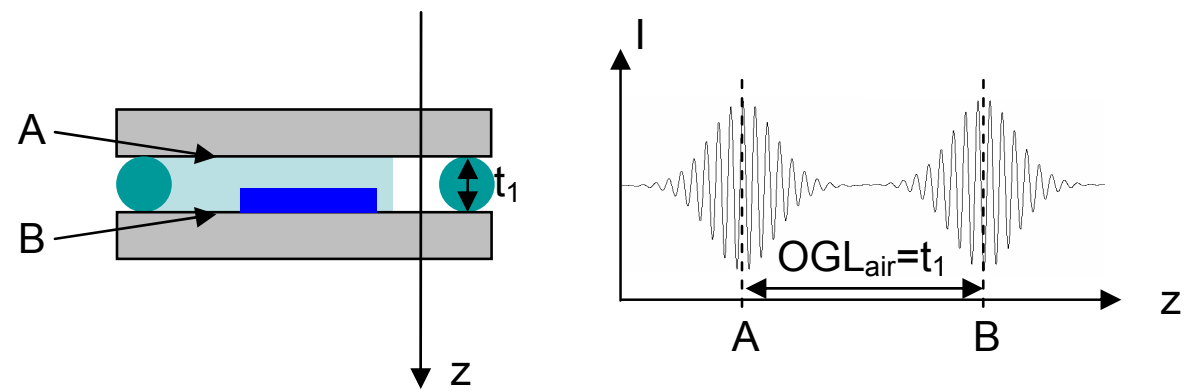

FIGURE 2.9 - Determining $\mathrm{t}_{1}(\mathrm{x}, \mathrm{y})$, the cuvette thickness, using LCI. 
Technically, the separation measured is the product of the physical thickness and the group index of air, $\mathrm{N}_{\mathrm{g} \text {,air }}$.

$$
O G L=t N_{g}
$$

The term OGL is defined as the optical group length, or the product of thickness and group index. In the wavelength region of the source $(\lambda=680 \mathrm{~nm})$, the group index of air is the same as the phase index to 7 parts in the $6^{\text {th }}$ decimal place.

$$
\begin{array}{cc}
N_{g, \text { air }}=n_{\lambda=680, \text { air }}-\left.\lambda \frac{d n_{\text {air }}}{d \lambda}\right|_{\lambda} & \text { Equation } 2.8 \\
N_{g, \text { air }}-n_{\text {air }}=-680 \frac{1.00027114-1.00027124}{685-675}=6.7 \times 10^{-6} & \text { Equation } 2.9
\end{array}
$$

Therefore, we can conclude:

$$
t_{1}=\frac{O G L_{\text {air }}}{N_{g, a i r}} \approx \frac{O G L_{\text {air }}}{n_{\text {air }}}=\frac{O G L_{\text {air }}}{1}=O G L_{\text {air }}
$$

The error in determining the physical thickness of the cuvette due to assuming $\mathrm{N}_{\mathrm{g} \text {,air }}=\mathrm{n}_{\text {air }}$ is about $2.3 \mathrm{~nm}$ for $\mathrm{t}_{1}=350 \mu \mathrm{m}$, which can be neglected relative to other errors, as will be shown later.

$$
\varepsilon=3.5 \times 10^{5} \mathrm{~nm} \cdot 6.7 \times 10^{-6}=2.3 \mathrm{~nm}
$$

An important note is that all index values reported by Engage are relative to $n_{\text {air }}=1.000$, which is the standard for most optics since most optical systems are used in air, not vacuum. 

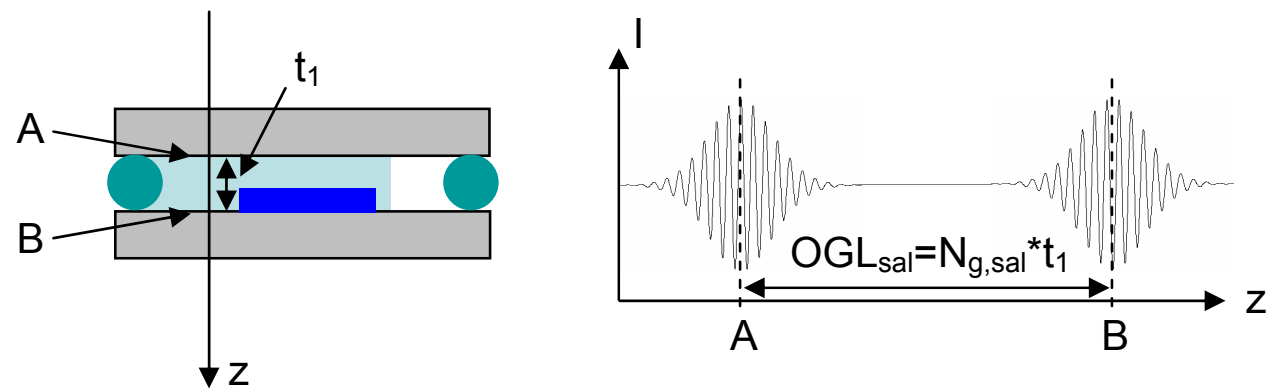

FIGURE 2.10 - Second LCI scan, to determine the group index of saline, $\mathrm{N}_{\mathrm{g} \text {,sal }}$.

Next, the saline column is measured. The separation between the top and bottom surfaces in the saline region is the product of $t_{1}$ and $\mathrm{N}_{\mathrm{g}, \text { sal }}$, the group index of the saline (Figure 2.10). Since $t_{1}$ is known, the value of $\mathrm{N}_{\mathrm{g}, \mathrm{sal}}$ is calculated:

$$
N_{g, s a l}=\frac{O G L_{s a l}}{t_{1}}
$$

Now, the reference mirror is scanned to locate the three surfaces in the column containing the test sample (Figure 2.11). The three surfaces are, in order from top to bottom; saline1 (the top plate/saline interface); saline2 (saline/top of sample interface); and saline3 (bottom of sample/top of bottom plate interface).
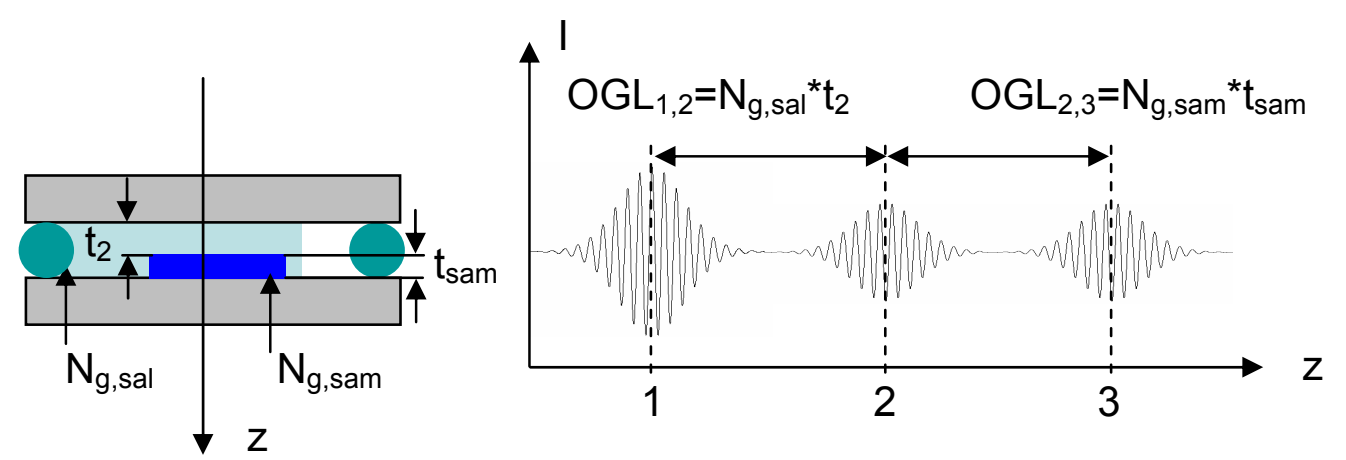

FIGURE 2.11 - Third LCI scan, to determine the sample thickness, $\mathrm{t}_{\mathrm{sam}}(\mathrm{x}, \mathrm{y})$. 
The separation between 1 and 2 is the product $\mathrm{N}_{\mathrm{g} \text {,sal }}$ and $\mathrm{t}_{2}$. Since $\mathrm{N}_{\mathrm{g} \text {,sal }}$ is known from the previous measurement, the thickness $t_{2}$ is easily found:

$$
t_{2}(x, y)=\frac{O G L_{1,2}(x, y)}{N_{g, s a l}}
$$

We want to use the difference between $t_{1}(x, y)$ and $t_{2}(x, y)$ to solve for the physical thickness of the sample, $\mathrm{t}_{\mathrm{sam}}(\mathrm{x}, \mathrm{y})$, so it is critical that the sample is in contact with the bottom glass plate. The sample thickness is simply:

$$
t_{\text {sam }}(x, y)=t_{1}(x, y)-t_{2}(x, y)
$$

The difference between 2 and 3 in this scan is the product of $\mathrm{N}_{\mathrm{g}, \mathrm{sam}}$ and $\mathrm{t}_{\mathrm{sam}}$. Therefore the group index of the test sample is determined. Note that this value is not the overall goal of Engage, just a useful byproduct of the measurement process.

$$
N_{g, s a m}=\frac{O G L_{2,3}(x, y)}{t_{\text {sam }}(x, y)}
$$

This is the summary of how the thickness measurement is done using low coherence interferometry. The next section discusses the details and consideration necessary to realizing this measurement technique.

\subsection{Detailed Theory of LCI}

The purpose of this section is to discuss the various necessary considerations for making the thickness measurement using low coherence interferometry. In this section, the need for these considerations will be discussed from a theoretical standpoint. A later section will talk about the physical realization of the fixes for these problems. 
Before the thickness measurement technique was proposed to the project sponsor as part of a workable refractive index measurement, it was important to simulate the measurement to try to spot potential problems that might be encountered. By creating a simulated signal that included the expected amount of noise, algorithms for finding the center of the pattern could be written and tested before building any hardware. The center of the pattern, or the maximum of the coherence envelope, coincides with the location of the physical boundary creating the signal.

\subsubsection{Origins of the Low Coherence Signal}

The interferometer for the low coherence thickness measurement is a Twyman-Green configuration, as is shown in Figure 2.12 below. The signal that is incident on the detector, in this case a CCD camera, comes from reflections in the test and reference arms of the interferometer. 


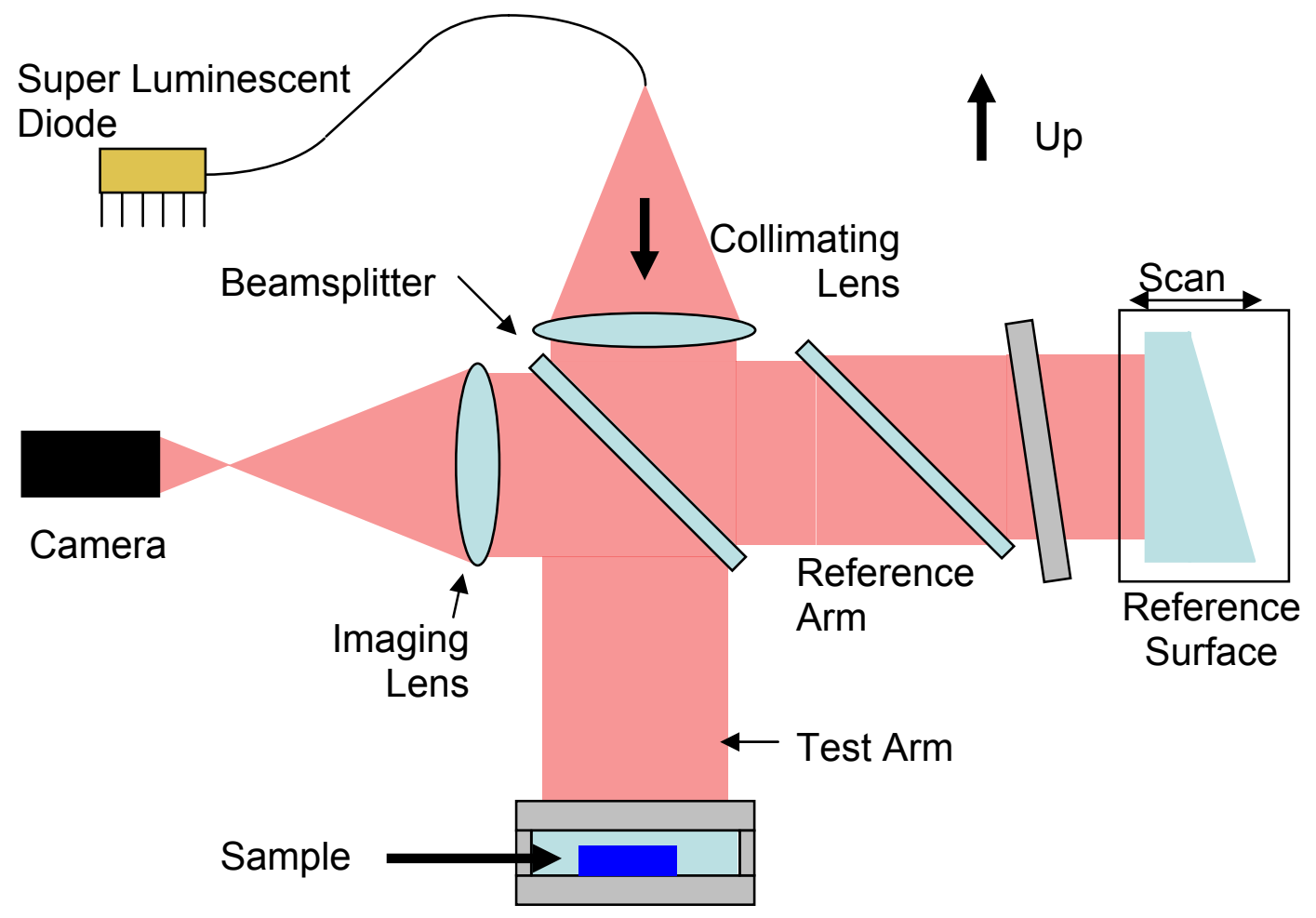

FIGURE 2.12 - The LCI used in Engage, indicating the test and reference arms of the interferometer.

Light is reflected whenever there is boundary between two regions with different refractive indices. In the test arm of Engage, the test beam is normally incident on the sample and the cuvette. The amount of light reflected from a boundary increases as the index difference increases (neglecting any coatings), according to the Fresnel equation for normal incidence.

$$
R=\frac{\left(n_{1}-n_{2}\right)^{2}}{\left(n_{1}+n_{2}\right)^{2}}
$$

Here $\mathrm{R}$ is the ratio of reflected light, and $\mathrm{n}_{1}$ and $\mathrm{n}_{2}$ are the refractive indices of the two materials forming the boundary. The value of $\mathrm{R}$ varies slightly with wavelength depending on the dispersion of the two materials, but this effect is minor. If the cuvette 
windows are made of BK7 glass and the incident wavelength is $680 \mathrm{~nm}$, the percent reflectance is summarized in Table 2.1 below for the various interfaces in the cuvette (Figure 2.13).

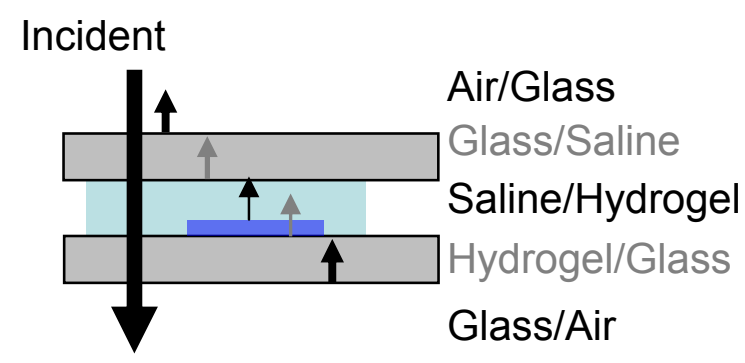

FIGURE 2.13 - Reflection from the five interfaces of the measurement cuvette.

\begin{tabular}{|c|c|c|c|}
\hline Interface & $\mathrm{n}_{1}$ & $\mathrm{n}_{2}$ & $\mathrm{R}$ \\
\hline Air/Glass & 1.000 & 1.514 & $4.18 \%$ \\
\hline Glass/Saline & 1.514 & 1.336 & $0.390 \%$ \\
\hline Saline/Hydrogel & 1.336 & 1.412 & $0.076 \%$ \\
\hline Hydrogel/Glass & 1.412 & 1.514 & $0.122 \%$ \\
\hline Glass/Air & 1.514 & 1.000 & $4.18 \%$ \\
\hline
\end{tabular}

TABLE 2.1 - Example reflectance coefficients for various interfaces in the sample cell.

The top surface of the top plate and bottom surface of the bottom plate are both antireflection coated to decrease the light reflected back to the camera. The details of the cuvette are discussed later. 
The development that follows considers the origins of the LCI signal in the absence of dispersive materials. The inclusion of dispersive materials in the model is important, and will be addressed in a later section.

Fringe visibility is maximized when the optical group difference (OGD) between the reference surface and an interface in the test arm is zero.

$$
O G D=O G L_{\text {Reference }}-O G L_{\text {Test }}
$$

For now, the irradiance signal at a single pixel will be considered. The distance away from $\mathrm{OGD}=0$ where fringes can still be observed is related to the coherence length $\left(1_{\mathrm{c}}\right)$ of the source, which depends on the center wavelength of the source, $\lambda_{\mathrm{c}}$, and the width of the spectral distribution, $\Delta \lambda$.

$$
l_{c}=\frac{\lambda_{c}^{2}}{\Delta \lambda}
$$

The spectral distribution with respect to frequency is commonly referred to as the power spectrum of the source (Figure 2.14).

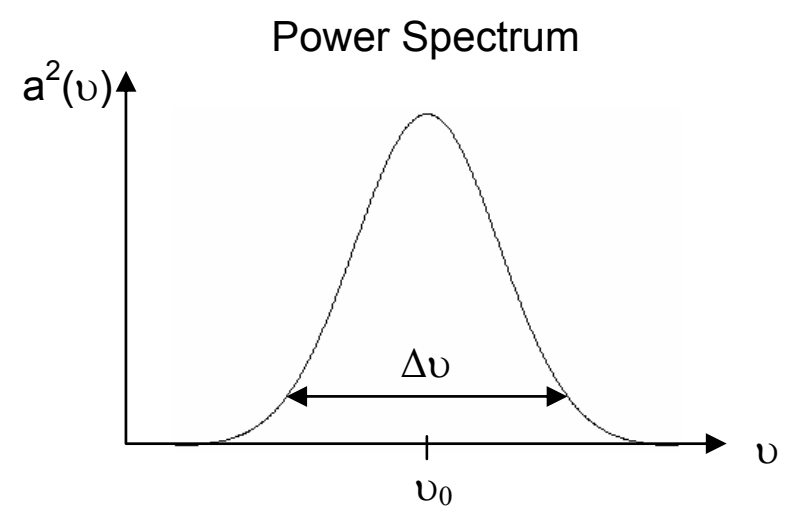

FIGURE 2.14 - An example of a power spectrum of an LCI source. 
A source with a Gaussian power spectrum in the frequency domain can be described as follows, where $\mathrm{a}^{2}$ is the power spectrum, $v$ is frequency and $v_{0}$ is the center frequency:

$$
\begin{gathered}
a^{2}(v)=e^{-\pi\left(\frac{v-v_{0}}{\Delta v}\right)^{2}}=\operatorname{Gaus}\left(\frac{v-v_{0}}{\Delta v}\right) \\
c=\lambda v \\
\Delta v=-\frac{c}{\lambda^{2}} \Delta \lambda
\end{gathered}
$$

Equation 2.21

For narrow source bandwidths (small $\Delta \lambda$, small $\Delta v$ ), a Gaussian spectral distribution versus frequency is very close to a Gaussian spectral distribution versus wavelength.

The irradiance as a function of $\mathrm{z}$ (reference mirror position) was shown in Figure 2.2 and is given as:

$$
I(z) \approx I_{w}+2 I_{w} m\left(\frac{z}{c}\right) \cos \left(\phi\left(\frac{z}{c}\right)\right)
$$

Here $\mathrm{c}$ is the speed of light and $v$ is the optical frequency of the light. $\mathrm{I}_{\mathrm{w}}$ is the integral of the source power spectrum (Equation 2.23) and is the DC offset of the irradiance signal. The function $\mathrm{m}()$ is the normalized Fourier transform of the source power spectrum (Equation 2.24), and is also the component of I(z) that is referred to as the coherence envelope. The function $\varphi(\mathrm{z} / \mathrm{c})$ is the phase of the Fourier transform of the power spectrum (Equation 2.25). The cosine of $\varphi(z / c)$ gives the fringes as a function of $z$ (Milster, 2007).

$$
I_{w}=\int_{-\infty}^{+\infty} a^{2}(v) d v
$$




$$
\begin{gathered}
m\left(\frac{z}{c}\right)=\frac{\left|F T\left\{a^{2}(v)\right\}_{\frac{z}{c}}\right|}{I_{w}} \\
\phi\left(\frac{z}{c}\right)=\arg \left[F T\left\{a^{2}(v)\right\}_{\frac{z}{c}}\right]
\end{gathered}
$$

Equation 2.24

Equation 2.25

In the case of the Gaussian power spectrum defined above, the Fourier transform, and hence the coherence envelope, is a Gaussian as well.

$$
\begin{gathered}
a^{2}(v)=e^{-\pi\left(\frac{\nu-v_{0}}{\Delta v}\right)^{2}}=\operatorname{Gaus}\left(\frac{v-v_{0}}{\Delta v}\right) \\
I_{w}=\int_{-\infty}^{+\infty} a^{2}(v) d v=\int_{-\infty}^{+\infty} e^{-\pi\left(\frac{v-v_{0}}{\Delta v}\right)^{2}} d v=|\Delta v| \\
\left|F T\left\{a^{2}(v)\right\}\right|=\left|\Delta v \cdot \operatorname{Gaus}\left(\Delta v \frac{z}{c}\right) e^{-i 2 \pi v_{0} \frac{z}{c} \mid}\right|=\left|\Delta v \cdot e^{-\pi\left(\Delta v \frac{z}{c}\right)^{2}} e^{-i 2 \pi v_{0} \frac{z}{c}}\right| \\
\phi\left(\frac{z}{c}\right)=\arg \left[\Delta v \cdot \operatorname{Gaus}\left(\Delta v \frac{z}{c}\right) e^{-i 2 \pi v_{0} \frac{z}{c}}\right]=2 \pi v_{0} \frac{z}{c}
\end{gathered}
$$

Equation 2.28

Combining equations 2.22 through 2.30 gives I(z) in the case of a source with a Gaussian power spectrum.

$$
I(z)=I_{w}+2 I_{w} m\left(\frac{z}{c}\right) \cos \left(\phi\left(\frac{z}{c}\right)\right)=\Delta v+2 \Delta v \cdot \operatorname{Gaus}\left(\Delta v \frac{z}{c}\right) \cos \left(2 \pi v_{0} \frac{z}{c}\right)
$$

Equation 2.31 


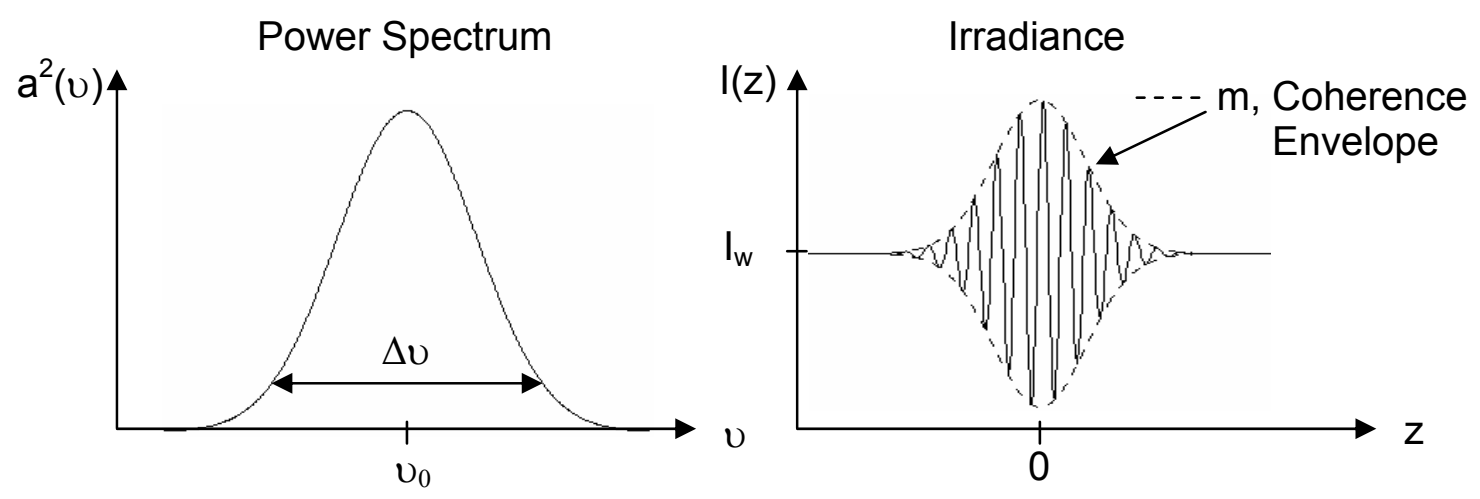

FIGURE 2.15 - Gaussian power spectrum and associated irradiance pattern, I(z). Superimposed dotted envelope indicates the coherence envelope.

Looking at the equation for irradiance shows that the frequency of the fringes is dictated by the center frequency (or wavelength) of the source distribution. Equation 2.31 neglects effects due to dispersive materials, so a bright fringe is coincident with the peak of the coherence envelope (Figure 2.15). In general, this coincidence cannot be assumed about a low coherence signal.

The coherence envelope is a Fourier transform of the power spectrum, so some basic properties of Fourier transforms provide insight. As the power spectrum narrows, the coherence envelope broadens. In the extreme case of a laser source with an infinitely narrow power spectrum (a delta function), the coherence envelope becomes infinitely wide, or can simply be represented as a constant value (Gaskill, 1978). This property is also contained in the expression for coherence length (Equation 2.18). A plot of I(z) for a laser source is shown in Figure 2.16. 


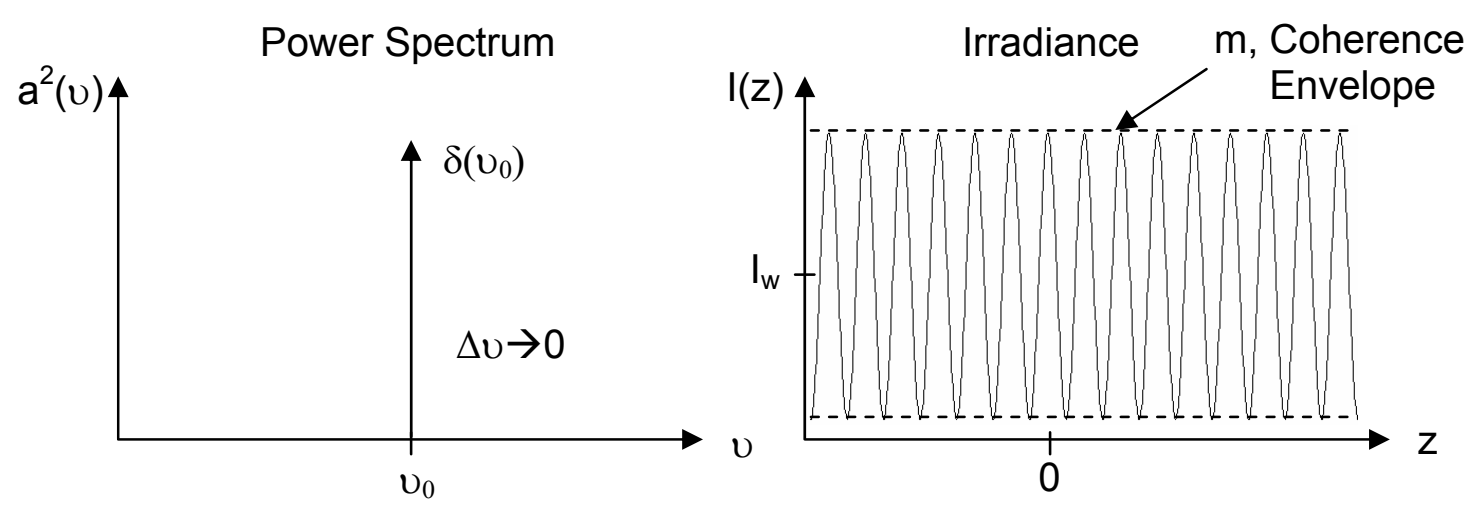

FIGURE 2.16 - Power spectrum and I(z) for a laser.

\subsubsection{Sources of LCI Signal Degradation}

The previous section discussed the origins of the low coherence signal and its dependence on the spectral characteristics of the source. This section will discuss some of the causes of signal degradation in a real system, such as one used to measure the physical thickness of hydrogel materials. The effect of these degradations on recovering the center of the coherence envelope depends on the algorithm used, so these effects will be discussed in the section on algorithms.

Perhaps the most obvious deviation from the ideal signal shown in Figure 2.2 is that the fringe visibility, $\mathrm{V}$, will not reach a maximum of 1 at the peak of the envelope.

$$
V=\frac{I_{\max }-I_{\min }}{I_{\max }+I_{\min }}
$$

This is due to the additional light reflected from other interfaces in the test arm of the interferometer. This reflected light contributes to the DC component of the signal $\left(\mathrm{I}_{\mathrm{w}}\right)$, 
but is incoherent and does not increase the visibility of the fringes. Allowing the camera to integrate the signal longer will only result in saturation near the peak of the coherence function. This problem is common to many interferometers.

Another consideration is sampling. The importance of sampling interval and its relationship to surface recovery will be discussed later, but it is important to consider that the low coherence signal must be discretely sampled along $\mathrm{z}$ and cannot be represented in a continuous form, as the figures show.

The signal discussed above does not consider effects from sending a spectrally broad signal through a dispersive medium. Conceptually, the problem can be described as follows. Consider a plate of glass that is in one arm of the Twyman-Green interferometer of Engage. The refractive index of that plate depends on wavelength. Since the low coherence source is made up of light over a finite range of wavelengths, each spectral component is delayed a different amount (Figure 2.17).

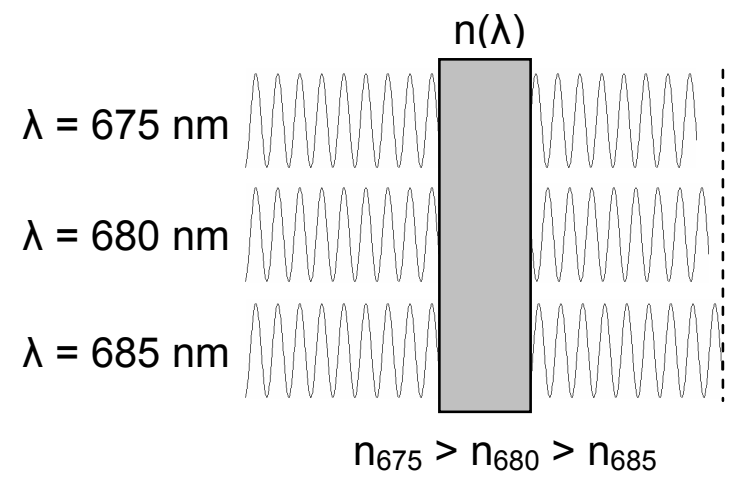

FIGURE 2.17 - Since $\mathrm{n}$ is larger at $\lambda=675 \mathrm{~nm}$, that wavelength has more OPL through the glass plate. 
If the refractive index varied linearly with wavelength, the coherence envelope would be delayed by a term dependent on the slope of the index with respect to wavelength. The difference between the delay experienced by the fringes and the delay experienced by the coherence envelope in this case is the same as the ratio between the group index of refraction, $\mathrm{N}_{\mathrm{g}}$ and the phase index, $\mathrm{n}$. In other words, the fringes are delayed by OPD $\left(\mathrm{S}_{\mathrm{OPD}}\right)$ while the coherence envelope is delayed by the OGD $\left(\mathrm{S}_{\mathrm{OGD}}\right)$ (Figure 2.18a-b). The difference depends on the slope of the refractive index with respect to wavelength. This will be shown explicitly in the following sections.

a) No Dispersive Material

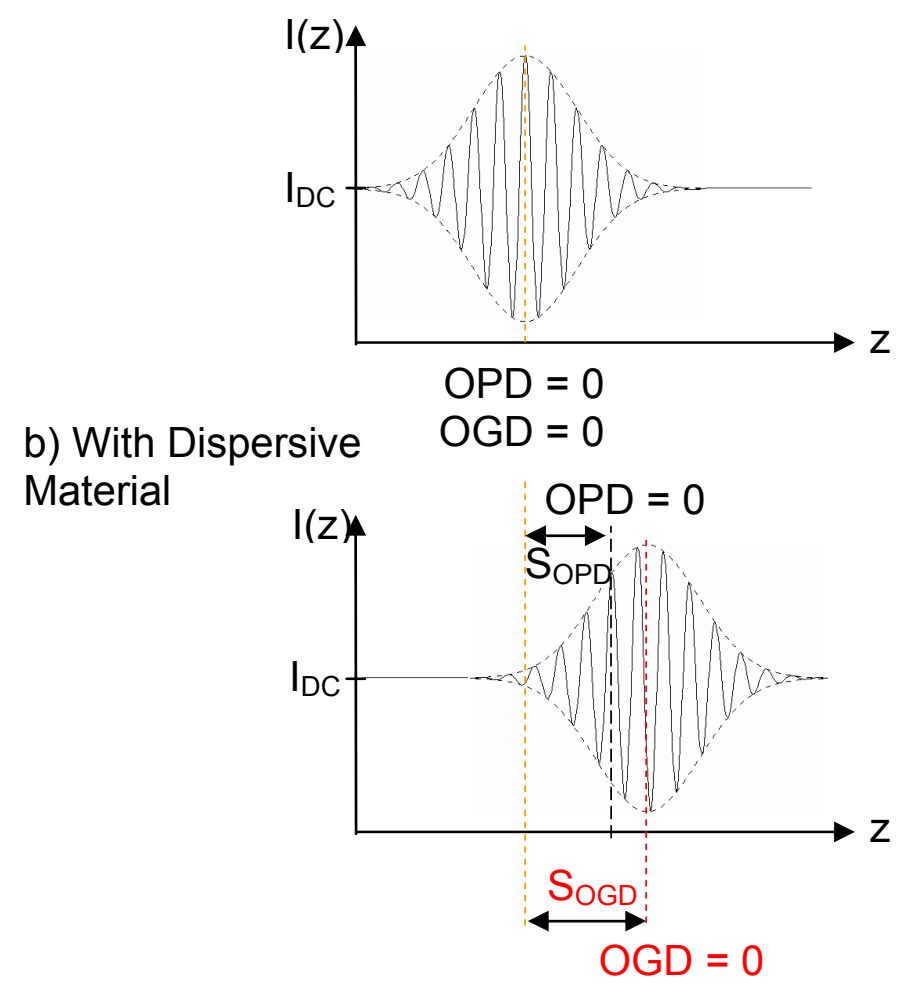

FIGURE 2.18 - The fringe pattern shifts according to the OPD while the coherence envelope shifts by the OGD. This is shown with a modeled LCI signal a) without dispersive material and $b$ ) with dispersive material. 
In low coherence interferometry, the surface location is found by determining the center of the coherence envelope. The irradiance recorded is modulated by the fringes with a wavelength equal to the center wavelength of the spectral distribution of the source. Therefore, the fringes are the available information for obtaining the coherence envelope. When the low coherence signal passes through a dispersive medium with index $\mathrm{n}$, the fringes are delayed according to OPL. The envelope, the feature containing the surface locations, is delayed according to the OGL.

$$
\begin{gathered}
O P L=n t \quad O G L=N_{g} t \\
N_{g}=n_{\lambda_{c}}-\left.\lambda_{c} \frac{d n}{d \lambda}\right|_{\lambda_{c}}
\end{gathered}
$$

For now, consider the refractive index as varying linearly with wavelength. The group index is also a function of wavelength. If the slope of the index goes to zero, as is approximately the case in air, then group index and phase index are equivalent. 


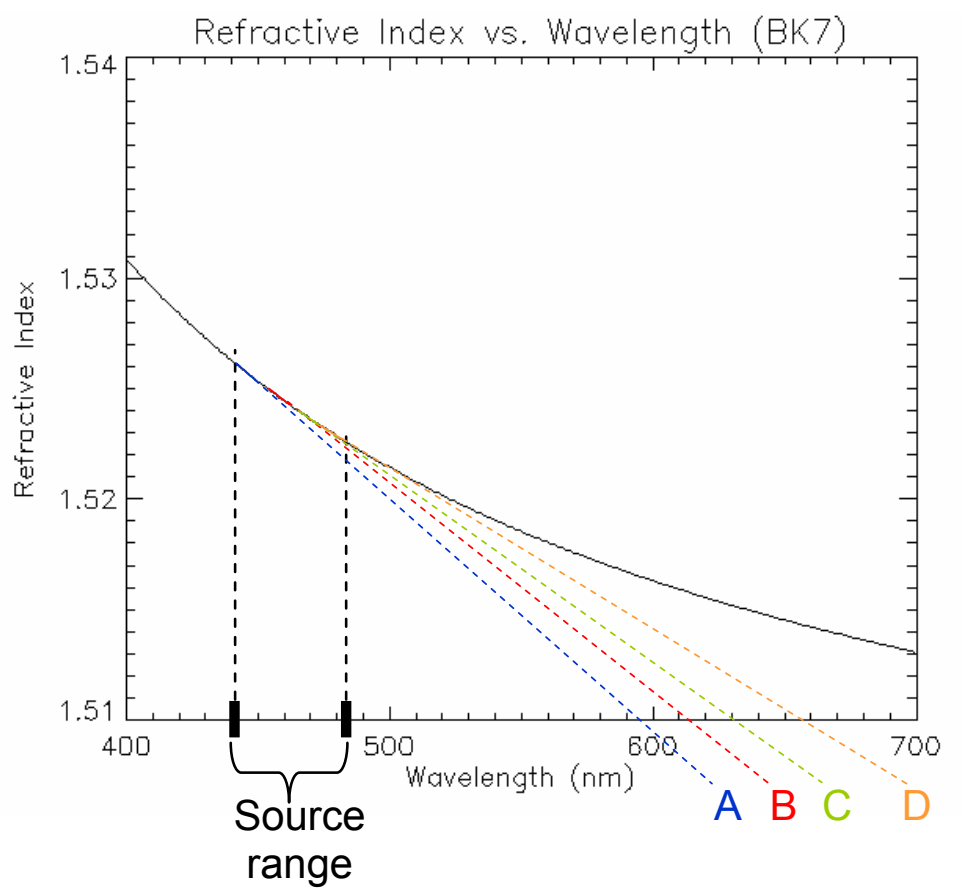

FIGURE 2.19 - Second order dispersion leads to variations in the local slope of refractive index which leads to a distortion of the coherence envelope.

$$
\left|\frac{d n}{d \lambda}\right|_{A}>\left|\frac{d n}{d \lambda}\right|_{B}>\left|\frac{d n}{d \lambda}\right|_{C}>\left|\frac{d n}{d \lambda}\right|_{D}
$$

Unfortunately, refractive index does not vary linearly with wavelength, but is better approximated by a higher order polynomial. First, consider a quadratic term for the index as a function of wavelength. If the source distribution is broken into smaller wavelength sections over which the index of refraction can be considered linear, the slope of the lines will vary for each small wavelength section (Figure 2.19). Therefore each portion of the packet will be delayed differently. The result is that the coherence envelope is no longer symmetric about the peak value after passing through a dispersive material (Figure 2.20). The coherence envelope also increases in width. 


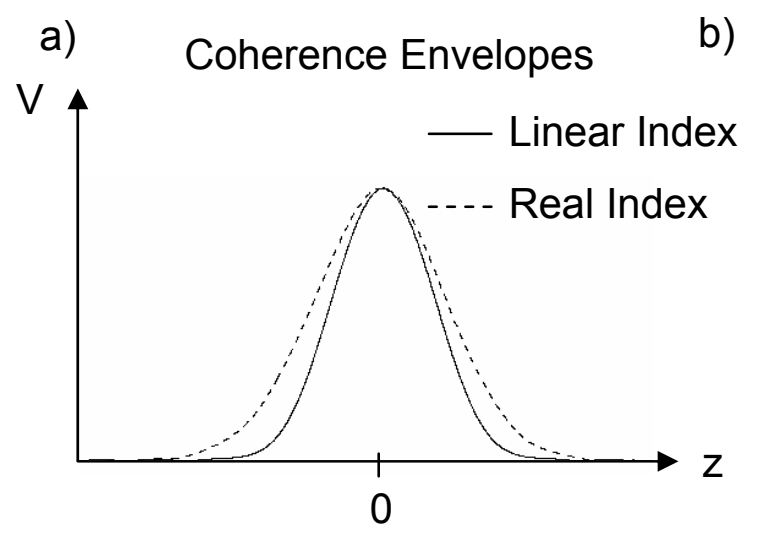

b) (Real Envelope) - (Linear Envelope)

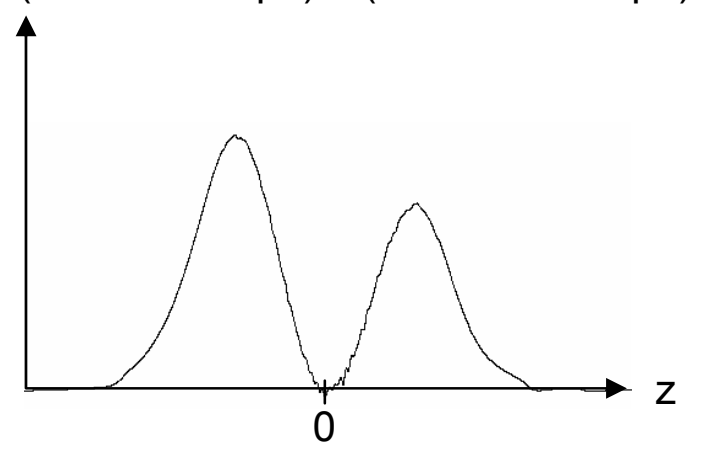

FIGURE 2.20 - Comparison of coherence envelope for linear and real refractive index versus wavelength: a) envelopes plotted together, and b) difference plot. Note the lack of symmetry for the real index.

Third order and higher terms will further distort the low coherence signal. The effects of second and higher order terms depend heavily on the spectral bandwidth of the source. The narrower the source, the lower the effects from higher orders of dispersion. The center wavelength of the source will change the effect from these higher order terms, as the higher order terms tend to have larger effects at shorter wavelengths. The linear effect $\left(\mathrm{N}_{\mathrm{g}}\right.$ vs. $\left.\mathrm{n}\right)$ will not diminish simply by narrowing the source bandwidth, so long as the center of the coherence envelope is the quantity being measured (Hopler, 1991). This argument summarizes the conclusions from the mathematical approach to follow.

\subsubsection{Derivation of the Effects of Dispersion on the LCI Signal}

For the examples following the mathematics, the dispersion of BK7 glass will be used to illustrate the problems caused by dispersive materials since the index of BK7 with respect 
to wavelength is well characterized. The effects from sending low coherent light through a hydrogel sample will be similarly disruptive.

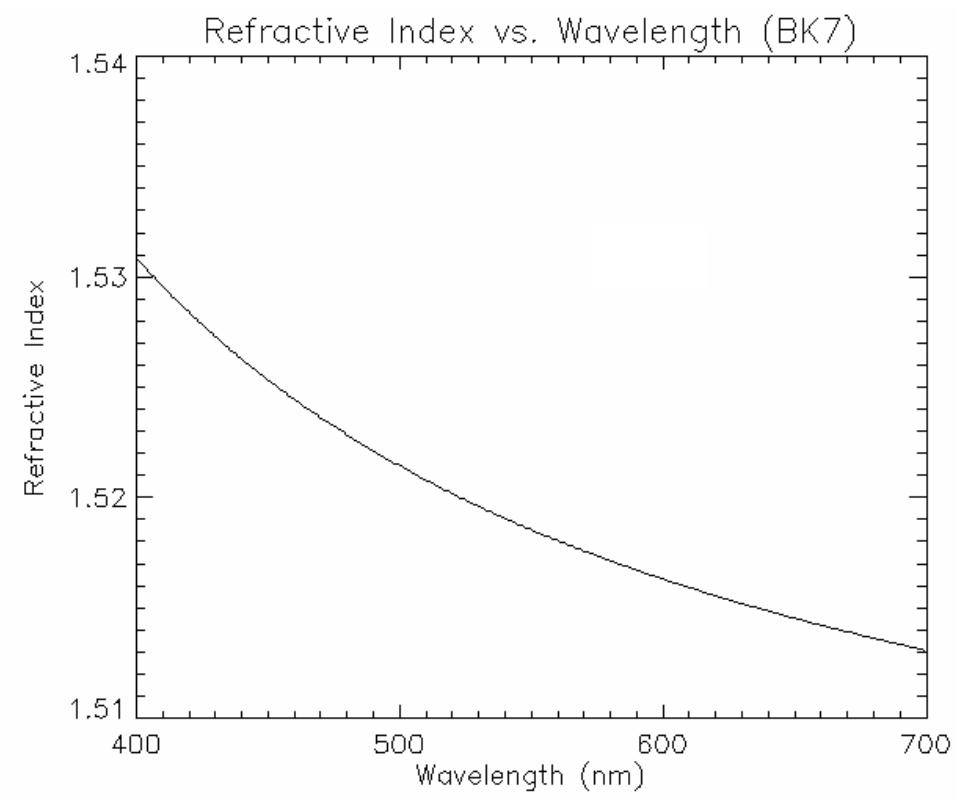

FIGURE 2.21 - Plot of index versus wavelength for BK7.

As can be seen for the plot of refractive index versus wavelength in Figure 2.21, index is not linear with respect to wavelength. A low coherence light source contains light from every frequency between the minimum and maximum frequency with varying amplitudes, $\mathrm{a}_{v}$ (Figure 2.15). Consider this source in a simple Twyman-Green interferometer, where there is a plate of glass in the test arm of thickness $t$ and index $n(\lambda)$ (Figure 2.22). 


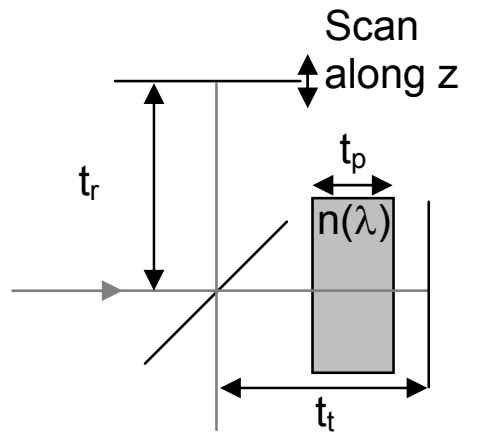

FIGURE 2.22 - Glass plate in test arm of a Twyman-Green Interferometer. The beamsplitter has zero thickness.

The goal here is to obtain a quantitative solution for the effects of dispersion on the coherence envelope. The irradiance from a single frequency of the power spectrum after the two beams recombine comes from the two-beam interference equation and is represented by $I_{v}$.

$$
I_{v}(z)=a_{v}^{2}\left[1+\cos \left(2 \pi \frac{v}{c} 2\left[t_{t}+\left(n_{v}-1\right) t_{p}-t_{r}+z\right]\right)\right]
$$

The factor of 2 comes from the double pass nature of this setup. The reference mirror moves in $z$, so $t_{r}$ has been replaced by $t_{r}+z$. Next, a sum over all frequencies is performed to determine the form of the coherence envelope as a function of mirror position, $\mathrm{I}_{\mathrm{env}}(\mathrm{z})$.

$$
I_{\text {env }}(z)=\sum_{0}^{\infty} I_{v}(z)=\int_{0}^{\infty} I_{v}(z) d v=\int_{0}^{\infty} a^{2}(v) d v+\int_{0}^{\infty} a^{2}(v) \cos \left(4 \pi \frac{v}{c}\left[t_{t}+\left(n_{v}-1\right) t_{p}-t_{r}+z\right]\right) d v
$$


The integral over all space of the power spectrum is simply $I_{w}$ (Equation 2.23). The cosine is now expressed as the real part of the complex exponential term via Euler's formula. Note the lower limit of integration changes to $-\infty$.

$$
\begin{gathered}
\int_{0}^{\infty} \cos (a) d a=\frac{1}{2} \int_{-\infty}^{\infty} e^{i a} d a \\
I_{e n v}(z)=I_{w}+\int_{-\infty}^{\infty} \frac{1}{2} a^{2}(v) e^{i 4 \pi \frac{v}{c}\left[t_{t}+\left(n_{v}-1\right) t_{p}-t_{r}+z\right]} d v
\end{gathered}
$$

Now, the index as a function of wavelength is expanded as a Taylor series. A second order fit of index over a narrow spectral band $(<20 \mathrm{~nm})$ is a good fit.

$$
n_{\lambda}=n_{0}+\left.\left(\lambda-\lambda_{0}\right) \frac{d n}{d \lambda}\right|_{\lambda_{0}}+\left.\frac{1}{2}\left(\lambda-\lambda_{0}\right)^{2} \frac{d^{2} n}{d \lambda^{2}}\right|_{\lambda_{0}}+\ldots
$$

Convert to $\mathrm{n}_{v}$ using $\mathrm{c}=\lambda v$.

$$
n_{v}=n_{0}+\left.c\left(\frac{1}{v}-\frac{1}{v_{0}}\right) \frac{d n}{d \lambda}\right|_{\lambda_{0}}+\left.\frac{c^{2}}{2}\left(\frac{1}{v}-\frac{1}{v_{0}}\right)^{2} \frac{d^{2} n}{d \lambda^{2}}\right|_{\lambda_{0}}+\ldots
$$

Insert equation 2.41 into equation 2.39:

$$
I_{\text {env }}(z)=I_{w}+\int_{-\infty}^{\infty} \frac{1}{2} a^{2}(v) e^{i 4 \pi \frac{v}{c}\left[t_{t}+\left(n_{0}+\left.c\left(\frac{1}{v}-\frac{1}{v_{0}}\right) \frac{d n}{d \lambda}\right|_{\lambda_{0}}+\left.\frac{c^{2}}{2}\left(\frac{1}{v}-\frac{1}{v_{0}}\right)^{2} \frac{d^{2} n}{d \lambda^{2}}\right|_{\lambda_{0}}-1\right) t_{p}-t_{r}+z\right]} d v
$$

Equation 2.42

Rearranging terms: 


$$
\begin{aligned}
& I_{\text {env }}(z)=I_{w}+\int_{-\infty}^{\infty} \frac{1}{2} a^{2}(v) e^{i 4 \pi \frac{v}{c}\left[t_{t}+\left(n_{0}+\left.c\left(\frac{1}{v}-\frac{1}{v_{0}}\right) \frac{d n}{d \lambda}\right|_{\lambda_{0}}+\left.\frac{c^{2}}{2}\left(\frac{1}{v}-\frac{1}{v_{0}}\right)^{2} \frac{d^{2} n}{d \lambda^{2}}\right|_{\lambda_{0}}-1\right) t_{p}-t_{r}+z\right]} d v
\end{aligned}
$$

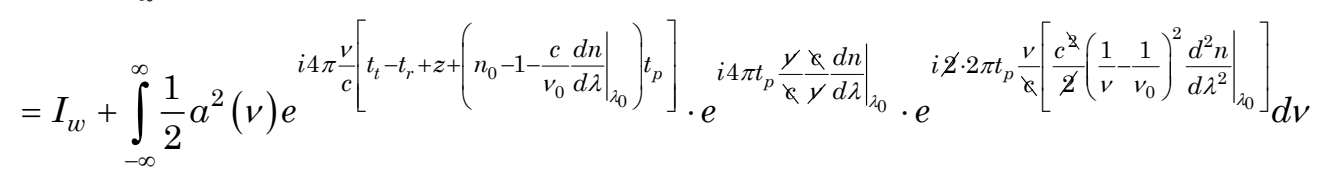

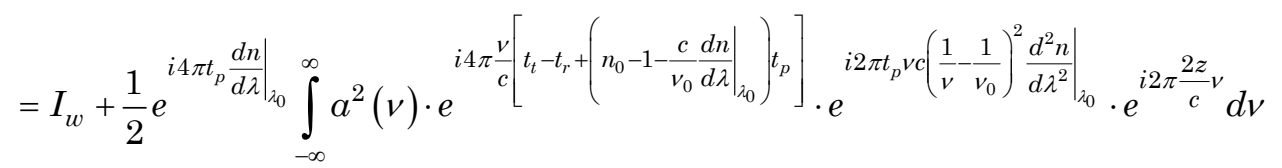

Equation 2.43

The last term that has been pulled out on the right is a Fourier transform kernel, simplifying the integral to a series of Fourier transforms. It is well known the Fourier transform of a product is equivalent to the convolution of the individual Fourier transforms (Gaskill, 1978).

$$
F T\{A \cdot B \cdot C\}=F T\{A\} * F T\{B\} * F T\{C\}
$$

The Fourier transform of the power spectrum depends on the power spectrum and for now will be left in the general form:

$$
A: F T\left\{a^{2}(v)\right\} \rightarrow A^{2}\left(\frac{2 z}{c}\right)
$$

The Fourier transform of the second term can be simplified by substituting $\mathrm{K}$ for the bulk of the terms: 


$$
\begin{aligned}
B: F T\left\{e^{i 4 \pi \frac{v}{c}\left[t_{t}-t_{r}+\left(n_{0}-1-\left.\frac{c}{v_{0}} \frac{d n}{d \lambda}\right|_{\lambda_{0}}\right) t_{p}\right]}\right\} & =F T\left\{e^{i 2 \pi v K}\right\} \rightarrow \delta\left(\frac{2 z}{c}-K\right) \\
& =\delta\left(\frac{2 z}{c}-\frac{2}{c}\left[t_{t}-t_{r}+\left(n_{0}-1-\left.\frac{c}{v_{0}} \frac{d n}{d \lambda}\right|_{\lambda_{0}}\right) t_{p}\right]\right)
\end{aligned}
$$

Where $K=\frac{2}{c}\left[t_{t}-t_{r}+\left(n_{0}-1-\left.\frac{c}{v_{0}} \frac{d n}{d \lambda}\right|_{\lambda_{0}}\right) t_{p}\right]$

Equation 2.46

The Fourier transform of the third component becomes much more difficult. The important thing to note about the resulting function is that it lacks symmetry and has a finite width along $\mathrm{z}$.

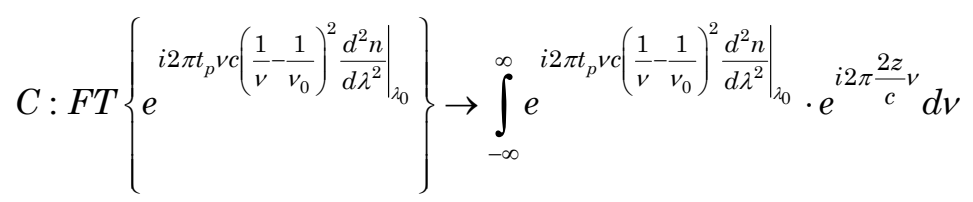

Equation 2.47

Now the final form of the coherence envelope can be expressed as:

$$
I_{\text {env }}(z)=I_{w}+\frac{1}{2} e^{\left.i 4 \pi t_{p} \frac{d n}{d \lambda}\right|_{\lambda_{0}}}\left[A^{2}\left(\frac{2 z}{c}\right) * \delta\left(\frac{2 z}{c}-\frac{2}{c}\left[t_{t}-t_{r}+\left(n_{0}-1-\left.\frac{c}{v_{0}} \frac{d n}{d \lambda}\right|_{\lambda_{0}}\right) t_{p}\right]\right) F T\{C\}\right]
$$

Equation 2.48

Equation 2.48 gives a general form of the effects the low coherence signal traversing through a plate of dispersive material, such as a liquid, glass, or hydrogel. The dispersive material has a refractive index as a function of wavelength that is approximated by a second order polynomial and has a thickness $t_{p}$. 


\subsection{Dispersion Effects versus Index, Material and Source Characteristics}

It is important to justify the assumption that a second order polynomial is sufficient for modeling the effects of dispersion. The model used depends on the spectral bandwidth of the light source. This model works well for bandwidths on the order of 30 nanometers or less. Figure 2.23 compares the real refractive index of BK7 glass with both a linear model and a quadratic model.
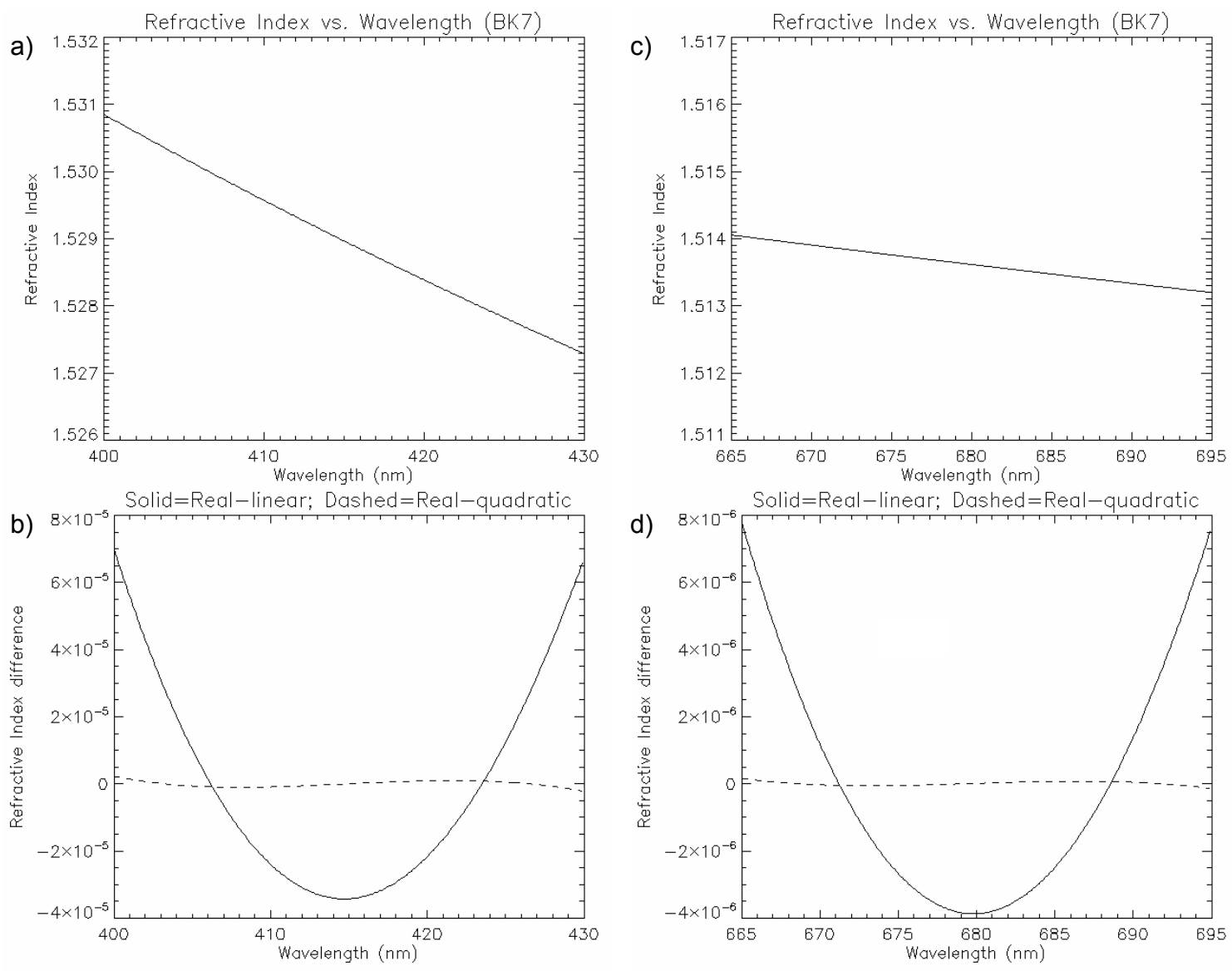

FIGURE 2.23 - Plots of a) $n$ vs. $\lambda(400-430 \mathrm{~nm})$; b) Difference for linear and quadratic models of index (400-430 nm); c) $n$ vs. $\lambda$ (665-695 nm); d) Difference for linear and quadratic models of index $(665-695 \mathrm{~nm})$. 
The residual index differences after fitting with a quadratic have maximum index differences of $2.2 \times 10^{-6}(400-430 \mathrm{~nm})$ and $1.6 \times 10^{-7}(665-695 \mathrm{~nm})$. So for a narrow bandwidth source, a second order fit is sufficient, especially at the red end of the visible spectrum.

For illustrative purposes, consider a source with a Gaussian power spectrum, which results in a Gaussian coherence envelope. Initially, the coherence envelope is centered at $\mathrm{z}=0$. The plate in the test arm of the interferometer is BK7. The exponential term out front in equation 2.48 is a phase term which does not affect the shape or location of the coherence envelope. The independent variable is changed from units of time $(\mathrm{z} / \mathrm{c})$ to units of distance (z). The first term to be convolved with the coherence envelope is a delta function at a location in $\mathrm{z}$, where $\mathrm{z} \neq 0$. The result of the convolution is that the coherence envelope is shifted away from $\mathrm{z}=0$.

$$
I_{\text {env }}(z)=I_{w}+\text { Phase }\left[A^{2}(2 z) * \delta\left(2 z-2\left[t_{t}-t_{r}+\left(n_{0}-1-\left.\frac{c}{v_{0}} \frac{d n}{d \lambda}\right|_{\lambda_{0}}\right) t_{p}\right]\right) * F T\{C\}\right]
$$

Equation 2.49

The shift depends on several factors: obviously, it depends on the relative position of the two mirrors $\left(t_{t}-t_{r}\right)$. In the case where the plate thickness, $t_{p}$, goes to zero, this is all it depends on. In the more interesting case of a plate with nonzero thickness and an index that is constant with respect to wavelength, the shift is:

$$
\text { If: } n(\lambda)=n_{0} \text {, then: Shift }=\left(n_{0}\right) t_{p}=O P L_{\text {plate }}
$$

Equation 2.50 
In this case, the envelope center would move according to OPL. For a refractive index that is linear with respect to wavelength, the envelope center shifts according to OGL, the optical group length.

$$
\text { If: } n(\lambda)=n_{0}+m \lambda \text {, then: Shift }=\left(n_{0}-\left.\frac{c}{v_{0}} \frac{d n}{d \lambda}\right|_{\lambda_{0}}\right) t_{p}=\left(N_{g}\right) t_{p}=O G L_{\text {plate }}
$$

Equation 2.51

For a center wavelength $\lambda_{0}=415 \mathrm{~nm}$, the difference between group and phase index is:

$$
-\left.\lambda_{0} \frac{d n}{d \lambda}\right|_{\lambda_{0}}=-415 \cdot\left(-1.18 \times 10^{-4}\right)=0.0491=N_{g}-n_{0} \quad \text { Equation } 2.52
$$

For a center wavelength $\lambda_{0}=680 \mathrm{~nm}$, the difference between group and phase index is:

$$
-\left.\lambda_{0} \frac{d n}{d \lambda}\right|_{\lambda_{0}}=-680 \cdot\left(-2.85 \times 10^{-5}\right)=0.0194=N_{g}-n_{0} \quad \text { Equation } 2.53
$$

It is important to again note that as long as the center of the coherence envelope is what is determined, no matter how narrow the source spectrum becomes, the linear term of index will always cause the product of thickness and group index to be measured. Narrowing the source spectrum will not result in a measurement of the product of thickness and phase index.

For the quadratic model of dispersion, the first order shift is still present. There is a secondary shift which is much smaller than the shift due to group index. The more important effect from this second order dispersion is that the coherence envelope broadens non-symmetrically. Simulated plots illustrated this in Figure 2.20. 


$$
\text { If: } n(\lambda)=n_{0}+m \lambda+p \lambda^{2} \text {, then: Shift }=\left(n_{0}-\left.\frac{c}{v_{0}} \frac{d n}{d \lambda}\right|_{\lambda_{0}}\right) t_{p}
$$

One way to understand this non-symmetric broadening is to think of it as a spectrally dependent group index variation. The dispersion of transparent materials in the visible has a positive second derivative. At shorter wavelengths, the slope of the group index versus wavelength is larger than for longer wavelengths. Therefore, shorter wavelength components are delayed slightly more than the longer wavelengths are advanced. This causes a weighting on one side of the coherence envelope. The magnitude of this effect depends on the magnitude of the second derivative and the bandwidth of the source. Figure 2.24 shows a plot of the second derivative of the index of BK7 with respect to wavelength, showing that the magnitude of the second derivative is smallest at longer wavelengths. This is a major benefit of using a source in the red part of the spectrum rather than in the blue.

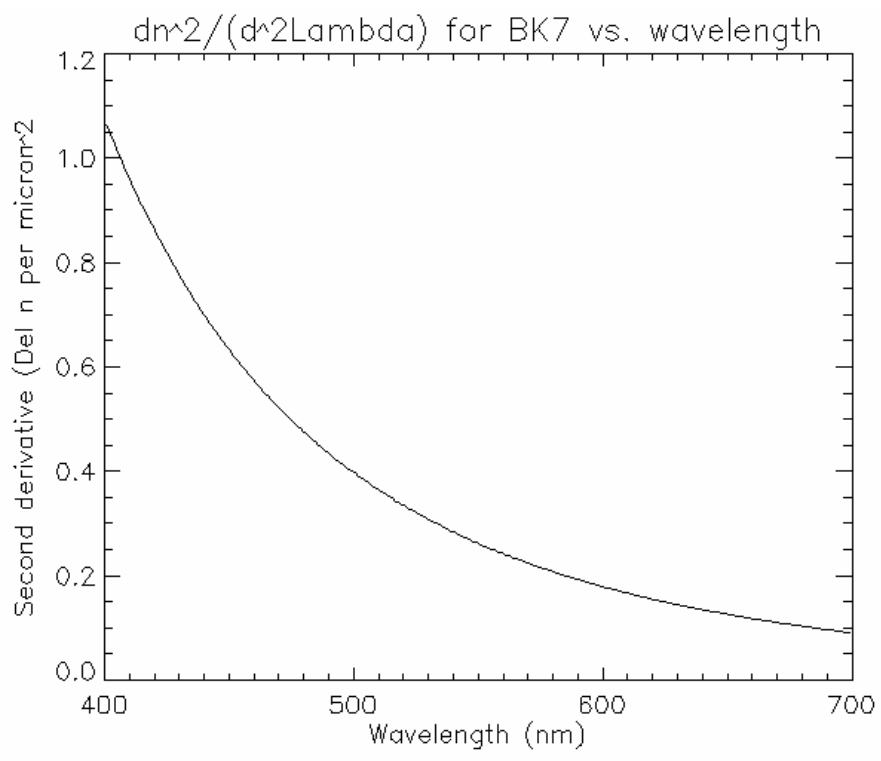

FIGURE 2.24 - Plot of second derivative of index $\left(\Delta \mathrm{n} / \mu \mathrm{m}^{2}\right)$ versus $\lambda$ for BK7. 
The fact that the broadening is non-symmetric can cause other problems. Depending on the algorithm used to recover the peak of the coherence envelope, the recovered surface location can be shifted due to this distortion. Extensive modeling of this effect on coherence envelope peak detection was performed prior to building the instrumentation. If the amount of dispersive material $\left(\mathrm{t}_{\mathrm{p}}\right)$ exclusive to one arm of the interferometer can be kept to a minimum, the effect from this distortion can be minimized.

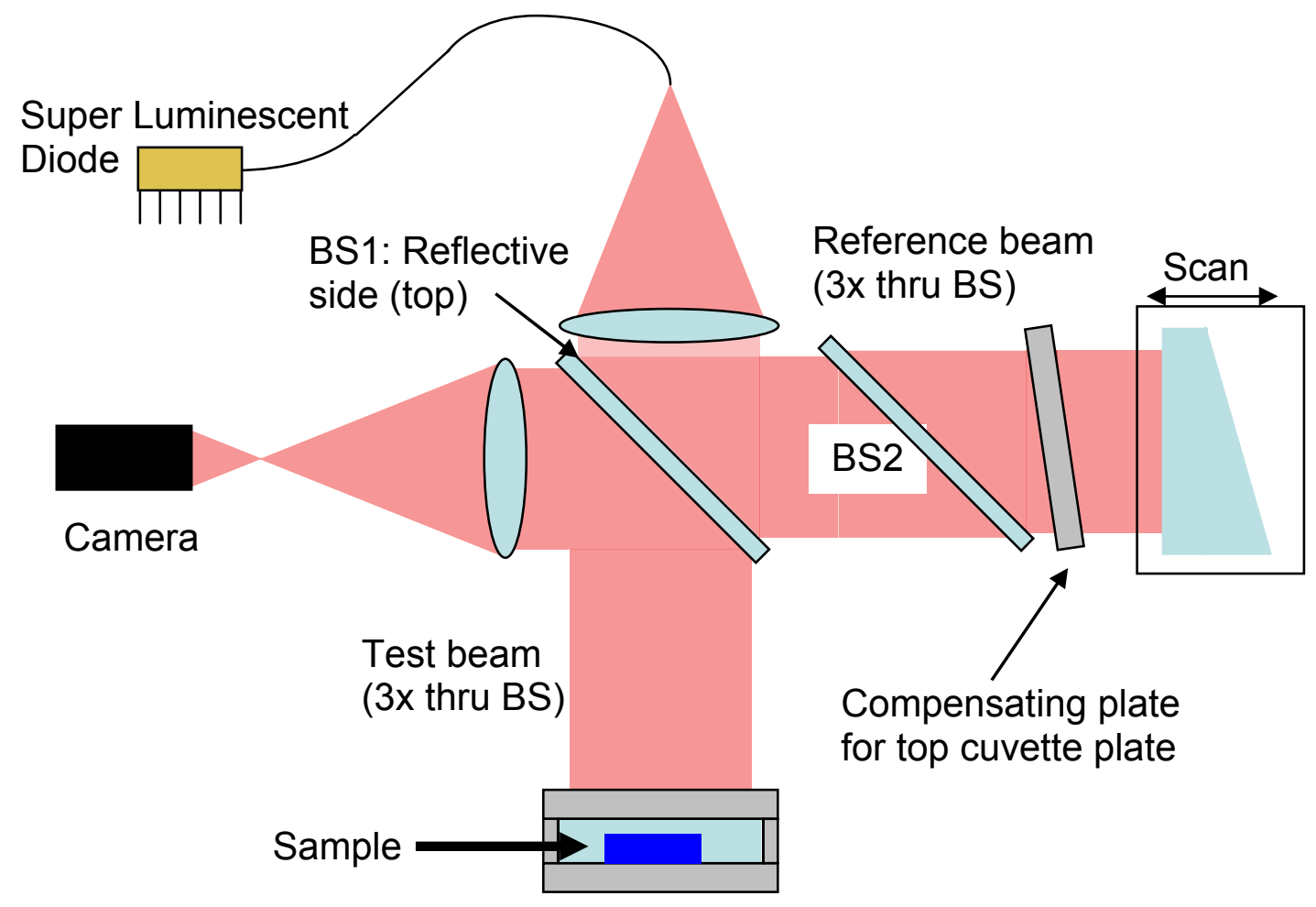

FIGURE 2.25 - By matching the dispersive material in the test and reference arms of the Twyman-Green interferometer, LCI signal due to dispersion can be compensated. 
In other words, if identical plates (same thickness, same material) are placed in both the test and reference arms of the interferometer, their effects will exactly cancel and the signal will remain symmetric. This fact was used in the design of the interferometer. Two nearly identical beamsplitters are used in the Twyman-Green interferometer, where the purpose of the second beamsplitter is to compensate for the dispersion in the other arm of the interferometer (Figure 2.25). With the inclusion of the second beamsplitter, both the test and reference beams traverse a beamsplitter 3 times.

The sample must be measured in a cuvette, and this cuvette contains a piece of glass as the top surface. The dispersion of this plate cannot be ignored and is also compensated for in the reference arm of the interferometer with a plate made of the same material and with nearly the same thickness. A simple technique for balancing the dispersion between the compensating plate and the top plate of the cuvette is discussed later.

Assuming the glass dispersion has been compensated for and the source distribution is symmetric with respect to frequency, the signal from the top interface (glass/air or glass/saline) in the sample cell should be symmetric about the center of the coherence envelope. For any of the other interfaces buried further in the cuvette beneath saline or hydrogel, this is no longer true. This is why it is important to minimize the amount of saline above the sample. The current cuvette cavity is about $350 \mu \mathrm{m}$ thick in order to minimize the envelope distortion. 
Another way to limit the signal distortion from second and higher order dispersion is to limit the spectral bandwidth of the source. The narrower the source spectrum, the better a linear fit to the index of refraction (over the part of the spectrum covered by the source) and the smaller the errors due to the higher order terms. This is a difficult thing to prove with measurements, so the effects will be modeled. Figure 2.26 shows a plot for the bias (error) of the surface location as a function of the spectral bandwidth of the source. The source is modeled with a Gaussian distribution, and the dispersive material is a plate of BK7 glass with a thickness of $0.5 \mathrm{~mm}$ ( $1 \mathrm{~mm}$ total in double pass). The second plot is the same model except that water is used as the dispersive material. Note that the noise in these curves is higher for smaller source bandwidths. This is because a narrower FWHM of the power spectrum leads to a wider coherence envelope. Naturally, it is a noisier operation to find the center of a broader envelope.
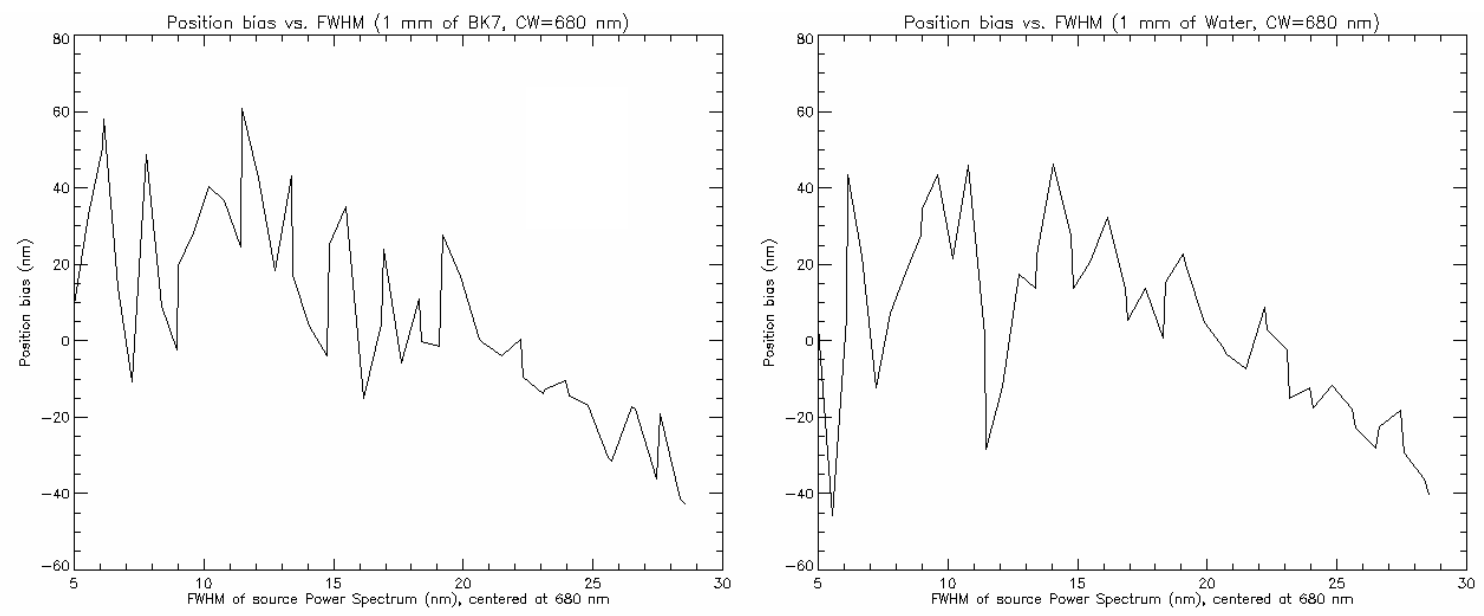

FIGURE 2.26 - Plot of position error in recovering the surface location using the Fourier transform algorithm (Chapter 3) from the modeled LCI signals for a fixed dispersive material thickness as a function of the FWHM of the Gaussian power spectrum of a modeled LCI source for BK7 (left) and water (right). 
A curious effect is seen in that there is an ideal bandwidth for which the higher order index terms causes no bias for a given source center wavelength and a given dispersive material. For a source centered at 680 nanometers and a plate of BK7, this ideal bandwidth is somewhere around 20 nanometers FWHM. This lack of bias should be independent of the plate thickness. This is simulated in Figure 2.27.

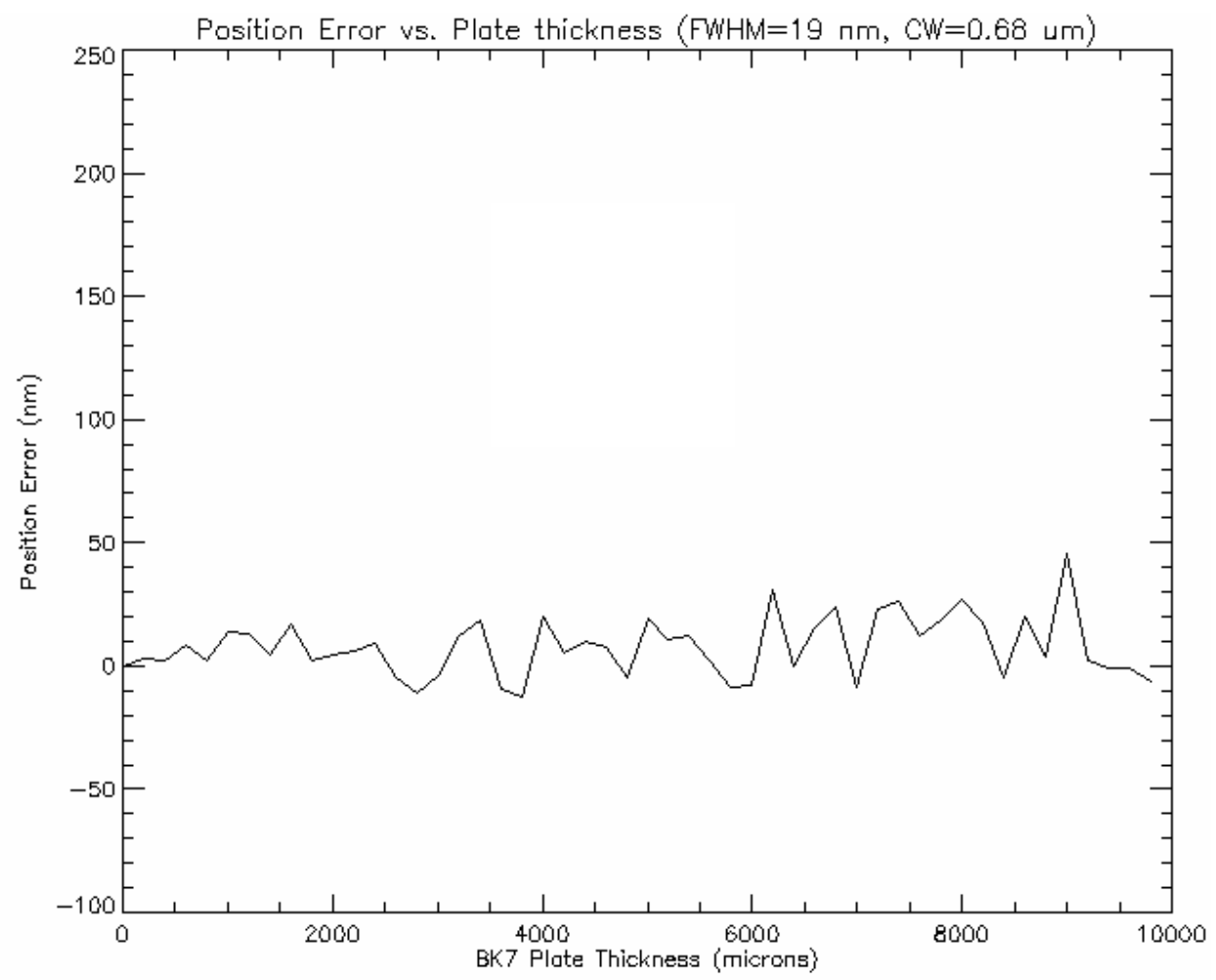

FIGURE 2.27 - Position bias vs. plate thickness of BK7 for a source centered at $680 \mathrm{~nm}$ with a FWHM of $19 \mathrm{~nm}$, using Fourier transform method.

The noise increases as the plate thickness increases. Even though there is no bias in this case, the envelope still broadens, making it more difficult to locate the center of the envelope accurately. Note that no sources with a $19 \mathrm{~nm}$ bandwidth centered at $680 \mathrm{~nm}$ that could be used to remove measurement bias could be located. The source used (SLD- 
680) is a Superluminescent diode from Superlum, LTD in Moscow, Russia. Its spectrum is centered at roughly 680 nanometers and it has an $8.5 \mathrm{~nm}$ FWHM (Figure 2.28). The source data sheet is included in Appendix A.

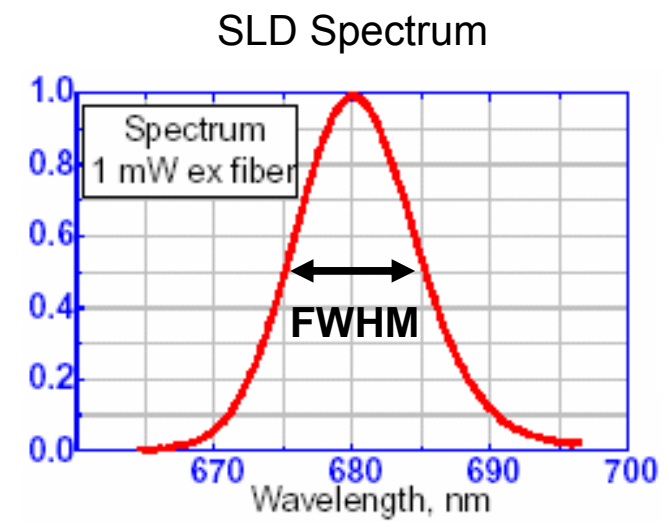

FIGURE 2.28 - SLD output versus wavelength.

As can be seen in Figure 2.29, the recovered position bias is relatively small at this FWHM and center wavelength for a $1 \mathrm{~mm}$ plate of BK7 glass. At larger FWHM values, the bias falls negative quickly, causing larger measurement errors. 


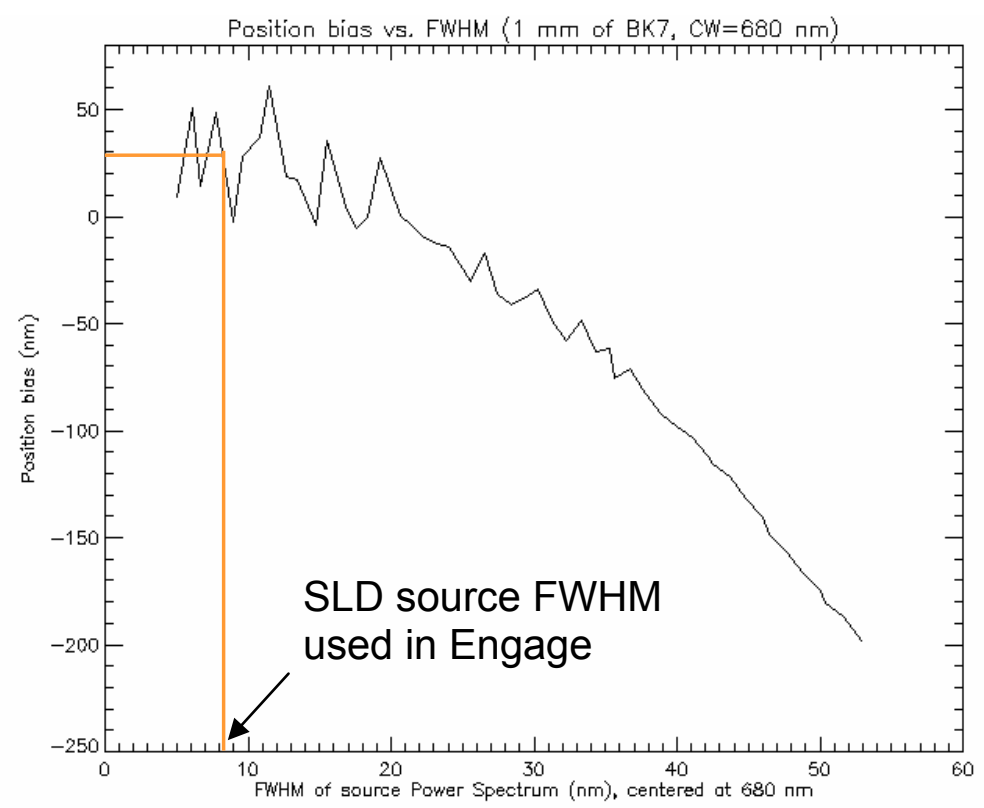

FIGURE 2.29 - Position error in recovering the surface location using the Fourier transform algorithm versus source FWHM, showing increasing measurement bias for larger spectral bandwidth sources. Note the positive error for the SLD used in Engage.

The narrower FWHM of the SLD source does make the envelope wider and make it more difficult to find the center, but at least the errors should have a minimal bias. By using a narrow source, the bias will be smaller than for a FWHM greater than about $25 \mathrm{~nm}$. For a FWHM of $8.5 \mathrm{~nm}$, Figure 2.30a shows the measurement bias as a function of plate thickness for BK7. Note that it fits well to a straight line with a slope of $22.1 \mathrm{~nm}$ of surface bias per millimeter of BK7 plate thickness. The bias for water is also shown (Figure 2.30b). 

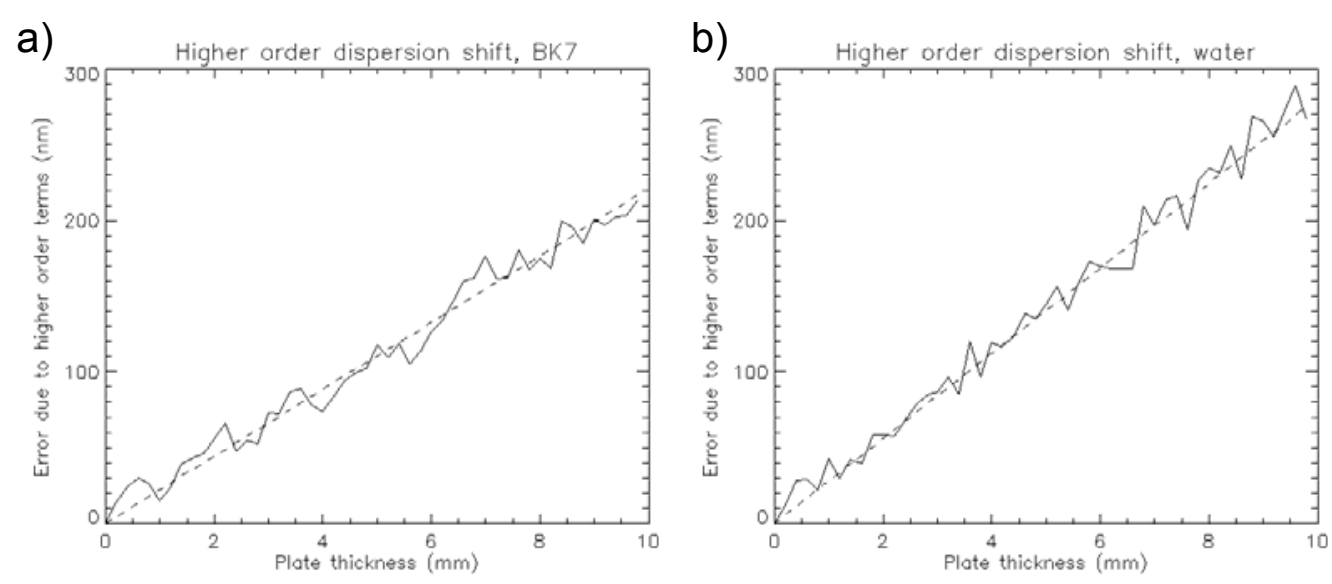

FIGURE 2.30 - Surface biases vs. plate thickness for SLD source used in Engage. Dashed line is linear fit to data with a slope of a) $22.1 \mathrm{~nm}$ bias $/ \mathrm{mm}$ plate thickness for BK7 and b) $28.1 \mathrm{~nm} / \mathrm{mm}$ for water.

These plots are based on plate thickness, taking into account the fact that the light travels through the plate twice in the Twyman-Green interferometer configuration. For a cuvette thickness of $\sim 350 \mu \mathrm{m}$ filled with saline (approximating the measurement bias from the water model), the bias should be about $9.8 \mathrm{~nm}$, assuming the Fourier transform method for finding the center of the coherence envelope is used.

$$
\varepsilon_{\text {bias }}=0.350 \mathrm{~mm} \cdot 28.1 \frac{\mathrm{nm}}{\mathrm{mm}}=9.835 \mathrm{~nm}
$$

Higher order dispersion distorting the LCI signals is an effect that should not be ignored.

All of the models presented in this chapter were processed using the Fourier transform technique, which is covered in depth in the next chapter. The models of I(z) were constructed from an incoherent superposition of monochromatic fringe patterns from appropriately weighted wavelengths within the modeled spectral distribution of the source. The index of refraction was modeled using the Sellmeier equation. 


\section{DETERMINING SURFACE LOCATION FROM THE LCI DATA CUBE}

Now that the essential form of the low coherence signal has been discussed, including the physical imperfections and some of their effects on signal quality, methods for determining the envelope center can be explored. The term LCI data cube refers to the data collected for a single surface in the measurement cell. At a series of axial positions (z), images are taken with the camera. Stacking up the images in a 3D array creates a data cube, $I(x, y, z)$, where each location is the irradiance for that pixel at that depth location (Figure 3.1).

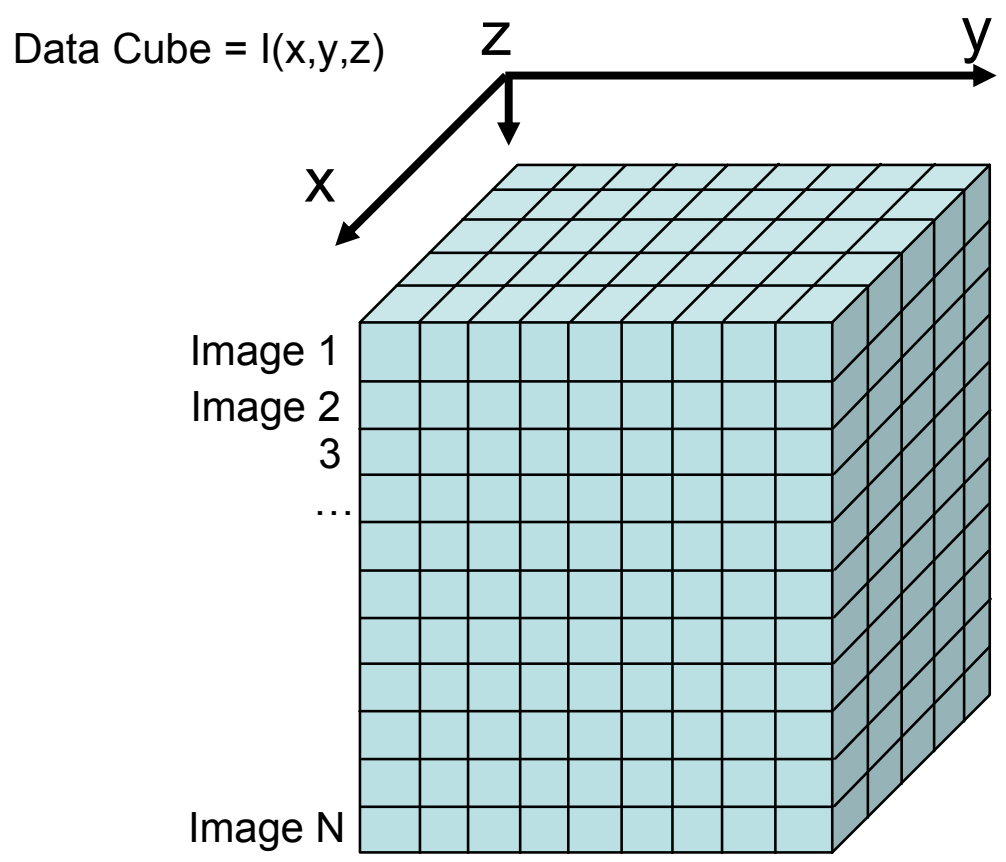

FIGURE 3.1 - Data cube for a single surface measured with LCI, I(x,y,z).

The data for a given pixel along $\mathrm{z}$ is an individual LCI signal, an example of which is shown in Figure 3.4. The center of the coherence envelope for this signal is the location 
of the surface for this pixel. The method for finding this center is the subject of this chapter as well as a large number of papers.

As always, the best technique depends on the mode of data collection and the characteristics of the hardware. During this project, the envelope detection technique evolved parallel to the evolution of the hardware and changed as necessary to minimize the error in finding the surfaces.

The measurement goal accuracy of 0.0001 in index dictates how well the thickness must be measured. In order to understand the required accuracy, the basic measurement equation in Equation 1.20 is expanded in Equation 3.1 to a more realistic form:

$$
n(\lambda)=\frac{\lambda_{\text {laser }}}{t_{\text {sample }}}\left[\left(m_{2}-m_{1}\right)+\frac{\left(\phi_{\text {con }}-\phi_{\text {sal }}\right)}{2 \pi}\right]+n_{\text {saline }}
$$

The spatial subscripts on $\mathrm{n}(\lambda), \mathrm{t}_{\text {sample }}, \varphi_{\text {con }}$ and $\varphi_{\text {sal }}$ have been omitted. The $\varphi_{\text {con }}$ and $\varphi_{\text {sal }}$ arrays are the phase measured by the Mach-Zehnder interferometer in the regions containing contact lens material and saline, respectively; $\mathrm{n}_{\text {saline }}$ is the index of the saline in which the sample is immersed; and the difference between the two m values is an integer number of wavelengths of the laser between the column with contact and the column with saline at the laser wavelength, $\lambda_{\text {laser }}$. The details of this expression will be covered in later chapters; it is shown here to help determine the required accuracy of the thickness measurement. 
For a 100 micron thick hydrogel sample $(\mathrm{n}=1.4100)$ in saline $(\mathrm{n}=1.3364)$, a thickness error of 70 nanometers corresponds to an index error of 0.00005 , or half the error budget. This assumes there are no errors in the measured values in this equation. For now, the thickness accuracy goal is 50 nanometers. This thickness measurement is the combination of 5 individual surface measurements (air1, air2, saline1, saline 2 and saline3). It is also important to remember that multiple measurements are made of the index; one for each pixel on the camera.

As the proposed test method was explored via simulations, several envelope detection techniques were explored. The source characteristics were determined early on simply due to the small number of available sources near the desired specifications. The coherence length of the source is roughly 35 microns with a center wavelength of 680 nanometers. Therefore, there are more then 50 fringes under the coherence envelope for an interface (Figure 3.2).

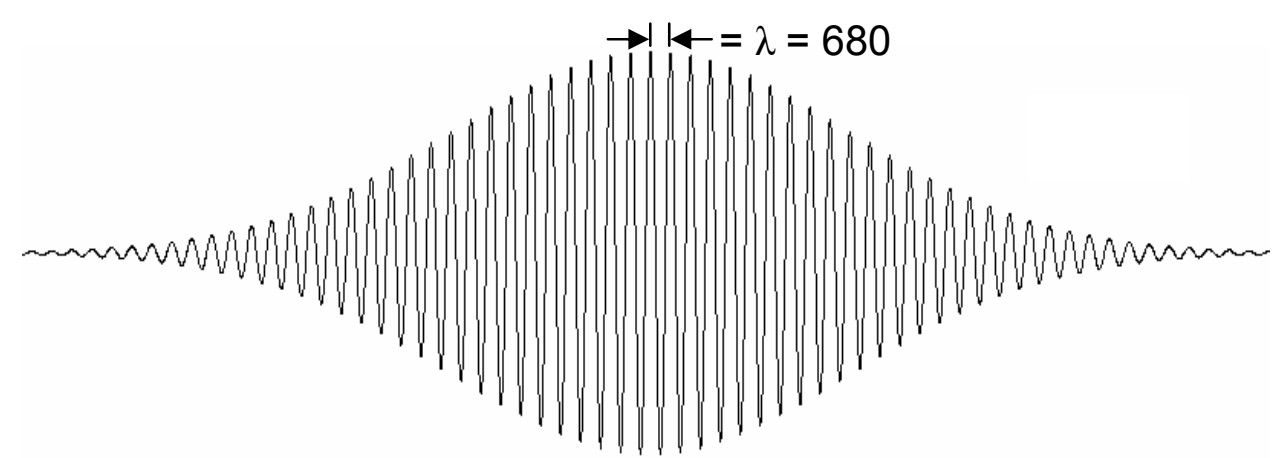

FIGURE 3.2 - Continuous representation of I(z) for Engage SLD. 
There are many publications that include algorithms for finding the center of a low coherence envelope, many of which report accuracies on the order of $5 \mathrm{~nm}$ or better (Danielson, 1990; Lee, 1990; Caber, 1993; de Groot, 1993; Hirabayashi, 2002). The biggest differences between their signals and the signals from Engage are the width of the coherence envelope and the overall fringe visibility. The signals measured in these papers are usually from a white light source and are only a few fringes wide ( $\sim 5$ fringes). Simply scaling the surface error by the ratio of the number of fringes (50:5) means finding the center of the signal from the SLD source could at best be around 50 nanometers. The SLD coherence envelope is further degraded by dispersion, as discussed earlier. Another difference is that the signal for Engage has such a low visibility due to the low reflectance coefficients in the measurement cell (Table 2.1), and so the noise levels are higher. An important consequence of the relatively wide coherence envelope is that the signal must be captured with sub-Nyquist sampling, or one image every $5 \lambda / 4$, or $450^{\circ}$ of phase. This is a factor of 5 fewer samples then the standard $\lambda / 4$ wave sampling, which reduces the number of required images per surface from about 625 images to around 125 images. Data collection is therefore faster (Figure 3.3). 

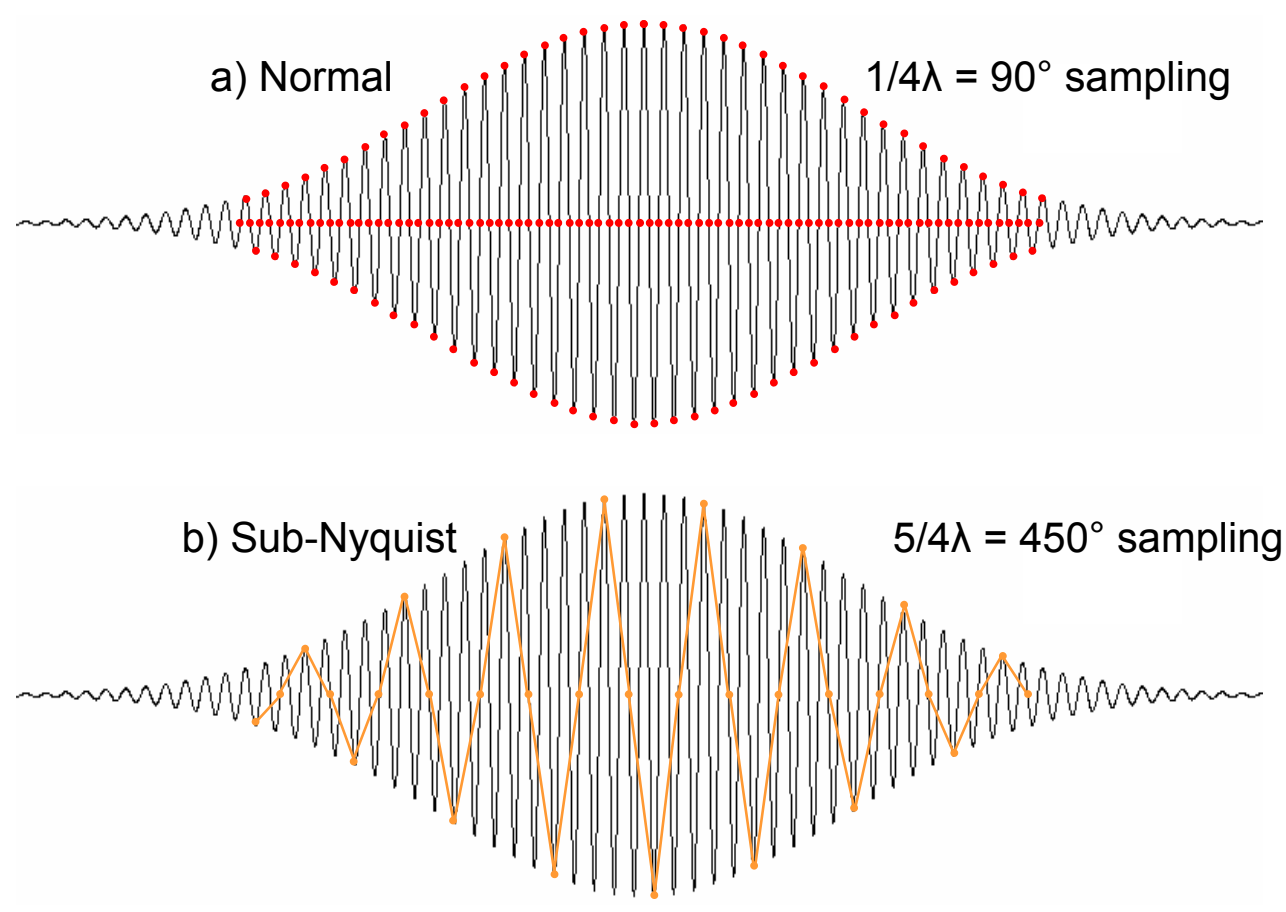

FIGURE 3.3 - Comparison of a) $\lambda / 4$ sampling and b) sub-Nyquist 5 $\lambda / 4$ sampling of the LCI signal.

\subsection{The Fourier Transform Method}

This first method is commonly found in the literature, and locates the surface by measuring the phase slope of the Fourier components of the recorded irradiance versus depth for a single pixel (de Groot, 1993; Larkin, 1996). Therefore, the signal from each spatial location on the camera is processed independently of any neighboring pixels. There are several advantages to processing the data in the Fourier domain over simply calculating the fringe contrast envelope point by point in the spatial domain.

The Fourier method does not require the data to be at exactly $90^{\circ}$ (or $450^{\circ}$ ) steps, but it does require evenly spaced data, i.e. identical step sizes between images. The irradiance 
as a function of position, $\mathrm{z}$, is given in equation 3.2 , where $\mathrm{z}_{0}$ is the center of the coherence envelope and the surface location.

$$
I(z)=I_{D C}+A \cdot G a u s\left(\frac{z-z_{0}}{c / \Delta v}\right) \cos \left(2 \pi v_{0} \frac{z}{c}+\varphi_{0}\right)
$$

The phase of the underlying fringe pattern is offset from the center of the coherence envelope by an arbitrary amount $\varphi_{0}$, which includes the dependence on $z_{0}$ and any path mismatch. The $I_{D C}$ term is equivalent to $I_{w}$ from equation 2.31. The phase of the Fourier transform of I(z), $\Phi$, is directly proportional to the shift of the Gaussian away from being centered at $\mathrm{z}=0$. By evaluating the derivative of the phase of the Fourier transform at the appropriate wave number, $\mathrm{k}$, the value of $\mathrm{z}_{0}$ can be found. Another way to look at I(z) is as an incoherent superposition of single-frequency interference patterns, as was used earlier to construct equation 2.37. Ignoring scaling, a single wavelength component $\mathrm{I}_{\lambda}(\mathrm{z})$ is expressed as:

$$
I_{\lambda}(z)=1+\cos (\Phi)=1+\cos (k z)=1+\cos \left(\frac{2 \pi}{\lambda} z\right)
$$

In the absence of dispersion effects, the value of $z_{0}$ is found from the derivative of the phase with respect to the wave number, $\mathrm{k}$, where $\mathrm{k}$ is the independent variable and $\Phi$ is the dependent variable:

$$
z_{0}=\frac{d \Phi}{d k}
$$

From an algorithm point of view, the phase of the Fourier transform is found as follows:

$$
\Phi(k)=\tan ^{-1}\left[\frac{\operatorname{Im}(F T\{I(z)\})}{\operatorname{Re}(F T\{I(z)\})}\right]
$$


The envelope center, $\mathrm{z}_{0}$, is found by evaluating the derivative of equation 3.5 and evaluating it at the correct values of $\mathrm{k}$. The locations of the valid points along the $\mathrm{k}$ axis are determined by a weighting function which is calculated from the magnitude of the Fourier transform. An example of this technique with real data follows (Figure 3.4).

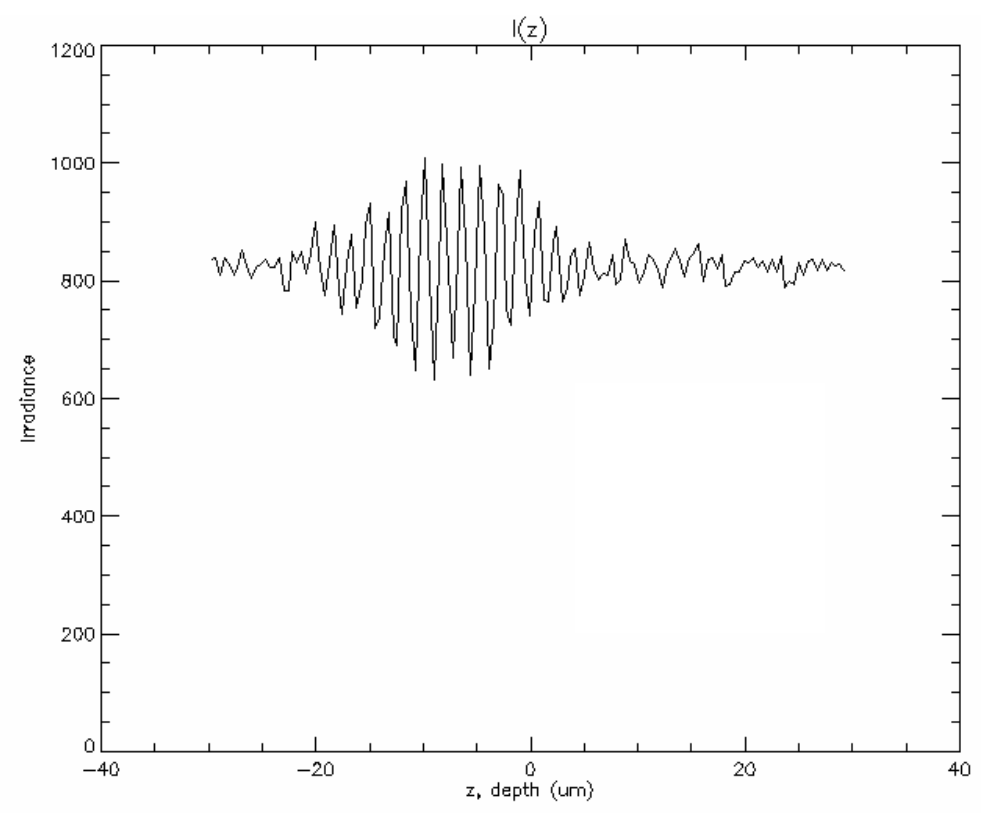

FIGURE 3.4 - Actual data of irradiance as a function of depth, I(z). The data has been shifted to be roughly centered at zero. 


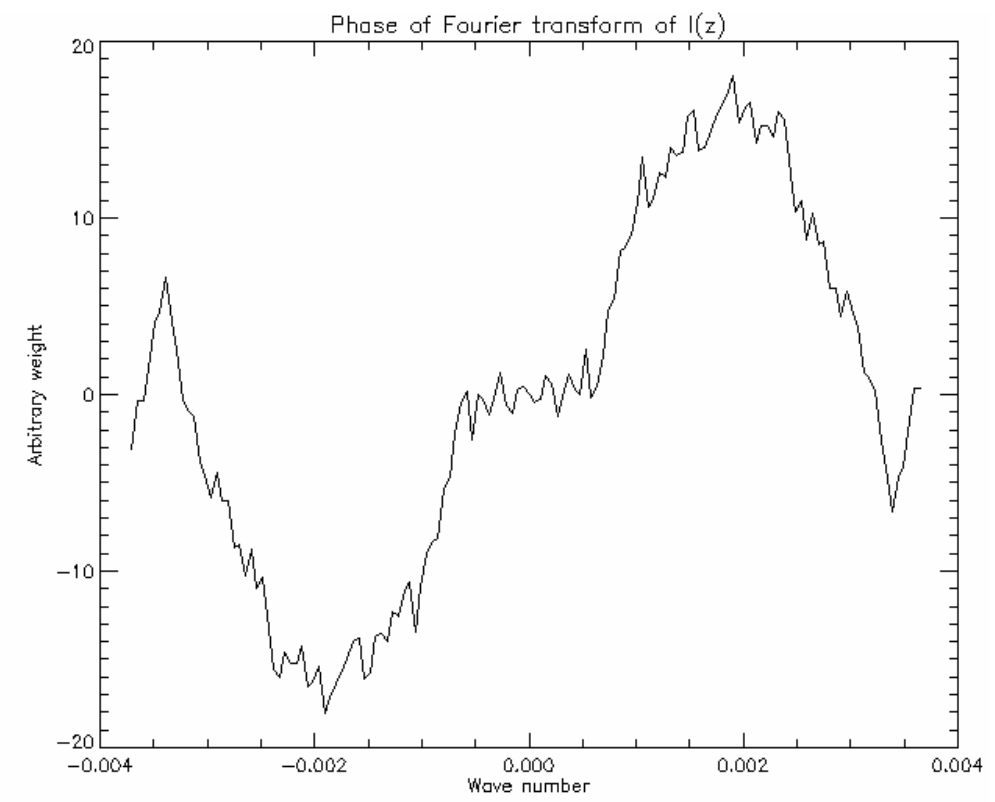

FIGURE 3.5 - Plot of $\Phi(\mathrm{k})$ as defined in equation 3.5, for the data in Figure 3.4.

The plot in Figure 3.5 shows the phase, $\Phi$, as a function of $k$, for the data shown in Figure 3.4. Figure 3.6 is the associated weighting plot, where any value above the dotted line represents valid data. It is the magnitude of the Fourier transform as a function of wave number, $\mathrm{k}$. 


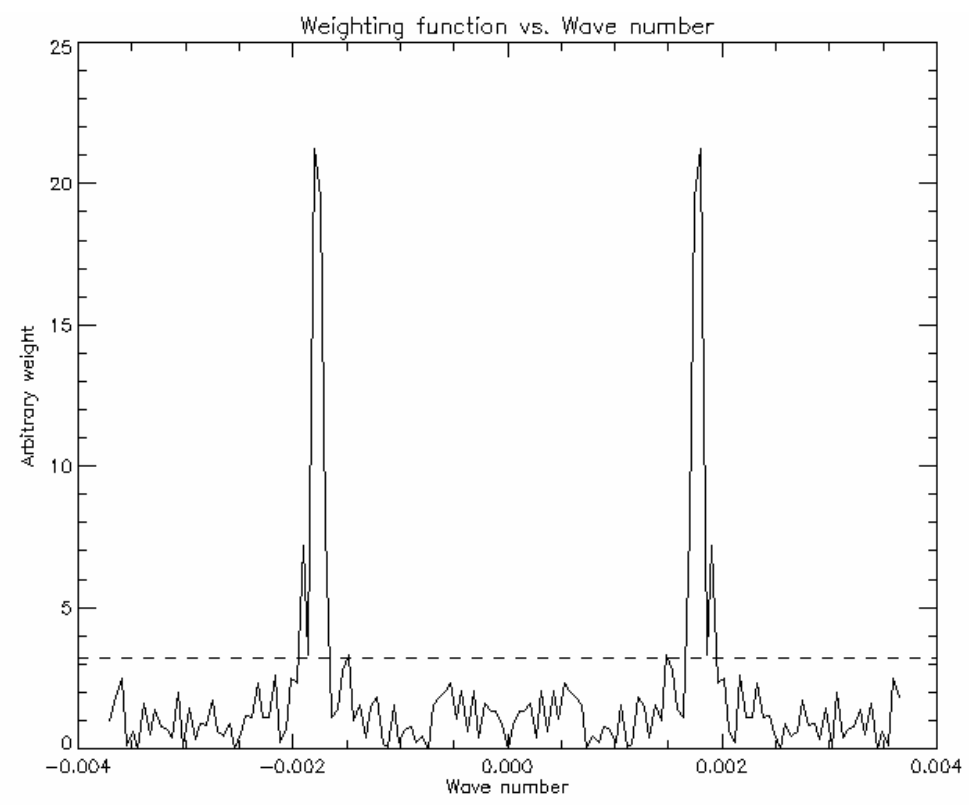

FIGURE 3.6 - Weighting function for valid points for calculating $\mathrm{z}_{0}$.

Note that this data is sampled at $5 / 4 \lambda$, so the approximate wave number for the underlying fringes is $0.00185 \mathrm{rad} / \mathrm{nm}$.

$$
k_{\text {center }}=\frac{2 \pi}{680 \cdot 5}=0.00185 \mathrm{rad} / \mathrm{nm}
$$

Equation 3.6

The derivative of the plot in Figure 3.6, multiplied by the proper sign and other constant parameters yields the plot in Figure 3.7, where only valid derivative values are plotted for simplicity. Only values for $\mathrm{k}>0$ are considered since the data is symmetric about 0 . 


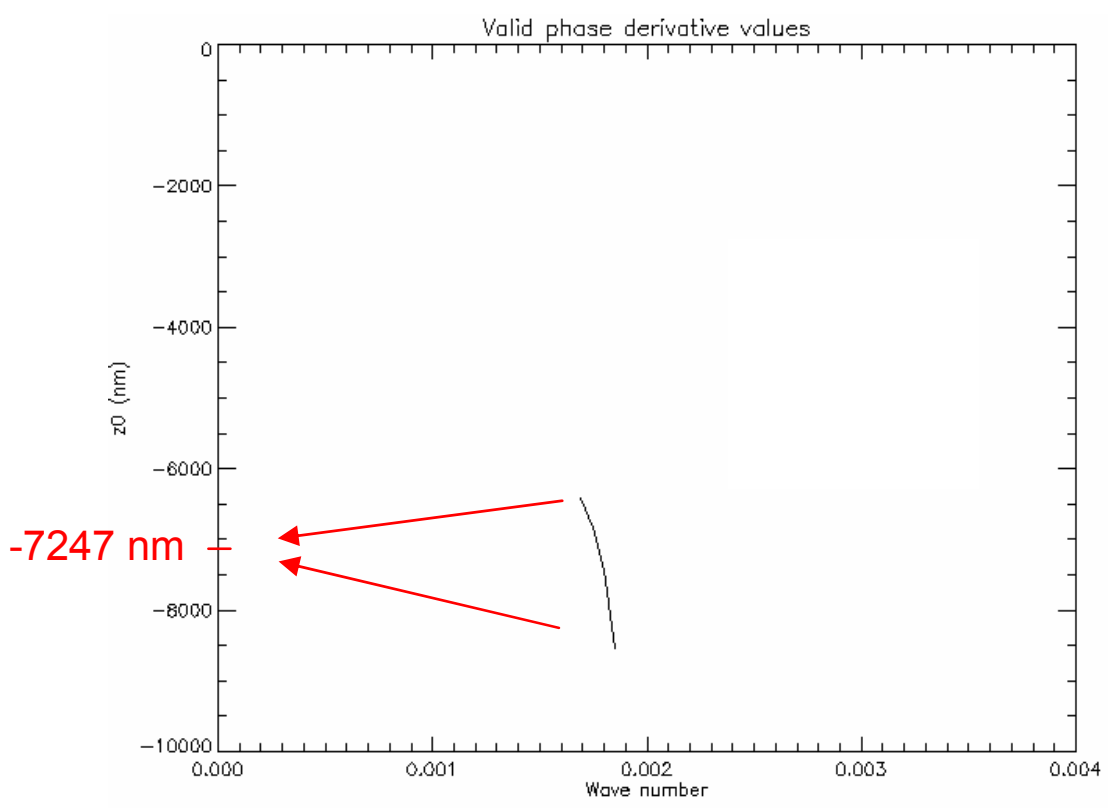

FIGURE 3.7 - Determination of $\mathrm{z}_{0}$ from phase of the Fourier transform of $\mathrm{I}(\mathrm{z})$.

The weighting function is used to determine the location of the surface, $\mathrm{z}_{0}$, which in this case was calculated as $-7247 \mathrm{~nm}$, which seems reasonable when compared with the irradiance signal in Figure 3.4.

To get a better idea of how well this technique works, a larger data set is required. The above example was for a single pixel in a surface, in this case a flat glass surface, such as interface air1. An image of the full recovered surface $\mathrm{z}_{0}(\mathrm{x}, \mathrm{y})$, with a best fit plane subtracted, is shown in Figure 3.8. 


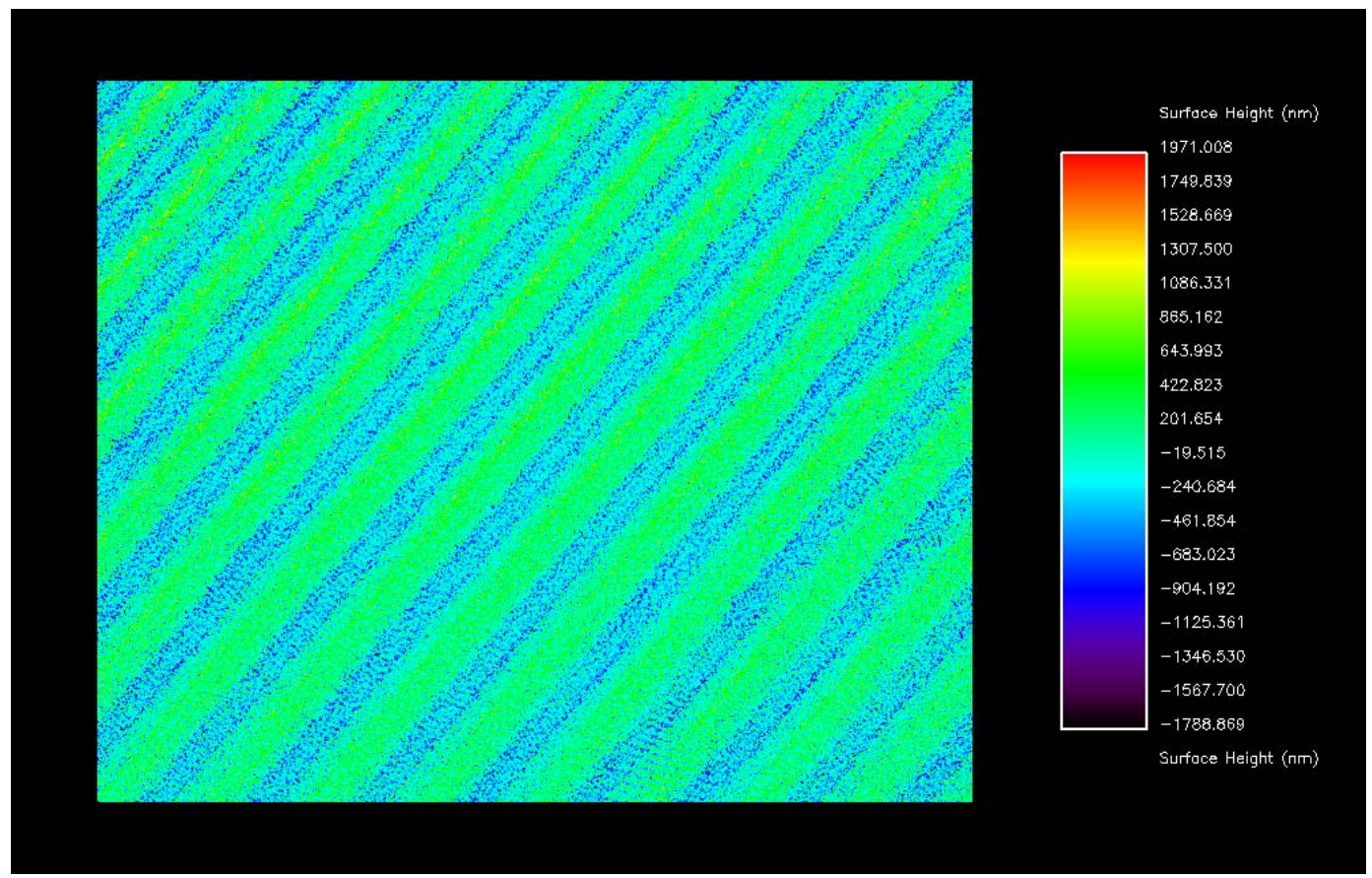

FIGURE 3.8 - Reconstructed surface, $\mathrm{z}_{0}(\mathrm{x}, \mathrm{y})$ for a glass/air interface (best fit plane removed).

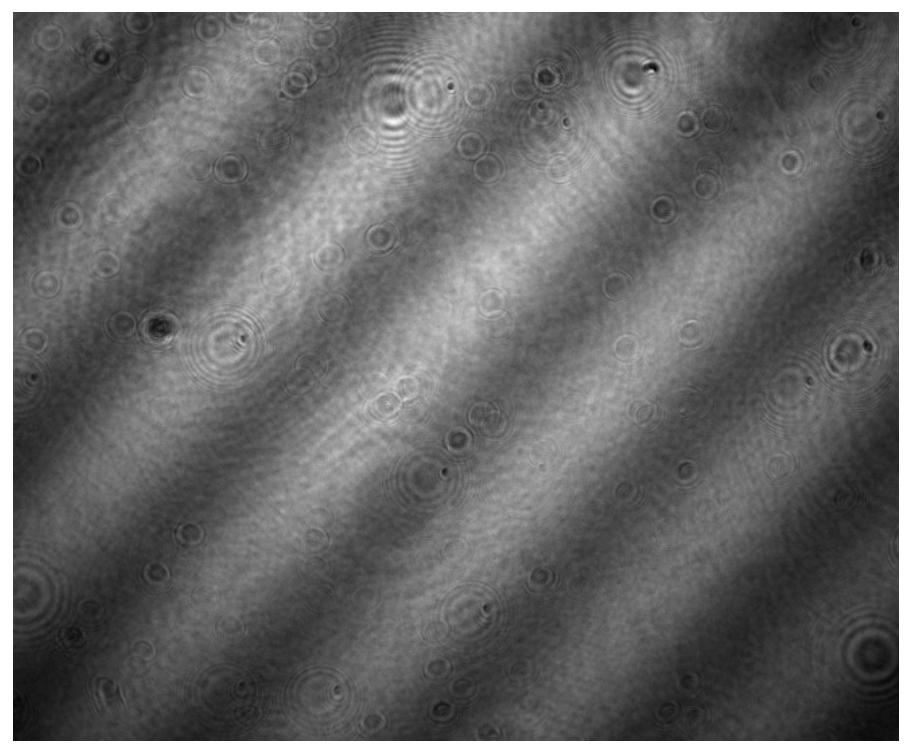

FIGURE 3.9 - Single LCI image from data cube, I(x,y), illustrating relationship between fringe orientation and features in the recovered surface in Figure 3.8. 
Figure 3.9 shows an image of the low coherence fringes used to reconstruct the surface. It is clear that the surface deviation from flatness in the reconstructed surface depends on twice the frequency of the phase of the underlying cosine fringes. Figure 3.10 shows a profile of the reconstructed surface in Figure 3.8.

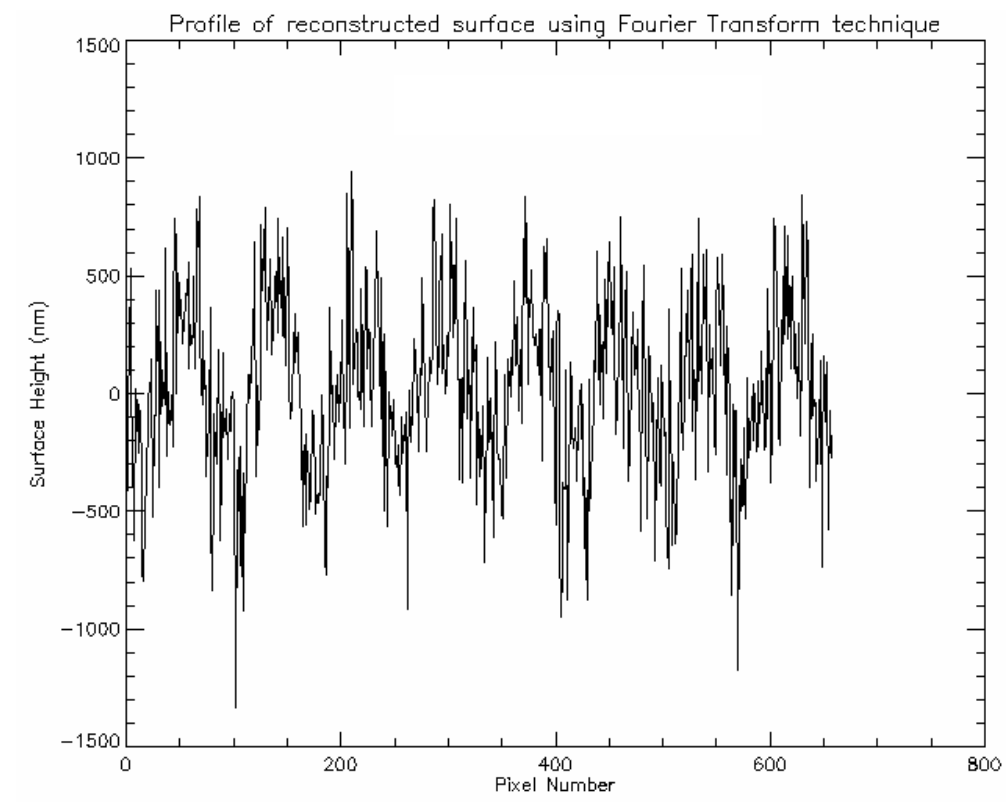

FIGURE 3.10 - Profile of surface shown in Figure 3.8. Note the scale of the error.

The standard deviation of the reconstructed surface with the best fit plane subtracted is 357.3 nanometers. The larger problem is the obvious structure of the noise and its dependence on the underlying fringe structure. The reason for this large structure is that the distance between each image along the scan varies. The distance varies because of imperfections in the stage hardware. Recall that a Fourier transform assumes uniform spacing of the data, or that the data is sampled at a constant rate. The result is similar to the classic error of twice the fringe frequency in phase shifting interferometry. An important advantage of the low coherence interferometer in Engage is that the positions 
for each image, while uneven, are known thanks to a careful stage calibration. This position information should be useful in improving the surface finding technique.

The analysis of the Fourier technique has been shown for a glass/air interface, which is the interface with the best fringe visibility. For comparison with other methods, the results here show a reconstructed surface for a saline/contact material sample interface (saline2). Figure 3.11 shows the irradiance as a function of depth for one pixel, I(z).

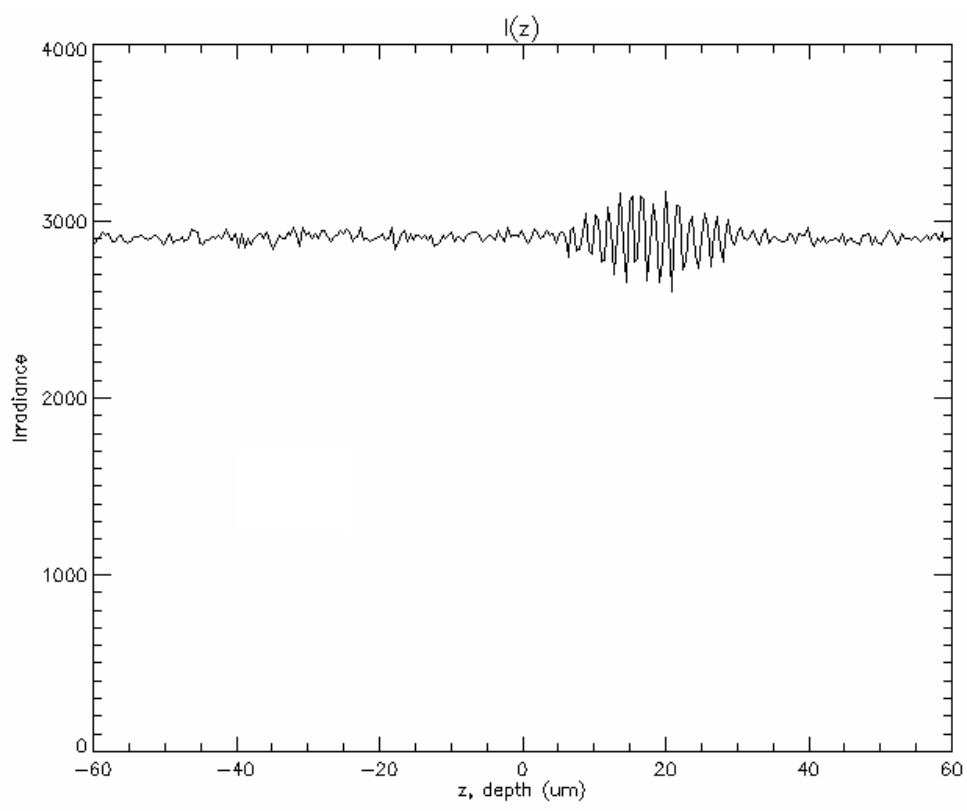

FIGURE 3.11 - Data for I(z) for the saline2 interface. Note the low fringe visibility.

The resulting valid slope values are plotted in Figure 3.12. When the calculated weighting function is applied to these points, the resulting surface location is $17,892 \mathrm{~nm}$. Due to the lower fringe visibility, the errors in this surface are larger then the case of a glass/air interface. 


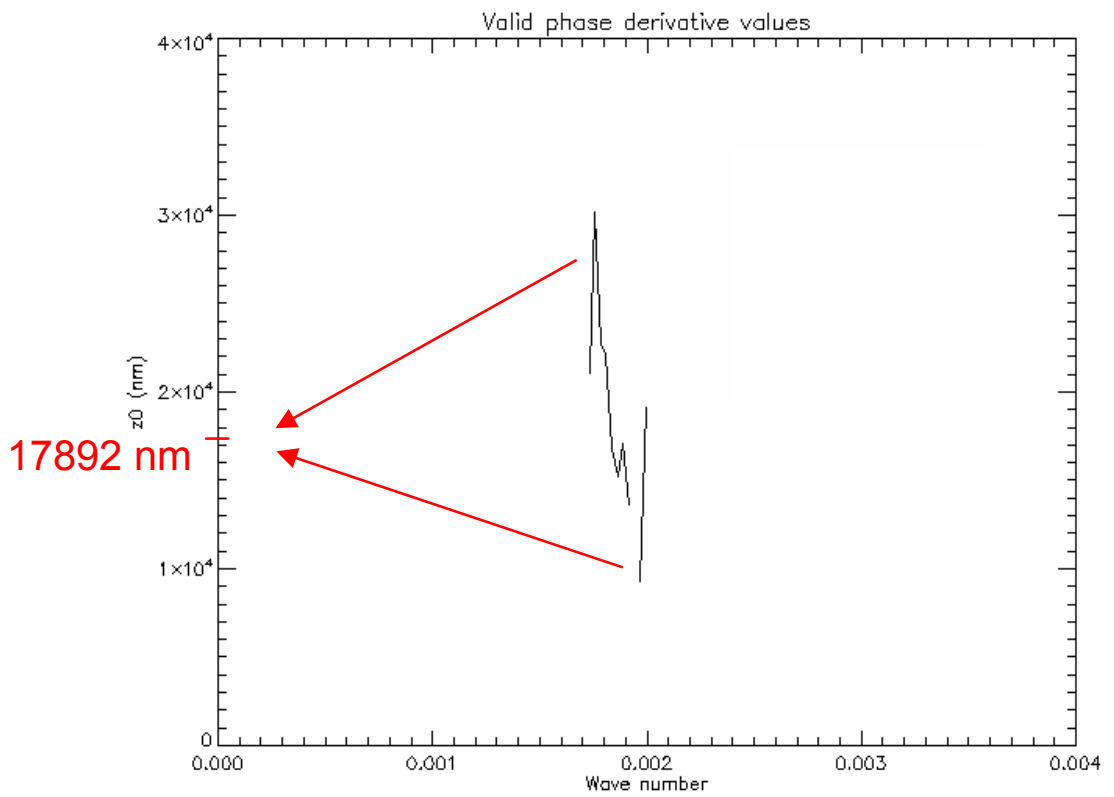

FIGURE 3.12 - Recovered $\mathrm{z}_{0}$ value for LCI data at interface saline2, weighted appropriately.

The reconstructed surface for a sample with regions of different thickness is shown in

Figure 3.13. The different heights are clearly seen. 


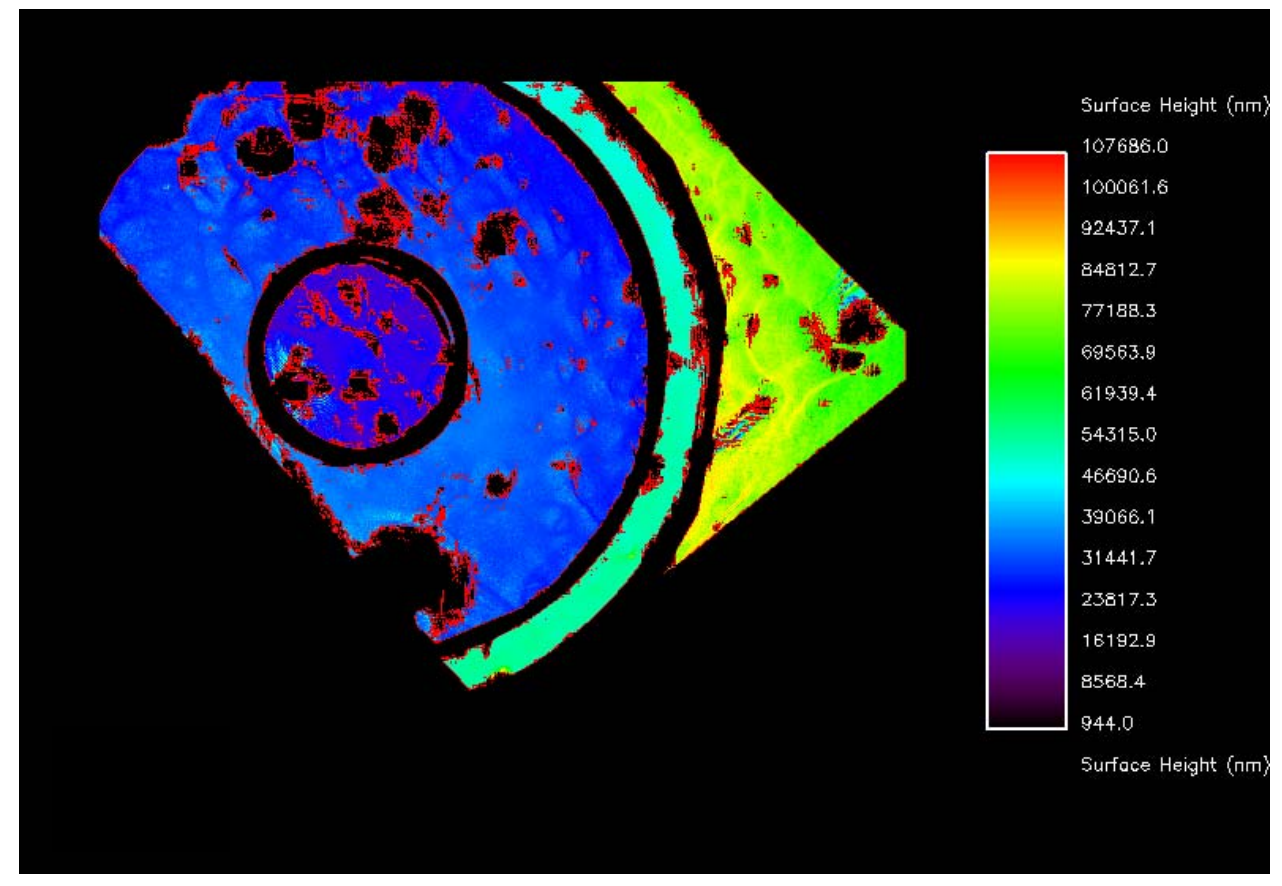

FIGURE 3.13 - Recovered saline2 surface using Fourier transform technique.

An image with higher axial resolution $(20 \mu \mathrm{m})$ on one sub-section of this image is shown in Figure 3.14. 


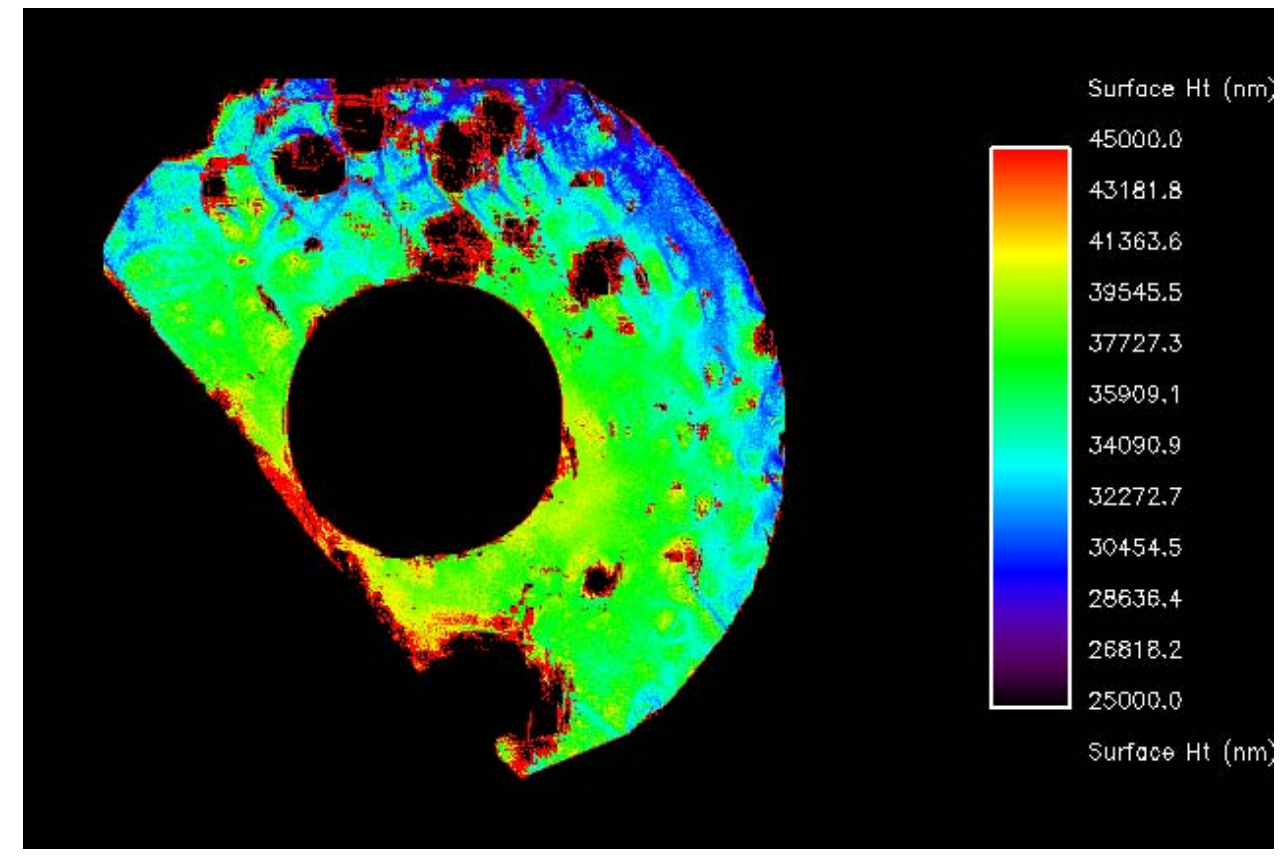

FIGURE 3.14 - Surface height of sub-section on a finer axial scale.

Finally, a profile through the subsection is plotted in Figure 3.15.

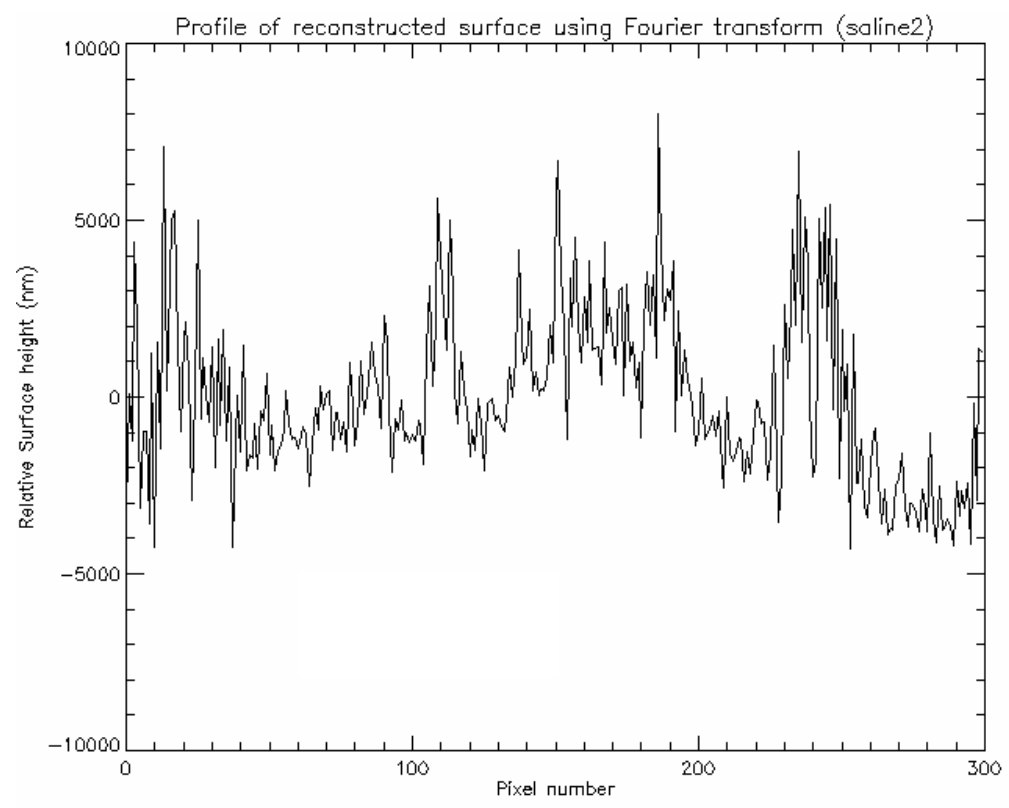

FIGURE 3.15 - Profile of the recovered surface in Figure 3.14. 
The standard deviation of this line is about $2300 \mathrm{~nm}$, or over six times worse then for a glass/air interface. Although some of the variation is actual surface shape, there is a large amount of random variation on top of the real variation. Clearly, an improvement must be made in order to reach the goals for measuring the physical thickness of the test material.

\subsection{The Curve Fitting Method}

The problem with the Fourier method is the unevenly spaced data and the fact that the $\mathrm{z}$ locations of the data are known, but unused. The curve fitting method is an attempt to use this axial information to improve the surface finding accuracy. As in the previous method, data for a single CCD pixel is used independently of any neighboring pixels, making the thickness measurement at each location unique.

The basic principle behind this measurement is that the form of the low coherence signal is known to be well approximated by the product of a cosine and a Gaussian. There are several variables in this equation, and this algorithm varies these parameters (including the location of the peak of the envelope) to match the recorded irradiance signal.

$$
I_{e s t}(z)=I_{D C}+A \cdot \operatorname{Gaus}\left(\frac{z-z_{0}}{b}\right) \cos \left(2 \pi \frac{z}{\lambda}+\varphi_{0}\right)=I_{D C}+A \cdot e^{-\pi\left(\frac{z-z_{0}}{b}\right)^{2}} \cos \left(2 \pi \frac{z}{\lambda}+\varphi_{0}\right)
$$

Equation 3.7

Here, $\mathrm{I}_{\mathrm{DC}}$ is the background value of the signal, which can be approximated as the mean of the signal value. A is the maximum fringe visibility for this pixel. The Gaussian 
envelope is centered at $\mathrm{z}_{0}$, which is also the value taken as the surface location once the best fit has been found. The width of the coherence envelope is given as $b$, which for this algorithm is fixed; it is primarily a function of the light source used. The center wavelength of the source spectrum, $\lambda$, can vary with time. The offset of the cosine at the center of the envelope, $\varphi_{0}$ also varies point to point, but is bound between 0 and $2 \pi$. The data recorded is a series of values for $\mathrm{I}(\mathrm{z})$ at a set of known positions, $\mathrm{z}$. The parameters $\mathrm{I}_{\mathrm{DC}}, \mathrm{A}, \mathrm{z}_{0}$, and $\varphi_{0}$ are all varied for each pixel to determine a best fit and the best estimate of $\mathrm{z}_{0}$.

A very rough estimate of the center of the envelope is found by averaging the axial locations of the smallest and largest values for I(z) for a given pixel, $\mathrm{z}_{\mathrm{st}}$. A 7-step phase shifting algorithm is applied to the closest $\mathrm{z}$ location +6 neighboring pixels and again for -6 neighboring pixels (Wyant, 2007). Recall that the sampling step along $\mathrm{z}$ is $5 \lambda / 4$ in OPD. Thus, the phase steps between neighboring axial locations is roughly $450^{\circ}$, which is equivalent to $90^{\circ}$ steps $\left(450^{\circ}-360^{\circ}=90^{\circ}\right)$. The two values for the phase are averaged and used as an initial value for $\varphi_{0}$.

$$
\begin{aligned}
\varphi_{0, \text { initial }} & =0.5\left(\tan ^{-1}\left\{\frac{4\left[I\left(z_{s t}+1\right)-2 I\left(z_{s t}+3\right)+I\left(z_{s t}+5\right)\right]}{-I\left(z_{s t}\right)+7 I\left(z_{s t}+2\right)-7 I\left(z_{s t}+4\right)+I\left(z_{s t}+6\right)}\right\}\right. \\
& \left.+\tan ^{-1}\left\{\frac{4\left[I\left(z_{s t}-1\right)-2 I\left(z_{s t}-3\right)+I\left(z_{s t}-5\right)\right]}{-I\left(z_{s t}\right)+7 I\left(z_{s t}-2\right)-7 I\left(z_{s t}-4\right)+I\left(z_{s t}-6\right)}\right\}\right)
\end{aligned}
$$

Equation 3.8

An initial value for $A$ is found from an average of $I(z)$. The next step is to calculate the simulated signal of the form in equation 3.7 for a range of values for $\mathrm{z}_{0}$. An error 
function is computed for each value of $\mathrm{z}_{0}$. The $\mathrm{z}_{0}$ value that corresponds to the minimum error value is stored.

$$
\operatorname{error}\left(z_{0}\right)=\sum\left(I(z)-\left[I_{D C}+A \cdot e^{-\pi\left(\frac{z-z_{0}}{b}\right)^{2}} \cos \left(2 \pi \frac{z}{\lambda}+\varphi_{0}\right)\right]\right)^{2}
$$

The next value to be adjusted is the maximum fringe visibility, A, to minimize the error function. The 7-step algorithm used to calculate $\varphi_{0, \text { initial }}$ has errors due to the variation of fringe visibility over those 7 images. Instead of trying to calculate this error, the algorithm is applied to the same axial locations in $\mathrm{I}_{\text {est }}$ and $\varphi_{0}$ is adjusted until the model phase matches the measured phase. The idea is that the phase errors will be duplicated in $\mathrm{I}_{\text {est }}$ by using the 7-step algorithm.

Finally, the value of $z_{0}$ is adjusted again until the error function is minimized. The minimum incremental change of $z_{0}$ is around $2.5 \mathrm{~nm}$. Figure 3.16 shows the collected data in black and the best fit curve in pink. 


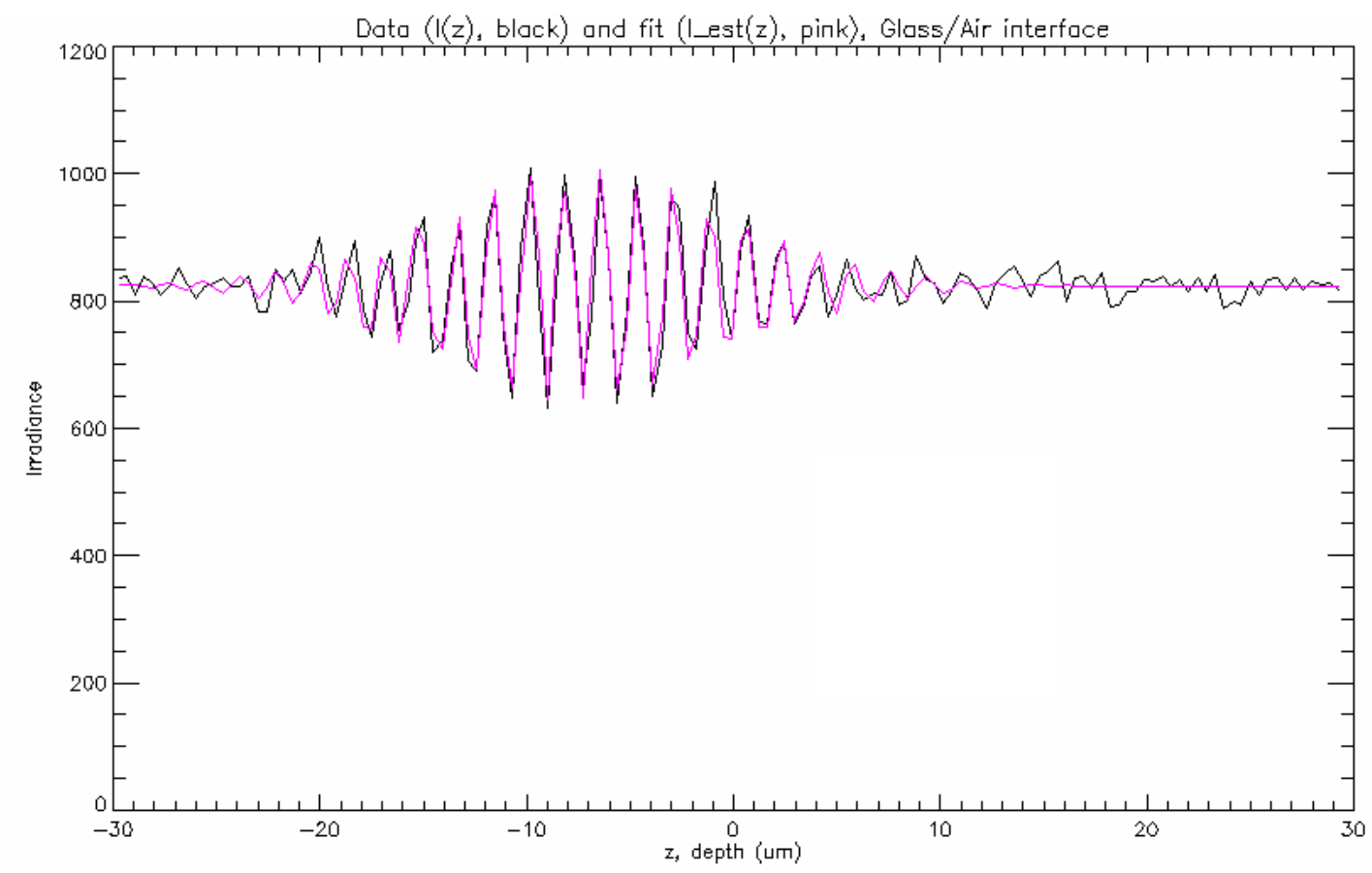

FIGURE 3.16 - Raw data I(z) (black) and estimated LCI curve, $\mathrm{I}_{\mathrm{est}}$, (pink) used to estimate $\mathrm{z}_{0}$.

This is a signal for a glass/air interface and is the same signal examined in detail for the Fourier transform routine, shown in Figure 3.4. The value recovered for the center of this signal, or the surface location, is $\mathrm{z}_{0}=-7371 \mathrm{~nm}$ (Fourier technique, $\mathrm{z}_{0}=-7247 \mathrm{~nm}$ ).

Figure 3.17 is a plot of the error function as the value of $z_{0}$ is varied; the final step of the algorithm. The minimum is clearly located at $-7371 \mathrm{~nm}$. 


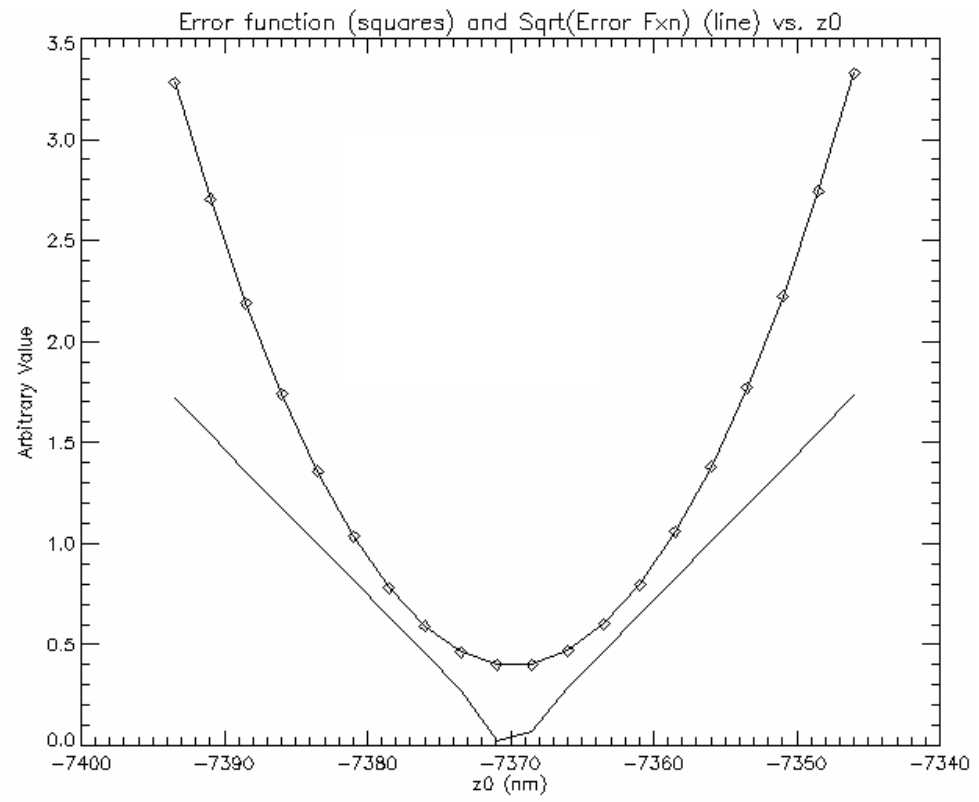

FIGURE 3.17 - Plot of error function (Equation 3.9) versus $z_{0}$ to determine best $\mathrm{z}_{0}$ value.

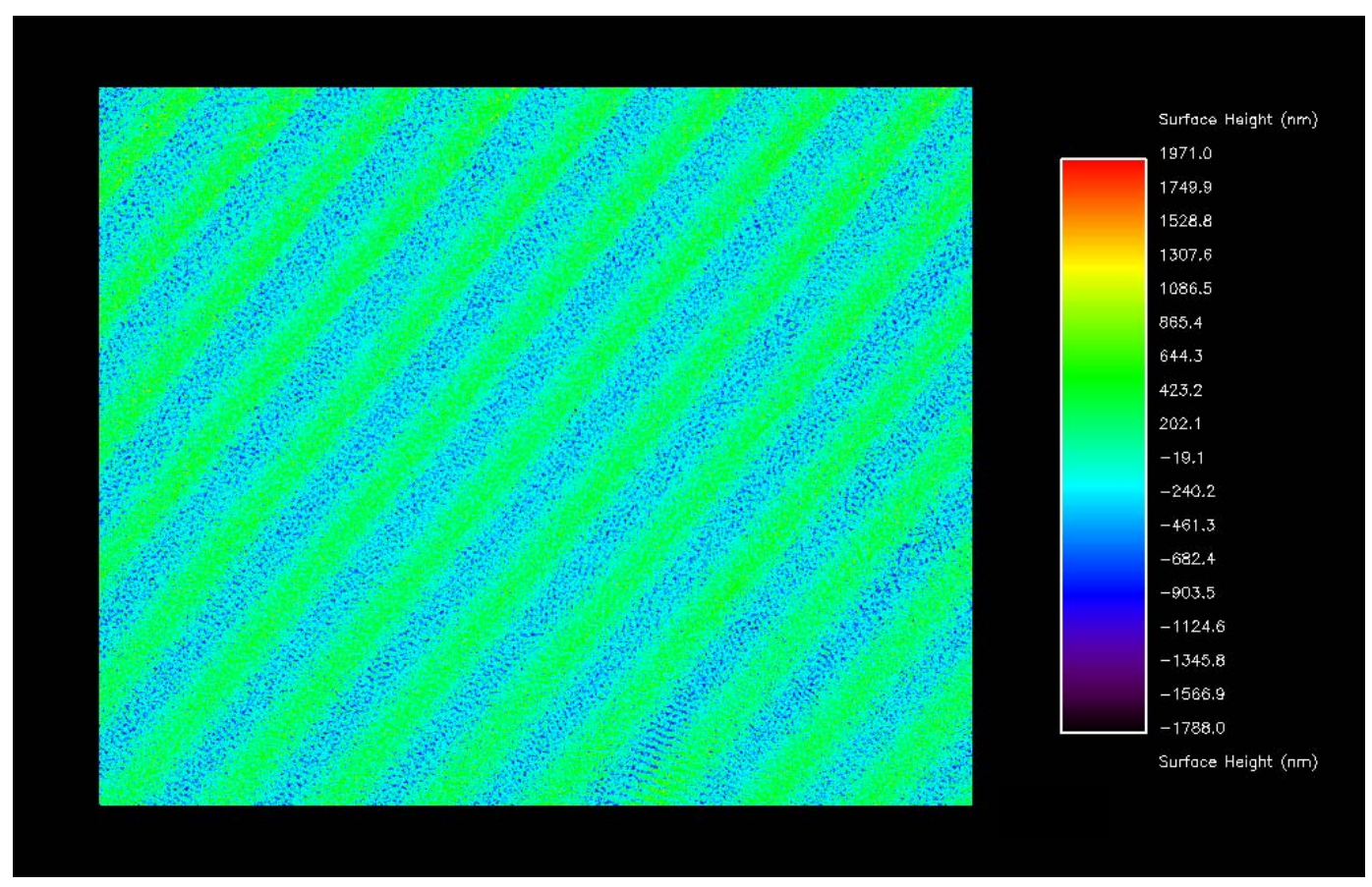

FIGURE 3.18 - Reconstructed surface, $\mathrm{z}_{0}(\mathrm{x}, \mathrm{y})$ for a glass/air interface using curve fitting technique. 
Figure 3.18 shows the full recovered surface with the best fit plane subtracted on the same scale as the surface for the first method. The standard deviation of this surface is $321.5 \mathrm{~nm}$, slightly better than the Fourier transform method $(357.3 \mathrm{~nm})$. A surface profile of the reconstructed surface is shown in Figure 3.19. It is clear that the predominant error is still a pattern at twice the frequency of the fringes. This technique is quite sensitive to the value of $\lambda$, or the underlying fringe frequency, and the amplitude of the fringe error changes with the value of $\lambda$.

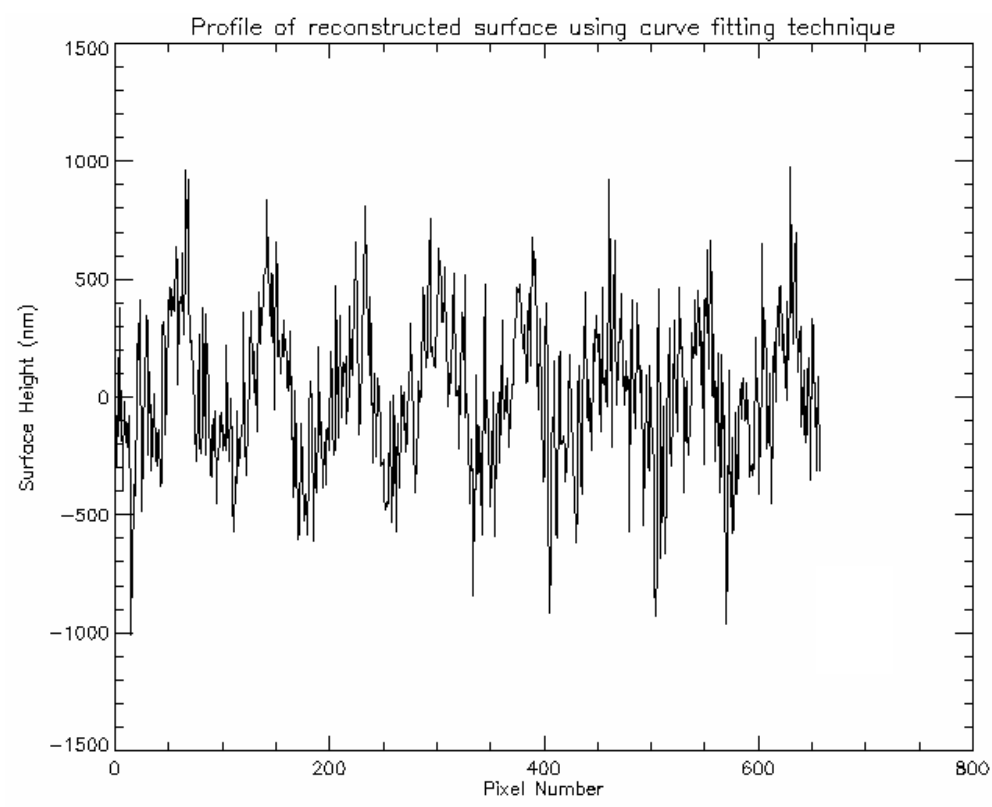

FIGURE 3.19 - Profile of reconstructed surface shown in Figure 3.18.

Another source of error for this particular algorithm is its sensitivity to the coherence envelope shape deviating from a Gaussian. Creating a non-symmetric function for curve fitting would be difficult and even more time consuming to run on a given surface. 
Perhaps the most interesting noise feature of this algorithm is the effect of coherent scatter. At first glance, the noise in the surface appears to be random. Upon closer inspection, the spatial regions of light (high) and dark (low) in the image are larger than a single pixel. A grayscale image of the reconstructed surface is included in Figure 3.20 as an additional reference for these features. The image on the right is a raw image taken during the scan to show the background features present in the image

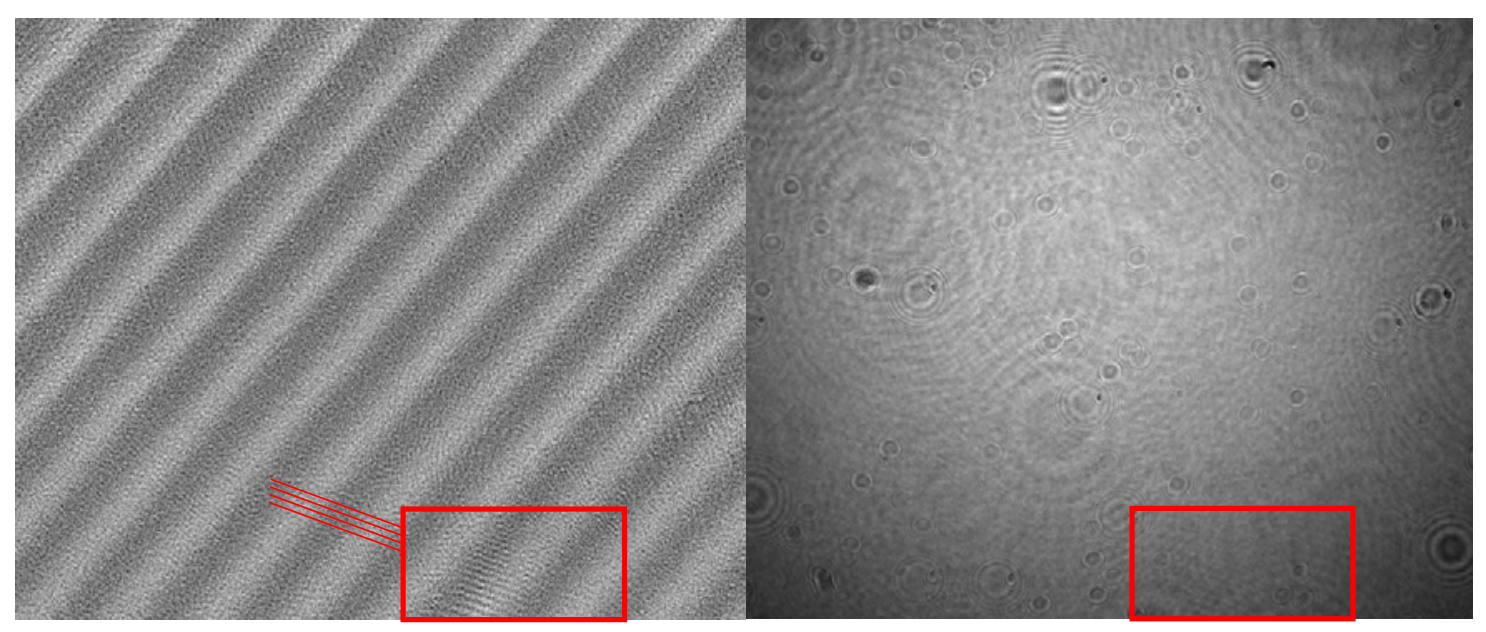

FIGURE 3.20 - Grayscale image of reconstructed surface (left) and single image (right), $\mathrm{I}(\mathrm{x}, \mathrm{y})$, from the scan to show the background features. Red box indicates the region that is magnified in Figure 3.21.

Many of these features are scatter from dust on the many optical interfaces in the system. Depending on their location, scattering efficiency and coherence properties, these features may or may not effect the reconstructed surface calculations. The box is indicating a region where the scatter was coherent; a pattern similar to the scattered light shows up in the surface. In this case, the pattern is a series of parallel lines, the orientation of which is indicated by the red lines superimposed on the surface image. There is so much scattered light in the raw image that it is difficult to see the scattered 
light that is effecting the surface calculation, and it is clear that much of the scatter does not affect the surface at all. In Figure 3.21, the region in the red box of Figure 3.20 is magnified for the two images.

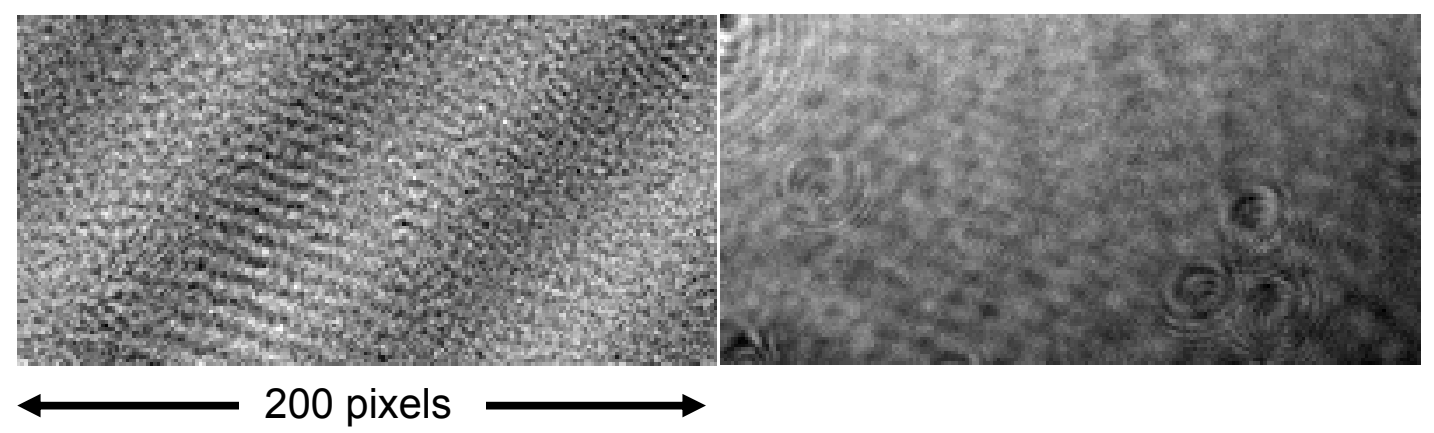

FIGURE 3.21 - Magnified regions from two images in Figure 3.20.

The surface noise that at first appears random is actually not so random. In fact, if the same surface is measured twice, the correlation $(\varsigma)$ between the noise structures of the two surfaces is as much as $97 \%$, indicating that the surface error is due to coherent scatter that is constant with time, not random irradiance fluctuations (Frieden, 2001).

$$
\varsigma=\frac{\left(s_{1}-\left\langle s_{1}\right\rangle\right)\left(s_{2}-\left\langle s_{2}\right\rangle\right)}{\sigma_{s_{1}} \sigma_{s_{2}}} ; \quad-1 \leq \varsigma \leq 1
$$

In the case of random noise, a comparison of the noise on the top and bottom surfaces of the measurement cavity should show independent noise features. This is not the case. For two independent surfaces, the standard deviation adds in quadrature, so the sum is larger then either of the components.

$$
\sigma_{\text {thickness }}=\sqrt{\sigma_{s_{1}}^{2}+\sigma_{s_{2}}^{2}}
$$

The fact that it is not indicates that the two surfaces are correlated. The correlation between the top and bottom surfaces in a single scan can be as much as $70 \%$. The 
standard deviation of the cavity thickness (difference of the two surfaces, air1 and air2) is actually less then the standard deviation of either of the two individual surfaces, which is important since the thickness is what the low coherence measurement is ultimately after.

Even with the improvement for a thickness value, the reconstructed surface is too noisy to reach the desired measurement goal of 0.0001 in refractive index. The largest noise is the features at twice the fringe frequency, which cause variations in the best fit plane that are much larger than the allotted error budget for the thickness extrapolation. This method is unacceptable for determining the surface location for the glass/air interfaces.

As in the Fourier method, the saline/sample interface is a more difficult surface to calculate using the curve fitting approach. Figure 3.22 shows the signal with the fit superimposed. The signal to noise ratio is so low that the curve fitting routine often has trouble with this interface. 


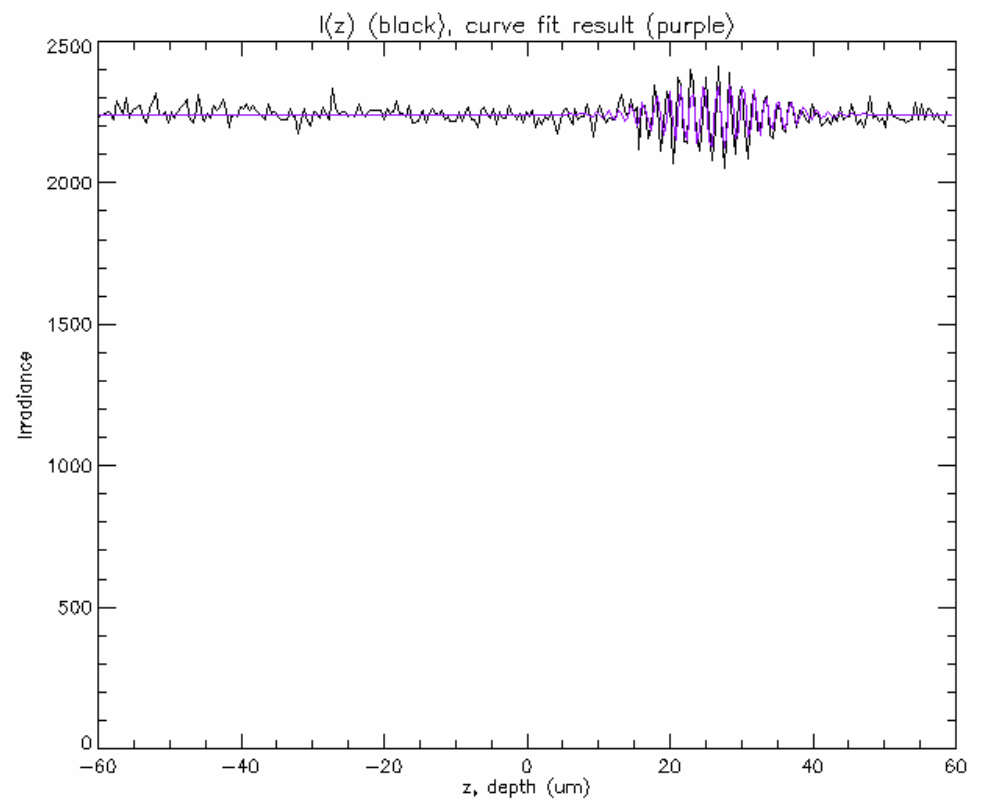

FIGURE 3.22 - Raw data I(z) (black) and estimated LCI curve, $\mathrm{I}_{\text {est }}$, (purple) for saline/contact interface (saline2).

The same region of the test sample for the same data set is displayed here on the same scale for comparison with the Fourier technique (Figure 3.23). 


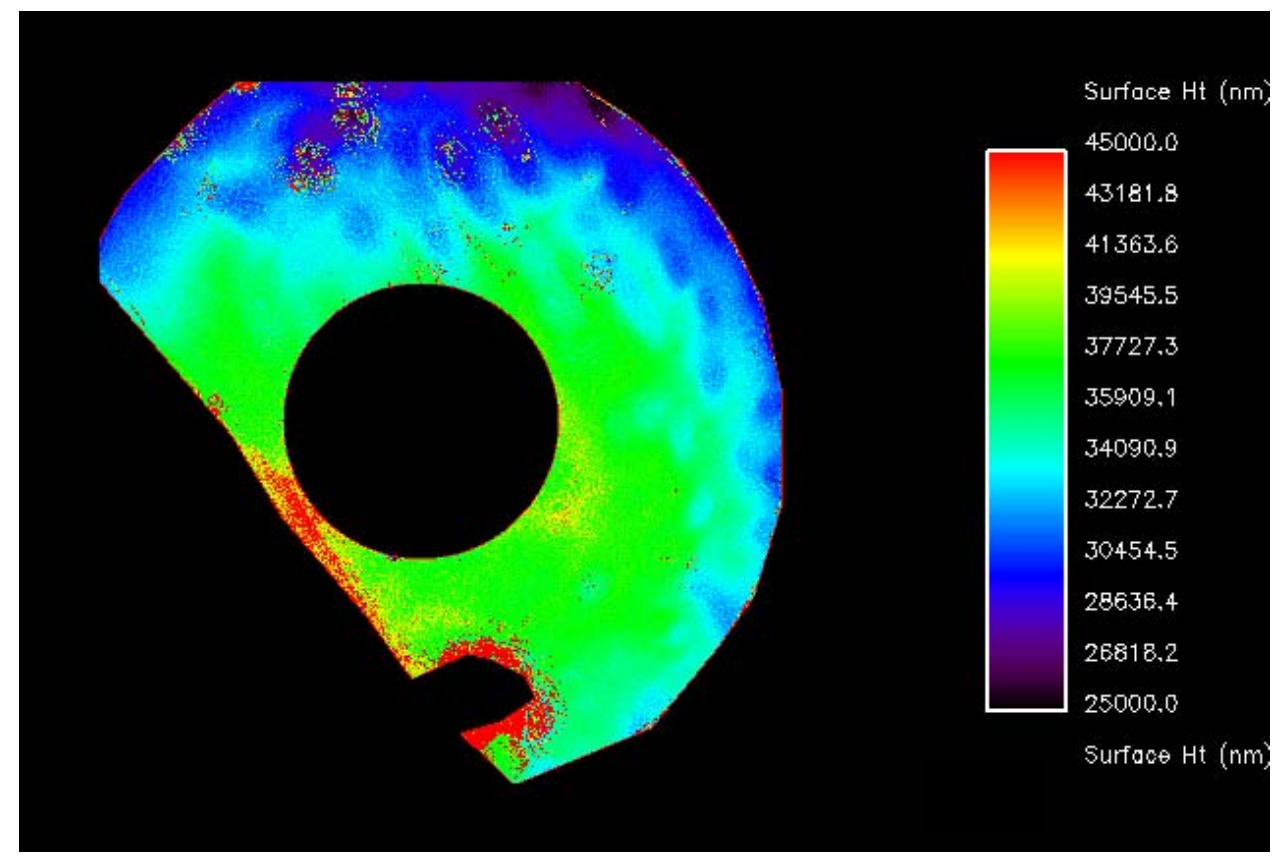

FIGURE 3.23 - Same surface sub-section from Figure 3.14 processed using the curve fitting technique, for comparison with the Fourier technique.

The standard deviation over the same region as in the Fourier technique is $1370 \mathrm{~nm}$, which is an improvement $\left(\sigma_{\mathrm{FT}}=2300 \mathrm{~nm}\right)$. The profile in the same region is shown in Figure 3.24. 


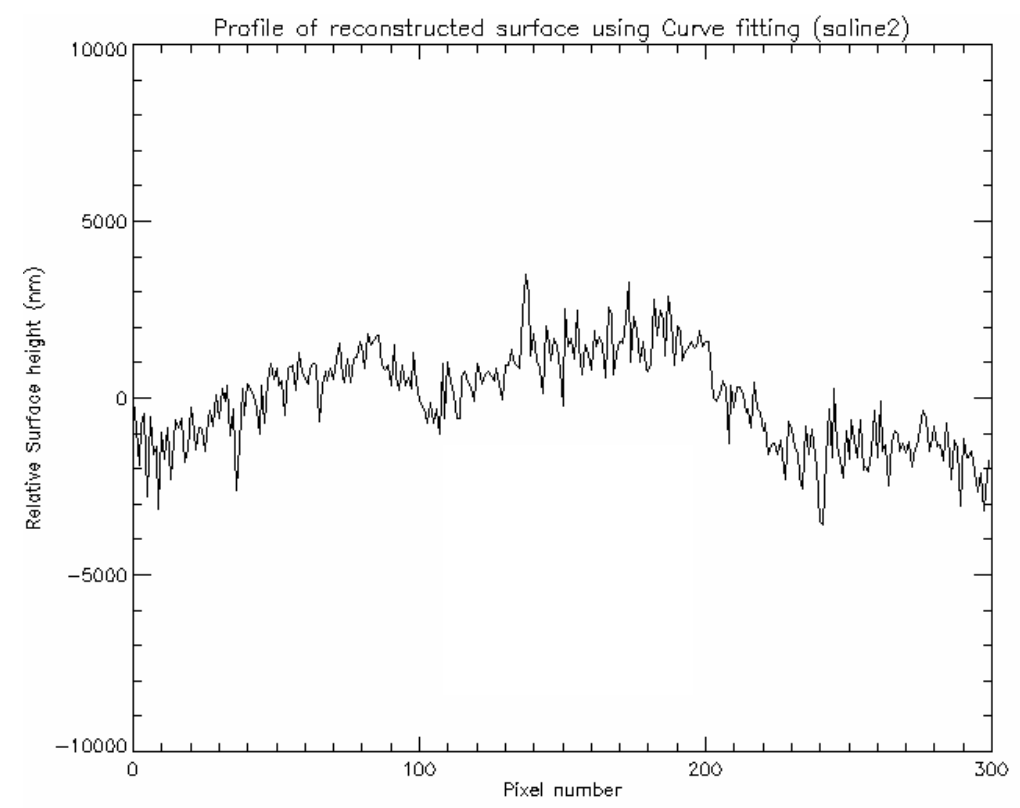

FIGURE 3.24 - Profile of the recovered surface using the curve fitting technique.

The above plot is on the same scale as Figure 3.15, and the spatial surface height variation is clearly lower. One important issue with the curve fitting technique is that there is an offset in the surface location, which leads to a bias in the physical thickness measurement. This bias in turn biases the value of refractive index.

The curve fitting algorithm offers improvement over the Fourier transform technique in terms of the standard deviation of the reconstructed surfaces, but the structured fringe pattern noise is still present and causes problems in surface extrapolation. In addition, there is often a bias in the recovered surfaces with lower contrast fringes. This technique is not sufficient for this measurement technique. 


\subsection{Least Squares Fit to Modulation}

The previously discussed techniques processed the low coherence signals for each pixel individually, providing as many measurements of the index, $\mathrm{n}$, as there were pixels in the region of interest. In general, the standard deviation of a measurement of a noisy process improves as more measurements are taken (Frieden, 2001).

$$
\sigma_{\bar{n}}=\frac{\sigma_{n}}{\sqrt{N}}
$$

Here, $\sigma_{\mathrm{n}}$ is the standard deviation of a single measurement of $n$. The estimate of the standard deviation of the index improves as the number of measurements, $\mathrm{N}$, increases. This equation assumes the repeated measurements are free of bias and are completely uncorrelated with each other. In the discussion of the two previous surface finding algorithms, it was clear that the multiple measurements of the surface suffered from both bias and spatial correlation. There is additional information to be gained by using the fact that the surfaces under test are continuous, and in the case of the glass plates, flat. If the information from neighboring pixels is combined such that this additional information is incorporated into finding surfaces, the recovered surfaces should be more accurate. The tradeoff is that the value at each pixel on the camera is no longer independent of all the other pixels and equation 3.12 no longer applies for averaging the data to get improved accuracy. Since equation 3.12 could not be applied in conjunction with the previous algorithms anyway, little is lost in this tradeoff.

In the previous techniques, the irradiance information used to determine a surface height was contained in a $1 \mathrm{xN}$ array, where $\mathrm{N}$ is the number of frames taken in depth for a scan. 
Using the spatial information changes the data used for a single height value to an array as large as $\mathrm{W}_{\mathrm{x}} \mathrm{x} \mathrm{W}_{\mathrm{y}} \mathrm{xN}$, where $\mathrm{W}_{\mathrm{x}}$ and $\mathrm{W}_{\mathrm{y}}$ are the widths of the spatial region of interest.

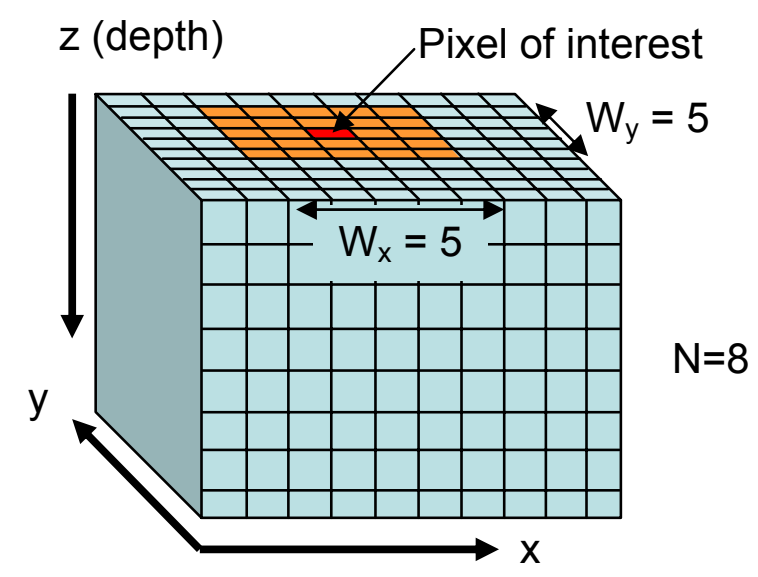

FIGURE 3.25 - Using spatial information from the data cube should improve surface finding accuracy.

As the pixel of interest shifts, so does the three-dimensional region of interest. The signal along the $\mathrm{z}$ axis is the low coherence signal shown previously; the product of a cosine and a Gaussian, I(z).

This new method approaches the problem with the goal of finding the highest fringe modulation $(\gamma)$ versus depth, which is also the peak of the coherence envelope. An earlier attempt was made to calculate $\gamma(\mathrm{z})$ from I(z) for a single pixel. This approach produced results similar to the Fourier and Curve Fitting techniques, so the results will not be discussed in detail. The problem is that the equations used for calculating the modulation require, in general, two things; 1 ) the distance (or phase) between the samples is constant, and 2), the modulation is constant over the samples. The low coherence signals violate both conditions. One such algorithm for calculating the modulation which 
is based off the 5-step Schwider-Hariharan algorithm additionally requires equal $90^{\circ}$ phase steps (Greivenkamp, 1992):

$$
\gamma=\frac{3 \sqrt{4\left(I_{4}-I_{2}\right)^{2}+\left(I_{1}+I_{5}-2 I_{3}\right)^{2}}}{2\left(I_{1}+I_{2}+2 I_{3}+I_{4}+I_{5}\right)}
$$

Equation 3.13 is applied to all groups of 5 pixels along the scan, and the peak of the resulting modulation plot corresponds to the surface location. Recall that the phase steps are actually $450^{\circ}$ phase steps, so this algorithm is five times more sensitive to errors in the actual phase step then if the steps were $90^{\circ}$.

There are other algorithms used to determine fringe modulation that require only that the phase shifts be known, not necessarily constant. One such algorithm is based off of a least squares fit to the irradiance signal, I(z) (Greivenkamp, 1984).

$$
\gamma=\frac{\sqrt{a_{1}^{2}+a_{2}^{2}}}{a_{0} \operatorname{sinc}(\Delta / 2)}
$$

The integration period for each image is $\Delta$, which in the case of Engage, is 0 . Since the value of interest is the relative modulation, as long as $\Delta$ is constant, it does not need to be known to determine the surface location. The three ' $a$ ' coefficients are defined by the following matrix operation (Greivenkamp, 1984).

$$
\left|\begin{array}{l}
a_{0} \\
a_{1} \\
a_{2}
\end{array}\right|=\mathbf{A}^{-1}\left(\delta_{i}\right) \mathbf{B}\left(\delta_{i}\right)=\left|\begin{array}{ccc}
N & \sum \cos \left(\delta_{i}\right) & \sum \sin \left(\delta_{i}\right) \\
\sum \cos \left(\delta_{i}\right) & \sum \cos ^{2}\left(\delta_{i}\right) & \sum \cos \left(\delta_{i}\right) \sin \left(\delta_{i}\right) \\
\sum \sin \left(\delta_{i}\right) & \sum \cos \left(\delta_{i}\right) \sin \left(\delta_{i}\right) & \sum \sin ^{2}\left(\delta_{i}\right)
\end{array}\right|^{-1}\left|\begin{array}{c}
\sum I_{i} \\
\sum I_{i} \cos \left(\delta_{i}\right) \\
\sum I_{i} \sin \left(\delta_{i}\right)
\end{array}\right|
$$

Equation 3.15 
Where $\mathrm{N}$ is the number of irradiance values, $\mathrm{I}_{\mathrm{i}}$ is the irradiance at the $\mathrm{i}^{\text {th }}$ depth location, and $\delta_{\mathrm{i}}$ is the phase shift for the $\mathrm{i}^{\text {th }}$ depth location. This allows different but known phase shifts to be used in an algorithm to determine the modulation. This algorithm still assumes a constant modulation for all $\mathrm{N}$ irradiance values, which is not the case for the irradiance as a function of depth. It can not be used effectively versus depth for this reason.

\subsubsection{Incorporating Spatial Information}

The modulation as a function of depth varies, by definition, for the low coherence signal. However, the data collected for a surface is a cube $I(x, y, z)$, with the spatial size and sampling period defined by the number of pixels and pixel spacing, respectively. Consider a single image, $\mathrm{I}(\mathrm{x}, \mathrm{y})$, from the cube. The fringe modulation calculated using neighboring locations in the cube in either of the spatial dimensions $(\mathrm{x}, \mathrm{y})$ will result in the same modulation as calculated along depth (z). The advantage is that the modulation varies much more slowly spatially. The slower modulation variation will drastically reduce the errors in calculating $\gamma$. Repeating the modulation calculation at every voxel in the cube will result in $\gamma(z)$ for each pixel. The center of this relatively noise-free calculated coherence envelope is the surface location. This summarizes the technique proposed in this section for calculating the surface location from the data cube.

In the case of a glass/air interface, the surface is known to be flat to better then $\lambda / 20$. Figure 3.26 is a single interferogram in depth $\left(\mathrm{z}=\mathrm{z}_{\mathrm{i}}\right)$ for a glass/air interface. 


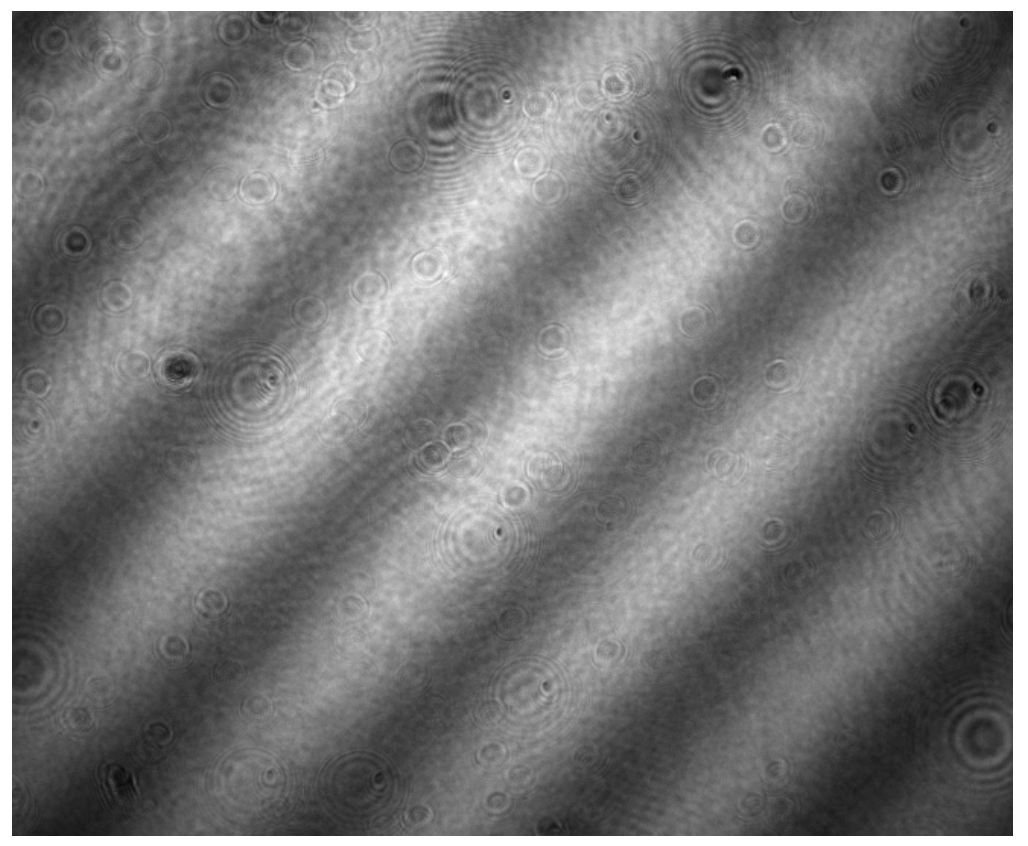

FIGURE 3.26 - Image from data cube showing tilt fringes from a glass/air interface.

The tilt fringes represent the difference in optical path length (OPL) between the reference and test surfaces, just as they do in a Twyman-Green interferometer with a laser light source. In the case of Engage, that path difference is a function of the two beamsplitters, the top plate of the cuvette, its corresponding compensating plate, the test and reference surfaces, and all of the air in between (Figure 3.27). All of the optical surfaces of these elements are flat to at least $\lambda / 20$, and there is no detectable index inhomogeneity. Therefore, the OPL between the two paths is a plane with some tilt that is a function of the wedge of the plates and the tilt of the test and reference surfaces. 


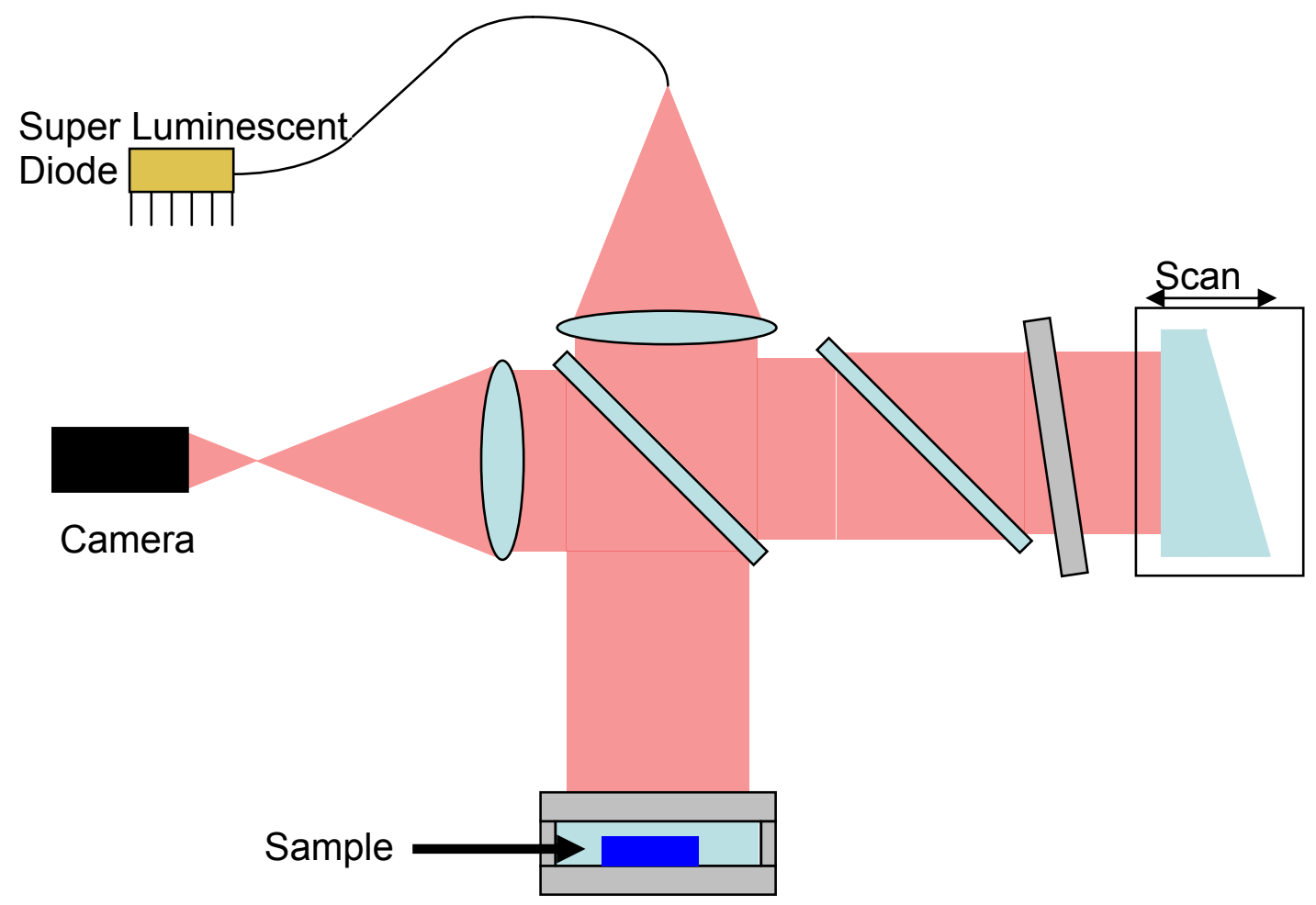

FIGURE 3.27 - Schematic of the LCI in Engage.

Note that this plane will not have the same slope as the height of the surface under test. This is because there will inevitably be some spatial variation of glass thickness in the interferometer. Recall that the phase index of glass does not equal the group index of glass. Since the fringe orientation depends on the OPD, which depends on phase index of the glass, and the surface location depends on the OGD, which depends on the group index of the glass, the OPD and OGD will only have the same $\mathrm{x}$ and $\mathrm{y}$ slopes when $\mathrm{t}_{\Sigma \text { glass }}(\mathrm{x}, \mathrm{y})$ becomes a constant across the detector.

$$
\begin{aligned}
& O P D(x, y)=t_{\sum \text { glass }}(x, y) n_{\bar{\lambda}} \\
& O G D(x, y)=t_{\sum \text { glass }}(x, y) N_{g, \bar{\lambda}}
\end{aligned}
$$


The fixed spacing of the pixels and the fact that the phase is a plane means that the phase difference between neighboring pixels along $\mathrm{x}$ (or $\mathrm{y}$ ) is a constant. These constant values can be determined by applying the Schwider-Hariharan phase shifting algorithm (Schwider, 1983) to the five frames with the best fringe visibility.

$$
\phi(x, y)=\tan ^{-1}\left[\frac{2\left(I_{2}(x, y)-I_{4}(x, y)\right)}{2 I_{3}(x, y)-I_{5}(x, y)-I_{1}(x, y)}\right]
$$

The resulting calculated phase will be modulo $2 \pi$ (Figure 3.28). The details of the unwrapping process, which removes these phase discontinuities, are discussed in Chapter 5. Finally a plane is fit to the unwrapped phase. The slope of the best fit plane to $\varphi(x, y)$ is the phase change between neighboring pixels, $\delta_{\mathrm{i}, \mathrm{x}}$ and $\delta_{\mathrm{i}, \mathrm{y}}$ along the $\mathrm{x}$ and y directions, respectively. These two values are constants across the detector for a planar surface.

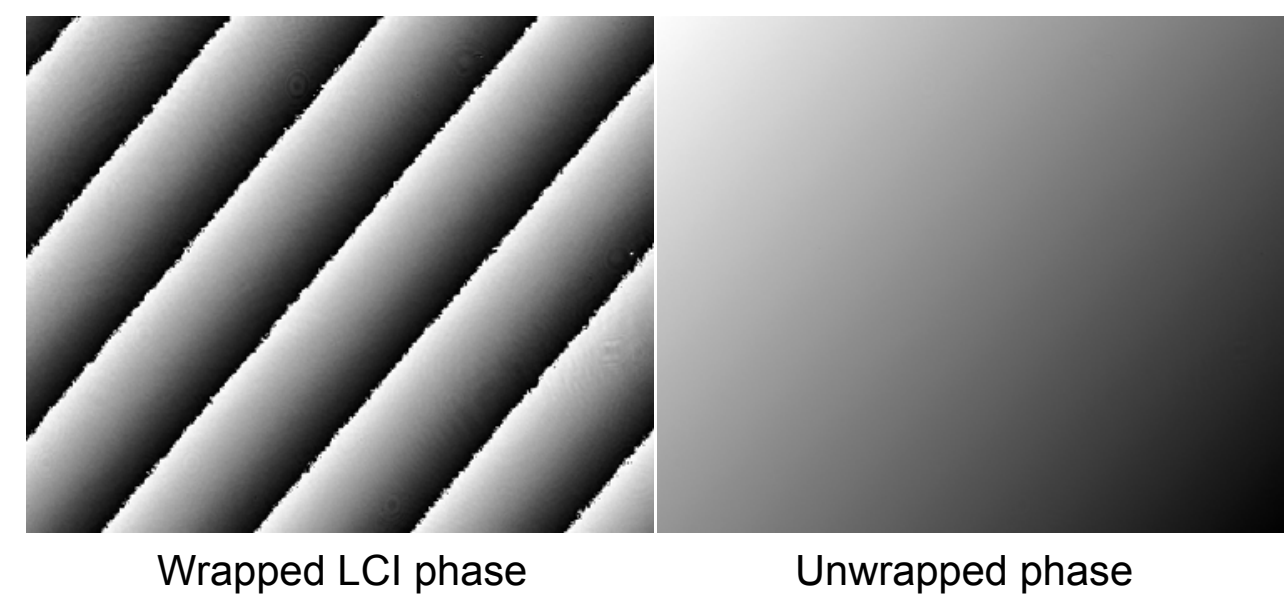

FIGURE 3.28 - Wrapped and unwrapped phase from the 5-step phase-shifting algorithm applied to LCI data. The slope of the phase surface is different than the slope of the actual surface in space.

For a planar surface, the matrix $\mathbf{A}$ is also a constant (along $\mathrm{x}$ or $\mathrm{y}$ ) since it only depends on the phase shift between adjacent pixels $\left(\delta_{\mathrm{i}, \mathrm{x}}, \delta_{\mathrm{i}, \mathrm{y}}\right)$ (Equation 3.18). 


$$
\mathbf{A}\left(\delta_{i}\right)=\left|\begin{array}{ccc}
N & \sum \cos \left(\delta_{i}\right) & \sum \sin \left(\delta_{i}\right) \\
\sum \cos \left(\delta_{i}\right) & \sum \cos ^{2}\left(\delta_{i}\right) & \sum \cos \left(\delta_{i}\right) \sin \left(\delta_{i}\right) \\
\sum \sin \left(\delta_{i}\right) & \sum \cos \left(\delta_{i}\right) \sin \left(\delta_{i}\right) & \sum \sin ^{2}\left(\delta_{i}\right)
\end{array}\right|
$$

Equation 3.18

Instead of applying the least squares algorithm to a series of pixels along $\mathrm{z}$, it can now be used on a series of pixels along $\mathrm{x}$ or $\mathrm{y}$. The modulation will still vary across these pixels, but the variation is much less since the glass/air interface is usually nearly perpendicular to the test beam. This variation will still cause some error, $\varepsilon$, in the calculated value of the modulation, $\gamma\left(\mathrm{z}_{\mathrm{i}}\right)$. For example, a group of pixels along $\mathrm{x}$ are used to calculate the modulation at pixel $\mathrm{x}_{\mathrm{c}}$ for each frame. Figure 3.29 shows $\mathrm{I}(\mathrm{x})$ after the spatial irradiance variation has been removed. The source has a non-uniform irradiance distribution, which can be found by averaging the frames in the data cube, $\mathrm{I}(\mathrm{x}, \mathrm{y}, \mathrm{z})$ at depth locations without any fringes. Every image in the cube is then divided by this 'baseline' image to remove the modulation variation due to the source distribution.

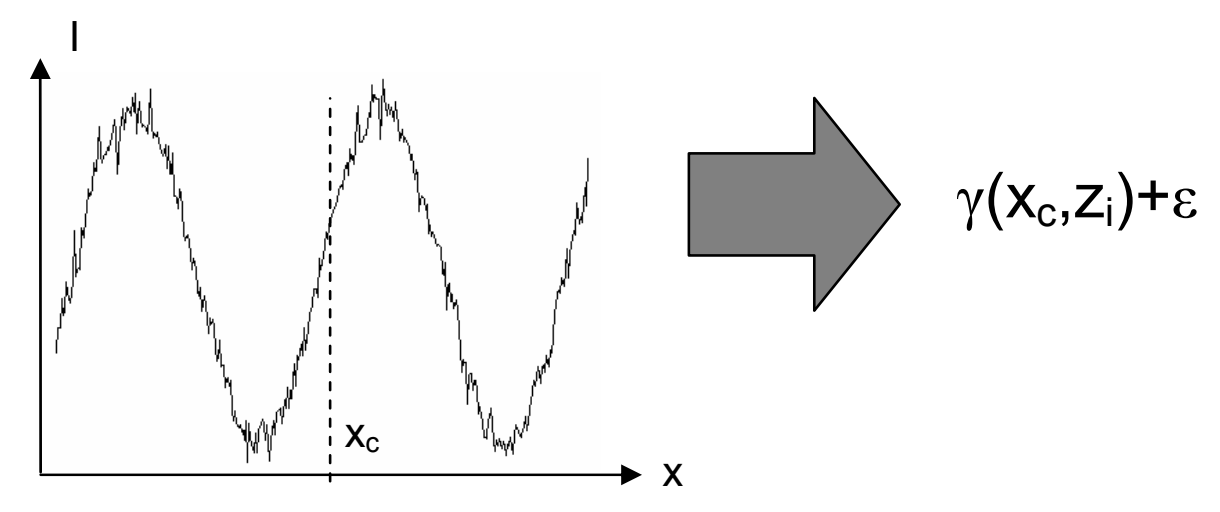

FIGURE 3.29 - I(x) for a single image after the baseline has been removed. This signal is used to calculate the modulation for the center pixel $\left(x_{c}\right)$ at this frame, $z_{i}$. 
This gives the modulation at the center pixel of the irradiance values $I(x)$, at the frame $z_{i}$. For a given pixel location $(\mathrm{x}, \mathrm{y})$, this is repeated for every frame, resulting in a plot of modulation versus depth $\gamma(\mathrm{z})$ (Figure 3.30).

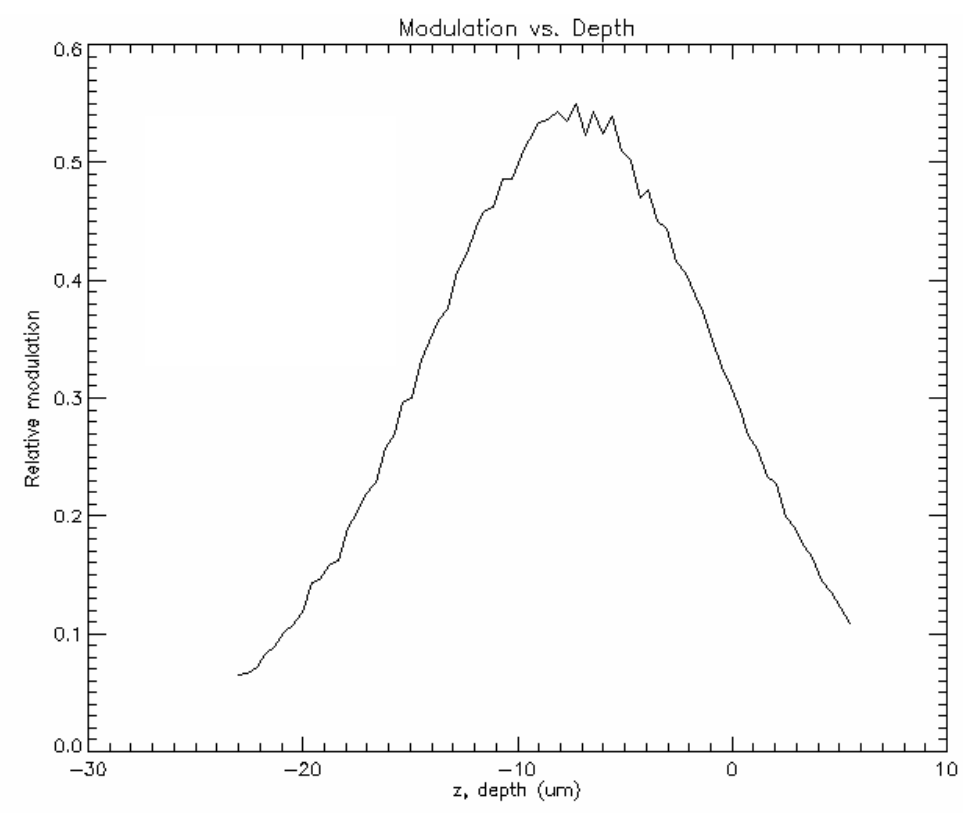

FIGURE 3.30 - Modulation versus depth $\gamma(\mathrm{z})$ for LCI signal in Figure 3.4.

The modulation error $\varepsilon$ comes from a few different sources. One component of the error depends on both the number of pixels used $(\mathrm{N})$ and which pixels are used. It is important to use the same group of pixels, $\left\{\mathrm{x}_{1} \ldots \mathrm{x}_{\mathrm{N}}\right\}$ for $\mathrm{x}_{\mathrm{c}}$ at each frame, $\mathrm{z}_{\mathrm{i}}$, so that the data used to calculate every modulation value versus depth is as uniform as possible.

The other major component of the error is the spatial modulation variation over the set of pixels used, $\left\{\mathrm{x}_{1} \ldots \mathrm{x}_{\mathrm{N}}\right\}$. This relationship will vary from frame to frame, depending on the location of the image relative to $\mathrm{OGD}=0$. Since the modulation variation varies as a 
function of frame, $\mathrm{z}_{\mathrm{i}}$, the error term $\varepsilon$ will vary as well. This error variation is much less than the error variation from calculating $\gamma$ from pixels along $\mathrm{z}$.

After the modulation has been calculated for every pixel $(x, y)$ at every frame $z_{i}$ that had sufficient modulation, the result is a curve like the one in Figure 3.30 for each pixel. The curve looks like the coherence envelope of the low coherence signal, in this case, a Gaussian. The center of this Gaussian is the location of the surface. By taking the natural logarithm of the modulation, $\gamma(\mathrm{z})$, the Gaussian shape becomes a quadratic (Hopler, 1991).

$$
\ln [\gamma(z)]=\ln \left[e^{-\pi\left(\frac{z-z_{0}}{b}\right)^{2}}\right]=-\pi\left(\frac{z-z_{0}}{b}\right)^{2}
$$

Finding the center of the quadratic is done by using a least squares fit (LSF) algorithm to fit a quadratic to the data in frames $\left\{z_{1} \ldots z_{N}\right\}$.

$$
\begin{gathered}
L S F\{\ln [\gamma(z)]\}=b_{0}+b_{1} z+b_{2} z^{2} \\
\left|\begin{array}{l}
b_{0} \\
b_{1} \\
b_{2}
\end{array}\right|=\mathbf{D}^{-1}\left(z_{i}\right) \mathbf{C}\left(z_{i}\right)=\left|\begin{array}{ccc}
N & \sum z_{i} & \sum z_{i}^{2} \\
\sum z_{i} & \sum z_{i}^{2} & \sum z_{i}^{3} \\
\sum z_{i}^{2} & \sum z_{i}^{3} & \sum z_{i}^{4}
\end{array}\right|^{-1}\left|\begin{array}{c}
\sum \ln \left(\gamma_{i}\right) \\
\sum z_{i} \ln \left(\gamma_{i}\right) \\
\sum z_{i}^{2} \ln \left(\gamma_{i}\right)
\end{array}\right|
\end{gathered}
$$

Once the coefficients of the quadratic fit have been calculated $\left(b_{0}, b_{1}, b_{2}\right)$, the center of the modulation signal is calculated using the following equation:

$$
z_{0}=\frac{-b_{1}}{2 b_{2}}
$$

The natural $\log$ of the modulation and the result of the LSF are plotted in Figure 3.31. 


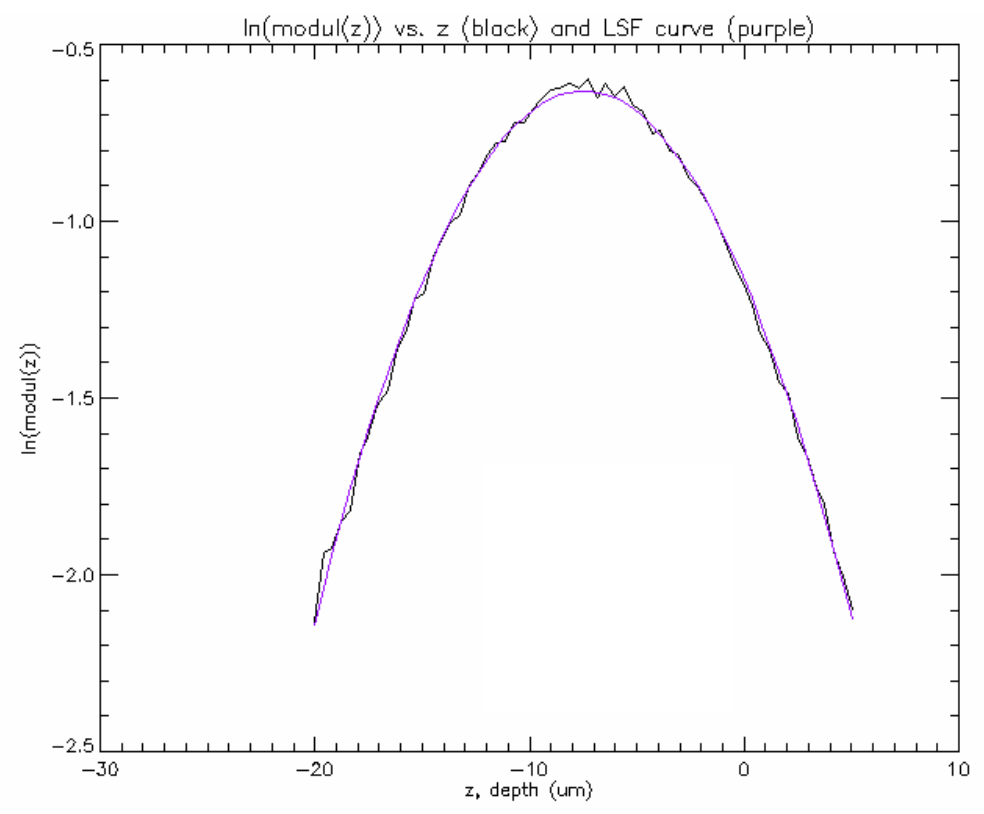

FIGURE 3.31 - Plots of calculated modulation, $\gamma(\mathrm{z})$ (black) and LSF to the natural log of the modulation (purple).

In this example, the resulting value for $\mathrm{z}_{0}$ is $-7479 \mathrm{~nm}$ (note this is a different $(\mathrm{x}, \mathrm{y})$

location then used in the previously discussed techniques). The modulation curves in this example are a result of spatial data taken along the $\mathrm{x}$-direction. The same procedure is repeated, using data along the y-direction, resulting in two values for $\mathrm{z}_{0}(\mathrm{x}, \mathrm{y}): \mathrm{z}_{0 \mathrm{x}}(\mathrm{x}, \mathrm{y})$ and $\mathrm{z}_{0 \mathrm{y}}(\mathrm{x}, \mathrm{y})$. The two calculated surfaces are displayed in Figure 3.32. 


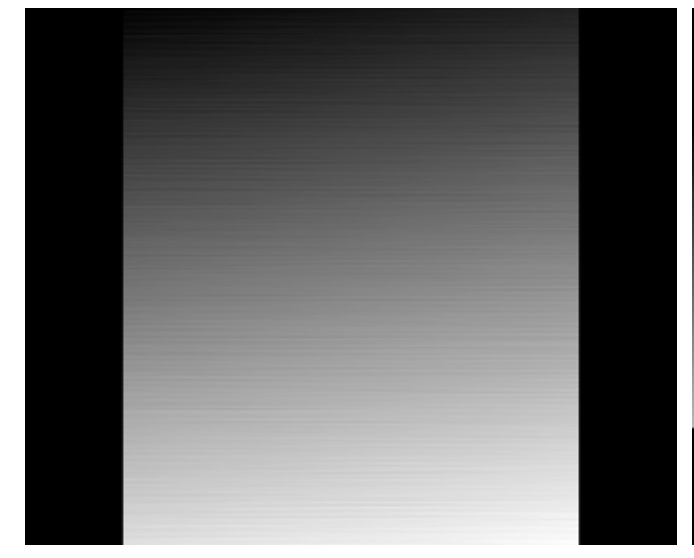

$\mathrm{Z}_{0}, \mathrm{x}$-direction

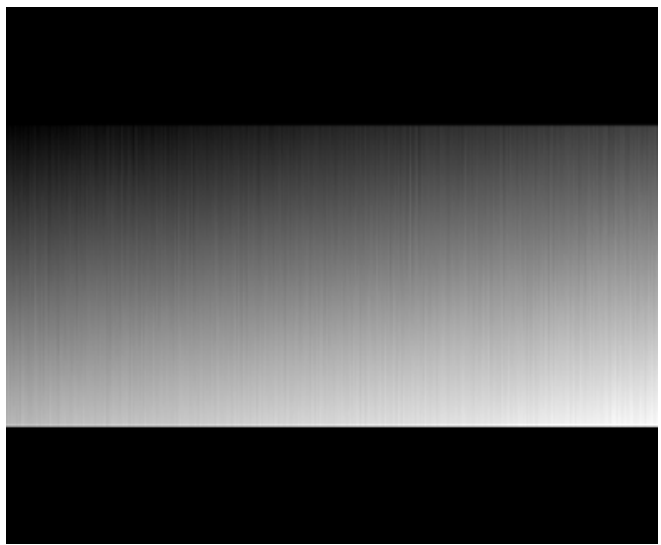

$\mathrm{Z}_{0}, \mathrm{y}$-direction

FIGURE 3.32 - Recovered surfaces using LSF to modulation with spatial information technique.

The black regions in the two images are where this algorithm was unable to determine a surface height. This is because of equation 3.15, which requires (N-1)/2 neighboring pixels along $\mathrm{x}$ (or $\mathrm{y})$ to compute the modulation at pixel $(\mathrm{x}, \mathrm{y})$. Since it calculates the modulation for the center pixel, the result is that edge pixels (left and right) are missing for the surface found using x-profiles. Many of these pixels can be found by looking at the y-profiles, but the corners are always lost (Figure 3.33). This loss of data is usually about $5 \%$ of the valid data points.

The average difference between $\mathrm{z}_{0, \mathrm{xprofile}}$ and $\mathrm{z}_{0 \text {,yprofile }}$ is $0.03 \mathrm{~nm}$; clearly they agree well. The point-to-point standard deviation of the difference between the $\mathrm{x}$ and $\mathrm{y}$ surfaces is 38 nanometers. These two surfaces are averaged over the area of common coverage, and the resulting surface after the best fit plane has been subtracted, is shown in Figure 3.33. 


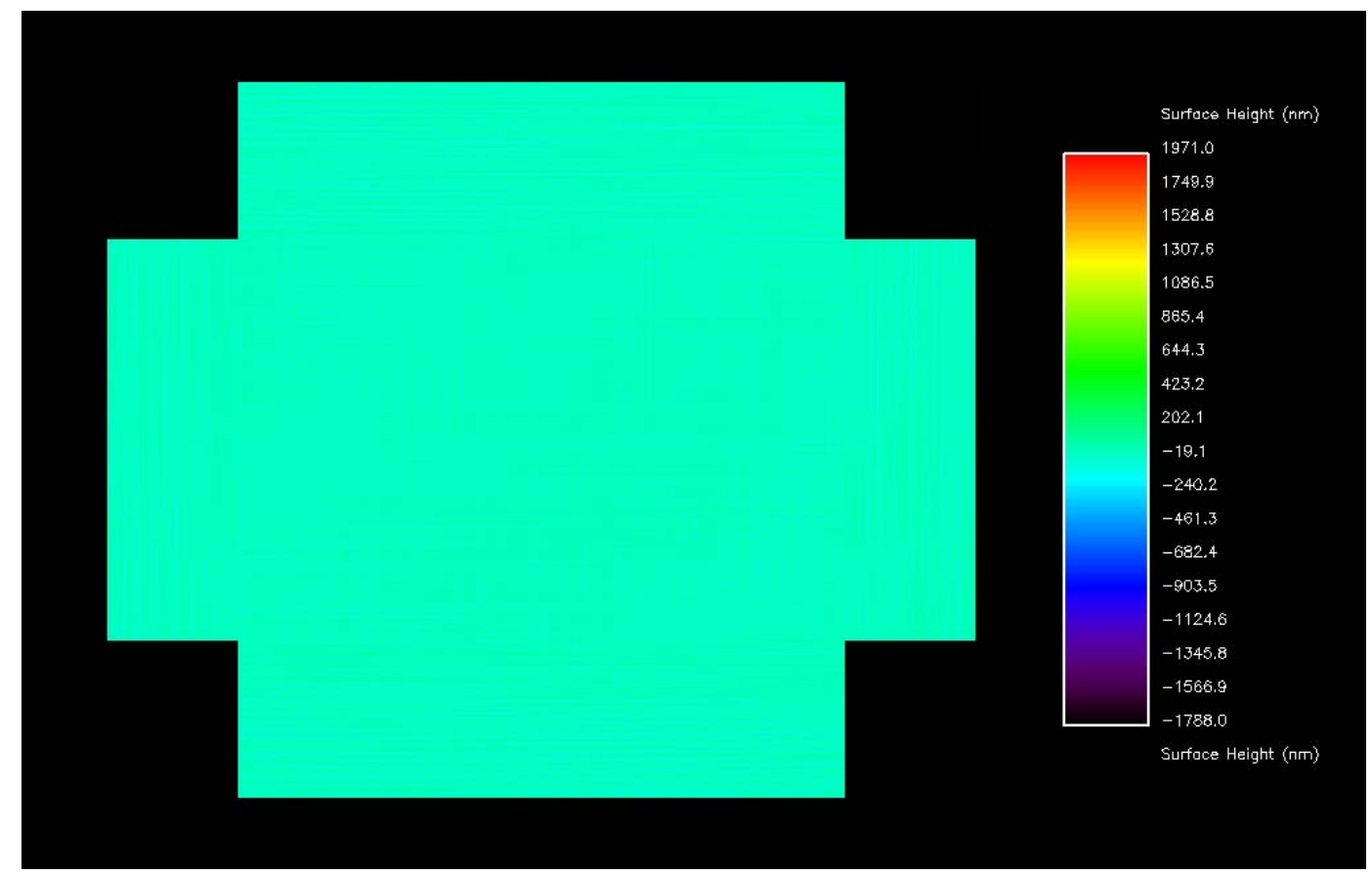

FIGURE 3.33 - Recovered air1 surface using spatial modulation technique (plane removed), shown on same axial scale as 3.8 and 3.18 for comparison.

This surface is displayed on the same scale as the previous technique, and the low standard deviation of the surface of $25.3 \mathrm{~nm}$ makes it hard to see any noise on the reconstructed surface. The noise of the surface reconstruction is an order of magnitude improvement over the previously discussed techniques. The same data is shown at a higher axial resolution in Figure 3.34 and Figure 3.35. 


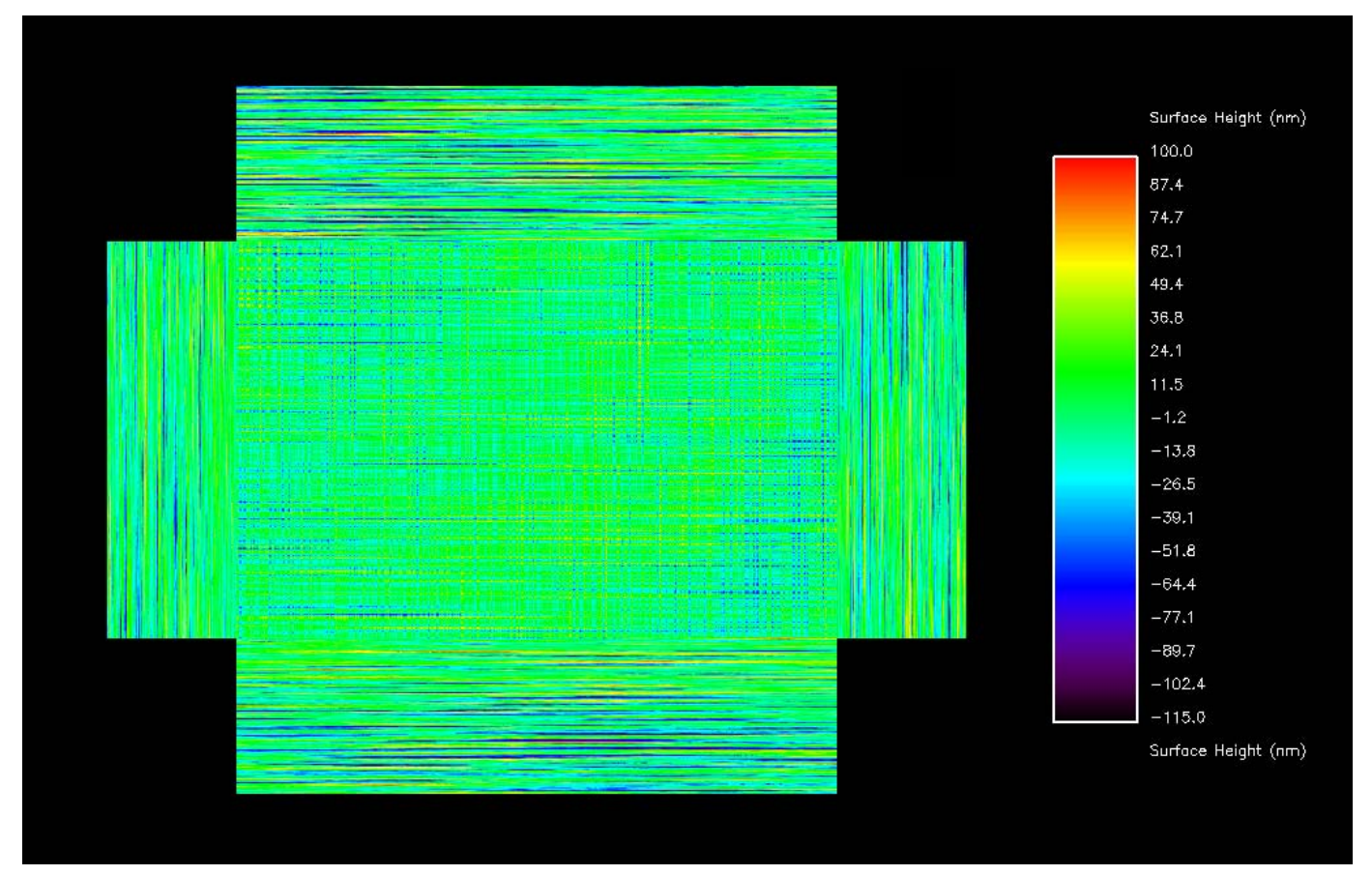

FIGURE 3.34 - Recovered air1 surface using spatial modulation technique (plane removed), at a finer scale $(\mathrm{P}-\mathrm{V}=225 \mathrm{~nm})$.

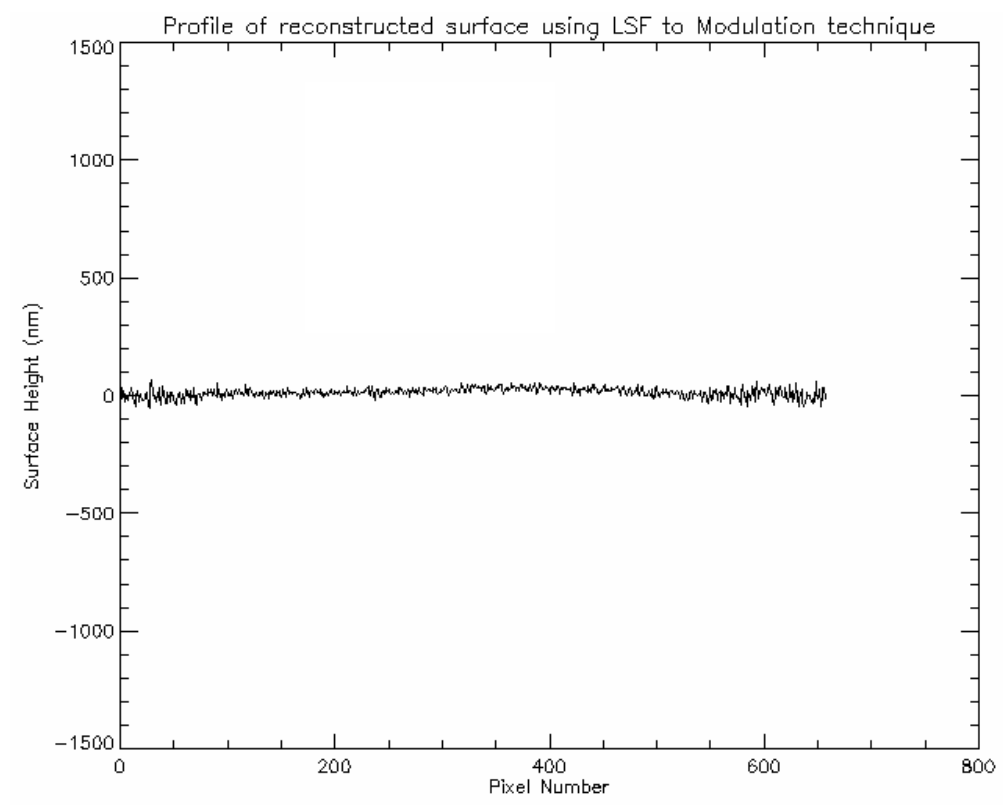

FIGURE 3.35 - Profile of reconstructed surface in Figure 3.34, same region and scale as Figures 3.10 and 3.19. Noise increases slightly at the edges since only the surface height is only calculated once at the edges (along y). 
The noise still has some structure to it in the form of streaks along $\mathrm{x}$ or $\mathrm{y}$, due to the fact that the recovered surface height for pixel $\mathrm{x}_{\mathrm{i}}$ is not found independently of pixel $\mathrm{x}_{\mathrm{i}+1}$.

$$
\begin{aligned}
& \text { Pixels used to find } \gamma_{x_{i}}(\mathrm{z}):\left\{x_{i}-\frac{N-1}{2}: x_{i}+\frac{N-1}{2}\right\} \\
& \text { Pixels used to find } \gamma_{x_{i+1}}(\mathrm{z}):\left\{x_{i+1}-\frac{N-1}{2}: x_{i+1}+\frac{N-1}{2}\right\}
\end{aligned}
$$

The value of $\mathrm{N}$ depends on the tilt in the interferograms and is therefore a dynamic parameter, within limits. The least squares algorithm used to calculate $\gamma$ gets noisy if the range of phase values used to fit the cosine is not at least $2 \pi$. If $N$ gets too large, the edge regions where no data is recovered increase. The limits on $\mathrm{N}$ are as follows, where $\delta_{\mathrm{i}, \mathrm{x}}$ $\left(\delta_{\mathrm{i}, \mathrm{y}}\right)$ is the phase change per pixel along the $\mathrm{x}(\mathrm{y})$-direction for the best fit plane to the unwrapped phase.

$$
\begin{aligned}
& \frac{2 \pi}{\left|\delta_{i, x}\right|} \leq N_{x} \leq \text { Image dimension along x-direction } \\
& \frac{2 \pi}{\left|\delta_{i, y}\right|} \leq N_{y} \leq \text { Image dimension along y-direction } \\
& N_{x}, N_{y} \text { must be odd }
\end{aligned}
$$

The nominal values used for $\mathrm{N}$ are shown here, as long as they fall within the above limits. Typically $\mathrm{N}$ is between 30 and 80 .

$$
N_{x}=\frac{2 \pi}{\delta_{i, x}} \frac{5}{4} \quad N_{y}=\frac{2 \pi}{\delta_{i, y}} \frac{5}{4}
$$

$\mathrm{N}$ must be odd so that the center pixel is the pixel of interest. In the case where there are too few tilt fringes to satisfy either the $\mathrm{x}$ or $\mathrm{y}$-directions, that surface is not calculated for that direction. In the rare case where no tilt is present, this algorithm cannot be used to 
determine the surface location. Therefore it is important to have some tilt in the image, as was the case in Figure 3.26, to insure that this algorithm can be used to determine the surface location. Fortunately, having tilt in the image is the rule rather then the exception.

Table 3.1 summarizes the steps necessary to calculate the surface location from an LCI data cube for a single surface. 


\begin{tabular}{|c|c|c|}
\hline Step: & Task & Equation \\
\hline 1 & $\begin{array}{l}\text { Find the standard deviation of } \\
\text { each frame. Large } \sigma \text { are } \\
\text { frames with good fringes that } \\
\text { should be used for calculating } \\
\qquad \text { in step } 2 .\end{array}$ & $\sigma\left(z_{i}\right)=$ Std. Dev. $[I(x, y, z=i)]$ \\
\hline 2 & $\begin{array}{c}\text { Apply Equation } 3.17 \text { to the } \\
\text { images with the highest } \\
\text { modulation to determine } \\
\varphi(x, y) .\end{array}$ & $\phi=\tan ^{-1}\left[\frac{2\left(I_{2}-I_{4}\right)}{2 I_{3}-I_{5}-I_{1}}\right]$ \\
\hline 3 & Unwrap $\varphi(\mathrm{x}, \mathrm{y})$. & Phase unwrapping algorithm \\
\hline 4 & $\begin{array}{l}\text { Fit a plane to the unwrapped } \\
\text { phase to determine } \delta_{i, x}, \delta_{i, y}\end{array}$ & $\phi_{\text {PlanarFit }}(x, y)=\delta_{i, x} x+\delta_{i, y} y+c$ \\
\hline 5 & $\begin{array}{l}\text { Calculate } \mathrm{N}_{\mathrm{x}} \text { and } \mathrm{N}_{\mathrm{y}} \text { from } \\
\text { Equations } 3.24,3.25 \text {. }\end{array}$ & $N_{x}=\frac{2 \pi}{\delta_{i, x}} \frac{5}{4} \quad N_{y}=\frac{2 \pi}{\delta_{i, y}} \frac{5}{4}$ \\
\hline 6 & Compute $\mathbf{A}$ for the $\mathrm{x}$-direction. & Equation 3.18 \\
\hline 7 & Compute $\mathbf{B}$ at location $(\mathrm{x}, \mathrm{y}, \mathrm{z})$. & $\mathbf{B}\left(\delta_{i, x}\right)\left|\begin{array}{c}\sum I_{i} \\
\sum I_{i} \cos \left(\delta_{i, x}\right) \\
\sum I_{i} \sin \left(\delta_{i, x}\right)\end{array}\right|$ \\
\hline 8 & Find coefficients $a_{0}, a_{1}$ and $a_{2}$. & $\left|\begin{array}{l}a_{0} \\
a_{1} \\
a_{2}\end{array}\right|=\mathbf{A}^{-1}\left(\delta_{i, x}\right) \mathbf{B}\left(\delta_{i, x}\right)$ \\
\hline 9 & $\begin{array}{l}\text { Calculate modulation for one } \\
\text { pixel. }\end{array}$ & $\gamma_{x}(x, y, z)=\frac{\sqrt{a_{1}^{2}+a_{2}^{2}}}{a_{0} \operatorname{sinc}(\Delta / 2)}$ \\
\hline 10 & Repeat steps 7-9 to fill in $\gamma_{x}$. & Steps 7 through 9. \\
\hline 11 & $\begin{array}{l}\text { Fit a quadratic to } \ln [\gamma(z)] \text {. Use } \\
\text { Eqn. } 3.21 \text { to find } b_{0}, b_{1} \text {, and } b_{2} \text {. }\end{array}$ & $L S F\{\ln [\gamma(z)]\}=b_{0}+b_{1} z+b_{2} z^{2}$ \\
\hline 12 & $\begin{array}{l}\text { Repeat step } 11 \text { for all } \\
\text { locations. }\end{array}$ & $z_{0, x}(x, y)=-b_{1} / 2 b_{2}$ \\
\hline 13 & $\begin{array}{l}\text { Repeat steps 6-12 for } y- \\
\text { direction. }\end{array}$ & $z_{0, y}(x, y)$ \\
\hline 14 & Combine $\mathrm{z}_{0, \mathrm{x}}, \mathrm{z}_{0, \mathrm{y}}$ to get $\mathrm{z}_{0}$. & $z_{0}(x, y)=0.5\left[z_{0, x}(x, y)+z_{0, y}(x, y)\right.$ \\
\hline
\end{tabular}

TABLE 3.1 - Outline of steps for finding surface location $z_{0}(x, y)$ using the spatial modulation algorithm. 


\subsubsection{Spatial Modulation Technique and the Saline/Contact Interface}

The LSF of the modulation technique just described works for the interfaces air1, air2, saline 1 and the part of saline 3 containing saline, since all of these surfaces are nominally plane surfaces and have fairly uniform modulation variations. Applying this technique to the saline/contact interface requires significant modifications. Defining the pixels over which there is sufficient modulation becomes more difficult, and the phase difference between pixels is different for every pixel along $\mathrm{x}$ and $\mathrm{y}$. The phase difference between neighboring pixels along $\mathrm{z}$ is still constant. So, $\delta_{\mathrm{i}, \mathrm{x}}$ becomes $\delta_{\mathrm{i}, \mathrm{x}}(\mathrm{x}, \mathrm{y})$. Additionally, for samples with different thickness regions, the surface is no longer continuous (Figure 3.36).

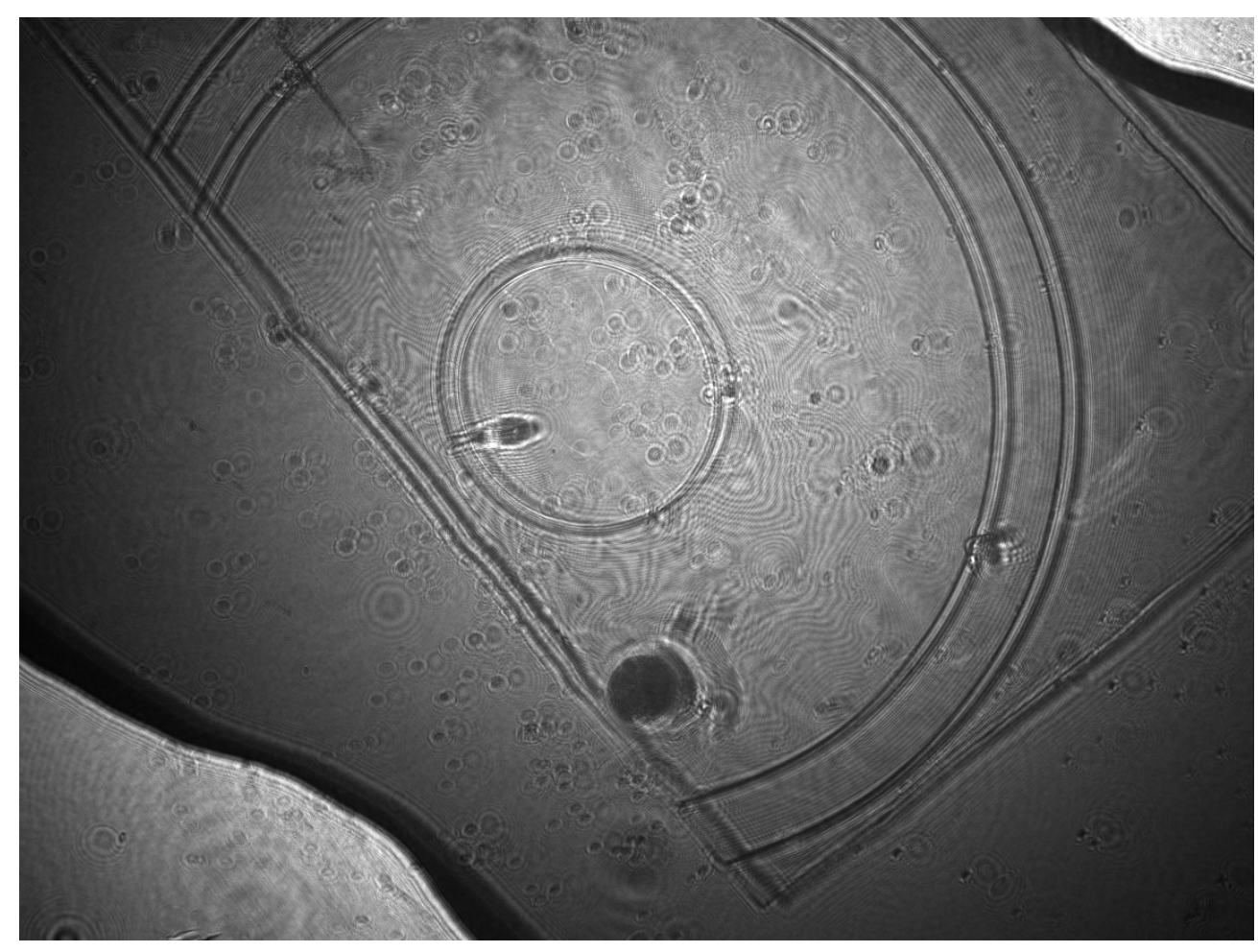

FIGURE 3.36 - Typical interferogram for saline2 interface. Dark lines indicate boundaries between regions with different thicknesses. 
The fringes are difficult to see in Figure 3.36, so Figure 3.37 shows this interferogram minus the previous image in the data cube in order to remove the background features.

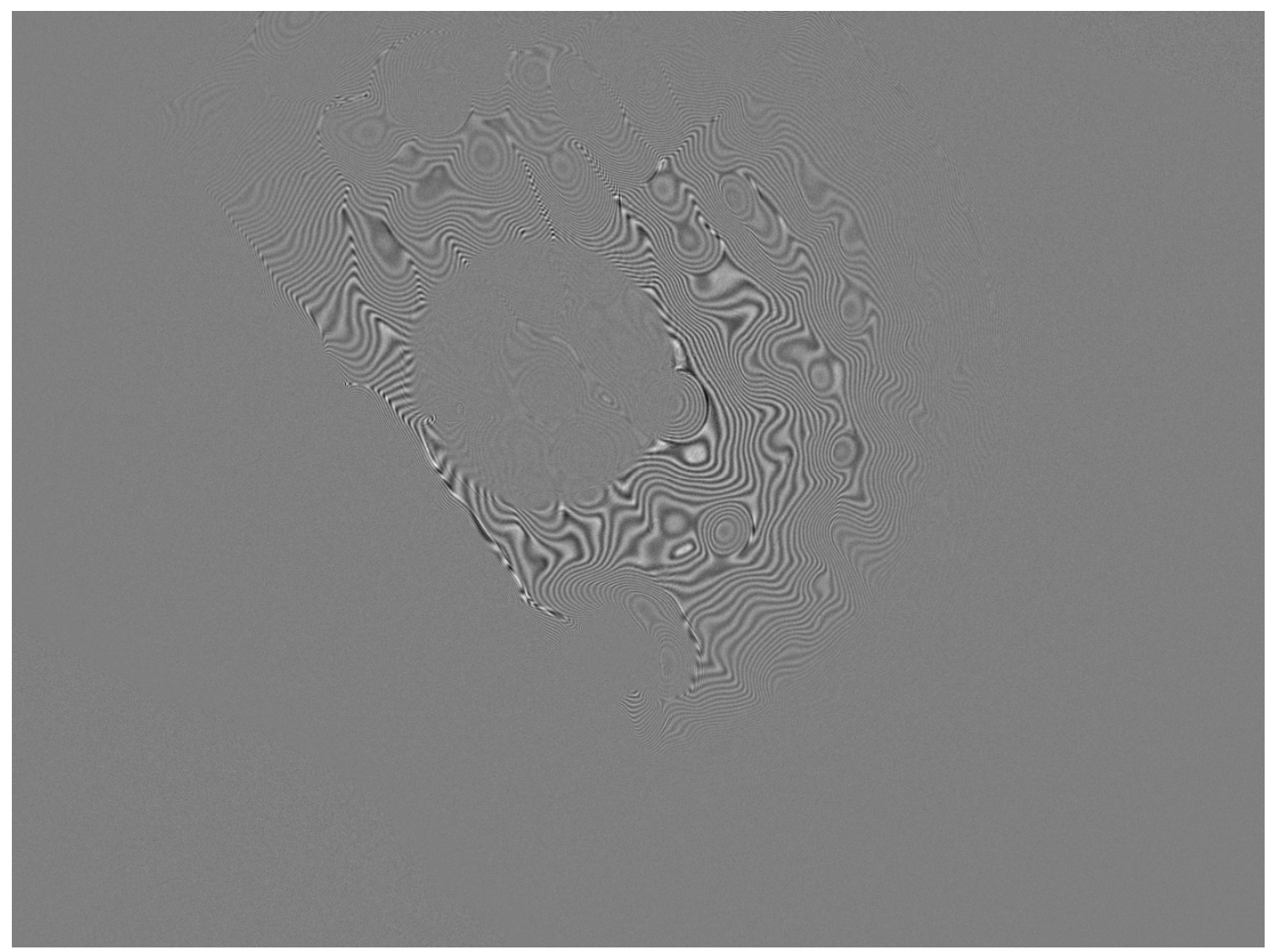

FIGURE 3.37 - Difference between neighboring frames in depth to show fringes.

It is clear that the phase difference between neighboring pixels is location dependent, and not all regions of the sample have fringes with sufficient modulation to calculate a surface. There is also a much larger range of surface heights, due to the steep slopes (high frequency fringes) and the discrete surface height steps.

The first modification to the algorithm outlined in Table 3.1 is the determination of the valid pixels, or those pixels with significant modulation. The phase difference between 
neighboring pixels is determined simultaneously $\left(\delta_{i, \mathrm{x}}(\mathrm{x}, \mathrm{y})\right)$. The data cube $\mathrm{I}(\mathrm{x}, \mathrm{y}, \mathrm{z})$ is stepped through along $\mathrm{z}$, and the phase $(\varphi(\mathrm{x}, \mathrm{y}))$ and modulation $(\gamma(\mathrm{x}, \mathrm{y}))$ are calculated for the five frames $\left\{\mathrm{z}_{\mathrm{i}-2} \ldots \mathrm{z}_{\mathrm{i}+2}\right\}$. All pixels $(\mathrm{x}, \mathrm{y})$ that have a modulation along $\mathrm{z}$ for these five frames above a certain threshold are considered valid for the frame $\mathrm{z}_{\mathrm{i}}$. All of the invalid pixels for this frame are multiplied by -1 . Since all of the values in the data cube are irradiance values $(I>0)$, negative values are now known to be invalid. The phase array, $\varphi(\mathrm{x}, \mathrm{y})$, is all between 0 and $2 \pi$. One option for determining $\delta_{\mathrm{i}, \mathrm{x}}\left(\mathrm{x}, \mathrm{y}, \mathrm{z}_{\mathrm{i}}\right)$ would be to unwrap this data and differentiate the result along x. Since the valid regions are irregularly shaped, this is a difficult and time consuming operation. All that is needed is the phase difference along $\mathrm{x}$ (and $\mathrm{y}$ ), which can easily be found by finding the difference between $\varphi(\mathrm{x}, \mathrm{y})$ and a copy of $\varphi(\mathrm{x}, \mathrm{y})$ shifted by 1 pixel in $\mathrm{x}($ or $\mathrm{y})$.

$$
\delta_{i, x}\left(x=0 \ldots x_{n}-1, y, z_{i}\right)=\left[\phi\left(x=1 \ldots x_{n}, y, z_{i}\right)-\phi\left(x=0 \ldots x_{n}-1, y, z_{i}\right)\right]
$$

Equation 3.26

This gives the phase difference as a function of position $(\mathrm{x}, \mathrm{y})$. The Nyquist limit and the optical layout dictate that the maximum phase difference between adjacent pixels will never be greater than $\pi$ radians. Therefore, $\delta_{\mathrm{i}, \mathrm{x}}$ is adjusted such that $-\pi<\delta_{\mathrm{i}, \mathrm{x}}<\pi$ everywhere by adding or subtracting $2 \pi$ from all values of $\delta_{\mathrm{i}, \mathrm{x}}$ outside the required range. Figure 3.38 shows the wrapped phase along with the phase difference $\delta_{\mathrm{i}, \mathrm{x}}$ along the $\mathrm{x}$ direction. The gray areas in the image on the right are regions with insufficient modulation for this particular frame, $\mathrm{z}_{\mathrm{i}}$. 


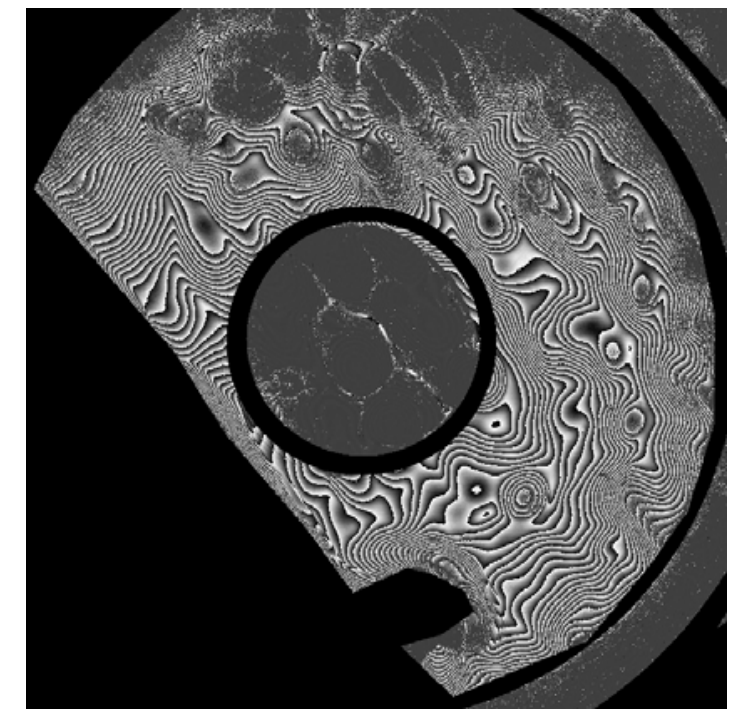

Wrapped phase, $\phi(\mathrm{x}, \mathrm{y})$

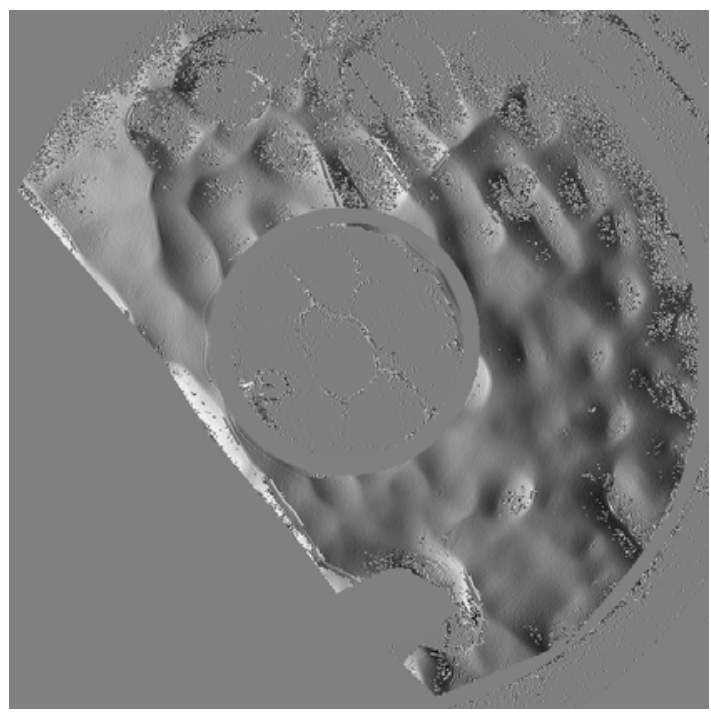

Phase difference along $x$

FIGURE 3.38 - Image of wrapped phase, $\varphi(\mathrm{x}, \mathrm{y})$ for a single frame, $\mathrm{z}_{\mathrm{i}}$ (left), and an image of the phase difference along $\mathrm{x}, \delta_{\mathrm{i}, \mathrm{x}}\left(\mathrm{x}, \mathrm{y}, \mathrm{z}_{\mathrm{i}}\right)$ (right). For each data cube, many such images are computed and averaged to determine $\delta_{\mathrm{i}, \mathrm{x}}(\mathrm{x}, \mathrm{y})$ for the whole surface.

As the algorithm steps through the frames, the sum of the phase differences is recorded and divided by the number of valid $\mathrm{z}$ locations to determine the phase difference along $\mathrm{x}$ (and y) for all the pixels with valid modulation (Equation 3.27). Any errors in calculating the phase differences are reduced due to the multiple calculations made for each pixel.

$$
\delta_{i, x}(x, y)=\frac{\sum \delta_{i, x}(x, y, z)}{\operatorname{Number} \operatorname{Valid}(x, y)}
$$

The valid locations of the data cube have now been defined and the phase differences between these valid pixels, $\delta_{i, x}(x, y)$ have been calculated. The value of $N_{x}$ still needs to be determined. Recall that there are two rules for calculating the modulation versus depth: first, for a given frame, $\mathrm{z}_{\mathrm{i}}$, the pixels used must be symmetric about the pixel of 
interest at $(\mathrm{x}, \mathrm{y})$. Second, the pixels used at all other $\mathrm{z}_{\mathrm{i}}$ locations for the spatial location $(\mathrm{x}, \mathrm{y})$ must be the same (Figure 3.39).

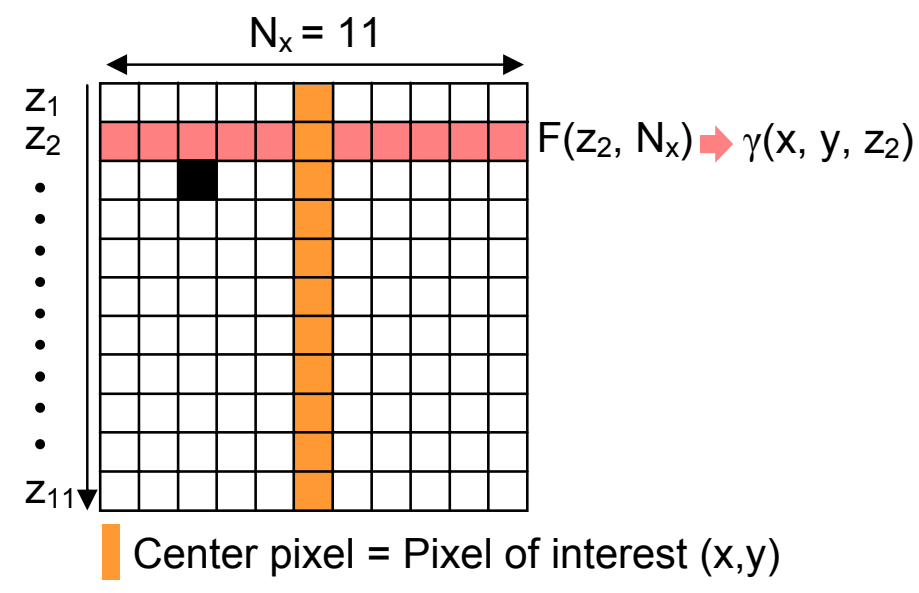

FIGURE 3.39 - Array of irradiance values $\mathrm{I}(\mathrm{x}, \mathrm{z})$. Each row is used to determine $\gamma$ at that depth $\mathrm{z}_{\mathrm{i}}$, for that pixel $(\mathrm{x}, \mathrm{y})$. Black pixel indicates a location with invalid data (insufficient fringe modulation). The software must determine which other pixel(s) must not be used to find $\gamma(z)$ for this location.

Pixels across the rows (red) are used to calculate the modulation, $\gamma$, for this pixel $(\mathrm{x}, \mathrm{y})$ at frame $\mathrm{z}_{\mathrm{i}}$. The orange column indicates the center pixel, for which the modulation is being calculated. The black pixel in the figure indicates a pixel that does not have sufficient modulation and is therefore invalid. Following the two rules listed, there are two routes to take in compensating for this invalid pixel to avoid biasing the resulting modulation calculation (Figure 3.40). 
a)

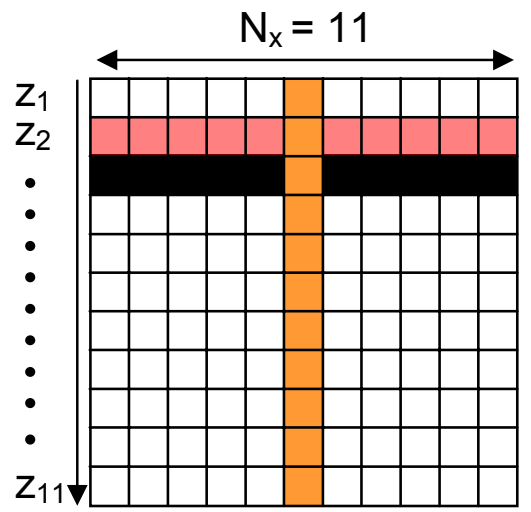

b)

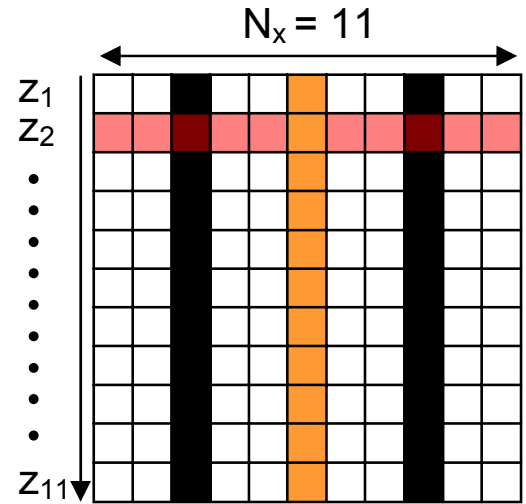

FIGURE 3.40 - Two methods for dealing with the bad pixel in Figure 3.39: a) remove the offending row, or b) remove the offending column and the column symmetric about the center column.

First, the row containing the invalid pixel can be removed, as in Figure 3.40a. Second, the column containing the bad pixel and the column symmetric about the center column can be removed (Figure 3.40b). This decision is made automatically in software for each group of data considered for determining the modulation curve for a given pixel. Figure 3.41 is a display of example data used for determining $\gamma(z)$ for one pixel as the rules are applied. 


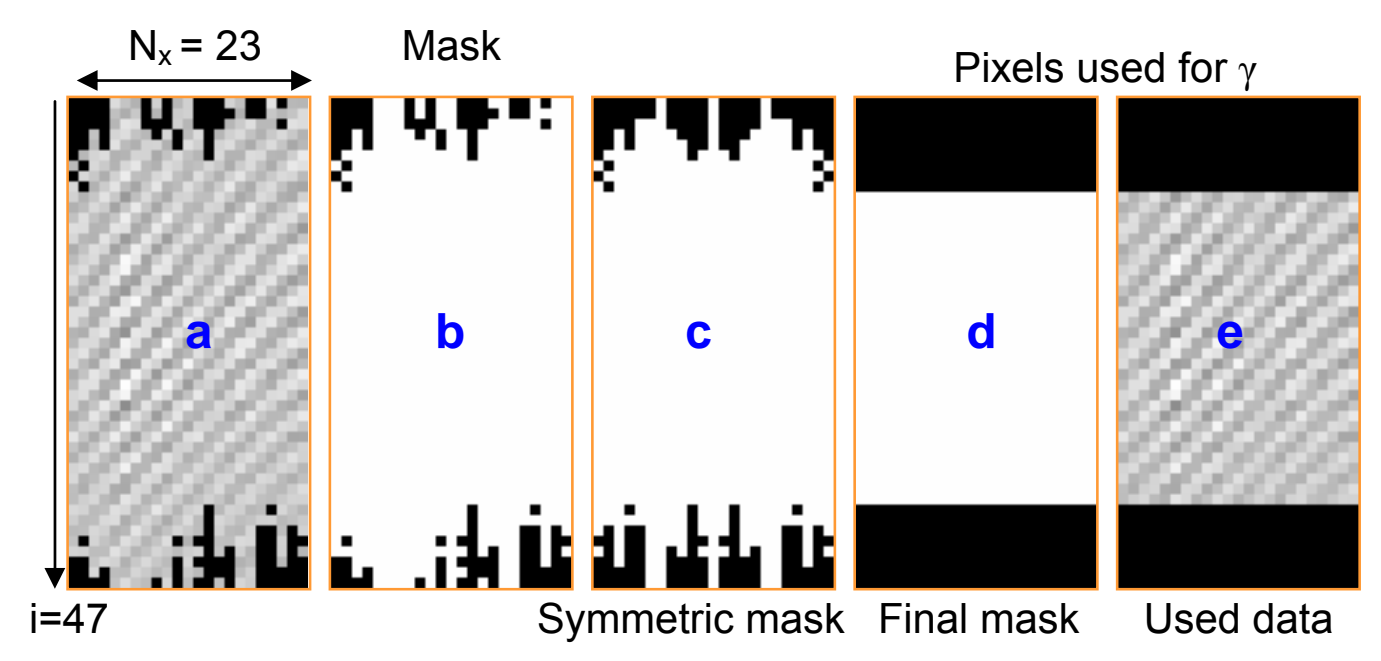

FIGURE 3.41 - Example data used to determine $\gamma(\mathrm{z})$ for a single pixel $(\mathrm{x}, \mathrm{y})$.

In Figure 3.41, there are a series of images with 23 pixels across $\mathrm{x}$ and 47 depth locations along z. Image a is the raw irradiance, where the invalid pixels (insufficient modulation) are negative (black). Image $\mathrm{b}$ is the mask that results from image a. Image $\mathrm{c}$ is the mask that is symmetric about the center row. Note that all of the pixels along $\mathrm{z}$ for the center row are valid (value of 1 in the mask). By applying the rules for using the same pixels at every location in $\mathrm{z}$, we obtain image $\mathrm{d}$. Image e is the final, used data for calculating $\gamma(\mathrm{z})$.

Again, there are limits that must be imposed on the data for the resulting surface location $\mathrm{z}_{0}(\mathrm{x}, \mathrm{y})$ to be considered valid. There are lower and upper bounds for $\mathrm{N}_{\mathrm{x}}$ and a lower bound for the number of frames, $i$, that are required. For this pixel, $\mathrm{N}_{\mathrm{x}}=23$ and $\mathrm{i}=30 \mathrm{in}$ the final mask (Figure 3.41e). For the case of a saline/contact interface:

$$
\begin{aligned}
& 14<N_{x(y)} \leq 23 \\
& 16 \leq i
\end{aligned}
$$


The number of frames depends on the number of frames with valid modulation data, and is typically around 45 . The initial value for $\mathrm{N}$ is 23 . The limits are less strict then for the flat surfaces since the fringe modulation is much lower for the saline2 interface. A mask is created that is symmetric about the center column such as the one in Figure 3.41c. The algorithm starts by determining all of the rows $\left(\mathrm{z}_{\mathrm{i}}\right)$ with all $\mathrm{N}_{\mathrm{x}}$ valid pixels. If the total of these is enough $(16 \leq i)$, the algorithm moves on to the least squares fit routine.

Otherwise, it continues searching the data for rows with (N-2) valid pixels, (N-4) valid pixels, etc, until the lower limit for $i$ is reached, in which case $z_{0}(x, y)$ can be found, or the lower limit for $\mathrm{N}_{\mathrm{x}}$ is reached, in which case the location is skipped and marked invalid.

After the valid pixels for a given pixel versus depth have been found, the matrices $\mathbf{A}$ and B must be calculated. Matrix A, which describes the phase shift from pixel to pixel, is different for every spatial location $(\mathrm{x}, \mathrm{y})$ but is constant along $\mathrm{z}$ for a single location once a mask similar to Figure $3.41 \mathrm{~d}$ has been determined. This is a major difference from the flat surface case, where A was constant for an entire surface in the $\mathrm{x}$ direction. Matrix B, which incorporates the irradiance information, must be recalculated for every location, $(\mathrm{x}, \mathrm{y}, \mathrm{z})$, as before. The mask (Figure 3.41d) is applied using $\mathrm{M}_{\mathrm{j}}$ :

$$
M_{j}=\left\{\begin{array}{l}
1 \text { for valid pixels in mask, along } \mathrm{x} \\
0 \text { for invalid pixels in mask }
\end{array}\right.
$$

Each summation in calculating $\mathbf{A}$ and $\mathbf{B}$ is from $\mathrm{j}=\mathrm{x}-\left(\mathrm{N}_{\mathrm{x}}-1\right) / 2$ to $\mathrm{j}=\mathrm{x}+\left(\mathrm{N}_{\mathrm{x}}-1\right) / 2$, where $\mathrm{x}$ is the center pixel for which $\mathbf{A}$ and $\mathbf{B}$ is calculated: 


$$
\begin{aligned}
& \mathbf{A}\left(\delta_{i, x}\right)= \\
& \left|\begin{array}{ccc}
\sum M_{j} & \sum M_{j} \cos \left[\delta_{i, x}(j)\right] & \sum M_{j} \sin \left[\delta_{i, x}(j)\right] \\
\sum M_{j} \cos \left[\delta_{i, x}(j)\right] & \sum M_{j} \cos ^{2}\left[\delta_{i, x}(j)\right] & \sum M_{j} \cos \left[\delta_{i, x}(j)\right] \sin \left[\delta_{i, x}(j)\right] \\
\sum M_{j} \sin \left[\delta_{i, x}(j)\right] & \sum M_{j} \cos \left[\delta_{i, x}(j)\right] \sin \left[\delta_{i, x}(j)\right] & \sum M_{j} \sin ^{2}\left[\delta_{i, x}(j)\right]
\end{array}\right|
\end{aligned}
$$

Equation 3.30

$$
\mathbf{B}\left(\delta_{i, x}\right)=\left|\begin{array}{c}
\sum M_{j} I_{i} \\
\sum M_{j} I_{i} \cos \left[\delta_{i, x}(j)\right] \\
\sum M_{j} I_{i} \sin \left[\delta_{i, x}(j)\right]
\end{array}\right|
$$

The modulation is calculated the same way as before:

$$
\begin{aligned}
& \left|\begin{array}{l}
a_{0} \\
a_{1} \\
a_{2}
\end{array}\right|=\mathbf{A}^{-1}\left(\delta_{i, x}\right) \mathbf{B}\left(\delta_{i, x}\right) \\
& \gamma_{x}(x, y, z)=\frac{\sqrt{a_{1}^{2}+a_{2}^{2}}}{a_{0} \operatorname{sinc}(\Delta / 2)}
\end{aligned}
$$

Once $\gamma_{x}(x, y, z)$ has been calculated following the above procedure, the same LSF to the natural $\log$ of the modulation curve is performed as for the flat surface case. Figure 3.42 is a plot of the natural log of the resulting modulation curve and the best fit parabola. 


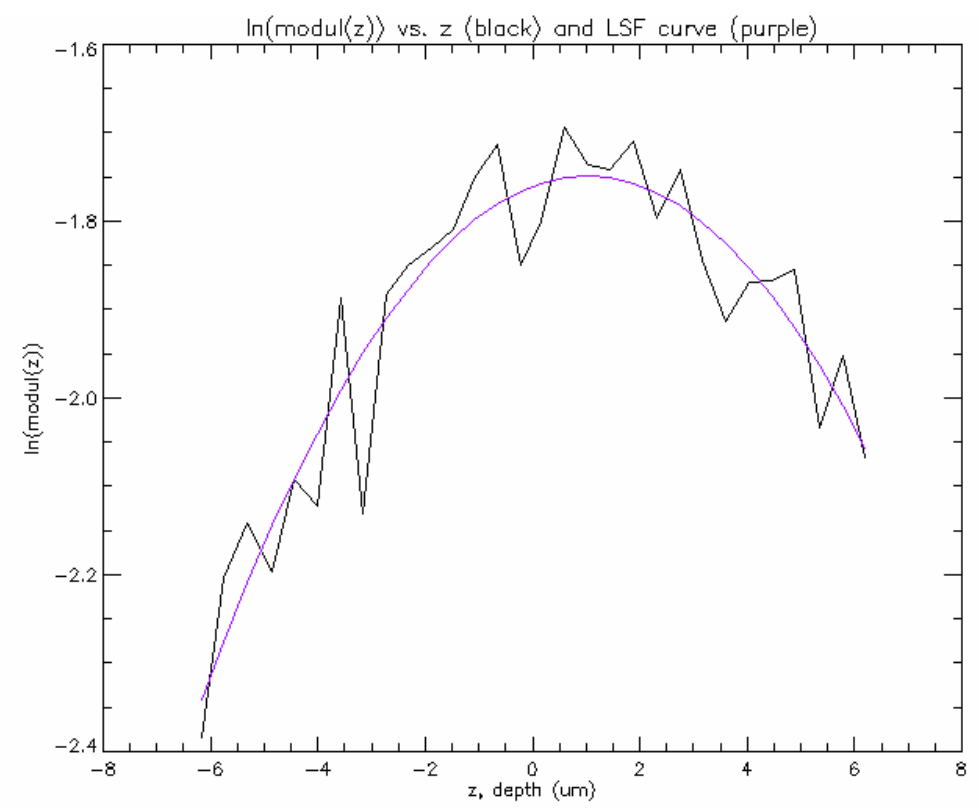

FIGURE 3.42 - Plot of the natural log of the modulation data (black) and the best fit parabola (purple).

The resulting surface location for this pixel, $\mathrm{z}_{0}$, is $1016 \mathrm{~nm}$. The noisiness of this curve is due to the low reflectance from the saline/contact interface. The reflected signal is about two orders of magnitude smaller then the signal from a glass/air interface. Just as in the case for planar surfaces, the regions at the edge are lost due to the $\mathrm{N}$ pixels used to calculate $\gamma$. The effect is often less noticeable due to the higher fringe frequencies. This process is repeated in the y-direction. The multiple regions in a discontinuous surface are processed individually, using masks, so that region boundaries do not cause problems.

It may not be clear how this technique works based on the preceding mathematics. The basic idea is that the surface is located where the fringe modulation is largest. Calculating the modulation using pixels along depth is a noisy operation because the 
modulation varies rapidly with depth. The modulation at a given voxel can also be calculated using laterally neighboring pixels on the detector, along $\mathrm{x}$ or $\mathrm{y}$. This is a less noisy process since the modulation varies slowly across the detector. An integral part of the algorithm requires the phase difference between adjacent pixels to be determined. For a given surface the phase difference between two pixels is constant with depth. This allows the phase difference to be calculated at each frame and averaged, improving the calculated phase differences. Once the modulation has been calculated at every voxel, the surface can be found by fitting a curve to the calculated modulation curve.

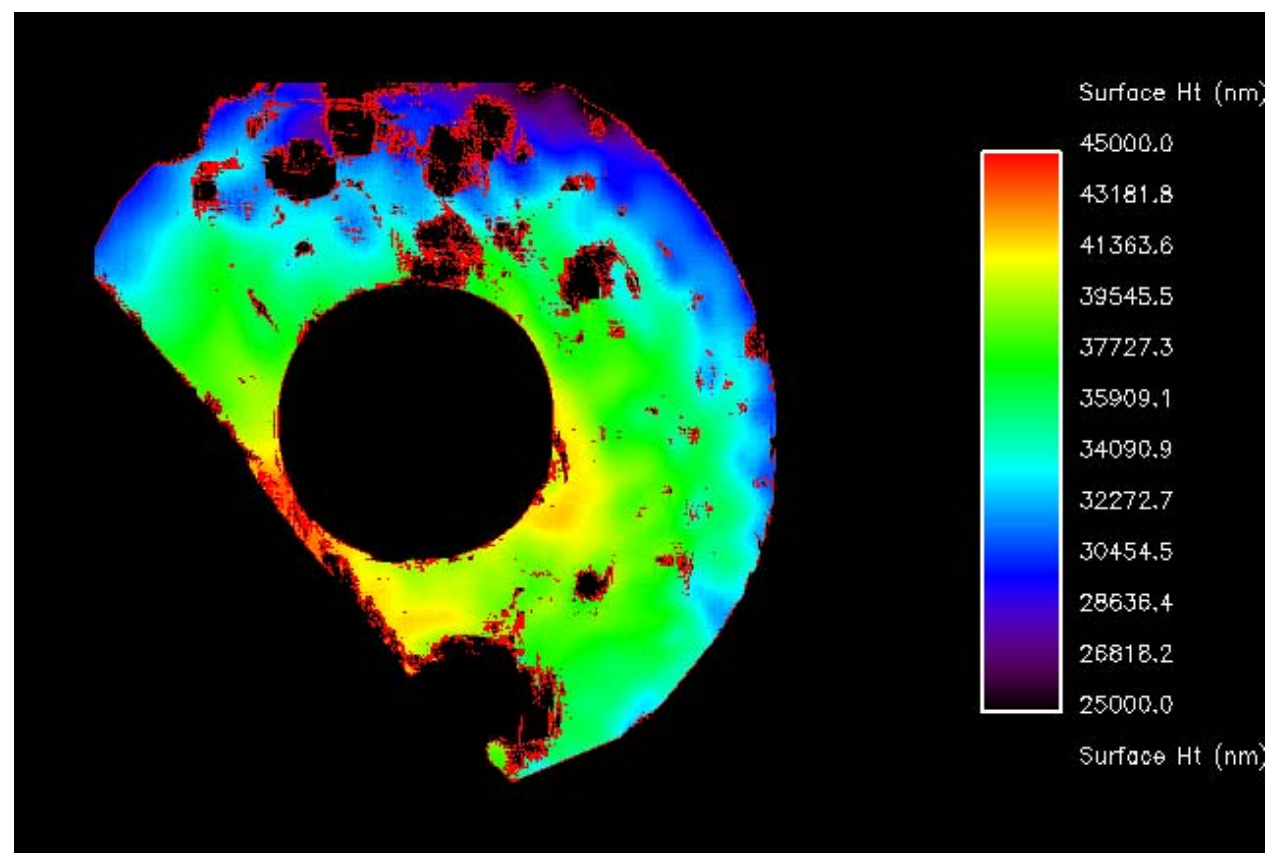

FIGURE 3.43 - Single region of saline2 interface using spatial modulation technique, shown on the same scale as Figures 3.14 and 3.23.

Figure 3.43 is the recovered top surface of the sample, or the saline/contact interface, for the same data set discussed in the previous techniques. The recovered surface is 
smoother and overall has less noise. A profile through the surface is displayed for comparison (Figure 3.44).

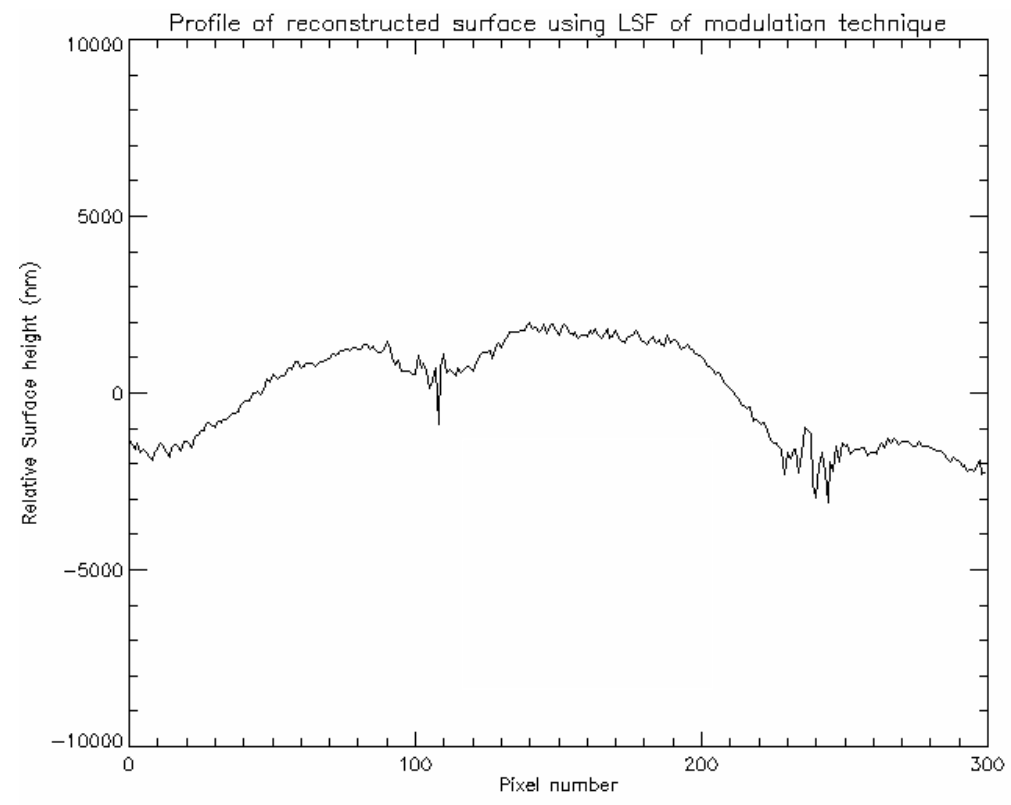

FIGURE 3.44 - Profile of reconstructed saline2 surface using the same region and scale as Figures 3.15 and 3.24.

Using a least squares fit algorithm to determine the modulation as a function of space and depth is the best technique for calculating surface locations, given the type of data available from the low coherence interferometer in Engage. Figure 3.45 shows the plots from the 3 algorithms discussed on the same scale. 


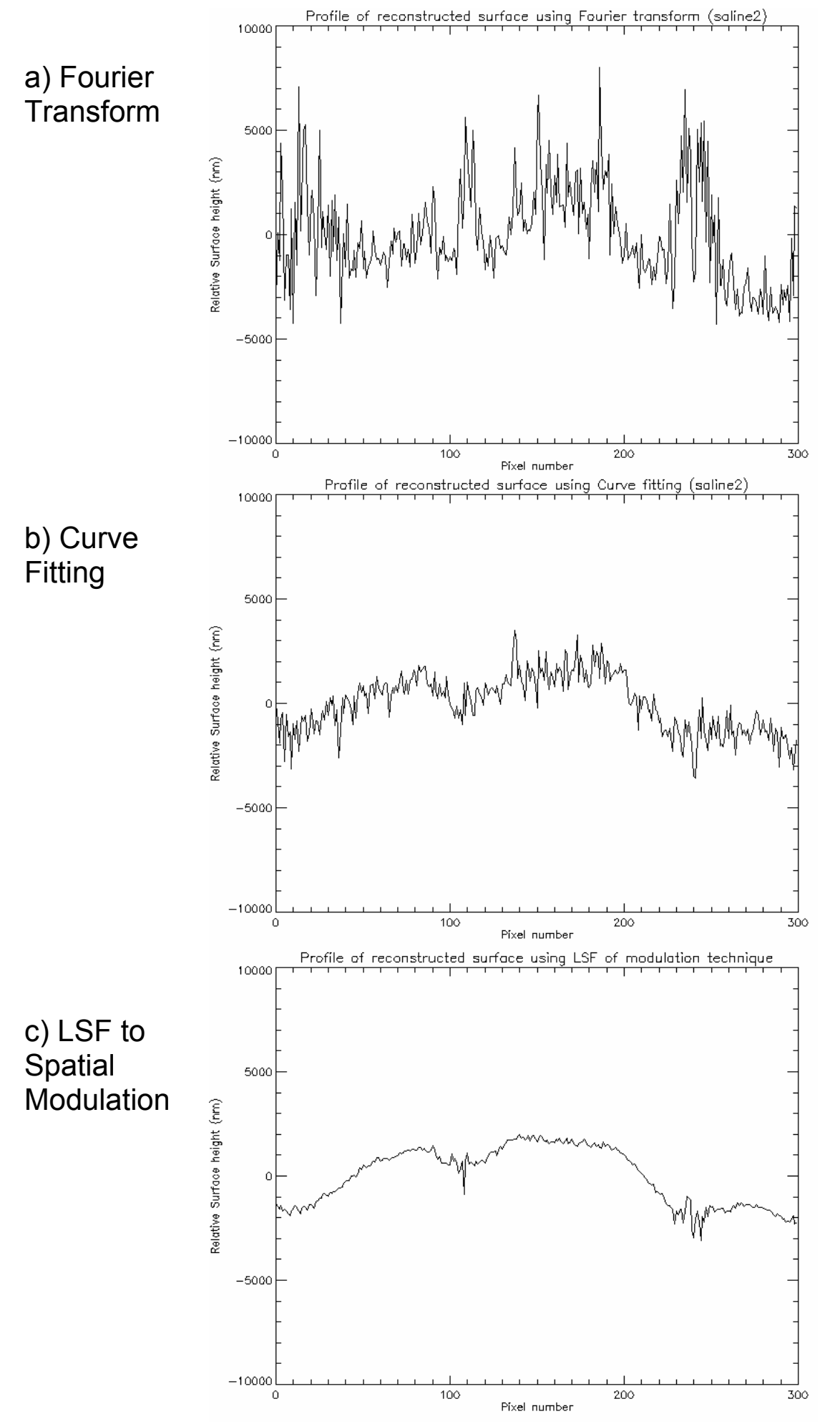

FIGURE 3.45 - Saline2 surface profile comparison for 3 methods. 


\subsubsection{Surface Finding Summary}

The technique for calculating the surface location from the low coherence data has gone through numerous changes as the measurement procedure was refined. The three techniques presented here illustrate the high points of the algorithmic evolution, and a variety of techniques were tried in between these methods. The method used in the final version of Engage is the least squares fit to the modulation technique, since it was shown to deliver the best overall results.

Due to the amount of computation necessary to locate the surfaces using this technique, the computation time is important to consider (Table 3.2). This is for a Windows XP machine with a Pentium 4 processor at $3.40 \mathrm{GHz}$.

\begin{tabular}{|c|c|c|}
\hline Interface & Time/1000 pixels & Time/surface (750x750 pixels) \\
\hline Glass/Air, Glass/Saline & 2.3 seconds & 22 minutes \\
\hline Saline/Sample & 3.8 seconds & 35 minutes \\
\hline Sample/Glass & 3.8 seconds & 35 minutes \\
\hline
\end{tabular}

TABLE 3.2 - Processing time for Spatial Modulation surface finding technique.

A full measurement requires five surfaces to be found, as shown in Figure 3.46. The total time to process these five low coherence data cubes to produce surfaces depends on the number of pixels in each image, but in the case of an average data set, is roughly 90 minutes. This time could be reduced by optimizing the processing code to reduce redundant calculations and parallelize as many operations as possible. 


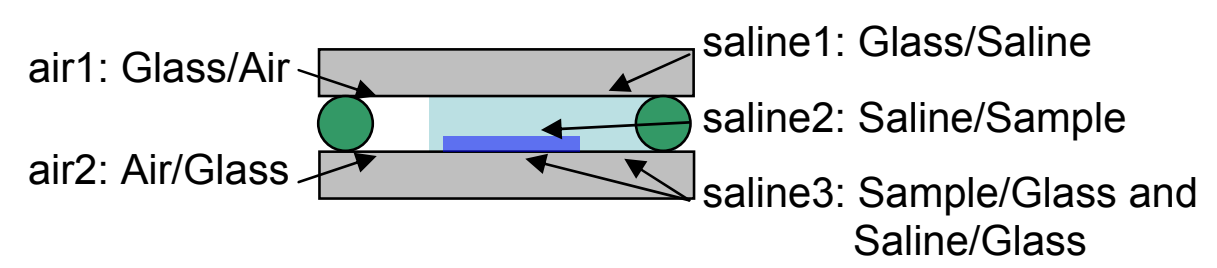

FIGURE 3.46 - Cartoon illustrating 5 required surfaces for measuring $\mathrm{t}_{\mathrm{sam}}(\mathrm{x}, \mathrm{y})$.

The last reconstructed surface, saline 3 , is processed twice; once in the region containing the sample and once in the region containing the saline. The two regions are combined to create the final surface.

\subsection{Determining the Sample Thickness}

The preceding discussion explains how the five required individual surfaces are calculated from the low coherence data. Turning a group of surfaces into the proper thickness values should be a simple subtraction:

$$
t_{1}(x, y)=z_{0, a i r 2}(x, y)-z_{0, a i r 1}(x, y)
$$

A plane is fit to $t_{1}$, and the validity of this fit depends somewhat on the noise levels in $t_{1}$. In the case of the plane that is fit to the thickness of the air column, additional data is available for determining $\mathrm{a}$ and $\mathrm{b}$ in Equation 3.35:

$$
h_{\text {plane }, \text { air }}(x, y)=a x+b y+c
$$

Recall that the fringes in the images captured for the low coherence interferometer represent the phase due to the OPD between the test and reference surfaces, which depends on $\mathrm{n}_{\text {air, }}$, but the coherence envelope represents the OGD, which depends on $\mathrm{N}_{\mathrm{g} \text {,air }}$. Due to wedge and tilt and imperfections in alignment, the OPD surface is different then 
the OGD surface for a single surface. In the special case of the thickness of the air column, $\mathrm{t}_{\text {air, }}$, the slopes of the thickness should be nearly identical for the OPD separation and the OGD separation, since $\mathrm{n}_{\text {air }} \approx \mathrm{N}_{\mathrm{g} \text {,air }}$.

$$
N_{g, \text { air }}-n_{\text {air }}=-680 \frac{1.00027114-1.00027124}{685-675}=6.7 \times 10^{-6} \quad \text { Equation } 3.36
$$

The OPD surfaces for air1 and air2 are easy to obtain given the low coherence data collected. The images are nominally separated by $450^{\circ}$ of phase, and a series of 5 consecutive images with high fringe visibility are similar to the data obtained in a phase shifting interferometer, with the only difference being the visibility variation with frame number. These five images are used in the Schwider-Hariharan algorithm to calculate the wrapped phase for the top surface, air1, and the bottom surface, air2.

$$
\phi(x, y)=\tan ^{-1}\left[\frac{2\left(I_{2}(x, y)-I_{4}(x, y)\right)}{2 I_{3}(x, y)-I_{5}(x, y)-I_{1}(x, y)}\right]
$$

The phase surface can be turned into the height (OPL) of the surface using the wavelength of the fringes, or the center wavelength of the low coherence source. The extra factor of two is from the double pass nature of the test.

$$
O P L=\phi \frac{\lambda_{\text {Low Coherence }}}{2 \cdot 2 \pi}
$$

These phase surfaces represent the OPL for each, while the surfaces recovered by processing the low coherence signals represent the OGL for each surface. For the top surface, these two will be different, as shown in Equations 3.39 and 3.40, where $\Delta \mathrm{t}_{\text {air }}$ and $\Delta \mathrm{t}_{\text {glass }}$ represent the difference of these materials between the test and reference arms of the low coherence interferometer. They vary spatially. 


$$
\begin{gathered}
O P L_{\text {top }}=n_{\text {air }} \Delta t_{\text {air }}(x, y)+n_{\text {glass }} \Delta t_{\text {glass }}(x, y) \\
O G L_{\text {top }}=N_{g, \text { air }} \Delta t_{\text {air }}(x, y)+N_{g, \text { glass }} \Delta t_{\text {glass }}(x, y)
\end{gathered}
$$

Since $\mathrm{N}_{\mathrm{g} \text {,glass }} \neq \mathrm{n}_{\text {glass }}$, the two top surfaces will not have the same slopes. Likewise, the OPL and OGL surfaces for the bottom surface (air2) will be different.

$$
\begin{gathered}
O P L_{\text {bottom }}(x, y)=n_{\text {air }}\left(\Delta t_{\text {air }}(x, y)+t_{1}(x, y)\right)+n_{\text {glass }} \Delta t_{\text {glass }}(x, y) \quad \text { Equation } 3.41 \\
O G L_{\text {bottom }}(x, y)=N_{g, \text { air }}\left(\Delta t_{\text {air }}(x, y)+t_{1}(x, y)\right)+N_{g, \text { glass }} \Delta t_{\text {glass }}(x, y)
\end{gathered}
$$

Equation 3.42

The quantity of interest here is of course $t_{1}(x, y)$, the thickness of the air gap inside the cuvette. Combining the above expressions to determine the OPD and OGD between the top and bottom surfaces will result in equations 3.43 and 3.44 .

$$
\begin{gathered}
O P D_{\text {air }}(x, y)=n_{\text {air }} t_{1}(x, y)+C \\
O G D_{\text {air }}(x, y)=N_{g, \text { air }} t_{1}(x, y)
\end{gathered}
$$

The phase calculations used to determine OPD are not capable of determining the distance between the top and bottom plates, so $\mathrm{C}$ is unknown. Since $\mathrm{N}_{\mathrm{g} \text {,air }} \approx \mathrm{n}_{\text {air }}$, the spatial derivatives of $\mathrm{OPD}_{\text {air }}$ and $\mathrm{OGD}_{\text {air }}$ are equal, which are the slopes of the plane describing $\mathrm{t}_{1}(\mathrm{x}, \mathrm{y})$.

$$
t_{1}(x, y)=a x+b y+c
$$

Ideally, the values of $\mathrm{a}$ and $\mathrm{b}$ are easily determined from the low coherence surfaces. As has been shown the typical standard deviations the thickness is on the order of 50 nanometers. The phase shifting algorithm (Equation 3.36), even in the presence of modulation variations, typically results in a thickness with a standard deviation of less 
then $5 \mathrm{~nm}$. These phase surfaces that represent the slopes of the thickness of the air column are useful for checking the validity of the thickness found using the low coherence data. Determining $\mathrm{t}_{\mathrm{sam}}(\mathrm{x}, \mathrm{y})$ follows the procedure outlined in Chapter 2 . 


\section{HARDWARE CONSIDERATIONS FOR LCI}

Understanding the origins of the low coherence signal, its relationship to the surface locations and thickness measurements, and the algorithms used to process these signals helps determine some of the hardware requirements for this measurement system. Some of the ideal hardware properties can not be satisfied due to the limits of the available optical components. Some of the ideal components of the measurement are contradictory, requiring a compromise. This section will discuss the hardware choices for constructing the low coherence interferometer portion of Engage, the factors that went into choosing that particular component, and where appropriate, alternatives that may be an improvement.

\subsection{The Light Source}

The first component in this optical system is the light source, which is a Superluminescent diode (SLD) from Superlum, Ltd. in Moscow, Russia.

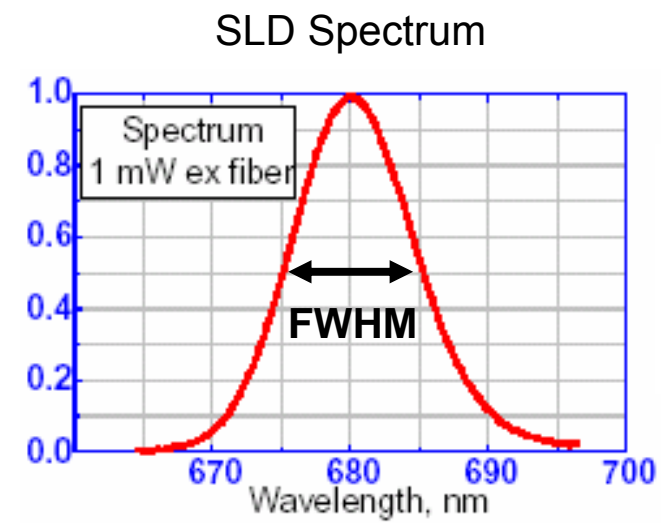

FIGURE 4.1 - SLD output versus wavelength. 
The standard spectral distribution is shown in Figure 4.1, with a center wavelength $\lambda_{c}=680 \mathrm{~nm}$, FWHM of $8.5 \mathrm{~nm}$, and a coherence length, $1_{c}$, of about $35 \mu \mathrm{m}$.

$$
l_{c}=k \frac{\lambda_{c}^{2}}{\Delta \lambda}=0.66 \frac{(680 \mathrm{~nm})^{2}}{8.5 \mathrm{~nm}} \approx 35 \mu \mathrm{m}
$$

The $\mathrm{k}$ factor is 0.66 for a Gaussian spectral distribution. At the time, this was the only SLD available in the visible spectrum, which eases alignment and allows a silicon based CCD to be used to capture the signal. The term 'superlumiscent diode' refers to a class of optical sources that are a combination of laser diode-like high output power and broad LED (light emitting diode) optical spectrum. This combination is achieved with high optical gain in a semiconductor material with very low reflectance at the two ends of the cavity. The semiconductor material is pumped into an excited state, and the spontaneous emission will propagate through the active region of the SLD in opposite directions, where it will be amplified by stimulated emission (Shidlovski, 2004).

As the spontaneous emission travels through the active region, it is amplified according to equation 4.2, which is an approximation of the bulk material gain as a function of carrier density $(\mathrm{N})$, the peak gain $\mathrm{g}_{0}$, the peak gain wavelength $\lambda_{\mathrm{p}}$, and the parabolicshaped gain profile width $\lambda_{\mathrm{w}}$ (Park, 2006).

$$
g=g_{0}(N)-g_{0}(N)\left(\frac{\lambda-\lambda_{p}(N)}{\lambda_{w}(N)}\right)^{2}
$$


If the reflection of the two end surfaces is 0 , then there is no optical feedback, and the gain medium is not an optical resonator as is used in a laser (Figure 4.2). This prevents spectral selection from mode competition that would occur with feedback.

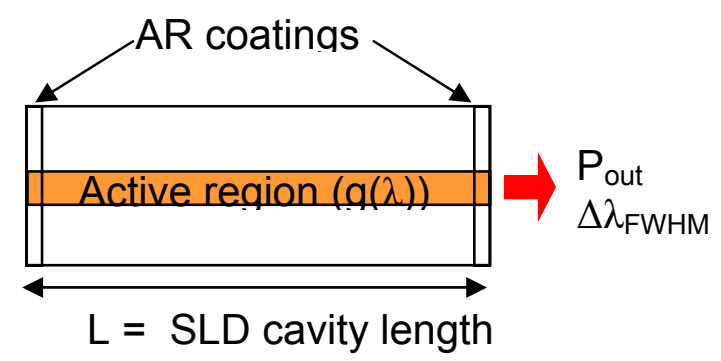

FIGURE 4.2 - Schematic of a SLD cavity (Park, 2006).

The effective device gain $\mathrm{G}$ depends on the confinement factor $\Gamma$ and the cavity length $\mathrm{L}$.

$$
G(N)=L \Gamma g_{0}(N)
$$

The total SLD output power under high injection current is $\mathrm{P}_{\text {out }}$, where the integral is performed over the spectral range $\Lambda$ and $\mathrm{P}_{\mathrm{p}}$ is the peak power spectral density.

$$
P_{\text {out }} \cong P_{p} \int_{\Lambda} e^{-\frac{\left(\lambda-\lambda_{p}(N)\right)^{2}}{\left(\lambda_{w}(N) / \sqrt{G(N)}\right)^{2}}}\left(\frac{\lambda_{p}(N)}{\lambda}\right)^{3} d \lambda
$$

The SLD output power spectrum is clearly approximately a Gaussian, not quadratic like the bulk material gain. This fact is important in how the LCI signals are processed (Chapter 3). The device spectral bandwidth is tied to the output power, and in general, an increase in power leads to a decrease in bandwidth. To raise the power-bandwidth product, material properties or SLD geometries need to be altered. 
In reality, there will always be some reflection from the facets of the SLD active region that is coupled into the optical fiber; these reflections can cause parasitic Fabry-Perot modulation. Their effect on the coherence envelope for this source is to produce secondary modulation peaks for $|\mathrm{OPD}|>>0$, near $+/-4 \mathrm{~mm}$ of OPD for this model of SLD (Figure 4.3).

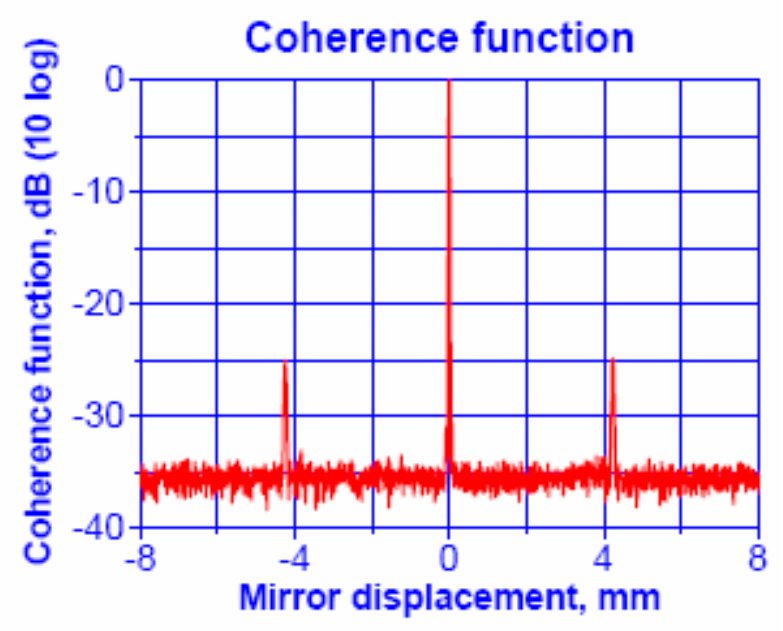

FIGURE 4.3 - Coherence function (vendor supplied) for SLD, showing secondary modulation peaks due to reflection from the facets of the active region of the SLD.

$$
O P D_{\text {Secondary Peak }}=2 L n \text { * }
$$

Here $\mathrm{n}^{*}$ is the effective refractive index for the optical mode in the cavity. These secondary peaks can cause unwanted fringes where there is no interface or can cause a small overlapping fringe pattern on top of fringes from a true interface.

The stability of the output power of the SLD is dependent on the stability of the DC power supply. This SLD works with a dedicated current and temperature controller called a PILOT. This model runs on an external 9V DC power supply, which in this case 
is a Lambda LP-521-FM. Newer models of the PILOT driver have an internal AC/DC converter and can be plugged into a standard wall outlet. In order to reach high thermal and output power stability, an hour of warm-up time is recommended by the manufacturer (Shidlovski, 2006). The spectral shift with temperature is quoted as less then $0.25 \mathrm{~nm} /{ }^{\circ} \mathrm{C}$.

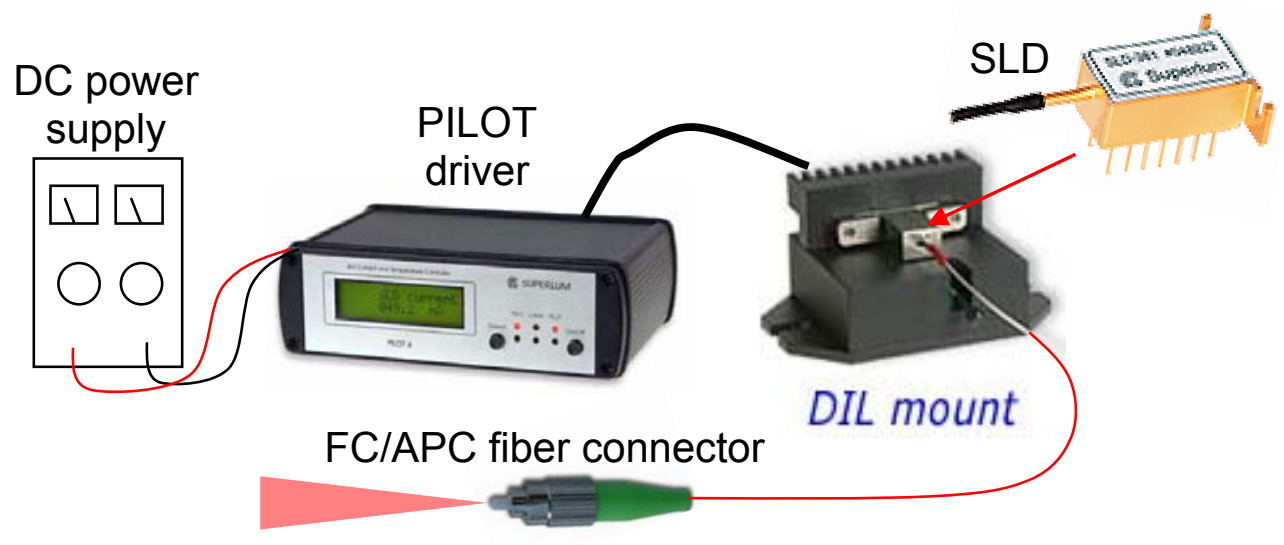

FIGURE 4.4 - Schematic of required components for SLD operation.

The SLD is mounted in a DIL mount, which has a heat sink in order to maintain a stable constant temperature (Figure 4.4). The SLD is controlled via a cable from the PILOT driver. The SLD output is coupled to a single mode optical fiber, which terminates with a $\mathrm{FC} / \mathrm{APC}$ fiber connector. A fiber in this type of connector is cleaved at $8^{\circ}$ to prevent the Fresnel reflection from coupling back into the single mode fiber and providing unwanted optical feedback in the SLD. For a fiber with $n=1.5$, the center of the output beam will be tilted at a deviation angle $4^{\circ}$ from the path of the fiber (Figure 4.5).

$$
\theta^{\prime}=\sin ^{-1}\left(1.5 \sin \left(8^{\circ}\right)\right)=12^{\circ}
$$




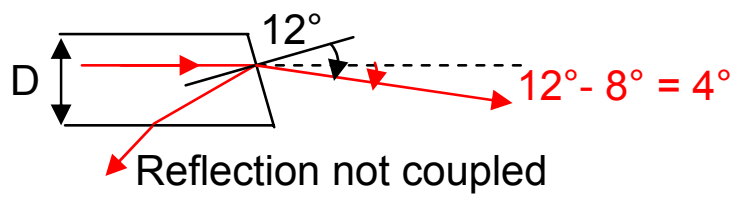

FIGURE 4.5 - Since the fiber output is cleaved at $8^{\circ}$, a stage with large tip/tilt capabilities is required for mounting the fiber.

This $4^{\circ}$ output angle dictates the range of travel required for the tip/tilt of the stage to which the fiber output will be mounted. The output of this fiber is small enough that additional spatial filtering is not required, as a single mode fiber diameter D is roughly 4 $\mu \mathrm{m}$, based on the measured NA of the output beam of 0.12 and the requirement that the normalized radius, $V$, of the core be less then 2.4 to be a single mode fiber for $\lambda=680 \mathrm{~nm}$.

$$
\begin{gathered}
V=D \frac{\pi}{\lambda} N A<2.4 \\
D<\frac{2.4}{\pi N A} \lambda=4.33 \mu \mathrm{m}
\end{gathered}
$$

Equation 4.8

This is smaller then the diameter of the pinhole for spatially filtering the laser in the Mach-Zehnder interferometer $(5 \mu \mathrm{m})$. The maximum output power for this model SLD is $1 \mathrm{~mW}$. Recall that the lowest reflectance surface is about $0.07 \%$ (Table 2.1 ). Combine that with two passes through the beamsplitter, and the best-case test beam signal for the low coherence interferometer measurement of the saline/sample interface is a little better then $1 / 1000^{\text {th }}$ of the initial power, or about $1 \mu \mathrm{W}$.

$$
0.07 \% \cdot(0.5)^{2}=0.017 \%
$$


The part number for this SLD is SLD-261-MP-DIL-SM-PD-FC/APC, which specifies the following: 261-MP: center wavelength of 680, medium power; DIL: packaging type for SLD (Figure 4.4); SM: couple to a single mode fiber; and FC/APC: specifies type of fiber connector that terminates the fiber.

The SLD light source is the start of the hardware components of the low coherence interferometer. The FC/APC connector screws into a fiber mount which sits on a small rail on a tip/tilt stage (561-tilt, Newport). A custom aluminum mount was machined to mount the tip/tilt stage on such that the optical axis is $76.2 \mathrm{~mm}$ above the vertically mounted breadboard (Figure 4.6). Details of this mount can be found in Appendix B.

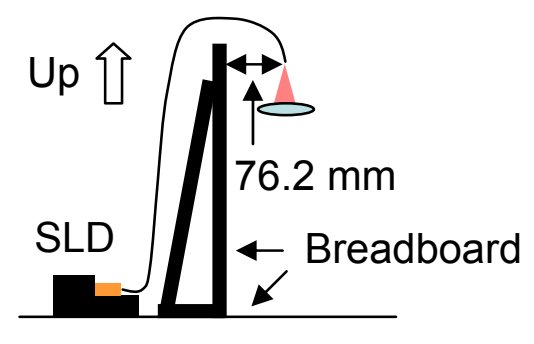

FIGURE 4.6 - Side view of Engage showing vertical orientation for SLD fiber.

The collimating lens used for the SLD light source will dictate the size of the illuminated portion of the cuvette, and will dictate the useable magnification for the imaging lens. The focal length of the lens, $\mathrm{f}$, depends on the NA of the beam emerging from the fiber and the size of the desired test sample. For now the lens chosen and the system magnification will be stated, leaving the justification for later in the discussion of the hardware. The collimating lens is a stock doublet (PAC070) with a focal length of 76.2 $\mathrm{mm}$ and a diameter of $38.1 \mathrm{~mm}$. The NA of the beam does not fill this lens (Figure 4.7). 


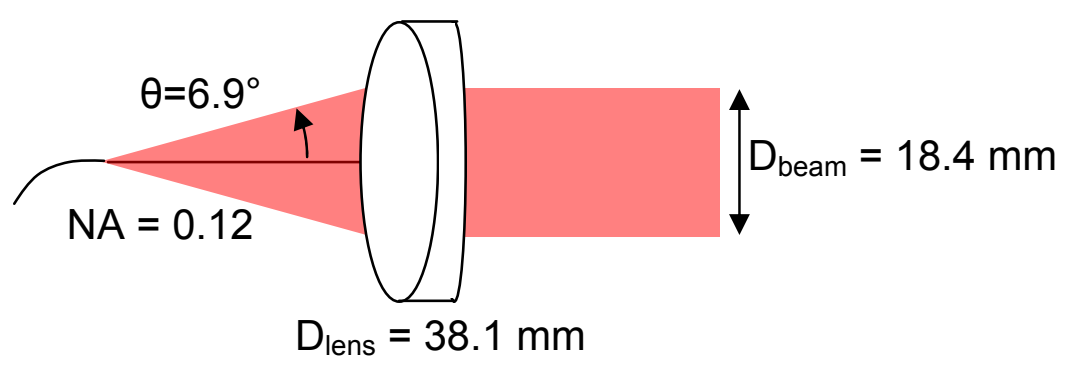

FIGURE 4.7 - Collimating lens for SLD source.

The irradiance falls off away from the optical axis, and the drop in relative irradiance limits the useable portion of the $18.4 \mathrm{~mm}$ diameter beam to about $10-12 \mathrm{~mm}$. This and the detector size will dictate a magnification of 0.5 for the imaging lens. The lens is coated with the standard Newport coating AR.14, which is a visible wavelength antireflection coating. The lens is mounted in a 5-axis mount (tip/tilt, $\mathrm{x}, \mathrm{y}$ and $\mathrm{z}$ ) for ease in alignment. The nominal height of the center of the lens is $76.2 \mathrm{~mm}$ from the breadboard, matching the mount for the SLD fiber output. 


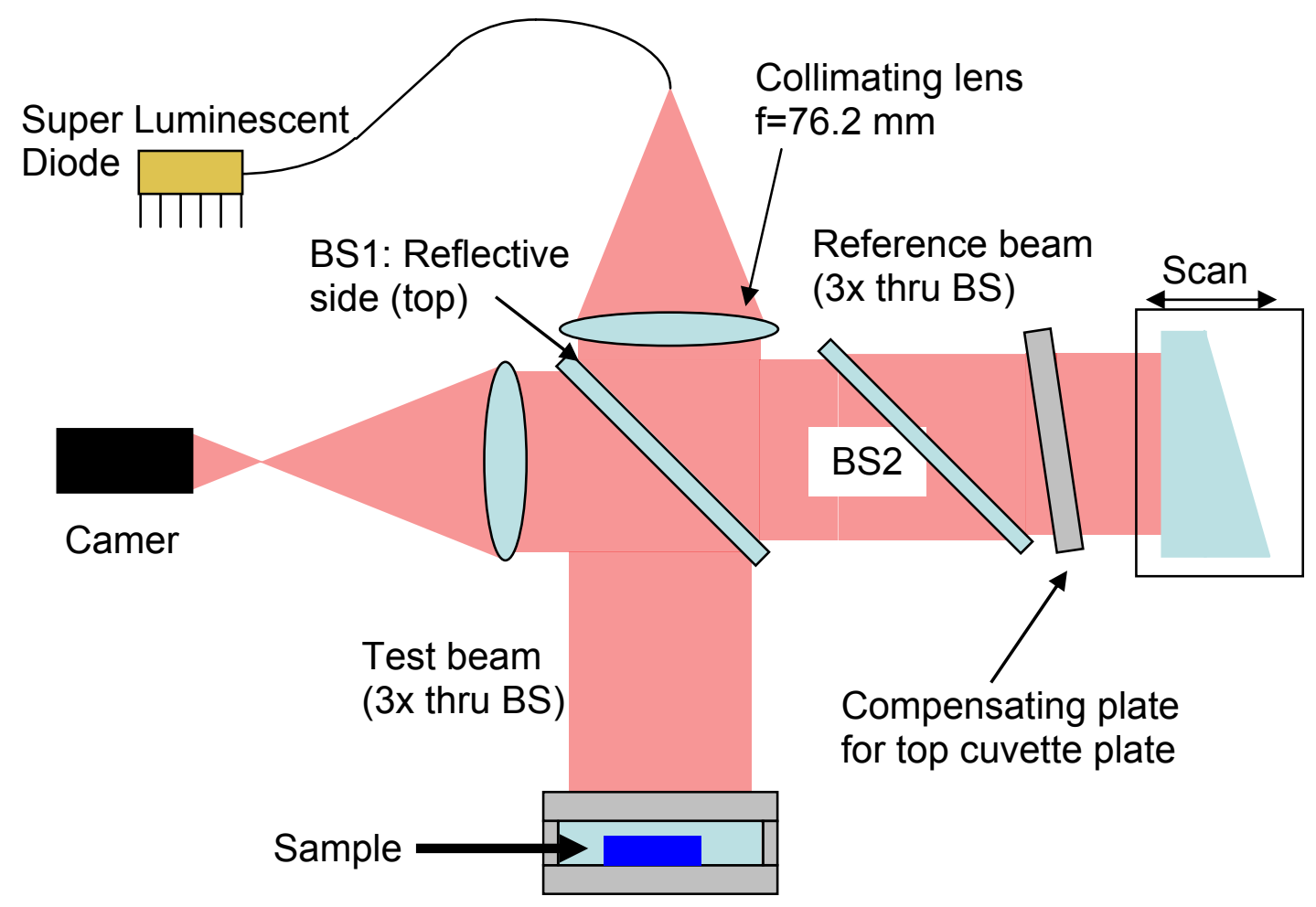

FIGURE 4.8 - Optical layout of the LCI portion of Engage.

The next optic is a 50/50, 2" diameter, plate beamsplitter (BS1) made of fused silica and oriented at a $45^{\circ}$ angle relative to the beam. The top surface is coated with the reflective coating, sending the reference beam off to the right in Figure 4.8. The average reflectance for unpolarized light at $\lambda=680 \mathrm{~nm}$ is about $45 \%$. There is a slight wedge of 30 arc-minutes to help prevent secondary reflections from the AR coated surface of the beamsplitter from contributing to the signal at the detector. The mount for this optic requires only tip/tilt, as the beamsplitter is somewhat larger then the size required to handle the $18 \mathrm{~mm}$ diameter beam. 


\subsection{Reference Arm Components of the LCI}

The first half of the interferometer that will be discussed is the reference arm of the LCI. The next optic in line is BS2, which has the same parameters as BS1, including the top surface having the reflective surface. This beamsplitter serves multiple purposes in Engage, but for the low coherence interferometer, it is simply compensating the reference beam for the OPL and dispersion of the test beam. After the two beams are recombined, both will have traversed three times through nominally identical beamsplitters, negating the dispersion contributions from the glass in the beamsplitters.

A compensating plate is required to match the top plate of the cuvette. The angle of this optic is used as a rough adjustment for dispersion matching the top plate of the cuvette in the test arm. The procedure for finding this angle is discussed in Appendix $\mathrm{C}$ on the system alignment.

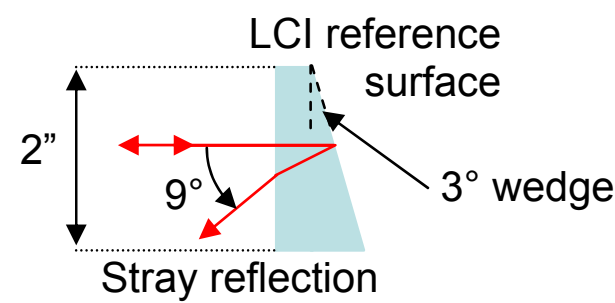

FIGURE 4.9 - The reference surface for LCI has a $3^{\circ}$ wedge.

The reference surface for the low coherence interferometer is a custom coated, 2" diameter, $3^{\circ}$ wedged window from Lambda Research Optics, Inc (Figure 4.9). The large wedge makes mounting this window difficult, and it is glued in place in the mount with UV curing cement to prevent unwanted motion of this window as the stage it is mounted 
to translates along the optical axis. Reflections off the back surface emerge from the plate tilted about $9^{\circ}$ from the incoming beam and can be ignored. The front surface of the plate is the reference surface, which in most interferometers is a mirror. In this case, it is actually anti-reflection coated. The goal of the coating is $1.5 \%$ reflectance at normal incidence, which is less then the $4 \%$ reflectance for an uncoated glass surface. For optimal fringe visibility between this signal and the variety of signal irradiances possible in the test arm, the reflectance is the median in order to get the best signal for all types of interfaces. In the expression, $\mathrm{T}$ and $\mathrm{R}$ are transmission and reflectance, respectively, for a given surface.

$$
\begin{aligned}
& \left(\frac{I_{\text {out }}}{I_{\text {in }}}\right)_{\text {Test }, \text { glass/air }}=T_{B S 1} R_{\text {glass /air }} R_{B S 1}=0.55 \cdot 0.04 \cdot 0.45=1 \% \\
& \left(\frac{I_{\text {out }}}{I_{\text {in }}}\right)_{\text {Test }, \text { glass } / \text { saline }}=T_{B S 1} R_{\text {glass } / \text { saline }} R_{B S 1}=0.55 \cdot 0.004 \cdot 0.45=0.1 \% \\
& \left(\frac{I_{\text {out }}}{I_{\text {in }}}\right)_{\text {Test, saline/sample }}=T_{B S 1} R_{\text {saline/sample }} R_{B S 1}=0.55 \cdot 0.00076 \cdot 0.45=0.019 \%
\end{aligned}
$$

Equation 4.12

For a $1.5 \%$ reflectance reference surface, the irradiance ratio for the test beam is:

$$
\left(\frac{I_{\text {out }}}{I_{\text {in }}}\right)_{\operatorname{Re} f}=R_{B S 1} T_{B S 2} R_{\operatorname{Re} f . S u r f} T_{B S 2} T_{B S 1}=0.45 \cdot 0.55 \cdot 0.015 \cdot 0.55 \cdot 0.55=0.1 \%
$$




\subsection{Moving the Reference Surface}

Aside from the light source, the motion of the reference surface is the most critical component for making successful thickness measurements of contact lens materials. Some of the requirements for motion were discussed during the theory section of the thickness measurement, as the chosen surface finding algorithm depends on the characteristics of the data collection, including axial image spacing. Recall that measuring the location of a surface requires collecting a series of images at known axial positions (z) to build up a coherence pattern for each pixel versus depth on the camera. In an ideal system, the locations of these images would be evenly spaced and known exactly. Mechanically, this is difficult if not impossible to achieve, and so the algorithms evolved to handle data that is not evenly spaced, but the positions are at least well known. The step sizes should also be within about $10 \%$ of the ideal constant step size. This section will discuss how this is achieved.

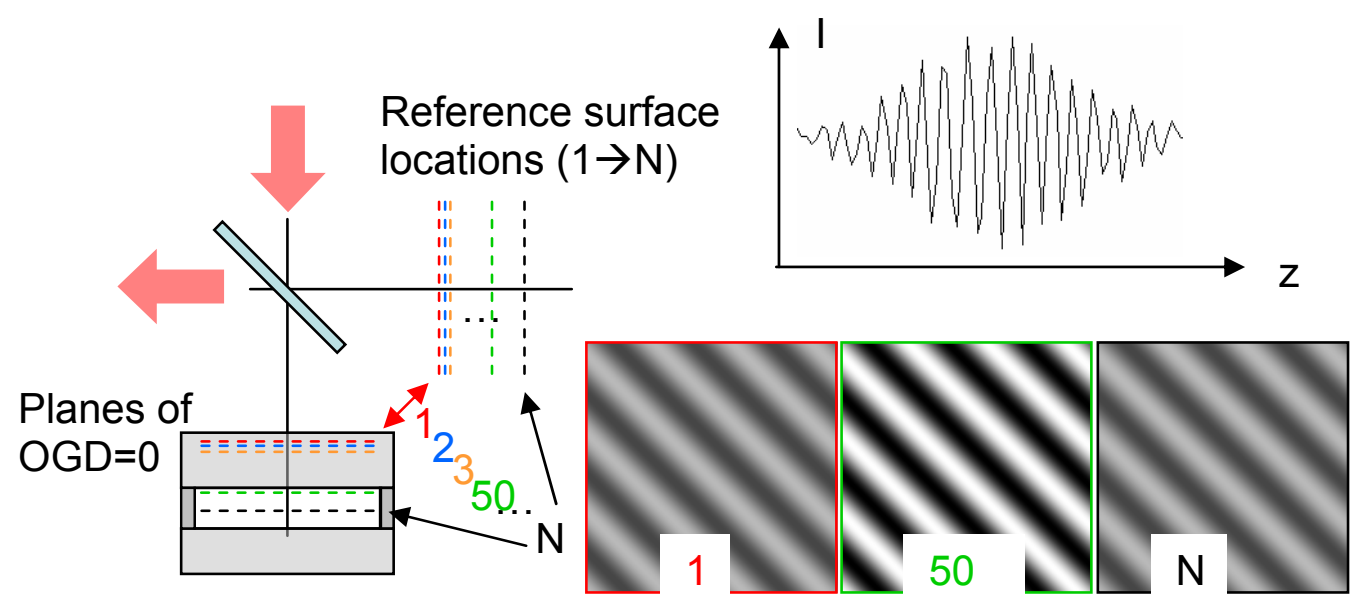

FIGURE 4.10 - Simulated fringe patters as the reference surface scans relative to the measurement cuvette. 
Figure 4.10 shows a few simulated images as a function of frame number (depth) of the scan. The dashed lines represent the locations of the reference surface and the corresponding plane of zero OGD in the test arm. Fringe contrast is higher when the zero OGD plane is closest to the interface. A signal versus depth is shown for all the depth location for a single CCD pixel as well (I(z)).

The motion system currently used to translate the mirror is a second generation system. The first generation utilized an open-loop motor and monitored the position of the stage with a distance measuring interferometer (DMI). The DMI was used to record the stage positions and not to close the positioning loop. There were several problems with this setup; the motor was extremely slow (1.2 $\mathrm{mm} /$ minute); it was unrepeatable, making it difficult to be within $10 \%$ of the desired step size; and, the hardware needed to duplicate it was expensive.

The current, second generation motion control system operates with a closed loop linear stage that moves by rotating a low friction ballscrew drive (VP-25XL, Newport). The components necessary to control this stage are shown in Figure 4.11. The computer controls an XPS Motion Controller/Driver from Newport via a crosswire Ethernet cable. The XPS is capable of controlling up to 8 axis of travel and read the associated 8 feedback positions for these stages. The XPS is essentially a chassis that accepts two types of boards: 1) motion control boards, in this case a XPS-DRVM5; and 2) position detection processing boards. Two cables are required for the VP-25XL stage; one to 
control the motion and one to read the position of the optical encoder. As the stage moves to the right, the plane of zero OGD moves through the measurement cuvette.

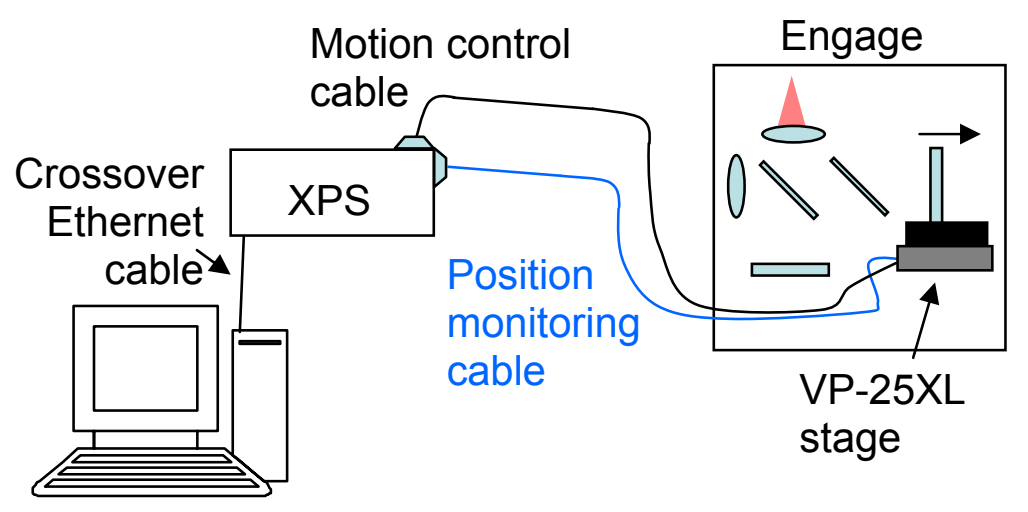

FIGURE 4.11 - Schematic of required components for achieving motion of reference surface.

This stage has a travel range of $25 \mathrm{~mm}$, much larger then the approximate required travel range of $800 \mu \mathrm{m}$, and reports the position to $+/-5 \mathrm{~nm}$. Target positions can be specified to the nearest $10 \mathrm{~nm}$. Capable of moving the entire travel range in 1 second, this stage has no problems moving fast enough for the purposes of this measurement system.

A hardware home allows all position measurement to be referenced to the same location for 0 , even after a power up/down cycle. The stage is required to find this home after being rebooted prior to attempting any other motion.

The position of the stage is monitored with an optical encoder that returns two analog sinusoidal signals (offset in phase by $90^{\circ}$ ) to a dedicated processing board for reporting positions to $+/-5 \mathrm{~nm}$. The stage uses the reported positions to close the loop and more 
accurately position the stage at the desired locations. The stability of the stage in terms of reported positions is usually about $+/-10 \mathrm{~nm}$. Even though the position monitoring is done on the side of the stage, it is not flawless. In fact the reported stage position has large errors compared to the desired measurement accuracy on the order of nanometers.

When the Newport stage was initially used to translate the reference surface, the low coherence signals recovered were poor. The desired step size in path difference for sampling the low coherence signal is to sample every $360^{\circ}+90^{\circ}=450^{\circ}$ in phase, or $5 / 4 \lambda$. For $\lambda_{\mathrm{c}}=680 \mathrm{~nm}$, this is a path difference of $850 \mathrm{~nm}$. Since the light is double pass off the reference surface, it only needs to move half of this distance, or $425 \mathrm{~nm}$. The initial reported positions were on a nearly ideal constant spacing (Figure 4.12).

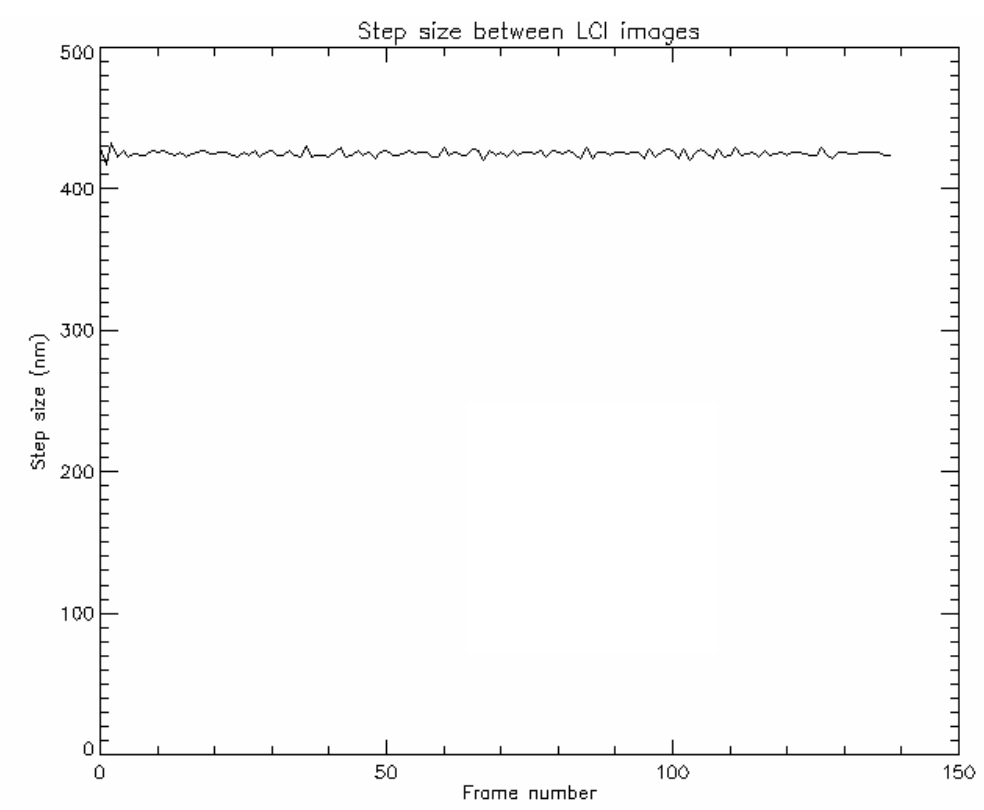

FIGURE 4.12 - Reported step distances for LCI stage, uncorrected. 
Given this reported spacing from the optical encoder on the side of the linear stage, a model signal could be created and compared to the recovered signal. As is shown in Figure 4.13, the modeled data (pink) does not match the signal (black). The phase of the underlying cosine matches at the black arrow, but about 4 microns to the left, the fringes are exactly out of phase (red arrow).

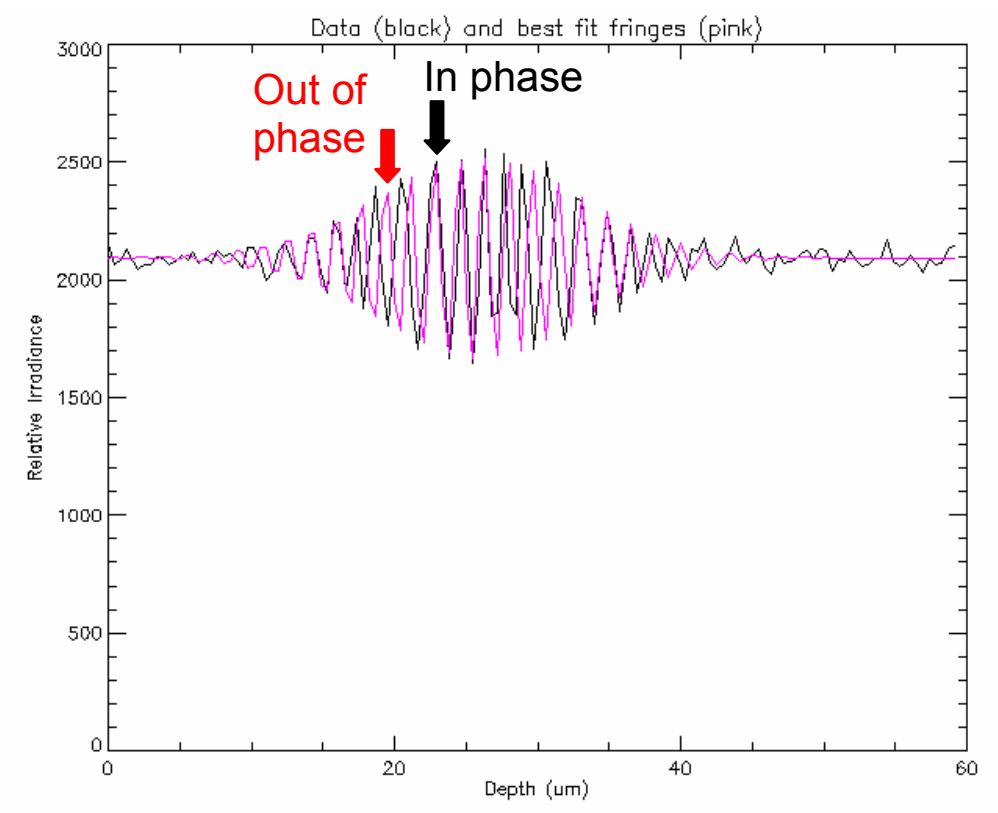

FIGURE 4.13 - Measured (black) and modeled (pink) I(z) for stage positions shown in Figure 4.12.

The most plausible explanation for this discrepancy is that the reported stage positions from the optical encoder are incorrect. One way to check the validity of the position measurements is to use a distance measuring interferometer (DMI) to track the position of the stage and compare these numbers those reported by the XPS optical encoder. 
The DMI used for this test, a Zygo ZMI-1000 has a single pass resolution of $2.47 \mathrm{~nm}$. A specialized laser emits two linear orthogonally polarized beams of with slightly different wavelengths (In a vacuum: $\lambda_{1}=632.991501 \mathrm{~nm}, \lambda_{2}=632.991528 \mathrm{~nm}$ ) with a frequency difference of $20.215 \mathrm{MHz}$.

$$
\Delta v=\frac{c}{\bar{\lambda}^{2}} \Delta \lambda
$$

One of these beams is the reference beam while the other is the test beam in an interferometer. The beamsplitter that separates the beams is a polarization beam splitter (PBS). The two beams are recombined and coupled to a single mode fiber that connects to a measurement board.

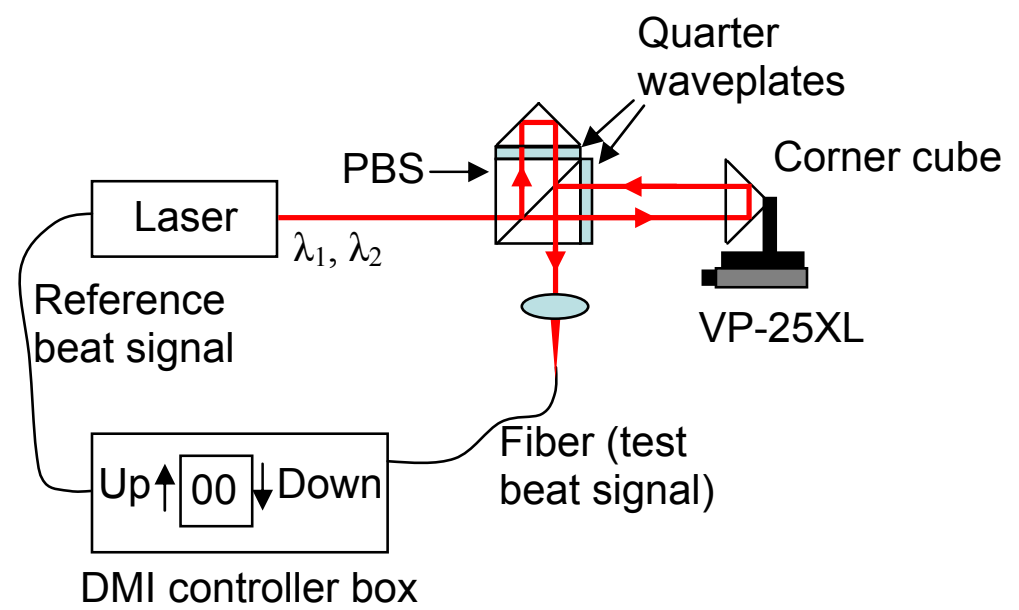

FIGURE 4.14 - Layout of the DMI for accurately monitoring stage position.

The test and reference beams, having slightly different wavelengths, will create a beat frequency that can be measured and compared to a similar reference beat frequency that is monitored directly in the laser source. The frequencies are monitored by means of a counter that counts up for each beat of the reference beat frequency (internal to laser) and 
down for each beat of the test beat frequency (from interferometer shown in Figure 4.14). If the path difference between the test and reference beams is constant, then the beat frequency is constant and equal to the beat frequency of the reference signal, and the counter value will remain constant. When the corner cube on the stage moves away from the cube beamsplitter, the test beam will be delayed and there will be a change in the number of beats recorded. The counter counts this difference as $\mathrm{N}$ counts and converts $\mathrm{N}$ to a distance moved $d$ that depends on the wavelength of the test beam.

$$
d=\frac{N \lambda_{\text {air }}}{256} ; \quad \lambda_{\text {air }}=\frac{\lambda_{1(2)}}{n_{\text {air }}}
$$

The accuracy of the distance measurement depends on the accuracy of the value for the refractive index of air, $\mathrm{n}_{\text {air }}$, which depends on temperature, atmospheric pressure, and humidity. The nominal value for $\mathrm{n}_{\text {air }}$ is 1.000271 , which translates into 2.47195 nanometers of corner cube displacement for a change in $\mathrm{N}$ of 1 count.

Using the setup in Figure 4.14, the stage was translated $+/-10 \mathrm{~mm}$ in roughly $200 \mathrm{~nm}$ steps (100,000 steps), and at each position, the optical encoder position, $\mathrm{z}_{\mathrm{OE}}$, and the DMI position, $\mathrm{z}_{\mathrm{DMI}}$, were recorded. Since the DMI is recording the position of the mirror, which is what is important in the low coherence measurement, it is considered to be the standard. Any difference between the DMI positions and the optical encoder positions can be attributed to errors in the stage. This position error, $\varepsilon_{\text {pos }}$, is a discrete function, with each error occurring at a position $\mathrm{z}_{\mathrm{OE}}$ that corresponds to a returned reading from the optical encoder. This creates a lookup table in an array of size $2 \times 100000$, where the first 
column is the returned optical encoder positions $\left(\mathrm{z}_{\mathrm{OE}}\right)$ and the second column is the error (difference) between the optical encoder positions and the DMI positions.

$$
\begin{aligned}
& \varepsilon_{p o s}\left(0,{ }^{*}\right)=z_{O E} \\
& \varepsilon_{p o s}(1, *)=z_{O E}-z_{D M I}
\end{aligned}
$$

The error depends on the distance per DMI count, which depends on the index of air. Any offset error in the index translates to a slope error in $\varepsilon_{\text {pos }}$ which leads to a bias in thickness measurements. A bias in thickness measurement causes a bias in refractive index measurement. The importance of $\varepsilon_{\mathrm{pos}}$ becomes clear. A plot of $\varepsilon_{\mathrm{pos}}$ as a function of reported optical encoder position is shown in Figure 4.15.

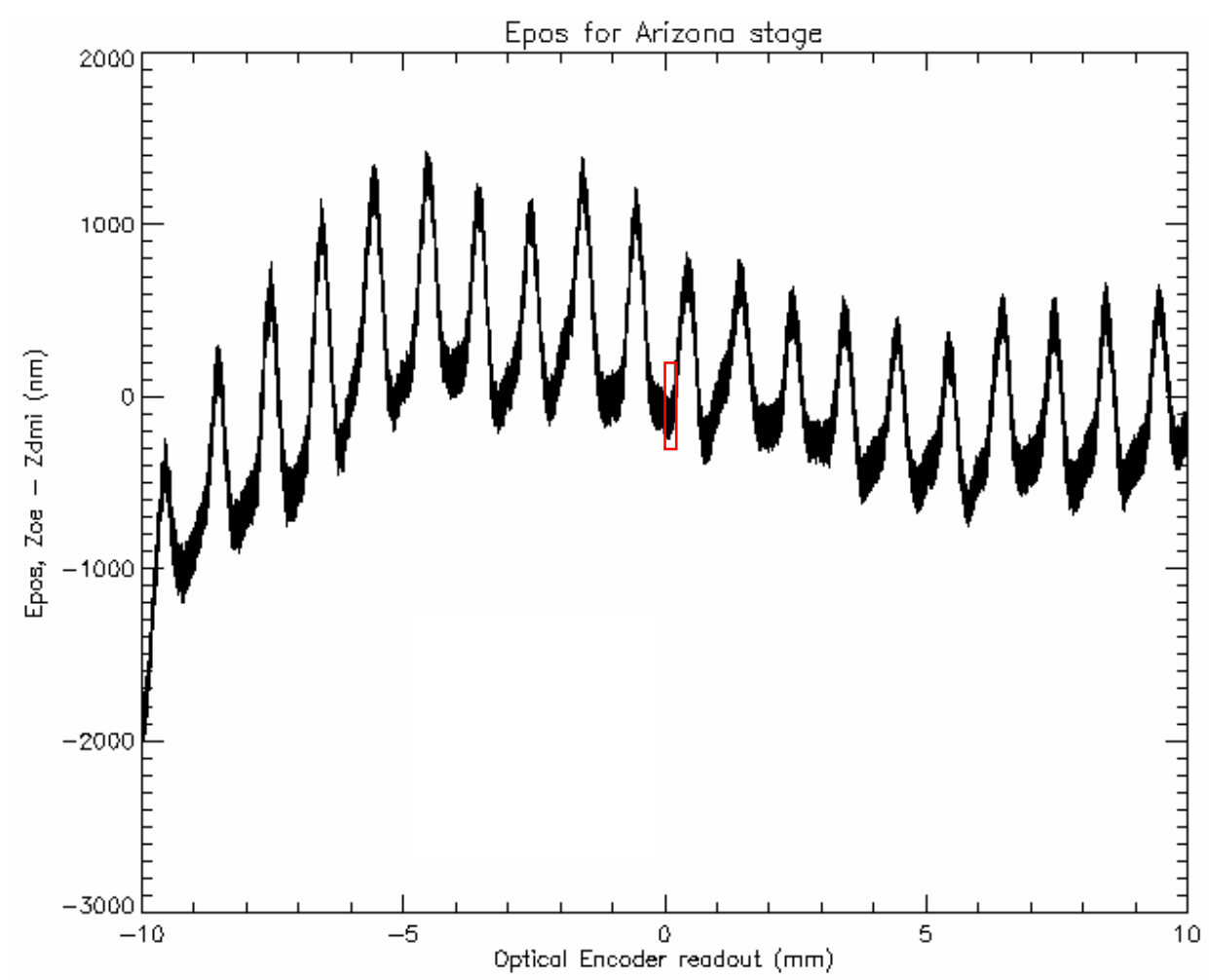

FIGURE 4.15 - Plot of $\varepsilon_{\text {pos }}$ for the stage used in the Arizona Engage system. 
This data is stored in a tiff file called "pos_map_az1_nm.tif” in the "config" folder for the Arizona Engage system. This file is created with the program IDL (Interactive Data Language) function POSITION_MAP_FILE.pro, which can be found in Appendix D. Figure 4.15 makes it obvious that this error mapping procedure is necessary. There are two major components of $\varepsilon_{\text {pos }}$ : a low frequency component with a period $\mathrm{T}_{\text {low }} \approx 1 \mathrm{~mm}$, which is clearly visible in Figure 4.15, and a high frequency component with a period $\mathrm{T}_{\text {high }} \approx 10 \mu \mathrm{m}$, that appears as thickening of the line above, but is clearly visible when zooming in on the region defined by the red box in Figure 4.15 (Figure 4.16).

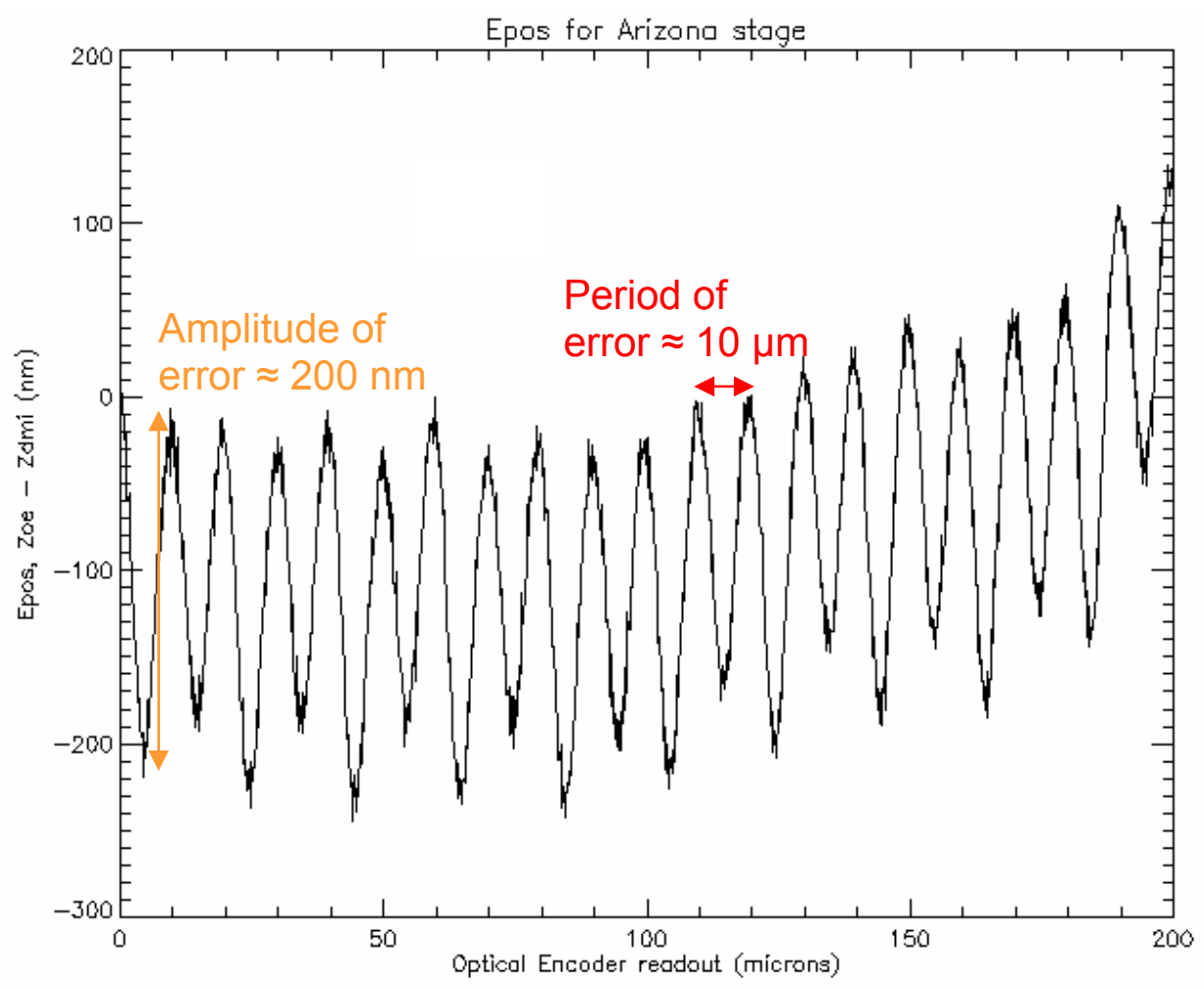

FIGURE 4.16 - Magnified view of the curve in Figure 4.15 showing the high frequency optical encoder error. 
The low frequency error is caused by something often referred to as a drunken lead screw. Physically, the threaded screw is not perfectly round. The result is a rotationally dependent position error. Since the screw repeats every $1 \mathrm{~mm}$ of axial travel, the drunken lead screw error also repeats (Figure 4.15). Since this error is from the physical position of the driving screw, the phase and form of the error is very consistent over multiple measurements of the stage error. The fact that all positions are relative to a hardware home that is fixed means that this error as a function of position is also fixed.

The high frequency error is analogous to a phase shifting error in phase shifting interferometry. The optical encoder reads two sinusoidal encoders, A and B, on the stage that should be offset from each other by $90^{\circ}$ in phase as shown in Figure 4.17.

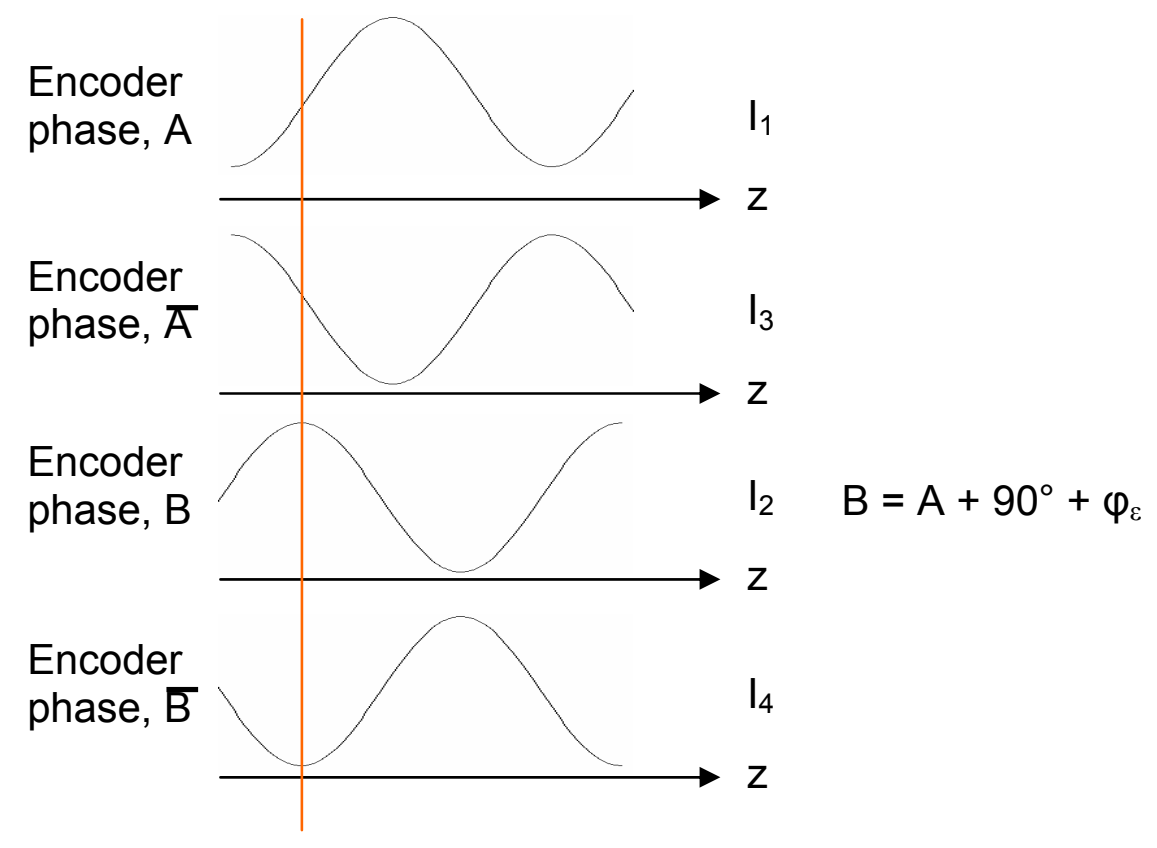

FIGURE 4.17 - Four signals recovered from the two optical encoders used to determine $\Phi_{\text {Encoder,Rec. }}$ 
The values for $I_{1}$ through $I_{4}$ are what is measured and relate to the two encoders as shown above. The calculated phase recovered from the two sinusoidal signals, $\Phi_{\text {Encoder,Rec, }}$ relates directly to a position measurement within the period of the encoders, which is 20 $\mu \mathrm{m}$ for the VP-25XL stage. The position within a period is calculated with the standard 4-step phase shifting algorithm.

$$
\begin{gathered}
\Phi_{\text {Encoder,Rec }}=\tan ^{-1}\left(\frac{I_{4}-I_{2}}{I_{1}-I_{3}}\right) \\
z_{O E}=\Phi_{\text {Encoder, } \operatorname{Rec} c} \frac{20 \mu m}{2 \pi}
\end{gathered}
$$

The accuracy of the recovered phase $\Phi_{\text {Encoder,Rec }}$ can be worse due to errors that are similar to those found in phase shifting interferometry. In the case of the optical encoder, the most common error is an additional phase offset between the two sinusoidal encoders, A and B. This additional offset phase $\varphi_{\varepsilon}$ is added to the phase used to calculate $\mathrm{I}_{2}$ and $\mathrm{I}_{4}$.

$$
\begin{array}{ll}
A: & I_{1}=1+\cos \left(\Phi_{\text {Encoder }}\right) \\
\bar{A}: & I_{3}=1-\cos \left(\Phi_{\text {Encoder }}\right)=1+\cos \left(\Phi_{\text {Encoder }}+180^{\circ}\right) \\
B: & I_{2}=1+\cos \left(\Phi_{\text {Encoder }}+90^{\circ}+\varphi_{\varepsilon}\right) \\
\bar{B}: & I_{4}=1-\cos \left(\Phi_{\text {Encoder }}+90^{\circ}+\varphi_{\varepsilon}\right)=1+\cos \left(\Phi_{\text {Encoder }}+270^{\circ}+\varphi_{\varepsilon}\right)
\end{array}
$$

Equation 4.19

This leads to an incorrect phase shift which results in an error in the recovered phase $\Phi_{\text {Encoder,Rec }}$ that oscillates at twice the frequency of the encoders, or with a period of 10 $\mu \mathrm{m}$. The high frequency error is $10 \mu \mathrm{m}$, as is clear from Figure 4.16.

$$
\Phi_{\text {Encoder }, \operatorname{Rec}}=\tan ^{-1}\left(\tan \left(\Phi_{\text {Encoder }}\right) \cdot \cos \left(\varphi_{\varepsilon}\right)+\sin \left(\varphi_{\varepsilon}\right)\right) \quad \text { Equation } 4.20
$$


The amplitude of this error depends on the magnitude of $\varphi_{\varepsilon}$; in fact, the amplitude of the error is the period times the ratio of the phase error to $2 \pi$.

$$
\varepsilon_{p o s}=\frac{\varphi_{\varepsilon}}{2 \pi} 20 \mu m
$$

The peak to valley of the amplitude of the error in Figure 4.16 is $200 \mathrm{~nm}$, which is $1 \%$ of the period. According to Equation 4.21, $\varphi_{\varepsilon}$ is 0.0628 radians, which is an equivalent offset of the encoder of $200 \mathrm{~nm}$.

The origin of this high frequency error is a slight misplacement of one of the optical encoders relative to the other, which is unlikely to change over time. Multiple measurements of the error with respect to stage position $\left(\varepsilon_{\mathrm{pos}}\right)$ show that the phase and amplitude of the error is indeed mostly constant. This consistency allows a reasonable level of correction to be achieved by storing $\varepsilon_{\mathrm{pos}}$ in a file and using that file as a lookup table to correct the positions reported by the optical encoder to their true locations.

\subsubsection{Post-correction of Stage Position Values}

A first level correction to the data is to simply post-correct the position data for a series of images, which for the step sizes shown in Figure 4.12, results in Figure 4.18. 


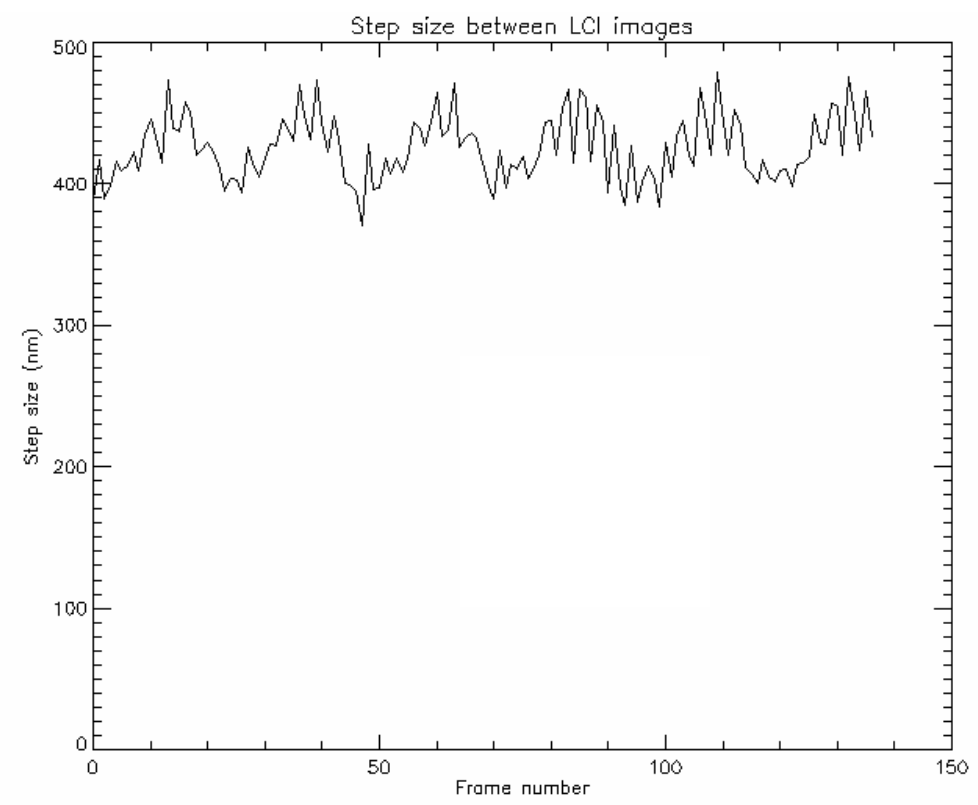

FIGURE 4.18 - Step distances for LCI stage, post-corrected using lookup table.

The reported positions will fall between two recorded positions with known errors. The error is assumed to be linear over this $200 \mathrm{~nm}$ sampling distance, and is linearly extrapolated to find the error at the reported position (Figure 4.19).

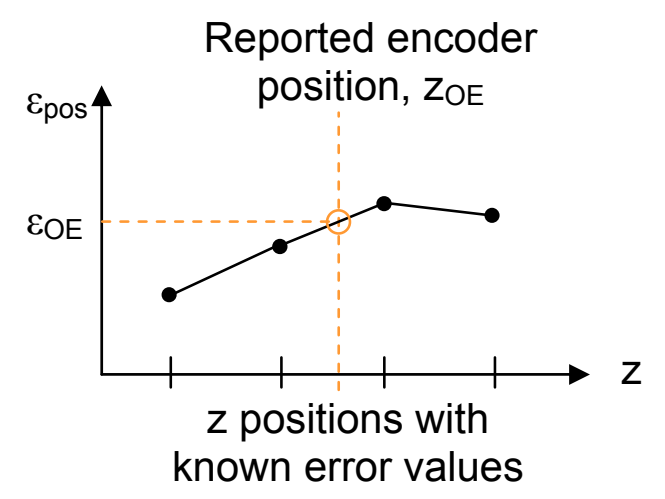

FIGURE 4.19 - Determining $\varepsilon_{\mathrm{OE}}$ for a $\mathrm{z}_{\mathrm{OE}}$ that falls between points with known $\varepsilon_{\mathrm{OE}}$.

This new position data can be used to improve the modeled low coherence signal to match the measured signal (Figure 4.20). 


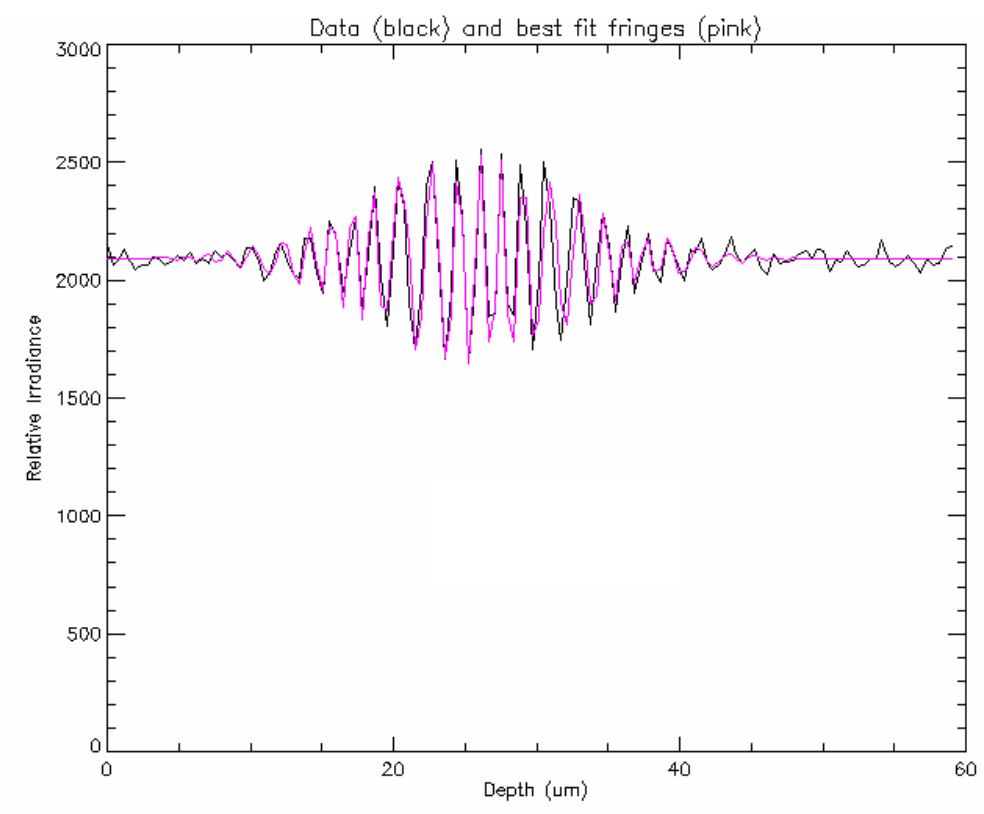

FIGURE 4.20 - Measured (black) and modeled (pink) I(z) for stage positions shown in Figure 4.18.

The phase relationship is now correct, with irradiance errors accounting for the majority of the mismatching between the data and the model. Applying this post correction is the difference between being able to use this VP-25XL stage to collect thickness data and having no ability to measure thickness accurately. However, as Figure 4.18 shows, there is now a periodic variation in the step sizes between images, which degrades the effectiveness of the surface calculation algorithms discussed earlier.

\subsubsection{Pre and Post-correction of Stage Position Values}

Once the start position for a surface scan has been determined, a series of evenly spaced locations for image capture is easily calculated. Since the stage uses the optical encoder to determine if it has reached the correct position, these 'evenly spaced' positions will 
become a curve like that shown in Figure 4.18. This problem can be fixed by applying a pre-correction of the axial positions for image capture.

The pre-correction uses the same lookup file containing $\varepsilon_{\text {pos }}$ to adjust the target positions based on the known position errors. Instead of feeding the stage controller the spacing shown on the left in Figure 4.21, the pre-correction positions might look something like the spacing values on the right.
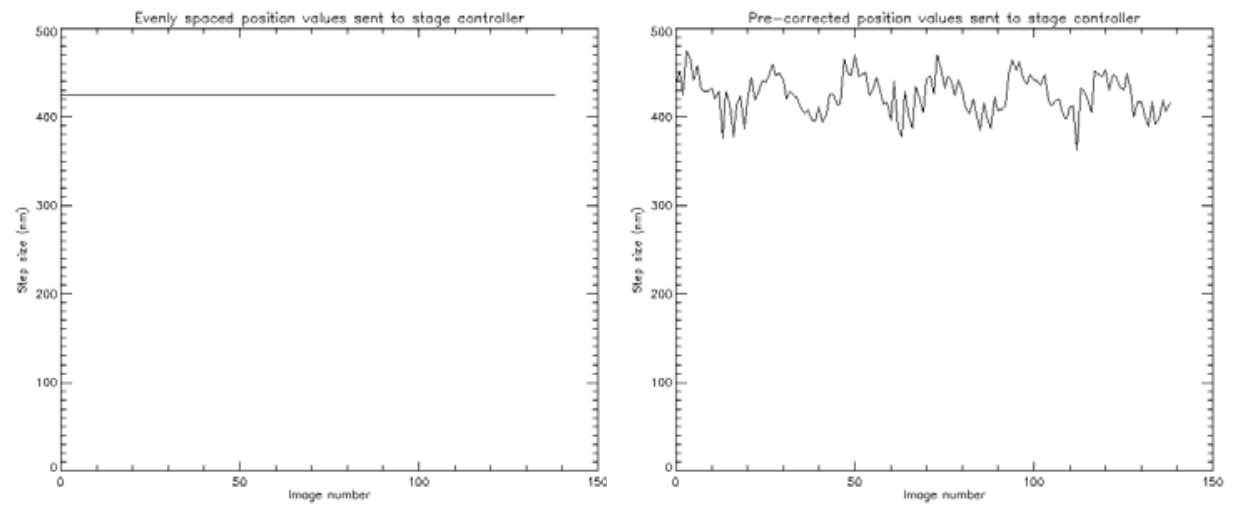

FIGURE 4.21 - Evenly spaced position goals (left) and pre-corrected position goals (right).

The pre-correction algorithm results in data that are more evenly spaced along the z-axis. Post-correction of the position values is still required since the stage does not position itself perfectly and since the reported positions from the stage are still wrong according to $\varepsilon_{\text {pos. }}$ The final used position data that was pre and post-corrected, resulting in evenly spaced images with correct position values, is shown in Figure 4.22. 


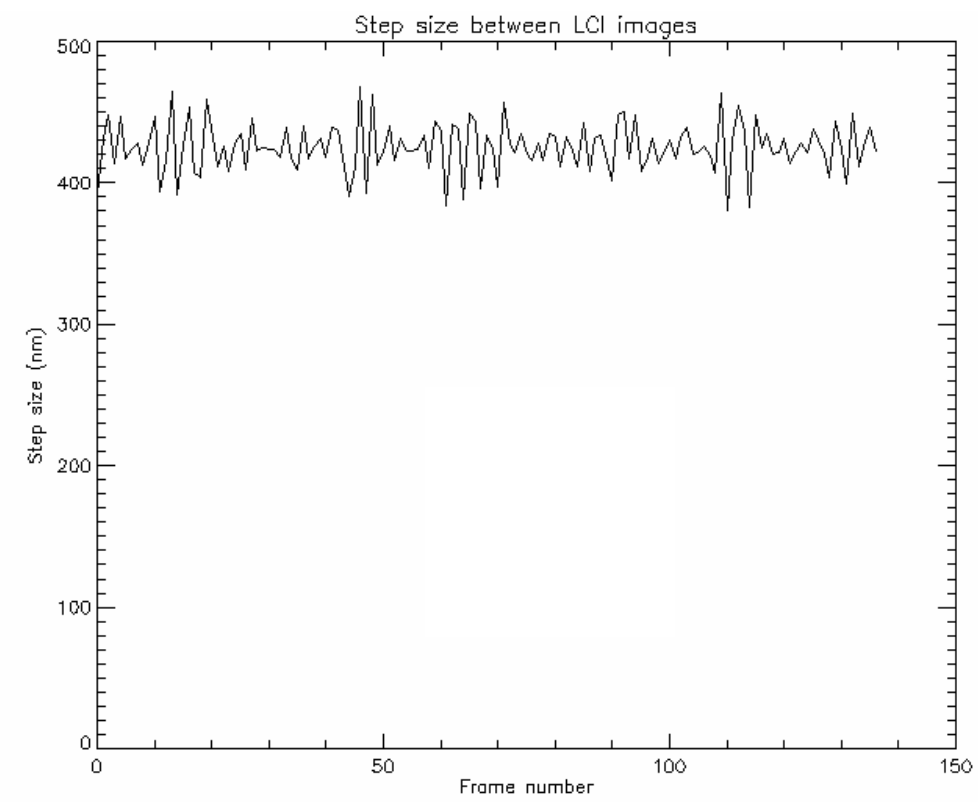

FIGURE 4.22 - Step distances for LCI stage, pre- and post-corrected using lookup table.

The resulting low coherence signal for a given pixel versus depth has a more consistent phase difference along depth because of the evenly spaced data collection due to the precorrection. The modeled curve matches this data well thanks to the post-correction (Figure 4.23). 


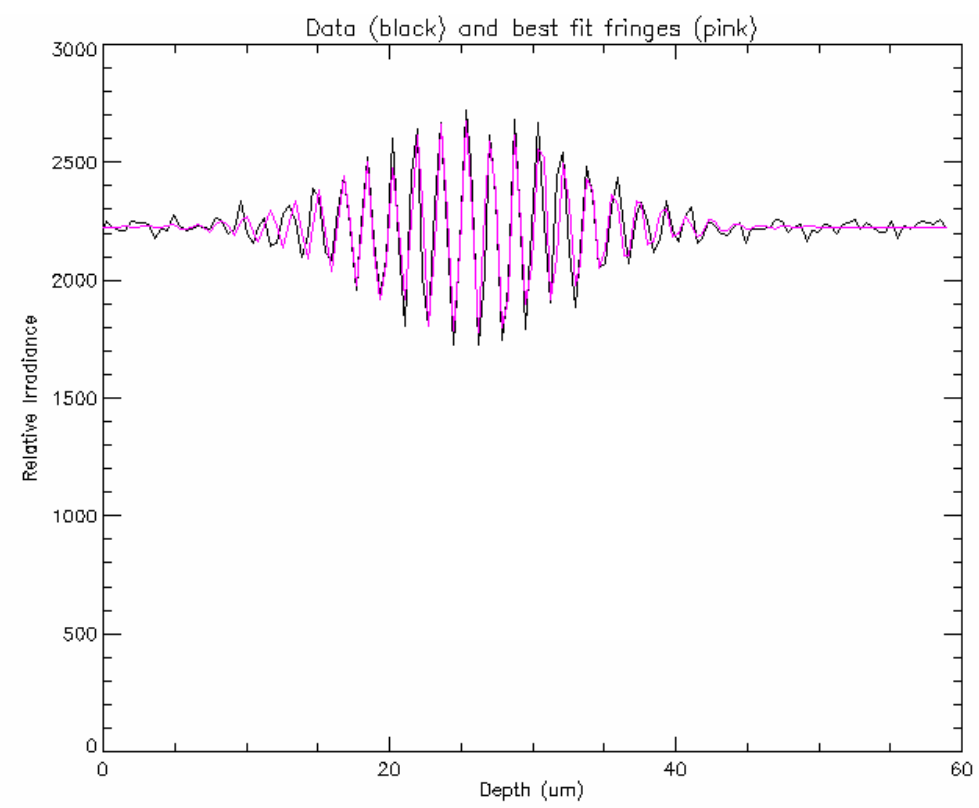

FIGURE 4.23 - Measured (black) and modeled (pink) I(z) for stage positions shown in Figure 4.22.

This process is applied to every stage position reported during data collection and is different for every data set collected.

\subsection{Test Arm Components of the LCI}

After the test beam transmits through BS1, the first optic it encounters is the measurement cuvette. At first glance, the cuvette is simply two glass plates separated by some distance $t_{1}$ (Figure 4.24). In fact, the cuvette is a critical component in the measurement of the contact lens materials. As has been mentioned before, the surfaces of the two plates must be flat to at least $\lambda / 20$ if not better, although small wedge between the top and bottom surfaces is allowable. 
The low coherence signal becomes non-symmetric when transmitting through dispersive materials, so the top plate of the cuvette is compensated for in the reference arm. The cavity thickness is measured when filled with saline, a dispersive medium, and that volume cannot be compensated for in the test arm. Since the signal degradation is dependent on the thickness of the dispersive medium, the cavity should be as thin as possible. The lower bound on the cavity thickness is dictated by the sample thickness and the required surface separation for the low coherence signals. The sample thickness ranges between 70 and $200 \mu \mathrm{m}$. The minimum OGL between surfaces such that the low coherence signals from the two do not overlap is roughly $50 \mu \mathrm{m}$. Given the test geometry where the sample is sitting on the bottom plate, the minimum thickness is about $250 \mu \mathrm{m}$ (Figure 4.24).

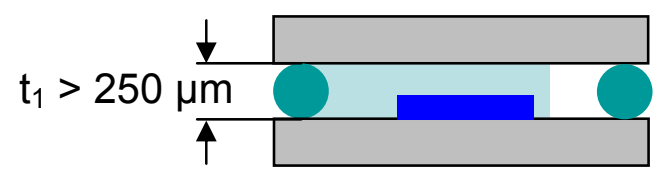

FIGURE 4.24 - Minimum thickness for the cavity of the cuvette.

The plate separation chosen is $350 \mu \mathrm{m}$, which is achieved using three $350 \mu \mathrm{m}$ diameter sapphire balls. Since the inside surfaces must be accessible for sample preparation and so that they can be cleaned between measurements, the cavity cannot be fixed. Therefore, the sapphire balls are glued to the top plate, arranged $120^{\circ}$ apart, allowing easy access to the cavity. They are attached to the plate using ultraviolet-curing cement (Figure 4.25). 


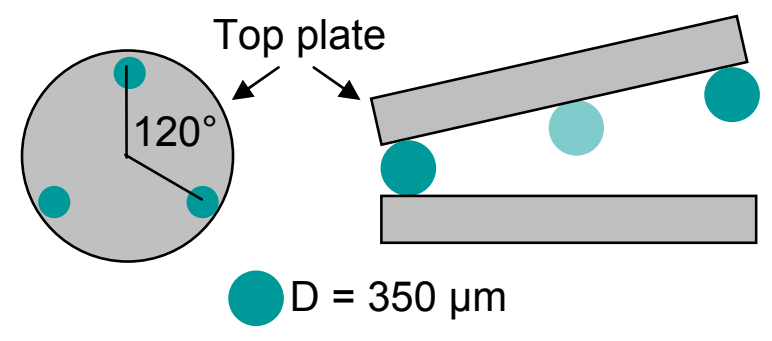

FIGURE 4.25 - Drawing of the current measurement cuvette where the cavity is formed by $350 \mu \mathrm{m}$ diameter sapphire balls glued to the top plate.

It should be noted that in the current cuvette, the bottom plate diameter is 2" while the top plate is $1.5 "$ in diameter. There is no reason the two plates cannot be the same physical dimensions, as is shown in many of the illustrations. Since the top plate is only supported in three points, the top plate has nothing restricting side to side motion, which should not occur during a measurement. Since the cavity is so thin, surface tension keeps the saline solution from spilling out the side of the cavity.

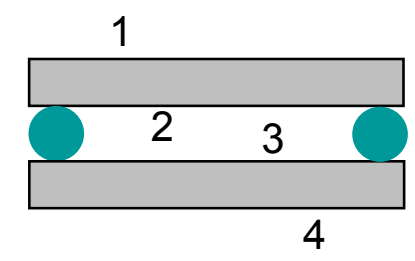

FIGURE 4.26 - Four optical surfaces of the cavity for coating designations.

There are four optical surfaces in the cavity; 1) top of the top plate, 2) bottom of the top plate, 3) top of the bottom plate, and 4) bottom of the bottom plate (Figure 4.26).

Surfaces 1 and 4 have identical broadband anti-reflection coatings to reduce the amount of light from the SLD that is reflected back to the camera from these two surfaces. Any light from these surfaces increases the irradiance offset for the low coherence signals, 
which decreases the attainable fringe visibility without saturating the camera. The coatings should cover the range of the SLD and of the laser wavelengths used $(543 \mathrm{~nm}<$ $\lambda<690 \mathrm{~nm}$ ) to prevent unwanted reflections and spurious fringes from the multiple cuvette surfaces. Surfaces 2 and 3 do not have AR coatings since the LCI measurement depends on some light being reflected from the glass interfaces inside the cuvette (Equation 4.10, 4.11).

Recall that the measurement technique requires the sample to be sitting on the bottom plate of the cuvette during measurement, both so that it is stable over the measurement time and so that the thickness of the sample can be calculated by subtracting the thickness of the saline from the overall cavity thickness. It should be apparent to anyone who has handled soft contact lens materials that the material, when hydrated, is slippery. Glass is somewhat hydrophobic, but not enough such that wet samples stick to glass. As the sample loses liquid content, it becomes tacky and quite sticky. Recall that one of the testing requirements is that the sample be tested while hydrated in solution. It became clear that a wet sample on bare glass was not going to work. If the sample does not stick to the glass plate, the index of refraction can not be measured.

\subsection{Test Sample Mounting}

Significant effort was put into solving the problem of sample mounting. The first approach was to use mechanical methods to mount the sample so that it was in contact with the bottom plate of the glass cuvette. One such attempt was with a metal washer 
used as an annular weight. The thought was that the weight would force the sample to stay down while only forcing out saline in the regions in which it was weighting down the sample, which would be blocked from view by the washer anyway (Figure 4.27a).

a) Desired Mounting:

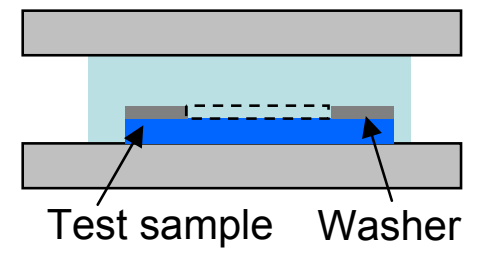

b) Actual Result:

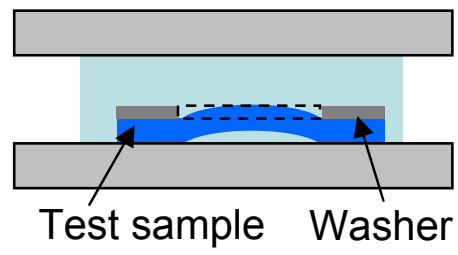

FIGURE 4.27 - Drawing of a) desired effects of the washer and b) actual results of the washer.

The result was that the middle region of the flat sample bowed upwards so that it was not in contact with the glass at all (Figure 4.27b). A few fringes were obtained in the center region, where the sample was normal to the test beam (Figure 4.28).

a) Single image

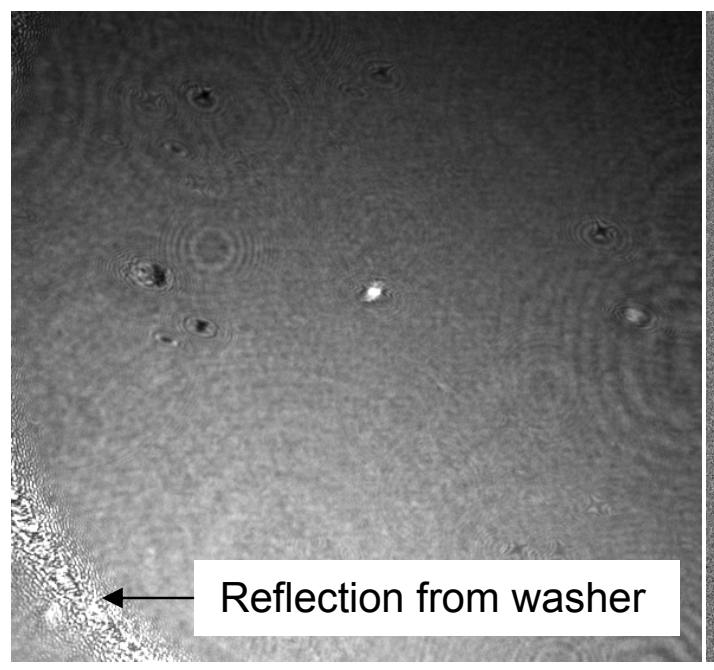

b) Image subtraction

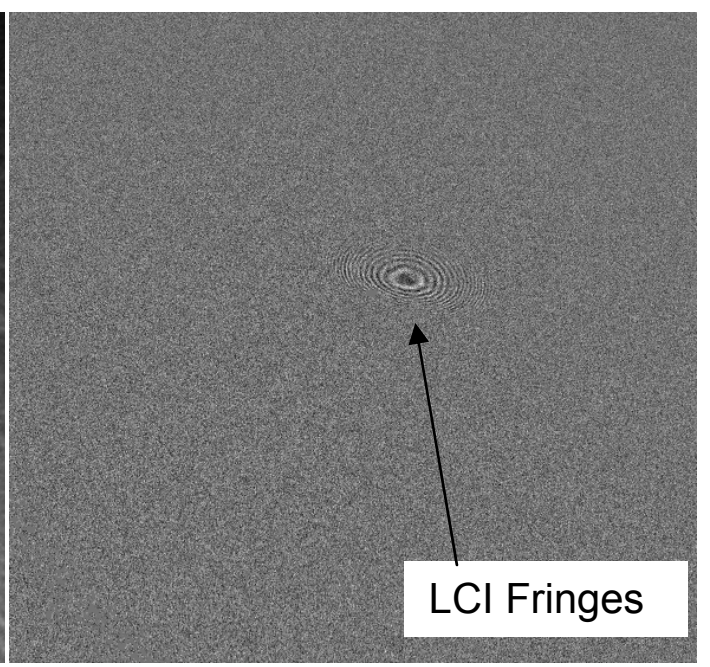

FIGURE 4.28 - Resulting a) raw image and b) image subtraction, showing LCI fringes, using a washer as a weight. 
Another attempt with an annular weight resulted in the image in Figure 4.29. The fringes are unfortunately not due to interference between the test and reference beams but are static fringes due to interference between two beams from the test arm of the interferometer: 1) reflection from the bottom surface of the sample/saline interface and 2) reflection from the interface between the saline and the top of the bottom glass plate (Figure 4.30). The sample is positioned closer then the coherence length to the glass plate, causing the static fringe pattern.

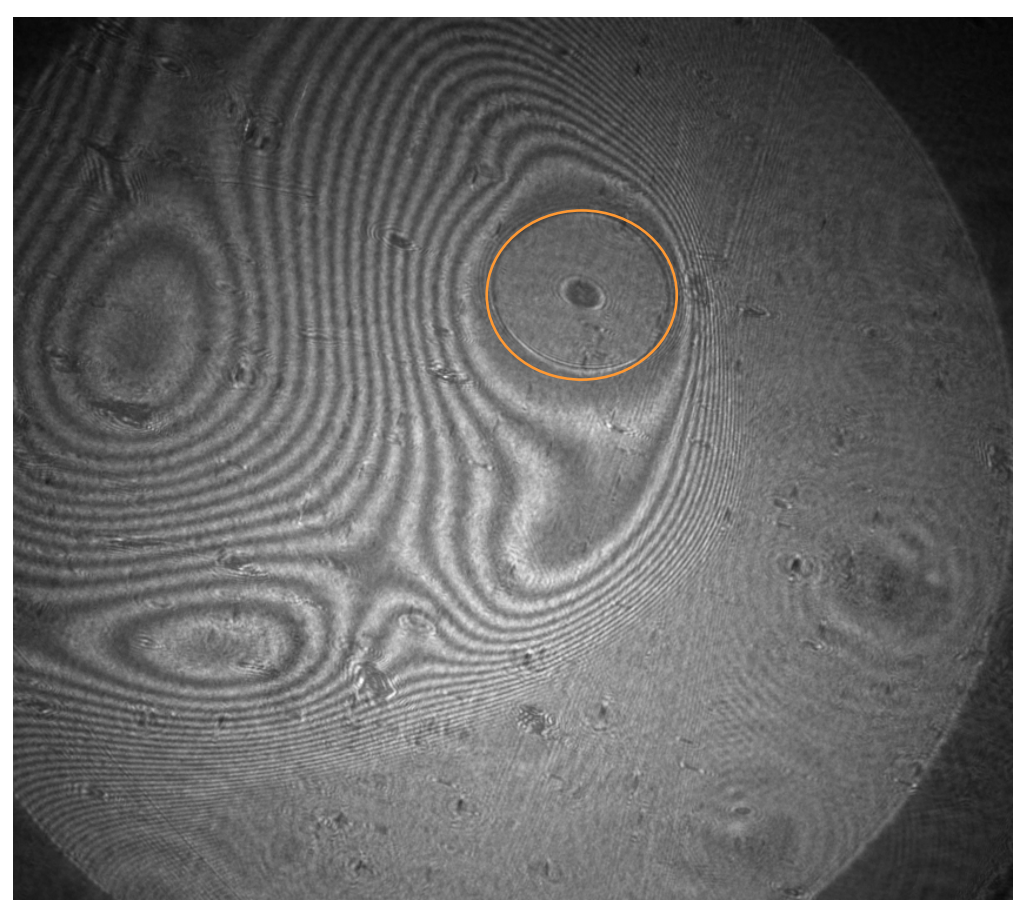

FIGURE 4.29 - Static fringe pattern from sample shown in Figure 4.30. Orange circle indicates the only region that is sticking to the glass plate. 


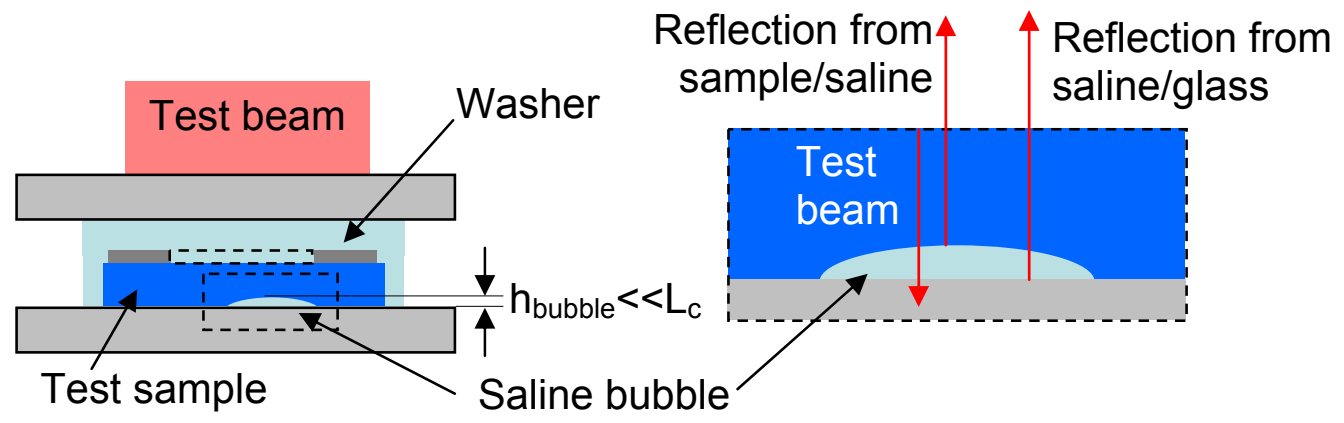

FIGURE 4.30 - For a small enough saline bubble beneath the sample, the reflections from the two interfaces are coherent, causing a static fringe pattern (does not vary as the reference surface is moved).

It became clear that mechanically forcing the sample to be in contact with the glass plate was difficult to achieve. Another option is to mount the sample some distance above the bottom plate, creating an extra interface to be measured along depth in the region containing the sample. The sample thickness could then be determined by subtracting the lengths of the two saline regions; one above the sample and one below. Several attempts were made to suspend the sample in the saline. One method involved gluing fishing line to the top and bottom plates to create a support structure for the sample (Figure 4.31).

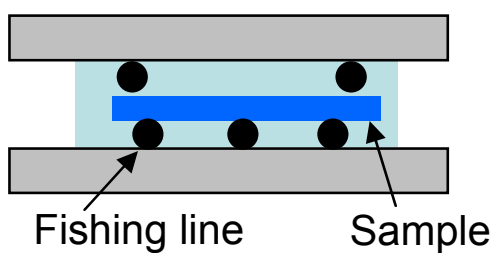

FIGURE 4.31 - Fishing line as a support structure for suspending the sample in saline.

This worked better then the washer methods, as larger regions were normal to the test beam, resulting in fringes (Figure 4.32). There remained several problems with this technique. 
First, it is very difficult to position the sample satisfactorily on the fishing line with any consistency. Second, the sample did not appear to be stable over the time required to take a measurement. One expected benefit of having the sample mounted to the glass plate is that it would be forced to stay stationary over the measurement time. Any movements will cause incorrect thickness measurements, which lead to incorrect refractive index values. Finally, the fishing line structure blocks light in the camera field of regard, reducing the number of valid measurement pixels. Many other mechanical techniques were attempted, but none of these worked any better then the techniques shown already.

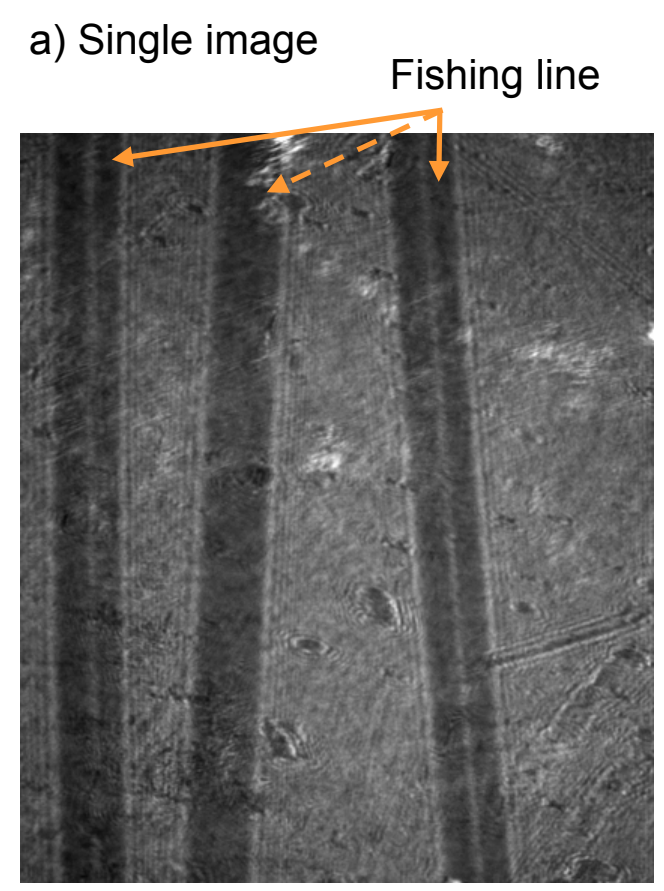

b) Image subtraction

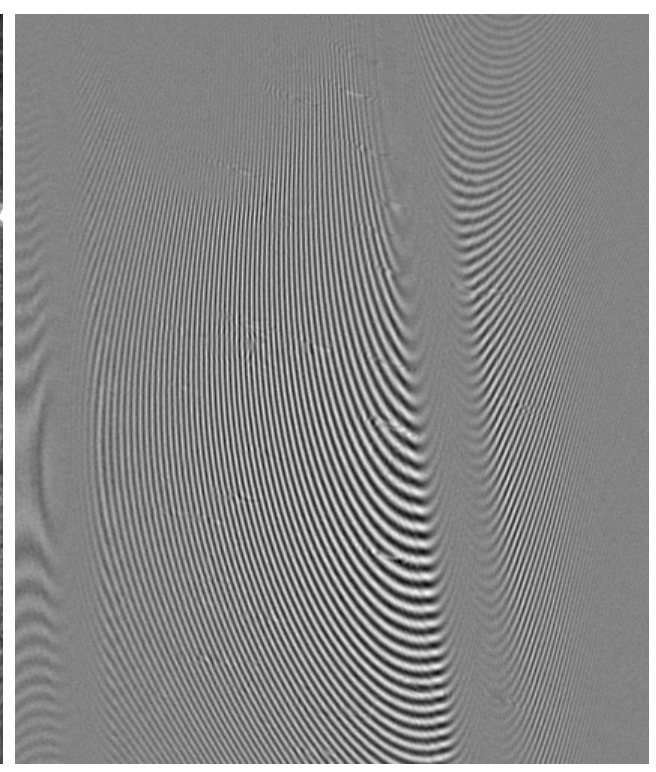

FIGURE 4.32 - Fishing line used to suspend the sample in the cuvette. In a), the dashed arrow indicates the fishing line on the bottom plate while b) shows the fringes obtained. 
The eventual solution to this problem is based in chemistry. Recall from Chapter 1 the definitions of hydrophilic and hydrophobic materials and the fact that the hydrogel materials become sticky when dried slightly. What if the bottom layer of the sample could somehow be kept slightly dry, and therefore sticky, while the sample remains submerged in solution? By changing the properties of the glass surface such that it becomes more hydrophobic, a thin layer of the sample would remain slightly drier and sticky, allowing the sample to be successfully mounted to the glass plate. This would allow Engage to measure the thickness of the contact lens materials.

Several chemical compounds exist for rendering glass hydrophobic, or water repellent. One use for these coatings is for a car windshield to repel rain, and one of the popular products for applying this coating is called Rain-X. This product is available at any hardware store. The Rain-X molecules adhere to the surface of the glass in an extremely thin layer. It is thin enough that it could not be measured using an interferometer. The glass surface becomes so hydrophobic that the contact angle of a water droplet approaches $90^{\circ}$. Surface 3 (Figure 4.26) of the current cuvette is coated with Rain-X so that the sample will adhere to the surface during testing. The bottom surface of the test sample is typically dabbed dry with a lint-free cloth to prevent small puddles of saline from being trapped beneath the sample, which would cause fringes similar to those in Figure 4.29. As long as there is no saline trapped beneath the sample, the altered surface chemistry of surface 3 keeps saline from migrating to that interface. The chemical repulsion of the saline affects a small layer of the test sample by keeping it dry. The 
thickness of this 'dried' layer is only the thickness of a few molecular layers of the polymer, so the change in index due to hydration changes is a negligible fraction of the bulk thickness of the material.

\subsection{Camera}

Both the test and reference beams are recombined at BS1 and sent to the imaging lens,

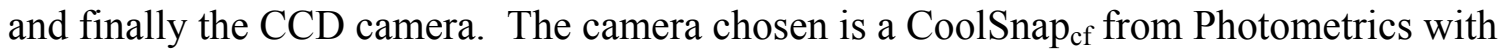
square pixels that are $4.65 \mu \mathrm{m}$ on a side. The CCD dimensions are $1392 \times 1040$ pixels, and the signals are digitized to $12 \mathrm{bits} /$ pixel $(0-4095)$. The exposure rate is continuously adjustable in one microsecond intervals between $1 \mu$ and several seconds with a linear full well of 9700 electrons. This high sensitivity camera was chosen because of the low light levels for the low coherence signals. A useful feature from a data reduction standpoint is that the region of interest on the camera is a continuously adjustable rectangle, so that only the desired region of the image is saved to disk. A full list of camera specifications for the CoolSnap $\mathrm{cf}$ is included in Appendix E.

Given a desired field of regard on the test plane of the cuvette of about $10 \mathrm{~mm}$ on a side and a small dimension of the detector of $4.8 \mathrm{~mm}(1040 * 4.65 \mu \mathrm{m})$, the magnification of the imaging lens should be about $\mathrm{m}=-0.5$. The lens chosen is a stock achromatic doublet from Newport with a focal length of $76.2 \mathrm{~mm}$ and a diameter of $38 \mathrm{~mm}$ (Clear Aperture $=$ $95 \%)$.

$$
f / \#=\frac{f}{D_{e p}}=\frac{76.2}{0.95 * 38.1}=2.1
$$


This focal length and magnification give the object distance $\mathrm{z}$ and the image distance $\mathrm{z}$ ':

$$
\begin{gathered}
m=-0.5=\frac{z^{\prime}}{z} \\
\frac{1}{z^{\prime}}=\frac{1}{z}+\frac{1}{f_{\text {lens }}} \\
z=-228.6 \mathrm{~mm} \quad z^{\prime}=114.3 \mathrm{~mm}
\end{gathered}
$$

The main reason a lens with a small f/\# was chosen was to limit the overall size of the interferometer. High fringe frequencies were not expected. Most of the expected fringes from the low coherence interferometer will be low frequency tilt fringes, with the exception of the saline/sample interface, which is highly unpredictable and varies spatially. This lens does not limit the fringe frequencies that can be recorded by the system.

The extra sensitivity gained by digitizing to 12 bits was a camera requirement so the low coherence signals could be recorded better. Given that the irradiance noise on the camera is quite large, it is a useful study to see if the full 12 bit digitization is critical to recovering the surfaces, or if the system could still function with 10 or even 8 bit camera. Fortunately, it is easy to edit data and remove the last 2 or 4 bits to test this theory. The potential gains from going to fewer bits are faster data collection and a less expensive camera. Another possible benefit from going to fewer bits per pixel is a reduction in disk space required to save the hundreds of images captured for a single measurement. 
The metric for comparing images with different bit depths must take into account the variation in fringe visibility for the different interfaces. The signal from glass/saline interfaces has a higher peak visibility then for glass/air (due to the higher background signal in the glass/air column), which is higher then a saline/sample interface. To be able to use less bit depth, all three surface types must be unaffected.

The first thing to consider is the amount of noise in the images, and in this case a good metric is to calculate the standard deviation of a frame by frame subtraction in a region of the scan without low coherence fringes. The noise for the example data set has a standard deviation of about 30 digital counts (on a 12-bit scale), which is between 4 and 5 bits worth of noise. The small spike at a difference of 0 in the histogram is due to a few saturated pixels that remained saturated for both frames (Figure 4.33).

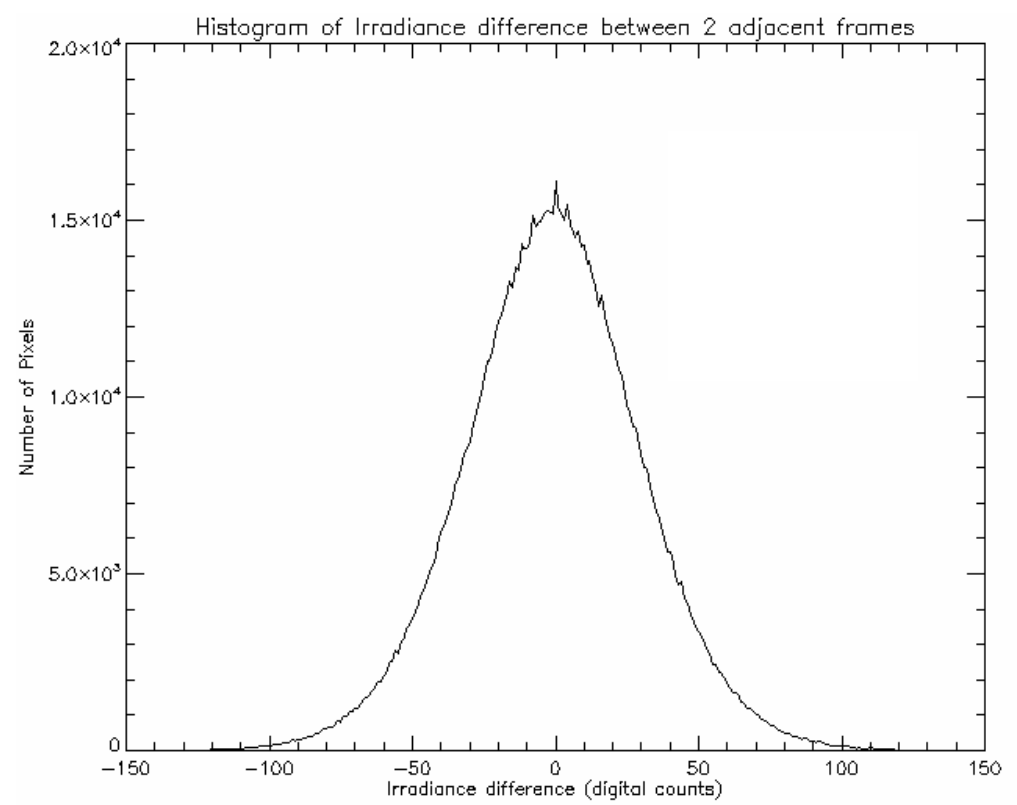

FIGURE 4.33 - Histogram of the difference in irradiance values between two frames during an LCI measurement ( $\sigma=30$ digital counts). 
Fringe visibility for the glass/saline is actually typically higher then visibility for glass/air, so it should be the least effected by reduced bit depth. The analysis to measure the effect was to process the same data for a glass/saline interface at three bit depths ( 8 , 10 and 12) and compare the standard deviations of the surface from a plane and the coefficients of that plane.

$$
h_{\text {plane }}(x, y)=a x+b y+c
$$

Equation 4.26

From Table 4.1, it is clear that the recovered surface is not affected by reducing the bit depth of the data. The average difference between the planes fit to the surfaces between the 8 and 12 bit surfaces is only 1 Angstrom. The difference in surface slope is at most 3 $\mathrm{pm} / \mathrm{pixel}$. There is no sacrifice going to 8-bit digitization for the glass/saline interface.

\begin{tabular}{|c|c|c|c|}
\hline & 8 -bit & 10-bit & 12 -bit \\
\hline Standard Deviation $(\mathrm{nm})$ & 36.9 & 36.4 & 36.3 \\
\hline $\mathrm{a}(\mathrm{nm} /$ pixel $)$ & -6.2555 & -6.2558 & -6.2558 \\
\hline $\mathrm{b}(\mathrm{nm} / \mathrm{pixel})$ & -6.1541 & -6.1535 & -6.1538 \\
\hline $\mathrm{c}(\mathrm{nm})$ & 264276.69 & 264276.69 & 264276.84 \\
\hline Average $\left(\mathrm{h}_{\left.\text {plane } 12-\mathrm{h}_{\text {plane8,10 }}\right), \mathrm{nm}}\right.$ & 0.10 & 0.02 & $\mathrm{~N} / \mathrm{A}$ \\
\hline
\end{tabular}

TABLE 4.1 - Summary of surface statistics for the same LCI data for a glass/saline interface processed at 8,10 and 12 bits. The differences in the surface coefficients $(a, b, c)$ are negligible.

The more difficult surface is the saline/sample interface, and the comparison of recovered surfaces become tougher since there is no ideal surface fit for these irregular interfaces. The comparison used was to examine a profile across the reconstructed surface and 
compare the recovered profiles for varying bit depth. The two plots in Figure 4.34 show the difference between the 12-bit profile and the 8-bit (left) and 10-bit (right).
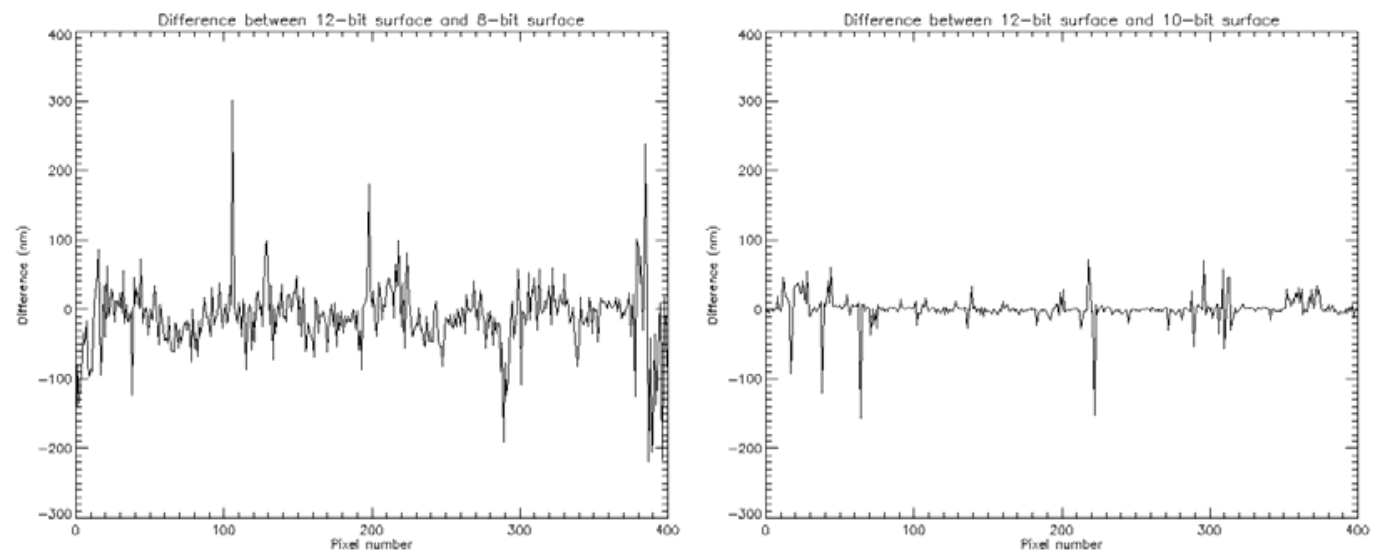

FIGURE 4.34 - Difference in recovered surface height between a) 8-bit and 12-bit, and b) 10-bit and 12-bit. The profiles are for a saline/sample interface (saline2).

The mean and standard deviation of the difference for the 8-bit profile are $-11.5 \mathrm{~nm}$ and $49 \mathrm{~nm}$, respectively. The mean and standard deviation of the 10-bit difference is better; $0.6 \mathrm{~nm}$ and $19 \mathrm{~nm}$, respectively. The surface degradation of the 10-bit data is probably small enough that 10-bit images would be sufficient while the 8-bit is too low. Since the camera used must be able to handle all the interfaces, it does not appear that an 8-bit camera would be sufficient for this system.

\subsection{Data Collection Procedures}

All of the hardware required to measure the physical thickness of the sample under test has been described in detail, which allows for a discussion of the data collection process. In order to measure the index of the sample, the physical thickness must be determined, which involves measuring the locations of 5 surfaces, denoted air1, air2, saline1, saline2 
and saline3. Each surface requires enough images to capture the full low coherence signal; the values in Table 4.2 can be changed using the Graphical User Interface (GUI) if necessary.

\begin{tabular}{|c|c|}
\hline Surface & Number of images \\
\hline Air1 & 140 \\
\hline Air2 & 140 \\
\hline Saline1 & 140 \\
\hline Saline2 & 280 \\
\hline Saline3 & 160 \\
\hline
\end{tabular}

TABLE 4.2 - Table of surface name and number of required images to capture the full LCI signal.

The data collected for a single interface is collected by recording the optical encoder position, capturing the image, writing the data to RAM so that it can be saved to a binary file after data collection is completed, initiating the move of the reference surface to the next image location, and allowing it to settle. The times for the image capture depends on the number of pixels in the region of interest selected by the user, but Figure 4.35 shows the typical time required for a single image in the data collection loop. All of the hardware control is done with a DLL (Dynamic Link Library) that is called by IDL. 


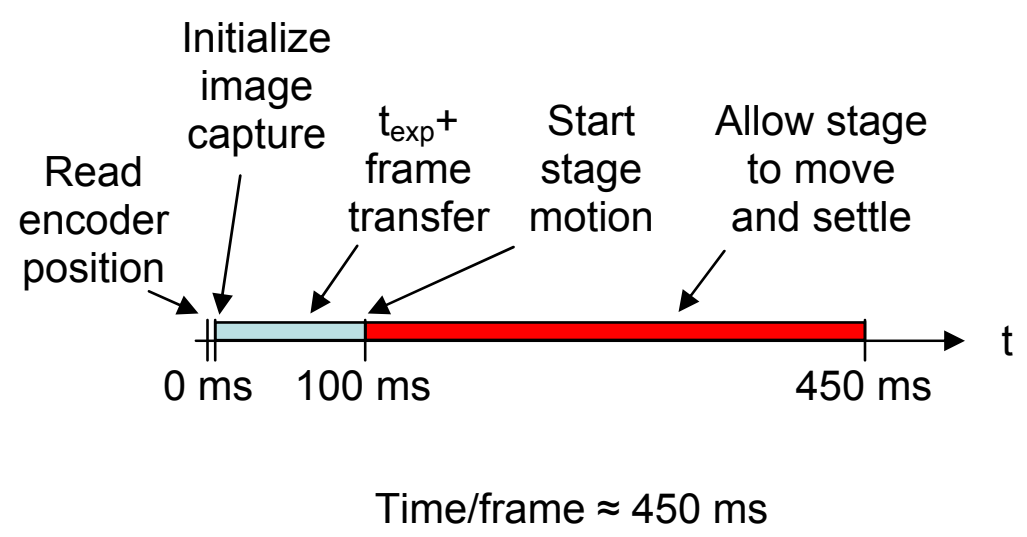

FIGURE 4.35 - Timing diagram for capturing a single image during an LCI scan.

For a 140 images, the data collection process takes about a minute. This time could be shortened by moving the stage at a constant speed and recording images at known encoder positions, as the typical exposure times are less then $10 \mathrm{~ms}$. The problem with this stage is that the encoder position is used in feedback loop to control the velocity of the stage to keep that velocity constant. As was shown earlier, the encoder has a cyclical error, which means a constant velocity indicated by the encoder is not actually a constant velocity of the reference mirror. For a constant exposure time, the fringe visibility degradation due to exposing while the mirror is moving will vary with position. Varying modulation degradation is a false modulation variation which will directly cause errors in the surface finding algorithm. This is an additional error that is avoided by taking images while the stage is stationary.

The drawback to taking the data slowly is that the surfaces have time to drift due to thermal expansion in the aluminum breadboard supporting the system. Thermal drift in 
the time between measurement of the top and bottom surfaces of an air column, for example, will result in a bias in the thickness measurement. The faster the data is collected, the smaller the effects of temperature.

It should be noted that frames are only captured in regions of known interference. Determining these locations for each surface is mostly automated, but requires some user interaction. To avoid thickness bias from thermal drift, the data for the 5 surfaces should be captured in rapid succession. Therefore, the locations for the beginning of each surface scan are determined before any data is collected. Once all 5 of these locations are known, the system automatically collects all the required data in about seven minutes.

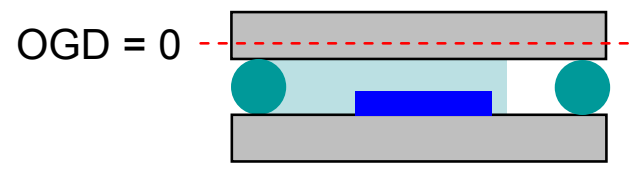

FIGURE 4.36 - The plane of OGD $=0$ should be here when the surface finding routine starts.

The regions containing the fringe information are found by scanning the reference mirror away from the beamsplitter such that the plane of zero OGD moves down through the cuvette. It is important that the search process starts with this zero OGD plane above the bottom surface of the top plate of the cuvette (Figure 4.36).

Sets of 10 images are gathered, spaced by $9 \mu \mathrm{m}$ along $\mathrm{z}$, and the differences between frames $\mathrm{N}$ and $\mathrm{N}-1$ are examined for fringes in software. The metric for fringe detection is to calculate the standard deviation of each frame subtraction and compare it to an 
interface-dependent threshold value. If the software finds an image greater then the threshold, the image is displayed to the user, who is prompted to decide if this image truly has fringes. Once the system has received a "Yes" answer, the starting scan position is calculated and stored in a structure for that particular surface. This is repeated for each surface (Figure 4.37). In addition, the exposure time and region of interest are different for "air" scans and "saline" scans. The difference in exposure time is necessary since the air region is much brighter then the regions with saline and sample. The exposure difference is also why there are two depth scans instead of only one.

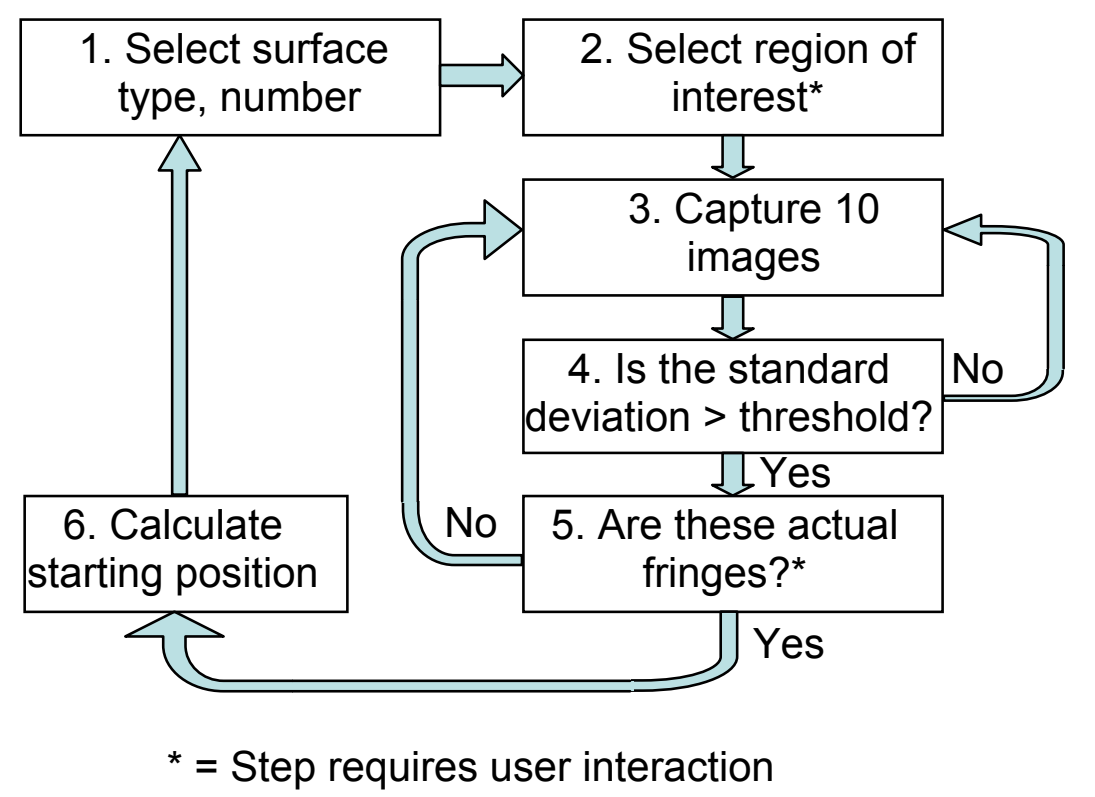

FIGURE 4.37 - Flowchart for finding the starting locations for the 5 LCI surfaces.

The computer used to control all of the hardware must be able to allocate enough RAM to store all of the images for a full LCI scan. The largest amount of data that must be stored in RAM at once during data collection is about 810 megabytes for a saline 2 interface: 
280 frames $\frac{1392 \times 1040 \text { pixels }}{1 \text { frame }} \frac{2 \text { bytes }}{\text { pixel }}=810.7 \mathrm{MB} \quad$ Equation 4.27

Each image is written as a binary file because of the speed at which this type of data can be written to disk by the DLL and the speed at which IDL can open data in this format. 


\section{OPL MEASUREMENTS}

The measurement procedure for determining the refractive index of the hydrogel materials is a two step process. The first step, determining the physical thickness of the sample, has been discussed. The second step is to determine the optical path length (OPL) of the sample in transmission. The index is then determined from Equation 5.1:

$$
n(\lambda)=\frac{O P L(\lambda)}{t}
$$

The OPL is measured as a function of wavelength in order to get a measurement of the dispersion of the test sample. A laser light source is used in a Mach-Zehnder interferometer configuration.

Figure 5.1 is a schematic of the beam paths for the test and reference beams of the MachZehnder interferometer. Many of the optics used are common to the two multiplexed interferometers, including the imaging lens, the camera and two of the beamsplitters (BS1, BS2). This was done to simplify the optical system as much as possible. The grey boxes indicate components used exclusively for the LCI interferometer. 


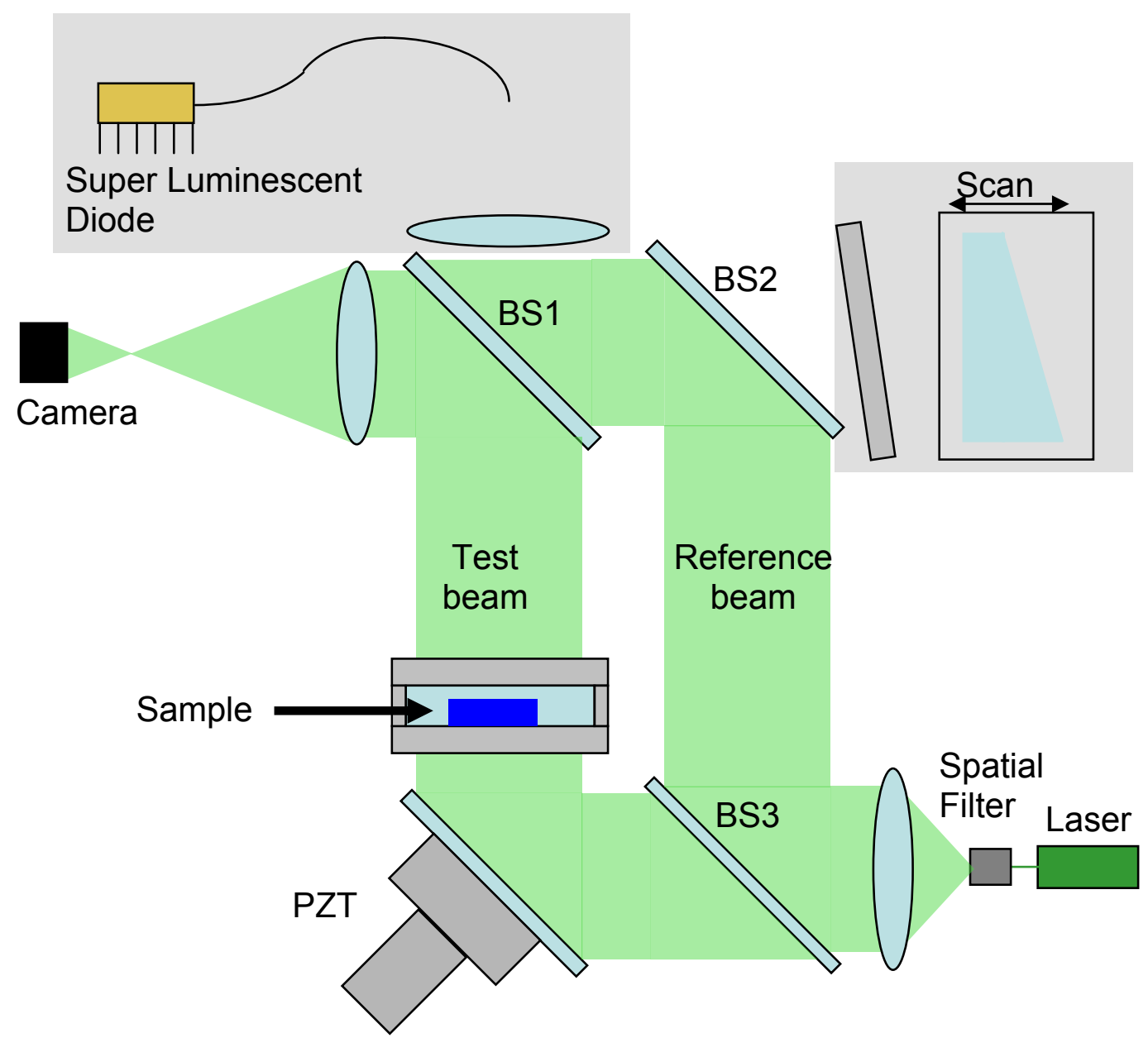

FIGURE 5.1 - Optical layout of Engage, illustrating the beam paths for the MachZehnder interferometer.

The OPL is not measured directly. Another equation for OPL relates to the optical phase in transmission, $0<\varphi<2 \pi$, and an integer number of wavelengths ( $2 \pi$ radians of phase), $\mathrm{m}$.

$$
O P L(\lambda)=\lambda\left(m+\frac{\varphi}{2 \pi}\right)=n(\lambda) t
$$

Here, $\lambda$ is the laser wavelength used in the Mach-Zehnder interferometer. Consider the measurement cuvette in Figure 5.2 that has a constant cavity thickness, t. Equations 5.3 and 5.4 compare the path length through this cuvette, where the path length in air is 
$\mathrm{OPL}_{\text {air }}$ and the path length in saline is $\mathrm{OPL}_{\text {saline. For now assume the two glass plates }}$ forming the cuvette are perfect plane parallel plates, so $t_{\text {plate }}$ is also constant (Figure 5.2).

$$
\begin{gathered}
O P L_{\text {air }}=\lambda\left(m_{\text {air }}+\frac{\varphi_{\text {air }}}{2 \pi}\right)=n_{\text {air }} t+2 \cdot n_{\text {plate }} t_{\text {plate }}=t+2 \cdot n_{\text {plate }} t_{\text {plate }} \\
O P L_{\text {saline }}=\lambda\left(m_{\text {saline }}+\frac{\varphi_{\text {saline }}}{2 \pi}\right)=n_{\text {saline }} t+2 \cdot n_{\text {plate }} t_{\text {plate }}
\end{gathered}
$$

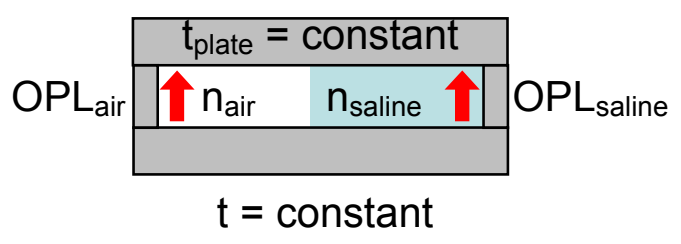

FIGURE 5.2 - Measuring OPL $\mathrm{O}_{\text {air }}$ and $\mathrm{OPL}_{\text {saline }}$ for a cuvette containing air and saline.

The difference between these two path lengths is due solely to the difference in index between air $\left(\mathrm{n}_{\text {air }}=1.0\right)$ and saline.

$$
\begin{array}{cc}
O P D=O P L_{\text {saline }}-O P L_{\text {air }} & \text { Equation } 5.5 \\
O P D=\left(n_{\text {saline }} t+2 \cdot n_{\text {plate }} t_{\text {plate }}\right)-\left(t+2 \cdot n_{\text {plate }} t_{\text {plate }}\right)=t\left(n_{\text {saline }}-1\right) & \text { Equation } 5.6 \\
O P D=\lambda\left(m_{\text {saline }}+\frac{\varphi_{\text {saline }}}{2 \pi}\right)-\lambda\left(m_{\text {air }}+\frac{\varphi_{\text {air }}}{2 \pi}\right)=\lambda\left[\left(m_{\text {saline }}-m_{\text {air }}\right)+\frac{\varphi_{\text {saline }}-\varphi_{\text {air }}}{2 \pi}\right]
\end{array}
$$

Equation 5.7

Setting Equation 5.6 equal to equation 5.7 results in Equation 5.8:

$$
\begin{gathered}
t\left(n_{\text {saline }}-1\right)=\lambda\left[\left(m_{\text {saline }}-m_{\text {air }}\right)+\frac{\varphi_{\text {saline }}-\varphi_{\text {air }}}{2 \pi}\right] \\
n_{\text {saline }}=\frac{\lambda}{t}\left[\left(m_{\text {saline }}-m_{\text {air }}\right)+\frac{\varphi_{\text {saline }}-\varphi_{\text {air }}}{2 \pi}\right]+1
\end{gathered}
$$


Equation 5.9 is the more useful form for calculating the refractive index from a path length measurement. A similar equation is determined for finding the index of the test sample. In the case of the test sample, the OPD is between the saline path length and the sample path length, and the thickness of the cuvette is replaced by the thickness of the sample, obtained through low coherence interferometry.

$$
n_{\text {sample }}=\frac{\lambda}{t_{\text {sample }}}\left[\left(m_{\text {sample }}-m_{\text {saline }}\right)+\frac{\varphi_{\text {sample }}-\varphi_{\text {saline }}}{2 \pi}\right]+n_{\text {saline }}
$$

Equation 5.10

The unknowns in Equation 5.9 and Equation 5.10 are the values for $m$ and for $\varphi$. The thickness values are determined from LCI and the wavelength of the source is known beforehand. It is clear that the index of saline must be found before the index of the material can be determined.

In practice, the thickness values and the phase values are functions of spatial position $(\mathrm{x}, \mathrm{y})$. Instead of assuming these values are constants, they are assumed to be planar. The justification for this assumption is discussed later in this chapter.

\subsection{Measuring the Phase in Transmission}

Of the two unknowns in the equation, the most straightforward value to determine is that of the phase, $\varphi$, which is found by phase shifting the test beam relative to the reference beam. A piezoelectric transducer (PZT) moves the mirror a distance equivalent to a $90^{\circ}$ 
phase shift, for a total of five images $\left(\mathrm{I}_{1}-\mathrm{I}_{5}\right)$ at these phase values: $\varphi, \varphi+90^{\circ}, \varphi+180^{\circ}$, $\varphi+270^{\circ}$, and $\varphi+360^{\circ}$. The distance moved per step is $d_{\mathrm{i}}$.

$$
d_{i}=\frac{\lambda}{8} \cos (\theta)
$$

Equation 5.11

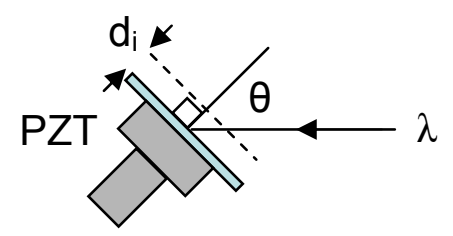

FIGURE 5.3 - The required mirror motion to achieve a $90^{\circ}$ phase shift is $d_{i}$.

In the case of the Mach-Zehnder interferometer, $\theta$ is $45^{\circ}$ (Figure 5.3). Once these five images are recorded, the phase, $\varphi(\mathrm{x}, \mathrm{y})$, at each pixel is calculated using the SchwiderHariharan phase shifting algorithm:

$$
\varphi(x, y)=\tan ^{-1}\left[\frac{2\left(I_{2}(x, y)-I_{4}(x, y)\right)}{2 I_{3}(x, y)-I_{5}(x, y)-I_{1}(x, y)}\right]
$$

Knowing the signs of the numerator and denominator allow the inverse tangent operation to give values for $\varphi$ between 0 and $2 \pi$ radians. An image containing fringes (left) and the phase as a result of Equation 5.12 (right) are shown in Figure 5.4. 


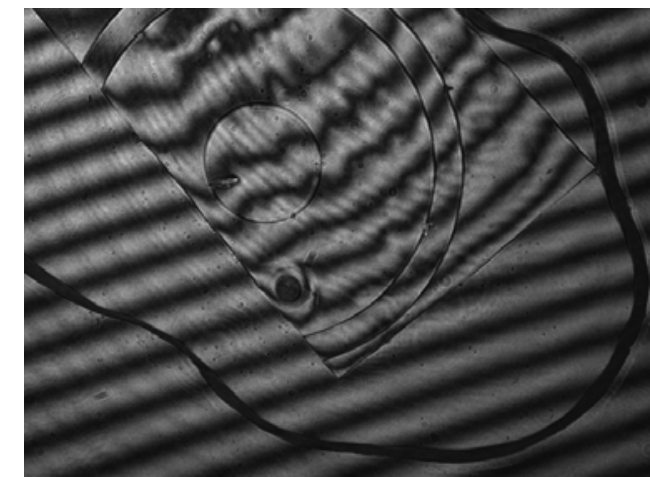

$\mathrm{I}_{1}, \varphi+0^{\circ}$

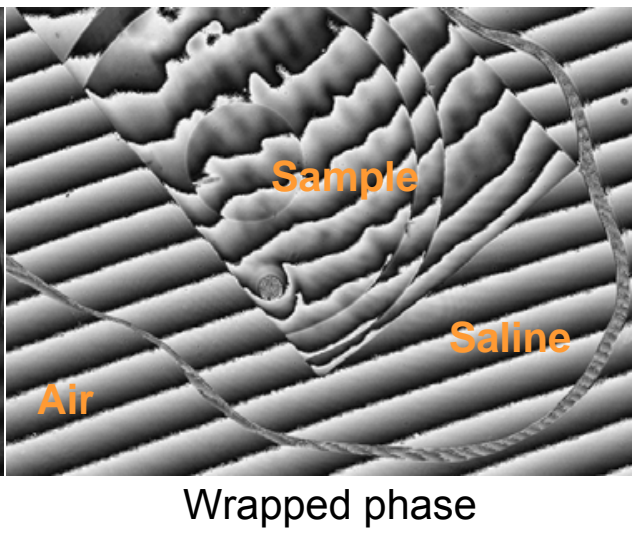

Wrapped phase

FIGURE 5.4 - Fringe image $\mathrm{I}_{1}$ (left) and the wrapped phase (right).

The phase map contains wrapped phase modulo $2 \pi$ that must be unwrapped. Recall that the field of view of the camera encompasses regions of air, saline and test sample, and that the phase across these boundaries is discontinuous. Since the phase can not be unwrapped across discontinuities, the unwrapping process must know the boundaries of a given region.

\subsubsection{Boundary Determination}

There are many methods for unwrapping the phase obtained from a phase shifting interferometer, but all have at least one thing in common, and that is that the region to be unwrapped must be continuous. Unwrapping across a step in phase, whether it is a discontinuous surface in reflection or a discrete boundary between materials of different index in transmission would result in an incorrect surface or OPD map. Therefore the boundaries of a continuous region must be defined before trying to unwrap the fringes. 
In some cases the definition of this boundary could be determined using features in the image, such as fringe modulation, but for the purposes of this interferometer, the regions are defined through user intervention. A function has been written in IDL which allows the user to create an $\mathrm{n}$-sided polygon to select a region of interest (ROI) containing, in the case of Figure 5.5, air. Note the red line drawn by the user in the lower portion of the left image of Figure 5.5. A mask file is saved for reference in determining the location of the air column for the particular data set.
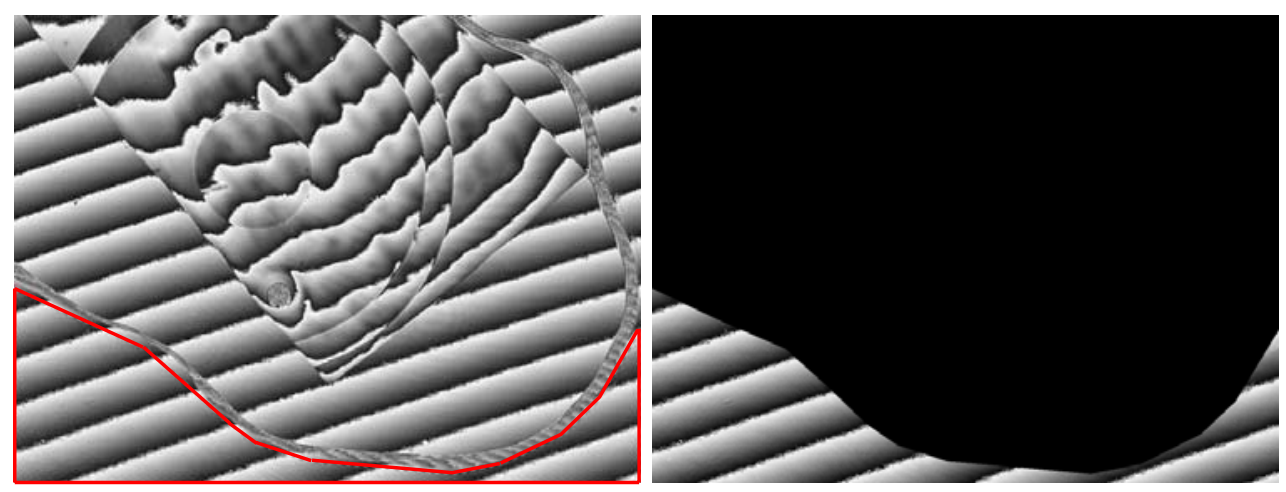

FIGURE 5.5 - User drawn polygon specifies the region of $\varphi(\mathrm{x}, \mathrm{y})$ containing air.

\subsubsection{Unwrapping the Phase}

Now that the ROI has been defined, the phase can be unwrapped. The most straightforward method for unwrapping phase, summarized in Equation 5.13, is a good place to start. One of the required assumptions is that the phase change between adjacent pixels will always be less then $\pi$ radians. This assumption follows from the Nyquist limit, requiring 2 pixels/fringe ( 1 fringe $=2 \pi$ radians $)$. 


$$
\begin{aligned}
& \text { If }\left[\varphi\left(x_{i}, y\right)-\varphi\left(x_{i-1}, y\right)\right]>\pi \text { then: } \varphi\left(x_{i}: x_{N}, y\right)=\varphi\left(x_{i}: x_{N}, y\right)-2 \pi \\
& \text { If }\left[\varphi\left(x_{i}, y\right)-\varphi\left(x_{i-1}, y\right)\right]<-\pi \text { then: } \varphi\left(x_{i}: x_{N}, y\right)=\varphi\left(x_{i}: x_{N}, y\right)+2 \pi
\end{aligned}
$$

Equation 5.13

An example profile in Figure 5.6a shows jumps of approximately $2 \pi$ radians in phase, which is greater then the maximum difference of $\pi$. Basic unwrapping adds or subtracts $2 \pi$ from the pixel violating this condition through the end of that line, depending on the sign of the difference. The result of the unwrapping process for this profile is also shown (Figure 5.6b).
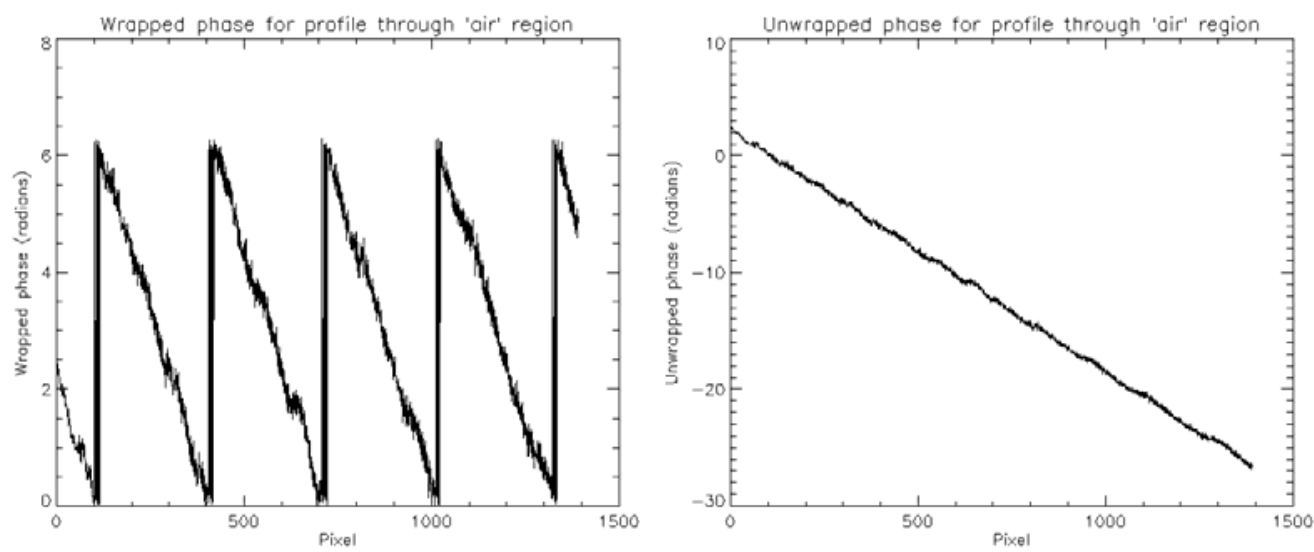

FIGURE 5.6 - Profile of a) wrapped phase and b) unwrapped phase.

This process is the repeated for every x-profile, resulting in a fully unwrapped surface. If the data had no noise and was defined by a rectangle, this is all that needs to be done.

Due to the nature of this measurement process, the ROI is rarely a rectangle. The only requirement is that all of the pixels in the ROI be connected, so that the region is continuous. Regions can be shaped like a donut, or they can have regions missing in the middle of valid data, or any other shape which is not known beforehand. Therefore the 
unwrapping code must be flexible enough to handle arbitrarily shaped regions of phase data. White regions represent valid data in the examples shown in Figure 5.7a-f.
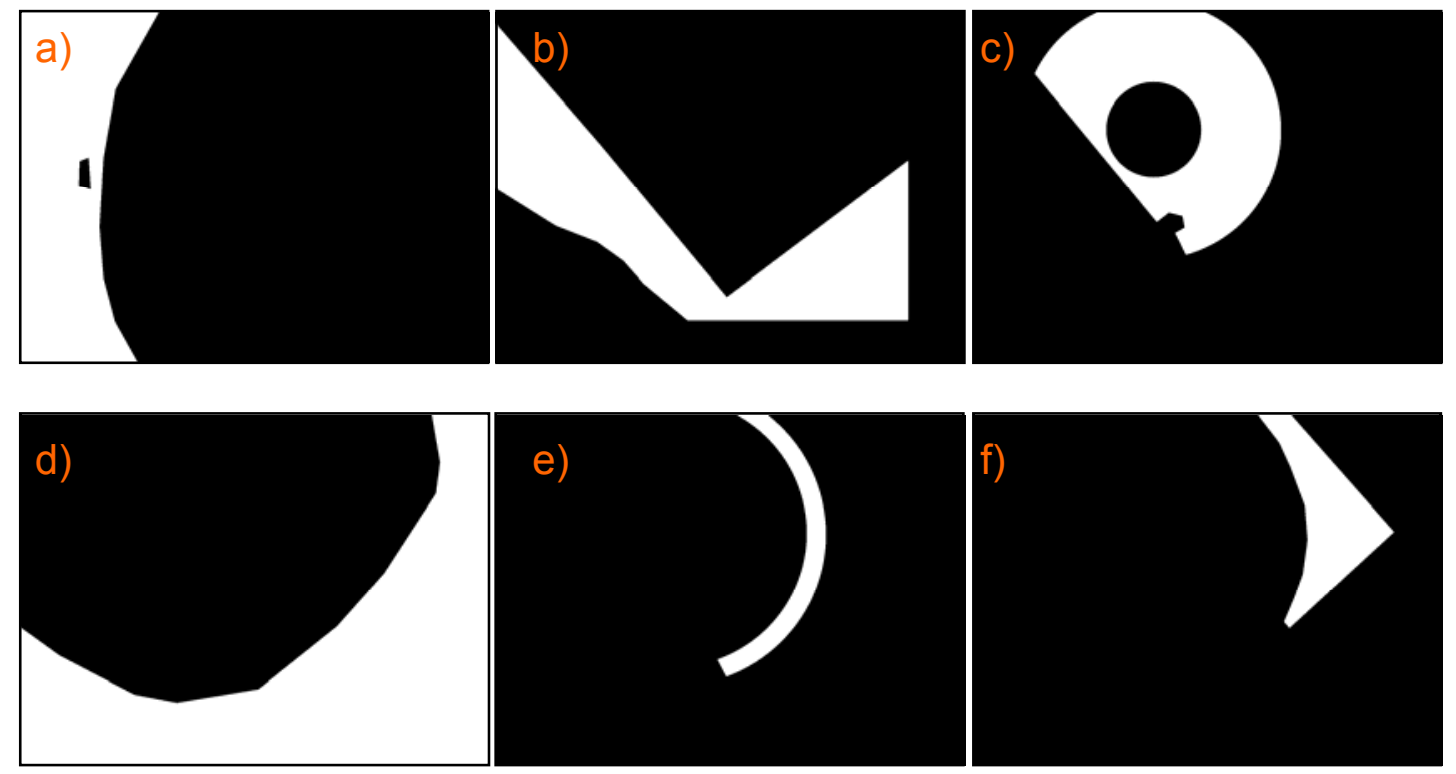

FIGURE 5.7 - Typical user defined ROI's based on sample geometry.

This flexibility is accomplished by partitioning the ROI into subsections that follow certain restrictions. Once these subsections are defined, each one is unwrapped separately. The resulting unwrapped subsections must then be put back together so that there are no $2 \pi$ ambiguities in the final unwrapped image.

The restrictions on regions are related to the unwrapping method, and are actually defined as the phase is unwrapped, not beforehand. Once a starting pixel has been defined (white ' $x$ ' in Figure 5.8), the phase is unwrapped along continuous data in the $+/-y$ direction until a boundary has been reached. A starting point for each y-profile (red line) in a valid region (gray area) is recalculated for each y-profile. Each y-profile is compared to the 
previously unwrapped line to see if the entire line needs to have any $2 \pi$ multiples added to it. This continues along $+\mathrm{x}$ and $-\mathrm{x}$ until the edges of the $\mathrm{ROI}$ are reached.

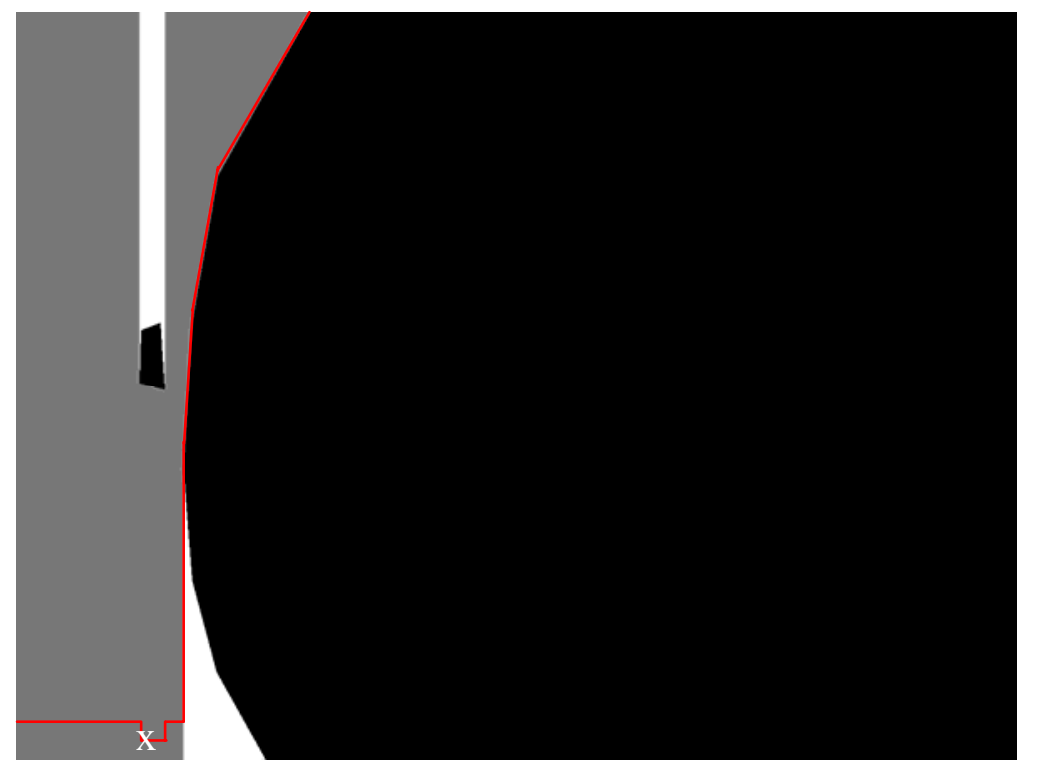

FIGURE 5.8 - Gray region is the first subsection to be unwrapped. Black regions are invalid data. White regions are areas yet to be unwrapped. White ' $\mathrm{x}$ ' is the overall starting point and each point on the red line is the starting point for that y-profile.

The gray region represents what was unwrapped by this first pass. This algorithm is unable to 'turn corners' in the ROI, and so some regions (white) were not unwrapped. These regions become additional subsections, which are found and unwrapped using the same procedure. For Figure 5.8, three subsections were unwrapped (Figure 5.9). 


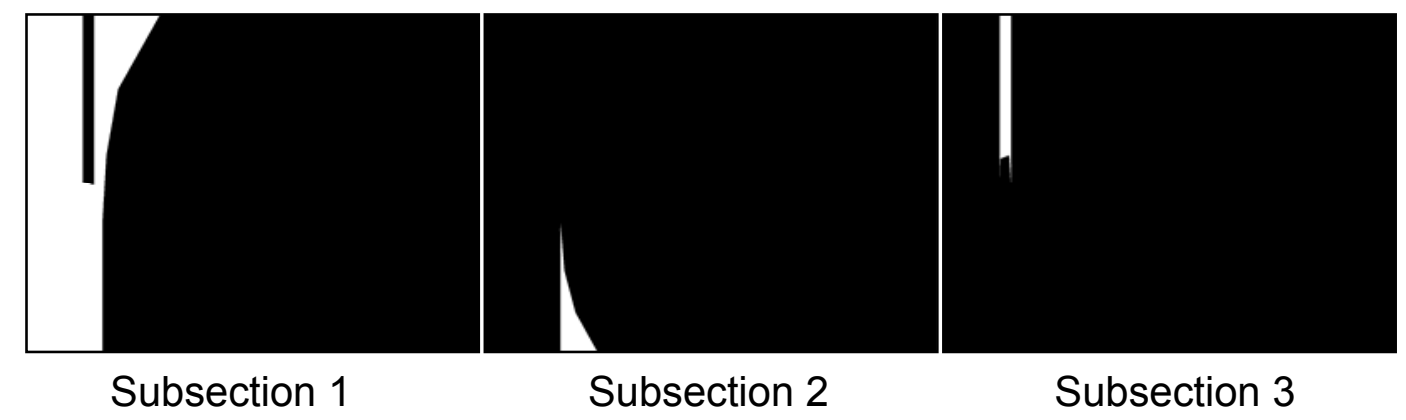

FIGURE 5.9 - Three subsections were required to unwrap the region shown in Figure 5.8.

Once all such regions have been unwrapped, the common edge points are determined and checked to see if there are phase discontinuities at the boundaries. In this example, the three regions do not line up with each other, so some of the subsections must be changed by multiples of $2 \pi$. Subsection 2 is too high (light) and subsection 3 is too low (dark) (Figure 5.10).
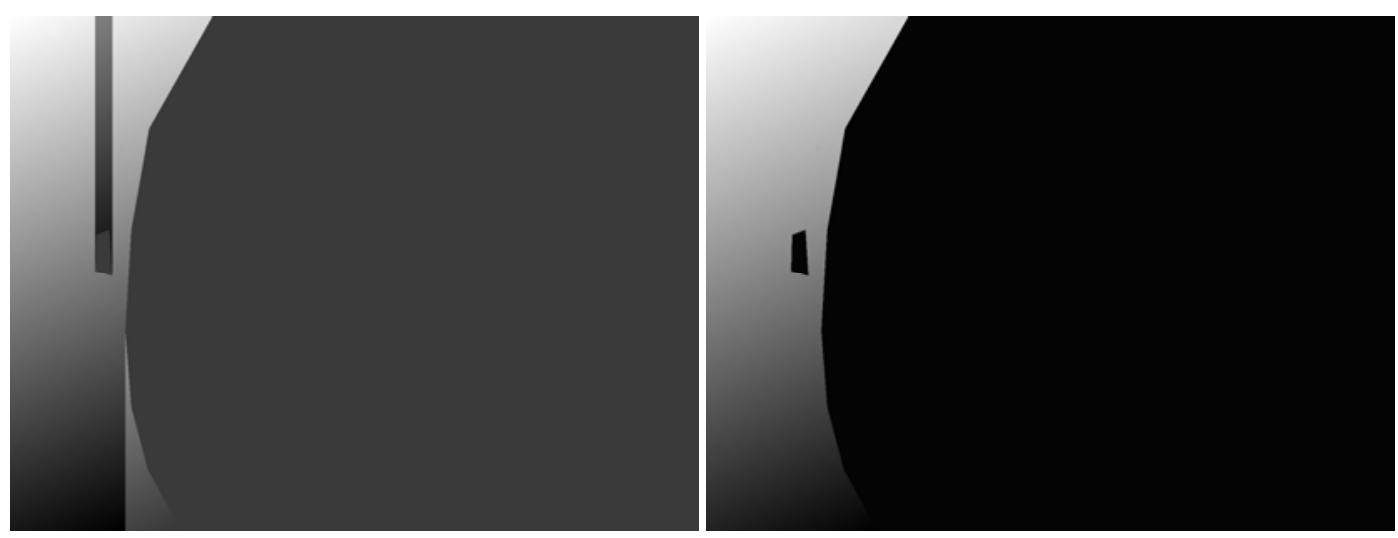

FIGURE 5.10 - The image on the left shows discontinuities between unwrapped subsections. The image on the right shows result of correcting the regions.

Another concern for unwrapping algorithms is the effect of noisy or bad regions. The most common artifact of a bad region is streaking in the image, or lines that have been unwrapped incorrectly. Ideally, the unwrapping algorithm would be able to detect these 
regions, mask them off, and continue to unwrap the phase map. Code has been written to reduce the propagation of errors from a bad region, but not to remove them. If a bad region causes problems during unwrapping, the user is prompted to remove these bad regions manually. A typical example of a bad region can be seen in Figure 5.11a, where saline droplets on the plates caused phase discontinuities. The image on the left shows the streaks as a result of the phase discontinuities. The majority of these streaks are corrected automatically by a correction algorithm, as shown in Figure 5.11b.

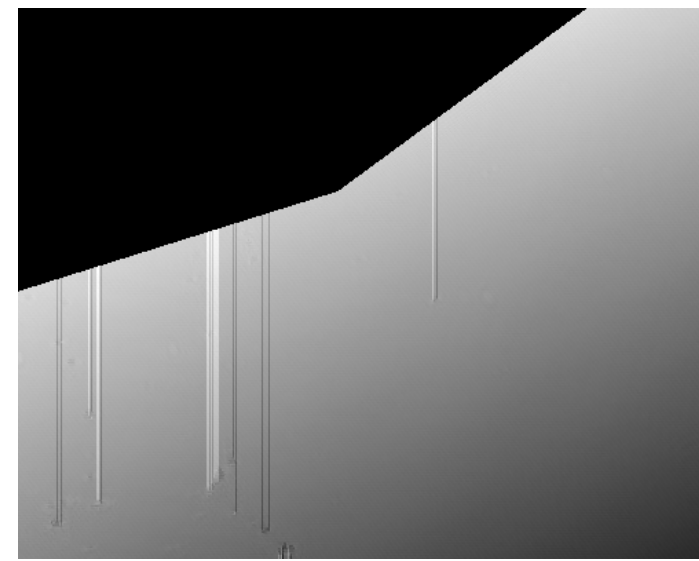

a) Raw unwrapped

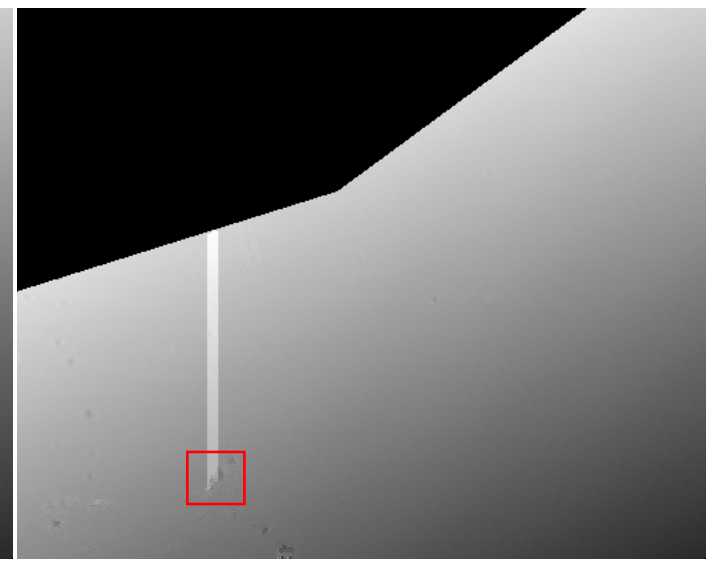

b) Automatically corrected results

FIGURE 5.11 - Image with a) raw unwrapped data and b) after most the errors have been removed automatically in software. The red box in b) indicates a suitable region for the user to remove for error-free unwrapping.

One region was not fully corrected, and at this point the user would be prompted to manually remove this region. The red box illustrates a reasonable region to remove to fix this unwrapping artifact. After the user selects out the red region, the final unwrapped product is shown in Figure 5.12. 


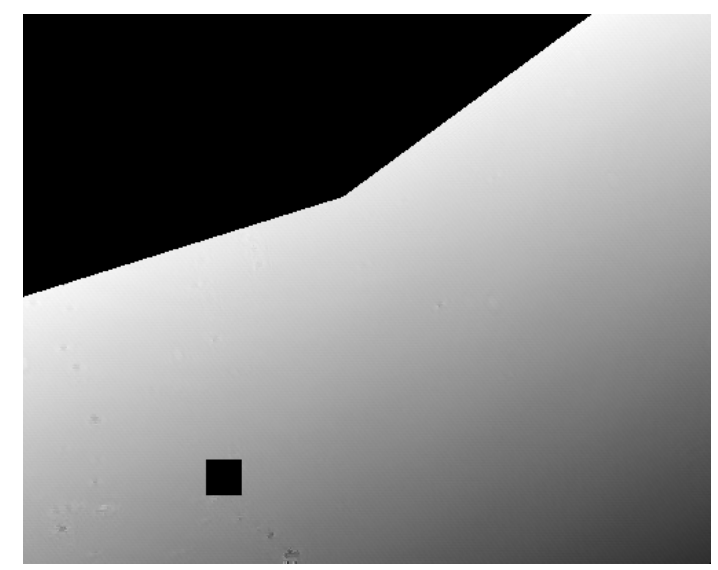

FIGURE 5.12 - Final unwrapped phase after the small region outlined by the red box in Figure 5.11 has been removed by the user.

For the relatively low volume of testing done with the preliminary version of the Engage system and the great variability from data set to data set in terms of the ROI's and the various noise sources, it was more efficient to occasionally require user interaction then it was to spend the time trying to make the unwrapping code flawless. Robust phase unwrapping algorithms do exist and are capable of handling this type of data (Ghiglia, 1998).

\subsubsection{Fitting a Plane to the Phase}

The phase for air is known over some portion of the camera, and from the tilt fringes it is clear that there is some wedge to the phase of the air column. The only elements of the interferometer that are not common path are plates with flat surfaces. There are several sources of tilt, such as the glass plates and the gap formed by those plates. Ideally, the phase only contains a wedge component. A plane can be fit to the unwrapped air phase, using the region shown in Figure 5.13. 


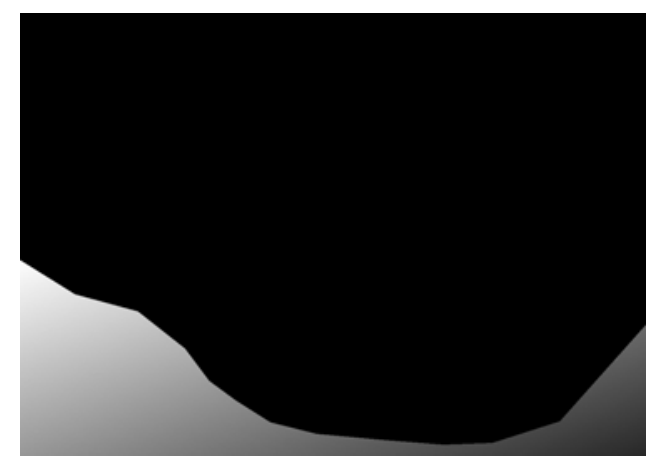

Unwrapped phase, $\varphi_{\text {air }}$

FIGURE 5.13 - A plane is fit to the phase data in the ROI containing air.

This plane, defined by the three coefficients in Equation 5.14, is extrapolated across the region containing saline. The phase with the saline is measured in these same images (Figure 5.4). Now both $\varphi_{\text {saline }}(\mathrm{x}, \mathrm{y})$ and $\varphi_{\text {air,fit }}(\mathrm{x}, \mathrm{y})$ have been determined. There are separate phase values for every laser wavelength.

$$
\varphi_{\text {air }, f i t}(x, y)=a x+b y+c
$$

The phase extrapolation process is repeated to determine the phase of the saline in the region containing the test material. This is allowable because the index of the saline is uniform, so the measured phase will also be a plane. A fit to this plane yields $\varphi_{\text {saline }}(\mathrm{x}, \mathrm{y})$ over the entire field of view.

\subsection{Interferometer Background Error}

Another consideration for determining $\varphi$ for the various columns of material in the cuvette is the deviations of the phase surfaces in the interferometer from ideal planes. Consider the optics that affect the laser beam in the Mach-Zehnder interferometer. Any common path optics prior to the beamsplitter, such as the collimating lens, will not cause 
a difference between the test and reference wavefronts. The reference wavefront, which reflects off the top surface of the beamsplitter 3, transmits twice through beamsplitter 2 (reflects off the top surface) and once through beamsplitter 1, ideally should only contain tilt due to wedge and tilt of the surfaces it interacts with. The test wavefront transmits through beamsplitter 3, reflects off the mirror in the PZT, and transmits twice through beamsplitter 1 (reflects off the top surface) in the absence of the sample cuvette (Figure 5.14). Again, these surfaces should only introduce tilt. The system is not ideal, and the resulting phase (OPD) of the difference between the reference and test wavefronts is not quite flat.

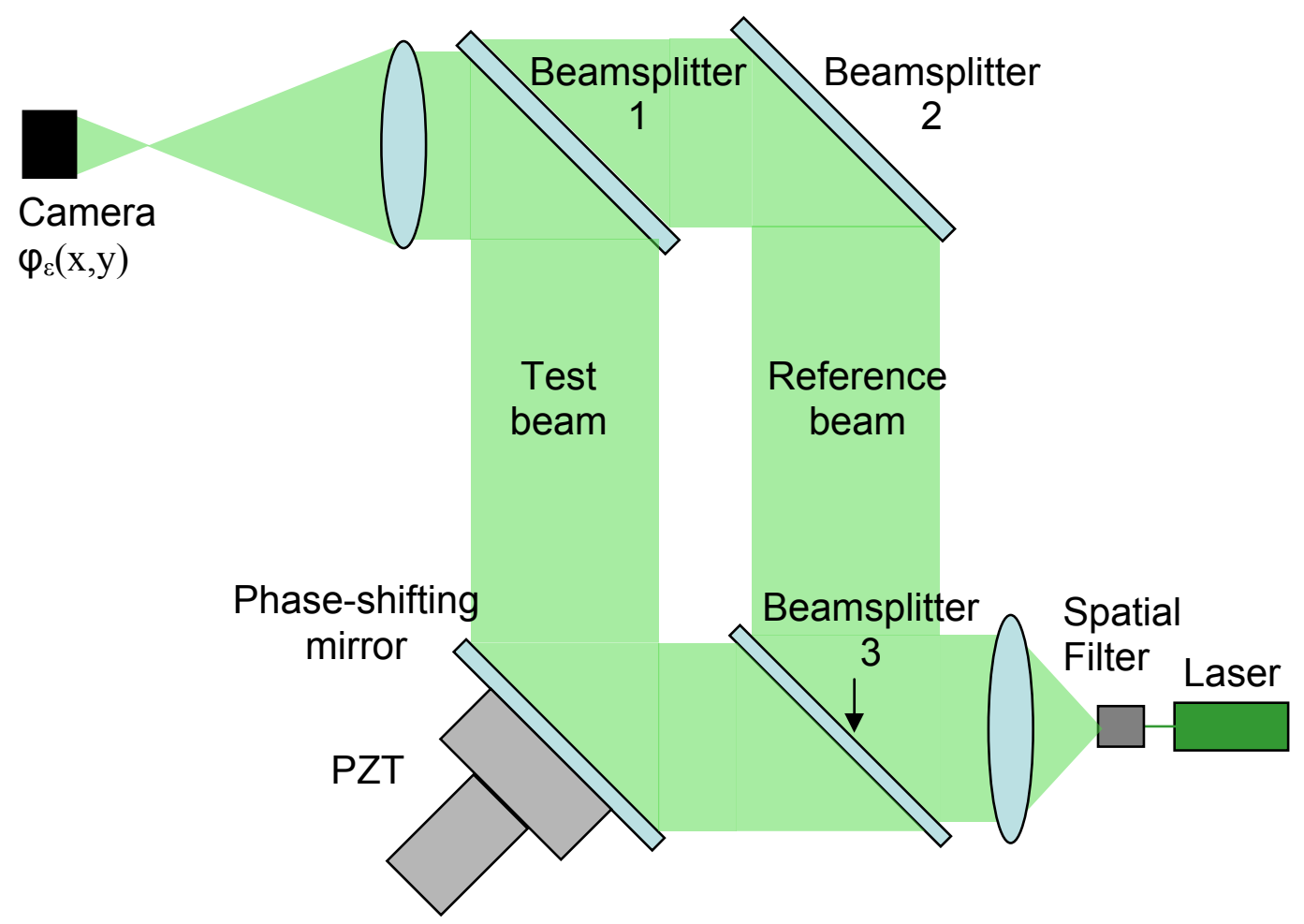

FIGURE 5.14 - Schematic of Mach-Zehnder interferometer without a cuvette. Ideally, the resulting interferogram should only contain tilt. 
The Mach-Zehnder interferometer configuration has the useful feature that a wavefront can be measured in the absence of a test part (i.e, the cuvette). A series of 5 phase shifted images are captured for such a purpose. The phase is calculated from 5 frames and unwrapped, as discussed earlier. This phase will be designated as $\varphi_{\varepsilon}(\mathrm{x}, \mathrm{y})$. After removing the best fit plane, a $5^{\text {th }}$ order polynomial surface is fit to $\varphi_{\varepsilon}(\mathrm{x}, \mathrm{y})$ (Figure 5.15).

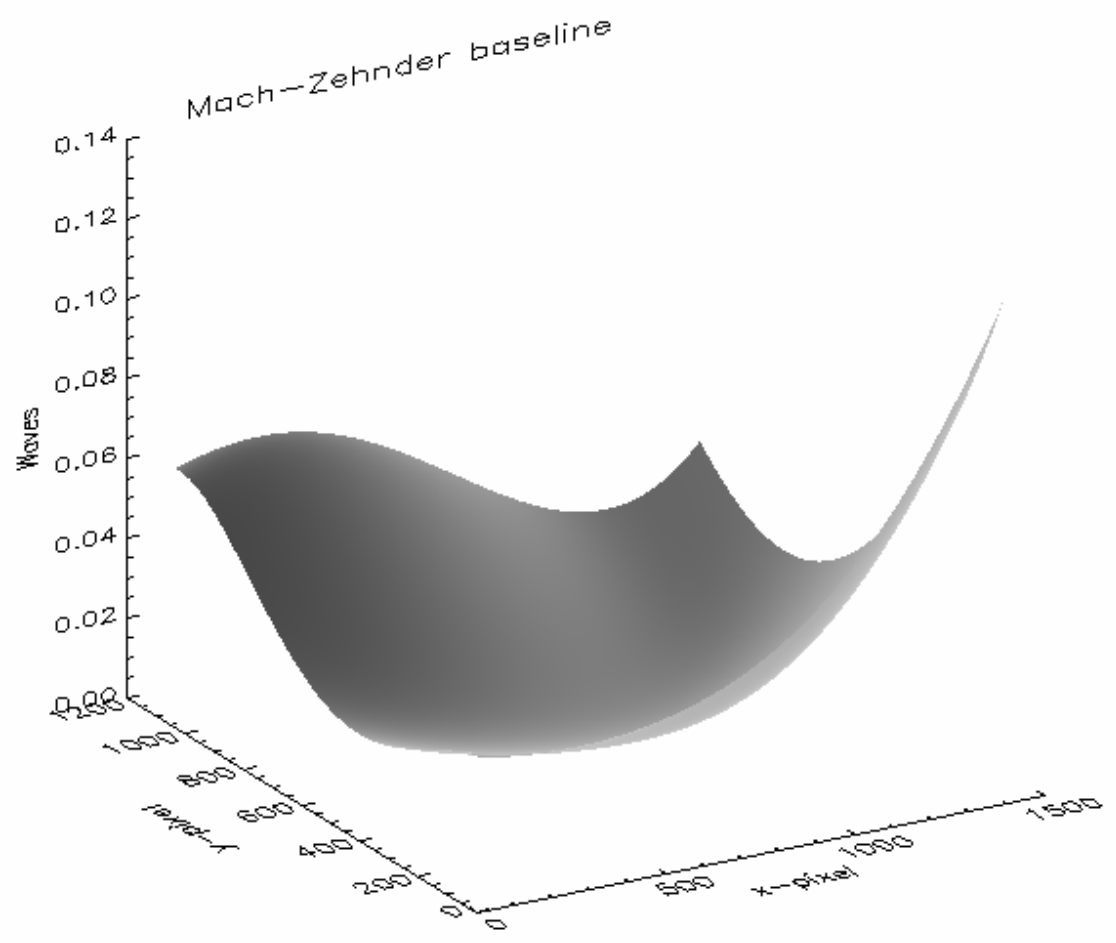

FIGURE $5.15-\mathrm{A} 5^{\text {th }}$ order polynomial fit to $\varphi_{\varepsilon}(\mathrm{x}, \mathrm{y})$, the Mach-Zehnder interferometer baseline error.

This is the Mach-Zehnder interferometer baseline surface, $\varphi_{\varepsilon, \text { fit }}(\mathrm{x}, \mathrm{y})$, and it represents the difference between the test and reference wavefronts due to imperfections in beamsplitters 1,2, and 3 and the phase shifting mirror. This error function is wavelength 
dependent. The phase error due to surface errors for reflected and transmitted beams are shown in Equations 5.15 and 5.16.

$$
\begin{gathered}
\Delta \varphi_{\operatorname{Re} \text { flection }}=\frac{2 \pi}{\lambda} \frac{2 h}{\cos (\theta)} \\
\Delta \varphi_{\text {Transmission }}=\frac{2 \pi}{\lambda}\left(\frac{n h}{\cos \left(\sin ^{-1}\left(\frac{1}{n} \sin \theta\right)\right)}-h \cos (\theta)\right)
\end{gathered}
$$

Here, $\theta$ is the angle of incidence of the beam on the surface. For plates $(\mathrm{n} \approx 1.5)$ or surfaces tilted at $45^{\circ}$, surface errors (h) on reflection cause about 3 times the wavefront distortion caused by that same surface error in transmission. The range of wavefront distortion in Figure 5.15 is about $0.11 \lambda$. Another view of the background error the MachZehnder interferometer is in Figure 5.16, where each fringe represents $1 / 50^{\text {th }}$ of a wave of OPD.

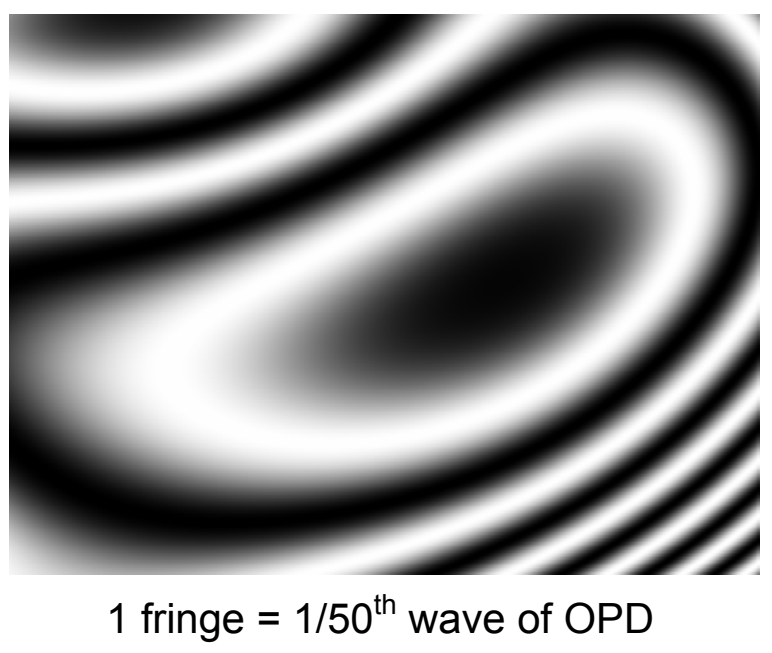

FIGURE 5.16 - Each fringe represents $1 / 50^{\text {th }}$ of a fringe in the collected images. 
This error is only important because a plane is fit to the unwrapped data in the air and saline regions. If this interferometer background is ignored, it can contribute to an incorrectly extrapolated phase for the full detector due to the variation of the slope of the OPD across the detector. The maximum peak-to-valley slope variation is about 0.28 $\mathrm{nm} /$ pixel. The worst case error caused by this background error over 1000 pixels is then $280 \mathrm{~nm}$. Uncompensated, a reasonable median magnitude for this error is roughly $50 \mathrm{~nm}$. In order to fix this problem, the wavelength dependent interferometer baseline is subtracted from the unwrapped phase before a best fit plane is calculated.

$$
\begin{array}{cc}
\text { FitPlane }\left\{\varphi_{\text {air }}(x, y)-\varphi_{\varepsilon}(x, y)\right\}=\varphi_{\text {air }, \text { fit }}(x, y) & \text { Equation 5.17 } \\
\text { FitPlane }\left\{\varphi_{\text {saline }}(x, y)-\varphi_{\varepsilon}(x, y)\right\}=\varphi_{\text {saline }, \text { fit }}(x, y) & \text { Equation 5.18 }
\end{array}
$$

For the calculation of refractive index, $\varphi_{\varepsilon}(\mathrm{x}, \mathrm{y})$ must also be subtracted from the unwrapped measured phases $\left(\varphi_{\text {saline }}\right.$ and $\left.\varphi_{\text {sample }}\right)$ so that the interferometer background error is accounted for in both phase arrays.

$$
\begin{array}{r}
n_{\text {saline }}=\frac{\lambda}{t(x, y)}\left[\left(m_{\text {saline }}-m_{\text {air }}\right)+\frac{\left[\varphi_{\text {saline }}(x, y)-\varphi_{\varepsilon}(x, y)\right]-\varphi_{\text {air }, \text { fit }}(x, y)}{2 \pi}\right]+1 \\
\quad \text { Equation } 5.19 \\
n_{\text {sample }}=\frac{\lambda}{t_{\text {sample }}(x, y)}\left[\left(m_{\text {sample }}-m_{\text {saline }}\right)+\frac{\left[\varphi_{\text {sample }}(x, y)-\varphi_{\varepsilon}(x, y)\right]-\varphi_{\text {saline }, \text { fit }}(x, y)}{2 \pi}\right]+n_{\text {saline }}
\end{array}
$$

Equation 5.20 


\subsection{Determining Correct Value for ' $m$ '}

The remaining unknowns in Equation 5.19 are the two values for m, both of which are integers. The subtraction can be replaced by a single $\Delta \mathrm{m}$ value, since the difference is all that is important. The problem is that for a single wavelength, the remaining single equation for determining refractive index has two unknowns: $\mathrm{n}_{\text {saline }}$ and $\Delta \mathrm{m}$.

$$
\begin{gathered}
n_{\text {saline }}=\frac{\lambda}{t_{\text {saline }}}\left[\Delta m+\frac{\varphi_{\text {saline }}-\varphi_{\text {air }}}{2 \pi}\right]+1 \\
n_{\text {sample }}=\frac{\lambda}{t_{\text {sample }}}\left[\Delta m+\frac{\varphi_{\text {sample }}-\varphi_{\text {saline }}}{2 \pi}\right]+n_{\text {saline }}
\end{gathered}
$$

Additional information is required to determine the correct values for $\Delta \mathrm{m}$. This problem is best approached in index space, and will be addressed in the next chapter.

\subsection{Hardware for the Mach-Zehnder Interferometer}

The phase data gathered using the Mach-Zehnder interferometer in Engage is done using phase shifting interferometry (PSI). As stated earlier, PSI requires a series of interferograms to be captured as the relative phase between the test and reference beams is changed in $90^{\circ}$ steps. This phase step is most common because the trigonometric functions in the phase shifting algorithms can be easily manipulated when $\delta=90^{\circ}$. 


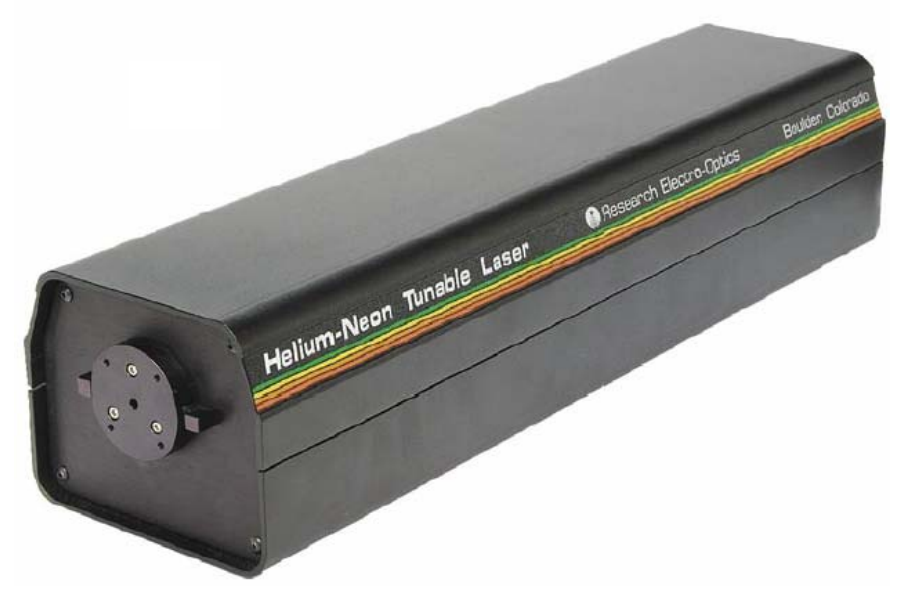

FIGURE 5.17 - Picture of the tunable HeNe laser used in Engage (REO website).

The first component of this interferometer is the light source, which is a 5-color tunable Helium-Neon gas laser from Research Electro-Optics, Inc (Figure 5.17). Table 5.1 lists the 5 wavelengths in air and their approximate optical power.

\begin{tabular}{|c|c|}
\hline Laser Wavelength $(\mathrm{nm})$ & Optical Power $(\mathrm{mW})$ \\
\hline 543.36 & 0.3 \\
\hline 593.93 & 0.6 \\
\hline 604.61 & 0.5 \\
\hline 611.80 & 2.5 \\
\hline 632.82 & 4.0 \\
\hline
\end{tabular}

TABLE 5.1 - Laser wavelengths and their optical powers for the tunable laser.

All 5 wavelengths operate in the TEM $_{00}$ mode. This wavelength range (543-632 nm) does not cover the entire visible spectrum $(400-700 \mathrm{~nm})$. This laser is relatively inexpensive and has been quite sufficient for proving the feasibility of the overall measurement concept. If it is found that more spectral coverage is required, additional laser wavelengths can easily be added to the system. 
The wavelength is selected by rotating a Littrow prism that is external to the plasma tube but within the laser feedback cavity; in fact, the back surface of the prism is the reflecting surface for the back of the cavity. This prism is rotated using a small micrometer on the back panel of the laser. A Littrow prism is nominally a $30^{\circ}-60^{\circ}-90^{\circ}$ prism, with the side opposite the $60^{\circ}$ angle coated with a highly reflective surface (Melles-Griot, 2007). The idea is that the angle of incidence, $\theta$, will refract the light to be normal to the reflective surface, causing it to retrace the same path through the laser cavity (Figure 5.18).

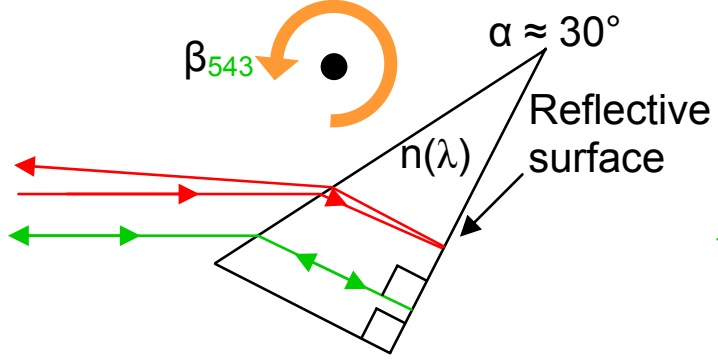

Amplified $\lambda=543 \mathrm{~nm}$

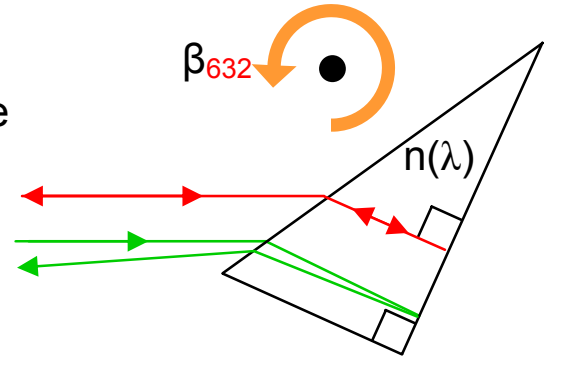

Amplified $\lambda=632 \mathrm{~nm}$

FIGURE 5.18 - Rotating the Littrow prism allows wavelength selection in the tunable laser.

Since the index of refraction of the prism varies with wavelength, only one wavelength will refract and retrace its path for a given prism rotation angle $\beta$. The other wavelengths will emerge from the prism at different angles and will not oscillate in the cavity or experience amplification.

In order to minimize reflection losses at the front surface of the Littrow prism, the angle $\alpha$ can be adjusted slightly such that the angle of incidence is near the Brewster angle. 


$$
\theta_{\text {Brewster }}=\tan ^{-1}\left(\frac{n_{\text {glass }}}{1}\right)=56.6^{\circ} \text { for } n=1.517
$$

At the Brewster angle, p-polarized light is completely transmitted (Fowles, 1989). The spolarized light is partially reflected (lost) on both passes, helping the laser to output linearly polarized light. A Brewster window on the other end of the cavity completes the laser cavity.

The output laser beam is steered through a $40 \mathrm{X}$ microscope objective and a $5 \mu \mathrm{m}$ pinhole, which is used as a spatial filter for the laser beam. The microscope objective has enough color correction that refocusing is not required as the laser wavelength is tuned. The output of the pinhole is collimated using a $38 \mathrm{~mm}$ diameter, $150 \mathrm{~mm}$ focal length cemented doublet from Newport (PAC075). The focal length difference for this lens between $543 \mathrm{~nm}$ and $632 \mathrm{~nm}$ wavelengths is 52 microns. The $\mathrm{f} / \#$ of this lens is 3.95 , so this focal length difference corresponds to a defocus of $417 \mathrm{~nm}$, or 0.66 waves at $\lambda=632 \mathrm{~nm}$.

$$
\begin{gathered}
f / \#=\frac{f}{D}=\frac{150 \mathrm{~mm}}{38 \mathrm{~mm}}=3.95 \\
\Delta W_{20}=\frac{\delta z}{8(f / \#)^{2}}=\frac{52 \mu \mathrm{m}}{8(3.95)^{2}}=417 \mathrm{~nm}
\end{gathered}
$$

This small defocus means that the collimating lens does not need to be refocused when the laser wavelength is tuned. 
Beamsplitter 3 splits the collimated input beam into the test (transmitted) and reference (reflected) beams. The usual phase shifting interferometer configuration is to have the reference phase shifted relative to the test phase, but in this case the test phase is changed. The reason for this is that the top right mirror in the interferometer configuration, which would typically be the phase shifting mirror, is a beamsplitter (BS 2). This surface is a beamsplitter because it is also used as a compensating optic for beamsplitter 1 in the low coherence interferometer. The phase shifting mirror is the fold mirror for the test arm of the interferometer, and the beam is incident on this surface before traversing the test cuvette. Therefore, the light is still collimated at the phase shifter.

The piezoelectric transducer (PZT) used is from EXFO, and it is capable of moving a 2" diameter mirror $2 \mu \mathrm{m}$. Recall that the mirror should be moved enough to induce a $90^{\circ}$ phase shift, and over the course of 5 images, should move the mirror $360^{\circ}$. For a mirror at a $45^{\circ}$ angle, the maximum motion required, $\mathrm{d}$, is given by:

$$
d=4 d_{i}=4\left[\frac{0.632 \mu m}{8} \cos (45)\right]=4[55.9 \mathrm{~nm}]=223.6 \mathrm{~nm} \quad \text { Equation } 5.26
$$

The PZT operates on high DC voltages, traveling the full $2 \mu \mathrm{m}$ range in 1000 Volts, or 2 $\mathrm{nm} / \mathrm{V}$. Table 5.2 shows the necessary voltages to obtain $90^{\circ}$ phase steps for the 5 laser wavelengths. 


\begin{tabular}{|c|c|}
\hline Laser Wavelength $(\mathrm{nm})$ & Voltage $(\mathrm{V})$ per $90^{\circ}$ \\
\hline 543.36 & 24.01 \\
\hline 593.93 & 26.25 \\
\hline 604.61 & 26.72 \\
\hline 611.80 & 27.04 \\
\hline 632.82 & 27.95 \\
\hline
\end{tabular}

TABLE 5.2 - Output voltages per $90^{\circ}$ for the five laser wavelengths.

The maximum required output voltage is $4 * 27.95=111.8 \mathrm{~V}$. It is a requirement that this output voltage is computer controlled, but this voltage is higher then what can be output by a standard digital to analog (D/A) PCI card. Recall that the motion of the reference mirror for the low coherence interferometer is controlled by an XPS Motion Controller/Driver from Newport. This controller box interfaces with the computer via a crosswire Ethernet cable, and this interface supports multiple channels of communication to operate simultaneously. In addition to the motion control and monitoring capabilities, the XPS control box has 4 channels of 16-bit D/A conversion on the DB-25 pin connector on the back panel of the box, labeled as GPIO2 (Figure 5.19).

\section{GPIO2 (DB-25, female)}

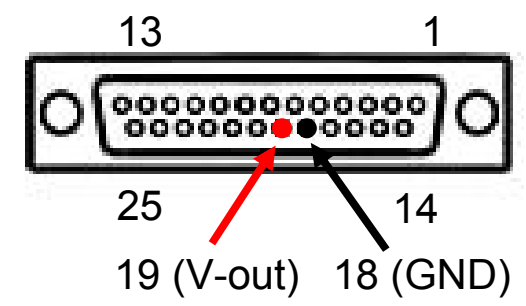

FIGURE 5.19 - Relevant pinout for GPIO2 connector of XPS controller. 
Using the appropriate XPS library functions, voltages from 0 to $10 \mathrm{~V}$ can be output. A DB-25 cable (Male-Female) is used to carry the voltage signal to a breakout board (BRK25M-R-FT, Winford Engineering) inside a small project box. The ground (pin 18) and the DC voltage (pin 19) are connected to a BNC cable, which is connected to the External Input port on the back of the RC-43 ramp generator (Figure 5.20).

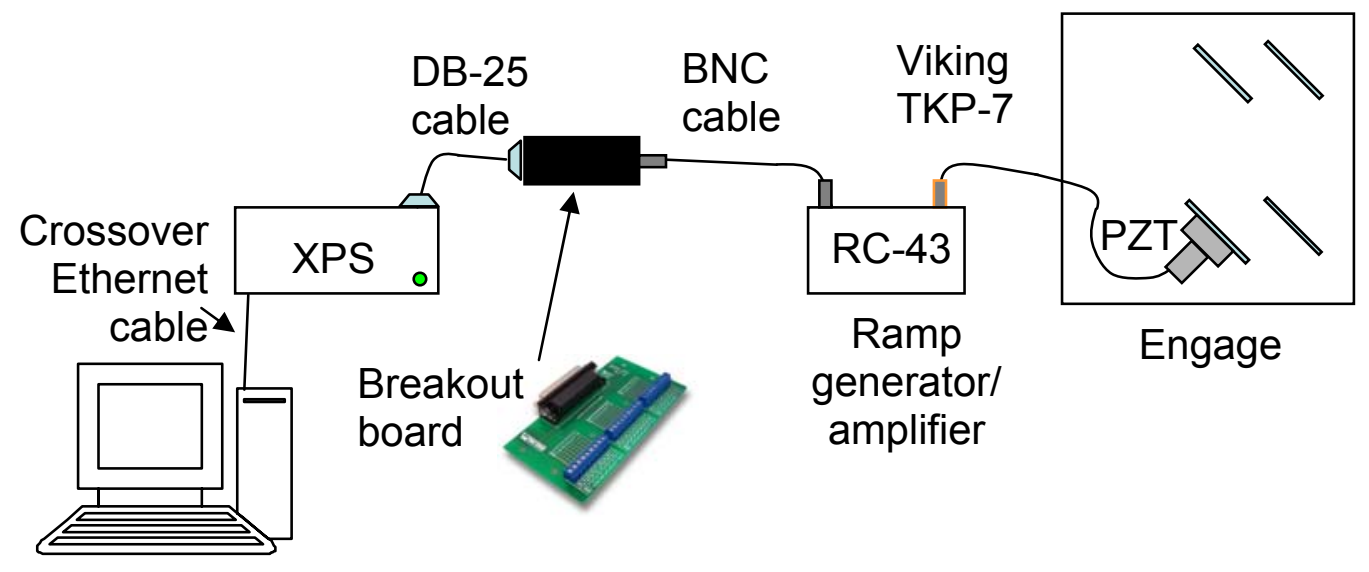

FIGURE 5.20 - Schematic of the components used to control the PZT.

The RC-43 ramp generator can be used to program various waveforms for the voltage signal applied to the PZT. In this application, it is used simply as an amplifier with a nominal amplification of 100 . Three identical voltages are applied to the three elements in the PZT to produce a piston motion; tip/tilt could also be introduced by varying the three voltages appropriately. The three channels require a 7-pin Viking Thorkom TKP-7 connector to interface with the PZT, which in this case is a model PZ-91. This is all the required hardware to control the motion of the phase shifter from the computer. 


\subsection{Data Collection}

There are two common methods for gathering the required frames in a phase shifting interferometer; phase-stepping and phase-ramping (Figure 5.21). Phase-stepping operates by moving the mirror $\mathrm{d}_{\mathrm{i}}$, allowing the mirror to settle, taking an image, and repeating (Goodwin, 2006).
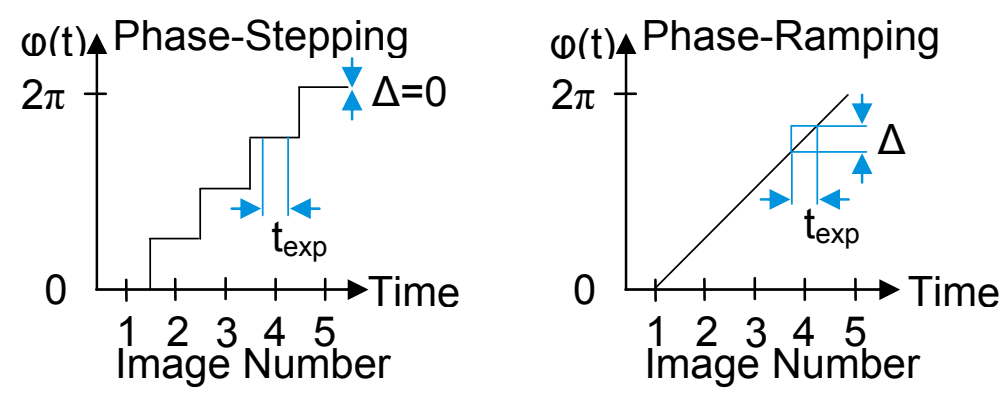

FIGURE 5.21 - Phase-stepping versus phase-ramping.

Phase-ramping moves the mirror continuously while the images are captured at a constant time interval. The images are captured over an integration time $\Delta$, which slightly reduces the fringe contrast but does not cause phase measurement errors.

$$
\begin{gathered}
I_{i}=I_{0}\left[1+\Gamma \operatorname{sinc}\left[\frac{\Delta}{2}\right] \cos \left[\varphi(x, y)+\varphi_{i}\right]\right] \\
\operatorname{sinc}[x]=\frac{\sin [x]}{x}
\end{gathered}
$$

Phase ramping setups can be more difficult because they require synchronization between the camera and the electronics controlling the PZT, but data can be collected faster, thus reducing the phase errors due to vibration. Engage uses phase-stepping to collect the data, reducing the complexity of the timing. The software waits about 100 milliseconds 
after the PZT has moved to allow it to settle before initiating the image capture sequence. Even with this delay, the full set of 5 images can be gathered in just a little over 1 second (Figure 5.22).

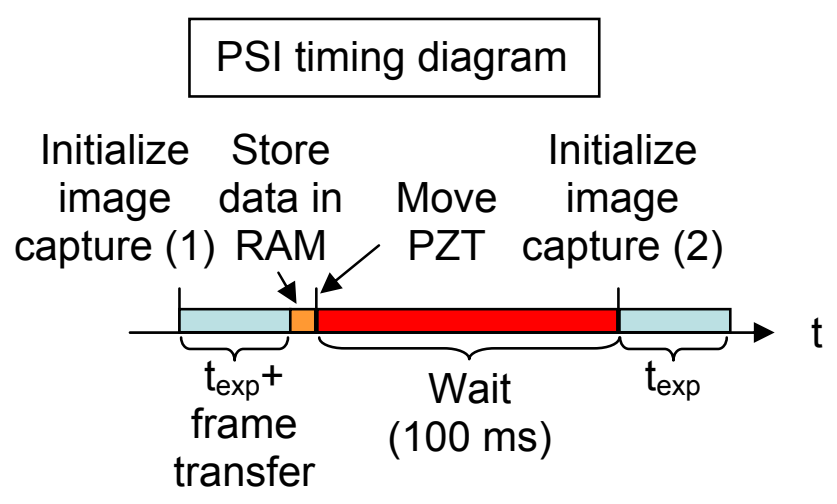

FIGURE 5.22 - Timing diagram for data collection with the Mach-Zehnder interferometer.

The exposure time varies with wavelength because of the change in relative irradiance (Table 5.3).

\begin{tabular}{|c|c|}
\hline Laser Wavelength $(\mathrm{nm})$ & Exposure time $(\mu \mathrm{s})$ \\
\hline 543.36 & 3000 \\
\hline 593.93 & 1050 \\
\hline 604.61 & 950 \\
\hline 611.80 & 450 \\
\hline 632.82 & 250 \\
\hline
\end{tabular}

TABLE 5.3 - Exposure times for the five laser wavelengths.

The speed at which the image data is transferred to the computer limits the speed of data collection, not the exposure time. The camera currently used is a CoolSnap $\mathrm{cf}$ from Photometrics; the same camera used to record images in the low coherence 
interferometer. Unbinned, full frames are always captured for the PSI measurements and are digitized at $20 \mathrm{MHz}$.

$$
(1392 \times 1040) \frac{\text { pixels }}{\text { frame }} \cdot \frac{1 \mathrm{sec}}{20 \times 10^{6} \text { pixels }}=72.4 \frac{\mathrm{ms}}{\text { frame }}
$$

The time required to digitize the frame is about 2 orders of magnitude longer then the actual exposure time, so even though the exposure time varies with wavelength, the data collection rate for the Mach-Zehnder interferometer is constant with respect to wavelength. 


\section{DETERMINING REFRACTIVE INDEX}

The overall goal of Engage is to build a system to measure the refractive index of contact lens materials. Recall that the method chosen to achieve this goal is based off the simple relationship between thickness, index and optical path length (OPL).

$$
n(\lambda)=\frac{O P L(\lambda)}{t}
$$

Equation 6.1

Chapter 2 described the theory of how the thickness of the material, $t$, is measured using low coherence interferometry as a function of position. Chapter 5 explained the second interferometer, a Mach-Zehnder interferometer, which is used to measure the OPL in transmission of the test sample at 5 different wavelengths, providing $\operatorname{OPL}(\lambda)$. Recall that the OPL is not fully determined using the Mach-Zehnder interferometer, and that the determination of the integer $\Delta \mathrm{m}$ is required to have enough information to determine the refractive index. The expanded equation for determining refractive index of saline is shown in Equation 6.2 .

$$
n_{\text {saline }}=\frac{\lambda}{t(x, y)}\left[\left(m_{\text {saline }}-m_{\text {air }}\right)+\frac{\left[\varphi_{\text {saline }}(x, y)-\varphi_{\varepsilon}(x, y)\right]-\varphi_{\text {air }, \text { fit }}(x, y)}{2 \pi}\right]+1
$$

Equation 6.2

Here, $\varphi_{\varepsilon}$ is the background phase error of the interferometer, $\varphi_{\text {air,fit }}$ is a plane that was fit to the phase of the air to determine the phase in the region containing saline for this measurement, and $\mathrm{t}(\mathrm{x}, \mathrm{y})$ is the thickness of the saline region for this measurement. The values for $\mathrm{m}_{\text {saline }}$ and $\mathrm{m}_{\text {air }}$ are positive integers; if the goal was to determine absolute OPL, their individual values would be required. Fortunately, all that is required for this 
technique is the difference, or $\Delta \mathrm{m}$, which will of course also be an integer. So the unknowns are $\Delta \mathrm{m}$ and $\mathrm{n}_{\text {saline. }}$. Clearly, additional information is required to get the index.

First, it is important to discuss the origins of the problem. Phase shifting interferometry (PSI) is used to determine the phase maps in transmission through the cuvette, as was explained in the previous chapter. The major benefit of PSI is its ability to measure surfaces in reflection with high precision, but one of the drawbacks of PSI is that it requires a continuous surface. The same is true in transmission, where the phase recovered can only be unwrapped along a continuous region. Due to the nature of this measurement, the phase through a column of air and the phase through a column of saline are separated by a boundary. The phase can be unwrapped on the air side of the boundary to determine $\varphi_{\text {air }}$, and on the other side to determine $\varphi_{\text {saline. }}$ In the absence of additional information, the height of the phase step at the boundary is impossible to determine because of the discontinuity. This unknown is shown by $\Delta \mathrm{m}$ in Equation 6.7. Figure 6.1 shows the measured phase prior to unwrapping, where the number of wavelengths, $2 \pi$ radians, or $\Delta \mathrm{m}$ between the air and saline regions cannot be determined. 


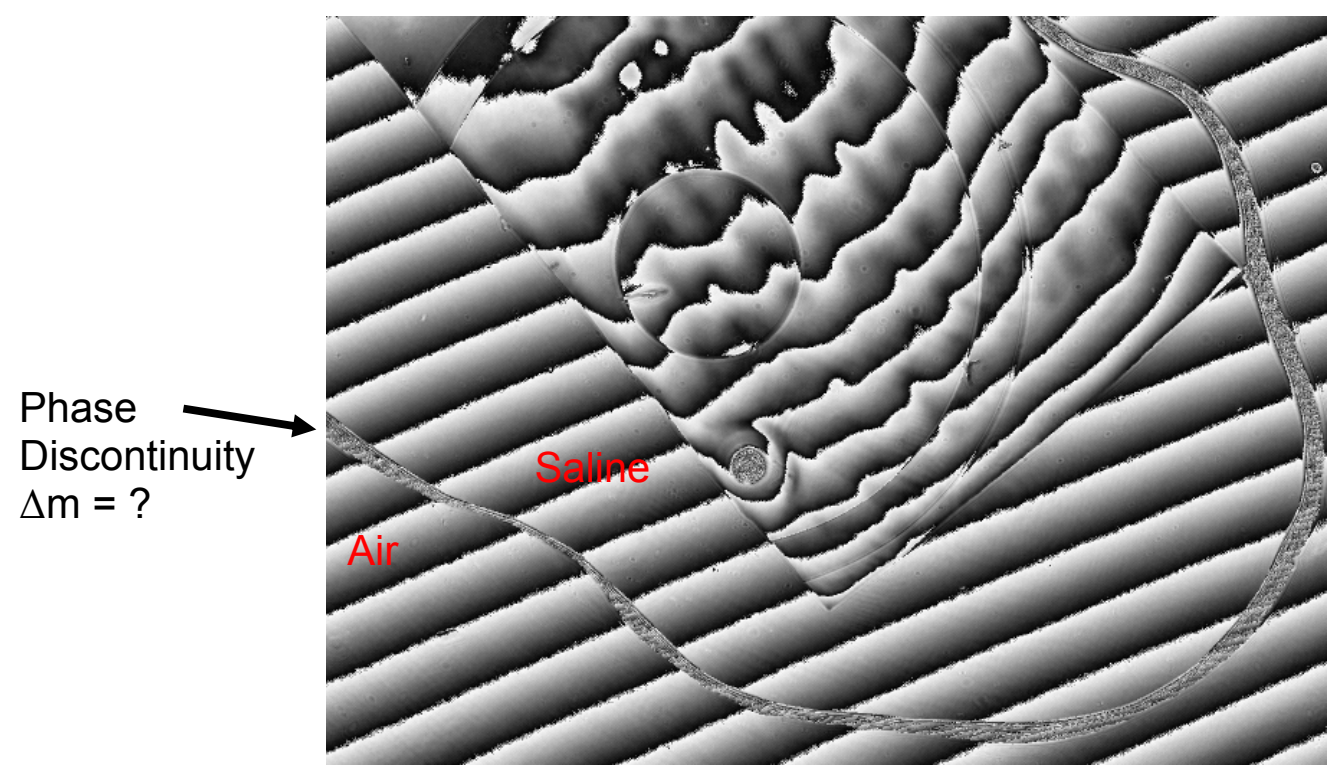

FIGURE 6.1 - Wrapped phase from Mach-Zehnder interferometer showing the discontinuity between the air and saline regions.

This issue is completely analogous to the determination of a step height on a surface in reflection, which is a problem that has been known for quite some time (Polhemus, 1973). Another way of looking at the problem is that it is unknown which fringe on the right is fringe ' $a$ ' from the left, since a single fringe represents a constant OPD (Figure $6.2)$.
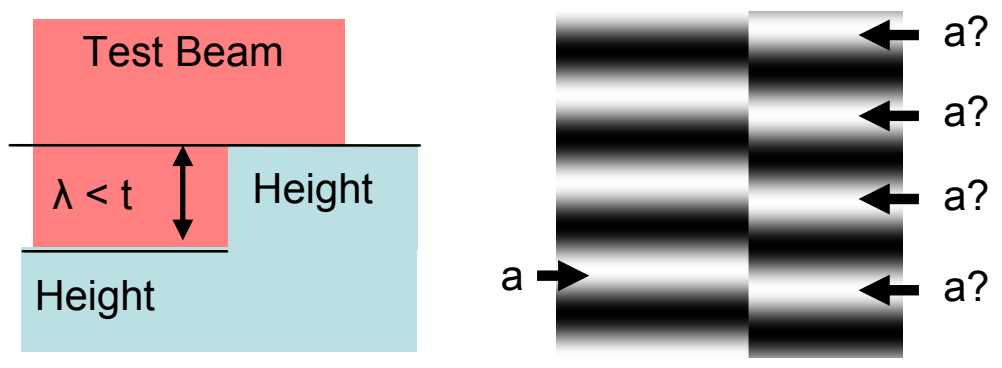

FIGURE 6.2 - The interferogram from a discrete step measured in reflectance will have a discontinuity at the boundary. Which fringe on the right is fringe ' $a$ ' from the left? 
The interferogram provides part of the information to determine the step height, or the fractional phase $\varphi$. Since the fringes are repetitive and indistinguishable, the pattern repeats every wavelength. In this case, the OPD across the boundary is:

$$
O P D_{\text {boundary }}=n t=\lambda\left(m+\frac{\varphi}{2 \pi}\right)
$$

Equation 6.3

Since the integer value of $\mathrm{m}$ is unknown, there is a family of possible solutions for the OPD $(\mathrm{m}=0,1 \ldots \infty)$. Of the available methods for determining $\mathrm{m}$, and hence the step height, the most applicable technique is to use multiple wavelengths. In the case of a step height, two phase measurements are made at two different wavelengths. Now there are two equations to use in solving for the two unknowns (Wyant, 1971).

$$
\begin{gathered}
\lambda_{e q}=\frac{\lambda_{1} \lambda_{2}}{\left|\lambda_{1}-\lambda_{2}\right|} \quad t<\frac{\lambda_{e q}}{4} \\
\lambda_{1}\left(m_{1}+\frac{\varphi_{2}}{2 \pi}\right)=O P D=t=\lambda_{2}\left(m_{2}+\frac{\varphi_{2}}{2 \pi}\right)
\end{gathered}
$$

There is only one solution $\left(\mathrm{m}_{1}, \mathrm{~m}_{2}\right)$ that gives the same value for OPD that satisfies the maximum thickness constraint that the step height is less then the a fourth of the equivalent wavelength. In the equation, the step is assumed to be in air, so the index $n$ is dropped from the equation.

The previous discussion was for a reflection test. The problem with a two wavelength test used in transmission is that the refractive index of saline, or any solid or liquid, varies with wavelength. So as soon as the second wavelength is added for a measurement of a 
discontinuity in a medium other then air, the OPD becomes a function of wavelength. Again, there are too many unknowns.

$$
\lambda_{1}\left(m_{1}+\frac{\varphi_{1}}{2 \pi}\right)=n_{1} t=O P D_{1} \neq O P D_{2}=n_{2} t=\lambda_{2}\left(m_{2}+\frac{\varphi_{2}}{2 \pi}\right) \quad \text { Equation } 6.6
$$

Since the existing technique using two wavelengths to determine $\Delta \mathrm{m}$ is not sufficient, a new technique must be developed. There is additional information obtained during the course of a full Engage measurement about the saline and the sample that is useful in narrowing down the possible values for $\Delta \mathrm{m}$ in Equation 6.7.

$$
n_{\text {saline }}=\frac{\lambda}{t(x, y)}\left[\Delta m+\frac{\left[\varphi_{\text {saline }}(x, y)-\varphi_{\varepsilon}(x, y)\right]-\varphi_{\text {air }, f i t}(x, y)}{2 \pi}\right]+1 \quad \text { Equation } 6.7
$$

Firstly, the group index of refraction, $\mathrm{N}_{\mathrm{g}}$, is calculated for both the saline and the test sample during the course of the low coherence thickness measurement at $\lambda=681.3 \mathrm{~nm}$. From the definition of $\mathrm{N}_{\mathrm{g}}$, it is clear that the value of $\Delta \mathrm{m}$ cannot result in a phase index that is higher than the group index.

$$
\begin{gathered}
N_{g}(\lambda)=n_{\lambda}-\left.\lambda \frac{d n}{d \lambda}\right|_{\lambda} \\
\left.\frac{d n}{d \lambda}\right|_{\lambda}<0 \quad N_{g}(\lambda)>n_{\lambda}
\end{gathered}
$$

This is unlikely to be enough to determine the correct index, but it is still useful information. Along the same line of reasoning relating to the expected behavior of materials with normal dispersion, the relationship between the indices for the 5 wavelengths must follow certain rules. First, as the wavelength increases from $543 \mathrm{~nm}$ to $632 \mathrm{~nm}$, the index of refraction decreases; the first derivative of the dispersion curve 
through the 5 values must be negative. Second, the index decreases at a decreasing rate, so the second derivative of the dispersion curve through the 5 values must be positive. Again, this helps to narrow the allowed values for $\Delta \mathrm{m}$ but it does not solve the problem completely. Figure 6.3 shows the dispersion curve along with the first and second derivatives for the model of distilled water.

a)

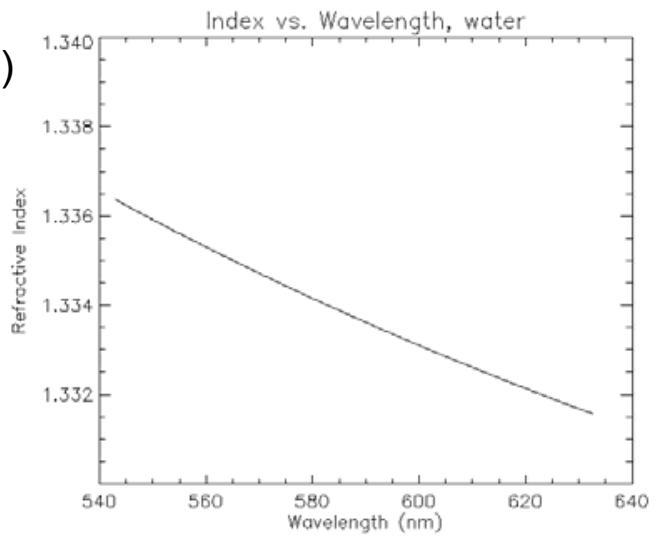

b) First derivative of Index vs. Wavelength, water

c)

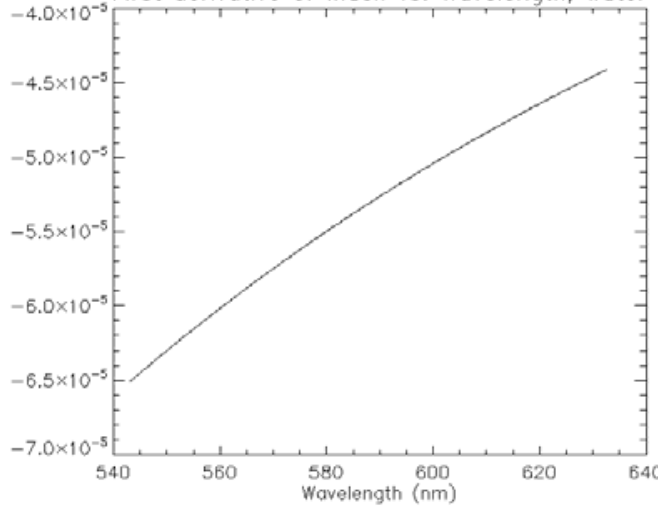

Second derivative of Index vs. Wavelength, water

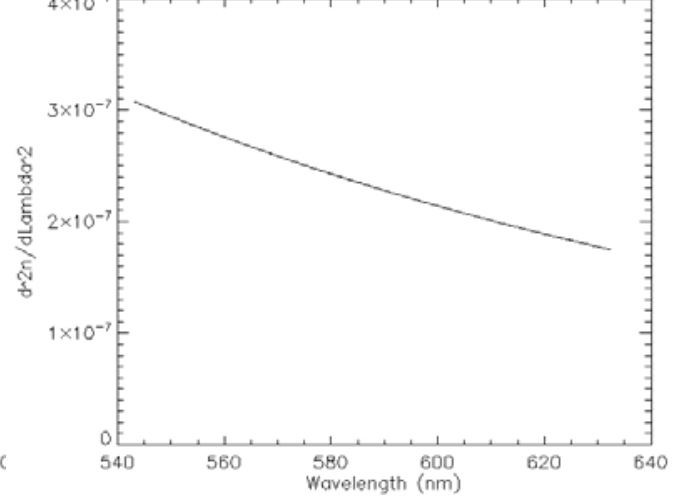

FIGURE 6.3 - Plots of a) refractive index vs. wavelength, b) first derivative of dispersion curve $(\Delta \mathrm{n} / \mathrm{nm})$ and c) second derivative of dispersion curve $\left(\Delta^{2} \mathrm{n} / \mathrm{nm}^{2}\right)$ for water. 


\subsection{Multiple Thickness Technique}

As the value of $\Delta \mathrm{m}$ is incremented by 1 , the resulting change in index is easy to determine:

$$
\begin{aligned}
n_{\text {sample }, \Delta m+1}-n_{\text {sample } \Delta m}= & \frac{\lambda}{t}\left[(\Delta m+1)+\frac{\left[\varphi_{\text {sample }}-\varphi_{\varepsilon}\right]-\varphi_{\text {saline,fit }}}{2 \pi}\right]+n_{\text {saline }}- \\
& \frac{\lambda}{t}\left[(\Delta m)+\frac{\left[\varphi_{\text {sample }}-\varphi_{\varepsilon}\right]-\varphi_{\text {saline,fit }}}{2 \pi}\right]+n_{\text {saline }}
\end{aligned}
$$

Equation 6.10

$$
n_{\text {sample }, \Delta m+1}-n_{\text {sample }, \Delta m}=\frac{\lambda}{t}=\Delta n_{\Delta m=1}
$$

This extremely simple expression is in fact quite useful. As the thickness of the test sample ( $\mathrm{t}$ ) increases, the change in index from a change in $\Delta \mathrm{m}$ of 1 , or $\Delta \mathrm{n}_{\Delta \mathrm{m}=1}$, decreases. At a test wavelength of $632 \mathrm{~nm}$ and a nominal sample thickness of $100 \mu \mathrm{m}$, the index step is 0.00632 in refractive index. This is about a factor of 50 larger then the desired system resolution, and therefore should be much larger then the noise in the system. However, it is not large enough to easily distinguish the correct index value for a previously unknown sample. From the simple relationship of Equation 6.11, only the laser wavelength and the sample thickness will change the index step size. As has been discussed, changing the wavelength changes the index, thanks to dispersion. This leaves the thickness of the sample. Consider a sample with two discrete sample heights, $t_{1}$ and $t_{2}$, tested at a single wavelength, $\lambda$.

$$
\Delta n_{t_{1}}=\frac{\lambda}{t_{1}}
$$




$$
\Delta n_{t_{2}}=\frac{\lambda}{t_{2}}
$$

Since $\mathrm{t}_{1} \neq \mathrm{t}_{2}, \Delta \mathrm{n}_{\mathrm{t} 1} \neq \Delta \mathrm{n}_{\mathrm{t} 2}$, and as $\Delta \mathrm{m}$ is incremented for both, there will only be one value of refractive index where the index in the region of $t_{1}$ and the index in the region of $t_{2}$ are identical (Figure 6.4).

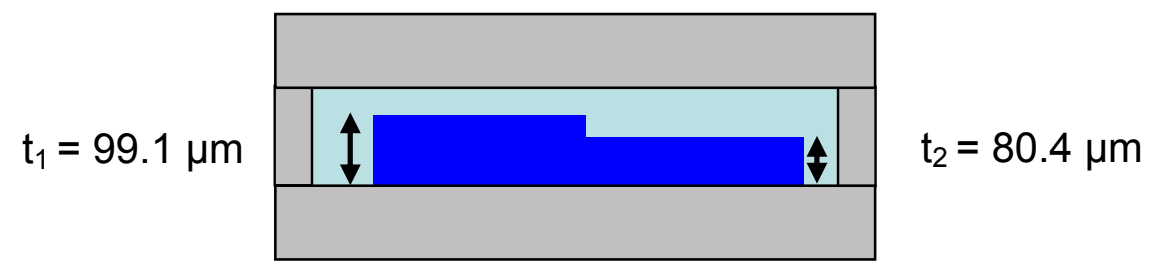

FIGURE 6.4 - A hypothetical sample with two different thicknesses.

Figure 6.5 illustrates the two 'families' of solutions for the two thickness regions, and the circle indicates where the two families agree, at a hypothetical index of refraction of 1.4063.

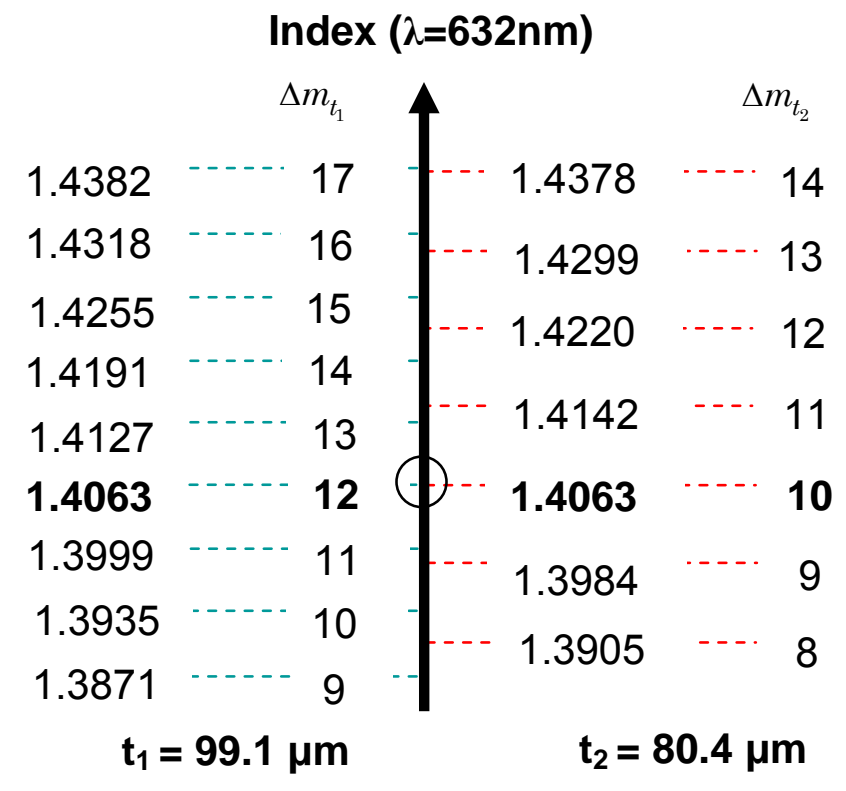

FIGURE 6.5 - The calculated index value for two thickness regions of the same material will only agree at one index value, indicated here by the circle. 
For a test sample with multiple thickness regions, the ambiguity in determining the value of $\Delta \mathrm{m}$ can be removed by comparing the two families of solutions associated with the two thickness regions. This technique will be referred to as the multiple thickness technique.

On further examination of Figure 6.5, it can be seen that the two thickness give nearly identical index values for $\Delta \mathrm{m}_{\mathrm{t} 1}=17$ and $\Delta \mathrm{m}_{\mathrm{t} 2}=14$, at indices of 1.4382 and 1.4378 , respectively. The two thickness regions will give identical index values to at least the resolution of the system $(0.0001)$ in a repetitive nature in index space. The distance in index over which this happens will be called $\mathrm{n}_{\text {periodic. }}$ Here $\mathrm{a}$ and $\mathrm{b}$ are integers.

$$
\begin{array}{cr}
\left|n_{t_{1} @ \Delta m_{1}}-n_{t_{2} @ \Delta m_{2}}\right|=\left|\left(n_{\text {correct }}+a \frac{\lambda}{t_{1}}\right)-\left(n_{\text {correct }}+b \frac{\lambda}{t_{2}}\right)\right|<0.0001 & \text { Equation 6.14 } \\
n_{\text {periodic }}=a \Delta n_{t_{1}}=b \Delta n_{t_{2}} & \text { Equation } 6.15
\end{array}
$$

For the two thicknesses in Figure 6.4, the two index values for the two thickness regions are equal to better than 0.0001 for $n=1.7447$, when $a=53$ and $b=43$, where $\mathrm{n}_{\text {periodic }}=0.3384$. If the criteria for the two index values to be the same is increased to 0.0002 , then the index values from the two thickness regions align for $n=1.5085$, when $\mathrm{a}=16$ and $\mathrm{b}=13$, and $\mathrm{n}_{\text {periodic }}$ is reduced to 0.1022 .

Ideally, $\mathrm{n}_{\text {periodic }}$ is large enough that all ambiguity in determining the correct refractive index has been removed. In general, a smaller thickness difference leads to a larger value 
for $\mathrm{n}_{\text {periodic, }}$ which might indicate a $1 \mu \mathrm{m}$ difference would be a good goal for an ideal test sample. The problem with very small thickness differences is that near the true value for the index, it becomes difficult to determine the correct value. Consider $t_{1}=100 \mu \mathrm{m}$ and $\mathrm{t} 2=99 \mu \mathrm{m}$ for a material with a true refractive index of 1.4000 being measured in a system with a resolution of 0.0001 in index, tested at $\lambda=632.8 \mathrm{~nm}$.

$$
\begin{aligned}
& \Delta n_{t_{1}}=\frac{0.6328}{100}=0.006328 \\
& \Delta n_{t_{2}}=\frac{0.6328}{99}=0.006392
\end{aligned}
$$

The two thicknesses may give the same index value at 1.4000 , but if $\Delta \mathrm{m}$ is incremented by 1 for both, the difference in index between the two regions is 0.000064 .

$$
n_{t_{1}, \Delta m_{1}+1}-n_{t_{2}, \Delta m_{2}+1}=1.406328-1.406392=0.000064<0.0001
$$

Equation 6.18

This difference is below the resolution of the system, and in the presence of a reasonable amount of noise, it is not always clear if $1.3936,1.4000$ or 1.4063 is the correct value. This example illustrates the origins of the lower bound for thickness difference, which is represented in index space by the noise ceiling (NC). The noise ceiling is calculated based on the wavelength and the two thicknesses, and it represents the maximum allowable uncertainty in any calculated value for index at a specific value of $\Delta \mathrm{m}$. If the resolution of the system is larger than the noise ceiling, the correct value for $\Delta \mathrm{m}$ cannot be determined.

$$
\text { Noise Ceiling }=N C=\lambda\left(\frac{\left|t_{1}-t_{2}\right|}{t_{1} t_{2}}\right)
$$


With any two thicknesses, there will be a tradeoff between increasing the noise ceiling and increasing $\mathrm{n}_{\text {periodic }}$; they cannot be done simultaneously. Table 6.1 summarizes this tradeoff for a variety of thicknesses, where the system resolution is assumed to be 0.0001 and the index is being measured at $\lambda=632.8 \mathrm{~nm}$.

\begin{tabular}{|c|c|c|c|}
\hline $\mathrm{t}_{1}(\mu \mathrm{m})$ & $\mathrm{t}_{2}(\mu \mathrm{m})$ & $\mathrm{n}_{\text {periodic }}$ & Noise Ceiling $(\mathrm{NC})$ \\
\hline 100 & 99 & 0.6328 & 0.000064 \\
\hline 100 & 95 & 0.1266 & 0.000333 \\
\hline 100 & 80 & 0.0316 & 0.001582 \\
\hline 100 & 50 & 0.0126 & 0.006328 \\
\hline 100 & 88.4 & 0.2720 & 0.000830 \\
\hline
\end{tabular}

TABLE 6.1 - For a sample with two thicknesses, $\mathrm{n}_{\text {periodic }}$ and the noise ceiling cannot be simultaneously large.

A good rule is that NC should be greater then 0.0005 .

All of the analysis thus far has considered two thickness regions, but the sample could have more than 2 different thickness regions. For example, three thickness values of 100, 99 and $80 \mu \mathrm{m}$ could be used. The two largely separated thicknesses will create a large noise ceiling while the 99 and $100 \mu \mathrm{m}$ regions can be used to achieve a large value for

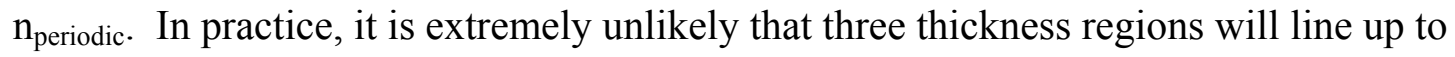
within the system resolution somewhere in index space other than the correct value. 


\subsection{Practical Considerations for the Multiple Thickness Technique}

The initial sample geometry that this system was designed to measure was a flat disk made of the same material used to make soft contact lenses. Ideally, index of refraction measurements would be performed on an actual lens, but recall that for glass lenses, prisms are made during the same melt and used to characterize the refractive index of the lenses. The Engage measurement procedure is analogous to using a prism for glass.

As it became clear that a sample with multiple thickness regions was required, the test samples were modified to incorporate 4 regions with different thicknesses, so that the index could be measured using the multiple thickness technique. Most of the data shown so far in this document are from these 'inverted wedding cake' samples, with concentric rings of varying thickness regions. The approximate geometry for these samples is shown in Figure 6.6.

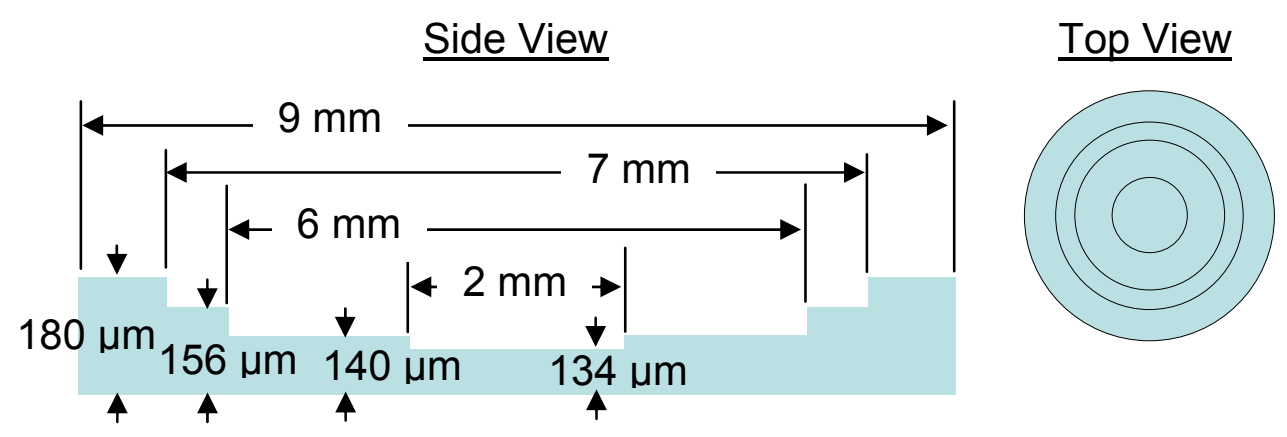

FIGURE 6.6 - Side and top views of an 'inverted wedding cake' sample.

In the discussion on the theory of the multiple thickness technique, each region was assigned a single thickness value (t), when in reality, even the best 'flat' will have a 
spatial thickness variation: $\mathrm{t} \rightarrow \mathrm{t}(\mathrm{x}, \mathrm{y})$. The index of refraction is calculated at each pixel in each region using the thickness and phase values at that pixel, creating a large number of measurements of index of refraction. To determine the index step for incrementing $\Delta \mathrm{m}$, the value of $\mathrm{t}$ is simply an average of all the $\mathrm{N}$ thickness values in the region.

$$
\Delta n_{t_{1}}=\frac{\lambda}{t_{1}} \quad t_{1}=\frac{\sum_{i=1}^{N} t(x, y)_{\text {Region } 1}}{N}
$$

So each region is not required to have a constant thickness, but simply to have a different average thickness than the other regions.

\subsubsection{Wedged Samples}

From a theoretical perspective, it is perhaps easier to analyze the desired properties for varying thickness regions in order to remove phase ambiguities and determine the refractive index of a test sample when discrete thickness regions are used, as in Figure 6.6 above. The same theory also applies to a sample with a continuous thickness gradient, also known as a wedge (Figure 6.7).

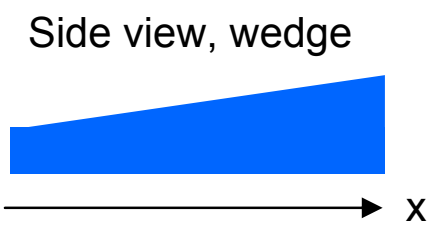

FIGURE 6.7 - A wedged sample with a thickness gradient only along $\mathrm{x}$.

All of the multiple thickness techniques assume that the refractive index is uniform throughout the test sample, which is a good assumption for homogenous materials. 
Consider measuring a wedged sample whose thickness varies along $\mathrm{x}$; the correct value for $\Delta \mathrm{m}$ will result in an index map that is constant across the material.

$$
\begin{gathered}
t_{\text {wedge }}(x, y) \rightarrow t_{\text {wedge }}(x)=a x+c \\
n_{\text {sample }}(x, y)=\frac{\lambda}{t_{\text {wedge }}(x)}\left[\Delta m_{\text {correct }}+\frac{\left[\varphi_{\text {sample }}(x, y)-\varphi_{\varepsilon}(x, y)\right]-\varphi_{\text {saline }, f i t}(x, y)}{2 \pi}\right]+n_{\text {saline }} \\
=\text { Constant }
\end{gathered}
$$

Equation 6.22

If $\Delta \mathrm{m}$ is increased by $1, \mathrm{n}_{\text {sample }}$ will increase, but it will not increase uniformly. Since the index step, $\Delta \mathrm{n}_{\mathrm{t} 1}$, depends on $\mathrm{t}$ which changes along $\mathrm{x}$, the index step will also change along $\mathrm{x}$. Therefore, the slope of the calculated refractive index will change as the value used for $\Delta \mathrm{m}$ gets farther away from the correct value. Under the assumption that the index is constant for a given sample, the presence of slope in the calculated index indicates that the wrong value for $\Delta \mathrm{m}$ has been chosen (Figure 6.8). 


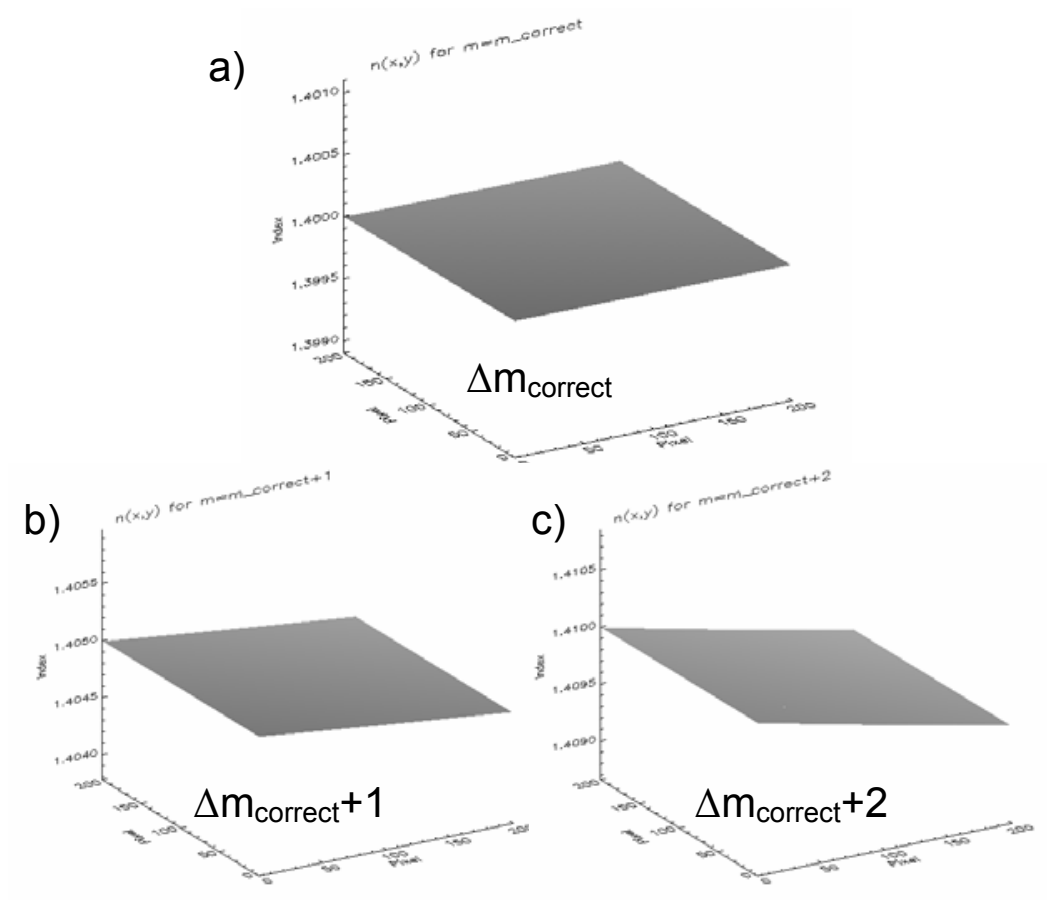

FIGURE $6.8-$ Calculated $\mathrm{n}(\mathrm{x}, \mathrm{y})$ for $\Delta \mathrm{m}=\mathrm{a}) \Delta \mathrm{m}_{\text {correct }}$ b) $\Delta \mathrm{m}_{\text {correct }}+1$ and c) $\Delta \mathrm{m}_{\text {correct }}+2$.

For a wedge, the slope of the index depends on the spatial thickness variation. The larger the variation, the larger the slope in index space and the easier it is to determine the correct $\Delta \mathrm{m}$. Once again, the slope cannot be increased indefinitely without running up against another physical restriction.

\subsubsection{Slope Limitations}

The low coherence interferometer in Engage is configured in a Twyman-Green configuration, and the surfaces of the test sample are located using a normally incident, reflected beam. The law of reflection dictates that any tilt of angle $\theta$ on the surfaces of the sample will reflect the return beam by $2 \theta$ with respect to the incoming beam. As the 
wedge of the test sample is increased, the reflected beam will eventually cause tilt fringes that violate the Nyquist limit of a minimum of two pixels per fringe (Figure 6.9).

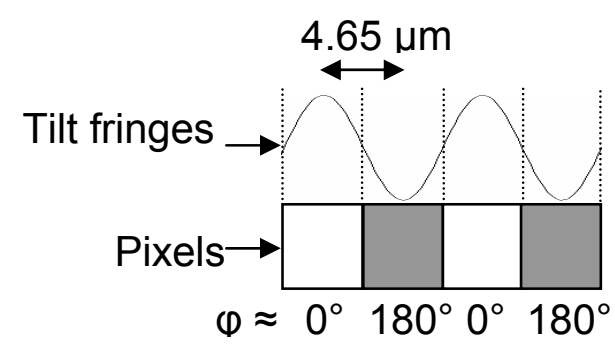

FIGURE 6.9 - A fringe pattern at the Nyquist limit has a phase change of $180^{\circ}$ or $\pi$ radians.

Based on the pixel size of $4.65 \mu \mathrm{m}$, the imaging lens magnification of -0.5 , and the center wavelength of the SLD, the maximum wedge angle $\theta$ of the test sample can be calculated (Figure 6.10). The maximum OPD between neighboring pixels is $\lambda_{\mathrm{c}} / 2$, which means the maximum height difference is $\lambda_{d} / 4$ since the test beam is double pass.

$$
\theta_{\max }=\tan ^{-1}\left(\frac{0.25 \lambda_{c}}{\text { PixelPitch } /|m|}\right)=\tan ^{-1}\left(\frac{0.25 \cdot 0.680 \mu m}{4.65 \mu m /|0.5|}\right)=1.04^{\circ}
$$

Equation 6.23

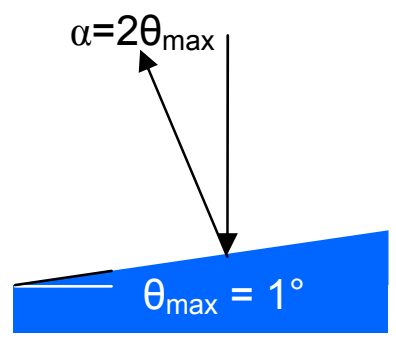

FIGURE $6.10-$ The maximum wedge angle for a test sample is about $1^{\circ}$. 
A more conservative limit on the part wedge of $0.5^{\circ}$ can be applied. A sample that covers 500 pixels with a wedge of $0.5^{\circ}$ will have about $42 \mu \mathrm{m}$ of height difference $(4.65$ $\mu \mathrm{m}$ pixels, $\mathrm{m}=-0.5)$. If the mean sample thickness is $100 \mu \mathrm{m}$, the slope of the refractive index for $\Delta \mathrm{m}=\Delta \mathrm{m}_{\text {correct }}+1$ at $\lambda=632 \mathrm{~nm}$ is a change in index per pixel of $5.58 \times 10^{-6}$. Over 500 pixels, the full index difference is 0.0028 . There are several possible ways to examine the index values for a wedged sample for slope variation; perhaps the simplest is to calculate the standard deviation of the calculated index at a series of values for $\Delta \mathrm{m}$ and find the minimum. This works for a sample with an arbitrarily varying thickness, including certain types of contact lenses.

\subsubsection{Contact Lenses as Test Samples}

From the standpoint of requiring thickness variation in a test sample, contact lenses offer many advantages. The thickness of the material varies radially, with an increasing rate of change. As long as the thickness variation is sufficiently low such that the Nyquist limit is not violated, the optical zone of a contact lens offers an excellent test sample. Small negative powers (-1.00 to -2.00 diopters) will provide enough thickness variation without violating $\theta_{\max }$. Based on the optical power, the surface curvatures must be different, resulting in a thickness variation. Equally important is the base thickness of the material. The plot in Figure 6.11a shows the noise ceiling for a fixed thickness difference $\left(t_{2}-t_{1}=\right.$ $20 \mu \mathrm{m})$ versus $t_{1}$. The thicker the sample is, the less effective the $20 \mu \mathrm{m}$ thickness difference becomes. If $\mathrm{t}_{2}$ is $20 \%$ greater than $\mathrm{t}_{1}$, a less drastic, yet similar trend is observed (Figure 6.11b). 

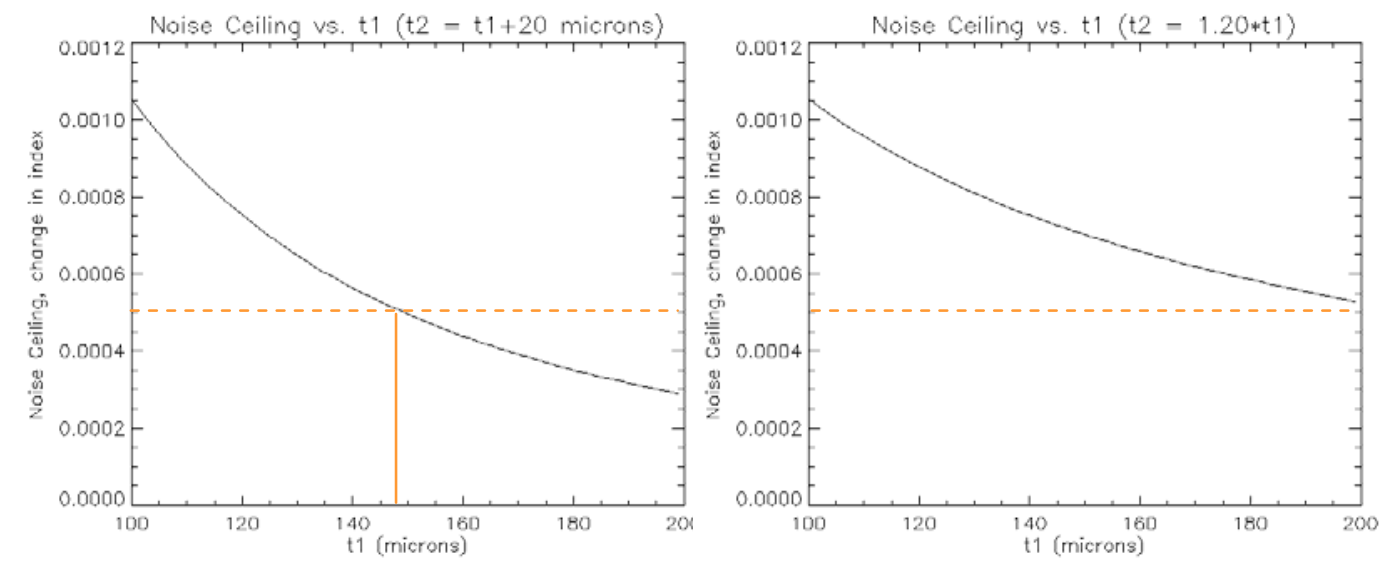

FIGURE 6.11 - Plots of a) NC vs. $t_{1}$, where $t_{2}=t_{1}+20$ (fixed thickness difference), and $b$ ) $\mathrm{NC}$ vs. $\mathrm{t}_{1}$, where $\mathrm{t}_{2}=1.2 * \mathrm{t}_{1}$. The minimum thickness difference needed to satisfy the NC limits depends on $\mathrm{t}_{1}$. The dashed orange line is $\mathrm{NC}=0.0005$.

The wavelength used for these plots is $\lambda=632 \mathrm{~nm}$. Following the rule that $\mathrm{NC} \geq 0.0005$ between any 2 height regions, a $20 \mu \mathrm{m}$ height difference is not enough for $\mathrm{t}_{1}>148 \mu \mathrm{m}$.

The difficult part of lens testing is that even though the thickness variation is good, the steep base curve of the lenses severely limits the testable area of a lens in its relaxed state (Figure 6.12).

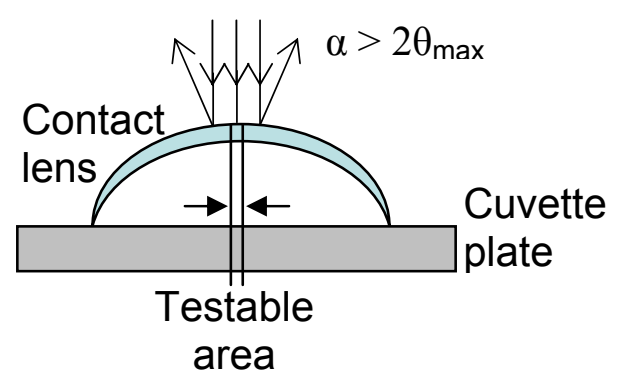

FIGURE 6.12 - A contact lens in its relaxed state has too large of a base curve. 
In order to make measurements on a lens or portion of a lens, it must be made to lie flat on the bottom plate of the cuvette, just as is the case for the stepped sample. Since the lens wants to return to a meniscus shape and the contact lens material does not stick well to the glass, it is difficult to mount a lens to meet the testing requirements. Finding a mounting procedure for contact lenses has been identified as an important next step for Engage. This may involve slicing out a part of the lens or otherwise manipulating into the desired configuration. Once a suitable method has been developed for mounting a portion of a contact lens, there is no need to make 'inverted wedding cake' samples in order to measure the refractive index of a contact lens material. This would give the Engage measurement system an advantage over the testing of the index of glass lenses in that the index of the actual lens can be measured. Unfortunately, the Engage testing geometry will likely require the destruction of the lens.

\subsection{Index Calculation - Saline}

This chapter is meant to show how the refractive index is calculated from the two sets of data collected; the thickness measurement using the low coherence interferometer, and the path length, or phase measurement from the Mach-Zehnder interferometer. Determining the sample thickness and the phase in transmission for the various materials in the camera field of view (air, saline and sample) has already been covered. The problem of determining the integer value of $\Delta \mathrm{m}$ has been covered from a theoretical viewpoint, and the background of the Mach-Zehnder interferometer has been incorporated in the form of $\varphi_{\varepsilon}(\mathrm{x}, \mathrm{y})$. Now that all of this information has been obtained, it 
must be combined to determine the refractive index of the sample. First, the index of the saline solution must be determined, using Equation 6.24.

$$
n_{\text {saline }}\left(\lambda_{\text {laser }}\right)=\frac{\lambda_{\text {laser }}}{t(x, y)}\left[\Delta m+\frac{\left[\varphi_{\text {saline }}(x, y)-\varphi_{\varepsilon}(x, y)\right]-\varphi_{\text {air }, f i t}(x, y)}{2 \pi}\right]+1
$$

Equation 6.24

The thickness of the cuvette is found in the air region and extrapolated across the field of view, as described in Chapter 2. The phase in the air region is unwrapped, and a plane is fit to the unwrapped phase minus the interferometer background, $\varphi_{\varepsilon}(\mathrm{x}, \mathrm{y})$, as discussed in Chapter 5. Next, the phase in the saline region is unwrapped and extrapolated in the same manner. The extrapolated saline phase, $\varphi_{\text {saline,fit }}(\mathrm{x}, \mathrm{y})$, is used in determining $\mathrm{n}_{\text {sample }}$. Everything in Equation 6.24 has been determined except for $\Delta \mathrm{m}$. All of the multiple thickness techniques apply to determining $\mathrm{n}_{\text {saline }}$ except that different limits must apply since the nominal thickness of the saline region is $350 \mu \mathrm{m}$. To obtain a NC of 0.0005 with $\mathrm{t}_{1}=350 \mu \mathrm{m}, \mathrm{t}_{2}$ must be greater than $516 \mu \mathrm{m}$ or less than $265 \mu \mathrm{m}$.

$$
t_{2}=\left(\frac{1}{t_{1}} \pm \frac{0.0005}{\lambda}\right)^{-1} \rightarrow t_{2}=265 \mu m \text { or } t_{2}=516 \mu m
$$

Clearly this would violate $\theta_{\max }$. Relaxing the constraint on NC to 0.0002 gives a more reasonable value for $t_{2}$ of about $400 \mu \mathrm{m}$, but this still is difficult to obtain over a varying region of interest containing saline without pushing the Nyquist limit of the detector.

There are multiple solutions to this problem, two of which are applicable in this context. The first is to measure the refractive index of the saline in an independent instrument 
such as an Abbe refractometer. An independent refractive index measurement can serve either of two purposes; a) it might only need to be accurate enough to determine the correct $\Delta \mathrm{m}$ in Equation 6.24, or b) it can be at least as accurate as Engage and used as a calibration tool. The requirements for a) are that it identify the index to better than half of $\Delta \mathrm{n}_{\Delta \mathrm{m}=1}$, which for $\mathrm{t}=350 \mu \mathrm{m}$ is 0.0015 , or an accuracy of at least 0.00075 (Figure 6.13).

$$
\Delta n_{\Delta m=1}=\frac{\lambda}{t_{\text {saline }}}
$$

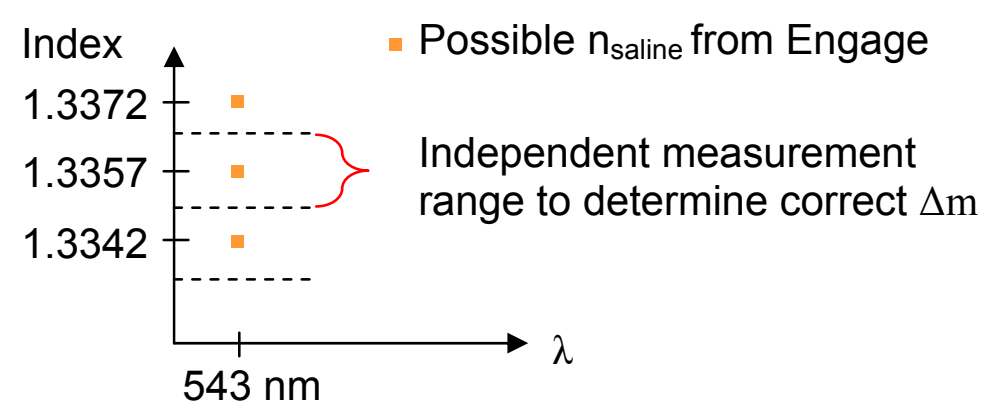

FIGURE 6.13 - If an independent measurement is used to determine the correct value of $\Delta \mathrm{m}$ and find $\mathrm{n}_{\text {saline, }}$ it must be more accurate then $\Delta \mathrm{n}_{\Delta \mathrm{m}=1} / 2$.

Many Abbe refractometers are good enough to satisfy requirement 1) for order determination, except that they typically measure $\mathrm{n}_{\mathrm{d}}$, which is the index at the Sodium $\mathrm{d}$ line $(\lambda=587.6 \mathrm{~nm})$. This is between the two of the wavelengths used in Engage, and could be used to determine which group of five index values has the correct dispersion curve and hence, the correct refractive index values. 
The second possible purpose of an independent index measurement is that it serves as a calibration instrument; an instrument that satisfies requirement b) will obviously satisfy a) as well. One such instrument is the Hilger-Chance Refractometer (HCR), which measures the refractive index of liquids by creating a prism out of the test liquid in a test block and measuring the beam deviation. This instrument was introduced in the late 1930's (Hughes, 1941), but was more recently built by Bruce Pixton in our lab partially for the purpose of verifying the measurements made by Engage. The same 5-color tunable $\mathrm{HeNe}$ laser is used as the source for this instrument so that all of the index values for saline are easily compared to values from Engage.

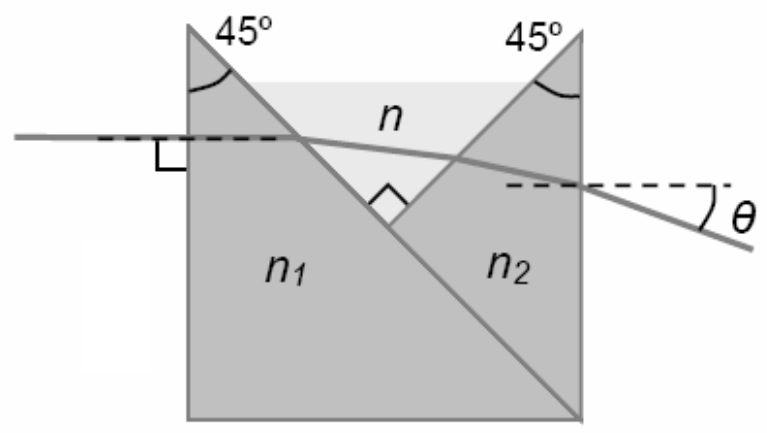

FIGURE 6.14 - Measuring $\theta$ in a well-known V-block allows $\mathrm{n}$ to be calculated.

Figure 6.14 illustrates the V-block which holds the liquid sample for testing with index $\mathrm{n}$. The beam output angle $\theta$ relative to the beam input angle is what is measured, which is related to refractive index by Equation 6.27 (Pixton, 2006).

$$
n_{\text {saline }}=\sqrt{\frac{n_{1}^{2}}{2}+\frac{n_{2}^{2}}{2}-\sin \theta \sqrt{n_{2}^{2}-\sin ^{2} \theta}}
$$

Accuracy is improved by carefully characterizing the two prisms that make up the Vblock, for both index and angles. With care, this instrument is capable of measuring the 
index of distilled water at multiple wavelengths with a standard deviation of 0.00005 , or about two times better than the goal for Engage. Measuring saline with the HCR is simpler than for Engage, resulting in values for saline that can be used as calibration data for Engage. Details of the Engage system calibration will be explained in Chapter 7.

In practice, data from the $\mathrm{HCR}$ is often used to determine the correct value for $\Delta \mathrm{m}$ and $\mathrm{n}_{\text {saline, }}$ but it is not required. The HCR measurement is made first. If this file is present during processing, the software will plot the HCR data on top of what is referred to as a "Christmas tree plot", shown in Figure 6.15, so that the HCR data can be used to determine the correct index values. 


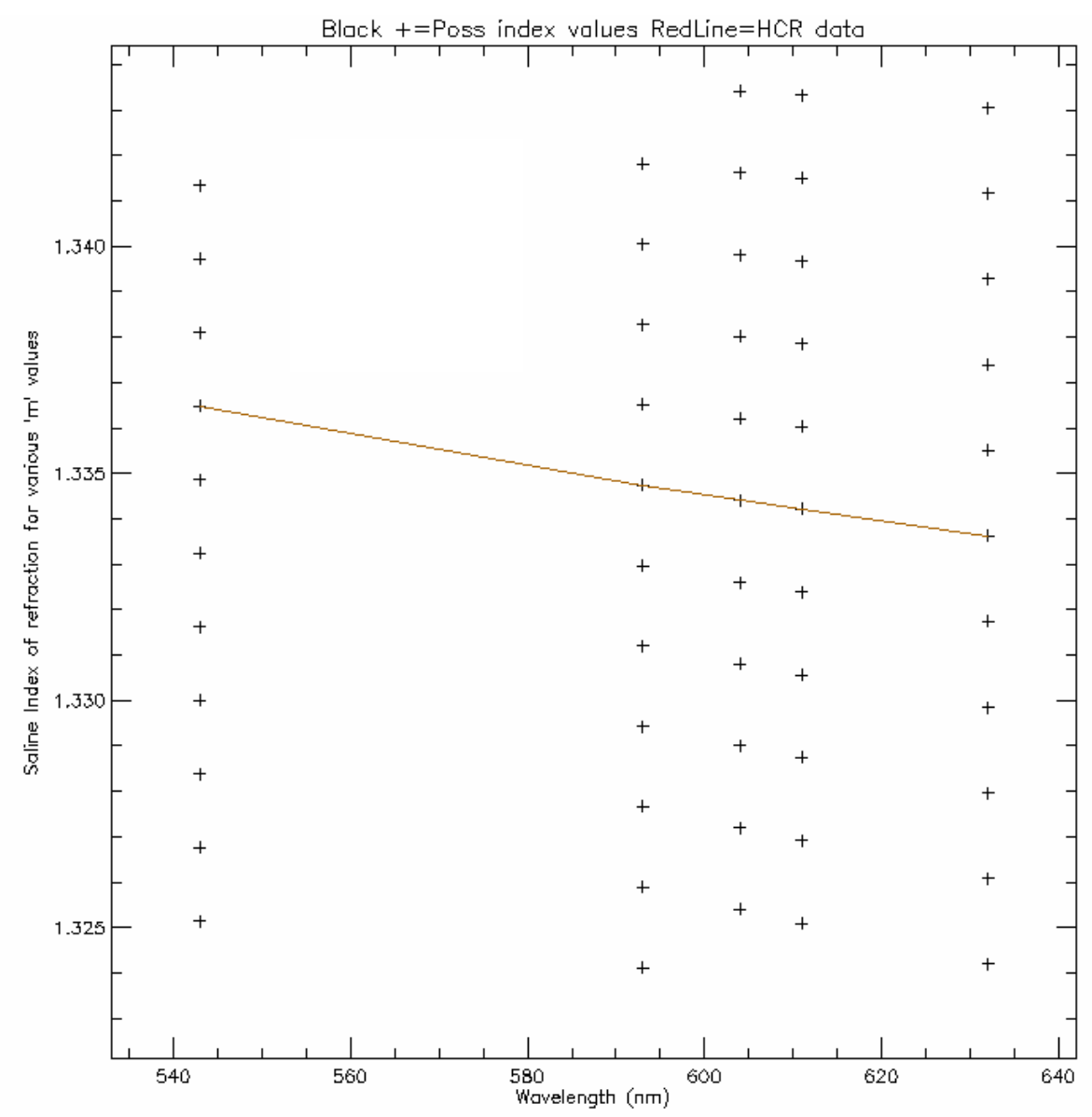

FIGURE 6.15 - Christmas tree plot for selecting correct values for $\mathrm{n}_{\text {saline. }}$.

This "Christmas tree plot" deserves some explanation. The x-axis values are the laser wavelengths used in the Mach-Zehnder interferometer, where the refractive index has been measured. The y-axis is refractive index. The multiple "+" symbols above each wavelength value represent the family of possible index values obtained by incrementing $\Delta \mathrm{m}$ at that wavelength. Similar groups occur for each wavelength. Note that although $\mathrm{t}$ is constant for every wavelength, the changing wavelength dictates that the index step, $\Delta \mathrm{n}_{\Delta \mathrm{m}=1}$, increases with increasing wavelength (Equation 6.26). 
The red line is the data from the HCR measurement for this data set, superimposed on the possible solutions. This makes determining the correct value of $\Delta \mathrm{m}$ trivial, as all 5 wavelengths agree quite well, as summarized in Table 6.2.

\begin{tabular}{|c|c|c|c|}
\hline Wavelength $(\mathrm{nm})$ & $\mathrm{n}(\mathrm{HCR})$ & $\mathrm{n}($ Engage $)$ & $1 \mathrm{e} 4 *\left(\mathrm{n}_{\text {Engage }}-\mathrm{n}_{\mathrm{NCR}}\right)$ \\
\hline 543.36 & 1.336483 & 1.336479 & -0.047 \\
\hline 593.93 & 1.334736 & 1.334739 & 0.028 \\
\hline 604.61 & 1.334434 & 1.334406 & -0.272 \\
\hline 611.80 & 1.334212 & 1.334208 & -0.038 \\
\hline 632.82 & 1.333604 & 1.333621 & 0.170 \\
\hline
\end{tabular}

TABLE 6.2 - Comparison of $n$ measured by HCR and Engage $\left(\mathrm{T}=24^{\circ} \mathrm{C}\right)$.

One important factor in the comparison of HCR and Engage data is the temperature of the sample, as the $\mathrm{dn} / \mathrm{dT}$ of saline is approximately $-0.0001 /{ }^{\circ} \mathrm{C}$, which is coincidentally the same as the goal of the measurement accuracy. The temperature of the sample must always be known to better then one degree Celsius in order to reach the desired accuracy. The above measurements were both made at $\mathrm{T}=24^{\circ} \mathrm{C}$, but temperature must always be monitored during calibration measurements. The temperature is currently monitored in both Engage and the HCR with thermocouples. These are connected to the computer with a USB-TC (Measurement Computing) so that the sample temperature can be automatically recorded for every measurement.

Clearly, the HCR data makes the determination of the index of saline from Engage measurements easy, but it would be advantageous to not require a HCR measurement with every contact lens sample measurement. In fact, other information from the 
"Christmas tree plot", the dispersion curve, and even the group index of refraction can be used to determine the correct $\Delta \mathrm{m}$.

As discussed earlier, the first derivative of the dispersion curve will always be negative while the second derivative should be positive. From Figure 6.15, it is clear that the nearest refractive index orders all have negative first derivatives. Over the spectral range of the visible, the second derivative is clearly positive (Figure 6.3c), but from the 5 values of the $\mathrm{HeNe}$ laser, the second derivative becomes extremely sensitive to $\mathrm{n}_{611}$ relative to $\mathrm{n}_{604}$. For example, if $\mathrm{n}_{611}$ is off by as little as 0.00002 , the sign of the point by point second derivative will be negative for one location. The second derivative test is actually too sensitive to be a reliable metric, due to the close spectral proximity of the laser wavelengths used.

The sign of the first derivatives of the possible dispersion curves is consistent while the magnitude changes due to the spectral dependence of $\Delta \mathrm{n}_{\Delta \mathrm{m}=1}$. In fact, the shape of the dispersion curve at the correct value for $\Delta \mathrm{m}$ is consistent, even if the offset of the curve changes due to effects such as temperature. Although $\mathrm{dn} / \mathrm{dT}$ does have some spectral dependence, the majority of the effect of a temperature change is an offset of the curve, not a change in the shape of the curve (Tilton, 1938).

$$
\begin{aligned}
& \frac{d n_{546}}{d T_{{ }^{\circ} \mathrm{C} @ 25^{\circ}}}=-0.0000978 \\
& \frac{d n_{656}}{d T_{{ }^{\circ} \mathrm{C} @ 25^{\circ}}}=-0.0000959
\end{aligned}
$$


Therefore, it should be possible to establish a baseline dispersion curve for saline based on a series of measurements with the HCR and with Engage, and compare the dispersion curves for the various orders of $\Delta \mathrm{m}$ until they are aligned. Having a reference dispersion curve means that an HCR measurement is not required for every Engage measurement. The file contains typical index values that are used to compare dispersion curves (Appendix F). In Figure 6.16, the green lines are this reference dispersion curve plotted on top of the data for three values of $\Delta \mathrm{m}$, where the indices at $\lambda=604 \mathrm{~nm}$ are always set equal for easier comparison of the curves. Based on this comparison, the center curve is the correct dispersion curve and hence the correct value for $\Delta \mathrm{m}$.

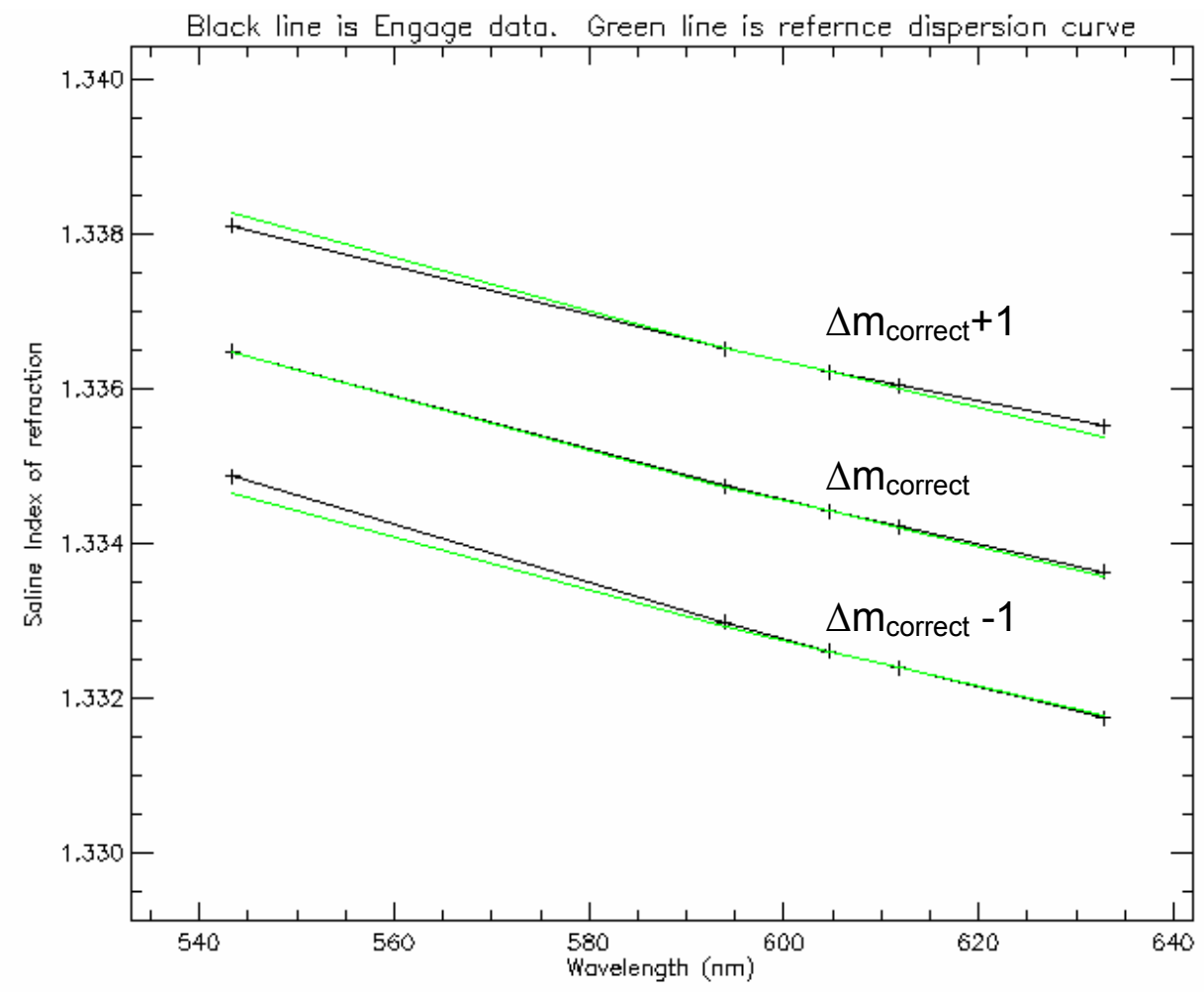

FIGURE 6.16 - A reference dispersion curve for saline can be used to determine $\Delta \mathrm{m}_{\text {correct. }}$ 
This comparison is typically done by the operator, although by comparing the quantity $\left(\mathrm{n}_{543}-\mathrm{n}_{632}\right)$ for the orders for $\Delta \mathrm{m}$ with the same quantity for the reference dispersion curve, the correct curve can be identified (Table 6.3). For the reference dispersion curve for saline, $\left(\mathrm{n}_{543}-\mathrm{n}_{632}\right)=0.002879$.

\begin{tabular}{|c|c|}
\hline$\Delta \mathrm{m}$ & $\mathrm{n}_{543}-\mathrm{n}_{632}$ \\
\hline$\Delta \mathrm{m}_{\text {correct }}+1$ & 0.002591 \\
\hline$\Delta \mathrm{m}_{\text {correct }}$ & 0.002857 \\
\hline$\Delta \mathrm{m}_{\text {correct }}-1$ & 0.003124 \\
\hline
\end{tabular}

TABLE 6.3 - Comparing $\left(\mathrm{n}_{543}-\mathrm{n}_{632}\right)$ can also yield $\Delta \mathrm{m}_{\text {correct }}$.

Once this reference file has been established, there is no need to independently measure the index of saline on another instrument in order to determine the correct value of $\Delta \mathrm{m}$ for saline.

\subsection{Index Calculation - Contact Lens Materials}

The equation used to calculate the index of refraction of the sample of contact lens material is similar to that used for $\mathrm{n}_{\text {saline }}$.

$$
n_{\text {sample }}\left(\lambda_{\text {laser }}\right)=\frac{\lambda_{\text {laser }}}{t_{\text {sample }}(x, y)}\left[\Delta m+\frac{\left[\varphi_{\text {sample }}(x, y)-\varphi_{\varepsilon}(x, y)\right]-\varphi_{\text {saline }, f i t}(x, y)}{2 \pi}\right]+n_{\text {saline }}
$$

Equation 6.30

Any errors in $\mathrm{n}_{\text {saline }}$ translate directly into errors for $\mathrm{n}_{\text {sample. }}$. The thickness of the sample is obtained through several steps with the low coherence surface location information, which depend on the physical thickness and the group index of refraction, $\mathrm{N}_{\mathrm{g}}$. One

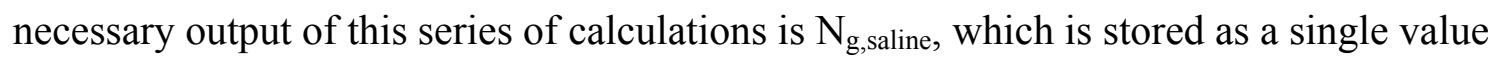




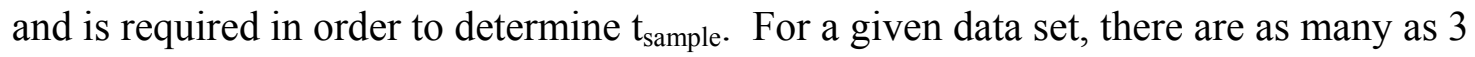
different methods for determining $\mathrm{N}_{\mathrm{g}, \text { saline }}$ at $\lambda_{\mathrm{SLD}}=681 \mathrm{~nm}$.

The first method only uses the low coherence data to determine $\mathrm{N}_{\mathrm{g} \text {,saline }}$ and was described earlier. First, the thickness of the air column is calculated from the LCI data and extrapolated across the field of view. The separation of the plates in a column of saline is

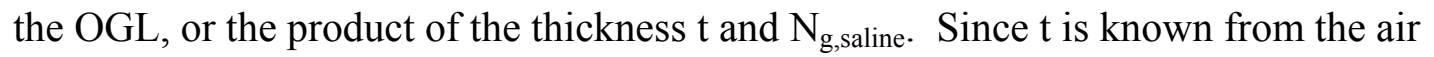
column, a simple division yields the group index of saline. This value is computed as an average of the valid data points, and an associated standard deviation is recorded as well. For this data set, $\mathrm{N}_{\mathrm{g}, \text { saline }}$ is 1.34744 with $\sigma=2.66 \times 10^{-4}$.

$$
N_{g, \text { saline }}=\operatorname{Mean}\left(\frac{O G L_{\text {saline }}(x, y)}{t_{\text {cuvette }}(x, y)}\right)
$$

The second method uses the values calculated for $n_{\text {saline }}$ as a function of wavelength. A dispersion curve is fit to these values using a power series and extrapolated out to $\lambda_{\mathrm{SLD}}=$ $681 \mathrm{~nm}$. Here, the phase index is found along with the local slope, allowing $\mathrm{N}_{\mathrm{g} \text {,saline }}$ to be calculated directly using the definition for $\mathrm{N}_{\mathrm{g}}$.

$$
N_{g, \text { saline }}=n_{\lambda}-\left.\lambda \frac{d n}{d \lambda}\right|_{\lambda}
$$

The curve fitting is fine for finding values between the extreme wavelengths of $543 \mathrm{~nm}$ and $632 \mathrm{~nm}$. Over this $90 \mathrm{~nm}$ span, the effects of the higher order terms of the dispersion curve are subtle and difficult to predict accurately. When this curve is extrapolated out to $681 \mathrm{~nm}$, the fit will not be perfect. The data used to compute the group index are the phase indices from the Engage measurement, which in turn depend on the air column 
thickness and the air and saline phase measurements. Therefore it is not an entirely independent method but can still serve as a useful comparison. The calculated group index for this data set using this method is 1.34728 .

The third method for determining $\mathrm{N}_{\mathrm{g}, \text { saline }}$ only uses data from the HCR, and so can only be calculated when HCR data has been collected. The same curve fitting routine is used as in the second method, and the resulting value for this data set is 1.34734 .

Table 6.4 summarizes the resulting saline group index values for the three methods and the data used to obtain them. Methods 1 and 2 are not entirely independent as both use $\mathrm{t}_{\text {air }}$ while method 3 is independent, coming from a different instrument. Methods 2 and 3 employ curve fitting routines to find values outside the available data, which are sensitive to index errors.

\begin{tabular}{|c|c|c|}
\hline Method & Data required & $\mathrm{N}_{\mathrm{g} \text { saline }}$ \\
\hline 1 & $\mathrm{t}_{\text {air }}, \mathrm{OGL}_{\text {saline }}$ & 1.34744 \\
\hline 2 & $\mathrm{t}_{\text {air }}, \varphi_{\text {air }}, \varphi_{\varepsilon}, \varphi_{\text {saline }}$ & 1.34728 \\
\hline 3 & HCR data & 1.34734 \\
\hline
\end{tabular}

TABLE 6.4 - Three methods are available for calculating $\mathrm{N}_{\mathrm{g}, \text { saline. }}$.

The group index of saline varies by as much as 0.00016 between the methods, but as will be shown, the final phase index, $\mathrm{n}_{\text {sample }}$, is less affected by errors in $\mathrm{N}_{\mathrm{g} \text {,saline }}$ than errors in $\mathrm{n}_{\text {saline. }}$ A large difference between the values found from methods 1 and 2 would be an indication of an error in the measurement. 
The thickness measurement in the column containing the sample will encounter 3 surfaces: saline1 (glass/saline), saline2 (saline/sample) and saline3 (sample/glass). The OGL between saline1 and saline2 is divided by $\mathrm{N}_{\mathrm{g}, \text { saline }}$ to determine the physical thickness of the saline. This is subtracted from the extrapolated cuvette thickness to determine the physical thickness of the sample.

$$
t_{\text {sample }}(x, y)=t_{\text {cuvette }}(x, y)-\frac{O G L_{\text {saline } 2}(x, y)-O G L_{\text {saline } 1}(x, y)}{N_{g, \text { saline }}}
$$
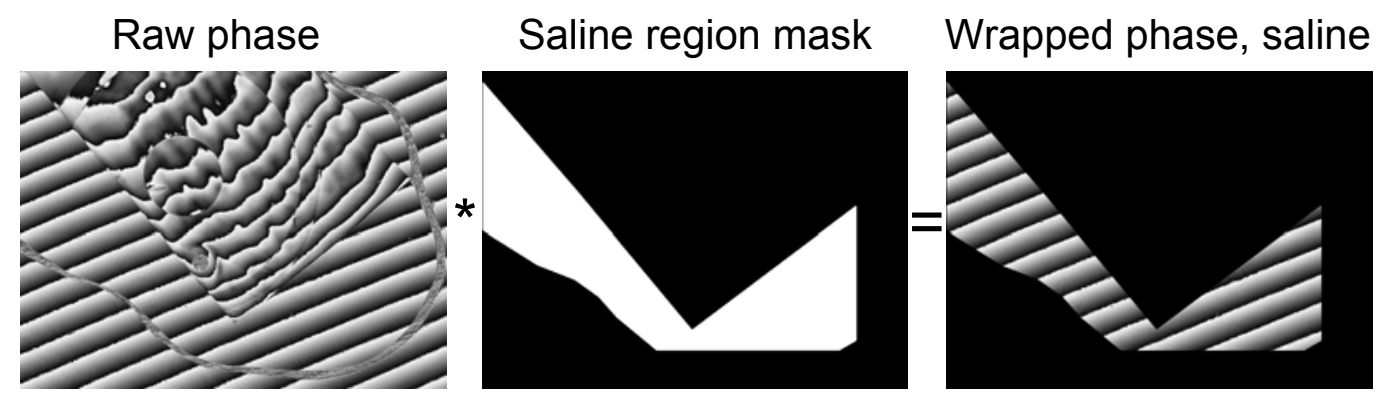

FIGURE 6.17 - The product of the raw phase and the saline region mask gives the saline region that must be unwrapped to obtain $\varphi_{\text {saline }}$.

The phase in the region containing saline (Figure 6.17) has already been unwrapped, but since $\mathrm{n}_{\text {saline }}$ is constant as a function of position (assuming the saline is homogenous) and the thickness of the column only varies linearly, a plane can be used to represent this phase. This planar fit to the saline phase, $\varphi_{\text {saline,fit }}$, is found after the interferometer baseline error $\varphi_{\varepsilon}$ has been subtracted (Figure 6.18). 


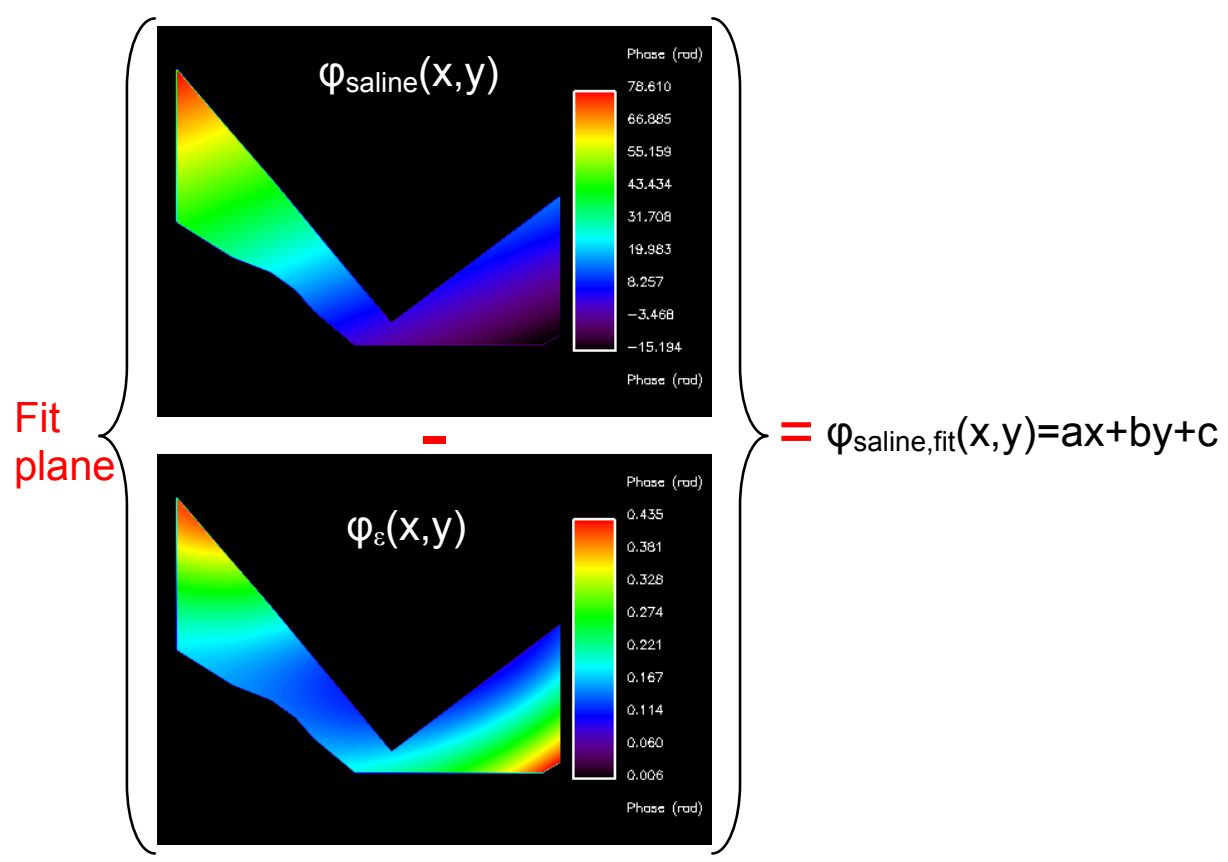

FIGURE 6.18 - A plane is fit to $\varphi_{\text {saline }}$ after $\varphi_{\varepsilon}$ has been subtracted.

The next component of Equation 6.30 that can be found is the phase in transmission through the sample, $\varphi_{\text {sample. }}$ In the case of a stepped height sample, the phase through each (in this case 4) thickness region must be unwrapped separately, since there are unknown phase jumps at the region boundaries. In addition, the phase must be unwrapped separately at each wavelength (5 laser wavelengths). The geometry in Figure 6.19 results in $4 * 5=20$ sample regions to unwrap. 


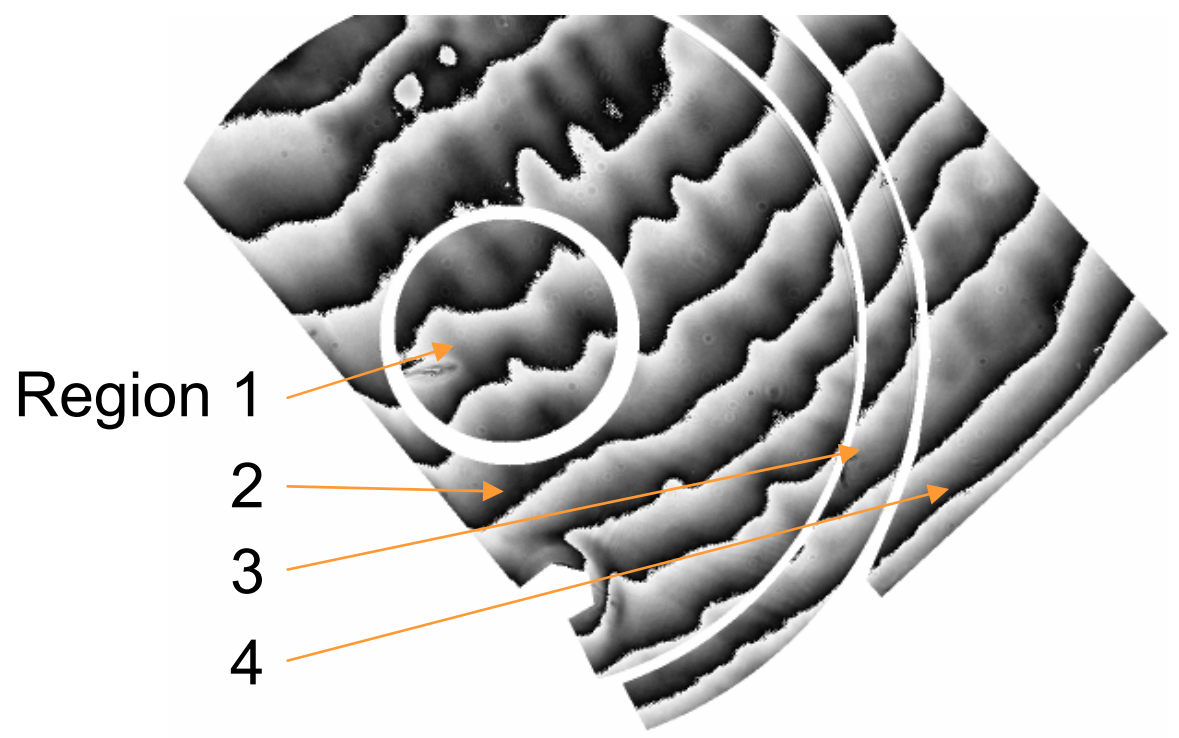

FIGURE 6.19 - The phase in each thickness region must be unwrapped individually.

Everything required for calculating $n_{\text {sample }}$ has now been found with the exception of $\Delta \mathrm{m}$, which is likely be different for each of the 4 regions. As was discussed in describing the multiple thickness technique, there will be a set of index values from the four regions that will all agree for a set of $\Delta \mathrm{m}$ values, and this will be the correct refractive index. Table 6.5 shows the average thicknesses of the 4 regions along with the index steps at $\lambda=543$ $\mathrm{nm}$.

\begin{tabular}{|c|c|c|}
\hline Region & Average $\mathrm{t}(\mu \mathrm{m})$ & $\Delta \mathrm{n}_{\Delta \mathrm{m}=1}(\lambda=543 \mathrm{~nm})$ \\
\hline 1 & 133.814 & 0.00406 \\
\hline 2 & 139.801 & 0.00389 \\
\hline 3 & 156.065 & 0.00348 \\
\hline 4 & 178.863 & 0.00304 \\
\hline
\end{tabular}

TABLE 6.5 - Average thicknesses of the 4 regions and $\Delta \mathrm{n}_{\Delta \mathrm{m}=1}$ for each. 
Four thickness regions offer many noise ceiling values, depending on the two regions used. The largest $\mathrm{NC}$ is between regions 1 and 4 , and is 0.00102 , or more than twice the required minimum of 0.0005 .

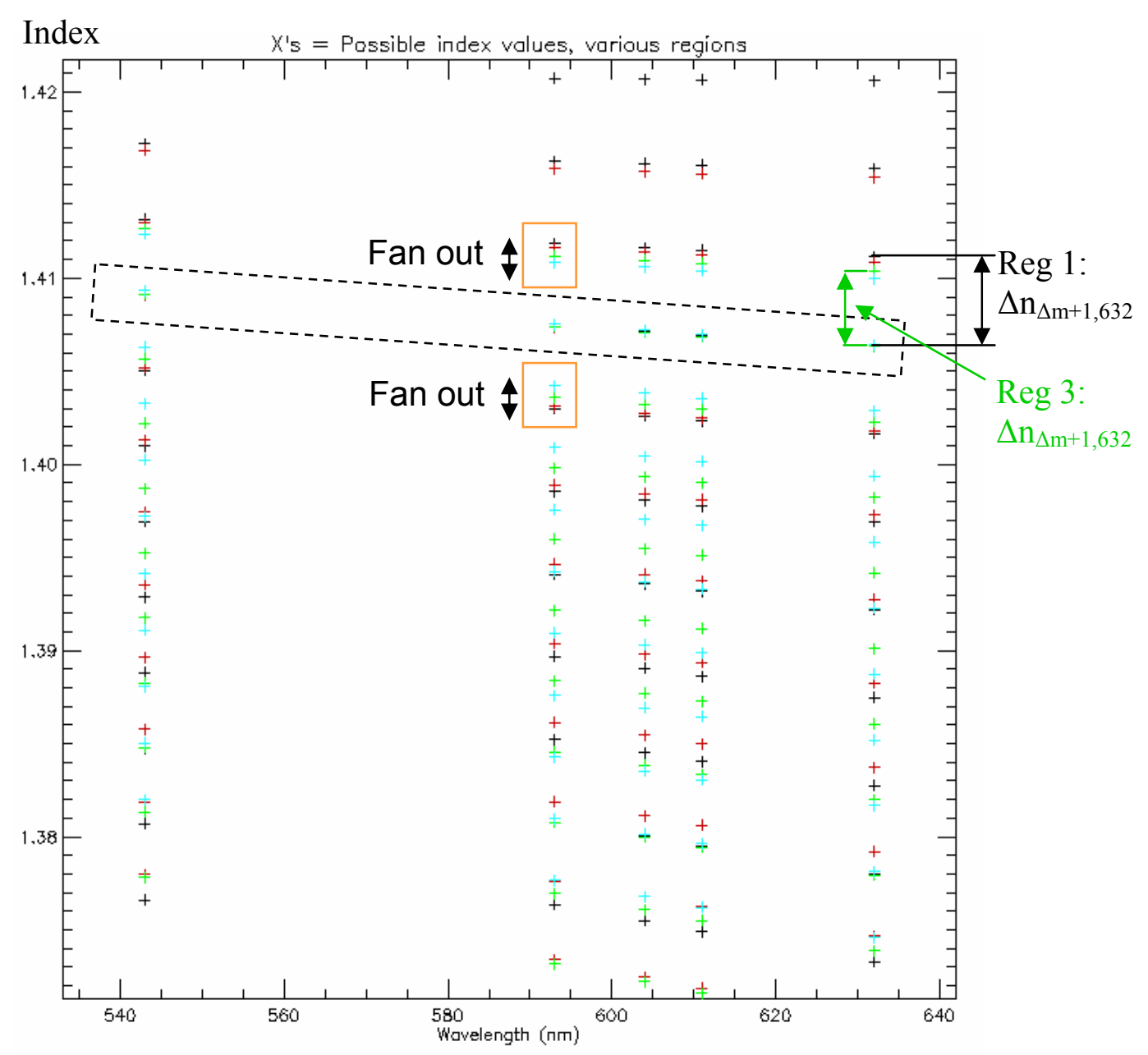

FIGURE 6.20 - Christmas tree plot for contact lens material. The orders outlined by the dashed box line up for all 4 thickness regions. Note how the index values for the multiple regions 'fan out' at $+/-1$ in $\Delta \mathrm{m}$.

The "Christmas tree plot" for the index of this 'inverted wedding cake' contact lens material sample is considerably more complicated than the plot for saline because there are now 4 regions represented simultaneously, distinguished by the color of the "+" 
symbol (black $=1, \mathrm{red}=2$, green $=3$, teal $=4)$ (Figure 6.20). There are no longer lines plotted representing data collected from other instruments. The dashed box indicates the correct orders of $\Delta \mathrm{m}$ lining up at the same refractive index values. It may be difficult to see that all 4 " $+"$ symbols are there at this index value, but looking at the orders above and below and observing that the "+" signs fan out with the thinnest regions fanning farthest indicates that they are all present.

By clicking the mouse on groups of "+" signs above $\lambda=543 \mathrm{~nm}$, two additional windows are displayed for closer examination of the data. The first window has a plot of the dispersion curves for the four regions to confirm that all 4 indeed line up at this value for refractive index (Figure 6.21).

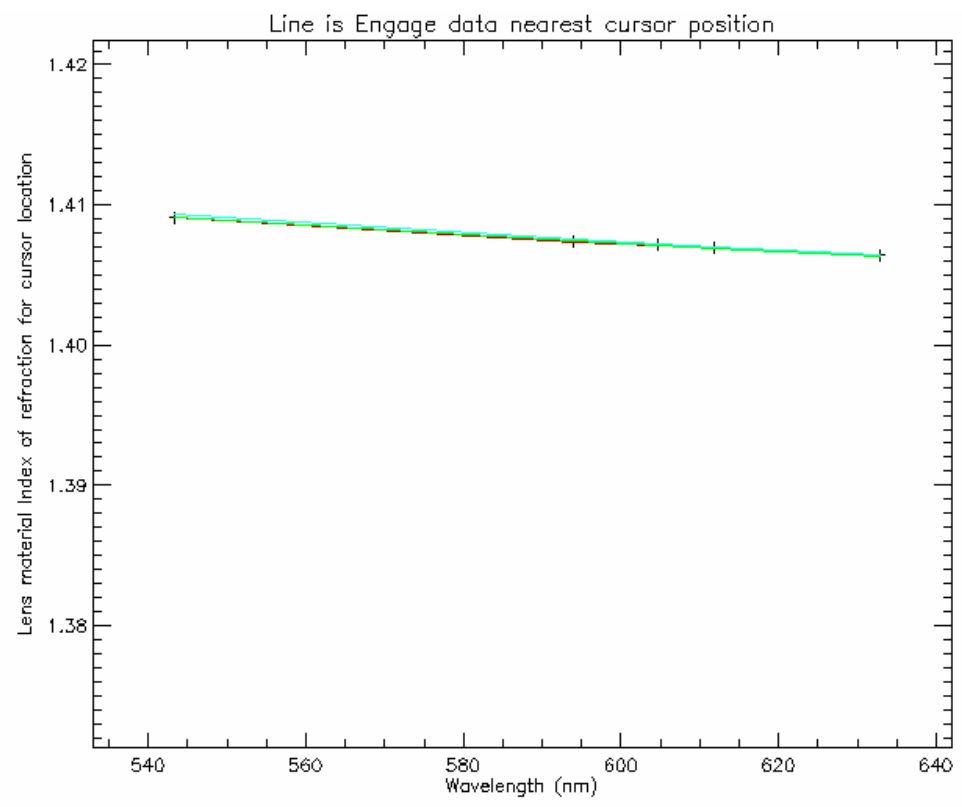

FIGURE 6.21 - First plot shown after user clicks near a set of refractive index values in Figure 6.20, which shows the dispersion curves for all four regions. 
The second window (Figure 6.22) contains many pertinent values about the location selected by the user, including the index at each of the 4 regions for each of the 5 wavelengths. Under each index value is the point by point standard deviation of the index of refraction values in that region multiplied by $1 \times 10^{4}$ to indicate how noisy the data is at each region/wavelength. The third value is the number of valid index measurements for each region/wavelength. The final line of data is the weighted average of the index over the multiple regions at every wavelength.

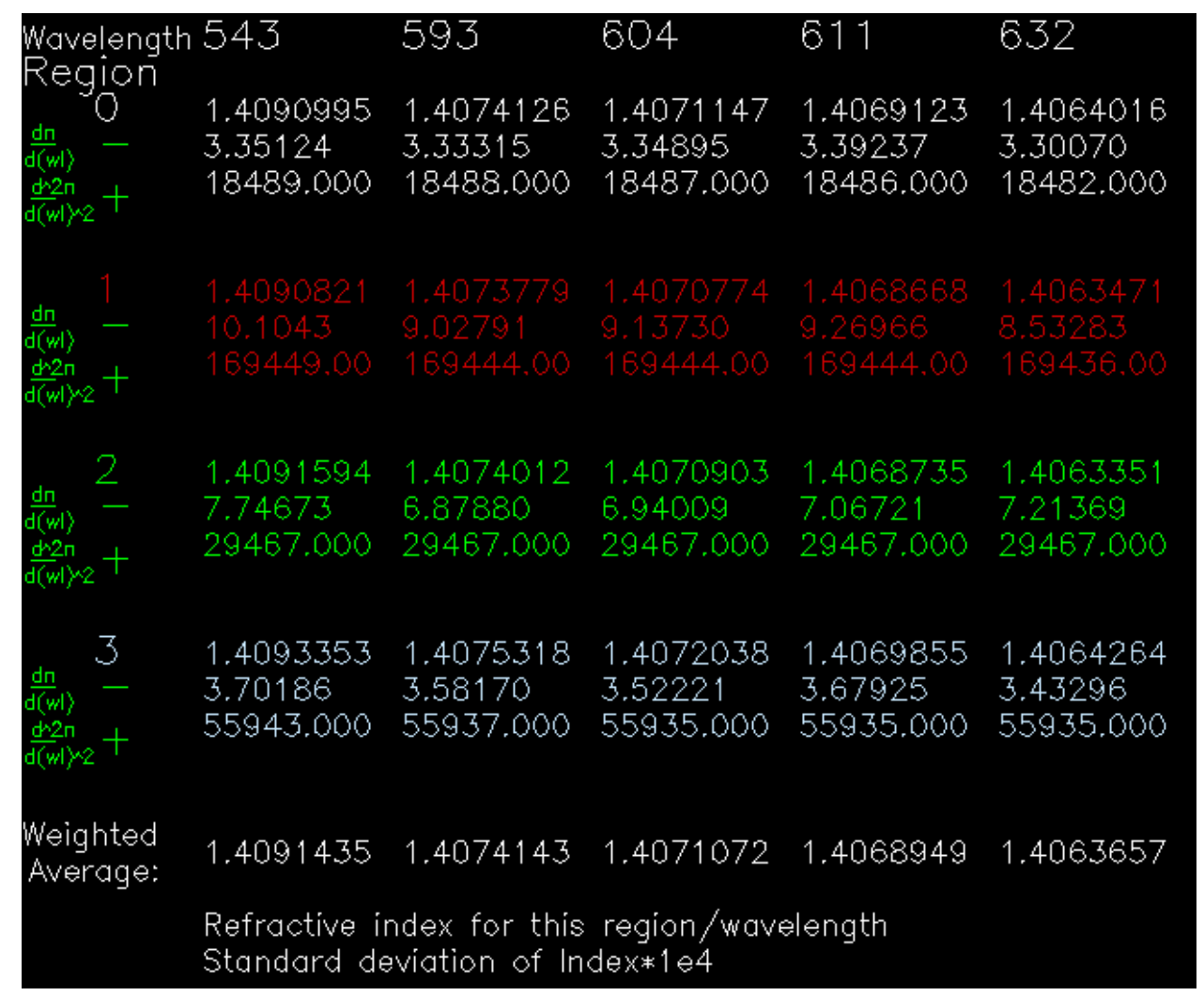

FIGURE 6.22 - Second window, showing calculated index values for each wavelength and region.

In addition, the first and second derivatives are calculated for the dispersion curve for each region, and the sign of both is shown in a column on the left. Recall that the first 
derivative should be negative and the second derivative positive, although the second derivative is too sensitive to noise and often suggests data is incorrect when it is not.

In the example data set shown here, the correct index of refraction values are easily chosen, and this process could occur without user interaction based on analyzing the data according to the various criteria outlined. The 4 regions extend $n_{\text {periodic }}$ large enough that there will not be another location where the index values all overlap.

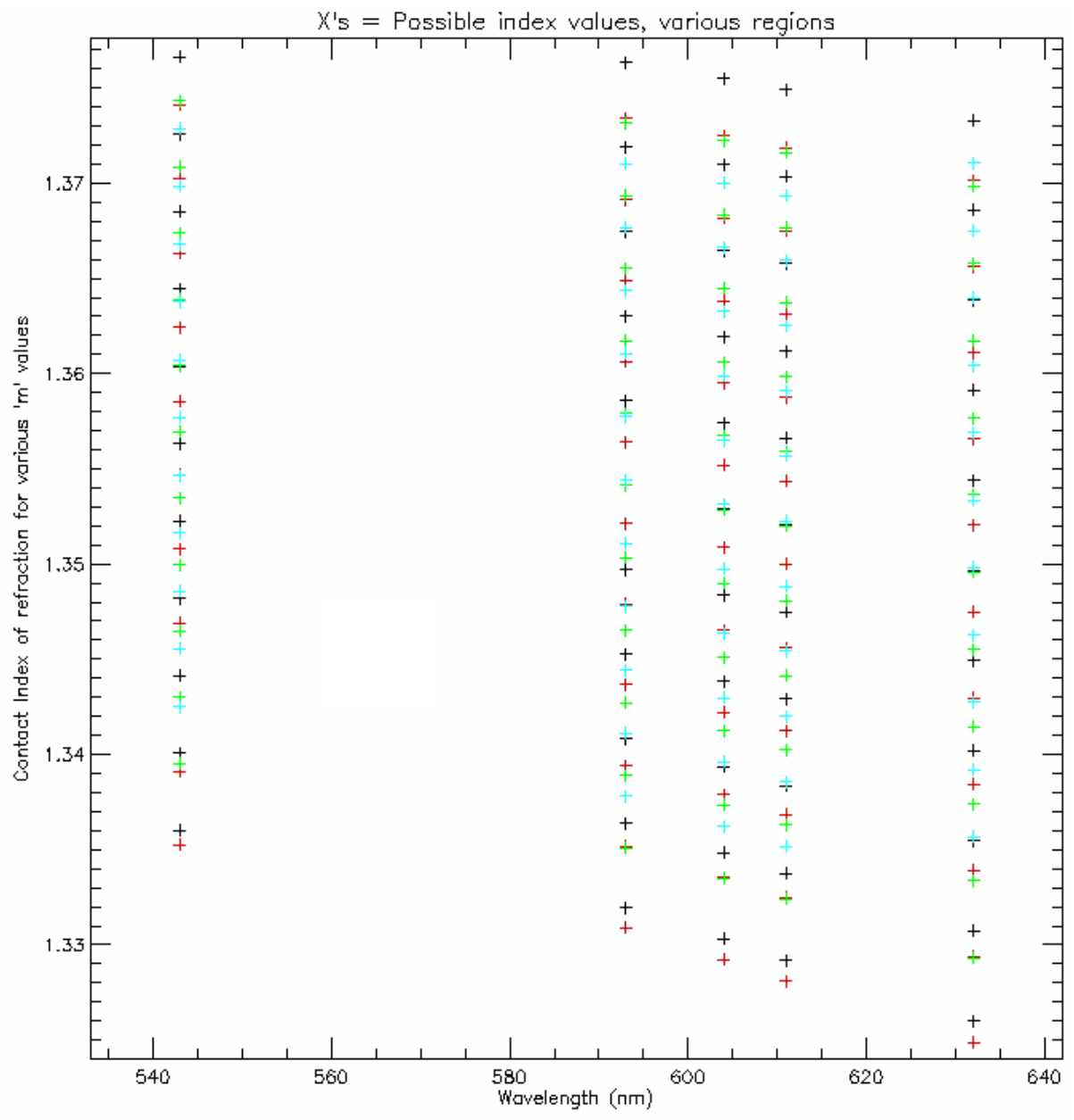

FIGURE 6.23 - Clicking below $\mathrm{x}$-axis recalculates possible index values at lower values for $\mathrm{n}$. The various $\mathrm{n}_{\text {periodic }}$ values for this data set prevent all of the orders from lining up at any value for $\mathrm{n}$ other than the correct one. 
Figure 6.23 is an example of this, showing another "Christmas tree plot" for the same data set at lower index of refraction values. Figure 6.23 appears to be a random scattering of "+" signs, as there are no places where more than 2 appear to line up. The second derivatives of the dispersion curves are also starting to change sign, which is another indication that this plot shows a region of possible index values that are incorrect.

Now that the correct set of values for $\Delta \mathrm{m}$ has been identified by the dashed box in Figure 6.20, they can be inserted into Equation 6.34 to solve for the refractive index of the soft contact lens test sample.

$$
n_{\text {sample }}\left(\lambda_{\text {laser }}\right)=\frac{\lambda_{\text {laser }}}{t_{\text {sample }}(x, y)}\left[\Delta m+\frac{\left[\varphi_{\text {sample }}(x, y)-\varphi_{\varepsilon}(x, y)\right]-\varphi_{\text {saline }, \text { fit }}(x, y)}{2 \pi}\right]+n_{\text {saline }}(\lambda)
$$

Equation 6.34

The weighted averages for the index of the soft contact lens material at the 5 wavelengths are shown in Table 6.6.

\begin{tabular}{|c|c|}
\hline Wavelength (nm) & Refractive Index \\
\hline 543.36 & 1.40914 \\
\hline 593.93 & 1.40741 \\
\hline 604.61 & 1.40711 \\
\hline 611.80 & 1.40689 \\
\hline 632.82 & 1.40637 \\
\hline
\end{tabular}

TABLE 6.6 - Measured index of soft contact lens material as a function of wavelength. 
Future testing may include measurement samples that are not sectioned into regions of different physical thickness, such as a portion of a contact lens. In this case, the user will be prompted to locate a minimum of 2 regions within the sample to be used as the 'multiple thickness' regions. As long as the average thickness difference varies between the regions, the noise ceiling will be greater then zero. The recommended geometry for region selection of a contact lens is concentric rings about the center of optic zone, as shown in Figure 6.24. This should ensure that the average thickness difference for the regions is as large as possible.
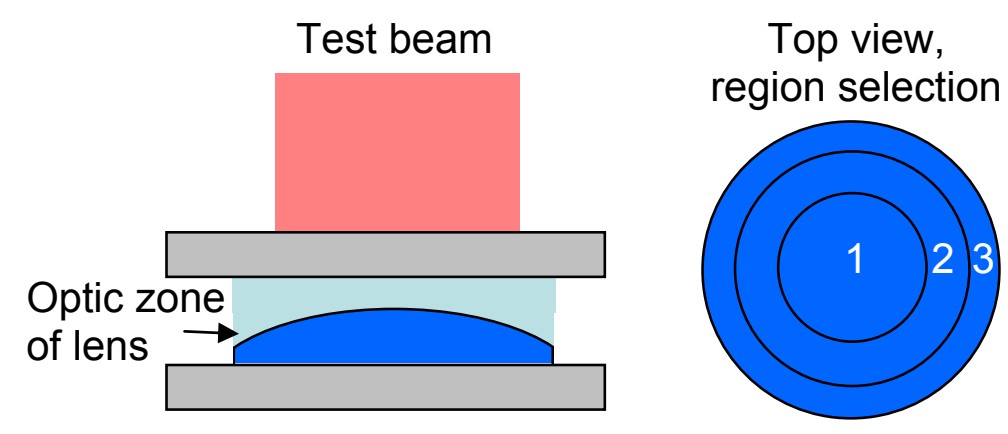

FIGURE 6.24 - The optic zone of a contact lens tested in Engage, along with the concentric ring structure for selecting multiple thickness regions.

\subsection{Removing Bad Regions from the Measurement}

Data from several different sources is used to make the final refractive index calculation, and at every step along the way, there are bound to be data points or regions that are invalid. Some of these regions can be removed automatically with software while others require some user interaction to select the regions, but all of them need to be removed in order to obtain the most accurate values for refractive index. A few of the region 
removal tests, such as regions in the LCI measurement with insufficient fringe visibility, have been discussed already, but most have not.

Of those that remain, one of particular importance is verification that the sample is truly in contact with the bottom plate of the cuvette. The physical thickness of the sample is found by subtracting the saline thickness above the sample from the cuvette thickness. If the sample is not touching the bottom plate, the thickness of the extra saline beneath the sample will be falsely included as part of the sample thickness, resulting in $t_{\text {sample }}$ being too large in the area of the saline bubble.

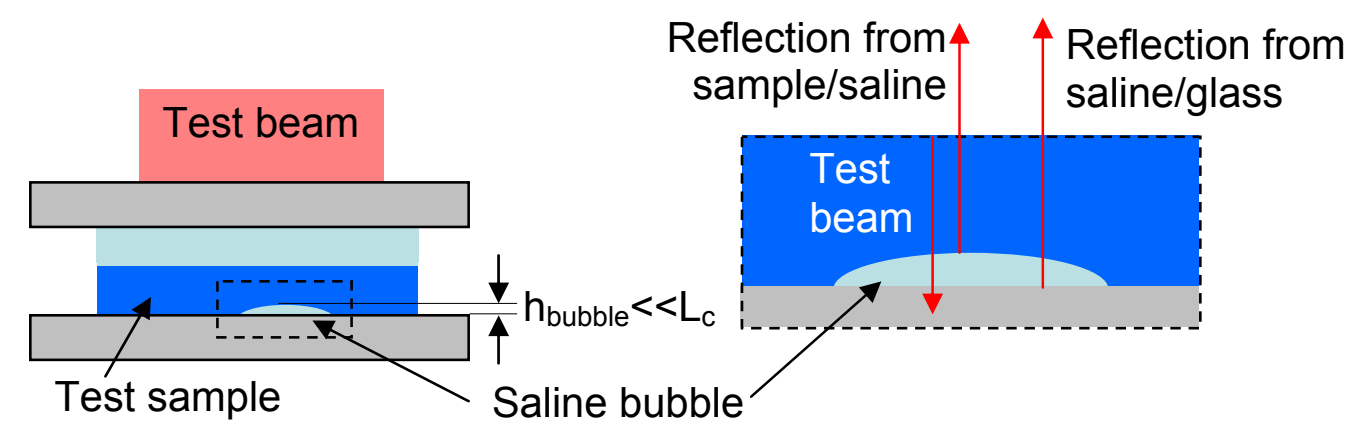

FIGURE 6.25 - A saline bubble trapped beneath the sample will make $t_{\text {sample }}$ too large.

Consider the case illustrated in Figure 6.25, where a section of the sample has lifted up off the surface, and a small amount of saline is between the sample and the glass. The height of the bubble is no more than a few microns high and is too small to be resolved in depth by the low coherence interferometer, which requires surfaces to be separated in OGD by at least $50 \mu \mathrm{m}$ (this value depends on the coherence length of the SLD). Instead of a single beam reflecting from the sample/glass interface, there are now two beams: one from the sample/saline interface and another from the saline/glass interface. Since the 
bubble height is much less then the SLD coherence length, $1_{c}$, these two beams are temporally coherent and will cause static fringes at the detector, regardless of the location of the reference surface of the low coherence interferometer. Figure 6.26 is a single image with the reference surface positioned such that there are no fringes between the test and reference beams. The circled regions have the static fringes indicative of a saline bubble.

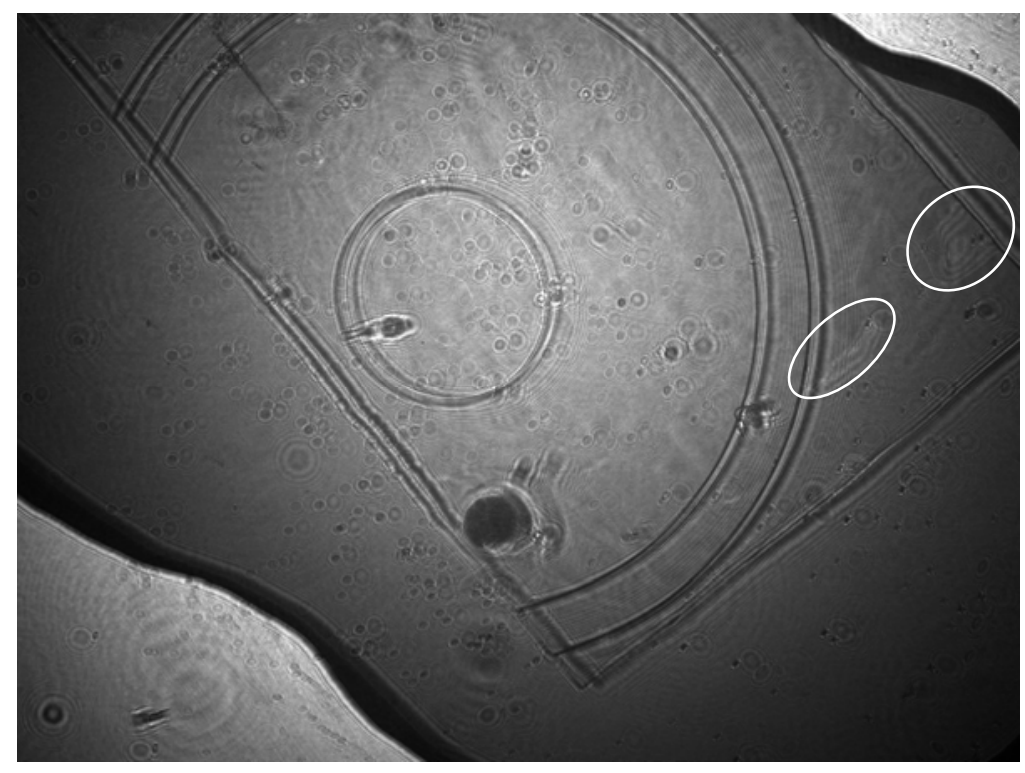

FIGURE 6.26 - Image from LCI data cube showing sample regions that are not sticking to the glass plate at the bottom of the cuvette.

Two regions are indicated as areas with these static fringes. The one on the right has about 4 waves of OGD, so the saline bubble height is about $1 \mu \mathrm{m}$.

$$
h_{\text {bubble }}=\frac{1}{2} \frac{(\# \text { fringes }) \lambda_{S L D}}{N_{g, \text { saline }}}=\frac{1}{2} \frac{(4) 681}{1.347}=1.01 \mu \mathrm{m}
$$

In order to determine the thickness of the sample in these regions, the height of the bubble must be known to a few tens of nanometers. It is difficult if not impossible to 
determine the height of these bubbles to the nearest $20 \mathrm{~nm}$ from a single image, so any region in a data set containing these static fringes must be removed. The presence of too many saline bubble features can be detected before any data is actually collected, and is reason enough to remount the sample prior to measuring it. These details are discussed in Appendix G. Almost every data set will have some region that is not sticking to the glass, and there needs to be an easier method for finding their location than from images like Figure 6.26.

Another result of a location in the image where the sample is not sticking to the glass is that more light is reflected there than in nearby areas. The irradiance difference is small compared to the overall background irradiance, and is not enough to use as a consistent metric.

As the reference mirror scans the plane of zero OGD through this depth region (saline3), two LCI signals will be generated simultaneously in regions with a saline bubble; one from each interface. These two LCI signals will also interfere with each other, but their phase relationship is fixed by the height of the saline bubble. If the two LCI signals are in phase, the maximum visibility of the combination will be larger then the LCI signals from nearby regions without saline bubbles. The extra fringe visibility increases the standard deviation of the signal at that pixel. A series of 160 images are captured at this interface, and by simply calculating the standard deviation of all the values for each pixel versus depth, the regions that are not sticking become clear (Figure 6.27). 


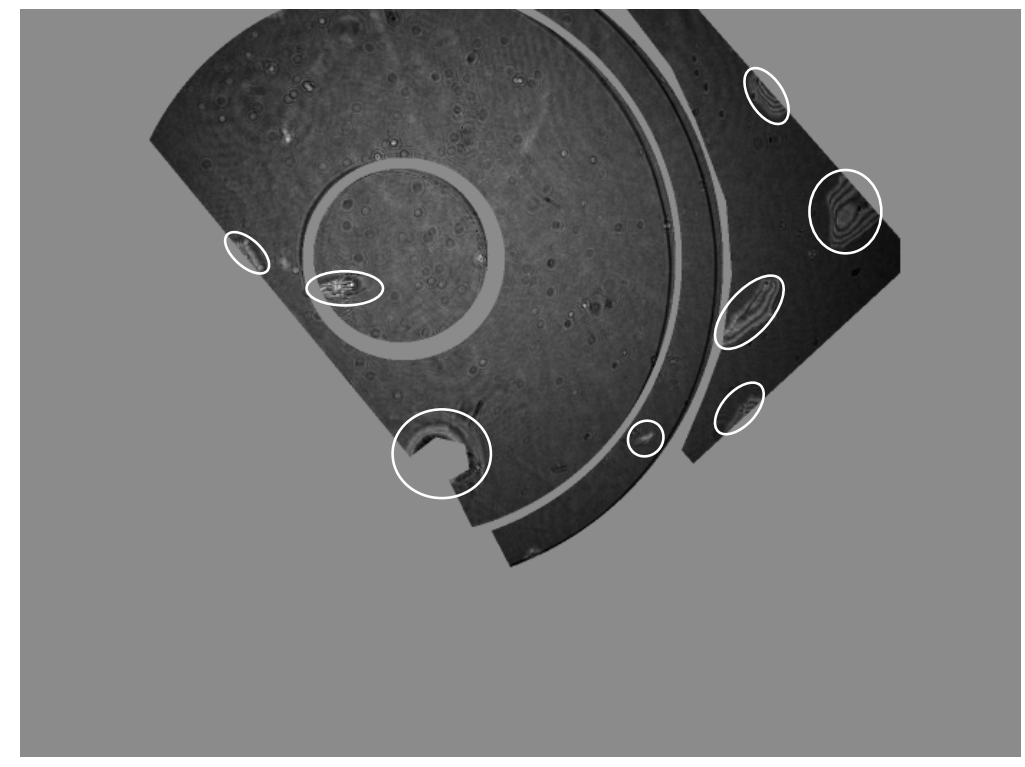

FIGURE 6.27 - Masked standard deviation image. The circled regions show rapid variations in the standard deviations consistent with the varying phase relationship for the two reflected beams from a saline bubble.

In fact, more regions are evident in this view then the trained eye can find on a raw image. These regions are removed by the user using on an image similar to Figure 6.27.

\subsection{Graphical User Interface (GUI)}

Up to this point of this dissertation, there has been a full description of the theory and the hardware required to measure the refractive index of soft contact lens materials. When appropriate, there has been some discussion of the software required to keep all of the various measurement tasks organized. At the top of the software flow chart is the Graphical User Interface, or GUI, that controls data collection and analysis. A screenshot of the GUI used for Engage is shown in Figure 6.28. 


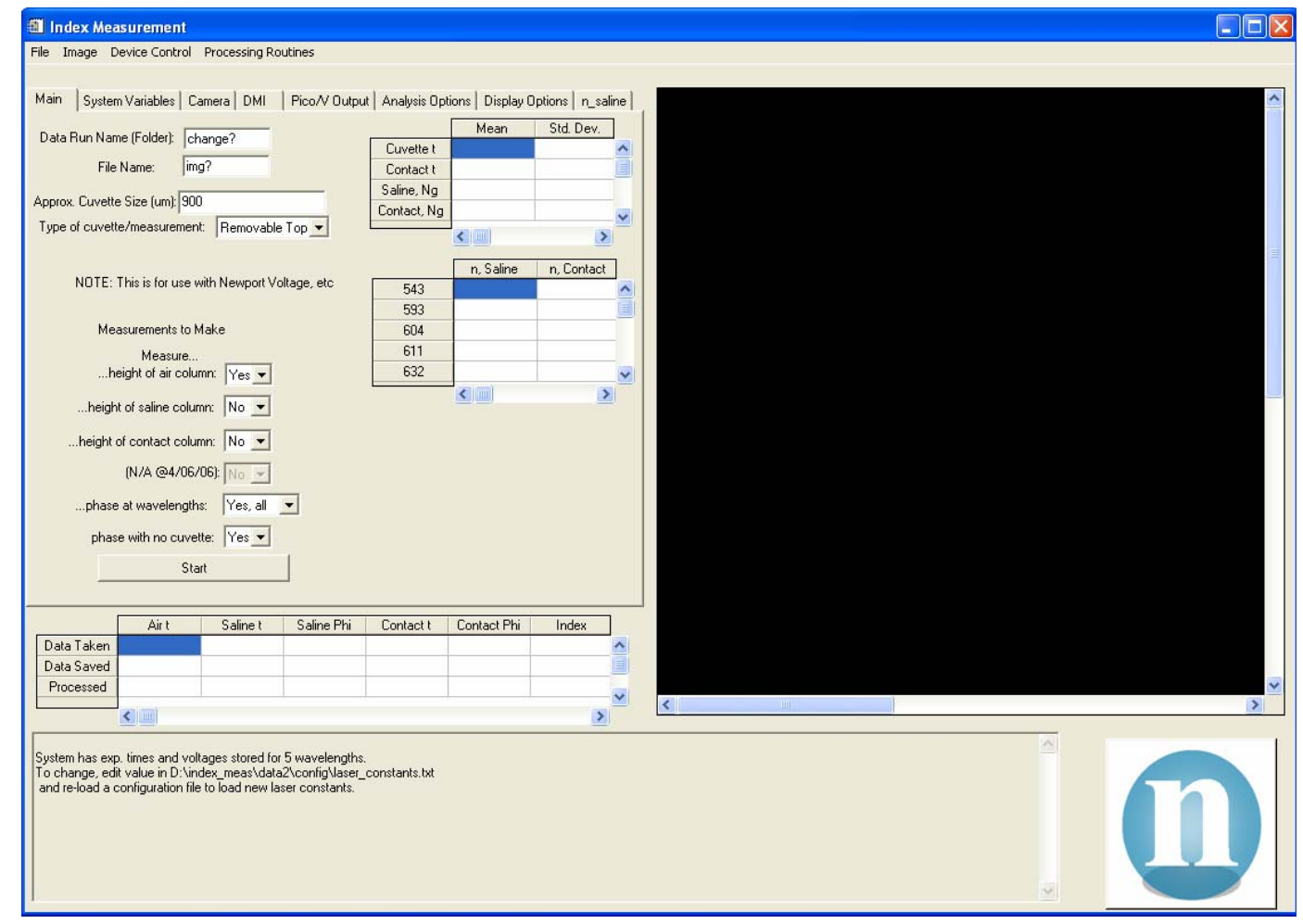

FIGURE 6.28 - GUI used for both data collection and analysis for Engage.

Every function required to complete a measurement is called from this GUI which was written in IDL. From a software perspective, the measurement is broken down into 3 major steps: Step 1 is the data collection, Step 2 is the processing of the LCI signals, and Step 3 is the final data processing required to obtain the index. For the purposes of this discussion, we will cover the parameters that must be specified using the GUI along with the basic controls used to run a measurement. These controls are accessed via menu items or by using the fields and buttons on the various tabs, like those in Figure 6.28. 


\subsubsection{GUI Menu Items}

When the GUI is created, the first thing the user must do is open a configuration file using the menu item File $\rightarrow$ Open config file... . This function will prompt the user to select the text file containing the necessary information. The information in this file is used to set many of the text boxes and dropdown menus to appropriate values so that the user does not need to set up everything manually. Once this procedure has been done, all other GUI options are valid. The line by line contents of "config2.txt" are shown in Table 6.7.

\begin{tabular}{|c|c|c|}
\hline Parameter & Starting Value & Range \\
\hline Folder/measurement name & change? & Different for each data set \\
\hline LCI image file prefix & img & No need to be changed \\
\hline $\begin{array}{c}\text { Estimate of cuvette } \\
\text { thickness }\end{array}$ & $900 \mu \mathrm{m}$ & No longer used \\
\hline $\begin{array}{l}\text { System path to system data } \\
\text { folder }\end{array}$ & $\begin{array}{c}\text { D: } \begin{array}{c}\text { lindex_meas } \\
\mid \text { data } 2 \backslash\end{array} \\
\end{array}$ & Computer dependent \\
\hline $\begin{array}{l}\text { System path to system data } \\
\text { folder (Double 'l') }\end{array}$ & $\begin{array}{l}\text { D: } \| \text { index meas } \\
\| \text { data } 2 \backslash\end{array}$ & $\begin{array}{c}\text { Identical to above except } \\
\text { ' } \mid \text { ' replaces ' } \mid \text { ' }\end{array}$ \\
\hline $\begin{array}{c}\text { System path to folder } \\
\text { containing system DLL's }\end{array}$ & $\begin{array}{l}\text { D: } \mid \text { index_meas } \\
\mid \text { Main2lc_code } \mid\end{array}$ & Computer dependent \\
\hline $\begin{array}{l}\text { System path to file with } \\
\text { LCI stage correction, } \varepsilon_{\mathrm{pos}}\end{array}$ & $\begin{array}{c}\text { D: }: \text { index_meas } \backslash \\
\text { data2 } 2 \text { config } \backslash \\
\text { pos_map_az1_nm.tif }\end{array}$ & $\begin{array}{c}\text { Computer and LCI stage } \\
\text { dependent }\end{array}$ \\
\hline Name of main system DLL & index_control_NPT.dll & Depends on DLL name \\
\hline Camera DLL & roper1.dll & Varies with camera \\
\hline DMI DLL & call_zygo2.dll & No longer used \\
\hline Old stage control DLL & move_pico.dll & No longer used \\
\hline Old stage control .exe & move_pico5.exe & No longer used \\
\hline Old phase shift controller & pzt_drive1.dll & No longer used \\
\hline First $\mathrm{x}$-pixel defining $\mathrm{ROI}_{1}$ & 300 & 0-1390 (even) \\
\hline 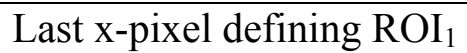 & 799 & $1-1391$ (odd) \\
\hline
\end{tabular}




\begin{tabular}{|c|c|c|}
\hline Binning, $\mathrm{x}$-direction & 1 & 1 \\
\hline First y-pixel defining $\mathrm{ROI}_{1}$ & 200 & $0-1038$ (even) \\
\hline Last y-pixel defining $\mathrm{ROI}_{1}$ & 699 & 1-1039 (odd) \\
\hline Binning, $y$-direction & 1 & 1 \\
\hline $\begin{array}{l}\text { Exposure time for LCI } \\
\text { images (microseconds) }\end{array}$ & $500 \mu \mathrm{s}$ & $1-32000 \mu \mathrm{s}$ \\
\hline $\begin{array}{l}\text { Number of LCI images at } \\
\text { each interface (airl, etc) }\end{array}$ & 140 & $\begin{array}{l}\text { 100-180 (automatically } \\
\text { changed for saline2, 3) }\end{array}$ \\
\hline $\begin{array}{l}\text { Images to average at each } \\
\text { LCI location }\end{array}$ & 1 & $\begin{array}{l}\text { 1-16 (significant time cost } \\
\text { if }>4 \text { ) }\end{array}$ \\
\hline First $\mathrm{x}$-pixel defining $\mathrm{ROI}_{2}$ & 0 & $0-1390$ (even, typically 0) \\
\hline Last $\mathrm{x}$-pixel defining $\mathrm{ROI}_{2}$ & 1391 & 1-1391(odd, typically 1391) \\
\hline Binning, $\mathrm{x}$-direction & 1 & 1 \\
\hline First y-pixel defining $\mathrm{ROI}_{2}$ & 0 & 0-1038 (even, typically 0) \\
\hline Last y-pixel defining $\mathrm{ROI}_{2}$ & 1039 & 1-1039(odd, typically 1039) \\
\hline Binning, y-direction & 1 & 1 \\
\hline $\begin{array}{l}\text { Number of PSI steps for } \\
\text { determination of } \varphi(x, y)\end{array}$ & 5 & $\begin{array}{l}5 \text { (only value supported in } \\
\text { software) }\end{array}$ \\
\hline Measure LCI thickness? & 0 (Yes) & 0 or 1 (Yes or No) \\
\hline Unused & $1(\mathrm{No})$ & $1(\mathrm{No})$ \\
\hline Unused & 1 (No) & $1(\mathrm{No})$ \\
\hline Unused & $1(\mathrm{No})$ & 1 (No) \\
\hline $\begin{array}{l}\text { Measure } \varphi(x, y) \text { with } \\
\text { cuvette? }\end{array}$ & $\begin{array}{c}0 \text { (Yes, all } \\
\text { wavelengths) }\end{array}$ & $\begin{array}{l}0 \text { (All), } 1 \text { (None), } 2-6 \text { (only } \\
543,593,604,611 \text { or } 632 \text { ) }\end{array}$ \\
\hline $\begin{array}{l}\text { Measure } \varphi_{\varepsilon}(\mathrm{x}, \mathrm{y}), \text { without } \\
\text { cuvette? }\end{array}$ & $\begin{array}{c}0 \text { (Yes, all } \\
\text { wavelengths) }\end{array}$ & $\begin{array}{l}0 \text { (All), } 1 \text { (None), } 2-6 \text { (only } \\
543,593,604,611 \text { or } 632 \text { ) }\end{array}$ \\
\hline $\begin{array}{l}\text { Measurement type (contact } \\
\text { material or glass) }\end{array}$ & $\begin{array}{l}\text { (1) Removable top } \\
\text { (contact lens sample) }\end{array}$ & $\begin{array}{l}\text { (1) Removable top or }(0) \\
\text { glass standard measurement }\end{array}$ \\
\hline COM port number & COM4 & No longer used (DMI) \\
\hline COM port baud rate & 9600 & No longer used (DMI) \\
\hline COM port bits & 8 & No longer used (DMI) \\
\hline DMI command \#1 & EXSA $1,1, T$ & No longer used (DMI) \\
\hline DMI commnd \#2 & READ? 1, M & No longer used (DMI) \\
\hline $\begin{array}{l}\text { Step size during fringe } \\
\text { finding }(\mathrm{FF}), \mathrm{LCI}\end{array}$ & $9.0 \mu \mathrm{m}$ & $\begin{array}{c}6-12 \mu \mathrm{m} \text { (no need to vary } \\
\text { this parameter) }\end{array}$ \\
\hline $\begin{array}{l}\text { Steps to take during FF at } \\
\text { one time }\end{array}$ & 10 & $8-20$ \\
\hline
\end{tabular}




\begin{tabular}{|c|c|c|}
\hline $\begin{array}{c}\text { Maximum velocity of LCI } \\
\text { stage during FF }\end{array}$ & $1 \mathrm{~mm} / \mathrm{s}$ & $0.01-2 \mathrm{~mm} / \mathrm{s}$ \\
\hline $\begin{array}{c}\text { Acceleration of LCI stage } \\
\text { during FF }\end{array}$ & $5.0 \mathrm{~mm} / \mathrm{s}^{2}$ & $0.1-10.0 \mathrm{~mm} / \mathrm{s}^{2}$ \\
\hline $\begin{array}{c}\text { Distance/frame, LCI data } \\
\text { collection (DC) }\end{array}$ & $425 \mathrm{~nm}$ & $=\frac{1}{2} \frac{5 \lambda_{c, S L D}}{4}$ \\
\hline $\begin{array}{c}\text { Optical encoder position } \\
\text { samples/frame, LCI DC }\end{array}$ & 12 & $1-25$ \\
\hline $\begin{array}{c}\text { Maximum velocity of LCI } \\
\text { stage during DC }\end{array}$ & $0.1 \mathrm{~mm} / \mathrm{s}$ & $0.01-0.5 \mathrm{~mm} / \mathrm{s}$ \\
\hline Acceleration LCI stage DC & $10.0 \mathrm{~mm} / \mathrm{s}^{2}$ & $0.1-10.0 \mathrm{~mm} / \mathrm{s}^{2}$ \\
\hline $\begin{array}{c}\text { Distance to move LCI } \\
\text { stage after final image }\end{array}$ & $0 \mu \mathrm{m}$ & $0-100 \mu \mathrm{m}$ \\
\hline $\begin{array}{c}\text { Stage settling wait time per } \\
\text { LCI image location }\end{array}$ & $355 \mathrm{~ms}$ & $\begin{array}{c}\text { Stage dependent }(355-600 \\
\text { ms for VP-25XL) }\end{array}$ \\
\hline Board \# of output voltage & 1 & No longer used \\
\hline $\begin{array}{c}\text { Channel name for XPS } \\
\text { D/A output (LCI) }\end{array}$ & GPIO2.DAC2 & $\begin{array}{c}\text { Hardware dependent. No } \\
\text { longer used }\end{array}$ \\
\hline Voltage out, DMI trigger & $5000 \mathrm{mV}$ & No longer used \\
\hline Time to leave voltage high & $1 \mathrm{~ms}$ & No longer used \\
\hline Unused & N/A & N/A \\
\hline Channel for output & GPIO.DAC1 & Hardware dependent \\
\hline voltage, PZT control & 192 & Fixed by vendor \\
\hline IP address of XPS (1) & 168 & Fixed by vendor \\
\hline IP address of XPS (2) & 0 & Fixed by vendor \\
\hline IP address of XPS (3) & 254 & Fixed by vendor \\
\hline IP address of XPS (4) & & \\
\hline
\end{tabular}

TABLE 6.7 - Guide to the values in Engage configuration file, "config2.txt".

As noted in the table, many of the configuration file parameters are no longer used, or are legacy items. Many never need to be changed for a given hardware setup. 
File $\rightarrow$ Save Current Config as... : This can be used to take the current contents of the parameters shown in Table 6.7 and save them in a new configuration file with the title entered by the user.

File $\rightarrow$ Quit : Closes down the Engage GUI and IDL.

Image $\rightarrow$ Display data image...: Allows user to select an image for display.

Image $\rightarrow$ Take image...: Unused.

Image $\rightarrow$ Play movie...: Allows user to select a surface (air1, etc) from data set named in

"Data Run Name (Folder):" text box on main GUI panel (Figure 6.28).

Image $\rightarrow$ Display data image...: Unused.

Device Control $\rightarrow$ Initialize Newport Box: Resets XPS controller and homes the LCI stage.

Device Control $\rightarrow$ Return Current Newport Stage Position...: Returns current position of LCI stage in GUI output window (Figure 6.28).

Device Control $\rightarrow$ Return Newport stage to 0...: Return LCI stage to hardware home. Device Control $\rightarrow$ Move Newport stage...: Prompts user to enter a new position for the LCI stage, either absolute (relative to home) or relative (relative to current). Device Control $\rightarrow$ Output Test Voltage...: Send test voltage to channel used for PZT. Device Control $\rightarrow$ Report temperature...: Returns current thermistor temperature in GUI output window.

Device Control $\rightarrow$ Unload Main DLL...: Unloads main DLL from system memory. Device Control $\rightarrow$ Clear text display: Deletes output in GUI output window. Device Control $\rightarrow$ RESET DMI...: Unused 
Device Control $\rightarrow$ Set PZT to 0 Volts: Unused.

Device Control $\rightarrow$ Return Current Picomotor Position...: Unused.

Device Control $\rightarrow$ Return Picomotor to 0...: Unused.

Device Control $\rightarrow$ Move pico...: Unused.

Processing Routines $\rightarrow$ Remove bad regions...: Unused.

\subsubsection{GUI Tabs}

As can be seen from Figure 6.28, there are eight tabs available to access various measurement parameters: Main, System Variables, Camera, DMI, Pico/V Output, Analysis Options, Display Options, and n_saline. As the hardware and software evolved, some of the controls on the various tabs became obsolete. In the following discussions, only the controls that are used in the final system configuration will be discussed. There are three elements of the GUI that are not part of any one tab and that are used: the black window, in which images are displayed; the text output window, which displays useful information during a measurement, and the Engage system logo in the lower right hand corner (Figure 6.28). 


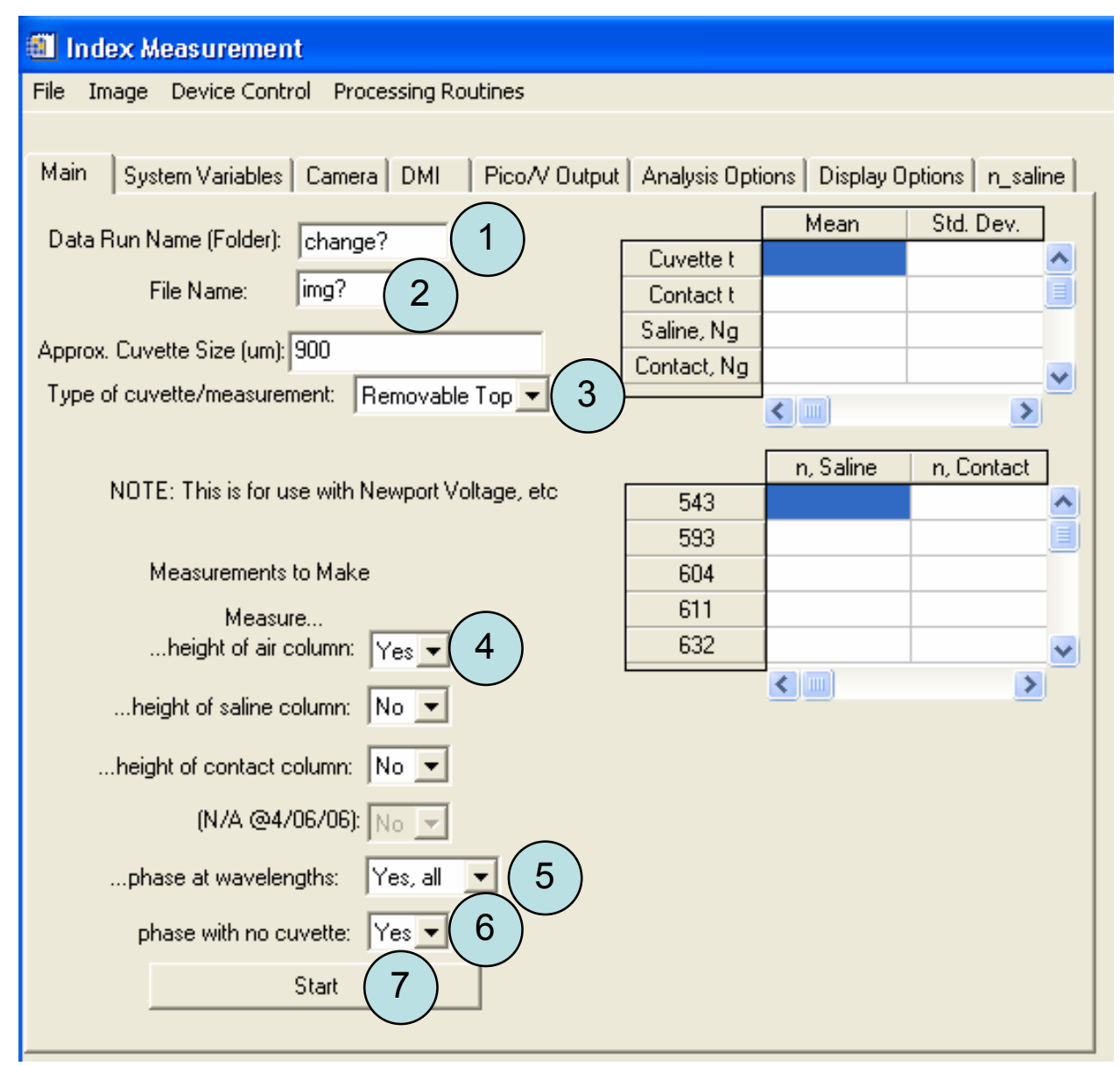

FIGURE 6.29 - Screenshot of "Main" tab of Engage control GUI.

Main (Figure 6.29): The unique name of the current data set goes in the box labeled "Data Run Name (Folder):" (1) along with the file prefix name in the box "File Name:" (2), which does not need to be unique. The "Type of cuvette/measurement" (3) will be "Removable Top" for any contact lens material and "Glass Standard" when measuring a glass calibration sample. To measure the sample thickness using LCI, select "Yes" in the dropdown menu (4). To measure the phase of the cuvette, select "Yes" for (5), and to measure the phase without the cuvette $\left(\varphi_{\varepsilon}(\mathrm{x}, \mathrm{y})\right)$, select "Yes" for (6). To initiate data collection, click the "Start" button (7). 


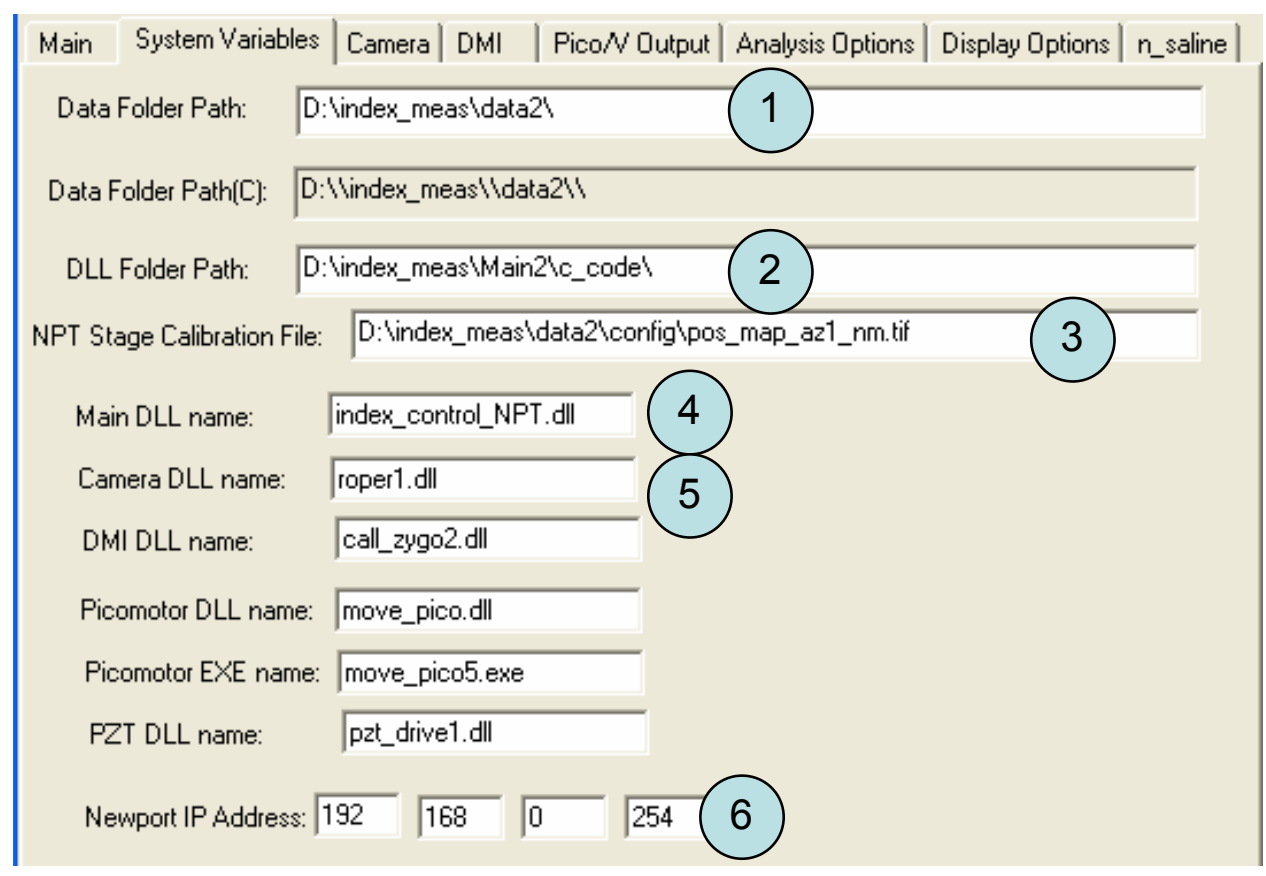

FIGURE 6.30 - Screenshot of "System Variable" GUI tab.

System Variables (Figure 6.30): This tab is used to specify system paths to the main data folder (1), the folder containing the DLL's (2), and the LCI stage correction file (3). Two DLL's are used in the system: the main DLL (4) and the camera DLL (5). The IP address of the XPS stage controller is also specified in this tab (6). These values will normally not change.

Camera (Figure 6.31): The camera control tab allows the user to specify a ROI for the LCI data collection (1) and for the Mach-Zehnder interferometer (2). The exposure time for both can also be varied $(3,4)$. Test images are captured using the camera test buttons $(5,6)$ below each column. All of the values in (1-4) are redefined during the course of a measurement, so their initial values are not critical. The number of LCI images per 
surface is specified here (7) along with the number of images to average at each LCI image location (8) to reduce the shot noise in the collected images.

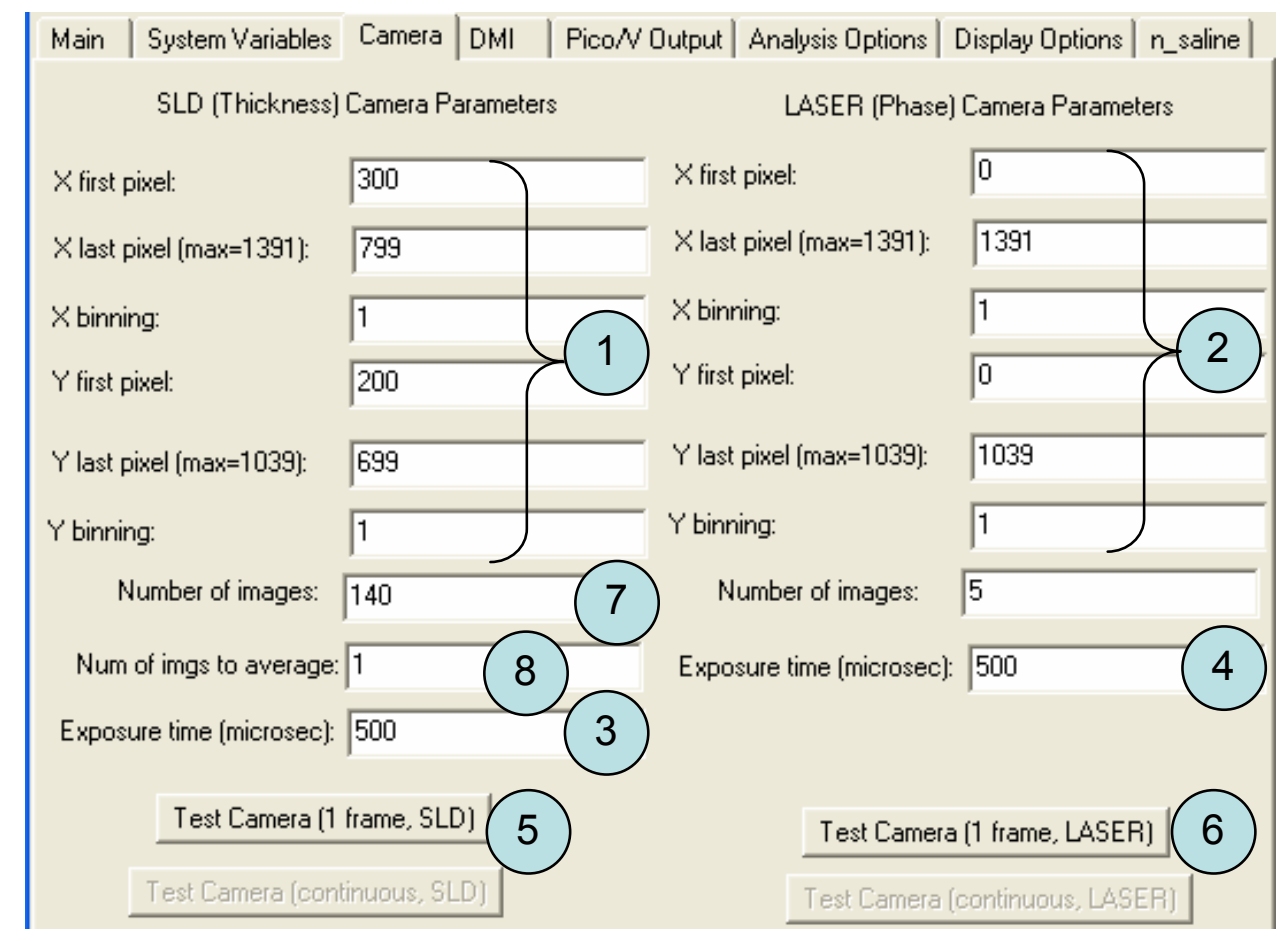

FIGURE 6.31 - Screenshot of "Camera" tab of the Engage GUI.

Pico/V Output (Figure 6.32): The top half of this tab controls the motion parameters for the LCI reference mirror stage. The values under the "Fringe Finding Routine:" heading define the distance between fringe finding images (1), the number of such images to capture in a group (2), along with the target velocity and acceleration (3). Under the "Data Collection Parameters:" heading are specified the distance between data collection frames (4), the number of optical encoder positions to average at each of those images (5), stage velocity and acceleration (6), the distance to move after the final image (7), and the wait time after a stage motion has completed before an LCI image is captured (8). 
The values under the "PZT" heading are the D/A output channel for the XPS controller (9) and the required voltage steps for each wavelength, 543 through $632 \mathrm{~nm}$ (10).

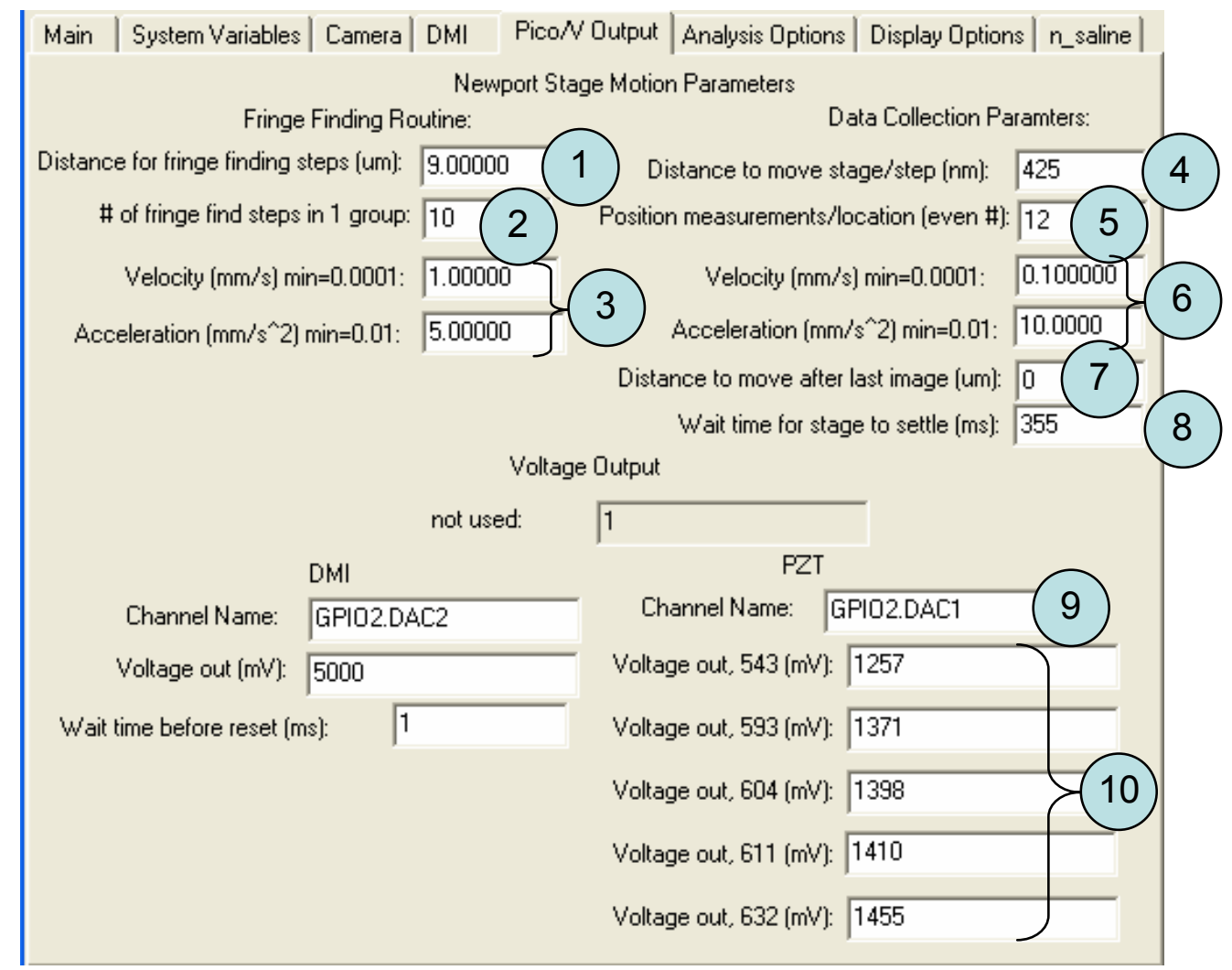

FIGURE 6.32 - Screenshot of the "Pico/V output" tab of the GUI.

Analysis Options (Figure 6.33): The tabs in previous four tabs specify values required for Step 1, the data collection. All of the necessary values are stored in header files during Step 1 so that the necessary information can be accessed during the data processing (Steps 2, 3). In the "Analysis Options" tab, up to four data sets can be specified for processing of Step 2, which is the calculation of the surface locations from the LCI data. Three values are defined for each: (1) the folder name of the data set, (2) the file prefix for the LCI images and (3) the time to wait before starting processing, in 
seconds. Each data set takes between 60 and 120 minutes to process, so this allows data sets to be processed overnight by pushing the button titled "Process Data (Step 2, surface calculation)" (4). Once Step 2 has completed, Step 3 is initiated using the button titled "Process Data (Step 3, Calculated Index)" (5), which uses the values in (1) through (3) to define the data set to process.

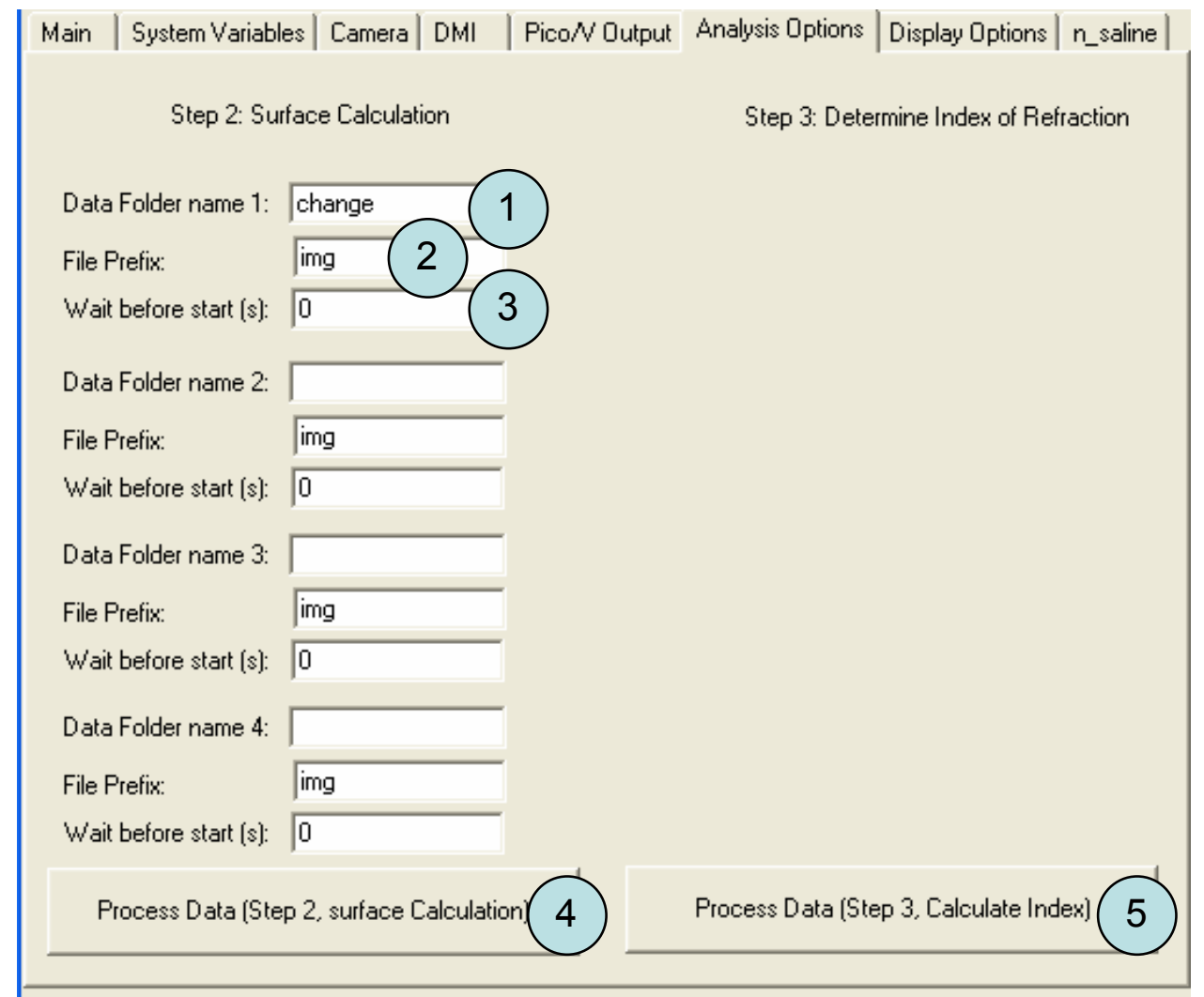

FIGURE 6.33 - Screenshot of the "Analysis Options" tab of the Engage GUI.

During all three measurement steps, the user is guided through a series of prompts necessary for completion of a measurement. This user interaction is all software manifestations of the implementation of the theory outline in Chapters 2 through 6 of this document. 


\section{SYSTEM CALIBRATION PROCEDURES}

In any measurement system, it is necessary to have a method of calibration. The required accuracy of a given measurement system dictates how accurate this calibration must be. In the case of Engage, which is meant to measure the refractive index of sample of soft contact lens material, the accuracy goal is $+/-0.0001$ in refractive index. Since this accuracy is a significant improvement on any existing measurement technique for this type of material, there is no other independent instrument that can be used to directly validate measurements made on soft contact lens materials. Therefore, another method must be used for system calibration.

\subsection{Glass Calibration Samples}

Refractive index as a material property is also important for different types of glass. In order to measure the refractive index of glass, a prism is made and characterized using a prism spectrometer. The details of the prism spectrometer were discussed in Chapter 1. Glass has a much smaller dn/dT, holds its shape better then a hydrogel, and is in general a more stable measurement sample. Given these properties, the index of glass can be determined to better then $1 \times 10^{-5}$.

Since a calibration sample cannot be made from hydrogel, glass would appear to be the best candidate to be used as a calibration sample. If a sample of glass with a well known refractive index and the correct geometry could be created, it could then be measured in 
Engage. Comparing those results to the known data of the calibration sample gives a measure of the overall system accuracy. Repeating these measurements will determine the system repeatability.

Although the refractive index of glass is stable, it is difficult to measure in any geometry other then a prism. Unfortunately, a prism is about the worst possible geometry for testing in Engage. Lookup tables listing refractive indices for common glasses are not good enough to be used as calibration data, as the index of BK7 inevitably varies from melt to melt. Therefore the calibration samples must be individually characterized.

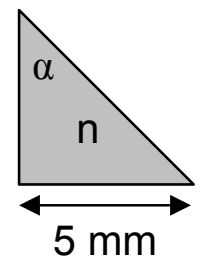

FIGURE 7.1 - Initial prism geometry of glass calibration samples.

Seven small right angle prisms were purchased ( 5 of BK7, 2 Fused silica), with $5 \mathrm{~mm}$ sides (Figure 7.1). Using the prism spectrometer, both the apex angle $(\alpha)$ and minimum deviation angles $\left(\delta_{\min }\right)$ were measured for each prism at each of the wavelengths of the 5color tunable HeNe laser. These numbers are used to determine the refractive index of the prisms using Equation 7.1.

$$
n(\lambda)=\frac{\sin \left(\frac{\alpha+\delta_{\min }(\lambda)}{2}\right)}{\sin \left(\frac{\alpha}{2}\right)}
$$


These angles were measured multiple times, and the standard deviations of the apex angle $\alpha$ and the minimum deviation angle $\delta_{\min }$ were 1.8 arc-seconds and 3.1 arc-seconds, respectively. This leads to a worse case error of 0.00003 in index. The change in index with respect to temperature was obtained from a standard data sheet as $3 \times 10^{-6} /{ }^{\circ} \mathrm{C}$ at room temperature, so minor adjustments to the prism data can be made depending on the relative temperature of the Engage measurements.

Now that the refractive index of these 7 prisms had been well characterized, they needed to be made into a testable geometry for Engage. Since the thickness measurement tests by reflecting light off of the two surfaces of thin, nearly parallel plates, the prisms must be machined into a similar geometry. In a typical measurement system, it is generally true that the more material available, the easier it is to measure accurately. In contrast, the problem of determining the fringe order $(\Delta \mathrm{m})$ in the Mach-Zehnder interferometer is easier for thinner samples. More accurately, if the OPD through the test sample is too large relative to the air or liquid column, the multiple orders of $\Delta \mathrm{m}$ get too close together.

$$
n_{\text {sample }, \Delta m+1}-n_{\text {sample }, \Delta m}=\frac{\lambda}{t}=\Delta n_{\Delta m=1} \quad \text { Equation } 7.2
$$

For example, for a $1 \mathrm{~mm}$ thick piece of BK7 glass at $\lambda=543 \mathrm{~nm}$, the index step is only 0.0005. The challenge becomes turning a $5 \mathrm{~mm} \mathrm{BK} 7$ prism into a thin plate with specularly reflective surfaces that still has some thickness variation. This task was assigned to an external fabrication company. The resulting calibration triangles are thin right triangles, roughly $160 \mu \mathrm{m}$ thick, with a wedge angle of about 2.3 arc-minutes (prism BK7-1) (Figure 7.2). 


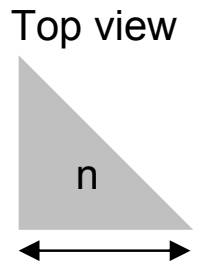

$5 \mathrm{~mm}$
Side view

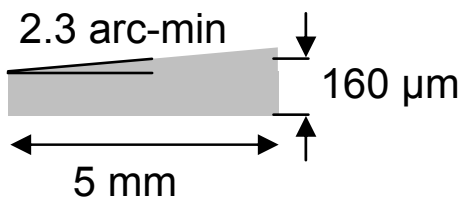

FIGURE 7.2 - Two views of the final geometry of the glass calibration samples.

Contact lens samples can be manipulated to stick to the bottom plate of the cuvette, as their surfaces are flexible. The calibration samples cannot be made to fit perfectly to the bottom plate of the cuvette, so the testing modality must be modified slightly by positioning the glass calibration sample in the measurement cuvette suspended on three pieces of plastic shim stock. This creates four surfaces in the column containing the test sample that must be measured. In order to determine the thickness of the sample, the thicknesses above and below the sample ( $a$ and $b$ ) are subtracted from the cuvette thickness found from the air column (Figure 7.3).

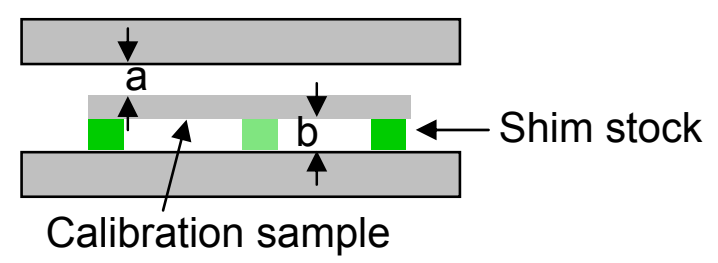

FIGURE 7.3 - Testing geometry of the glass calibration samples.

Figure 7.4 shows a single image (a) and a frame by frame subtraction (b) for the saline2 interface, which is the top of the glass sample. Figure 7.5 shows the wrapped phase in transmission, where it is clear from the curved fringes that there is some thickness variation in the calibration sample. Two thickness regions can be selected from this 
continuous sample to simulate a sample with multiple thickness regions, as described in Chapter 6.

a)

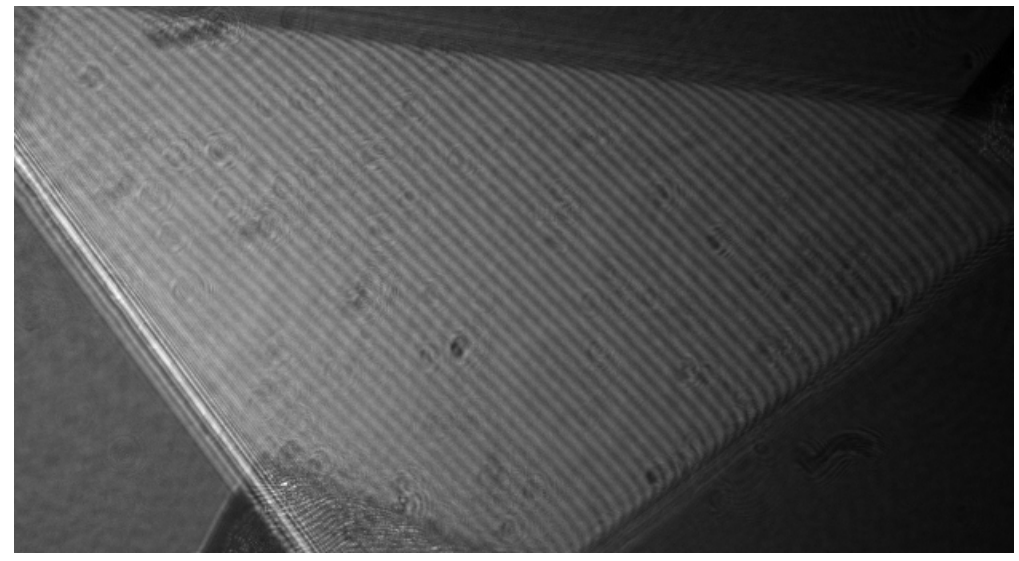

b)

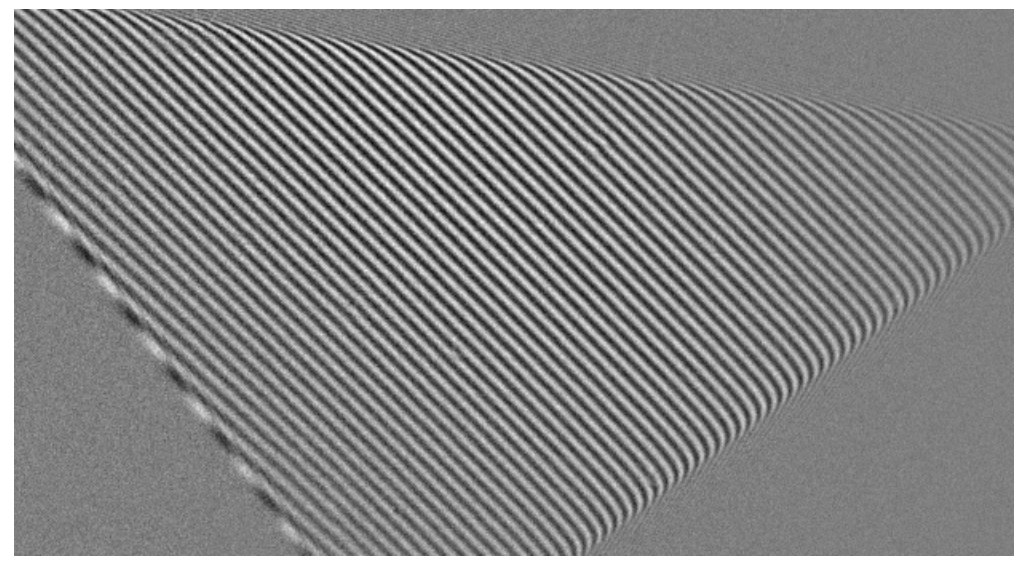

FIGURE 7.4 - An a) LCI image and b) a frame-by-frame subtraction to show the fringes of data from the saline 2 interface of a calibration measurement. 


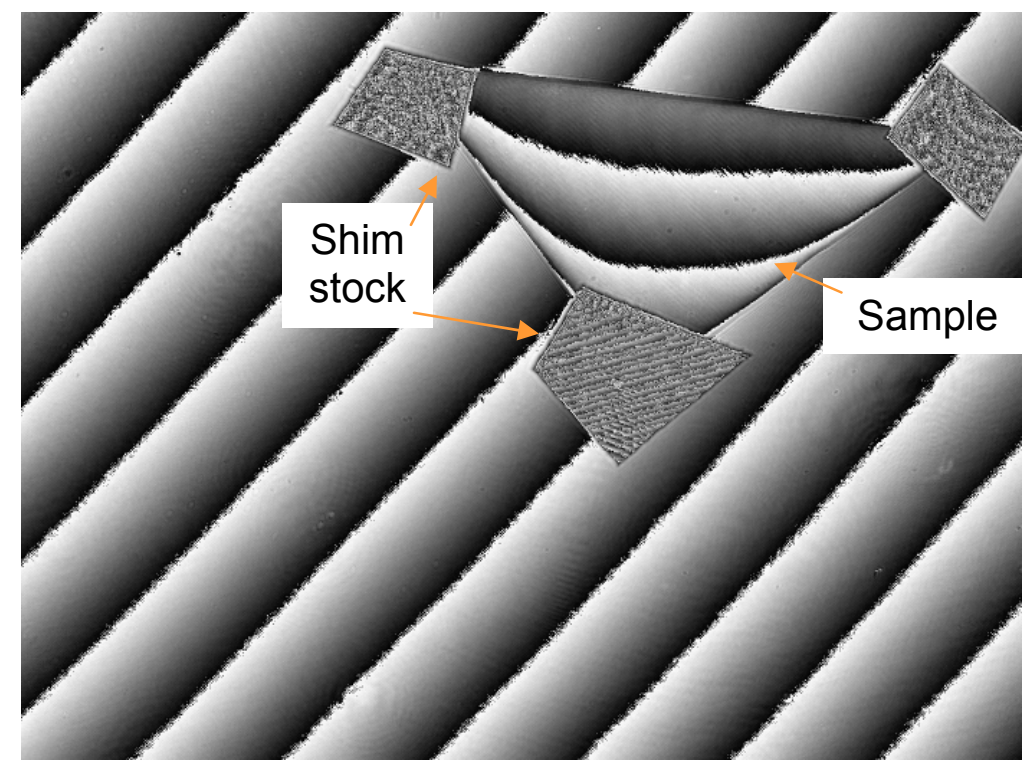

FIGURE 7.5 - Wrapped phase in transmission for a glass calibration sample.

The mean thicknesses of the two regions shown in Figure 7.6 are $t_{\text {Reg1 }}=163593 \mathrm{~nm}$ and $\mathrm{t}_{\operatorname{Reg} 2}=164541 \mathrm{~nm}$.

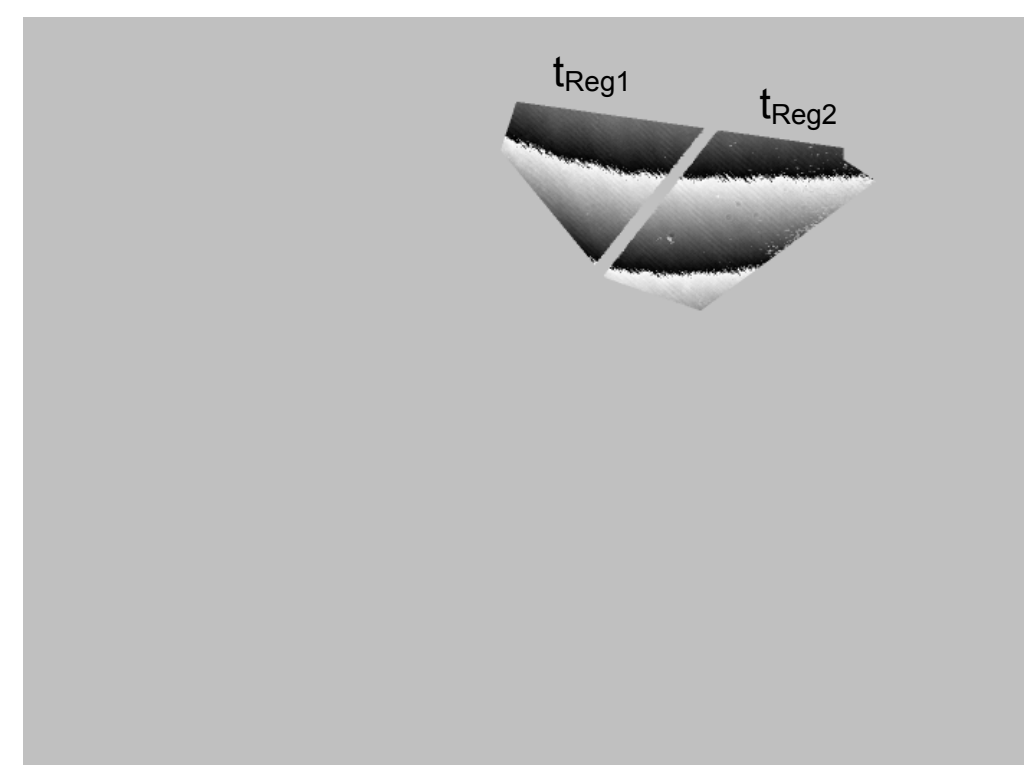

FIGURE 7.6 - Two thickness regions chosen from the area containing the glass calibration sample. 
The noise ceiling for $\lambda=543 \mathrm{~nm}$ is:

$$
N C=\lambda\left(\frac{\left|t_{1}-t_{2}\right|}{t_{1} t_{2}}\right)=543 \mathrm{~nm}\left(\frac{|948 \mathrm{~nm}|}{163593 \mathrm{~nm} \cdot 164541 \mathrm{~nm}}\right)=0.000019 \quad \text { Equation } 7.3
$$

The small amount of wedge in the sample is not enough to create a noise ceiling large enough to use the multiple thickness theory to determine the correct order for $\Delta \mathrm{m}$. As was the case for saline, the dispersion curve variation from an incremental change of $\Delta \mathrm{m}$ is enough to determine the correct order.

Table 7.1 summarizes the index of refraction of the calibration sample as measured by Engage and by the prism spectrometer for calibration prism

\begin{tabular}{|c|c|c|c|}
\hline Wavelength $(\mathrm{nm})$ & Engage & Prism Spectrometer & Difference \\
\hline 543 & 1.518933 & 1.518955 & -0.000022 \\
\hline 593 & 1.516599 & 1.516618 & -0.000019 \\
\hline 604 & 1.516168 & 1.516186 & -0.000018 \\
\hline 611 & 1.515887 & 1.515904 & -0.000017 \\
\hline 632 & 1.515152 & 1.515158 & -0.000006 \\
\hline
\end{tabular}

TABLE 7.1 - Refractive index of calibration sample BK7-1 as measured with Engage and with the prism spectrometer.

Clearly, the agreement is excellent; roughly 5 times better then the measurement accuracy goal of 0.0001 . All of the index values from Engage are slightly lower then the values measured with the prism spectrometer, which suggests that the thickness of the sample is causing the error, since $t_{\text {sample }}$ is used to calculate the index at all wavelengths. As will be shown, errors in finding the sample thickness are the dominant measurement errors in the system. 
Several (13) measurements of calibration sample BK7-1 were taken over the course of several weeks to verify the repeatability of Engage. The variation in the measured refractive index values is actually larger then the accuracy goal of 0.0001 , as is shown in Figure 7.7, where the index of BK7-1 at $\lambda=543 \mathrm{~nm}$ is plotted versus measurement number. The variation is too large to attribute to environmental variations such as temperature fluctuations.

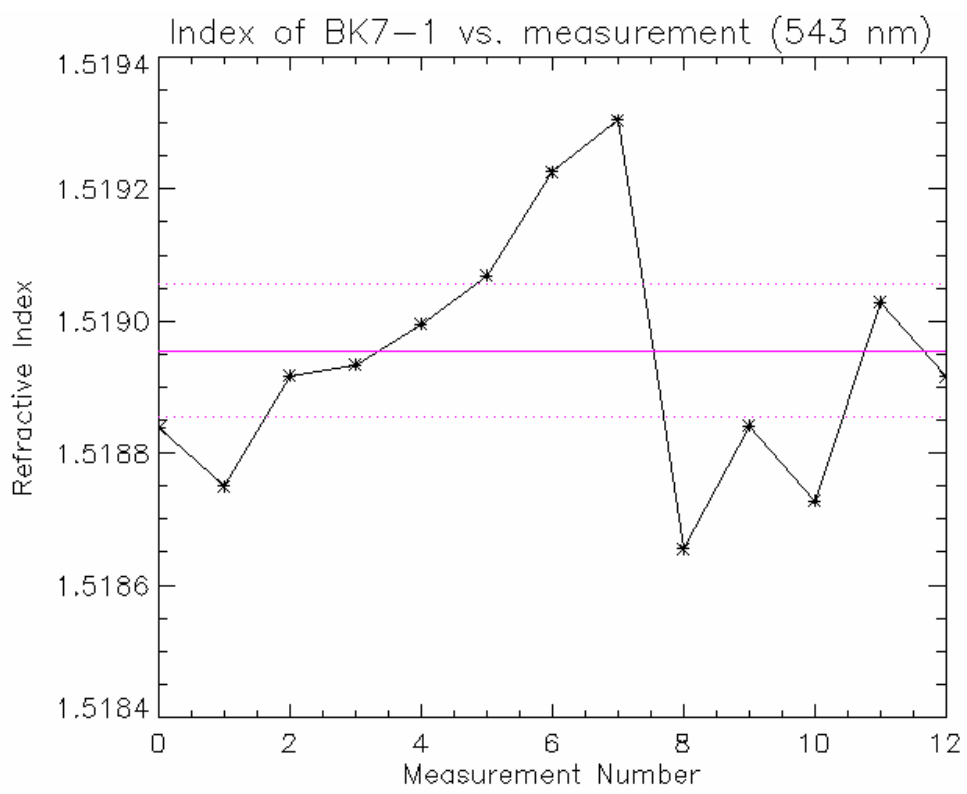

FIGURE 7.7 - The measured index of prism BK7-1 varies with measurement. The solid pink line is the value measured with the prism spectrometer and the dotted lines are $+/-$ 0.0001 from this accepted value.

The standard deviation of the refractive index plotted in Figure 7.7 is 0.00019 . The shape of the curve is nearly identical for all 5 wavelengths (Figure 7.8), which makes it clear that the index variation is likely entirely due to variations in the measured thickness of the glass calibration sample. 


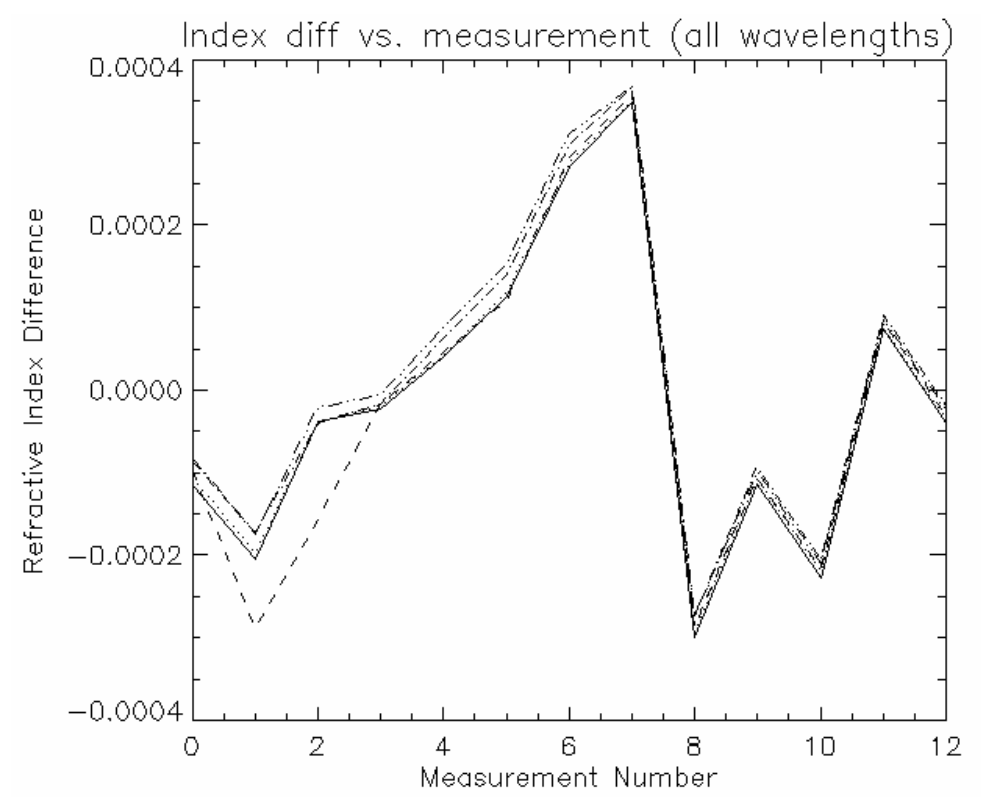

FIGURE 7.8 - Index difference versus measurement for all 5 laser wavelengths. Note each curve (wavelength) follows a similar trend.

The refractive index is inversely proportional to the measured thickness (Equation 7.4).

$$
n_{\text {glass }}\left(\lambda_{\text {laser }}\right)=\frac{\lambda_{\text {laser }}}{t_{\text {glass }}(x, y)}\left[\Delta m+\frac{\left[\varphi_{\text {glass }}(x, y)-\varphi_{\varepsilon}(x, y)\right]-\varphi_{\text {air }, f i t}(x, y)}{2 \pi}\right]+1
$$

Equation 7.4

Figure 7.9 is a plot of the measured thickness versus measurement number. The sample is positioned differently for each measurement, so the region on the test sample over which the average thickness is computed varies slightly for each data set. 


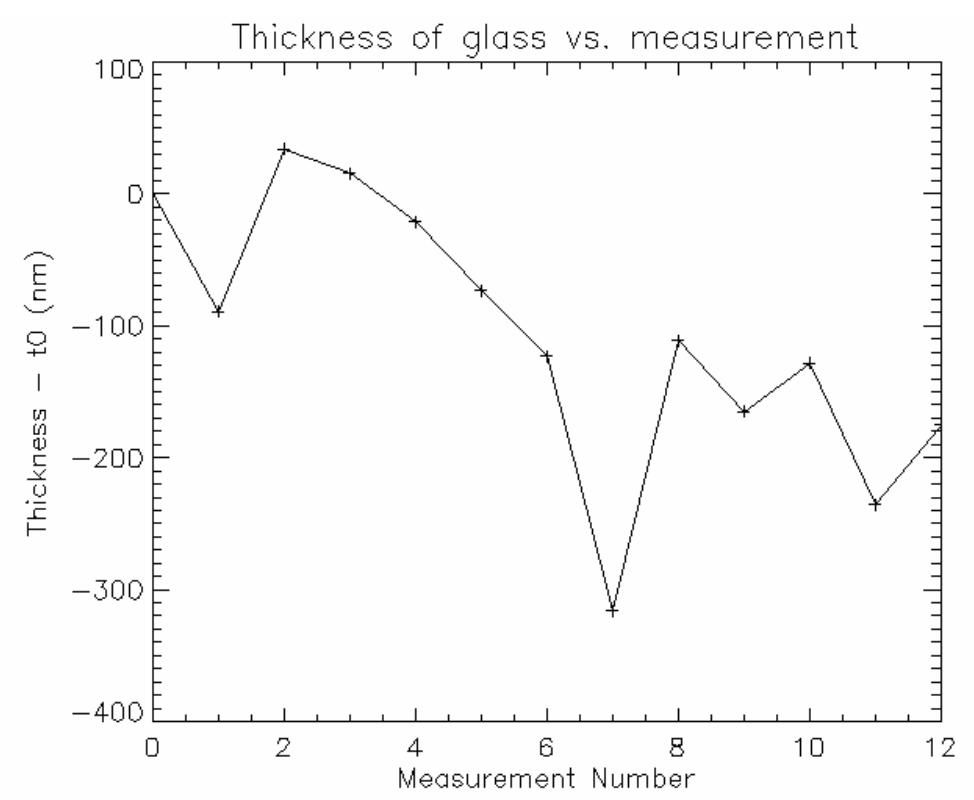

FIGURE 7.9 - Mean measured thickness of glass sample (relative to measurement 0 ) versus measurement number.

The inverse correlation is evident, especially at the large outlying values, such as measurement 7. The magnitude of the index error from a known thickness difference can be determined by examining a single data set by artificially varying $t_{\text {glass }}$ and calculating the index difference. For this sample geometry (glass prism in air, $t_{\text {glass }}=164 \mu \mathrm{m}$ ), a 31.6 $\mathrm{nm}$ thickness difference changes the index by the desired accuracy of 0.0001 . Contrast this with measuring a hydrogel sample immersed in saline with a nominal thickness of $134 \mu \mathrm{m}$. The immersion reduces the sensitivity to errors in the thickness measurements, so that a 0.0001 index error results from a thickness error of $187 \mathrm{~nm}$. The sensitivity improves by a factor of 5.9. A thorough analysis of the relationship between various errors and the refractive index error is discussed in Chapter 9. 
If we apply this factor to the standard deviation of the measured index of the calibration samples, we obtain a standard deviation of $3.2 \times 10^{-5}$ in index.

$$
\frac{0.00019}{5.9}=3.2 \times 10^{-5}
$$

Regardless of this reduction, the source of the error should be investigated. Clearly it is due to errors in the thickness measurement, which is a combination of 6 surface locations for a glass sample. The average of these 13 measurements of BK7-1 at $\lambda=543 \mathrm{~nm}$ is 1.518939, which is only $1.6 \times 10-5$ greater than the value from the prism spectrometer $\left(\mathrm{n}_{\text {prism }}=1.518955\right)$. This indicates a lack of bias in the measurement process as a whole.

Recall that every axial frame position recorded during the course of a measurement is adjusted using a lookup table of the errors in the LCI stage. The contents of this lookup table are plotted in Figure 7.10 for reference. The axial extent of a measurement covers a range of about $500 \mu \mathrm{m}$, which clearly covers a significant error correction range of roughly $500 \mathrm{~nm}$ of error correction (y-axis of the plot in Figure 7.10). 


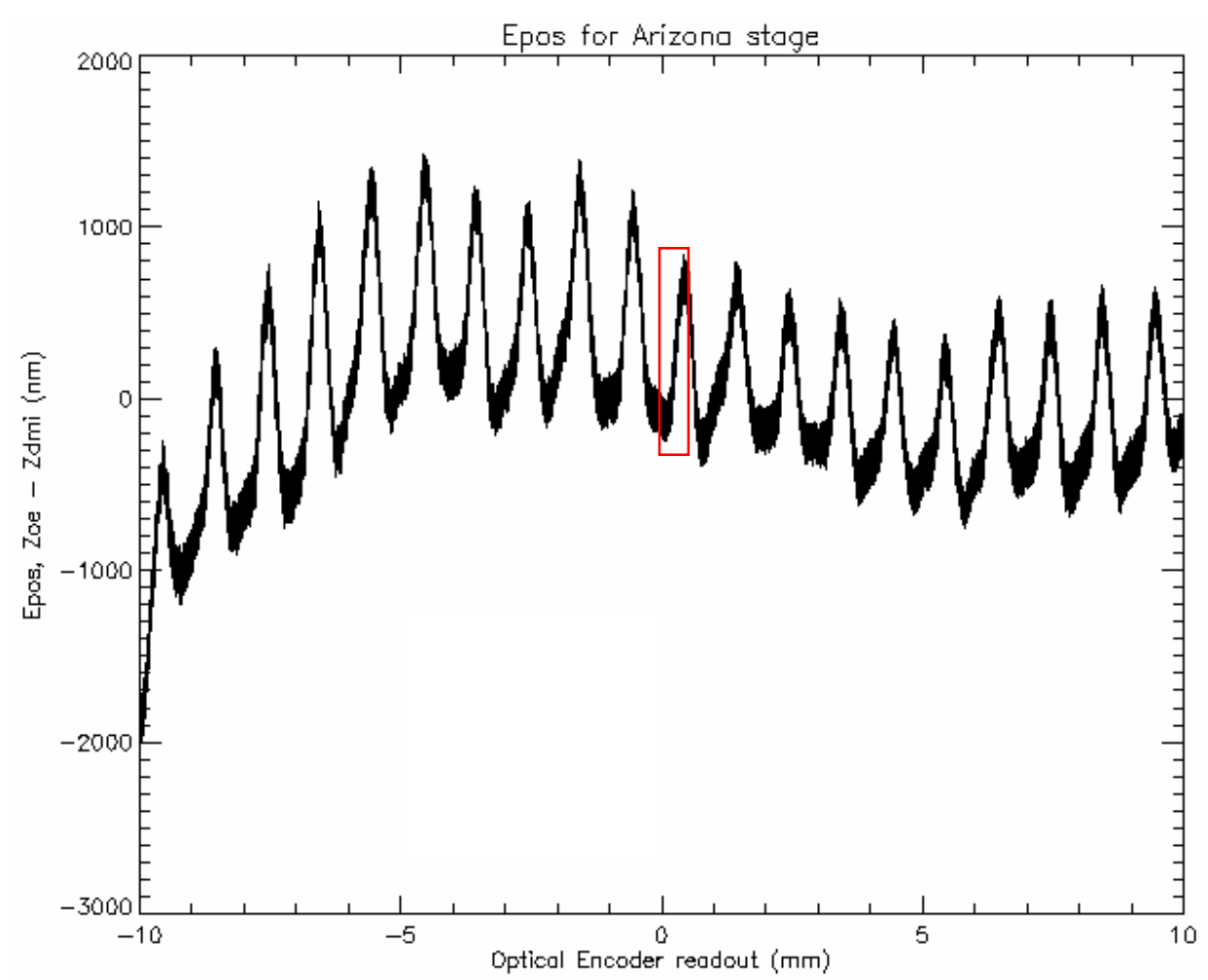

FIGURE 7.10 - Plot of $\varepsilon_{\text {pos }}$ for the stage used in the Arizona Engage system. The width of the red box corresponds to the typical travel range used for a single LCI measurement.

The assumption is that $\varepsilon_{\mathrm{pos}}$ is not changing over time, and that the magnitude and phase of the error is constant. Unfortunately, the measured variation of the index of the calibration samples indicates that this is probably not the case. The phase or the error, which is based on the physical construction of the LCI stage, is consistent. The amplitude of the error tends to vary slightly. A 5\% variation in the amplitude of the error at a given optical encoder position can mean that $\varepsilon_{\mathrm{pos}}$ is off by as much as $50 \mathrm{~nm}$. Recall that each surface results from about $140 \mathrm{LCI}$ images, each with a recorded position. If the mean of the error in $\varepsilon_{\text {pos }}$ is nonzero, the resulting surface location will be biased. This bias is 
position dependent, so the results obtained from a second measurement after translating the cuvette slightly along $\mathrm{z}$ will likely be different.

A more obvious manifestation of the problem is evident when calculating the separation of two surfaces, for example air1 and air2. Consider the situation where the high contrast fringes for the air 1 interface are near $0 \mathrm{~mm}$ on the LCI stage while the fringes for the air2 interface are centered near $0.4 \mathrm{~mm}$ (Figure 7.10). If the height of the jump in $\varepsilon_{\text {pos }}$ between these two regions changes from $800 \mathrm{~nm}$ to $750 \mathrm{~nm}$, the thickness of the air gap in the cuvette will be off by $50 \mathrm{~nm}$, regardless of how well the LCI processing algorithm works. The uncertainty of the relative positions of all of the LCI images in a data set is what causes the disagreement in the measured index of refraction values.

This data illustrates the current limit of this measurement system. The accuracy is only as good as the accuracy of the reported stage positions for each image. It is important to take images at a constant axial spacing $(\Delta z=5 \lambda / 4)$, but it is far more important to know the correct position of each image, even if the step size is varying $+/-5 \%$. As discussed in Chapter 3, the algorithm used to process the LCI signals does not require or assume constant image spacing.

Recall that each refractive index measurement is an average of index measured at hundreds of thousands of locations on the sample, one for each pixel in the ROI. These individual measurements are not completely independent for two reasons: first, the depth 
locations for the images are the same for each pixel, and second, the surface finding algorithm uses information from neighboring pixels to help determine the modulation curve and surface locations.

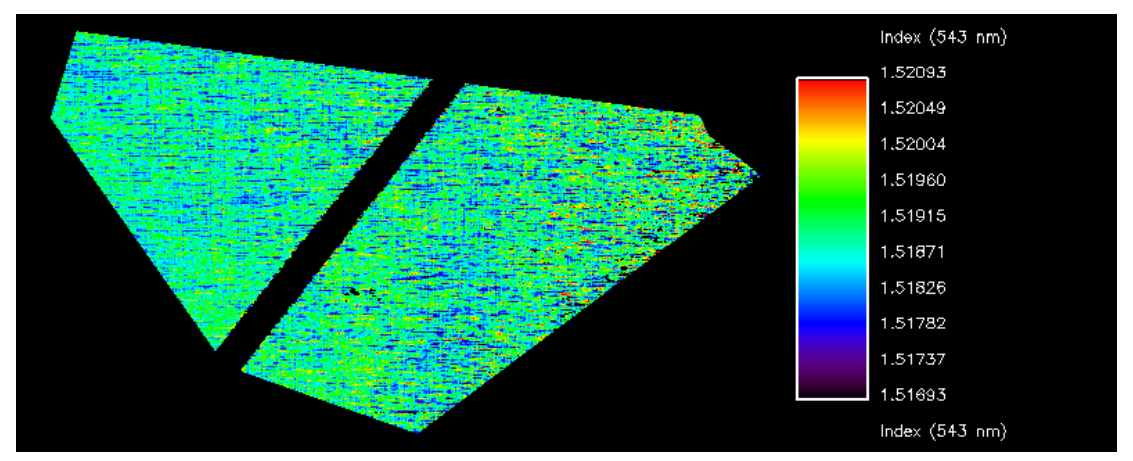

FIGURE 7.11 - Spatial plot of refractive index of calibration sample.

Figure 7.11 is the refractive index at $\lambda=543 \mathrm{~nm}$ of the calibration sample as a function of position. The standard deviation of the 80712 measurements of index is 0.0006 . This point-to-point standard deviation is much larger then the desired measurement goal of 0.0001. Since there are so many measurement of $\mathrm{n}$, the standard deviation of the entire measurement is much less. In the case of independent measurements, the reduction scales by the square root of the number of measurements, $\mathrm{N}$ :

$$
\sigma_{N}=\frac{\sigma}{\sqrt{N}}
$$

Since the measurements are not truly independent, the improvement from averaging the multiple values is not as dramatic as Equation 7.6. Recall that the number of spatial data points used to find $\gamma(z)$ depends on the number of tilt fringes in the images. If, for example, fifty pixels across $\mathrm{x}$ are used, that is not simply a reduction of the averaging improvement by fifty, since each pixel is used at multiple locations. In addition, the 
phase component of the measurement is independent. A conservative reduction to the improvement due to averaging would be an order of magnitude. For this particular data set, the standard deviation of the final reported index value $\left(\mathrm{n}_{543}=1.51893\right)$ is given as:

$$
\begin{array}{ll}
\sigma_{N}=\frac{0.0006}{0.1 \cdot \sqrt{80712}}=0.00002 & \text { Equation } 7.7
\end{array}
$$

This discussion is meant to illustrate that the variation in the measured refractive index is primarily due to uncertainties in the stage position during LCI data collection and not due to point-to-point index variations.

The refractive index as a function of wavelength for the seven calibration samples as measured in the prism spectrometer is included as Appendix H.

\subsection{Calibration with the Hilger-Chance Refractometer}

It was mentioned in Chapter 6 that the saline measurement can also be used as a means of calibration. The Hilger-Chance refractometer (HCR) was built partially as an external means for determining the correct $\Delta \mathrm{m}$ and $\mathrm{n}_{\text {saline }}$ at each of the 5 laser wavelengths, but also as a means of calibration. The refractive index of a liquid varies with temperature much more then glass; $\mathrm{dn} / \mathrm{dT}$ of distilled water at room temperature is approximately $0.0001 /{ }^{\circ} \mathrm{C}$. Saline is mostly water, so for small temperature variations near room temperature, the water model serves as a good approximation of $\mathrm{dn} / \mathrm{dT}$ for saline. This also requires that the temperature be monitored in both the HCR and Engage measurements in order to accurately correlate the values obtained from both techniques. 
Table 7.2 summarizes the data obtained from two samples of saline taken from the same sample, measured on the same day.

\begin{tabular}{|c|c|c|c|}
\hline Wavelength $(\mathrm{nm})$ & $\mathrm{n}(\mathrm{HCR})$ & $\mathrm{n}$ (Engage) & $1 \mathrm{e} 4 *\left(\mathrm{n}_{\text {Engage }}-\mathrm{n}_{\text {NCR }}\right)$ \\
\hline 543.36 & 1.336483 & 1.336479 & -0.047 \\
\hline 593.93 & 1.334736 & 1.334739 & 0.028 \\
\hline 604.61 & 1.334434 & 1.334406 & -0.272 \\
\hline 611.80 & 1.334212 & 1.334208 & -0.038 \\
\hline 632.82 & 1.333604 & 1.333621 & 0.170 \\
\hline
\end{tabular}

TABLE 7.2 - Comparison of the index of a saline sample measured by the HCR and Engage.

Both measurements were taken at $\mathrm{T}=24^{\circ} \mathrm{C}$. Index can also vary based on the salinity of the samples, which can vary if the saline is allowed to evaporate, for example. The absolute accuracy of these measurements is only as good as the absolute accuracy of the HCR, which was calibrated using distilled water. The distilled water was measured in a hollow glass prism on a prism spectrometer, using the same technique used to characterize the glass calibration samples. Based on this calibration, the absolute accuracy is of the HCR is about 0.00005 .

The agreement between the two instruments for this saline sample is excellent. The measured index of saline is susceptible to variations in the LCI stage calibration causing thickness measurement errors to about the same level as the measurement of the glass samples. Therefore, the variation in measured saline index does vary in a similar fashion to the variation for the glass calibration samples shown in Figure 7.7. A discussion of the 
effects of saline index on the measured values for contact lens materials is included in Chapter 8 .

Two calibration methods have been discussed in this chapter. Each offers benefits and drawbacks in terms of system calibration. When measuring the previously characterized glass samples, the advantages are the relative thermal stability along with the ease in which the calibration measurement can be made. If Engage were to be duplicated elsewhere, a system calibration can be made by measuring one of the glass samples. The downside to this technique is that an extra interface must be measured (saline4).

The saline calibration method has the advantage of using an intermediate output of a typical Engage measurement as a calibration tool. The drawback is that a second instrument (HCR) is required to perform the calibration, and the measurement must be made on the same sample, at the same temperature, and at nearly the same time. 


\section{MEASUREMENT DATA}

As in many research and development projects, the goal of this work has been to build an instrument to prove the validity of the proposed novel measurement technique. The data output is often used as feedback to indicate the parts of the measurement process that need improvement. The data presented here is meant to show the capabilities of the system and to report the values obtained for the soft contact lens material. It is easy to see how measurements could be made on other materials, and this will likely be a worthwhile task for any future users of this instrument.

In addition to soft contact lens material data, the saline measurements for each data set will be shown and compared to the data obtained from the Hilger-Chance Refractometer (HCR) for that sample.

As was indicated in the previous chapter, errors in the LCI stage are likely the cause of the variations in the thickness measurements. As a check of this theory, the distance measuring interferometer (DMI) was also used to record the positions of the LCI stage for each image. The LCI data cubes were processed twice; once using the positions from the optical encoder (OE) and once using the positions from the DMI.

Five measurements were taken on the same inverted wedding cake sample. The sample was removed from the cuvette, cleaned, and remounted for data sets 1,2 and 3. The 
sample was left mounted in the same configuration for data sets 3 through 5 so that the test-retest of just the instrument could be gauged. The initial saline packaging solutions contained a lot of particulates, so the sample was allowed to equilibrate in a clean saline solution after data set 2 but prior to data sets 3 through 5 . Both saline solutions were measured using the HCR in order to establish an absolute refractive index reference from another instrument. The temperature was also recorded for all data sets on both instruments.

\subsection{Thickness Measurements}

According to Chapter 7, the cause of the thickness variations of the glass sample is errors in the LCI stage that are insufficiently corrected with the lookup table. A simple metric for comparing the measured thickness of the physical thickness of the cuvette, $t_{1}$, is to examine the three coefficients that define the extrapolated thickness, $\mathrm{t}_{1, \mathrm{ext}}$ :

$$
t_{1, e x t}(x, y)=a x+b y+c
$$

Equation 8.1

If the stage positions are causing errors, it would most likely be evident by a change in c, which is the offset between the two surfaces used to calculate $t_{1}$ (air1, air2). The surface slopes $(a, b)$ should not be affected since they do not depend on the surface separation. The coefficients for the best fit plane to the cuvette thickness for data sets 3 through 5 are shown in Table 8.1, since these three measurements were taken sequentially without disturbing the cuvette. 


\begin{tabular}{|c|c|c|c|c|}
\hline Data Set & z values from: & $\mathrm{a}(\mathrm{nm} /$ pixel $)$ & $\mathrm{b}(\mathrm{nm} / \mathrm{pixel})$ & $\mathrm{c}(\mathrm{nm})$ \\
\hline 3 & DMI & -0.53080 & 0.14119 & 352555.0 \\
\hline 4 & DMI & -0.52590 & 0.14368 & 352511.0 \\
\hline 5 & DMI & -0.51938 & 0.14605 & 352519.2 \\
\hline 3 & Optical Encoder & -0.53076 & 0.14127 & 352349.3 \\
\hline 4 & Optical Encoder & -0.52639 & 0.14367 & 352272.0 \\
\hline 5 & Optical Encoder & -0.51961 & 0.14617 & 352332.1 \\
\hline
\end{tabular}

TABLE 8.1 - The coefficients of the best fit plane for 3 measurements using the axial positions from the DMI and the optical encoder.

First, the maximum slope difference between the DMI surface $\left(t_{1, D M I}\right)$ and the optical encoder surface $\left(\mathrm{t}_{1, \mathrm{OE}}\right)$ in either direction is $0.0005 \mathrm{~nm} /$ pixel for data set 4 , coefficient a. That is equivalent to 5 Angstroms of slope difference across 1000 pixels; a negligible difference. There are differences in the values for $\mathrm{c}$ between $\mathrm{t}_{1, \mathrm{DMI}}$ and $\mathrm{t}_{1, \mathrm{OE}}$ of about 211 nanometers, on average. The coefficients for $t_{1, \mathrm{OE}}$ are consistently lower than those for $t_{1, \text { DMI}}$, which illustrates a thickness bias. The standard deviation of $\mathrm{c}$ is also larger for $\mathrm{t}_{1, \mathrm{OE}}(40.6 \mathrm{~nm}$ versus $23.4 \mathrm{~nm})$, which indicates a slight change in the stage errors with time. The best way to determine which of the thickness measurements is more accurate is to compare the values for $n_{\text {saline }}$ found using these thicknesses against the values from the HCR.

\subsection{Saline Measurements}

Table 8.2 summarizes $n_{\text {saline }}$ for $\lambda=593.93 \mathrm{~nm}$ for the five data sets. The difference between the two columns of Engage values is purely due to the thickness differences in Table 8.1. 


\begin{tabular}{|c|c|c|c|}
\hline Data Set & $\mathrm{n}_{\text {saline, Engage, DMI }}$ & $\mathrm{n}_{\text {saline, Engage, }}$ OE & $\mathrm{n}_{\text {saline, }}, \mathrm{HCR}$ \\
\hline 1 & 1.334728 & 1.334914 & 1.334793 \\
\hline 2 & 1.334736 & 1.334951 & 1.334793 \\
\hline 3 & 1.334554 & 1.334749 & 1.334702 \\
\hline 4 & 1.334694 & 1.334921 & 1.334702 \\
\hline 5 & 1.334758 & 1.334936 & 1.334702 \\
\hline
\end{tabular}

TABLE 8.2 - Index of saline comparison for $\lambda=593.93 \mathrm{~nm}$.

The Engage values were taken over a 6 hour period. Recall that data sets 1 and 2 used the first saline sample while sets 3 through 5 used a second saline sample, which accounts for the difference in their index in the HCR $(\Delta=-0.00009)$. The index obtained using $t_{1, \text { OE }}$ is on average about 0.00015 too high while the index obtained using $t_{1, \text { DMI }}$ is on average $4.4 \times 10^{-5}$ too low. The bias is more than a factor of 3 lower for the DMI surfaces, indicating once again that the root of the bias is the LCI stage. The difference between DMI and OE index values is about 0.0002 for all wavelengths.

$$
n_{\text {sample }}(\lambda)=\frac{\lambda}{t_{\text {sample }}(x, y)}\left[\Delta m+\frac{\left[\varphi_{\text {sample }}(x, y)-\varphi_{\varepsilon}(x, y)\right]-\varphi_{\text {saline } f \text { fit }}(x, y)}{2 \pi}\right]+n_{\text {saline }}(\lambda)
$$

Equation 8.2

From Equation 8.2, which is used to calculate the refractive index of the contact lens materials, it is clear that an error in $\mathrm{n}_{\text {saline }}$ causes an equal error in $\mathrm{n}_{\text {sample. }}$. Neglecting other noise sources we should expect to see similar index variations for the contact lens material samples as are seen in Table 8.2. 


\subsection{Contact Lens Material Measurements}

Table 8.3 contains the measured refractive index of a stepped soft contact lens material sample measured in Engage at the 5 wavelengths of the tunable HeNe laser, where the LCI stage positions used were from the DMI.

\begin{tabular}{|c|c|c|c|c|c|c|}
\hline$\lambda(\mathrm{nm})$ & Set 1 & Set 2 & Set 3 & Set 4 & Set 5 & Stnd dev \\
\hline 543.36 & 1.407462 & 1.407463 & 1.407595 & 1.407537 & 1.407624 & $7.42 \times 10^{-5}$ \\
\hline 593.93 & 1.405383 & 1.405407 & 1.405471 & 1.405405 & 1.405501 & $5.01 \times 10^{-5}$ \\
\hline 604.61 & 1.405013 & 1.404994 & 1.405125 & 1.405057 & 1.405140 & $6.52 \times 10^{-5}$ \\
\hline 611.80 & 1.404765 & 1.404790 & 1.404845 & 1.404793 & 1.404881 & $4.71 \times 10^{-5}$ \\
\hline 632.82 & 1.404101 & 1.404083 & 1.404179 & 1.404121 & 1.404199 & $5.02 \times 10^{-5}$ \\
\hline
\end{tabular}

TABLE 8.3 - Index of refraction of soft contact lens material for 5 data sets using DMI positions.

Table 8.4 is the refractive index for the same five measurements with LCI stage positions reported by the optical encoder that were pre and post-corrected using the stage error lookup table.

\begin{tabular}{|c|c|c|c|c|c|c|}
\hline$\lambda(\mathrm{nm})$ & Set 1 & Set 2 & Set 3 & Set 4 & Set 5 & Stnd dev \\
\hline 543.36 & 1.407671 & 1.407694 & 1.407812 & 1.407796 & 1.407821 & $7.07 \times 10^{-5}$ \\
\hline 593.93 & 1.405591 & 1.405636 & 1.405687 & 1.405663 & 1.405697 & $4.27 \times 10^{-5}$ \\
\hline 604.61 & 1.405222 & 1.405223 & 1.405340 & 1.405315 & 1.405335 & $5.96 \times 10^{-5}$ \\
\hline 611.80 & 1.404972 & 1.405019 & 1.405060 & 1.405051 & 1.405076 & $4.19 \times 10^{-5}$ \\
\hline 632.82 & 1.404310 & 1.404311 & 1.404393 & 1.404379 & 1.404393 & $4.30 \times 10^{-5}$ \\
\hline
\end{tabular}

TABLE 8.4 - Index of refraction of soft contact lens material for 5 data sets using OE positions.

Recall that the sample was removed from the cuvette and completely remounted between data sets 1, 2 and 3. For data sets 3 through 5, the sample was hydrated in a different saline solution that had a slightly higher refractive index $\left(9 \times 10^{-5}\right.$, from Table 8.2$)$, so we 
should expect the index to be higher by roughly half of this value, based on a water content of $50 \%$. Both tables show a slight index increase from data set 2 to 3 , although at about $8.7 \times 10^{-5}$ averaged over the 5 wavelengths, it is larger than the predicted value.

There is clearly an index bias between the DMI and OE data in the two tables of roughly 0.0002 across all wavelengths and data sets. This is the difference between data set 1 DMI and set $1 \mathrm{OE}$, for example. Nearly all of the bias can be accounted for by the index difference for the DMI and OE surfaces shown in Table 8.2. One might expect $\mathrm{n}_{\text {sample }}$ to have a bias similar in magnitude to that of $\mathrm{n}_{\text {saline, }}$ since they are both the products of multiple surface measurements, which has been identified as the source of the bias. Chapter 9 will examine this in detail, but it turns out that based on the typical sample geometry, $\mathrm{n}_{\text {sample }}$ is about half as sensitive to thickness measurement errors as $\mathrm{n}_{\text {saline }}$. Along the same lines, $\mathrm{n}_{\text {saline }}$ is only a third as sensitive to thickness errors as the glass calibration samples discussed in Chapter 7.

The standard deviation in the final column of the Tables 8.3 and 8.4 is of all five measurements. Since the saline index changes after data set 2 and the sample refractive index is dependent on the index of the hydrating solution, we should not expect the same index for sets 3 through 5 as sets 1 and 2 . The sample set is probably too small to make any definitive conclusions about the expected standard deviation of the system, but it is reasonable to expect it is no worse than $8 \times 10^{-5}$ when the stage positions are accurately known. This is larger than any value in Table 8.3. If the stage positions are not 
accurately known, the system standard deviation will likely be as much as twice this value. This is based on the standard deviation of 0.00019 for the glass samples in Chapter 7 and the relative sensitivity of $\mathrm{n}_{\text {sample }}\left(\right.$ and $\left.\mathrm{n}_{\text {saline }}\right)$ compared to the index of the calibration samples. Of greater concern is the system bias that occurs from measurement to measurement, which was discussed in detail in Chapter 7. There appears to be no bias when using the DMI.

Based on the data presented here, the system seems to meet the measurement accuracy goal of 0.0001 in index at five wavelengths in the visible for a carefully mounted hydrogel sample immersed in saline solution if the stage positions are accurately known with the help of an external instrument. Without the DMI, Engage will be limited by the position errors of the optical encoder. 


\section{ANALYSES OF SYSTEM ERRORS}

The desired system accuracy for a measurement of refractive index was defined in Chapter 1 as $+/-0.0001$ in index at each of 5 laser wavelengths. Chapter 7 examined the data taken on glass calibration samples over time as a means of determining the absolute accuracy and repeatability of Engage. From the 13 measurements of sample BK7-1, the measurement to measurement standard deviation was found to be 0.00019 . The mean of these measurements $\left(\mathrm{n}_{543}=1.518939\right)$ was quite close to the value obtained from the prism spectrometer $\left(\mathrm{n}_{543}=1.518955\right)$. This indicates that the absolute accuracy over several measurements is better than the stated goal, while the absolute accuracy of a single measurement is subject to variations. The main cause of these variations was attributed to errors in the stage calibration. These errors cause a bias in one or more of the surfaces found during data collection which introduces a bias in the calculated thickness. In turn, this introduces a bias for the refractive index.

The goal of this chapter is to quantify the magnitude of various Engage errors in terms of the net index of refraction error. From this analysis, it will be clear which components of the measurement process are critical.

\subsection{Allowable Thickness Errors}

There are many causes of thickness errors, many of which have been discussed at appropriate locations in this document. Now that all of the typical system dimensions 
have been established, the allowable magnitude of the errors can be defined. The cuvette thickness is $350 \mu \mathrm{m}$, which means the thickness of saline measured is also $350 \mu \mathrm{m}$.

$$
n_{\text {saline }}(\lambda)=\frac{\lambda}{t(x, y)}\left[\Delta m+\frac{\left[\varphi_{\text {saline }}(x, y)-\varphi_{\varepsilon}(x, y)\right]-\varphi_{\text {air }, f i t}(x, y)}{2 \pi}\right]+1
$$

Equation 9.1

If $t_{E}$ is the thickness error that causes a refractive index error $\Delta n$, and $t_{E}<<t$, Equation 9.2 can be obtained:

$$
t_{E}=\Delta n \frac{t^{2}(x, y)}{\lambda\left[\Delta m+\frac{\left[\varphi_{\text {saline }}(x, y)-\varphi_{\varepsilon}(x, y)\right]-\varphi_{\text {air }, f i t}(x, y)}{2 \pi}\right]}
$$

From Equation 9.2, a 0.0001 index error is caused by a $99 \mathrm{~nm}$ thickness error. A similar expression can be found from Equation 9.3 for the relationship between sample thickness error and the change in index.

$$
n_{\text {sample }}(\lambda)=\frac{\lambda}{t_{\text {sample }}(x, y)}\left[\Delta m+\frac{\left[\varphi_{\text {sample }}(x, y)-\varphi_{\varepsilon}(x, y)\right]-\varphi_{\text {saline }, \text { fit }}(x, y)}{2 \pi}\right]+n_{\text {saline }}(\lambda)
$$

Equation 9.3

For a contact lens material sample with a nominal thickness of $134 \mu \mathrm{m}$, a $187 \mathrm{~nm}$ thickness error results in a 0.0001 index error. The fact that the contact lens material index is only half as sensitive to thickness errors is important, since surface saline 2 is the most difficult surface to calculate. Recall from Chapter 7 that the glass calibration sample $(\mathrm{t}=164 \mu \mathrm{m})$ index has an index error of 0.0001 for a thickness error of $31.6 \mathrm{~nm}$. 
Another important part of Equation 9.3 is that any errors in $n_{\text {saline }}$ directly translate to errors in $\mathrm{n}_{\text {sample. }}$ This illustrates the usefulness of using the HCR as a method for measuring $\mathrm{n}_{\text {saline }}$ to verify measurement results. Table 9.1 summarizes the thickness errors that cause 0.0001 index errors for the various materials.

\begin{tabular}{|c|c|c|}
\hline Material & Nominal Thickness & $\mathrm{t}_{\mathrm{E}}$ equivalent of $\Delta \mathrm{n}=0.0001$ \\
\hline Saline & $350 \mu \mathrm{m}$ & $99 \mathrm{~nm}$ \\
\hline Hydrogel Sample & $134 \mu \mathrm{m}$ & $187 \mathrm{~nm}$ \\
\hline Glass Calibration & $164 \mu \mathrm{m}$ & $31.6 \mathrm{~nm}$ \\
\hline
\end{tabular}

TABLE 9.1 - Thickness errors that cause a $\Delta \mathrm{n}=0.0001$ for various materials.

Recall that each thickness is the result of combining at least 2 surfaces, where each surface is calculated separately as described in Chapter 3. The independent surfaces are combined through addition, so their errors can be combined in quadrature (Equation 9.4). In the case of measuring the index of saline, only two surfaces are required: air1 and air2.

$$
t_{E}=\sqrt{h_{E, \text { air } 1}^{2}+h_{E, \text { air } 2}^{2}}
$$

Here, $h_{\mathrm{E}}$ is the height error for each of the two surfaces. If each of the surfaces is allowed an equal error budget, then we can solve for $h_{E}$. Equation 9.5 assumes an equal error for each of the two surfaces used to determine $\mathrm{n}_{\text {saline. }}$

$$
h_{E, S A L I N E}=\sqrt{\frac{t_{E}^{2}}{2}}=70 \mathrm{~nm}
$$

In the case of the hydrogel sample, 5 surfaces are combined to determine the thickness. The saline2 surface (saline/top of sample interface) is the most difficult surface to find, so it will be allowed twice the height error budget of the other surfaces. 


$$
h_{E, S A M P L E}=\sqrt{\frac{t_{E}^{2}}{6}}=\sqrt{\frac{187^{2}}{6}}=76.3 \mathrm{~nm}
$$

Equation 9.6

Therefore, the height error for the saline/top of sample interface is $2 * 76.3=152.6 \mathrm{~nm}$ for an index error of 0.0001 .

Each of the six surfaces found when measuring the glass calibration samples has an allowed height error of $12.9 \mathrm{~nm}$ :

$$
h_{E, S A M P L E}=\sqrt{\frac{t_{E}^{2}}{6}}=\sqrt{\frac{31.6^{2}}{6}}=12.9 \mathrm{~nm}
$$

The LCI signal strength, coherent scattered light, the algorithm chosen and stage errors all influence the calculated surface locations. One error source that has not been discussed to this point is the influence of the random irradiance noise in each of the LCI images.

\subsection{Detector Noise}

In Chapter 4 it was shown that the standard deviation of the random detector noise in the LCI images was about $30 \mathrm{DN}$ on a 12-bit scale. If these recorded irradiance fluctuations are truly random (and independent), then the magnitude of the errors can be decreased by averaging several images at the each depth location. For truly independent random noise, the improvement of the standard deviation of the noise scales as in Equation 9.8:

$$
\sigma_{N}=\frac{\sigma}{\sqrt{N}}
$$


The question is how this error effects the surfaces calculated using the least squares fit to the modulation algorithm for determining the surface locations. The first step was to see if the noise does reduce by averaging frames according to Equation 9.8 .

The plot in Figure 9.1 shows the theoretical reduction of the standard deviation of the noise versus the number of averaged images (black line) along with the actual measured reduction from four actual data sets (blue line). The data follows the theory quite well, so it is fair to say that the detector noise improves with averaging.

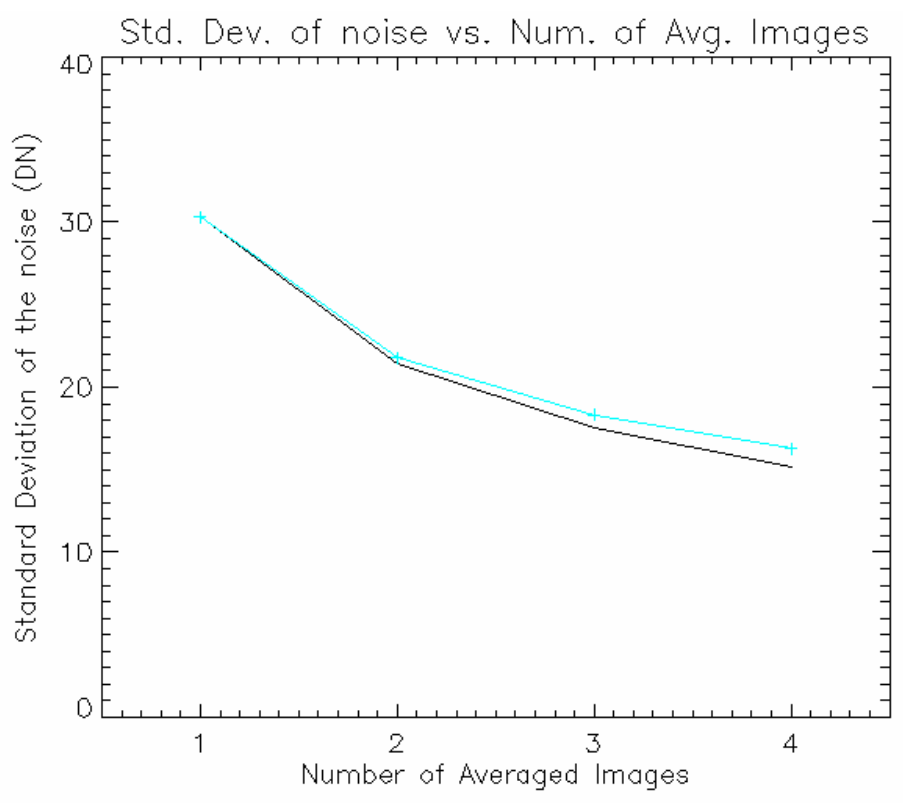

FIGURE 9.1 - Standard deviation of irradiance noise versus number of averaged images, theoretical and measured.

The LCI images for the four data sets were used to calculate the surface location of surface air 1 for each data set. After the best fit plane was subtracted from recovered surface, the standard deviation of the surfaces were calculated and plotted versus the 
number of images averaged at each depth location of the LCI scan. As the number of images increase, the standard deviation does decrease, but only at about $1 \mathrm{~nm} / \mathrm{image}$ averaged (Figure 9.2).

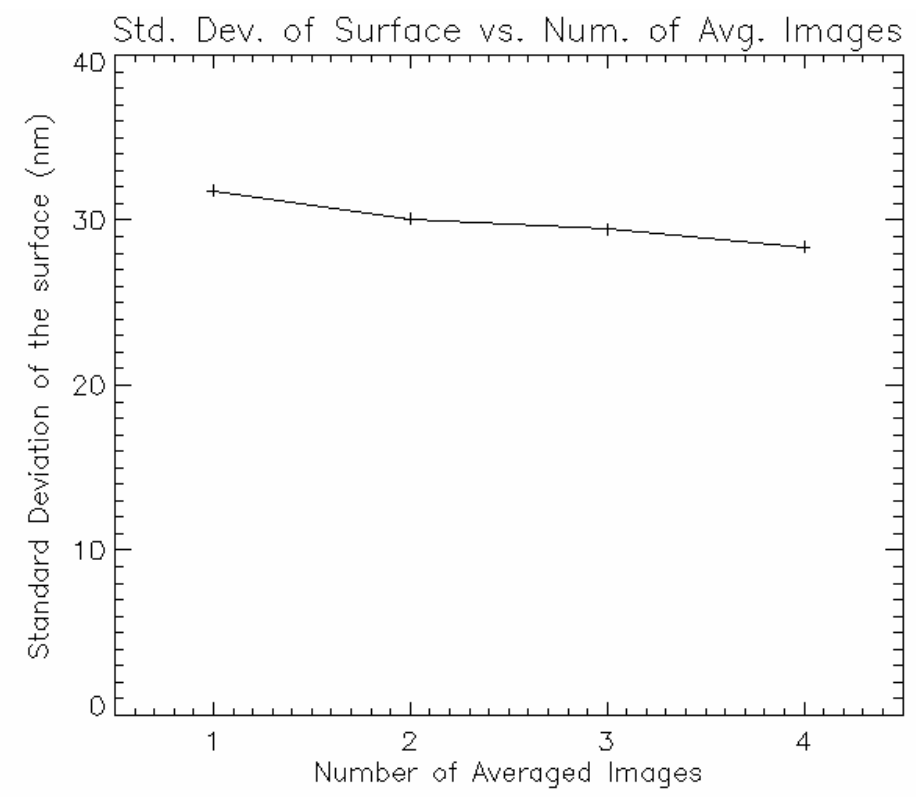

FIGURE 9.2 - Standard deviation of the surface error as a function of the number of images averaged during the LCI scan.

There are two conclusions to draw from this exercise. First, the improved surface standard deviation gain of $1 \mathrm{~nm} / \mathrm{image}$ does not warrant the extra time required to average multiple images at each location. Second, the standard deviation in the recovered surface is not primarily due to this detector noise. In fact, the primary error source within a calculated surface is due to coherent stray reflections that cause a spatially varying height error. As has been shown, the algorithm chosen tends to average out this spatial error by using spatial information to calculate the surface location. The error within a given surface is not the limiting noise for the thickness measurement 
portion of Engage. The errors in the optical encoder on the LCI stage are the limiting error source for the thickness measurement.

\subsection{Group Index Errors}

The group index of saline $\left(\mathrm{N}_{\mathrm{g}, \mathrm{sal}}\right)$ is an intermediate output that is necessary to arrive at the index of the sample. It is stored as a single value, and is calculate using four LCI surface locations (Chapter 2). It influences the thickness of the sample as shown in Equation 9.9.

$$
t_{\text {sam }}(x, y)=t_{1}(x, y)-\frac{O G L_{1,2}(x, y)}{N_{g, s a l}}
$$

Since the thickness error of the test sample required to cause a $\Delta \mathrm{n}$ of 0.0001 is known to be approximately $187 \mathrm{~nm}$, Equation 9.9 can be used to calculate the group index error which causes an error $t_{\text {sam }}$ of $t_{E}=187 \mathrm{~nm}$. This group index error is 0.00115 . This is quite large compared to the desired sample index accuracy. In practice, the group index error is just another way of expressing the effect of thickness errors.

\subsection{Phase Errors}

The differential OPL through the air, saline and sample columns of the cuvette at a given wavelength are all measured with a single set of 5 phase-shifted images (Chapter 5). Stray fringes, incorrect phase shifts and vibrations are all known to cause errors in $\varphi(x, y)$, along with other error sources. Rather than discuss the hypothetical effects due to each error source, the magnitude of the phase error required to cause an error in $\mathrm{n}$ of 0.0001 
will be found for the common test configurations. Each index of refraction calculation utilizes a phase difference, so it makes sense to express the phase errors as phase difference errors. From a $350 \mu \mathrm{m}$ thick saline sample, Equation 9.1 shows that a phase error of 0.39 radians will cause an index error of $0.0001(\lambda=543 \mathrm{~nm})$. For a hydrogel sample that is $134 \mu \mathrm{m}$ thick, a phase error of 0.15 radians is equivalent to an index error of 0.0001 . Finally, a $\Delta \mathrm{n}=0.0001$ for a glass calibration sample occurs for a phase error of 0.19 radians.

Since the laser has a fairly long coherence length, stray reflections are often coherent with the test and reference beams at the detector. These stray reflections are the dominant noise source for determining the phase using Engage. The typical point-topoint standard deviation of an unwrapped phase region ranges from 0.15 to 0.20 radians. For the most part, the phase measured at each pixel is independent of the neighboring pixels. By averaging the errors of all of these phase measurements, the phase error for each region is drastically reduced. A typical measurement of $\varphi_{\text {air }}$ contains about 200,000 values, reducing the phase error by $\sqrt{ } 200,000=447$. Even small regions will reduce the error by two orders of magnitude (10,000 points). The errors in the phase measurements contribute a negligible amount to the overall errors in calculating refractive index. This is evident in every plot of index versus wavelength shown thus far. It also allows the slope of the dispersion curve to be used for selecting the correct order $(\Delta \mathrm{m})$ since errors in the dispersion curve would be primarily influenced by phase errors. 


\subsection{Limiting Errors}

From the preceding discussion, it is clear that the system accuracy is limited by the thickness measurement half of Engage. The phase measurement results are consistently an order of magnitude better than required. The component of the thickness measurement that limits the system accuracy is the knowledge of the relative LCI stage positions which are the axial positions for the LCI images. Variations within a surface (Figure 9.2) are small enough that they are not limiting the system accuracy. Still, limiting the coherent stray reflections will reduce the surface variations and lower the risk of introducing a shift in the apparent surface location.

Another important error source is the sample temperature. Recall that distilled water has a change in index of 0.0001 per degree Celsius. The saline solution is primarily made up of water, and the water content of the hydrogel materials is approximately $50 \%$. From this simple argument, a best case $\mathrm{dn} / \mathrm{dT}$ for hydrogel materials is half that of water, or 0.00005 . This is at best half the accuracy goal taken up by a change in temperature of one degree. Proposed solutions for the limiting system errors are presented in the next chapter. 


\section{FUTURE WORK AND CONCLUSIONS}

A novel metrology system called Engage for measuring the refractive index of thin, transparent optical materials has been developed specifically for characterizing soft contact lens materials. The system combines the output from an LCI interferometer that measures the physical thickness of the sample with the output of a Mach-Zehnder interferometer that measures the differential OPL of the sample in transmission. One goal of this chapter is to summarize the current system capabilities and limitations. Another is to discuss possible future improvements and measurement applications such as testing a piece of a contact lens.

\subsection{System Capabilities and Limitations}

The original system goals are summarized in Table 10.1:

\begin{tabular}{|l|l|}
\hline Refractive index accuracy & 0.0001 \\
\hline Wavelengths & Visible $(\sim 400-700 \mathrm{~nm})$ \\
\hline Sample geometry & $\begin{array}{l}\text { Round disk }(\mathfrak{\imath} 200 \mu \mathrm{m}, \mathrm{D} \sim 10 \mathrm{~mm}), \\
\text { hydrated during testing }\end{array}$ \\
\hline Other considerations & Non-contact method \\
\hline
\end{tabular}

TABLE 10.1 - Original refractive index measurement goals.

Engage is capable of measuring thin, transparent optical materials. Although the system was designed for contact lens materials, Chapter 7 showed that it is capable of measuring glass samples as well as fluids. The range of testable thicknesses is limited on the thin 
side by the coherence length of the source. It is more appropriate to define the thickness limits in terms of the OGL, or the product of the physical thickness and the group index.

$$
1.5 \cdot l_{c}<O G L_{\text {sample }}
$$

The test sample OGL must be at least 1.5 times as large as the coherence length of the low coherence source. If it is less, the LCI signals from the two sample boundaries will start to overlap and it will be impossible to accurately determine the surface locations.

$$
n_{\text {sample }}(\lambda)=\frac{\lambda}{t_{\text {sample }}(x, y)}\left[\Delta m+\frac{\left[\varphi_{\text {sample }}(x, y)-\varphi_{\varepsilon}(x, y)\right]-\varphi_{\text {saline }, f i t}(x, y)}{2 \pi}\right]+n_{\text {saline }}(\lambda)
$$

Equation 10.2

The sample thickness upper limit is related to the index of refraction step for incrementing $\Delta \mathrm{m}$ by 1 in Equation 10.2. As the sample thickness increases, the change in index $\left(\Delta \mathrm{n}_{\mathrm{t}}\right)$ decreases (Equation 10.3).

$$
\Delta n_{t}=\frac{\lambda}{t}
$$

Equation 10.3

For a $1 \mathrm{~mm}$ sample measured at $\lambda=543 \mathrm{~nm}, \Delta \mathrm{n}_{\mathrm{t}}$ becomes 0.000543 . The "Christmas tree plot" becomes dense and it becomes difficult to choose between multiple orders. For a sample with two thickness regions, $\mathrm{t}_{1}=1000 \mu \mathrm{m}$ and $\mathrm{t}_{2}=900 \mu \mathrm{m}$, the noise ceiling is only $6 \times 10^{-5}$, or almost an order of magnitude below the noise ceiling rule of thumb of 0.0005 .

$$
\text { Noise Ceiling }=N C=\lambda\left(\frac{\left|t_{1}-t_{2}\right|}{t_{1} t_{2}}\right)
$$

Finally, the LCI signal distortion from that much dispersive material starts to become a problem (Chapter 2). Note that signal distortion would not be a problem for a sample 
that can adhere to the bottom plate of the cuvette like the hydrogel samples do, since no scan through the material would be required to measure the thickness. The upper bound on $\mathrm{OGL}_{\text {sample }}$ is set at $1000 \mu \mathrm{m}$ based on the limitations outlined here.

$$
1.5 \cdot l_{c}<O G L_{\text {sample }}=t N_{g, \text { sample }}<1000 \mu m
$$

The test sample must also be measured in the sample cuvette. For solid samples, the surfaces must be specularly reflective such that fringes are obtained from the interfaces. For a liquid, the glass/liquid interface provides the specular reflection.

$$
\theta_{\max }=\tan ^{-1}\left(\frac{0.25 \lambda_{c}}{\text { PixelPitch } /|m|}\right)=\tan ^{-1}\left(\frac{0.25 \cdot 0.680 \mu m}{4.65 \mu m /|0.5|}\right)=1.04^{\circ}
$$

Equation 10.6

The maximum surface angle for which the fringe frequency can be resolved by the camera is about $1^{\circ}$ from normal. Areas on the sample that are within this constraint can be measured.

The measured refractive index averaged over several measurements does not have a bias and agrees well with the calibration data for glass samples. There are two primary error sources which both relate to the thickness measurement using the LCI. First, the LCI stage errors are not perfectly constant so the calibration file contents to not completely correct for the errors with every measurement. This error causes thickness biases within a single measurement, resulting in biased values for $n(\lambda)$. The second is the point-topoint thickness variation which results from coherent stray reflections from dust and other scatters sites in the interferometer. 


\subsection{Future Work}

The Engage instrumentation that has been designed and built has facilitated a new index of refraction measurement technique. New theories and algorithms were developed as well, all of which have been implemented for use with the current hardware. The results prove the validity of the theory behind the instrument, which was the goal of this work. This does not mean that the full potential of this measurement technique has been achieved.

\subsubsection{Accuracy Improvement}

The first step towards improving the measurement accuracy of Engage is to accurately determine the axial locations of every frame taken during the LCI data collection. This can be done by replacing the current stage with a more reliable, precise stage, or by integrating an additional means of tracking the position of the current stage. Either way, this will improve the overall system accuracy and repeatability.

After the LCI reference mirror position errors have been corrected, it is likely that the next limiting factor will be temperature. The current instrumentation is only capable of monitoring the temperature near the measurement cuvette. Since the $\mathrm{dn} / \mathrm{dT}$ of saline is roughly $-0.0001 /{ }^{\circ} \mathrm{C}$, which is the measurement accuracy goal, it is important to know the sample temperature accurately. Another future item might be to incorporate temperature control of the cuvette. This would decrease the errors due to temperature fluctuations as 
well as provide the ability to measure $\mathrm{dn} / \mathrm{dT}$ of contact lens materials or other test samples.

It is usually important to collect data quickly in a temperature critical measurement because this reduces the temperature variation within a single measurement. It takes a little under 20 minutes to collect all of the required data with the current system. Of the 20 minutes, the first 10 minutes is required user interaction while the last 10 minutes is when the data is collected. Any temperature variations or other system drift during this time can cause index errors.

\subsubsection{Decreasing Data Collection Time}

Decreasing the data collection time is important from the perspectives of improved accuracy and improved throughput. The current time restrictions on Step 1 come from the stage settling time. After the stage tries to step a certain distance, it corrects that step in the case of an overshoot. This is done using feedback from the optical encoder. This process could not be turned off, so the system must wait $355 \mathrm{~ms}$ after each stage motion to be sure that the stage is stationary before capturing an image. This accounts for more than half the 10 minutes of data collection.

$$
0.355 \frac{\mathrm{sec}}{\mathrm{image}} \cdot(140 \cdot 3+280+160) \text { images } \cdot \frac{1}{60} \frac{\mathrm{min}}{\mathrm{sec}}=5.1 \mathrm{~min} \quad \text { Equation } 10.7
$$

The feedback loop is unnecessary since it is more important to accurately know the axial location of each image rather than to drive the stage to an exact, predetermined location. 
Adapting the hardware to this requirement would drastically decrease the time required for Step 1.

\subsubsection{Decreasing Data Processing Time}

The current realization of Engage was designed to be a research instrument. The amount of data collected for a single measurement is enormous ( $>1 \mathrm{~GB})$, so it is not too surprising that it takes about two hours to complete the data processing of steps 2 and 3 . As the LCI surface finding algorithms evolved into more complicated procedures, the time required also increased. All of the data processing code for steps 2 and 3 is written in IDL and comprises over 17,000 lines of code. There are two repetitive data processing steps which require the most time.

The first is calculating a surface location from the LCI data cubes for each interface (Step 2). Chapter 3 explained the steps. The current code finds $\gamma(z)$ for each pixel individually and then repeats the process for every pixel (up to 500,000 or more). Step 2 takes so long that it is usually run overnight for a group of 4 data sets. If the repetitive steps could be parallelized, the code could execute much faster, decreasing the processing time for a single sample and increasing the instrument throughput. If this instrument is ever meant to be used in a production environment, improving the code for Step 2 is critical.

The second time consuming data processing routine is the phase unwrapping that happens during Step 3. In an effort to reduce the required user interaction, this code was written 
to be nearly entirely self correcting. The phase is unwrapped at least twice in an effort to check for unwrapping errors and correct them automatically. A single, typically complex surface is unwrapped in about 30 to 45 seconds. This may not seem like much, but recall that a wedding cake sample measured at 5 wavelengths requires about 30 different surfaces to be unwrapped. Each of the air, saline and four sample regions must be unwrapped separately due to the phase boundaries between them. All of this is currently done with the help of a user, requiring approximately 20 minutes of their time. Again, there is no fundamental limitation in the software that is preventing this function from executing quickly. It is simply a matter of removing the unnecessary complexities and optimizing the function for speed.

\subsubsection{Ease of Use-Software}

The girth of the theory of this measurement should have indicated the complexity of this test along with many of the things that can go wrong for a measurement. One result of this complexity is the amount of user interaction required to navigate a measurement. In addition, a single measurement produces almost 1100 text and image files. Significant effort was put into removing the system user from this complexity. As a result, the user is not required to move, open or otherwise interact with any one of these 1100 files manually during a measurement; the software does it all. Part of this is done through a series of prompts to the user to select regions of interest, remove bad regions that could not be removed reliably with software, and click on the Christmas tree plots. Some of this user interaction could be replaced by automatic software selections, but this would 
require more code to assure that mistakes are not made that would have been prevented by a trained user. The current software is far too sophisticated to be considered research code yet it is not nearly automated enough to be on par with a commercial software package. The goal of the software was to allow a trained user to make a measurement without having to fight the computer, since there are plenty of physical foes to worry about.

\subsubsection{Ease of Use-Sample Mounting}

The largest physical hurdle to making measurements with Engage is mounting the material samples. Chapter 4 covered some of the failed attempts at mounting that were attempted prior to the current mounting scheme. Even for a trained user, mounting the sample can be extremely tedious. It can easily take four or five iterations to acceptably mount the sample. Surface chemistry plays a critical role in the current mounting setup and can probably be used to improve it further. Sample geometry also affects the mounting method.

\subsubsection{Contact Lens Testing}

Contact lens or pieces of contact lens are a testing geometry of considerable interest. Chapter 6 explained that a contact lens will probably need to be cut into a custom shape in order to be tested in Engage. As long as the lens thickness variation falls under the limits imposed by Equation 10.6, the lens can be tested as long as it can be mounted. Clearly, testing contact lens offers several advantages over testing custom stepped 
samples. Testing the final product removes the intermediate step of creating a custom sample, material could be measured that was created using the same process as the final product (a contact lens), and lenses from several different manufacturers could be characterized. The only thing preventing contact lens testing is a suitable mounting configuration. This should definitely be addressed in any future work on this project.

\subsection{Conclusions}

This dissertation started by explaining the need for a method for testing the refractive index of soft contact lens materials. What followed was a detailed description of the theory behind a novel measurement technique, an explanation of the hardware and software considerations necessary to implement that theory in a working instrument, and the measurement results of that instrument. This project has been challenging in many ways. From the theory through the final hardware and software versions, breakthroughs have often been required. As a student, the benefit of these challenges has been invaluable experience in design, development and troubleshooting of an optical system. The current form of Engage represents the result of vast improvements in every aspect discussed in this dissertation, but as outlined above, there is still work that can be done to further improve the system. 


\section{APPENDIX A: SUPERLUMINESCENT DIODE DATA SHEET}

Data sheet on Superluminescent diode used in Engage LCI, from SuperlumDiodes, LTD.

\section{SUPERLUM

Acceptance Test Report Device \# 108840

Test Date: $\quad 28.08 .03$

LIGHT-CURRENT and CURRENT-VOLTAGE CHARACTERISTICS @ T=25 C
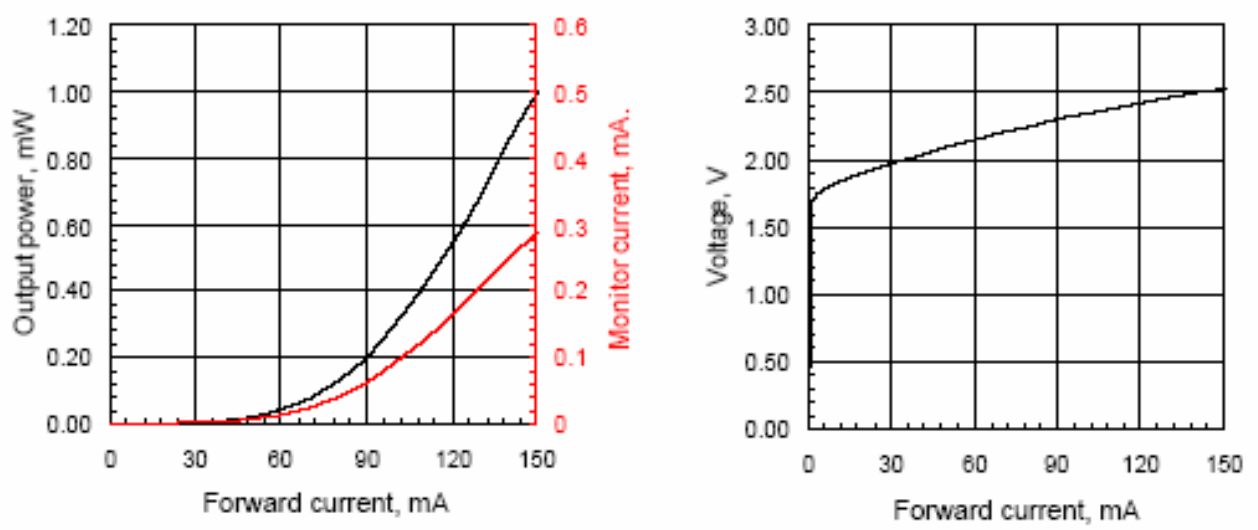

MAXIMUM SLD CURRENT IS $150 \mathrm{~mA}$

PERFOMANCE PARAMETERS @ 150 mA PUMPING CURRENT and T=25 C

\begin{tabular}{|l|c|}
\hline Spectral center, nm: & 677.2 \\
\hline Spectral bandwidth, FWHM, nm: & 13.8 \\
\hline Spectral Ripple, \%: & 0.6 \\
\hline Photodiode current, mA: & 0.289 \\
\hline
\end{tabular}




\section{APPENDIX B: CUSTOM MOUNT FOR SLD FIBER OUTPUT}

This appendix covers the schematics for duplicating the aluminum mount used to mount the 561-tilt (Figures B.1, B.3) which holds the 561-FCH (Figure B.2), which holds the optical fiber output of the SLD.

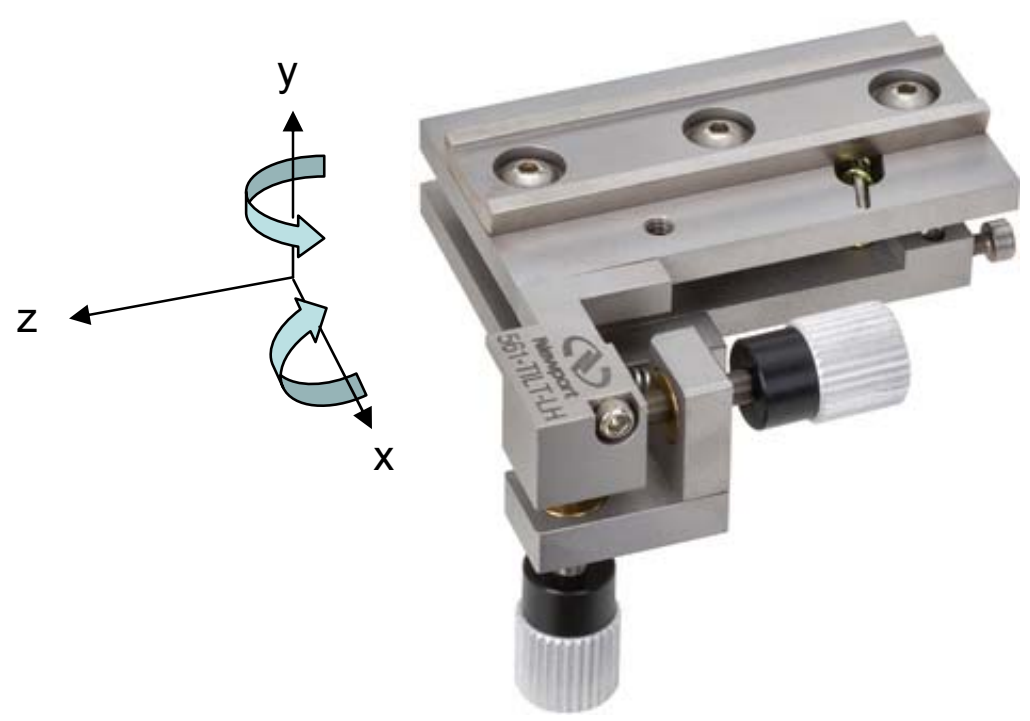

FIGURE B.1 - Photograph of the 561-tilt from Newport.

The 561-tilt has a total of $18^{\circ}$ of tilt around the $y$-axis and $4.7^{\circ}$ around the $x$-axis. The $y$ axis tilt is enough to overcome the $4^{\circ}$ beam deviation from the cleaved fiber. Rough ztranslation of the fiber position is achieved by translating the 561-FCH along the small rail on the 561-tilt. 

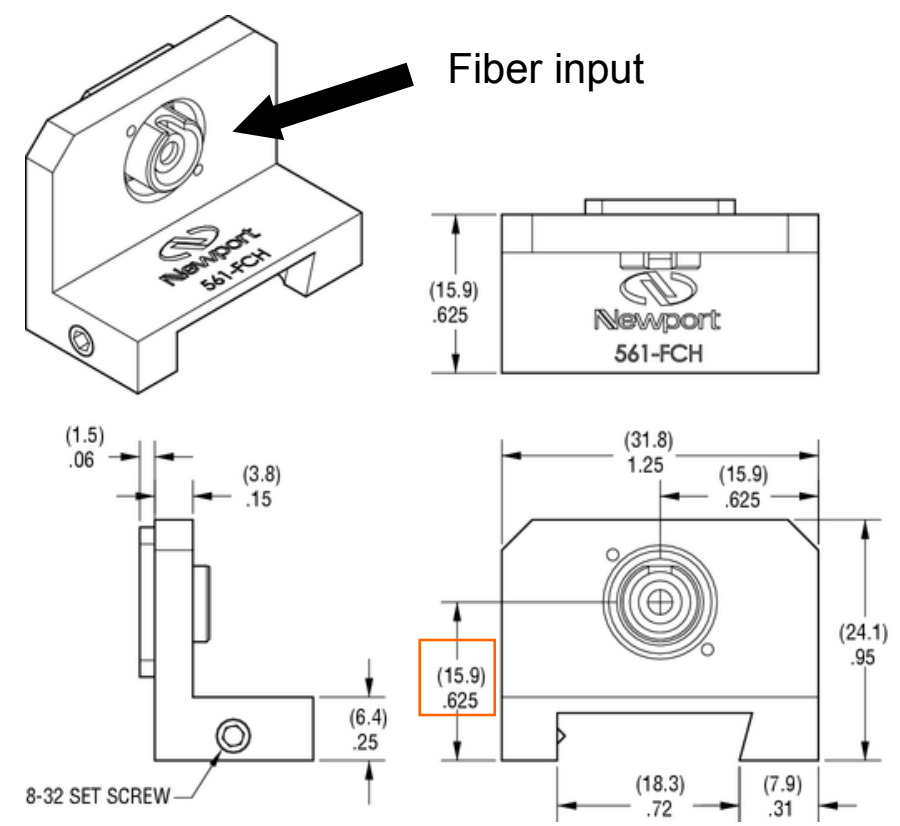

FIGURE B.2 - Drawings of the 561-FCH for holding the fiber.

The mount to hold the 561-tilt will be a static mount and will not add any ability to change the $\mathrm{x}$ or $\mathrm{y}$ position of the fiber output. Since the light source is the first component to be positioned during alignment, this adjustment is not necessary as long as the optical components down the line have the necessary adjustments. The desired height of the optical axis is 3", or $76.2 \mathrm{~mm}$. The distance from the bottom of the 561 tilt to the center of the fiber is $15.2 \mathrm{~mm}+15.9 \mathrm{~mm}=31.1 \mathrm{~mm}$. 


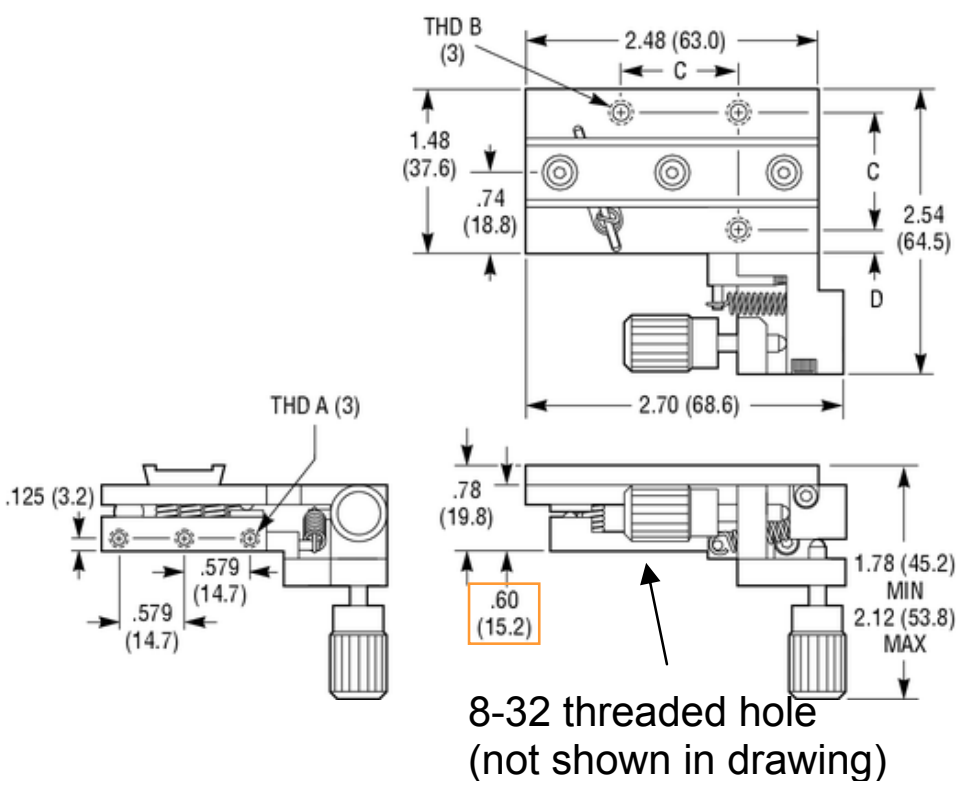

FIGURE B.3 - Drawings of 561-tilt.

Using these diagrams, the required height of the aluminum mount is $76.2-31.1=45.1 \mathrm{~mm}$ high. The distances in the drawings for the mount are all in inches, since this is what is commonly used in a machine shop. This mount is machined from a solid block of aluminum that has starting dimensions of at least: 1.9"x2.8"x2.0". Three clearance holes on the front lip are included for three 4-40 screws, which are used to mount the 561-tilt to this mount. There is also a clearance hole for an 8-32 screw to attach to the bottom of the 561-tilt. There is a spring for the rotation about y that requires a 0.5 " wide, 0.1 " deep trough for clearance. The base of this mounts is attached to the vertically mounted breadboard using 1/4-20 screws through clearance holes and a clearance slit to allow for some rough translation along x. The schematics are shown in Figure B.4. 


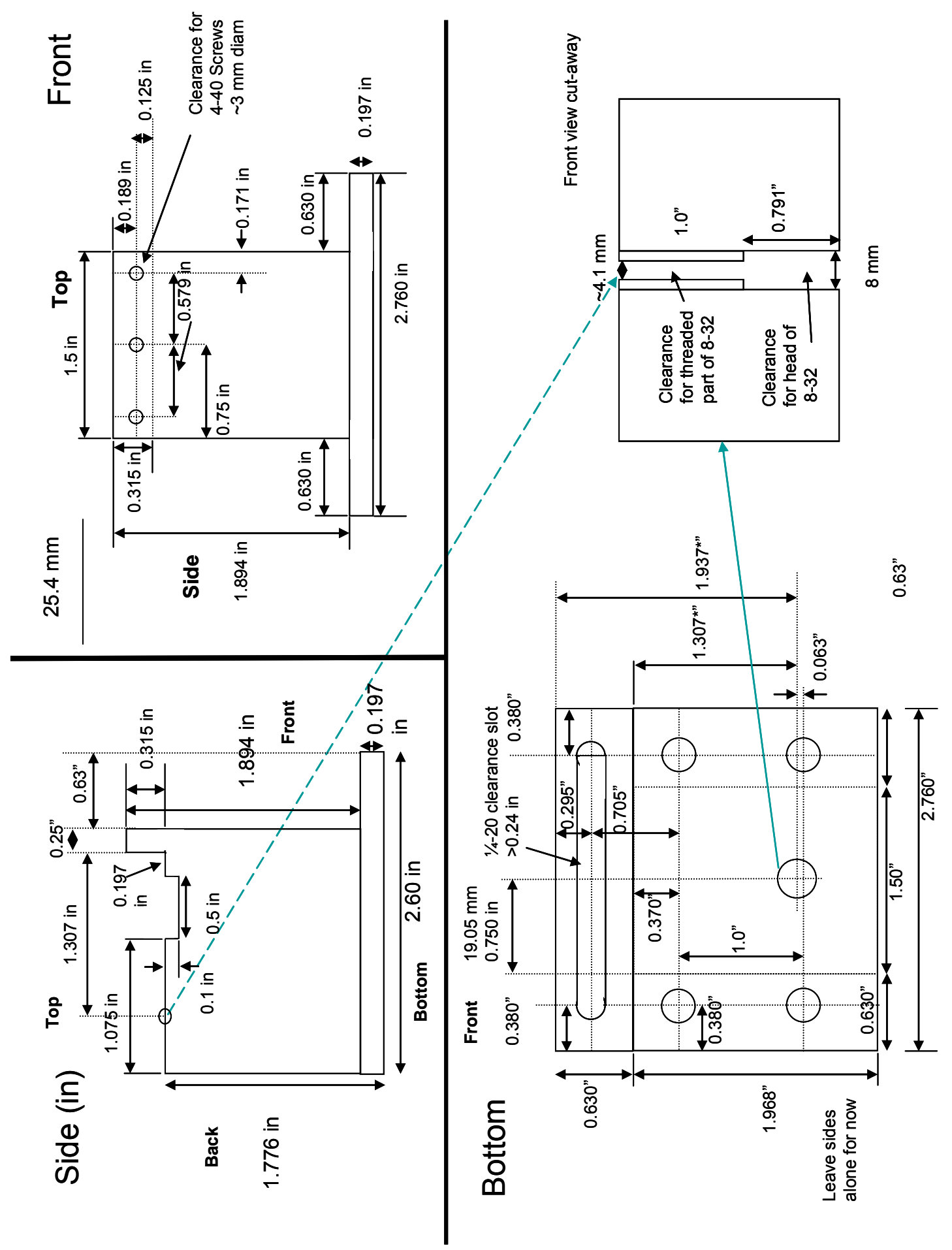

FIGURE B.4 - Schematic for custom mount. 


\section{APPENDIX C: DETERMINING CORRECT TILT OF COMPENSATING PLATE}

The purpose of the compensating plate is to balance the amount and type of dispersive material in the top plate of the cuvette. Two plates are ordered that are nominally identical, but inevitable one will be slightly thinner. The thinner plate will be the compensating plate, which can be found using calipers. Align the system without the top plate of the cuvette and without the compensating plate in place, and position the stage at the region with highest contrast LCI fringes from the top surface of the bottom plate of the cuvette. Assuming the sapphire spacers are already fixed to the top plate of the cuvette, place the top plate in place to complete the cuvette, after placing a piece of tissue paper inside the cavity to block the reflection from the bottom cuvette plate. The fringes should disappear due to the increased path length in the test arm.

Next, move the reference surface towards the beamsplitter a distance $350 \mu \mathrm{m}$; the height of the cavity. This positions the plane of $\mathrm{OGD}=0$ coincident with the bottom surface of the top plate of the cuvette except that the paths are mismatched, so no fringes will be observed yet.

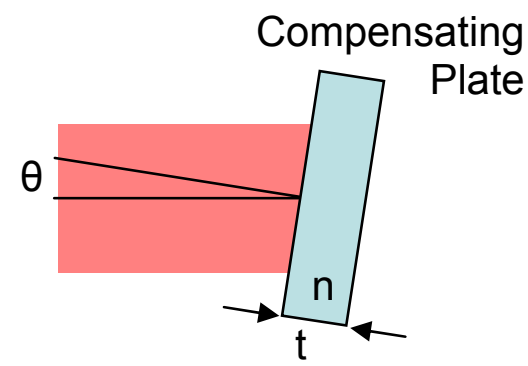

FIGURE C.1 - Tilt the compensating plate out of the beam to increase OGL. 
Place the compensating plate in the opticlaw mount, as close to normal to the reference beam as possible. By rotating the compensating plate as shown in Figure C.1, the OGL of the plate will increase as a function of tilt angle, $\theta$. By observing the fringes on the camera, the tilt of the plate is adjusted until the fringe contrast has reached a maximum. At this tilt angle, the paths through glass plates (top of cuvette and compensating plate) will be matched to within about half the coherence length of the source, or about $18 \mu \mathrm{m}$. 


\section{APPENDIX D: IDL FUNCTION POSITION_MAP_FILE.PRO}

Below is the code from the IDL function, POSITION_MAP_FILE.PRO:

pro position_map_file

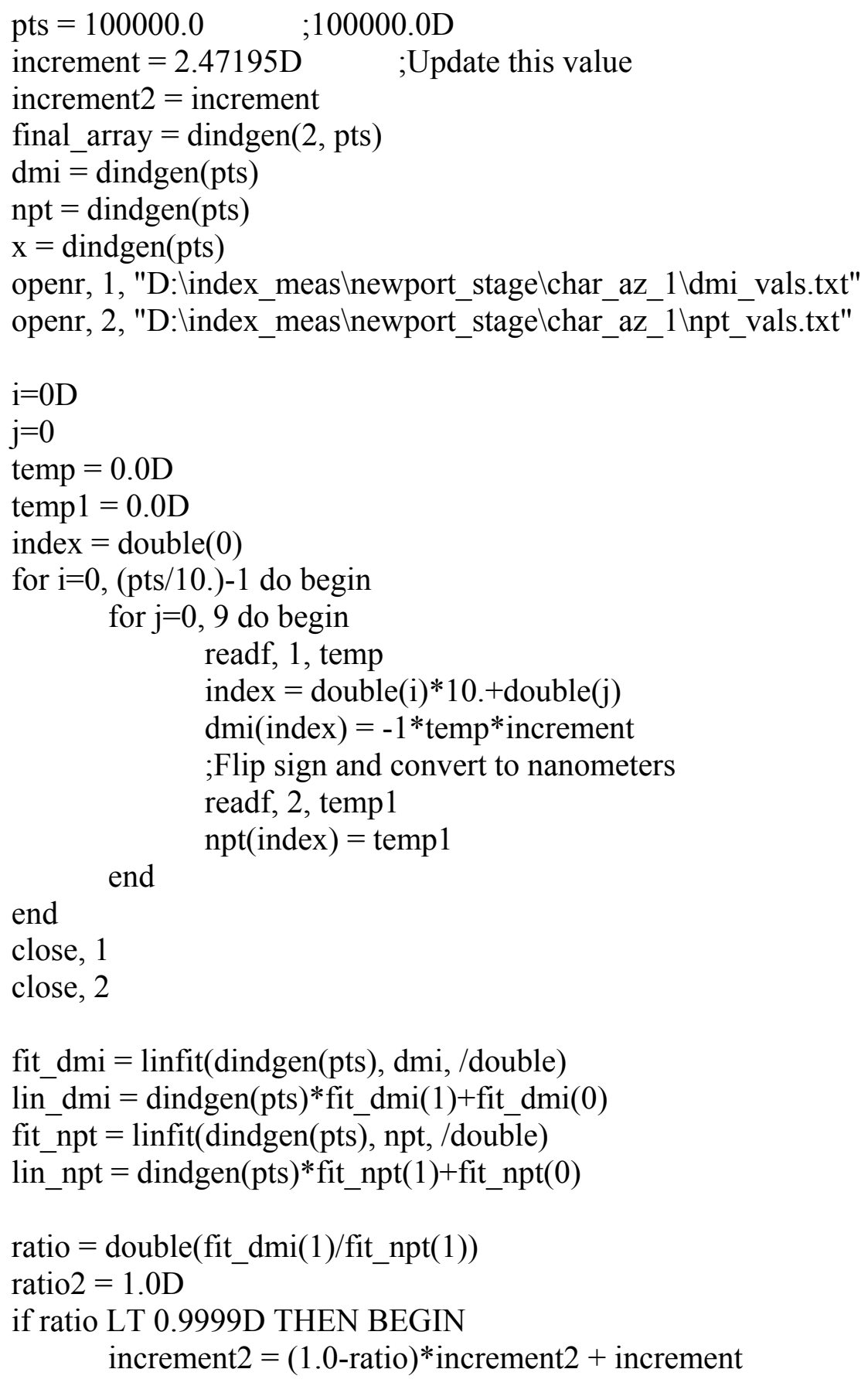

end

close, 1

close, 2

fit_dmi = linfit(dindgen(pts), dmi, /double $)$

lin_dmi $=$ dindgen $(\mathrm{pts}) *$ fit_dmi $(1)+$ fit_dmi $(0)$

fit_npt $=$ linfit(dindgen(pts), npt, /double)

lin_npt $=\operatorname{dindgen}(\mathrm{pts}) *$ fit_npt $(1)+\mathrm{fit} \_n p t(0)$

ratio $=$ double(fit_dmi(1)/fit_npt(1))

ratio2 $=1.0 \mathrm{D}$

if ratio LT 0.9999D THEN BEGIN

$$
\text { increment } 2=(1.0 \text {-ratio }) * \text { increment } 2+\text { increment }
$$




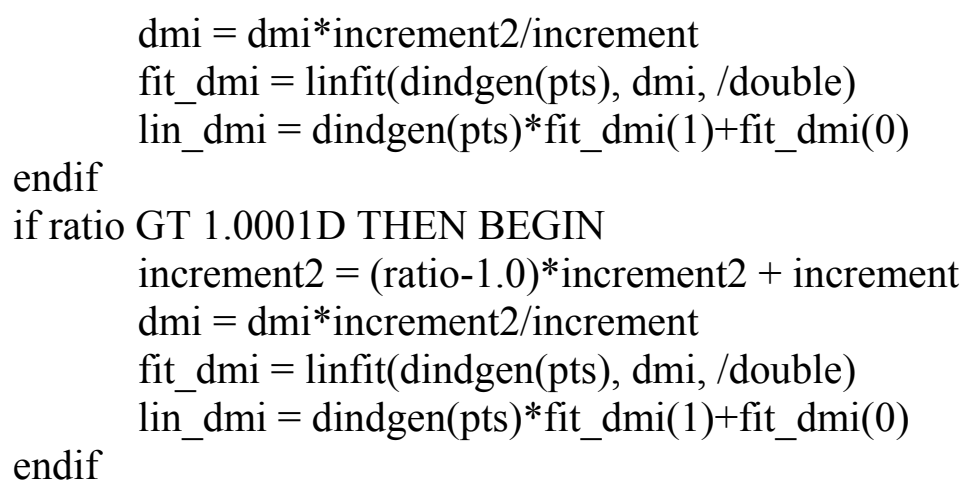

;now, find the 0 point for the npt, then adjust the offset of the ; dmi data accordingly.

zero_pt $=0.0 \mathrm{D}$

start_pt $=\min (\operatorname{abs}(\mathrm{npt})$, min_loc $)$

start pt $=0$

WHILE abs(npt(min_loc-10+start_pt)) GT 30 DO BEGIN

start_pt $=$ start $\_\mathrm{pt}+1$

print, npt(min_loc-10+start_pt)

endwhile

;Adjust the DMI data by $(0,0)$ offset

zero_pt $=$ min_loc-10+start_pt

dmi_off $=$ dmi $($ zero_pt $)$

$\mathrm{dmi}=\mathrm{dmi}-\mathrm{dmi}$ off

fit_dmi $(0)=$ fit_d $\bar{d}$ mi $(0)-$ dmi_off

print, "DMI min value:", dmi(zero_pt-1:zero_pt+1)

;Adjust the NPT data

npt_off $=$ npt(zero_pt)

$n p t=n p t-n p t \_o f f$

fit_npt $(0)=$ fit_npt(0) - npt_off

print, "NPT min value:", npt(zero_pt-1:zero_pt+1)

final_array $(1, *)=n p t-\mathrm{dmi}$

final_array $(0, *)=\mathrm{npt} \quad ; \mathrm{dmi}$

;Above, i think i need to use the npt positions to define where the errors are...

print, "errors: these get subtracted from NPT reported positions: ",

transpose(final_array(1, zero_pt-1:zero_pt+1))

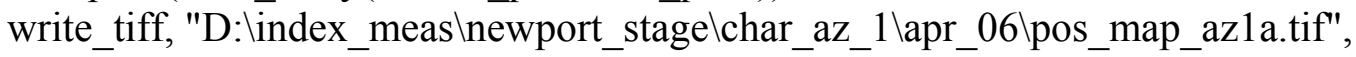
final_array, compression $=0, /$ long 


\section{APPENDIX E: CAMERA SPECIFICATION SHEET}

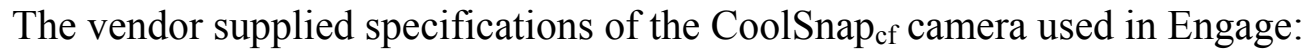

\section{PHOTOMETRICS \\ a division of Roper Scientific, Inc.}

\section{CoolSNAP cf Monochrome}

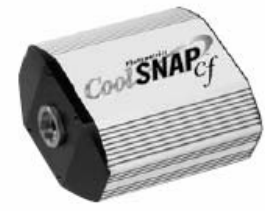

1392 x 1040 imaging array | $4.65 \times 4.65-\mu \mathrm{m}$ pixels

The CoolSNAP cf Monochrome camera from Photometrics incorporates low-noise electronics and moderate CCD cooling to achieve good low-light sensitivity. A megapixel sensor with small, square elements ensures that each image shows extraordinary detail This feature, along with a high-speed digitizer, shutterless operation, and an interline transfer $C C D$, makes the CoolSNAP $f$ Monochrome camera ideal for high-resolution microscopy and inspection applications.

\begin{tabular}{|c|c|}
\hline Features & Benefits \\
\hline 20-MHz digitization & Fast image readout for high-speed focus and image capture \\
\hline $\begin{array}{l}1392 \times 1040 \text { imaging array } \\
4.65 \times 4.65 \text { - pm pixels }\end{array}$ & Resolves fine detail \\
\hline \multicolumn{2}{|c|}{ Interline-transfer, progressive-scan CCD Full resolution in every frame } \\
\hline Flexible binning and readout & Increases light sensitivity while increasing the frame rate \\
\hline 12-bit digitization & Quantifies bright and dim signals in the same image \\
\hline Thermoelectric cooling & Increases integration times for higher sen sitivity \\
\hline C-mount & Easily attaches to microscopes, standard lenses, or optical equipment \\
\hline Subcompad, fanless design & Low profile allows easy setup \\
\hline Aoquisition soffware & Captures, analyzes, and saves high-resolution images \\
\hline Video output (optional) & Compatible with standard video equipment \\
\hline $\mathrm{PQ}$ interface & High-bandwidth, uninterrupted data transfer \\
\hline $\begin{array}{l}\text { PVCAM० } \\
\text { Circular buffers } \\
\text { Device sequencing }\end{array}$ & $\begin{array}{l}\text { Supported by numerous third-party software packages } \\
\text { Realtime focus } \\
\text { Precise integration with shutters, filler wheels, etc. } \\
\text { Compatible with Windows } 2000 / X P \text {, Mac OS X, and Red Hat" Linux } 9.0 \text { (kernel version 2.4) }\end{array}$ \\
\hline
\end{tabular}

FIGURE E.1 - Page 1 of camera data sheet (Photometrics, 2006). 


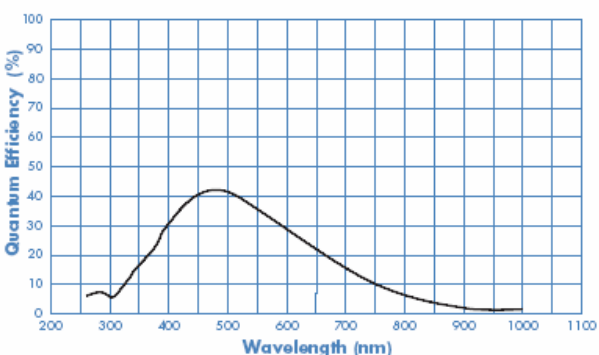

\begin{tabular}{llll}
\multicolumn{5}{c}{ Region } \\
\hline & $1392 \times 1040$ & $512 \times 512$ & $256 \times 256$ \\
\hline $1 \times 1$ & 11 & 21 & 38 \\
\hline $2 \times 2$ & 21 & 36 & 56 \\
\hline $3 \times 3$ & 28 & 46 & 71 \\
\hline $4 \times 4$ & 36 & 56 & 83 \\
\hline $8 \times 8$ & 56 & 83 & 125 \\
\hline \multicolumn{4}{c}{ (Frames per second) }
\end{tabular}

Note: Frame rates are mecsured at $20 \mathrm{MHz}$ with 0-second exposure times.

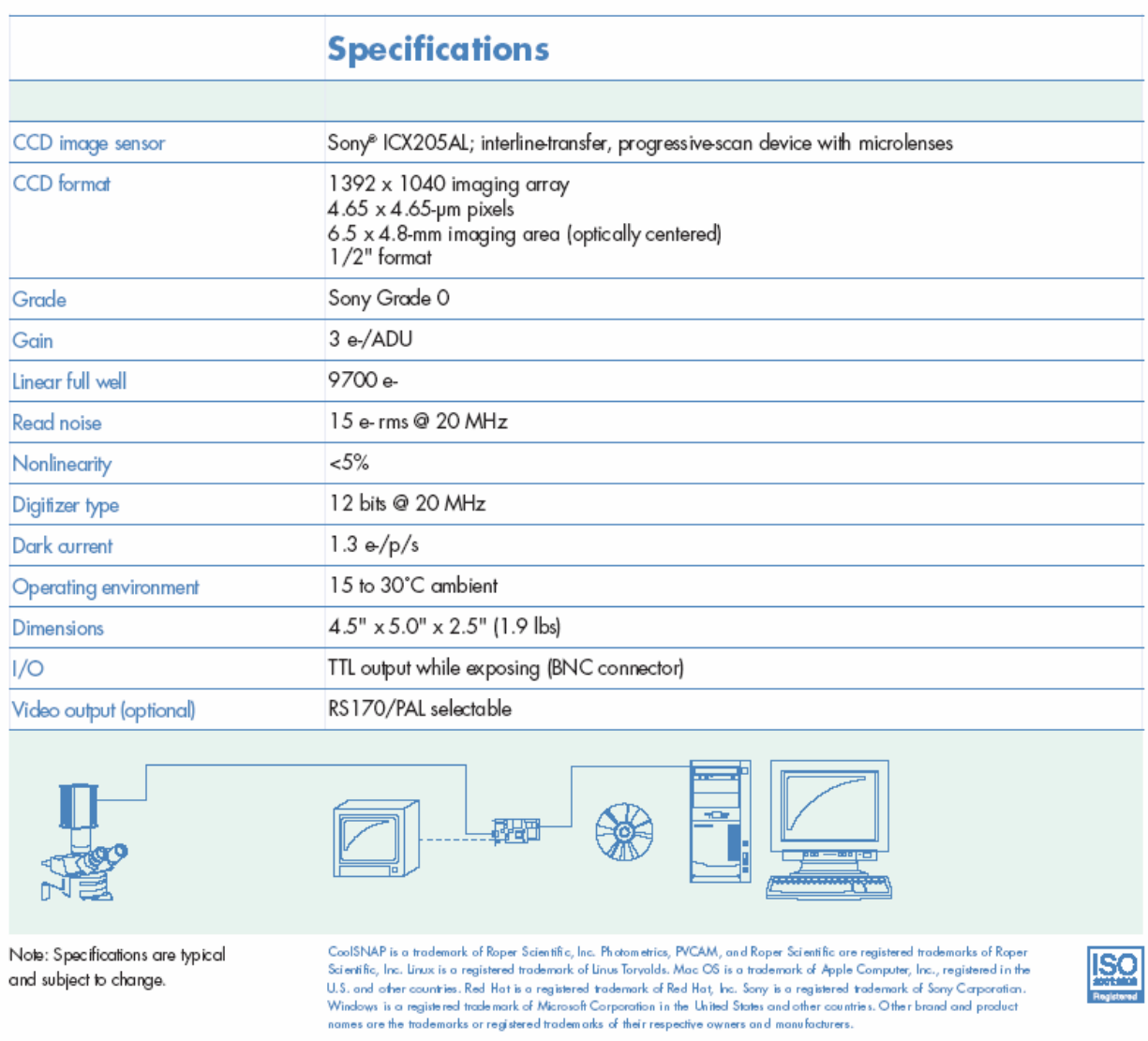

PHOTOMETRICS

USA 520.889.9933 | Benelux +31.347.324989 | France +33.160.86.03.65

Germany +49.89.660.779.3 I Japan +81.43.274.8022 I info@photomet.com I www. photomet.com CoolsNaPf Momo RovCl

FIGURE E.2 - Page 2 of camera data sheet (Photometrics, 2006). 


\section{APPENDIX F: REFERENCE DISPERSION CURVE FOR SALINE}

Chapter 6 describes the usefulness of a reference dispersion curve in determining the correct value for $\Delta \mathrm{m}$ for saline. This data is stored in "generic_n_sal_HC.dat" and stored in the "|config|" directory of the data folder for Engage. The contents of this file are shown here.

\begin{tabular}{ll}
$10 \quad 53.6982$ & \multicolumn{1}{c}{23.0000} \\
0.45789999 & 0.36128479 \\
0.47650000 & 0.36048254 \\
0.48800001 & 0.36020693 \\
0.49649999 & 0.35949636 \\
0.51450002 & 0.35918537 \\
0.54334998 & 0.35814318 \\
0.59391999 & 0.35716021 \\
0.60460001 & 0.35699362 \\
0.61179000 & 0.35676262 \\
0.63279998 & 0.35644987 \\
458.00000 & 1.3413075 \\
477.00000 & 1.3401375 \\
488.00000 & 1.3394011 \\
497.00000 & 1.3391373 \\
515.00000 & 1.3380980 \\
543.00000 & $\mathbf{1 . 3 3 6 4 8 3 4}$ \\
594.00000 & $\mathbf{1 . 3 3 4 7 3 5 8}$ \\
605.00000 & $\mathbf{1 . 3 3 4 4 3 3 7}$ \\
612.00000 & $\mathbf{1 . 3 3 4 2 1 1 5}$ \\
633.00000 & $\mathbf{1 . 3 3 3 6 0 4 3}$
\end{tabular}

The data includes the number of wavelengths (10), the Abbe number (53.69), and the temperature $\left(23^{\circ} \mathrm{C}\right)$. For the 10 wavelengths, the measured angle in radians is recorded along with the calculated index of refraction values. The bold values are used to plot the baseline dispersion curve. 


\section{APPENDIX G: EXAMPLE SAMPLE MOUNTING IMAGES}

One of the trickiest aspects of Engage is mounting the test samples. It is important to be able to distinguish between a correctly and incorrectly mounted test samples by looking at a raw image from the camera prior to executing a measurement. By not proceeding with the data collection of an improperly mounted sample, the user saves time by not capturing useless data.

There are several requirements to look for in an image of a contact lens material that is mounted in the cuvette. This appendix is meant to briefly describe these requirements and provide example images as a reference.

The first requirement is that there are substantial regions of air, saline and the sample within the field of regard of the camera. The air and saline regions must be continuous, or only one section of each material can be used since the phase can only be unwrapped over a continuous region. Figure G.1a-d shows four examples of acceptable spatial multiplexing. In all cases, the saline region surrounds the sample while the air region is near the perimeter of the image. 

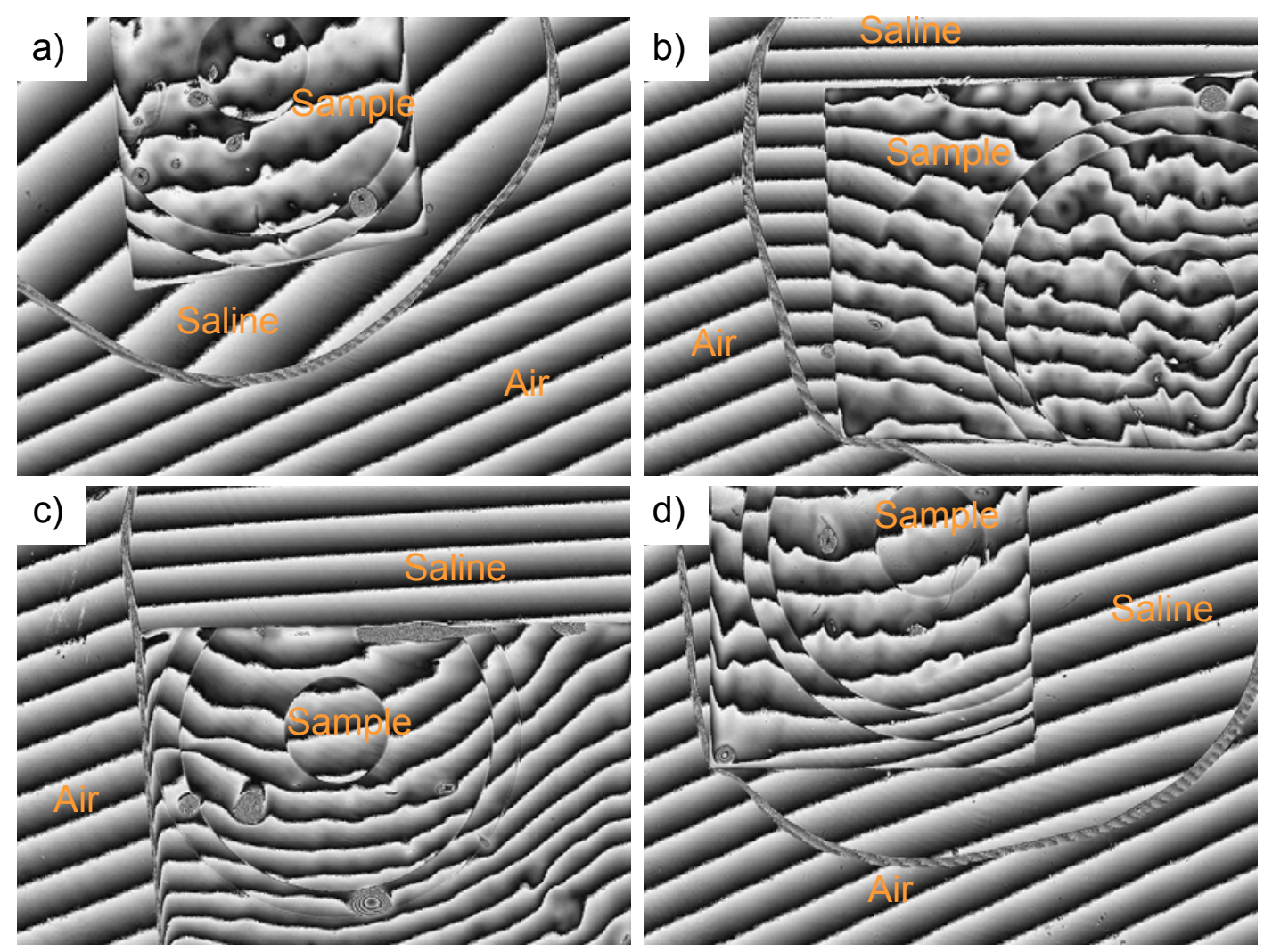

FIGURE G.1 - Four example images of suitable spatial multiplexing.

The more difficult aspect of mounting is obtaining a sample that is completely in contact with the bottom plate of the cuvette. If a small amount of saline, an air bubble, or a piece of debris gets between the sample and the glass plate, then Engage will not be able to test that region. It is difficult to obtain a mounted sample without any of these features, but the key is to limit the area that they render useless. All of the figures shown below are single images captured with the SLD light source and are enough to decide whether or not to proceed with the measurement based on the quality of mounting. 
The bright regions in Figure G.2 circled in orange are air bubbles. The high Fresnel reflection between air and glass is responsible for the brightness.

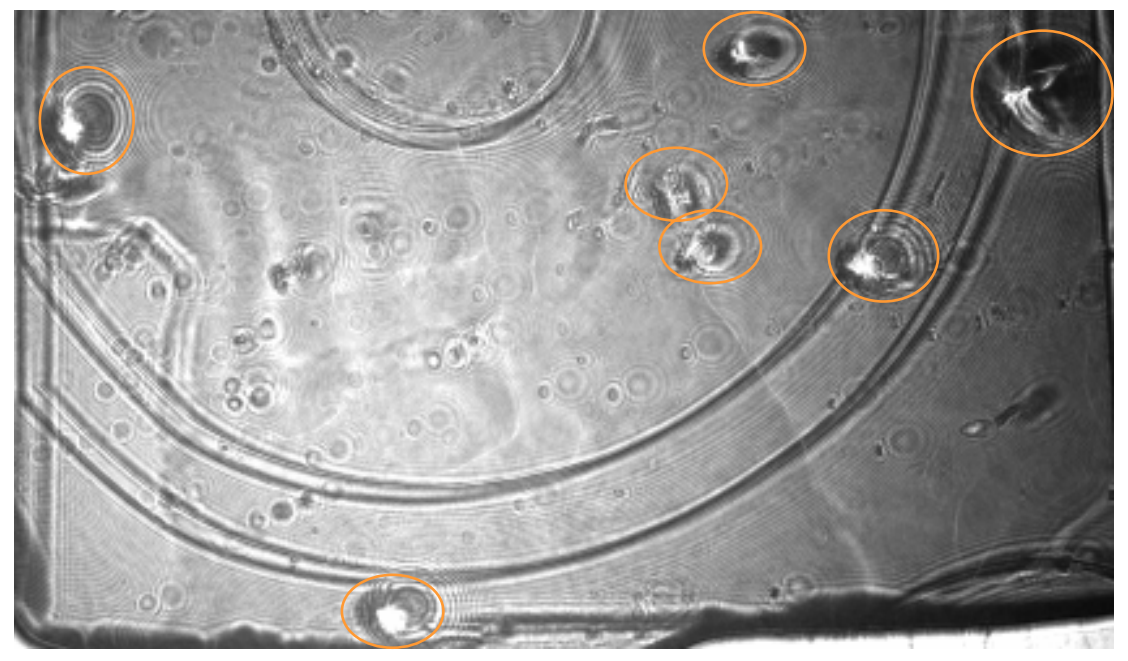

FIGURE G. 2 - Air bubbles appear as bright regions often surrounded by a circular diffraction pattern.

The most common and most difficult thing to detect is the presence of saline under the sample. The saline creates an extra interface. If the saline bubble is small enough, the two reflected beams (glass/saline and saline/sample) are coherent and form a static fringe pattern (Figure G.3, blue circles). The orange oval indicates a pieces of debris in the cuvette. 


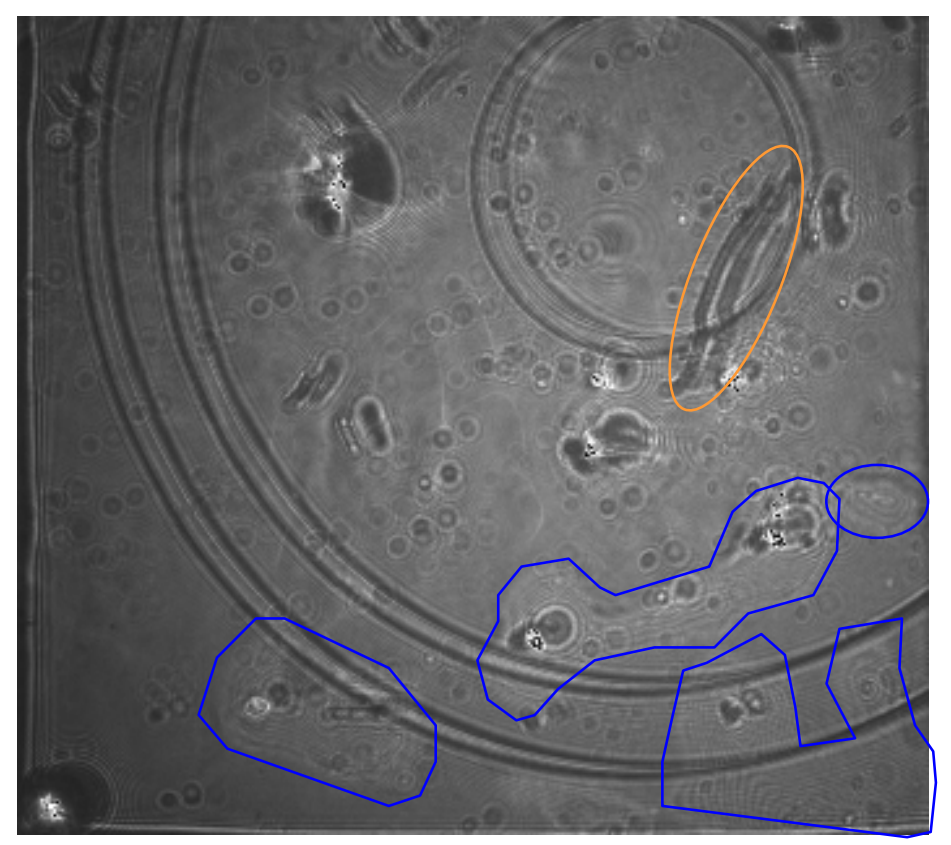

FIGURE G.3 - Blue polygons outline the static fringe patterns indicative of saline bubbles. The orange oval indicates debris.

The majority of the sample in Figure G.4 is not sticking to the bottom plate. There are static fringes throughout the image, but the bottom third of the image does not have these static fringes. At first glance, it would be easy to decide that the sample is sticking in this region. If we examine the boundaries of this region (blue polygon), we see that the static fringe contrast decreases as we move into the lower third of the image. In fact, this region is not sticking; it is so far from the glass that the two fringe patterns are no longer temporally coherent with each other. Another clue that this part of the sample is floating in saline is the relative brightness. The region in the black oval is one of the few portions of the sample that is sticking. Note the uniform gray level (neglecting the region boundaries) relative to the gray level inside the blue polygon. The extra light comes from the extra interface. 


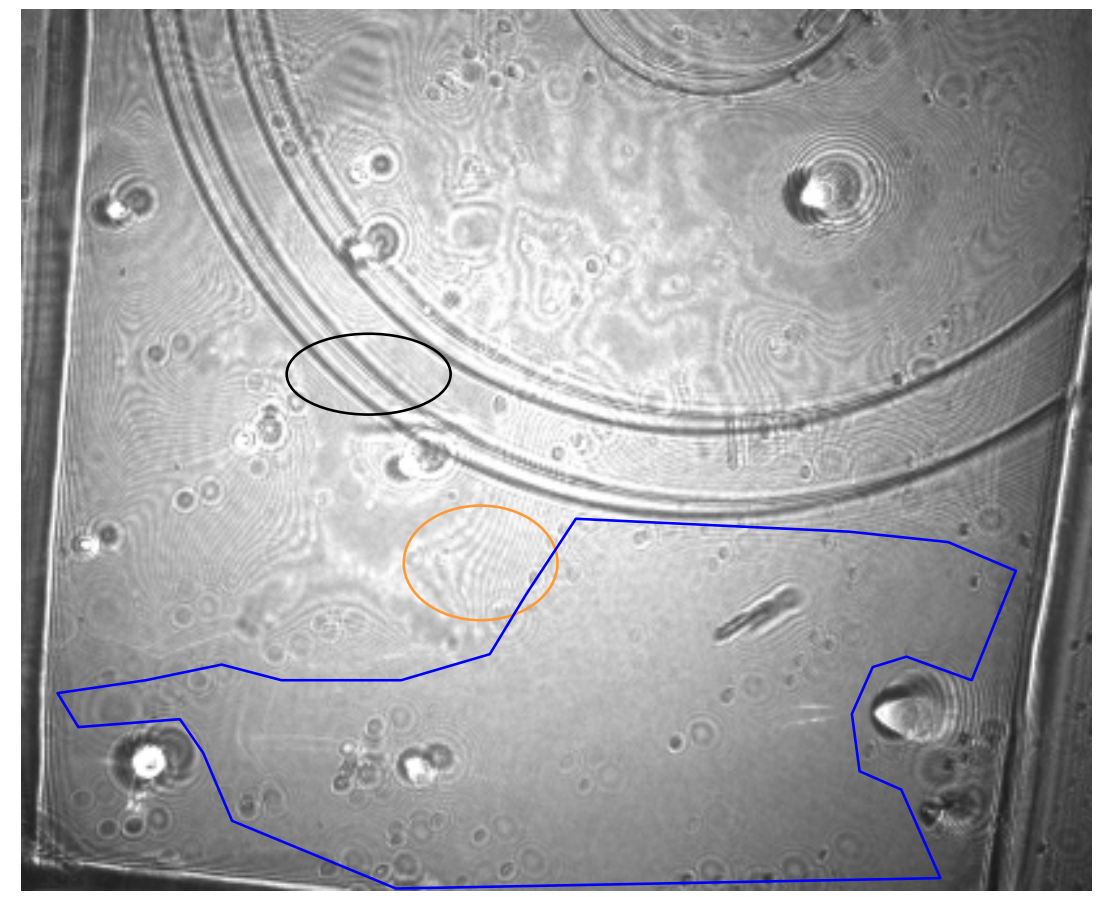

FIGURE G.4 - At first glance, the region outlined in blue is sticking. The loss of static fringe visibility and the brightness of the region indicate that this area is not sticking.

A similar sample image is shown in Figure G.5 where the brightness difference is clearer.

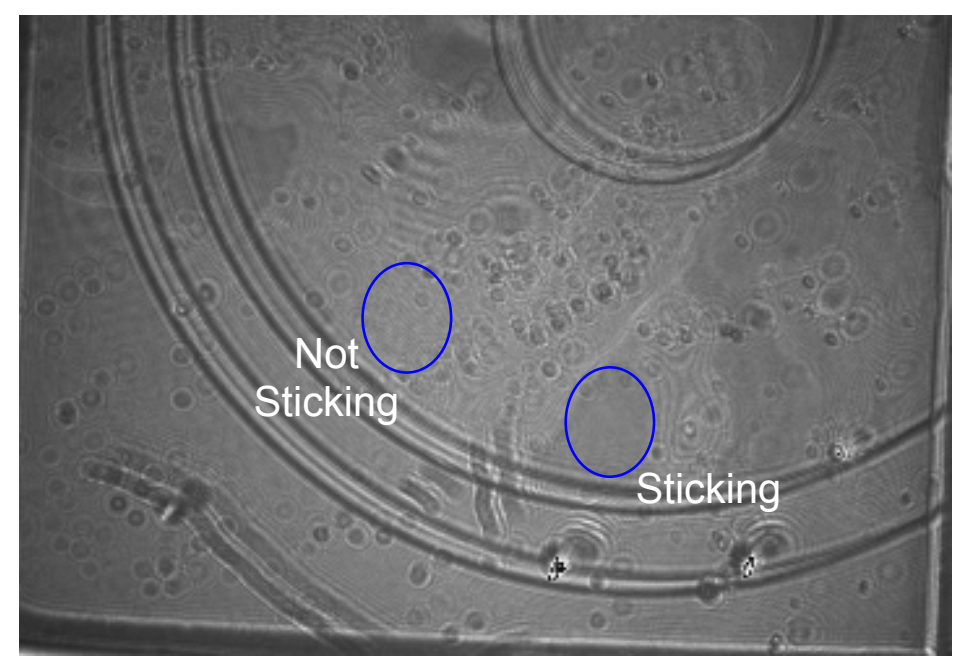

FIGURE G.5 - Another example of brightness variations. 
Finally, Figure G.6 shows a sample that is mounted adequately. There are still undesired features, such as debris and air bubbles (orange) and a few saline bubbles (blue). Rarely will a sample be mounted without some defects.

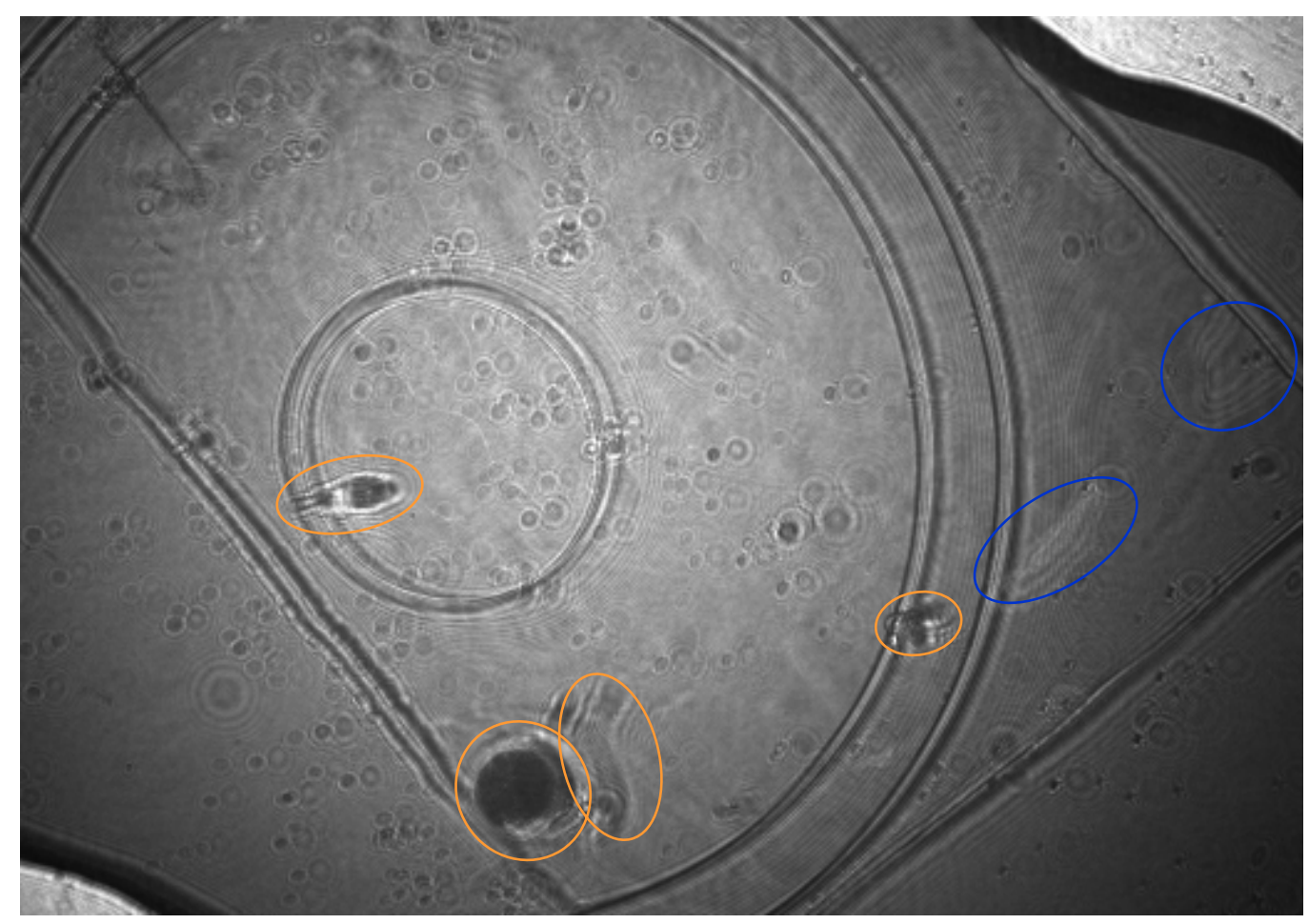

FIGURE G.6 - This sample is mounted correctly, even though there are regions with defects.

Differentiating between a sample that is mounted correctly or incorrectly is an important user-dependent aspect of measuring refractive index with Engage. 


\section{APPENDIX H: REFRACTIVE INDEX OF GLASS CALIBRATION SAMPLES}

This table summarizes the refractive index of the seven calibration samples as measured on the prism spectrometer.

\begin{tabular}{|c|c|c|c|c|c|}
\hline Sample & $\mathrm{n}_{543}$ & $\mathrm{n}_{593}$ & $\mathrm{n}_{604}$ & $\mathrm{n}_{611}$ & $\mathrm{n}_{632}$ \\
\hline BK7-1 & 1.518955 & 1.516618 & 1.516186 & 1.515904 & 1.515158 \\
\hline BK7-2 & 1.518963 & 1.516640 & 1.516211 & 1.515926 & 1.515182 \\
\hline BK7-3 & 1.518969 & 1.516643 & 1.516202 & 1.515939 & 1.515188 \\
\hline BK7-4 & 1.519111 & 1.516767 & 1.516340 & 1.516062 & 1.515307 \\
\hline BK7-5 & 1.518759 & 1.516415 & 1.515989 & 1.515719 & 1.514962 \\
\hline FS-1 & 1.460252 & 1.458277 & 1.457908 & 1.457695 & 1.457062 \\
\hline FS-2 & 1.460250 & 1.458291 & 1.457932 & 1.457695 & 1.457058 \\
\hline
\end{tabular}

TABLE H.1 - Prism spectrometer data for the 7 glass calibration samples.

Prism FS-2 was destroyed while being converted to the thin geometry required for testing in Engage and is therefore no longer available for use as a calibration sample. All of the prisms were measured at $\mathrm{T}=23^{\circ} \mathrm{C}$. 


\section{REFERENCES}

Azzam, R.M.A. and Bashara, N.M., Ellipsometry and Polarized Light, North-Holland, New York, 1988.

Blanca, C. and Hell, S., "Axial superresolution with ultrahigh aperture lenses," Opt. Express 10, 893-898 (2002).

Born, M. and Wolf, E., Principles of Optics, Seventh (Expanded) Edition, Cambridge University Press, Cambridge, UK, 1999.

Brumfield, R. B., "Measuring the Refractive Index of Hydrogel Material," Master's Report, University of Arizona, 2003.

Caber, P. J., "Interferometric profiler for rough surfaces," Appl. Opt. 32, 3438- (1993).

Danielson, B. L. and Boisrobert, C. Y., "Absolute optical ranging using low coherence interferometry," Appl. Opt. 30, 2975- (1991).

Deck, L. and de Groot, P., "High-speed noncontact profiler based on scanning white-light interferometry,” Appl. Opt. 33, 7334- (1994).

Fowles, G. R., Introduction to Modern Optics, Second Edition, Dover, New York, 1989.

Frieden, R. B., Probability, Statistical Optics, and Data Testing; A Problem Solving Approach, Third Edition, Springer-Verlag, New York, 2001.

Fukano, T. and Yamaguchi, I., "Separation of measurement of the refractive index and the geometrical thickness by use of a wavelength-scanning interferometer with a confocal microscope," Appl. Opt. 38, 4065 (1999).

Gaskill, J. D., Linear Systems, Fourier Transforms, and Optics, John Wiley \& Sons, New York, 1978.

Ghiglia, D. C. and Pritt, M. D., Two-Dimensional Phase Unwrapping; Theory, Algorithms and Software, John Wiley \& Sons, New York, 1998.

Goodwin, E. P. and Wyant, J. C., Field Guide to Interferometric Optical Testing, (FG 10) SPIE Press, 2006.

Greivenkamp, J. E. Field Guide to Geometrical Optics, (FG 1) SPIE Press, 2004. 


\section{REFERENCES - Continued}

Greivenkamp, J. E. “Generalized data reduction for heterodyne interferometry," Opt. Engr., Vol. 23, 350- (1984).

Greivenkamp, J. E. and Bruning, J. H., "Phase Shifting Interferometers," in Optical Shop Testing, Second Edition, D. Malacara, ed, John Wiley \& Sons, New York, 1992.

de Groot, P. and Deck, L., "Three-dimensional imaging by sub-Nyquist sampling of white-light interferograms," Opt. Lett. 18, 1462- (1993).

Guo, S. et. al. "Determination of refractive index and thickness of thick transparent films by variable-angle spectroscopic ellipsometry: application to benzocyclobutene films," Appl. Opt. 35, 1963- (1996).

Haruna, M. et. al. "Simultaneous measurement of the phase and group indices and the thickness of transparent plates by low-coherence interferometry," Opt. Lett. 23, 966- (1998).

Hecht, E., Optics, Third Edition, Addison-Wesley, Reading, MA, 1998.

Hirabayashi, A., Ogawa, H. and Kitagawa, K., "Fast surface profiler by white-light interferometry by use of a new algorithm based on sampling theory," Appl. Opt. 41, 4876- (2002).

Hirai, A. and Matsumoto, H., "Low-coherence tandem interferometer for measurement of group refractive index without knowledge of the thickness of the test sample," Opt. Lett. 28, 2112- (2003).

Hirai, A. and Matsumoto, H., "Measurement of group refractive index wavelength dependence using a low-coherence tandem interferometer," Appl. Opt. 45, 5614(2006).

Hopler, M. D. and Rogers, J. R., "Interferometric measurement of group and phase refractive index," Appl. Opt. 30, 735- (1991).

Hughes, J.V., “A new precision refractometer,” J. of Sci. Instruments 18, 234- (1941).

Larkin, K. G., "Efficient nonlinear algorithm for envelope detection in white light interferometry," J. Opt. Soc. Am. A 13, 832- (1996).

Lee B. S., and Strand T. C., "Profilometry with a coherence scanning microscope," Appl. Opt. 29, 3784- (1990). 


\section{REFERENCES - Continued}

Leica Mark II Abbe Refractometer photograph, ecomir-leica.com/refract/mark2.html, Accessed February 14, 2007.

Maruyama, H., et. al, "Low-coherence interferometer system for the simultaneous measurement of refractive index and thickness," Appl. Opt. 41, 1315- (2002).

Melles Griot, www.mellesgriot.com/glossary/wordlist/glossarydetails.asp?wID=367, Accessed February 20, 2007.

Milster, T. D. and Beaudry, N. A., "Coherence and Fringe Localization," Class notes, www.optics.arizona.edu/milster $/ 505 \% 20$ Lecture/Lecture $\% 20$ Notes $\% 20$ and $\% 20 \mathrm{Sl}$ ides/Chapter $\% 205 \% 20-\% 20$ Coherence/ch5 H H 20 basic $\% 20$ coherence \%20S2007.pdf, Accessed March 19, 2007.

Murphy, D. F. and Flavin, D. A., "Dispersion-insensitive measurement of thickness and group refractive index by low-coherence interferometry," Appl. Opt. 39, 4607(2000).

Park J. and Li, X., "Theoretical and numerical analysis of superluminescent diodes," J. Lightwave Technology 24, 2473- (2006).

Photometrics website, www.photomet.com/files/PDF/datasheets/cscf_mono.pdf, Accessed February 20, 2007.

Pixton, B. M. and Greivenkamp, J. E., "Automated Hilger-Chance refractometer for index measurement of liquids," in Optical Fabrication and Testing, OSA Technical Digest (Optical Society of America, 2006), paper OFMC7.

Polhemus, C., “Two-wavelength interferometry,” Appl. Opt. 12, 2071- (1973).

Research Electro-Optics, Inc (REO), www.reoinc.com/pdf/may2003/5_color_laser.pdf, Accessed February 20, 2007.

Schwider, J., et. al., "Digital wave-front measuring interferometry: some systematic error sources," Appl. Opt. 22, 3421- (1983).

Sears, F. W. Optics, Addison-Wesley, Reading, MA, 1949.

Seong, K. Private communication, February 14, 2007. 


\section{REFERENCES - Continued}

Sheppard, C. J. R., “Confocal imaging through weakly aberrating media,” Appl. Opt. 39, 6366- (2000).

Sheppard, C. J. R. and Gu, M., “Aberration compensation in confocal microscopy,” Appl. Opt. 30, 3563- (1991).

Shidlovski, V., SuperlumDiodes, Ltd. Personal correspondence via email, July 10, 2006.

Shidlovski, V., "Superluminsecent diodes: short overview of device operation principles and performance parameters," SuperlumDiodes Ltd. Online reference document (2004).

SuperlumDiodes Ltd., "SLD-26-MP: Medium power SLD at $680 \mathrm{~nm}$ ", www.superlumdiodes.com/pdf/26mp.pdf, Accessed February 19, 2007.

Synowicki, R. et. al. "Refractive index measurements of photoresist and antireflective coatings with variable angle spectroscopic ellipsometry," Proc. SPIE 3332, 384(1998).

Tighe, B. J., "Contact lens materials," in Contact Lenses, Fourth Edition, A. J. Phillips and L. Speedwell, eds., Butterworth-Heinermann, Oxford, UK, 1997.

Tilton, L. W. and Taylor, J. K., "Refractive index and dispersion of distilled water of visible radiation, at temperatures 0 to $60{ }^{\circ} \mathrm{C}$," J. of Research of National Bureau of Standards 20, 419- (1938).

Williby, G. A., “Transmitted Wavefront Testing of Complex Optics," PhD Dissertation, University of Arizona, 2003.

Woollam, J. et al. "Overview of variable angle spectroscopic ellipsometry (VASE), Part I: Basic theory and typical applications,” Proc. SPIE CR72, p. 3- (1999).

Wyant, J. C., class notes, www.optics.arizona.edu/jcwyant/Optics513/ChapterNotes/ Chapter02/QualOfOpticalMaterials.pdf, Accessed March 19, 2007.

Wyant, J. C., class notes, www.optics.arizona.edu/jcwyant/Optics513/ChapterNotes/ Chapter05/3.PrintedVersionPhaseShiftingInterferometry.pdf, Accessed March 19, 2007.

Wyant, J. C., “Tesing aspherics using two-wavelength holography,” Appl. Opt. 10, 2113 (1971). 


\section{REFERENCES - Continued}

Wyko NT1100 website, www.veeco.com/products/Metrology and Instrumentation/ Optical Profilers/Wyko NT1100, Accessed February 15, 2007.

Yoder, P.R., Opto-Mechanical Systems Design, Second Edition, Marcel Dekker Inc., New York, 1993. 